\title{
Introduction to RNAi and miRNA pathways
}

Petr Svoboda 


\section{KAROLINUM PRESS}

Karolinum Press is a publishing department of the Charles University www.karolinum.cz

C 2020 by Petr Svoboda

First edition

ISBN 978-80-246-4372-4 (pdf)

https://doi.org/10.14712/9788024643724 


\section{CONTENTS}

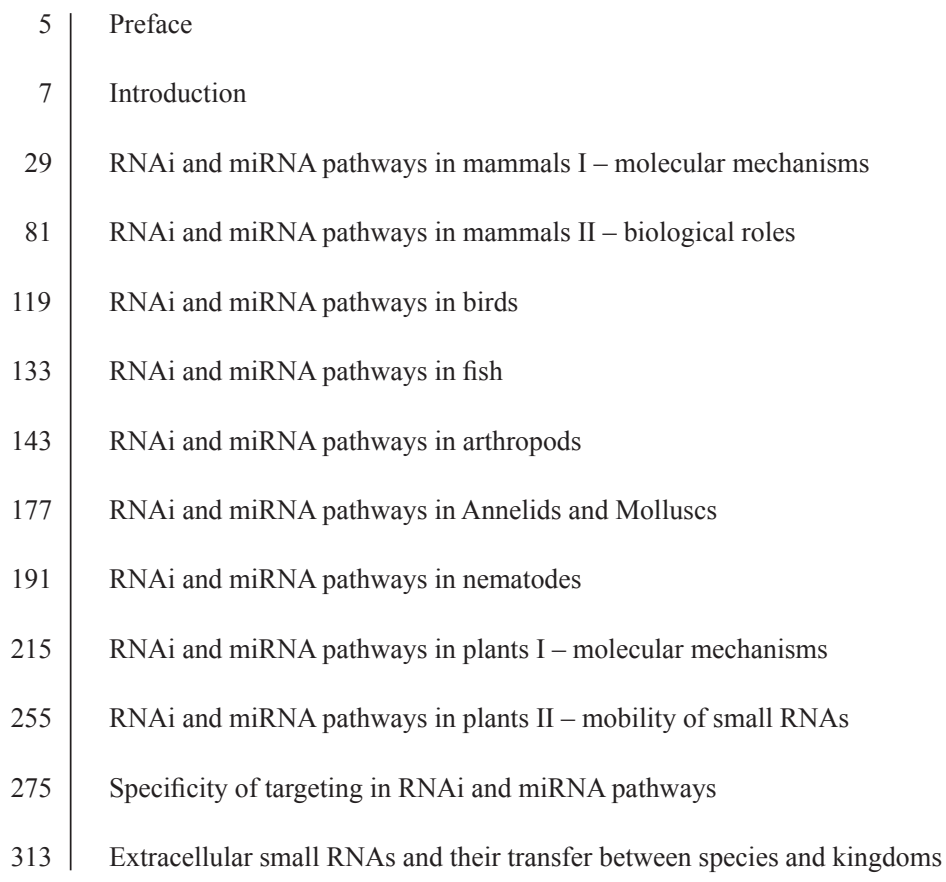





\section{PREFACE}

Small RNA pathways or RNA silencing is a group of pathways, which utilize small (20$30 \mathrm{nt}$ ) RNAs as guides for sequence-specific repression. This collection of texts on small RNA pathways originates from a report I prepared for the European Food and Safety Authority (EFSA) in 2016 and 2017. EFSA requested literature survey as it was concerned because of several works reporting that small RNAs, which naturally exist in plants, can enter into a mammalian body and affect gene expression. The main issue was that RNA interference (one of a small RNA pathways), represented a promising way for developing sequence-specific pesticides. If plant small RNAs could enter into a mammalian organism and regulate genes, RNAi-based pesticides could represent a potential health hazard.

With help of my colleagues, who set up a systematic literature search, I wrote an extensive systematic literature review for which I inspected $~ 10,000$ titles and abstracts during one year of writing. The original EFSA report had a number of technical sections describing search methodology and was compiling information from published works for specific tasks defined by EFSA. Once the report was completed and presented to EFSA, I became interested in converting the scientific part of the report into a more coherent overview of RNA silencing (particularly of RNAi and microRNA pathways) across different animal groups and plants. I thought it would be better if the scientific part would be revised, and provided as a coherent collection of chapters for studying RNAi and related pathways.

I subsequently contacted EFSA and discussed possible options for producing a set of chapters based on the report, which could serve as a study material for my lecturing. EFSA representatives agreed that I could produce a set of study materials from the report that would be published by a university publishing house under conditions that EFSA would be credited and the book would not be sold - as a solution, the material is provided as an open access collection of chapters. To satisfy the second EFSA requirement, I acknowledge hereby that the contents have been produced under a contract with EFSA (OC/EFSA/ GMO/2015/01-CT 01) and that the opinions expressed are those of the contractor only and do not represent EFSA's official position.

Regarding the conversion, the original text was reorganized into twelve chapters, which were reformatted and revised in order to remove copyrighted material from third parties and provide a introductory parts for stand alone chapters. Nine of the chapters focus on small RNA pathways in animals (mammals, birds, fish, arthropods, nematodes, molluscs, and annelids) and plants. The remaining three chapters include a general introduction and reviews of important phenomena - off-targeting and extracellular small RNAs. I hope that this collection will serve as a useful source for many.

\section{Petr Svoboda}





\title{
INTRODUCTION
}

Keywords: dsRNA, siRNA, miRNA, Dicer, TARBP2, PACT, Argonaute

\author{
PETR SVOBODA \\ Institute of Molecular Genetics, Academy of Sciences of the Czech Republic, \\ Videnska 1083, 14220 Prague 4, Czech Republic \\ Correspondence to: Petr Svoboda, Institute of Molecular Genetics ASCR, \\ Videnska 1083, 14220 Prague 4, Czech Republic, tel. \#+420 241063147, \\ e-mail: svobodap@img.cas.cz.
}

\begin{abstract}
RNA silencing denotes a group of pathways, which utilize small RNAs as sequence-specific guides for repressing gene expression. Two related RNA silencing pathways exist in animals and plants: RNA interference (RNAi) and microRNA (miRNA) pathway. While the miRNA pathway regulates endogenous protein-coding gene expression, RNAi serves as a form of innate immunity targeting viruses and mobile elements, although it occasionally also acquired function in protein-coding gene regulation. The aim of the following text is to provide an elementary introduction into RNAi and miRNA pathways for a series of taxon-specific and feature-specific reviews, which follow. The idea is to bring up common general principles allowing the reader to better navigate through common and derived mechanisms and functions of RNA silencing that are presented in taxon-oriented reviews. The entire review series was derived from an expert report for the European Food and Safety Agency, which was reorganized to be more accessible for the scientific community.
\end{abstract}

\section{Introduction to mechanistic principles and roles of RNA silencing}

The volume of the RNA silencing and double-stranded RNA (dsRNA)-related published data is stunning. In 2016, me and my colleagues did literature assessment for the European Food and Safety Agency, which identified over 200000 publications (Paces et al., 2017) and which served as a foundation for this article series. While selected taxons are reviewed separately, I thought the collection would benefit from introducing the core molecular mechanisms of RNAi and miRNA pathways (admittedly animal-centric).

As mentioned in the abstract, RNA silencing (reviewed in Ketting, 2011) designates repression guided by small RNA molecules (20-30 nucleotides long) and includes diverse silencing mechanisms including RNA degradation, translational repression, induction of repressive chromatin, and even DNA deletions. RNA silencing research evolved from parallel studies in several different model systems, primarily flowering plant models and animal 


\section{RNAi}

viruses, convergent transcription, inverted

repeat transcription, RdRp activity, artificial

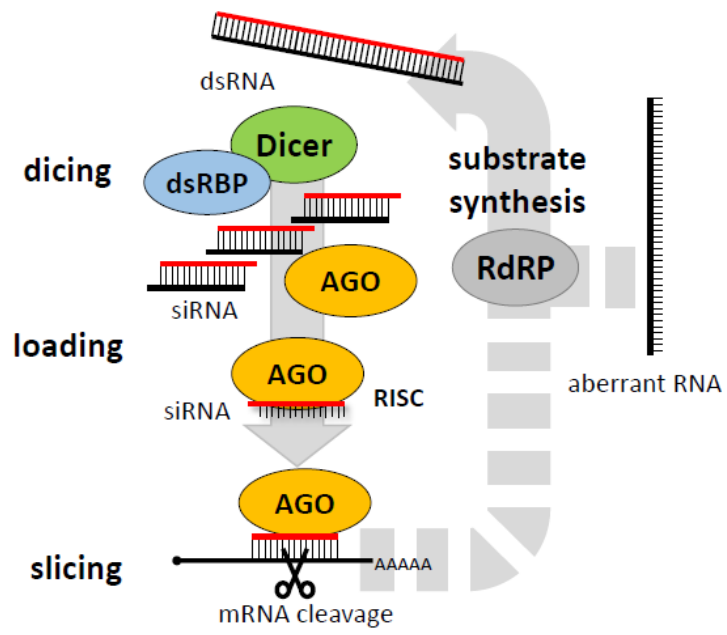

Figure 1 RNAi pathway overview

Canonical RNAi is triggered by some form of long dsRNA. dsRNA can originate from various sources including viruses and their replication intermediates or base pairing if RNAs transcribed in the genome (either as an intramolecular duplex (hairpin dsRNA), or by base pairing RNAs transcribed in cis (convergent transcription) or in trans (from interspersed elements, pseudogenes etc.). The core mechanism of RNAi has three steps: dicing - cleavage of long dsRNA into siRNA duplexes by RNase III Dicer, loading - where one strand of siRNA duplex is selected and loaded onto an Argonaute protein from AGO subfamily forming the RNA-induced silencing complex (RISC), and slicing - where siRNA guides RISC to cognate RNAs. Upon making a perfect duplex with a cognate RNA, AGO proteins performs endonucleolytic cleavage of the cognate RNA in the middle of the base-paired sequence. In some species, RNAi also involves an RNA-dependent RNA polymerase (RdRp), which may generate initial substrates or participate in amplification of the response by converting cognate RNAs into dsRNA.

models including Caenorhabditis elegans, Drosophila melanogaster, zebrafish, mouse, and humans. Some form of RNA silencing exists in almost every eukaryote. Here, the primary focus will be on the RNA interference (RNAi) and microRNA (miRNA) pathways (Fig. 1 and 2). The term RNAi has been originally used for sequence-specific mRNA degradation induced by long dsRNA (Fire et al., 1998). This mechanism, which employs small RNAs produced from long dsRNA, is the canonical RNAi. However, the term RNAi is also used as a common name for a broad range of RNA silencing pathways (Ketting, 2011). Here, I will use the term RNAi strictly in its original connotation. miRNAs are genome-encoded short RNAs that regulate gene expression by translational repression and/or degradation of cognate mRNAs.

Historically, the first discovered RNA silencing pathway was plant co-suppression, which appeared as sequence-specific silencing of endogenous genes induced by transgene expression (Napoli et al., 1990). The miRNA pathway was first found in 1993 in 


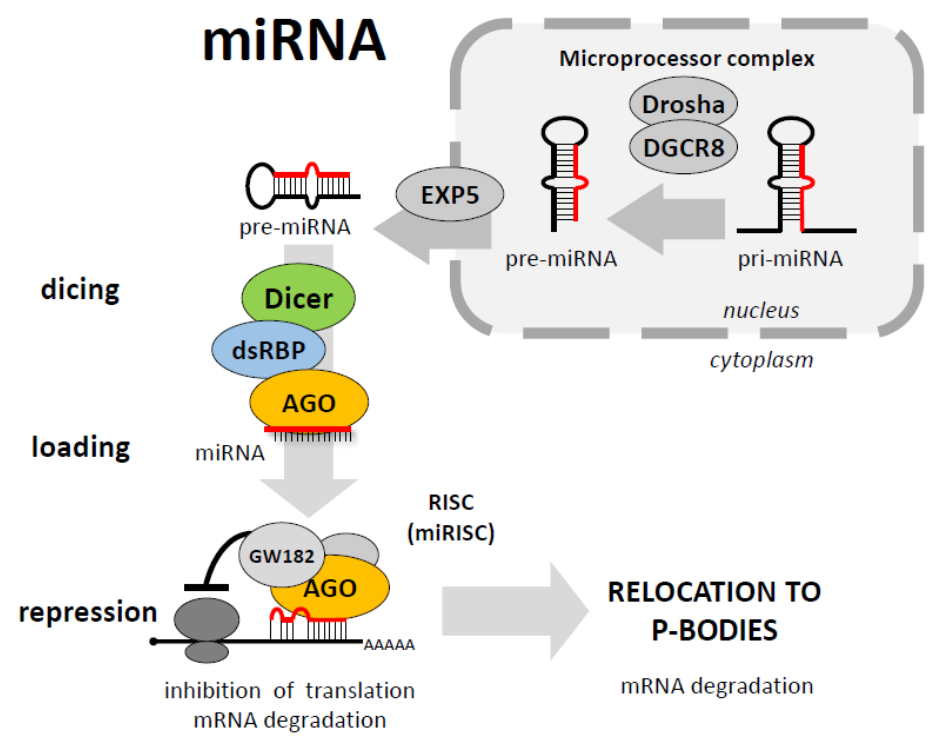

Figure 2 Canonical animal miRNA pathway overview

miRNAs are genome-encoded. Their synthesis starts with Pol II-mediated transcription of long primary miRNA transcripts (pri-miRNAs), which carry one or more local short hairpins, which are released as precursor miRNAs (pre-miRNAs) by the activity of the nuclear "Microprocessor complex". Pre-miRNAs are transported into the cytoplasm via Exportin 5. In the cytoplasm, Dicer cleaves a pre-miRNA and one strand of the duplex is loaded onto an AGO protein, which forms the core of the effector complex (RISC or miRISC). The effector complex contains additional proteins, which mediate translational repression and RNA degradation. The key bridge between AGO and proteins mediating deadenylation and decapping is GW182 protein. Targeted mRNAs usually localize to P-bodies, which are cytoplasmic foci associated with RNA metabolism

the nematode Caenorhabditis elegans (Lee et al., 1993). The idea of a conserved miRNA pathway emerged upon discovery of Let-7 miRNA in 2000, which was is conserved from Caenorhabditis elegans to mammals (Pasquinelli et al., 2000). In the meantime, RNAi was found in Caenorhabditis as well (Fire et al., 1998). Around the year 2000, it became apparent that earlier observations, which included the aforementioned plant co-suppression, quelling in fungi, and animal RNAi and miRNA pathways (Lee et al., 1993; Napoli et al., 1990; Romano and Macino, 1992; van der Krol et al., 1990), belong to one group of related molecular mechanisms commonly called RNA silencing.

The core principle of RNA silencing (repression mediated by a ribonucleoprotein complex guided by a small RNA) was deciphered during 1998-2004 using a combination of genetic and biochemical approaches. Key steps in understanding how RNA silencing works were biochemical studies in Drosophila embryo lysates (Tuschl et al., 1999; Zamore et al., 2000) and genetic studies in Caenorhabditis elegans and plants (e.g. (Bohmert et al., 1998; Dalmay et al., 2000; Fagard et al., 2000; Grishok et al., 2000; Lynn et al., 1999; Mourrain et al., 2000; Smardon et al., 2000; Tabara et al., 1999)). The last discovery, which arguably closed the era of deciphering the key principles of RNA silencing, was the structural 


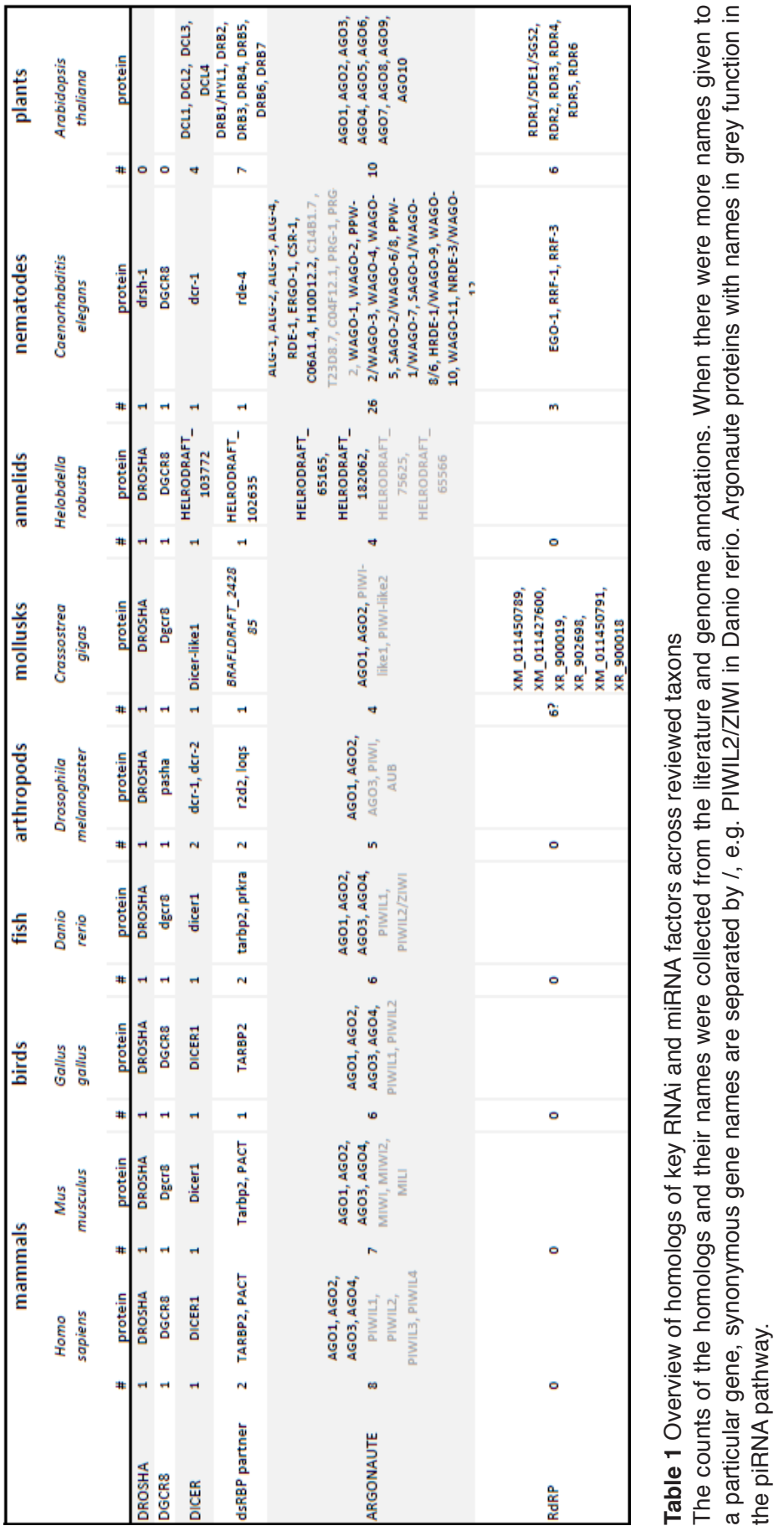


analysis of Argonaute 2, which revealed the last missing piece of the big puzzle: this protein carries the endonucleolytic activity executing (slicing) targeted mRNAs (Liu et al., 2004; Meister et al., 2004; Song et al., 2004).

\section{RNAi and miRNA pathway components}

\section{Substrates}

RNA silencing employs different types of substrates, which give rise to different kinds of small RNA populations (Fig. 3). Canonical precursors of miRNAs contain short hairpin structures, which are released by the Microprocessor complex and processed by Dicer in the cytoplasm (reviewed in Kim et al., 2009; Winter et al., 2009). However, additional substrates can produce non-canonical miRNAs, which can be generated in Drosha- and/or Dicer-independent manner. Long dsRNA substrates for RNAi may come in different forms; viral replication (and RdRP activities in general) often yields blunt-end dsRNA whereas hybridization of sense and antisense RNAs yields dsRNA with single-strand overhangs. Sense and antisense transcripts can be produced in cis by convergent transcription or in trans at separate loci. Another type of dsRNA are intramolecular duplexes of dsRNA hairpins, which form upon transcription of inverted repeats. The most efficient long dsRNA formation comes from RdRP synthesis followed by efficient forming intramolecular duplexes. Sense and antisense base pairing is, in principle, less likely to occur in cis, especially when sense and antisense RNAs are produced at distant loci. The reason is that RNAs are usually forming intramolecular secondary structures and are bound by RNA binding proteins, which may reduce probability of base pairing of complementary RNAs.

\section{Dicer - structure and function}

Dicer is the central enzyme for producing small RNAs in miRNA and RNAi pathways. It was discovered in 2001 as the enzyme processing long dsRNA into siRNAs (Bernstein et al., 2001). Dicer generates small RNAs in RNAi and many other (but not all) RNA silencing pathways (reviewed for example in Jaskiewicz and Filipowicz, 2008). Dicer is a large ( $200 \mathrm{kDa})$, multi-domain RNase III endonuclease cleaving various forms of duplex dsRNA. It carries two RNase III domains and several other domains that are typically found in Dicer-like proteins in eukaryotes (Fig. 4). These include an N-terminal helicase domain, piwi/Argonaute/zwille (PAZ) domain, domain of unknown function DUF283, and a C-terminal dsRNA binding domain (dsRBD, duplicated in some plant Dicers).

The ribonuclease activity of Dicer requires magnesium ions. Dicer preferentially cleaves dsRNA at the termini but it can also cleave internally with low efficiency (Provost et al., 2002; Zhang et al., 2002). Cleavage produces small (21-27 nt long) RNA duplexes with two nucleotide 3' overhangs and 5' monophosphate and 3' hydroxyl groups at RNA termini. Dicer structure sets the length of the substrate from the PAZ domain to RNase III domains where it is cleaved. The PAZ domain binds the end of dsRNA, with high affinity to 3 ' protruding overhangs (Lingel et al., 2003; Ma et al., 2004; Song et al., 2003; Yan et al., 2003); 


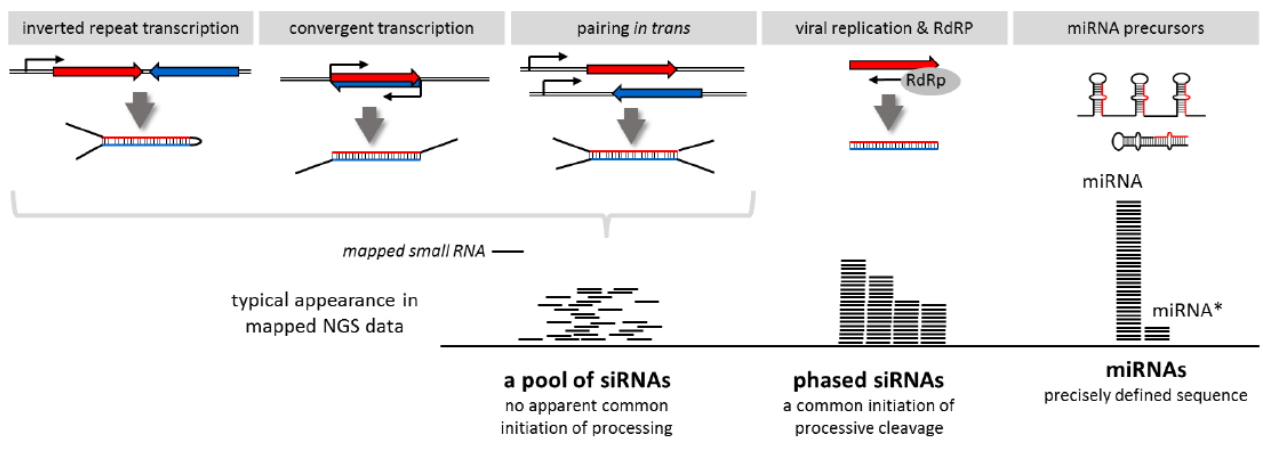

Figure 3 Substrate types in RNA silencing pathways.

The lower part schematically depicts typical appearance of small RNAs in mapped RNA-sequencing data.

these termini are typical for canonical miRNA precursors and processive cleavage of long dsRNA. RNase IIIa and IIIb domains form a single processing center containing two catalytic „half-sites”, each cleaving one strand of the duplex and producing short dsRNA with 2 nt 3' overhang. RNase IIIa domain is processing the protruding 3'-OH-bearing strand and RNase IIIb cuts the opposite 5'-phosphate-containing strand (Zhang et al., 2004).

The crystal structure of the full length Dicer from Giardia intestinalis, which represents a minimal Dicer model (MacRae et al., 2007; MacRae et al., 2006), showed that the RNase III domains form a catalytic center connected with the PAZ domain by a long $\alpha$-helix (,connector“ helix), which is implicated in determining the product length. The connector helix is supported by a platform-like structure containing the DUF283 domain, which has a dsRBD-like fold (Dlakic, 2006) and perhaps mediates protein-protein interaction (Qin et al., 2010). Full-length Dicer proteins from animals and plants were not crystalized. However, recent advances in cryo-electron microscopy (cryo-EM) provide additional insights into Dicer structure and function in other models (Fig. 4), which utilize more complex Dicer proteins. These results will be summarized in corresponding taxon-specific reviews.

Some organisms, like mammals, Caenorhabditis, or Trypanosoma, utilize a single Dicer protein to produce both siRNAs and miRNAs. In contrast, Drosophila utilizes two Dicer paralogs, DCR-1 to produce miRNAs and DCR-2 to produce siRNAs. Some species utilize even more paralogs with distinct functions and different cleavage product lengths (e.g. four Dicer paralogs in Arabidopsis thaliana (reviewed in Meins et al., 2005). Animal Dicer proteins are typically found in the cytoplasm. In plants, some Dicer proteins have a dedicated nuclear localization.

\section{dsRNA binding proteins (dsRBPs)}

Dicer interacts with many proteins of which two protein types stand out: (I) Argonaute proteins, which receive small RNAs produced by Dicer, and (II) dsRNA-binding proteins 


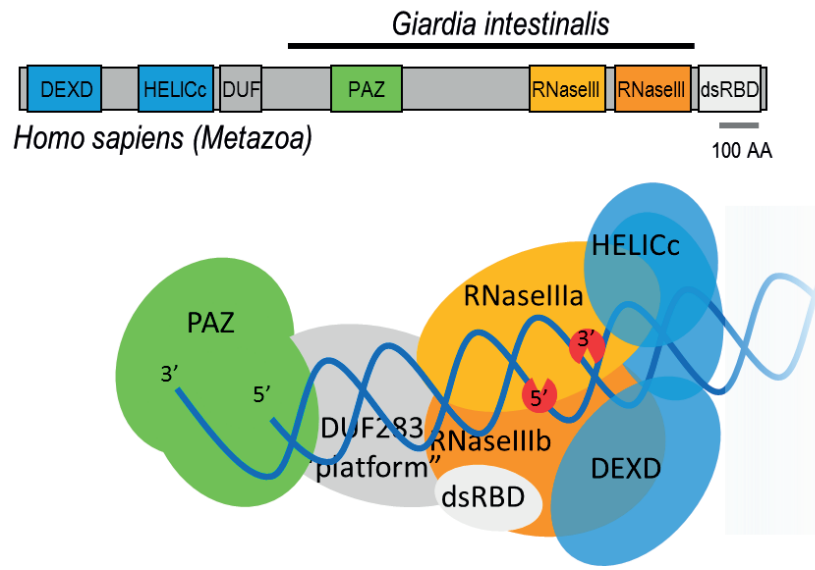

Figure 4 Dicer structure

The first Dicer structure was inferred from the crystal structure of Giardia intestinalis, which corresponds to a fragment of larger metazoan Dicer proteins. The schematic structure of human Dicer was subsequently derived from cryo-electron microscopy analysis. Dicers typically cleave dsRNA from an end, which are bound by the PAZ domain. Each of the RNase III domains cleaves one strand at a defined distance from the PAZ domain.

(dsRBPs) with tandemly arrayed dsRBDs. dsRBPs facilitate substrate recognition, cleavage fidelity, and Argonaute loading. However, despite a similar domain organization, these proteins evolved distinct roles in small RNA biogenesis by Dicer and sorting onto Argonautes in different model organisms. These roles will be discussed for each taxon separately.

\section{Argonaute proteins - structure and function}

Argonaute proteins have a molecular weight of $\sim 100 \mathrm{kDa}$ and carry four distinct domains: the central PAZ domain, the C-terminal PIWI (P-element induced wimpy testis), the N-terminal domain, and the MID domain between PAZ and PIWI domains (Fig. 5). The PAZ domain binds the 3 ' end of a short RNA in a sequence-independent manner (Lingel et al., 2003, 2004; Ma et al., 2004; Song et al., 2003). Structural studies of archaeal Argonaute homologs showed that the PIWI domain has an RNase H-like fold (Ma et al., 2005; Parker et al., 2004; Song et al., 2004; Yuan et al., 2005). A small RNA is anchored with its 3' end in the PAZ domain. The 5' phosphate of the small RNA is buried in a pocket at the interface between the MID domain and the PIWI domain (reviewed in Jinek and Doudna, 2009). The 5 ' end of the base pairing cognate RNA enters between the N-terminal and PAZ domains and its 3' end exits between the PAZ and MID domains.

Argonaute proteins can be divided into three distinct groups (reviewed in Faehnle and Joshua-Tor, 2007): (1) AGO proteins, found in all kingdoms, (2) PIWI proteins found in animals, and (3) WAGO proteins found only in nematodes. The WAGO subfamily was described last, so it is not recognized in the older literature, which typically divides 

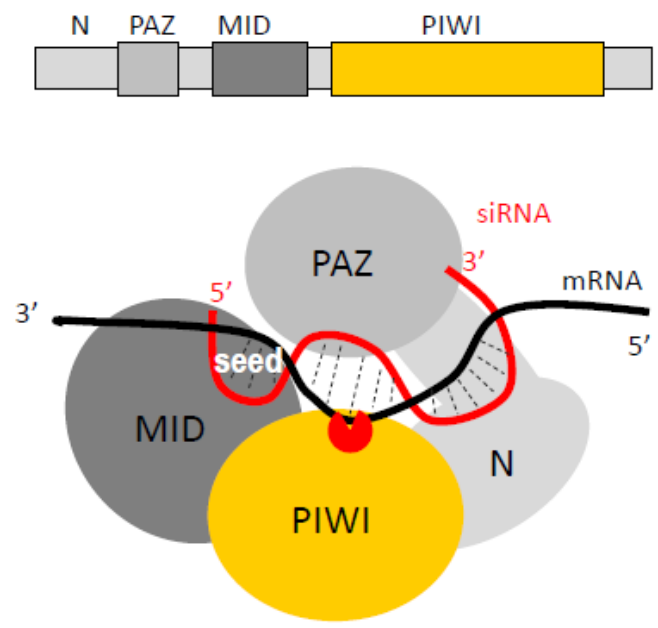

Figure 5 Argonaute protein structure

The order of domains in an Argonaute protein. The scheme shows how Argonaute cleaves a perfectly complementary RNA, which becomes accessible by the catalytical center in the PIWI domain upon base pairing with a small RNA. Nucleotides 2-8 of the small RNA initiate the interaction with the cognate RNA and form the so-called "seed", which has a highly predictive value for miRNA binding sites and siRNA off-targeting.

Argonaute proteins into AGO and PIWI subgroups (e.g. Carmell et al., 2002). Metazoan Argonaute proteins functioning in the RNAi pathway include RDE-1 (exogenous RNAi) and ERGO-1 (endogenous RNAi) in Caenorhabditis elegans, AGO-2 in Drosophila, and AGO2 in mammals. Other Argonautes act in the miRNA and other pathways employing small RNAs.

In RNAi, one strand of a siRNA produced by Dicer serves as a sequence-specific guide in RNA-induced silencing complex (RISC), which is the effector complex of RNAi. The key component of RISC is an Argonaute family protein (AGO), which binds the selected siRNA strand and uses it as a sequence-specific guide recognizing mRNAs that will be degraded. Argonaute is the „slicer” (Liu et al., 2004; Meister et al., 2004; Song et al., 2004), i.e. the enzyme catalyzing the cleavage of the cognate mRNA in the canonical RNAi pathway (Fig. 1). The active site in the PIWI domain is positioned such that it cleaves the mRNA opposite the middle of the siRNA guide (Song et al., 2004). However, only some Argonautes function as slicers. In other cases, silencing is mediated by additional proteins forming a complex with an Argonaute. There is a long list of Argonaute-interacting protein factors; they will be described separately in the taxon-dedicated reviews.

While the minimal active RISC contains only the ,slicing“ Argonaute protein and the guide strand of siRNA, RISC activity was found in different models and cell types to reside in $\sim 200 \mathrm{kDa}, \sim 500 \mathrm{kDa}$, or 80S complexes (Martinez et al., 2002; Mourelatos et al., 2002; Nykanen et al., 2001; Pham et al., 2004). Multiple proteins either contribute to RISC formation or might regulate RISC activity, stability, target selection, mode of repression or otherwise contribute to RISC function. 


\section{RNA-dependent RNA polymerase (RdRPs) proteins}

RdRPs can contribute to RNA silencing in two ways - either by converting single-stranded RNA to dsRNA (Fig. 1) or by synthesizing short RNAs, which could function as guides. RdRP is an ancestral component of RNA silencing since RdRP orthologs were identified in RNA silencing pathways in plants, fungi and some animals: QDE-1 in Neurospora crassa (Cogoni and Macino, 1999), EGO-1 and RRF-1 in Caenorhabditis elegans (Grishok et al., 2001; Smardon et al., 2000), SDE1/SGS2 in Arabidopsis (Dalmay et al., 2001; Mourrain et al., 2000), and Rdp1 in Schizosaccharomyces pombe (Hall et al., 2002; Volpe et al., 2002). Homologs of these RdRPs exist in numerous metazoan taxons, including Nematoda (e.g. Caenorhabditis elegans), Cnidaria (hydra), Chelicerata (tick), Hemichordata (acorn worm), Urochordata (sea squirt) but appear absent in others, including Platyhelminthes (planaria), Hexapoda (Drosophila), or Craniata (vertebrates). Phylogenetic analysis suggests that RdRPs in RNA silencing pathways have a monophyletic origin, i.e. evolved from a single ancestral RdRP (Cerutti and Casas-Mollano, 2006; Murphy et al., 2008). The fact that RdRP orthologs are found in other protostomes and deuterostomes but not in Drosophila or mammals suggests a repeated loss of the ancestral RdRP component of RNA silencing. Whether RdRP activity completely disappeared from RNAi in Drosophila and mammals is unclear but transitive RNAi generating secondary sequences upstream of the region targeted by siRNAs was not observed in Drosophila or mouse (Roignant et al., 2003; Schwarz et al., 2002; Stein et al., 2003).

\section{Diversity of RNAi and miRNA pathway functions}

Some taxons, exemplified by vertebrates, utilize a relatively simple setup of RNA silencing pathways, which is also reflected by the low numbers of homologs of the key genes, listed in the Table 1. Other pathways diversified, often at the level of Argonaute protein adaptation for different pathways (Table 2). In mammals, the dominant somatic RNA silencing pathway in vertebrates is the miRNA pathway, which employs a single Dicer, one or two associated dsRBPs, and four AGO proteins, one of which retains the slicing activity. There is no protein uniquely dedicated to RNAi and no RdRP. The other three Argonaute proteins in vertebrates function in the piRNA pathway, present in the germline. This simple setup contrasts with that in Caenorhabditis elegans where 26 Argonaute proteins and three RdRPs exist, as well as with that in A. thaliana where there are four Dicer proteins, seven dsRBPs, ten Argonaute proteins and six RdRPs. Consequently, nematodes and plants have highly complex RNA silencing system adapted to many different biological roles.

\section{RNAi pathway}

The RNAi pathway (Fig. 1) has three mains steps: (1) the cleavage of long dsRNA by Dicer into siRNAs, (2) loading of small RNAs on the RISC, and (3) recognition and cleavage of cognate RNAs by the RISC. In addition to this core pathway, two extensions of the pathway, which are restricted to some animal species, should be mentioned: (1) an amplification 


\begin{tabular}{|c|c|c|c|c|c|c|c|c|}
\hline \multirow[t]{2}{*}{ model organism } & \multirow[t]{2}{*}{ common name } & \multirow[t]{2}{*}{ subfamily } & \multirow[t]{2}{*}{ slicer } & \multicolumn{5}{|c|}{ associated small RNA } \\
\hline & & & & type & length & $5^{\prime} \mathrm{nt}$ & $5^{\prime}$ end & $3^{\prime}$ end \\
\hline \multirow{8}{*}{ Homo sapiens } & AG01 & AGO & - & miRNA, siRNA & $21-23$ & $\mathrm{~N}(\mathrm{U})$ & mono-P & $-\mathrm{OH}$ \\
\hline & $\mathrm{AGO2}$ & AGO & + & miRNA, siRNA & $21-23$ & $\mathrm{~N}(\mathrm{U})$ & mono-P & $-\mathrm{OH}$ \\
\hline & AGO3 & AGO & - & miRNA, siRNA & $21-23$ & $N(U)$ & mono-P & $-\mathrm{OH}$ \\
\hline & AGO4 & AGO & - & miRNA, siRNA & $21-23$ & $N(U)$ & mono-P & $-\mathrm{OH}$ \\
\hline & PIWLL1 & PIWI & + & piRNA & $29-31$ & $\mathrm{~N}$ & mono-P & 2'-0-met \\
\hline & PIWLL2 & PIWI & + & piRNA & $24-28$ & U & mono-P & 2'-0-me \\
\hline & PIWLI3 & PIWI & + & PiRNA & $? ?$ & $? ?$ & mono-P & 2'-O-met \\
\hline & PIWIL4 & PIWI & + & PiRNA & $27-29$ & $u$ & mono-P & 2'-0-me \\
\hline \multirow{7}{*}{ Mus musculus } & AGO1 & AGO & - & miRNA, siRNA & $21-23$ & $N(U)$ & mono-P & $-\mathrm{OH}$ \\
\hline & $\mathrm{AGO} 2$ & AGO & + & miRNA, siRNA & $21-23$ & $N(U)$ & mono-P & $-\mathrm{OH}$ \\
\hline & AGO3 & AGO & - & miRNA, siRNA & $21-23$ & $N(U)$ & mono-P & $-\mathrm{OH}$ \\
\hline & AGO4 & AGO & - & miRNA, siRNA & $21-23$ & $N(U)$ & mono-P & $-\mathrm{OH}$ \\
\hline & MIWI (PIWIL1) & PIWI & + & PiRNA & $29-31$ & $\mathrm{~N}$ & mono-P & 2'-0-me \\
\hline & MIWI2 (PIWIL) & PIWI & + & PIRNA & $27-29$ & $\mathbf{u}$ & mono-P & 2'-0-me \\
\hline & MIL (PIWIL2) & PIWI & + & PiRNA & $24-28$ & u & mono-P & 2'-O-met \\
\hline \multirow{5}{*}{$\begin{array}{l}\text { Drosophila } \\
\text { melanogaster }\end{array}$} & AGO1 & AGO & $(+)$ & miRNA & $21-23$ & $N(U)$ & mono-P & $-\mathrm{OH}$ \\
\hline & AGO2 & AGO & + & SiRNA & $\sim 21$ & $\mathrm{~N}$ & mono-P & $-\mathrm{OH}$ \\
\hline & AGO3 & PIWI & + & PiRNA & $24-27$ & $\mathrm{~N}$ & mono-P & 2'-O-met \\
\hline & PIW & PIWI & + & piRNA & $24-29$ & $u$ & mono-P & 2'-O-met \\
\hline & AUB & PIWI & + & piRNA & $23-27$ & u & mono-p & 2'-O-met \\
\hline \multirow{26}{*}{$\begin{array}{c}\text { Caenorhabditis } \\
\text { elegans }\end{array}$} & ALG-1, F48F7.1 & AGO & + & MiRNA & $22-23$ & & mono-P & $-\mathrm{OH}$ \\
\hline & ALG-2, unknown & AGO & + & miRNA & $22-23$ & & mono-p & $-\mathrm{OH}$ \\
\hline & ALG-3, T22B3.2 & AGO & $+?$ & $26 G$ siRNA & 26 & G & mono-P & $-\mathrm{OH}$ \\
\hline & ALG-4, ZK757.3 & AGO & $+?$ & $26 G$ siRNA & 26 & G & mono-p & $-\mathrm{OH}$ \\
\hline & RDE-1, K08H10.7 & AGO & + & primary siRNA & $22-23$ & & mono-P & $-\mathrm{OH}$ \\
\hline & ERGO-1, R09A1.1 & AGO & + & $26 \mathrm{G}$ siRNA & 26 & G & mono-P & 2'-O-met \\
\hline & CSR-1, F20D12.1 & AGO & + & $22 \mathrm{G}$ siRNA & 22 & G & tri-p & $-\mathrm{OH}$ \\
\hline & C06A1.4 & AGO? & - & & & & & \\
\hline & H10D12.2, M03D4.6 & AGO? & - & & & & & \\
\hline & $\mathrm{C} 14 \mathrm{~B} 1.7$ & PIWI? & - & & & & & \\
\hline & T23D8.7, HPO-24 & PIWI? & - & & & & & \\
\hline & C04F 12.1 & PIWI? & $+?$ & & & & & \\
\hline & PRG-1, D2030.6 & PIWI & + & $21 U$ piRNA & 21 & u & mono-P & 2'-0-mer \\
\hline & PRG-2, C01G5.2 & PIWI & + & 210 piRNA & 21 & $u$ & & \\
\hline & WAGO-1, R06C7.1 & WAGO branch 1 & - & $22 \mathrm{G}$ siRNA & 22 & G & tri-P & $-\mathrm{OH}$ \\
\hline & WAGO-2, F55A12.1 & WAGO branch 1 & - & $22 \mathrm{G}$ siRNA & 22 & G & tri-P & $-\mathrm{OH}$ \\
\hline & PPW-2, WAGO-3, Y110A7A.18 & WAGO branch 1 & - & $22 \mathrm{G}$ siRNA & 22 & G & tri-P & $-\mathrm{OH}$ \\
\hline & WAGO-4, F58G1.1 & WAGO branch 1 & - & $22 \mathrm{G}$ siRNA & 22 & G & tri-P & $-\mathrm{OH}$ \\
\hline & WAGO-5, ZK1248.7 & WAGO branch 1 & - & $22 \mathrm{G}$ siRNA & 22 & G & tri-P & $-\mathrm{OH}$ \\
\hline & SAGO-2, WAGO-6/8, F56A6.1 & WAGO branch 2 & - & $22 \mathrm{G}$ siRNA & 22 & G & tri-P & $-\mathrm{OH}$ \\
\hline & PPW-1, WAGO-7, C18E3.7 & WAGO branch 2 & - & $22 \mathrm{G}$ SiRNA & 22 & G & tri-P & $-\mathrm{OH}$ \\
\hline & SAGO-1, WAGO-8/6, K12B6.1 & WAGO branch 2 & - & $22 \mathrm{G}$ siRNA & 22 & G & tri-P & $-\mathrm{OH}$ \\
\hline & HRDE-1, WAGO-9, C16C10.3 & WAGO branch 3 & - & $22 \mathrm{G}$ siRNA & 22 & G & tri-P & $-\mathrm{OH}$ \\
\hline & WAGO- 10, T22 H9.3 & WAGO branch 3 & - & $22 \mathrm{G}$ siRNA & 22 & G & tri-P & $-\mathrm{OH}$ \\
\hline & WAGO-11, Y49F6A.1 & WAGO branch 3 & - & $22 \mathrm{G}$ siRNA & 22 & G & tri-P & $-\mathrm{OH}$ \\
\hline & NRDE-3, WAGO-12, R04A9.2 & WAGO branch 3 & - & $22 \mathrm{G}$ siRNA & 22 & G & tri-p & $-\mathrm{OH}$ \\
\hline \multirow{10}{*}{$\begin{array}{c}\text { Arabidopsis } \\
\text { thaliona }\end{array}$} & AGO1 & plant AGO clade I & + & MiRNA & 21 & U & mono-P & 2'-O-met \\
\hline & $\mathrm{AGO} 2$ & plant AGO clade II & + & miRNAs, tasiRNAs, rasiRNAs & 21 & A & mono-p & 2'-O-met \\
\hline & AGO3 & plant AGO clade II & + & SiRNAs & 24 & A & mono-P & 2'-O-met \\
\hline & AGO4 & plant AGO clade III & + & intergentic siRNAs, rasiRNAs & $23-24$ & A & mono-P & 2'-O-met \\
\hline & AGO5 & plant AGO clade I & + & intergenic siRNA & $21,22,24$ & $c$ & mono-p & $2^{1}$-O-met \\
\hline & AG06 & plant AGO clade III & + & siRNAs & 24 & A & mono-P & 2'-O-met \\
\hline & AGO7 & plant AGO clade II & + & miRNA (miR390), ta-siRNA & 21 & A & mono-P & 2'-O-met \\
\hline & AGOB & plant AGO clade III & $(+)$ & - & - & - & - & - \\
\hline & AGO9 & plant AGO clade III & $(+)$ & rasiRnAs & 24 & A & mono-P & 2'-0-met \\
\hline & AGO10 & plant AGO clade I & + & miRNA (mir165/166) & 21 & $\mathrm{u}$ & mono-P & 2'-0-mer \\
\hline
\end{tabular}

Table 2 Overview of Argonaute proteins and associated RNAs in key model organisms

The table was compiled form the following literature (Batista et al., 2008; Buckley et al., 2012; Das et al., 2008; Duran-Figueroa and Vielle-Calzada, 2010; Fischer et al., 2011; Forstemann et al., 2007; Iwasaki et al., 2015; Liu et al., 2009; Tijsterman et al., 2002a; Tijsterman et al., 2002b; Vasale et al., 2010; Vourekas et al., 2012; Wang and Reinke, 2008; Yigit et al., 2006; Zhang et al., 2016; Zheng et al., 2007). Slicer activity "+" indicates that a given Argonaute protein has potential to act as a slicer, not that slicing is its primary mode of action. In some case, slicing potential has been inferred from the sequence, i.e. it is not supported with experimental evidence. 
step, in which RdRPs generate secondary siRNAs and (2) systemic RNAi where an RNAi response can spread across cellular boundaries.

Because dsRNA often originates from viruses, the role of RNAi has been viewed as a form of native immunity. While this role is experimentally supported in some models, RNAi may also have other roles in maintaining genome integrity, and control of gene expression.

RNA viruses generate dsRNA during their replication cycle in host cells. DNA viruses often produce complementary sense and antisense transcripts, which can form dsRNA upon annealing. Thus, dsRNA is a common marker of viral infection and it is recognized by different mechanisms mediating an innate immune response. The idea that RNA silencing may function as a form of innate immunity is supported by several lines of evidence, which were first found in plants and later also in invertebrates (reviewed in Marques and Carthew, 2007; Xie and Guo, 2006): 1) siRNAs derived from viral sequences were found in infected organism (Hamilton and Baulcombe, 1999), 2) inhibition of RNA silencing resulted in increased viral replication (Mourrain et al., 2000), and 3) some viruses produce suppressors of RNA silencing (Voinnet et al., 1999).

The role of RNAi varies among different organisms. Vertebrates replaced the antiviral defense system provided by RNAi by an array of innate immune sensors of dsRNA molecules, whose activation converges on a sequence-independent interferon response. Thus, the canonical RNAi is generally not a ubiquitous primary mechanism in response to dsRNA in vertebrates although it is observed in specific cases. In species, which still use RNAi as the primary antiviral immunity pathway (such as plants, nematodes, arthropods), it is frequently observed that viruses overcome the RNAi response with various protein inhibitors. Whether the more complex interferon system in vertebrates provides a stronger defense barrier is unclear as the interferon pathway is just a part of a highly complex immune system. One interesting aspect of RNAi and interferon response evolution is the rewiring of the RIG-I helicase family, which is associated with RNAi in Caenorhabditis elegans and interferon response in mammals. Understanding the role of the RNAi module in immunity of molluscs and annelids requires further research. Molluscs are a particularly interesting case, because their genome carries homologs of the genes involved in the interferon response, and studying them might provide an insight into how the interferon response has replaced RNAi, as the main antiviral response.

\section{Systemic and environmental RNAi}

RNAi can either act in a cell autonomous manner, i.e. affecting only cells directly exposed to dsRNA, or can propagate across cell boundaries. Two modes of non-cell autonomous RNAi are recognized: (1) environmental RNAi involves processes where dsRNA is taken up by a cell from the environment. (2) systemic RNAi includes processes where a silencing signal spreads from a cell across cellular boundaries into other cells. Both modes can be combined and systemic RNAi can follow environmental RNAi. Two pathways for dsRNA uptake were described: (1) a specific transmembrane channel-mediated uptake and (2) an alternative endocytosis-mediated uptake (reviewed in Huvenne and Smagghe, 2010; Whangbo and Hunter, 2008). 
The non-cell autonomous RNAi was observed already during the first RNAi experiments in Caenorhabditis elegans (Fire et al., 1998). When animals were microinjected with dsRNA into head, tail, intestine or gonad arm, or even just soaked in dsRNA solution or fed by bacteria expressing dsRNA, these treatments induced a specific null phenotype in the whole animal and even in its progeny, demonstrating a surprising ability of dsRNA to cross cellular boundaries (Fire et al., 1998; Tabara et al., 1998; Timmons and Fire, 1998). Noncell autonomous RNAi has been discovered also in parasitic nematodes (Geldhof et al., 2007), hydra (Chera et al., 2006), planaria (Newmark et al., 2003; Orii et al., 2003), insects (Tomoyasu et al., 2008; Xu and Han, 2008), or plants (Himber et al., 2003).

\section{miRNA pathway}

Unlike siRNAs, miRNAs are genome-encoded short RNAs with defined sequences that regulate gene expression by mediating translational repression and/or degradation of cognate mRNAs. miRNAs play important roles in many processes and are one of the most common small RNAs found in animal and plant cells. miRNAs have been implicated in countless cellular and developmental processes; in some cases are changes in their expression linked to pathological conditions. Bioinformatics estimates suggest that miRNAs might directly target over $60 \%$ of mammalian genes (Friedman et al., 2009); miRNA-dependent regulation in invertebrates and plants are less extensive.

Thousands of miRNAs have been annotated. The central miRNA database miRBase (http://www.mirbase.org, (Kozomara and Griffiths-Jones, 2014) includes 2654 human, 1978 murine, 469 Drosophila melanogaster , 437 Caenorhabditis elegans, and 428 Arabidopsis thaliana mature miRNAs (release 22.1). Remarkably, there are only a few miRNAs conserved between Drosophila and mammals and it is not clear if there are any conserved miRNA genes between plants and animals. Animal miRNAs seem to emerge from random formation of Drosha/Dicer substrates (discussed in detail in (Svoboda and Cara, 2006). Newly evolving miRNAs likely form a considerable portion of annotated miRNAs, especially in species where miRNAs were intensely studied by next generation sequencing (NGS), which can identify low-abundance miRNAs. The newly emerging miRNAs either acquire significant repressive functions and become retained during evolution or they become lost. Furthermore, target repertoire of individual miRNAs can evolve fast since a single point mutation can weaken an existing regulation or create a new one.

Animal miRNAs biogenesis starts with long primary transcripts (pri-miRNAs), which are processed by the nuclear "Microprocessor" complex, into short hairpin intermediates (pre-miRNAs). Pre-miRNAs are transported to the cytoplasm where they are further processed by Dicer into a small RNA duplex, from which is one RNA strand loaded onto an Argonaute protein where it guides recognition and repression of cognate mRNAs (Fig. 2).

The AGO-containing effector complex has been given different names; here it will be referred to as miRNA-Induced Silencing Complex (miRISC). The mechanism of action of an AGO-containing effector complex varies and may include either translational repression and/or RNAi-like endonucleolytic cleavage. Functional base pairing of animal miRNAs with their mRNA targets appears to involve little beyond the "seed" region comprising nucleotides 2 to 8 of the miRNA (Brennecke et al., 2005; Sontheimer, 2005). Pairing between 
miRNAs and mRNAs in plants is typically much more extensive and results in direct endonucleolytic cleavage.

Imperfect miRNA:mRNA base pairing in animals generally results in translational repression (Doench et al., 2003; Hutvagner and Zamore, 2002), which is coupled with mRNA degradation (Bagga et al., 2005; Lim et al., 2005). The molecular mechanism of mRNA degradation induced by imperfect base pairing differs from the RNA-like cleavage described above (Schmitter et al., 2006) and involves mRNA deadenylation and decapping activities (Chen et al., 2014; Djuranovic et al., 2012; Nishihara et al., 2013; Rouya et al., 2014). RNA degradation might actually be the dominant component of cognate gene repression (Eichhorn et al., 2014). Repressed mRNAs, miRNAs, and AGO proteins localize to cytoplasmic foci known as P-bodies (Liu et al., 2005; Pillai et al., 2005), which contain mRNA degrading enzymes such as the decapping complex, deadenylases, and the exonuclease XRN1 (reviewed in Decker and Parker, 2012).

There are only minor differences in miRNA pathways across animals. The main one is genetic separation between miRNA and RNAi pathways in arthropods, which utilize miRNA-dedicated Dicer, dsRBP, and AGO while other animals use one Dicer to produce miRNAs and siRNAs. There is a clear difference between animals and plants. Plants employ a single RNase III, one of their Dicer paralogs, to process pri-miRNA into pre-miRNA and then into miRNA duplex in the nucleus. These miRNAs are 2'-O-methylated at their 3' termini. This modification is absent in animal miRNAs (but found in piRNA small RNAs in the germline). In addition, animals employ two distinct RNase III enzymes - Drosha in the Microprocessor complex in the nucleus, which releases pre-miRNA from pri-miRNA, and Dicer, which produces miRNA duplex in the cytoplasm.

\section{Other relevant pathways in Metazoa}

\section{Adenosine deamination}

A-to-I editing is mediated by Adenosine Deaminases Acting on RNA (ADAR) enzymes, which contain dsRBD domains and recognize both inter- and intramolecular dsRNAs longer than 20-30 bp (Nishikura et al., 1991). ADARs convert adenosines to inosines, which translation and reverse transcription interpret as guanosines. ADARs were found in animals (including earliest branching groups) but not plants, yeasts or protozoa (Grice and Degnan, 2015; Nishikura, 2010). It was predicted that more than $85 \%$ of pre-mRNAs could be edited, predominantly in the non-coding regions (Athanasiadis et al., 2004).

RNA editing can negatively influence RNAi in several ways. First, ADARs can compete with RNAi for dsRNA substrates including siRNAs. A change of a single base in a sequence may result either in destabilization of dsRNA structure (inosine-uridine pair) or in its stabilization (inosine-cytidine pair) (Nishikura, 2010). This transition in the local and global stability of dsRNA structure can influence further processing of dsRNA, such as the selection of the effective miRNA strand (Bartel, 2004; Du and Zamore, 2005; Meister and Tuschl, 2004). While moderate deamination (one I-U pair per siRNA) does not prevent Dicer processing to siRNAs (Zamore et al., 2000), hyperediting ( $\sim 50 \%$ of deaminated adenosines) 
can make dsRNA resistant to Dicer processing (Scadden and Smith, 2001). Hyperedited dsRNA is also degraded by Tudor-SN (TSN) nuclease (Scadden, 2005). ADAR mutants in Caenorhabditis elegans exhibit defective chemotaxis while the phenotype can be rescued by RNAi-deficiency(Tonkin and Bass, 2003). In mammalian cells, ADAR1 limits siRNA efficiency (Yang et al., 2005). Editing can affect target recognition; a mismatch between siRNA and target mRNA can reduce RNAi efficacy (Scadden and Smith, 2001) or modify target specificity (Kawahara et al., 2007b). Several pri-miRNAs (e.g. miR-142) undergo editing, which inhibits miRNA biogenesis or causes even degradation of pri-miRNA by TSN (Kawahara et al., 2007a; Nishikura, 2010; Scadden, 2005; Yang et al., 2006).

\section{Interferon pathway}

Mammalian somatic cells can respond to dsRNA in a sequence-independent manner. A pioneering work by Hunter et al. showed that different types of dsRNA can block translation in reticulocyte lysates (Hunter et al., 1975). Analysis of the phenomenon identified protein kinase R (PKR) that is activated upon binding to dsRNA and blocks translation by phosphorylating the alpha subunit of eukaryotic initiation factor 2 (eIF2 $\alpha$ ) (Meurs et al., 1990). Activation of PKR represents a part of a complex response to foreign molecules known as the interferon response (reviewed in Sadler and Williams, 2007), which includes activation of the NFKB transcription factor and many interferon-stimulated genes (ISGs) (Geiss et al., 2001). In addition to PKR, several other proteins recognizing dsRNA induce the interferon response, including helicases RIG-I and MDA5, which sense cytoplasmic dsRNA and activate interferon expression, and the 2',5'-oligoadenylate synthetase (OAS), which produces 2',5'-linked oligoadenylates that induce general degradation of RNAs by activating latent RNase L, and specific Toll-like receptors (TLRs) (reviewed in Gantier and Williams, 2007; Sadler and Williams, 2007).

There is an evolutionary connection between RNAi and the interferon response. Mammalian RNA helicases $D d x 58, D h x 58$ and Ifih1, which are involved in immune response, are the closest homologs of helicases involved in processing of long dsRNA during RNAi in Caenorhabditis elegans. Notably, DDX58, also known as RIG-I, is an established component of the interferon response to long dsRNA (Yoneyama et al., 2004). This suggests that the interferon response, which has a common trigger and evolved after the RNAi pathway, adopted several components from the latter pathway. Notably, there is also connection between interferon pathway and A-to-I editing; analysis of mutant mice showed mice suggested that Adarl targets dsRNA and prevents MDA5-mediated interferon response (Liddicoat et al., 2015).

\section{Acknowledgement}

I would like to thank my colleagues Jan Paces, Miloslav Nic, and Tomas Novotny for help with collecting literature for the review. The review content was produced under a contract OC/EFSA/GMO/2015/01-CT 01 with European Food Safety Authority (EFSA); the opinions expressed are those of the contractor only and do not represent EFSA's official 
position. Publication of the review was funded by LO1220 and LM2015063 by the Ministry of Education, Youth and Sports.

\section{References}

Athanasiadis, A., Rich, A., and Maas, S. (2004). Widespread A-to-I RNA editing of Alu-containing mRNAs in the human transcriptome. PLoS Biol 2, e391.

Bagga, S., Bracht, J., Hunter, S., Massirer, K., Holtz, J., Eachus, R., and Pasquinelli, A.E. (2005). Regulation by let-7 and lin-4 miRNAs results in target mRNA degradation. Cell 122, 553-563.

Bartel, D.P. (2004). MicroRNAs: genomics, biogenesis, mechanism, and function. Cell 116, 281-297.

Batista, P.J., Ruby, J.G., Claycomb, J.M., Chiang, R., Fahlgren, N., Kasschau, K.D., Chaves, D.A., Gu, W., Vasale, J.J., Duan, S., et al. (2008). PRG-1 and 21U-RNAs interact to form the piRNA complex required for fertility in C-elegans. Molecular Cell 31, 67-78.

Bernstein, E., Caudy, A.A., Hammond, S.M., and Hannon, G.J. (2001). Role for a bidentate ribonuclease in the initiation step of RNA interference. Nature 409, 363-366.

Bohmert, K., Camus, I., Bellini, C., Bouchez, D., Caboche, M., and Benning, C. (1998). AGO1 defines a novel locus of Arabidopsis controlling leaf development. EMBO J 17, 170-180.

Brennecke, J., Stark, A., Russell, R.B., and Cohen, S.M. (2005). Principles of microRNA-target recognition. PLoS Biol 3, e85.

Buckley, B.A., Burkhart, K.B., Gu, S.G., Spracklin, G., Kershner, A., Fritz, H., Kimble, J., Fire, A., and Kennedy, S. (2012). A nuclear Argonaute promotes multigenerational epigenetic inheritance and germline immortality. Nature 489, 447-451.

Carmell, M.A., Xuan, Z., Zhang, M.Q., and Hannon, G.J. (2002). The Argonaute family: tentacles that reach into RNAi, developmental control, stem cell maintenance, and tumorigenesis. Genes Dev 16, 2733-2742.

Cerutti, H., and Casas-Mollano, J.A. (2006). On the origin and functions of RNA-mediated silencing: from protists to man. Current genetics 50, 81-99.

Chen, Y., Boland, A., Kuzuoglu-Ozturk, D., Bawankar, P., Loh, B., Chang, C.T., Weichenrieder, O., and Izaurralde, E. (2014). A DDX6-CNOT1 complex and W-binding pockets in CNOT9 reveal direct links between miRNA target recognition and silencing. Mol Cell 54, 737-750.

Chera, S., de Rosa, R., Miljkovic-Licina, M., Dobretz, K., Ghila, L., Kaloulis, K., and Galliot, B. (2006). Silencing of the hydra serine protease inhibitor Kazall gene mimics the human SPINK1 pancreatic phenotype. J Cell Sci 119, 846-857.

Cogoni, C., and Macino, G. (1999). Gene silencing in Neurospora crassa requires a protein homologous to RNA-dependent RNA polymerase. Nature 399, 166-169.

Dalmay, T., Hamilton, A., Rudd, S., Angell, S., and Baulcombe, D.C. (2000). An RNA-dependent RNA polymerase gene in Arabidopsis is required for posttranscriptional gene silencing mediated by a transgene but not by a virus. Cell 101, 543-553.

Dalmay, T., Horsefield, R., Braunstein, T.H., and Baulcombe, D.C. (2001). SDE3 encodes an RNA helicase required for post-transcriptional gene silencing in Arabidopsis. EMBO J 20, 2069-2078.

Das, P.P., Bagijn, M.P., Goldstein, L.D., Woolford, J.R., Lehrbach, N.J., Sapetschnig, A., Buhecha, H.R., Gilchrist, M.J., Howe, K.L., Stark, R., et al. (2008). Piwi and piRNAs act upstream of an 
endogenous siRNA pathway to suppress Tc3 transposon mobility in the Caenorhabditis elegans germline. Mol Cell 31, 79-90.

Decker, C.J., and Parker, R. (2012). P-bodies and stress granules: possible roles in the control of translation and mRNA degradation. Cold Spring Harb Perspect Biol 4, a012286.

Djuranovic, S., Nahvi, A., and Green, R. (2012). miRNA-mediated gene silencing by translational repression followed by mRNA deadenylation and decay. Science 336, 237-240.

Dlakic, M. (2006). DUF283 domain of Dicer proteins has a double-stranded RNA-binding fold. Bioinformatics 22, 2711-2714.

Doench, J.G., Petersen, C.P., and Sharp, P.A. (2003). siRNAs can function as miRNAs. Genes Dev $17,438-442$.

$\mathrm{Du}, \mathrm{T}$, and Zamore, P.D. (2005). microPrimer: the biogenesis and function of microRNA. Development 132, 4645-4652.

Duran-Figueroa, N., and Vielle-Calzada, J.P. (2010). ARGONAUTE9-dependent silencing of transposable elements in pericentromeric regions of Arabidopsis. Plant Signal Behav 5, 1476-1479.

Eichhorn, S.W., Guo, H., McGeary, S.E., Rodriguez-Mias, R.A., Shin, C., Baek, D., Hsu, S.H., Ghoshal, K., Villen, J., and Bartel, D.P. (2014). mRNA destabilization is the dominant effect of mammalian microRNAs by the time substantial repression ensues. Mol Cell 56, 104-115.

Faehnle, C.R., and Joshua-Tor, L. (2007). Argonautes confront new small RNAs. Curr Opin Chem Biol 11, 569-577.

Fagard, M., Boutet, S., Morel, J.B., Bellini, C., and Vaucheret, H. (2000). AGO1, QDE-2, and RDE-1 are related proteins required for post-transcriptional gene silencing in plants, quelling in fungi, and RNA interference in animals. Proc Natl Acad Sci U S A 97, 11650-11654.

Fire, A., Xu, S., Montgomery, M.K., Kostas, S.A., Driver, S.E., and Mello, C.C. (1998). Potent and specific genetic interference by double-stranded RNA in Caenorhabditis elegans. Nature 391, 806-811.

Fischer, S.E.J., Montgomery, T.A., Zhang, C., Fahlgren, N., Breen, P.C., Hwang, A., Sullivan, C.M., Carrington, J.C., and Ruvkun, G. (2011). The ERI-6/7 Helicase Acts at the First Stage of an siRNA Amplification Pathway That Targets Recent Gene Duplications. Plos Genetics 7, e1002369-e1002369.

Forstemann, K., Horwich, M.D., Wee, L., Tomari, Y., and Zamore, P.D. (2007). Drosophila microRNAs are sorted into functionally distinct argonaute complexes after production by dicer-1. Cell 130, 287-297.

Friedman, R.C., Farh, K.K., Burge, C.B., and Bartel, D.P. (2009). Most mammalian mRNAs are conserved targets of microRNAs. Genome Res 19, 92-105.

Gantier, M.P., and Williams, B.R. (2007). The response of mammalian cells to double-stranded RNA. Cytokine Growth Factor Rev 18, 363-371.

Geiss, G., Jin, G., Guo, J., Bumgarner, R., Katze, M.G., and Sen, G.C. (2001). A comprehensive view of regulation of gene expression by double-stranded RNA-mediated cell signaling. J Biol Chem 276, 30178-30182.

Geldhof, P., Visser, A., Clark, D., Saunders, G., Britton, C., Gilleard, J., Berriman, M., and Knox, D. (2007). RNA interference in parasitic helminths: current situation, potential pitfalls and future prospects. Parasitology 134, 609-619.

Grice, L.F., and Degnan, B.M. (2015). The origin of the ADAR gene family and animal RNA editing. BMC evolutionary biology 15,4 . 
Grishok, A., Pasquinelli, A.E., Conte, D., Li, N., Parrish, S., Ha, I., Baillie, D.L., Fire, A., Ruvkun, G., and Mello, C.C. (2001). Genes and mechanisms related to RNA interference regulate expression of the small temporal RNAs that control C. elegans developmental timing. Cell 106, 23-34.

Grishok, A., Tabara, H., and Mello, C.C. (2000). Genetic requirements for inheritance of RNAi in C. elegans. Science 287, 2494-2497.

Hall, I.M., Shankaranarayana, G.D., Noma, K., Ayoub, N., Cohen, A., and Grewal, S.I. (2002). Establishment and maintenance of a heterochromatin domain. Science 297, 2232-2237.

Hamilton, A.J., and Baulcombe, D.C. (1999). A species of small antisense RNA in posttranscriptional gene silencing in plants. Science 286, 950-952.

Himber, C., Dunoyer, P., Moissiard, G., Ritzenthaler, C., and Voinnet, O. (2003). Transitivity-dependent and -independent cell-to-cell movement of RNA silencing. EMBO J 22, 4523-4533.

Hunter, T., Hunt, T., Jackson, R.J., and Robertson, H.D. (1975). The characteristics of inhibition of protein synthesis by double-stranded ribonucleic acid in reticulocyte lysates. J Biol Chem 250, 409-417.

Hutvagner, G., and Zamore, P.D. (2002). A microRNA in a multiple-turnover RNAi enzyme complex. Science 297, 2056-2060.

Huvenne, H., and Smagghe, G. (2010). Mechanisms of dsRNA uptake in insects and potential of RNAi for pest control: a review. J Insect Physiol 56, 227-235.

Iwasaki, S., Sasaki, H.M., Sakaguchi, Y., Suzuki, T., Tadakuma, H., and Tomari, Y. (2015). Defining fundamental steps in the assembly of the Drosophila RNAi enzyme complex. Nature 521, 533-U274.

Jaskiewicz, L., and Filipowicz, W. (2008). Role of Dicer in posttranscriptional RNA silencing. Curr Top Microbiol Immunol 320, 77-97.

Jinek, M., and Doudna, J.A. (2009). A three-dimensional view of the molecular machinery of RNA interference. Nature 457, 405-412.

Kawahara, Y., Zinshteyn, B., Chendrimada, T.P., Shiekhattar, R., and Nishikura, K. (2007a). RNA editing of the microRNA-151 precursor blocks cleavage by the Dicer-TRBP complex. EMBO Rep 8, 763-769.

Kawahara, Y., Zinshteyn, B., Sethupathy, P., Iizasa, H., Hatzigeorgiou, A.G., and Nishikura, K. (2007b). Redirection of silencing targets by adenosine-to-inosine editing of miRNAs. Science 315, 1137-1140.

Ketting, R.F. (2011). The many faces of RNAi. Dev Cell 20, 148-161.

Kim, V.N., Han, J., and Siomi, M.C. (2009). Biogenesis of small RNAs in animals. Nat Rev Mol Cell Biol 10, 126-139.

Kozomara, A., and Griffiths-Jones, S. (2014). miRBase: annotating high confidence microRNAs using deep sequencing data. Nucleic Acids Res 42, D68-73.

Lee, R.C., Feinbaum, R.L., and Ambros, V. (1993). The C. elegans heterochronic gene lin-4 encodes small RNAs with antisense complementarity to lin-14. Cell 75, 843-854.

Liddicoat, B.J., Piskol, R., Chalk, A.M., Ramaswami, G., Higuchi, M., Hartner, J.C., Li, J.B., Seeburg, P.H., and Walkley, C.R. (2015). RNA editing by ADAR1 prevents MDA5 sensing of endogenous dsRNA as nonself. Science 349, 1115-1120.

Lim, L.P., Lau, N.C., Garrett-Engele, P., Grimson, A., Schelter, J.M., Castle, J., Bartel, D.P., Linsley, P.S., and Johnson, J.M. (2005). Microarray analysis shows that some microRNAs downregulate large numbers of target mRNAs. Nature 433, 769-773. 
Lingel, A., Simon, B., Izaurralde, E., and Sattler, M. (2003). Structure and nucleic-acid binding of the Drosophila Argonaute 2 PAZ domain. Nature 426, 465-469.

Lingel, A., Simon, B., Izaurralde, E., and Sattler, M. (2004). Nucleic acid 3'-end recognition by the Argonaute2 PAZ domain. Nat Struct Mol Biol 11, 576-577.

Liu, J., Carmell, M.A., Rivas, F.V., Marsden, C.G., Thomson, J.M., Song, J.J., Hammond, S.M., Joshua-Tor, L., and Hannon, G.J. (2004). Argonaute2 is the catalytic engine of mammalian RNAi. Science 305, 1437-1441.

Liu, J., Valencia-Sanchez, M.A., Hannon, G.J., and Parker, R. (2005). MicroRNA-dependent localization of targeted mRNAs to mammalian P-bodies. Nat Cell Biol 7, 719-723.

Liu, Q.L., Yao, X.Z., Pi, L.M., Wang, H., Cui, X.F., and Huang, H. (2009). The ARGONAUTE10 gene modulates shoot apical meristem maintenance and establishment of leaf polarity by repressing miR165/166 in Arabidopsis. Plant Journal 58, 27-40.

Lynn, K., Fernandez, A., Aida, M., Sedbrook, J., Tasaka, M., Masson, P., and Barton, M.K. (1999). The PINHEAD/ZWILLE gene acts pleiotropically in Arabidopsis development and has overlapping functions with the ARGONAUTE1 gene. Development 126, 469-481.

Ma, J.B., Ye, K., and Patel, D.J. (2004). Structural basis for overhang-specific small interfering RNA recognition by the PAZ domain. Nature 429, 318-322.

Ma, J.B., Yuan, Y.R., Meister, G., Pei, Y., Tuschl, T., and Patel, D.J. (2005). Structural basis for 5'-end-specific recognition of guide RNA by the A. fulgidus Piwi protein. Nature 434, 666-670.

MacRae, I.J., Zhou, K., and Doudna, J.A. (2007). Structural determinants of RNA recognition and cleavage by Dicer. Nat Struct Mol Biol 14, 934-940.

MacRae, I.J., Zhou, K., Li, F., Repic, A., Brooks, A.N., Cande, W.Z., Adams, P.D., and Doudna, J.A. (2006). Structural basis for double-stranded RNA processing by Dicer. Science 311, 195-198.

Marques, J.T., and Carthew, R.W. (2007). A call to arms: coevolution of animal viruses and host innate immune responses. Trends Genet 23, 359-364.

Martinez, J., Patkaniowska, A., Urlaub, H., Luhrmann, R., and Tuschl, T. (2002). Single-stranded antisense siRNAs guide target RNA cleavage in RNAi. Cell 110, 563-574.

Meins, F., Jr., Si-Ammour, A., and Blevins, T. (2005). RNA silencing systems and their relevance to plant development. Annu Rev Cell Dev Biol 21, 297-318.

Meister, G., Landthaler, M., Patkaniowska, A., Dorsett, Y., Teng, G., and Tuschl, T. (2004). Human Argonaute2 mediates RNA cleavage targeted by miRNAs and siRNAs. Mol Cell 15, 185-197.

Meister, G., and Tuschl, T. (2004). Mechanisms of gene silencing by double-stranded RNA. Nature 431, 343-349.

Meurs, E., Chong, K., Galabru, J., Thomas, N.S., Kerr, I.M., Williams, B.R., and Hovanessian, A.G. (1990). Molecular cloning and characterization of the human double-stranded RNA-activated protein kinase induced by interferon. Cell 62, 379-390.

Mourelatos, Z., Dostie, J., Paushkin, S., Sharma, A., Charroux, B., Abel, L., Rappsilber, J., Mann, M., and Dreyfuss, G. (2002). miRNPs: a novel class of ribonucleoproteins containing numerous microRNAs. Genes Dev 16, 720-728.

Mourrain, P., Beclin, C., Elmayan, T., Feuerbach, F., Godon, C., Morel, J.B., Jouette, D., Lacombe, A.M., Nikic, S., Picault, N., et al. (2000). Arabidopsis SGS2 and SGS3 genes are required for posttranscriptional gene silencing and natural virus resistance. Cell 101, 533-542.

Murphy, D., Dancis, B., and Brown, J.R. (2008). The evolution of core proteins involved in microRNA biogenesis. BMC evolutionary biology 8, 92 . 
Napoli, C., Lemieux, C., and Jorgensen, R. (1990). Introduction of a Chimeric Chalcone Synthase Gene into Petunia Results in Reversible Co-Suppression of Homologous Genes in trans. Plant Cell 2, 279-289.

Newmark, P.A., Reddien, P.W., Cebria, F., and Sanchez Alvarado, A. (2003). Ingestion of bacterially expressed double-stranded RNA inhibits gene expression in planarians. Proc Natl Acad Sci U S A 100 Suppl 1, 11861-11865.

Nishihara, T., Zekri, L., Braun, J.E., and Izaurralde, E. (2013). miRISC recruits decapping factors to miRNA targets to enhance their degradation. Nucleic Acids Res 41, 8692-8705.

Nishikura, K. (2010). Functions and regulation of RNA editing by ADAR deaminases. Annu Rev Biochem 79, 321-349.

Nishikura, K., Yoo, C., Kim, U., Murray, J.M., Estes, P.A., Cash, F.E., and Liebhaber, S.A. (1991). Substrate specificity of the dsRNA unwinding/modifying activity. EMBO J 10, 3523-3532.

Nykanen, A., Haley, B., and Zamore, P.D. (2001). ATP requirements and small interfering RNA structure in the RNA interference pathway. Cell 107, 309-321.

Orii, H., Mochii, M., and Watanabe, K. (2003). A simple "soaking method" for RNA interference in the planarian Dugesia japonica. Dev Genes Evol 213, 138-141.

Paces, J., Nic, M., Novotny, T., and Svoboda, P. (2017). Literature review of baseline information to support the risk assessment of RNAi-based GM plants. EFSA Supporting Publications 14, 315.

Parker, J.S., Roe, S.M., and Barford, D. (2004). Crystal structure of a PIWI protein suggests mechanisms for siRNA recognition and slicer activity. EMBO J 23, 4727-4737.

Pasquinelli, A.E., Reinhart, B.J., Slack, F., Martindale, M.Q., Kuroda, M.I., Maller, B., Hayward, D.C., Ball, E.E., Degnan, B., Muller, P., et al. (2000). Conservation of the sequence and temporal expression of let-7 heterochronic regulatory RNA. Nature 408, 86-89.

Pham, J.W., Pellino, J.L., Lee, Y.S., Carthew, R.W., and Sontheimer, E.J. (2004). A Dicer-2-dependent 80s complex cleaves targeted mRNAs during RNAi in Drosophila. Cell 117, 83-94.

Pillai, R.S., Bhattacharyya, S.N., Artus, C.G., Zoller, T., Cougot, N., Basyuk, E., Bertrand, E., and Filipowicz, W. (2005). Inhibition of translational initiation by Let-7 MicroRNA in human cells. Science 309, 1573-1576.

Provost, P., Dishart, D., Doucet, J., Frendewey, D., Samuelsson, B., and Radmark, O. (2002). Ribonuclease activity and RNA binding of recombinant human Dicer. EMBO J 21, 5864-5874.

Qin, H., Chen, F., Huan, X., Machida, S., Song, J., and Yuan, Y.A. (2010). Structure of the Arabidopsis thaliana DCL4 DUF283 domain reveals a noncanonical double-stranded RNA-binding fold for protein-protein interaction. RNA 16, 474-481.

Roignant, J.Y., Carre, C., Mugat, B., Szymczak, D., Lepesant, J.A., and Antoniewski, C. (2003). Absence of transitive and systemic pathways allows cell-specific and isoform-specific RNAi in Drosophila. RNA 9, 299-308.

Romano, N., and Macino, G. (1992). Quelling: transient inactivation of gene expression in Neurospora crassa by transformation with homologous sequences. Molecular microbiology 6, 3343-3353.

Rouya, C., Siddiqui, N., Morita, M., Duchaine, T.F., Fabian, M.R., and Sonenberg, N. (2014). Human DDX6 effects miRNA-mediated gene silencing via direct binding to CNOT1. RNA 20, 1398-1409.

Sadler, A.J., and Williams, B.R. (2007). Structure and function of the protein kinase R. Curr Top Microbiol Immunol 316, 253-292.

Scadden, A.D. (2005). The RISC subunit Tudor-SN binds to hyper-edited double-stranded RNA and promotes its cleavage. Nat Struct Mol Biol 12, 489-496. 
Scadden, A.D., and Smith, C.W. (2001). RNAi is antagonized by A-->I hyper-editing. EMBO Rep 2, 1107-1111.

Schmitter, D., Filkowski, J., Sewer, A., Pillai, R.S., Oakeley, E.J., Zavolan, M., Svoboda, P., and Filipowicz, W. (2006). Effects of Dicer and Argonaute down-regulation on mRNA levels in human HEK293 cells. Nucleic Acids Res 34, 4801-4815.

Schwarz, D.S., Hutvagner, G., Haley, B., and Zamore, P.D. (2002). Evidence that siRNAs function as guides, not primers, in the Drosophila and human RNAi pathways. Mol Cell 10, 537-548.

Smardon, A., Spoerke, J.M., Stacey, S.C., Klein, M.E., Mackin, N., and Maine, E.M. (2000). EGO-1 is related to RNA-directed RNA polymerase and functions in germ-line development and RNA interference in C. elegans. Curr Biol 10, 169-178.

Song, J.J., Liu, J., Tolia, N.H., Schneiderman, J., Smith, S.K., Martienssen, R.A., Hannon, G.J., and Joshua-Tor, L. (2003). The crystal structure of the Argonaute2 PAZ domain reveals an RNA binding motif in RNAi effector complexes. Nat Struct Biol 10, 1026-1032.

Song, J.J., Smith, S.K., Hannon, G.J., and Joshua-Tor, L. (2004). Crystal structure of Argonaute and its implications for RISC slicer activity. Science 305, 1434-1437.

Sontheimer, E.J. (2005). Assembly and function of RNA silencing complexes. Nat Rev Mol Cell Biol 6, 127-138.

Stein, P., Svoboda, P., Anger, M., and Schultz, R.M. (2003). RNAi: mammalian oocytes do it without RNA-dependent RNA polymerase. Rna 9, 187-192.

Svoboda, P., and Cara, A.D. (2006). Hairpin RNA: a secondary structure of primary importance. Cell Mol Life Sci 63, 901-908.

Tabara, H., Grishok, A., and Mello, C.C. (1998). RNAi in C. elegans: soaking in the genome sequence. Science 282, 430-431.

Tabara, H., Sarkissian, M., Kelly, W.G., Fleenor, J., Grishok, A., Timmons, L., Fire, A., and Mello, C.C. (1999). The rde-1 gene, RNA interference, and transposon silencing in C. elegans. Cell 99, 123-132.

Tijsterman, M., Ketting, R.F., Okihara, K.L., Sijen, T., and Plasterk, R.H.A. (2002a). RNA helicase MUT-14-dependent gene silencing triggered in C-elegans by short antisense RNAs. Science 295, 694-697.

Tijsterman, M., Okihara, K., Thijssen, K., and Plasterk, R. (2002b). PPW-1, a PAZ/PIWI Protein Required for Efficient Germline RNAi, Is Defective in a Natural Isolate of C. elegans. Curr Biol 12,1535 .

Timmons, L., and Fire, A. (1998). Specific interference by ingested dsRNA. Nature 395, 854.

Tomoyasu, Y., Miller, S.C., Tomita, S., Schoppmeier, M., Grossmann, D., and Bucher, G. (2008). Exploring systemic RNA interference in insects: a genome-wide survey for RNAi genes in Tribolium. Genome Biol 9, R10.

Tonkin, L.A., and Bass, B.L. (2003). Mutations in RNAi rescue aberrant chemotaxis of ADAR mutants. Science 302, 1725.

Tuschl, T., Zamore, P.D., Lehmann, R., Bartel, D.P., and Sharp, P.A. (1999). Targeted mRNA degradation by double-stranded RNA in vitro. Genes Dev 13, 3191-3197.

van der Krol, A.R., Mur, L.A., Beld, M., Mol, J.N., and Stuitje, A.R. (1990). Flavonoid genes in petunia: addition of a limited number of gene copies may lead to a suppression of gene expression. Plant Cell 2, 291-299. 
Vasale, J.J., Gu, W., Thivierge, C., Batista, P.J., Claycomb, J.M., Youngman, E.M., Duchaine, T.F., Mello, C.C., and Conte, D., Jr. (2010). Sequential rounds of RNA-dependent RNA transcription drive endogenous small-RNA biogenesis in the ERGO-1/Argonaute pathway. Proc Natl Acad Sci U S A 107, 3582-3587.

Voinnet, O., Pinto, Y.M., and Baulcombe, D.C. (1999). Suppression of gene silencing: a general strategy used by diverse DNA and RNA viruses of plants. Proc Natl Acad Sci U S A 96, 14147-14152.

Volpe, T.A., Kidner, C., Hall, I.M., Teng, G., Grewal, S.I., and Martienssen, R.A. (2002). Regulation of heterochromatic silencing and histone H3 lysine-9 methylation by RNAi. Science 297 , 1833-1837.

Vourekas, A., Zheng, Q., Alexiou, P., Maragkakis, M., Kirino, Y., Gregory, B.D., and Mourelatos, Z. (2012). Mili and Miwi target RNA repertoire reveals piRNA biogenesis and function of Miwi in spermiogenesis. Nat Struct Mol Biol 19, 773-781.

Wang, G., and Reinke, V. (2008). A C. elegans Piwi, PRG-1, regulates 21U-RNAs during spermatogenesis. Current Biology 18, 861-867.

Whangbo, J.S., and Hunter, C.P. (2008). Environmental RNA interference. Trends Genet 24, 297-305.

Winter, J., Jung, S., Keller, S., Gregory, R.I., and Diederichs, S. (2009). Many roads to maturity: microRNA biogenesis pathways and their regulation. Nat Cell Biol 11, 228-234.

Xie, Q., and Guo, H.S. (2006). Systemic antiviral silencing in plants. Virus Res 118, 1-6.

$\mathrm{Xu}, \mathrm{W}$., and Han, Z. (2008). Cloning and phylogenetic analysis of sid-1-like genes from aphids. J Insect Sci 8, 1-6.

Yan, K.S., Yan, S., Farooq, A., Han, A., Zeng, L., and Zhou, M.M. (2003). Structure and conserved RNA binding of the PAZ domain. Nature 426, 468-474.

Yang, W., Chendrimada, T.P., Wang, Q., Higuchi, M., Seeburg, P.H., Shiekhattar, R., and Nishikura, K. (2006). Modulation of microRNA processing and expression through RNA editing by ADAR deaminases. Nat Struct Mol Biol 13, 13-21.

Yang, W., Wang, Q., Howell, K.L., Lee, J.T., Cho, D.S., Murray, J.M., and Nishikura, K. (2005). ADAR1 RNA deaminase limits short interfering RNA efficacy in mammalian cells. J Biol Chem 280, 3946-3953.

Yigit, E., Batista, P.J., Bei, Y., Pang, K.M., Chen, C.C., Tolia, N.H., Joshua-Tor, L., Mitani, S., Simard, M.J., and Mello, C.C. (2006). Analysis of the C. elegans Argonaute family reveals that distinct Argonautes act sequentially during RNAi. Cell 127, 747-757.

Yoneyama, M., Kikuchi, M., Natsukawa, T., Shinobu, N., Imaizumi, T., Miyagishi, M., Taira, K., Akira, S., and Fujita, T. (2004). The RNA helicase RIG-I has an essential function in double-stranded RNA-induced innate antiviral responses. Nat Immunol 5, 730-737.

Yuan, Y.R., Pei, Y., Ma, J.B., Kuryavyi, V., Zhadina, M., Meister, G., Chen, H.Y., Dauter, Z., Tuschl, T., and Patel, D.J. (2005). Crystal structure of A. aeolicus argonaute, a site-specific DNA-guided endoribonuclease, provides insights into RISC-mediated mRNA cleavage. Mol Cell 19, 405-419.

Zamore, P.D., Tuschl, T., Sharp, P.A., and Bartel, D.P. (2000). RNAi: double-stranded RNA directs the ATP-dependent cleavage of mRNA at 21 to 23 nucleotide intervals. Cell 101, 25-33.

Zhang, H., Kolb, F.A., Brondani, V., Billy, E., and Filipowicz, W. (2002). Human Dicer preferentially cleaves dsRNAs at their termini without a requirement for ATP. EMBO J 21, 5875-5885.

Zhang, H., Kolb, F.A., Jaskiewicz, L., Westhof, E., and Filipowicz, W. (2004). Single processing center models for human Dicer and bacterial RNase III. Cell 118, 57-68. 
Zhang, Z., Liu, X., Guo, X., Wang, X.J., and Zhang, X. (2016). Arabidopsis AGO3 predominantly recruits 24-nt small RNAs to regulate epigenetic silencing. Nat Plants 2, 16049.

Zheng, X.W., Zhu, J.H., Kapoor, A., and Zhu, J.K. (2007). Role of Arabidopsis AGO6 in siRNA accumulation, DNA methylation and transcriptional gene silencing. EMBO J 26, 1691-1701. 


\title{
RNAi AND miRNA PATHWAYS IN MAMMALS I - MOLECULAR MECHANISMS
}

\author{
Keywords: dsRNA, siRNA, miRNA, Dicer, TARBP2, PACT, Argonaute
}

\section{PETR SVOBODA}

Institute of Molecular Genetics, Academy of Sciences of the Czech Republic, Videnska 1083, 14220 Prague 4, Czech Republic

Correspondence to: Petr Svoboda, Institute of Molecular Genetics ASCR, Videnska 1083, 14220 Prague 4, Czech Republic, tel. \#+420 241063147, e-mail: svobodap@img.cas.cz.

\begin{abstract}
RNA silencing denotes sequence-specific repression mediated by small RNAs. In mammals, there are two closely related pathways, which share several protein factors: RNA interference (RNAi) and microRNA (miRNA) pathway. The miRNA pathway regulates endogenous protein-coding gene expression. It has been implicated in many biological processes and majority of mammalian genes appear to be directly or indirectly exposed to miRNA-mediated regulations. RNAi generally serves as a form of innate immunity targeting viruses and mobile elements, although it occasionally also acquired function in protein-coding gene regulation. The function of RNAi in mammals is still poorly understood but it is clear that proteins supporting RNAi are also involved in miRNA biogenesis and function. Because of the volume of information, the review of mammalian miRNA and RNAi pathways was divided into two parts, where first one presented here reviews components of the pathways and the second one reviews function and significance of the pathways.
\end{abstract}

\section{Introduction}

Mammals belong (with birds and fishes) to the group Craniata of the phylum Chordata. Mammals are homeothermic animals distinguished by mammary glands, hair, middle ear bones, and neocortex. There are $\sim 5000$ extant mammalian species, most of which are placental mammals \{Margulis, $1998 \# 2572$ \}. The synapsid animal lineage leading to mammals branched of the sauropsid lineage leading to dinosaurs and birds. The mammalian miRNA, RNAi and other dsRNA-responding pathways are among the most studied dsRNA-responding pathways. Because of the large volume of the literature, I divided the mammalian material into two articles. In this first one, I will define molecular components and will review key functional implications in the second article. The first reviewed pathway will be the miRNA pathway (Fig. 1). 


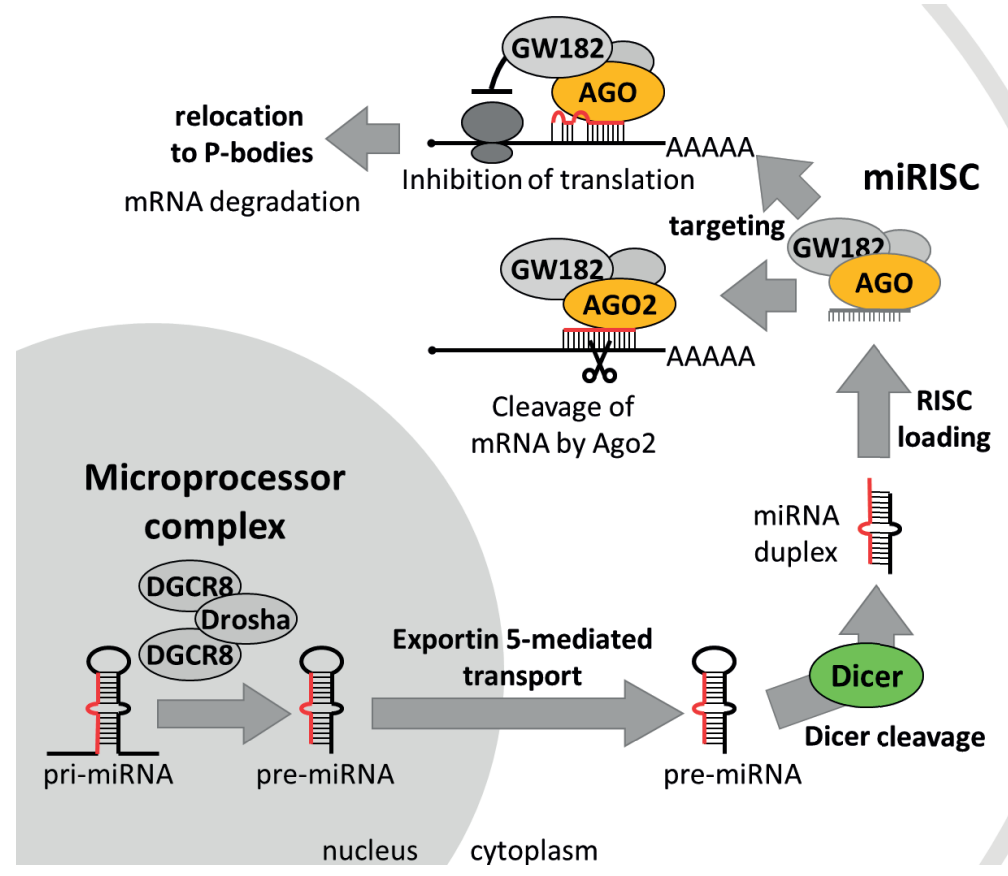

Figure 1 Overview of the molecular mechanism of the mammalian miRNA pathway. See text for details.

\section{The Microprocessor complex - nuclear initiation of miRNA processing}

The canonical primary miRNAs (non-canonical precursors are described separately further below) are transcribed by polymerase II (polymerase III-transcribed miRNA precursors are uncommon (Borchert et al., 2006; Canella et al., 2010)) and carry local hairpins, which are released in the nucleus as stem-loop precursors of approximately 70 nucleotides (pre-miRNAs) by the activity of the so-called Microprocessor complex, which is composed of RNase III Drosha and DGCR8 proteins (Gregory et al., 2004; Han et al., 2004; Landthaler et al., 2004).

\section{Drosha}

Drosha, the active component of the Microprocessor complex, was discovered in 2000 as a new member of RNase III in Drosophila with a conserved human homolog (Filippov et al., 2000). Structurally, Drosha protein carries a single C-terminal dsRNA-binding motif (dsRBM), tandem catalytic domains, a proline-rich region (PRR) and an RS domain (Fortin et al., 2002). Drosha was recognized as the nuclease that executes the initiation step of miRNA processing in the nucleus (Lee et al., 2003) and the Microprocessor complex was reported in a series of papers in 2004 (Gregory et al., 2004; Han et al., 2004; Landthaler et al., 2004). The current understanding of the Microprocessor complex includes the 
resolved X-ray structure of Drosha with the C-terminal helix of DGCR8 (Kwon et al., 2016). Drosha contains two DGCR8-binding sites, one on each RNase III domain, which mediate the assembly of the Microprocessor complex. The overall structure of Drosha is similar to that of Dicer (see further below) despite no sequence homology except of the C-terminal part. This suggests that Drosha could have evolved from a Dicer homolog and would be consistent with the proposed classification as a non-canonical Dicer in a single RNase III family (Jaskiewicz and Filipowicz, 2008). In addition, Drosha exhibits unique features, including non-canonical zinc-finger motifs, a long insertion in the first RIIID, and a kinked link between a Connector helix and RIIID explaining the 11-bp-measuring "ruler" activity of Drosha. The structural analysis is consistent with biochemical characterization of the complex, which suggested that the Microprocessor complex exists as a heterotrimeric complex (Herbert et al., 2016; Nguyen et al., 2015). In addition, Drosha and DGCR8, respectively, recognize the basal UG and apical UGU motifs in pri-miRNAs, which determines orientation of the complex (Nguyen et al., 2015). These results clarified inconsistencies and unknowns existing in the earlier literature regarding the stoichiometry of the complex and the mode of binding (Barr and Guo, 2014; Gregory et al., 2006; Gregory et al., 2004; Han et al., 2004; Landthaler et al., 2004; Ma et al., 2013).

\section{DGCR8}

While Drosha provides the catalytic site for cleavage, DGCR8 anchors substrate pri-miRNAs. A crystal structure of the human DGCR8 core (residues 493-720) showed two double-stranded RNA-binding domains (dsRBDs) arranged with pseudo two-fold symmetry tightly packed against the C-terminal helix (Sohn et al., 2007). Interestingly, DGCR8 uses heme as a co-factor. DGCR8 contains a previously uncharacterized heme-binding motif that is also required for its activity. Heme availability and biosynthesis in $\mathrm{HeLa}$ cells positively affect pri-miRNA processing and production of mature miRNAs while heme-binding-deficient DGCR8 mutants are defective in pri-miRNA processing (Barr and Guo, 2014; Barr et al., 2015; Weitz et al., 2014). Pri-miRNA recognition by DGCR8 seems to involve $\mathrm{N}(6)$-methyladenosine mark deposited by the methyltransferase-like 3 (METTL3) (Alarcon et al., 2015). DGCR8 is also regulated by phosphorylation. There are 23 possible phosphorylation sites mapped on the full-length human DGCR8 expressed in insect or mammalian cells (Herbert et al., 2013). Subsequent analysis showed that DGCR8 phosphorylation may increase DGCR8 stability but not processing activity in response to extracellular cues (Herbert et al., 2013). DGCR8 phosphorylation by the tyrosine kinase ABL has been observed after DNA damage stimulating the processing of selective primary miRNAs (Tu et al., 2015).

\section{Additional Microprocessor co-factors}

Apart from the ABL kinase, several additional co-factors of the Microprocessor complex were identified. One of them is the RNA-Binding Protein DDX1, which appears to be a regulatory protein promoting expression of a subset of miRNAs, majority of which is induced after DNA damage (Han et al., 2014). A peculiar Microprocessor complex component is 
Methyl-CpG-binding protein MECP2 (Cheng et al., 2014; Tsujimura et al., 2015), which is known to stably bind methylated DNA. According to one report, MECP2 promotes the posttranscriptional processing of particular miRNAs including miR-199a, which stimulates mTOR signalling (the key pathway regulating cell metabolism, growth, and survival) by targeting inhibitors of mTOR signalling (Tsujimura et al., 2015). In contrast, Cheng et al reported that MECP2 binds directly to DGCR8 and interferes with the assembly of the Microprocessor complex, thus affecting gene expression posttranscriptionally via relieving repression of miRNA targets (Cheng et al., 2014).

\section{Microprocessor complex localization and function(s)}

The Microprocessor complex shows apparent nuclear compartmentalization. While transiently expressed pri-miRNAs accumulate in nuclear foci with splicing factor SC35 and Microprocessor components, Drosha and DGCR8. (Pawlicki and Steitz, 2008), these foci do not appear to be major sites of pri-miRNA processing, which seems to be coupled to transcription (Pawlicki and Steitz, 2009). This is consistent with live-imaging, which revealed that a large fraction of Microprocessor resides with unspliced pri-miRNAs in close proximity to their genes. This analysis also provided a direct visual evidence that DGCR8 and Drosha are targeted to pri-miRNAs as a preformed complex (Bellemer et al., 2012).

Importantly, literature review identified also reports describing additional roles of the Microprocessor complex and its components beyond miRNA biogenesis although Microprocessor expression seems to be tuned according to pri-miRNA substrates (Barad et al., 2012). Non-canonical roles of Microprocessor (or Drosha) include: 1) mRNA cleavage (Chong et al., 2010), exemplified by Drosha-dependent cleavage of Hoxd4 RNA (Phua et al., 2011) or destabilization of Neurog2 mRNA, which supports neural stem cell maintenance by blocking accumulation of differentiation and determination factors (Knuckles et al., 2012), 2) processing of long non-coding RNAs restricted to the nucleus (Ganesan and Rao, 2008), 3), ribosomal RNA biogenesis (Liang and Crooke, 2011), and 4) cleavage of viral RNA (Shapiro et al., 2014). While immunoprecipitation of the Microprocessor complex followed by next-generation sequencing showed that precursors of canonical miRNAs and miRNA-like hairpins are the major substrates of the Microprocessor complex (Seong et al., 2014), high-throughput sequencing and cross-linking immunoprecipitation (HITS-CLIP) analysis of RNAs bound to DGCR8 suggest that miRNAs may not be the most abundant targets. DGCR8-bound RNAs also comprised several hundred mRNAs, small nucleolar RNAs (snoRNAs), and long noncoding RNAs (Macias et al., 2012). Interestingly, DGCR8-mediated cleavage of snoRNAs was independent of Drosha, indicating participation of DGCR8 in other RNA processing complexes (Macias et al., 2012). One of such complexes is the exosome (an hRRP6-containing nucleolar form), where DGCR8 is essential for its recruitment to snoRNAs and to the human telomerase RNA component (hTR/TERC) (Macias et al., 2015). Thus, DGCR8 acts as an adaptor recruiting the exosome complex to structured RNAs and inducing their degradation. (Macias et al., 2015). 


\section{Microprocessor complex crosstalk with other pathways}

In terms of a crosstalk with other pathways, it has been established that some miRNA precursors are edited by ADARs (Alon et al., 2012; Garcia-Lopez et al., 2013; Peng et al., 2012; Tomaselli et al., 2015; Vesely et al., 2014; Vesely et al., 2012; Yang et al., 2006) apparently as early as pri-miRNAs (Bahn et al., 2015; Chen et al., 2015). According to one model, ADAR1 interacts with Drosha and DGCR8 in the nucleus and possibly outcompetes DGCR8 in primary miRNA binding, thus enhancing mature miRNA expression. This appears dependent on ADAR1 editing activity, at least for a subset of targets (Bahn et al., 2015). According to the selective elimination model, miRNAs, such as miR-151, are edited and eliminated by Tudor-SN (a ribonuclease specific to inosine-containing dsRNAs and a reported component of RISC) during mouse preimplantation development (Garcia-Lopez et al., 2013). Similarly, pri-miR-142 editing results in suppression of its processing by Drosha while the edited pri-miR-142 is degraded by Tudor-SN. Consequently, mature miRNA-142 expression substantially increases in ADAR1 ${ }^{-/}$or ADAR2 $2^{--}$mice (Yang et al., 2006). According to the stimulation model, exemplified by miR-497, abundant editing event promotes processing by Drosha of the corresponding pri-miRNA (Vesely et al., 2014).

\section{Dicer - cytoplasmic production of miRNA from pre-miRNA}

A pre-miRNA produced by the Microprocessor complex is transported to the cytoplasm via Exportin 5 in a RanGTP-dependent manner. The next pre-miRNA processing step is Dicer mediated cleavage, which takes place the cytoplasm.

\section{Structure of Dicer}

The full length mammalian Dicer has not been crystallized. The current understanding of the mammalian Dicer structure has thus been inferred from several different sources, which can be divided into four groups:

(I) Biochemical studies of recombinant Dicer and individual domains (Ma et al., 2008; Park et al., 2011; Provost et al., 2002; Zhang et al., 2002; Zhang et al., 2004).

(II) The crystal structure of Giardia intestinalis Dicer (serving as a comparative scaffold) (MacRae et al., 2007; MacRae et al., 2006b).

(III) Crystallographic studies on mammalian Dicer fragments (Du et al., 2008; Wilson et al., 2015) or on individual domains (Ma et al., 2004; Takeshita et al., 2007; Tian et al., 2014; Wilson et al., 2015)

(IV) Cryo-EM studies of human Dicer and its complexes with other proteins (Lau et al., 2012; Lau et al., 2009; Taylor et al., 2013; Wang et al., 2009; Wilson et al., 2015).

Dicer is an siRNA-producing RNase III enzyme conserved across eukaryotes (Bernstein et al., 2001). Mammalian Dicer proteins are $\sim 220 \mathrm{kDa}$ multidomain proteins, which are composed of domains ordered from the $\mathrm{N}$ - to the C-terminus as follows: $\mathrm{N}$-terminal DExD and helicase superfamily C-terminal domains, a domain of unknown function DUF283, 

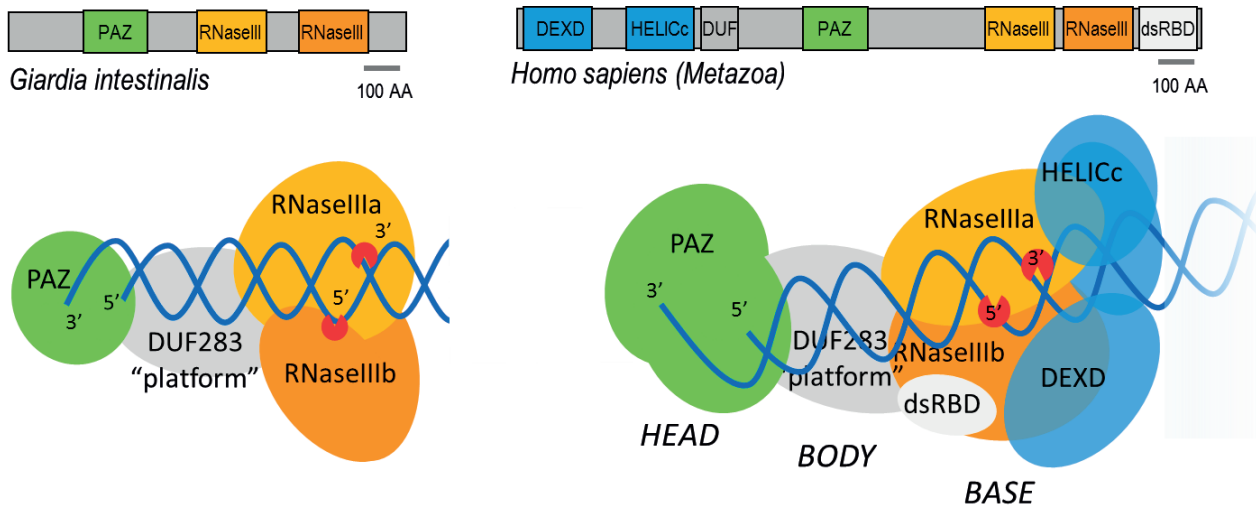

Figure 2 Domain architectures of Dicers from Giardia and humans.

a PAZ domain, RNase IIIa and RNase IIIb domains, and the C-terminal dsRBD (Fig. 2) (Nicholson and Nicholson, 2002). In contrast to the simplest RNase III family members (exemplified by E. coli RNase III), which carry only one RNase III domain and dimerize when cleaving dsRNA (Johanson et al., 2013; Lamontagne et al., 2001). Dicer proteins carry two RNase III domains, which form an intramolecular dimer (Zhang et al., 2004).

Giardia Dicer structure revealed spatial organization of the core part of eukaryotic Dicer proteins and explained how Dicer generates small RNAs of specific lengths (MacRae et al., 2006a). This crystal structure then served as a framework for deciphering the structure of other Dicer proteins, including mammalian Dicers. The front view of the Giardia Dicer structure resembles an axe. The blade is formed of an intramolecular duplex of two RNase III domains, which are connected by a bridging domain constituting the back end of the blade. The platform domain is adjacent to the RNase IIIa domain and makes up the upper part of the handle. The PAZ domain is connected by a long helix to the RNase IIIa domain and forms the base of the handle (MacRae et al., 2006b). Altogether, the Giardia Dicer is formed of three rigid regions, which are linked by flexible hinges. One region is formed by RNase III domains and the bridging domain, the second by the platform domain and the connector helix, and the third by the PAZ domain. These three parts can swing relative to each other and possibly ensure accommodation of Dicer to the structure of its substrate (MacRae et al., 2006a). This conformational flexibility likely enables binding of dsRNAs with non-canonical base pairing as well as imperfect duplexes of pre-miRNAs (MacRae et al., 2006a). In addition, dsRNA binding is presumably stabilized by several positively charged patches on the surface of Giardia Dicer between the processing center and the PAZ domain, which are in contact with dsRNA (MacRae et al., 2006a; MacRae et al., 2007).

Mammalian Dicers are much larger and contain domains absent in the Giardia Dicer but follow the same organizational and functional principles (Fig. 2). The crystal structure of Giardia Dicer confirmed an earlier biochemical analysis predicting that the two RNase III domains of the human Dicer form an intramolecular dimer resulting in a single processing center placed at a specific distance from the PAZ domain (Zhang et al., 2004). 
A structural component defining this distance is an $\alpha$ helix (connector helix), which directly links PAZ and RNase III domains (MacRae et al., 2006b). Thus, the key functional aspect that emerged from Dicer's structural analysis was that it functions as a molecular ruler, measuring the length of the substrate from the PAZ domain to RNase III domains where each domain cleaves one strand. Importantly, the mammalian Dicer (and metazoan Dicers in general) differ from Giardia's in two main aspects. The first is the general topology reflecting the fact that the typical mammalian Dicer product is shorter (21-23 nt). The second one is that the mammalian Dicer contains additional functional domains important for substrate recognition and processing.

As indicated above, the architecture of the human Dicer and positions of its domains and interacting partners have been inferred by cryo-EM of the full length protein and its mutants (Lau et al., 2012; Lau et al., 2009; Taylor et al., 2013; Wang et al., 2009; Wilson et al., 2015). The overall shape of the human Dicer resembles the letter $L$; the shape is further divided into a head, a body and a base (Fig. 2). The PAZ domain is adjacent to the platform domain in the head of the protein while the RNase IIIb is located in the body. Thus, the head of the human Dicer is a topological equivalent of the base of the handle in Giardia's Dicer. The helicase domain constitutes the base, which has no equivalent in Giardia's Dicer. The position of the processing center relative to the PAZ domains differs between human and Giardia Dicers, which explains the fact that the human Dicer produces siRNA about four nucleotides shorter than the Giardia Dicer, which corresponds to $\sim$ one-third of a dsRNA helical turn (Lau et al., 2012). Therefore, the processing center has to access the cleavage site of dsRNA from the different angle relative to the dsRNA helical end in comparison with Giardia Dicer (Lau et al., 2012).

For understanding substrate selection and processing, two areas of Dicer's structure deserve special attention: the PAZ and the N-terminal domains, which are described below. The following text represents exhaustive literature survey focused on the structural and functional aspects of the two domains.

\section{The PAZ domain}

The PAZ domain found in Dicer and Argonaute proteins is a dsRNA-terminus binding module (Ma et al., 2004; MacRae et al., 2006b). The PAZ domain has a 3' overhang binding pocket but only the PAZ domain of Dicer has an extra loop enriched in basic amino acids, changing electrostatic potential and molecular surface of the pocket. These changes may influence RNA binding by Dicer and handing-off the substrate to other proteins complexes (MacRae et al., 2006b). The PAZ domain of metazoan Dicers also recognizes phosphorylated 5' end of a pre-miRNA. A mutation of the 5' binding pocket leads to dysregulation of miRNA biogenesis in vivo (Park et al., 2011). The 5' binding pocket is conserved in Drosophila DCR-1 and human Dicer but not in Giardia Dicer (Park et al., 2011). Importantly, the 5' binding pocket appears conserved in Dicer proteins functioning in miRNA biogenesis (human Dicer, Drosophila DCR-1) but not in Dicer proteins dedicated to long dsRNA processing (Giardia, Schizosaccharomyces, Drosophila DCR-2). Accordingly, simultaneous fixing of 3 ' and 5 ' ends emerges as a feature important for fidelity of miRNA biogenesis but not for siRNAs (Park et al., 2011). 


\section{The N-terminal helicase domain}

The N-terminus of metazoan Dicers harbours a complex helicase structure, which is adjacent to RNase III catalytic domains (Lau et al., 2012). Although the helicase must come into contact with the substrate, its functional significance is still only partially understood. However, it is clear that the N-terminal helicase region is the key for the substrate preference. In mammals (and in most metazoan phyla), a single gene encodes Dicer, which has to process both: miRNA precursors into miRNAs as well as long double-stranded RNAs into small interfering RNAs (siRNAs). Different taxons apparently differ in how much they employ both types of Dicer activities; the mammalian Dicer is mainly dedicated to the miRNA pathway while its natural production is very limited.

The N-terminal helicase belongs to the RIG-I-like helicase family (Zou et al., 2009) and consists of a proximal DExD/H domain and an adjacent helicase superfamily c-terminal domain (Fig. 2). A conventional helicase domain has an ATPase activity. Indeed, invertebrate Dicers bind and hydrolyze ATP (Bernstein et al., 2001; Ketting et al., 2001; Nykanen et al., 2001; Zamore et al., 2000). However, despite the N-terminal helicase with conserved motifs important for ATP binding and hydrolysis is present in mammalian Dicers, there is no evidence of ATP requirement for the human Dicer activity (Provost et al., 2002; Zhang et al., 2002). The human Dicer has the same processing efficiency in the presence or absence of ATP. Moreover, the rate of cleavage is not influenced by addition of other nucleotides, non-cleavable ATP analogues or a mutation in the Walker A motif of ATPase/ helicase domain (Provost et al., 2002; Zhang et al., 2002). Notably, these experiments were performed using a long dsRNA substrate with blunt ends, whose processing by invertebrates Dicers is ATP-dependent (Bernstein et al., 2001; Ketting et al., 2001; Nykanen et al., 2001; Zamore et al., 2000; Zhang et al., 2002). Remarkably, deletion of the helicase domain results in high cleavage rate of long dsRNAs by human Dicer in vitro (Ma et al., 2008) as well as in vivo in murine and human cells (Flemr et al., 2013; Kennedy et al., 2015). Thus, the N-terminal helicase in mammalian Dicers has a different role in substrate recognition and processing than the helicase in invertebrate Dicers although the overall shapes of human and Drosophila Dicer proteins are similar (Lau et al., 2012).

The crystal structure of the N-terminal helicase has not been obtained. Thus, based on the cryo-EM-based modelling, the N-terminal helicase is composed of three globular subdomains (HEL1, HEL2, HEL2i) where the DExD/H domain corresponds to HEL1 and the helicase superfamily c terminal domain to HEL2 and HEL2i. All three parts of the helicase form a clamp near the RNase III domain active site. Interestingly, the N-terminal helicase was found in two distinct conformations, with respect to the body of the enzyme (Lau et al., 2012), similar to the RIG-I helicase which was used as a template for modelling (Kowalinski et al., 2011).

Analysis of substrate-specific structural rearrangements proposed that human Dicer exists in three states depending on presence and type of substrate (Taylor et al., 2013). Unbound Dicer existing in "canonical state" rearranges upon substrate binding that involves the PAZ domain as well as the helicase domain. Substrate-bound Dicer exists either in an "open" or closed" state. The open state is cleavage-competent and it is typical for pre-miRNA binding. It is characterized by binding of a pre-miRNA along the platform, bending of the helicase 
domain, and access of RNase IIIa and IIIb sites to the substrate (Taylor et al., 2013). The closed state has been observed for a 35 bp A-form RNA duplex, which represents a siRNA precursor. In this state, the substrate is trapped between the PAZ and helicase domains away from the catalytic sites (Taylor et al., 2013). This provides a structural explanation for previous observations that Dicer poorly processes longer perfect duplexes in vitro and in vivo (Kim et al., 2005; Nejepinska et al., 2012b).

Taken together, it is apparent that miRNA biogenesis has been the preferred role for Dicer during vertebrate evolution. The helicase domain in mammalian Dicers provides a structural basis for substrate specificity, namely distinguishing pre-miRNAs as the preferred substrate. In addition, a natural Dicer isoform has been found in mouse oocytes, which lacks the N-terminal helicase domain, can efficiently generate siRNAs from long dsRNAs, and is sufficient for enhancing RNAi in cultured cells. This isoform is a consequence of a rodent-specific retrotransposon insertion and is present in Muridae family (Flemr et al., 2013). This demonstrates that, while the mammalian Dicer primarily dedicated to the miRNA pathway, a small change in a mammalian Dicer gene can restore RNAi activity.

\section{Substrates and their processing by mammalian Dicer proteins}

The first in vitro studies of recombinant human Dicer showed that substrate cleavage is dependent on Mg2+ but not on ATP presence (Provost et al., 2002; Zhang et al., 2002). Subsequently, it was reported that Dicer can cleave long dsRNAs and pre-miRNAs with different efficiency, which stems from substrate's structural properties (Chakravarthy et al., 2010; Feng et al., 2012; Flores-Jasso et al., 2009; Ma et al., 2008). Therefore, cleavage of miRNA precursors and long dsRNAs will be discussed in separate sections.

\section{Canonical miRNA substrates}

Canonical miRNAs of $\sim 22 \mathrm{nt}$ in length (Fig. 3) are the dominant Dicer products in mammalian cells. Dicer mutagenesis showed that inactivation of the RNase IIIA domain results in complete loss of 3p-derived mature miRNAs, but only partial reduction in 5p-derived mature miRNAs (Gurtan et al., 2012). Conversely, inactivation of the RNase IIIB domain by mutation of D1709, a residue mutated in some cancers, produced complete loss of 5p-derived mature miRNAs, but only partial reduction in 3p-derived mature miRNAs (Gurtan et al., 2012). Mutation of the PAZ domain caused global reduction of miRNA processing, while mutation of the Walker A motif in the helicase domain of Dicer did not alter miRNA processing (Gurtan et al., 2012). These results are consistent with the above mentioned structural features of Dicer.

Pre-miRNAs are the most efficiently cleaved Dicer substrates in vitro. In contrast to long dsRNA, a canonical pre-miRNA is cleaved only once and releases a single small RNA duplex. Human Dicer alone cleaves pre-miRNAs much faster than pre-siRNA substrates under both single and multiple turnover conditions; with more than 100-fold difference in maximal cleavage rates (Vmax) under multiple turnover conditions (Chakravarthy et al., 2010). This indicates that the mammalian Dicer is optimized for miRNA biogenesis and 


\section{miRNA size distribution in Mus musculus}

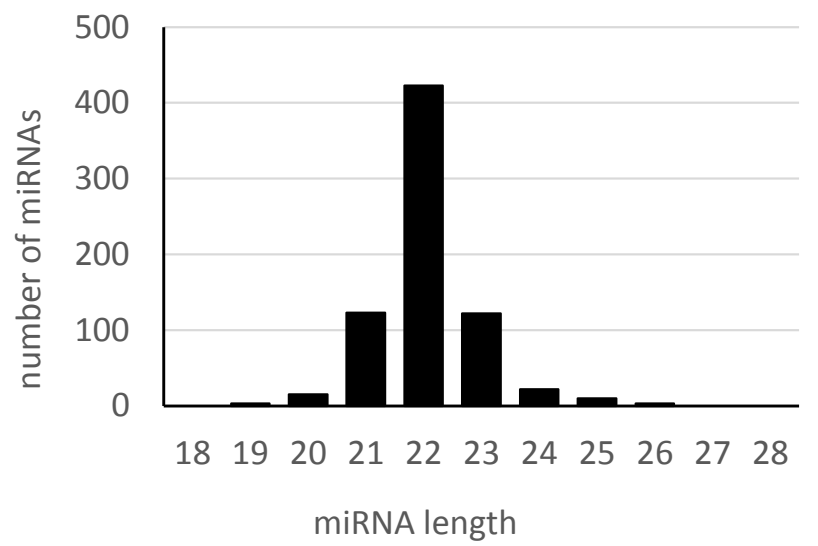

Figure 3 Mammalian miRNA size distribution

Distribution of mature murine miRNA lengths according to miRNA annotations in miRBase (release 21)

several specific structural adaptations discussed below support this notion. Dicer seems to interact directly with the terminal loop region of a pre-miRNA (Feng et al., 2012; Gu et al., 2012b) while a large pre-miRNA terminal loop further enhances pre-miRNA cleavage (Feng et al., 2012). A large-scale in vitro analysis and mutagenesis study of 161 human pre-miRNAs showed that human Dicer tolerates remarkable structural variation in pre-miRNA substrates (Feng et al., 2012). The dsRNA structure in the stem region and the 2-nt 3'-overhang structure in a pre-miRNA contribute to binding and cleavage by Dicer (Feng et al., 2012).

A characteristic feature of the pre-miRNA hairpin, which is accessed by the PAZ domain of Dicer, is a 2 nt 3' overhang generated by the nuclear Microprocessor complex (Gregory et al., 2004). Pre-miRNAs with the 2 nt 3 ' overhang at the 3 ' terminus are bound by Dicer with higher affinity than pre-miRNAs with different ends (Feng et al., 2012). Moreover, the $2 \mathrm{nt} 3$ 'end overhang leads to a higher substrate processing, which was shown on both, pre-miRNAs and perfect duplexes (Feng et al., 2012; Park et al., 2011; Zhang et al., 2004). Such preference is likely conferred by/due to simultaneous binding of pre-miRNA end by both 5' and 3' binding pockets in the PAZ domain (Park et al., 2011). Importantly, fidelity of miRNA biogenesis is critical for miRNA functionality because a single nucleotide shift at the 5 ' end of a miRNA would redefine its target repertoire. In contrast, RNAi, which typically involves perfect complementarity between a small RNA and its target, would be essentially insensitive to a precise cleavage positioning as long as it would not affect Argonaute loading. Thus, the simultaneous recognition of both strands at the $2 \mathrm{nt} 3$ ' overhang terminus by Dicer can be seen as an adaptation driven by miRNA biogenesis (Park et al., 2011).

The second structural adaptation of mammalian Dicer supporting miRNA biogenesis is the N-terminal helicase, which forms a clamp-like structure adjacent to RNase III domains, hence it is positioned to bind the stem loop of a pre-miRNA (Lau et al., 2012). While the loss of the entire $\mathrm{N}$-terminal helicase only slightly increases pre-miRNA processing activity 
in vitro (Ma et al., 2008), pre-miRNA-processing by recombinant Dicer in vitro is much faster than that of a perfect duplex (Chakravarthy et al., 2010; Ma et al., 2008). In vivo, a naturally occurring N-terminally truncated Dicer isoform can rescue miRNA biogenesis in Dicer/- embryonic stem cells (ESCs) (Flemr et al., 2013). This suggests that the N-terminal helicase domain in mammalian Dicers is not important for miRNA biogenesis per se; it rather provides constrains for substrate selectivity favouring pre-miRNAs.

This is consistent with the model where pre-miRNA binding is associated with the cleavage-competent open conformation. In the open state, a pre-miRNA is bound along the platform, the helicase domain is bent, and RNase IIIa and IIIb sites have access to the substrate (Taylor et al., 2013). It has been proposed that the loop of a pre-miRNA may prevent adoption of the closed conformation by Dicer by interacting with HEL1 and HEL2i domains and possibly stabilizing the open conformation of Dicer (Feng et al., 2012; Lau et al., 2012; Ma et al., 2012). This also indicates that the N-terminal helicase had acquired distinct roles in Dicer function in RNA silencing during evolution. In mammalian cells, the $\mathrm{N}$-terminal helicase has a gatekeeper function where pre-miRNA loops appear to be a key keeping the gate open.

\section{Dicer-dependent non-canonical miRNA substrates}

Apart from canonical miRNA substrates mentioned above, Dicer is processing additional miRNA-like substrates, which are independent of the Microprocessor complex (described in a separate section below). Some non-canonical miRNAs are produced by Dicer in a Microprocessor-independent fashion, including mirtrons, which utilize the splicing machinery to bypass the Microprocessor complex. Mirtrons are substantially longer than Microprocessor-generated pre-miRNAs and exhibit 3' uridylation and 5' heterogeneity (Wen et al., 2015). A recent analysis yielded $\sim 500$ novel mouse and human introns that generate Dicer-dependent small RNA duplexes (Wen et al., 2015). These represent nearly 1000 loci distributed in four splicing-mediated biogenesis subclasses, with 5'-tailed mirtrons being the dominant subtype (Wen et al., 2015). Another example of non-canonical miRNAs found in the literature are Microprocessor-independent miRNAs which were originally described as small interfering RNAs derived from a unique hairpin formed from short interspersed nuclear elements (SINEs) (Babiarz et al., 2008; Castellano and Stebbing, 2013).

While a typical pre-miRNA is a hairpin RNA with 2-nt 3' overhangs, production of a mature miRNA from an endogenous hairpin RNA with 5' overhangs has also been reported; mouse pre-mir-1982 is a mirtron with an $11 \mathrm{nt}$ tail at the 5' end (Babiarz et al., 2008). A possible mechanism for processing such templates has been provided by an in vitro study which showed that Dicer can produce such miRNAs in a two-step cleavage, which releases dsRNAs after the first cleavage and binds them again in the inverse direction for a second cleavage (Ando et al., 2011a).

\section{Long dsRNA substrates}

In addition to pre-miRNA, Dicer can process long dsRNAs coming from different sources. Exogenous sources of dsRNA include viral dsRNAs and imply function of RNAi in 
eukaryotic antiviral immune response (Vance and Vaucheret, 2001; Wang et al., 2006; Wilkins et al., 2005). Endogenous dsRNAs have variable length and termini, and are generated by transcription of inverted repeats, by convergent transcription or by pairing of complementary RNAs in trans. Importantly, mammals lack an ortholog of RNA-dependent-RNA polymerase (RdRP), which is a conserved component of RNAi-related mechanisms in plants, fungi and invertebrates (see the separate RdRP section). Endogenous RNAi in mouse oocytes, the best documented mammalian endogenous RNAi example, works independently of RdRP activity (Stein et al., 2003).

The human Dicer binds long dsRNA but not siRNAs in vitro (Provost et al., 2002). Long dsRNA binding is independent both on $\mathrm{Mg}^{2+}$ and ATP. The human Dicer preferentially binds and cleaves long dsRNA from the end, due to inefficient binding of internal regions of dsRNA (Zhang et al., 2002). In comparison to pre-miRNA processing, human Dicer exhibits lower cleavage activity on perfect dsRNA substrates (Ma et al., 2008). An explanation was proposed that a closed conformation of the $\mathrm{N}$-terminal helicase domain disturbs the RNase III catalytic core and inhibits cleavage of perfect dsRNAs (Lau et al., 2012). As it was mentioned, in vitro deletion of the $\mathrm{N}$-terminal helicase domain increases cleavage activity of human recombinant Dicer ( 65 -fold). Authors hypothesize that DExD/H-box domain mainly inhibits the functionality of the Dicer active site, but not RNA binding (Ma et al., 2008). This model is supported by previously mentioned structural data, where Dicer is in a closed state with a 35 bp A-form RNA duplex trapped between PAZ and helicase domains away from the catalytic center (Taylor et al., 2013).

The complexity of the differential substrate processing by Dicer is illustrated by a Dicer mutant carrying an in-frame 43-amino-acid insertion immediately adjacent to the DExH box. This Dicer exhibits defects in the processing of most, but not all, endogenous pre-miRNAs into mature miRNA but enhanced processing efficiency and concomitant RNA interference when thermodynamically stable, long-hairpin RNAs are used (Soifer et al., 2008). This result implies an important function for the helicase domain in the processing of thermodynamically unstable hairpin structures (Soifer et al., 2008).

Dicer-mediated cleavage of dsRNA can be stimulated in vitro by TARBP2. However, it is not clear if TARBP2 stimulation could be sufficient to induce endogenous RNAi in vivo (Chakravarthy et al., 2010). So far, the evidence for endogenous RNAi (including attempts to induce RNAi with exogenous substrates) is scarce (reviewed in detail in Nejepinska et al., 2012a; Svoboda, 2014). The only tissue type, where abundant endogenous siRNAs are present and where long dsRNA readily induces RNAi are mouse oocytes, which express an oocyte-specific Dicer isoform lacking a part of the N-terminal helicase domain (Flemr et al., 2013), thus mimicking some of the Dicer mutants tested in vitro (Ma et al., 2008). Taken together, long dsRNA, the typical endogenous RNAi substrate, is poorly processed by endogenous full-length Dicer. This is due to the gatekeeper role of the N-terminal helicase domain, which does not open upon binding long dsRNA.

Off note is that the human Dicer can bind 21-nt ssRNAs in vitro, independent of their sequence and secondary structure. Dicer binds ssRNAs having a 5'-phosphate with greater affinity versus those with a 5'-hydroxyl. (Kini and Walton, 2007). 


\section{Dicer-interacting dsRBPs: TARBP2 and PACT}

A common Dicer interacting partner found across Metazoa is a dsRBP with tandemly arrayed dsRBDs. Mammals have four dsRBP with tandemly arrayed dsRBDs proteins: trans-activation responsive RNA-binding protein 2 (TARBP2), protein activator of PKR (PACT), Staufen 1 (STAU1), and Staufen 2 (STAU2). However, only TARBP2 (also known as TRBP or TRBP2) and PACT were identified as Dicer binding partners (Chendrimada et al., 2005; Haase et al., 2005).

TARBP2 and PACT are paralogs, which evolved through a gene duplication event in an ancestral chordate (Daniels and Gatignol, 2012). The structure of human TARBP2 has been partially resolved (Benoit and Plevin, 2013). Each protein consists of three dsRBDs, where the first two domains can bind dsRNA (or miRNA) while the third domain has a partial homology to dsRBD and does not bind dsRNA. Instead, it mediates protein-protein interactions and is a part of a larger protein-protein interacting C-terminal region referred to as Medipal domain as it interacts with Merlin, Dicer, and PACT (reviewed in Daniels and Gatignol, 2012). TARBP2 and PACT can also form homodimers and heterodimers through the Medipal domain (Laraki et al., 2008).

The binding site of TARBP2 and PACT on Dicer was recently determined using cryoEM and crystallography (Wilson et al., 2015). Homology-based modelling showed that Dicer-binding residues are conserved in TARBP2 and PACT, implicating that binding of TARBP2 and PACT to Dicer is mutually exclusive (Wilson et al., 2015).

TARBP has a positive effect on Dicer activity. Human Dicer is much faster at processing a pre-miRNA substrate compared to a pre-siRNA substrate under both single and multiple turnover conditions. Maximal cleavage rates $\left(\mathrm{V}_{\max }\right)$ calculated by Michaelis-Menten analysis differed by more than 100 -fold under multiple turnover conditions. TARBP 2 was found in vitro to stimulate Dicer-mediated cleavage of both, pre-miRNA and pre-siRNA substrates; this stimulation requires the two N-terminal dsRBDs (Chakravarthy et al., 2010). Thus, while the structure of the substrate affects the rate at which Dicer generates small RNAs, TARBP2 stimulates dicing by presumably enhancing the stability of Dicer-substrate complexes (Chakravarthy et al., 2010).

When compared to Dicer and Dicer:TARBP2 complex, PACT inhibits Dicer processing of pre-siRNA substrates (Lee et al., 2013). The two N-terminal dsRBDs contribute to the observed differences in dsRNA substrate recognition and processing behaviour of Dicer:dsRNA-binding protein complexes (Lee et al., 2013). In addition, PACT and TARBP2 have non-redundant effects on the generation of different-sized miRNAs (isomiRs) (Kim et al., 2014; Lee et al., 2013; Wilson et al., 2015). Cells lacking TARBP2 exhibit altered cleavage sites in a subset of miRNAs but no effect on general miRNA abundance or Argonaute loading (Kim et al., 2014). Thus, impact of TARBP2 and PACT on miRNAs biogenesis in vivo seems to be relatively minor (Kim et al., 2014; Wilson et al., 2015). However, it should be pointed out that any change in the 5 ' end position of any miRNA will have a strong effect on its target repertoire. Taken together, TARBP2 and PACT are regulatory factors that contribute to the substrate specificity and cleavage fidelity during miRNA and siRNA production.

Moreover, TARBP2 and PACT have an additional role in a cross-talk of the interferon (IFN) response and small RNA pathways (reviewed in Daniels and Gatignol, 2012). The 
IFN response is the major antiviral branch of innate immunity in mammals, which deals with threats associated with long dsRNA. Among the key components sensing dsRNA in the IFN response are protein kinase R (PKR) and helicase RIG-I (reviewed in Gantier and Williams, 2007). The two N-terminal dsRBDs of PACT and TARBP2 bind PKR through the same residues (Wilson et al., 2015) while the (C-terminal) Medipal domain of PACT is needed for PKR activation (Huang et al., 2002). In contrast, the Medipal domain of TARBP2 has an inhibitory effect (Gupta et al., 2003). Furthermore, sequestering of PACT by TARBP2 has negative effect on PKR phosphorylation and activation. PKR inhibition by TARBP2 is released in stress conditions, leading to IFN response activation (Daher et al., 2009). Therefore, absolute and/or relative expression levels of TARBP2 and PACT might be buffering or sensitizing the IFN response to dsRNA. One could envision that suppression of the IFN response might result in increased RNAi. However, there is no evidence, so far that TARBP2 would redirect long dsRNA to Dicer and stimulate RNAi in vivo enough to achieve a robust sequence-specific mRNA knock-down.

Taken together, while TARBP2 and PACT are clearly associated with dsRNA binding, Dicer and the miRNA pathway. However, the full extent of biological roles of TARBP2 and PACT in dsRNA response, in the miRNA pathway, and elsewhere is still not fully understood. Since TARBP2 also interacts with and inhibits PKR (Cosentino et al., 1995; Park et al., 1994), it was speculated that TARBP2 could be a component of a network of protein-protein interactions underlying a reciprocal regulation of RNAi/miRNA and IFNPKR pathway (Haase et al. 2005). This notion is further supported by PACT, a paralog of TARBP2, which exerts the opposite role on PKR. PACT was shown to interact with TARBP2 and Dicer and to facilitate siRNA production (Kok et al., 2007). At the same time, the role of TARB2P in RNA silencing has been studied in cells where the physiological substrate for Dicer processing and RISC loading are miRNA precursors and where long dsRNA readily activates the protein kinase R (PKR) and interferons (IFN). Thus, while the RISC loading role of TARBP2 may be common for miRNA and RNAi pathway, it is not clear if an isoform of TARBP2 plays any specific role in recognition and processing of long dsRNA in the canonical mammalian RNAi pathway. Accordingly, one of the unexplored areas, which deserve further attention is the contribution of different splice variants to TARBP2 biology (Bannwarth et al., 2001). Likewise, it needs to be tested if TARBP2 plays a role in directing long dsRNA into RNAi. Finally, there is RNAi silencing-independent function of TARBP2 to explore. TARBP2 can also bind mRNA hairpins and it has been proposed that TARBP promotes metastasis by destabilizing metastasis suppressor through binding of mRNA structural elements (Goodarzi et al., 2014).

\section{(Absence of) mammalian RNA-dependent RNA polymerase}

RNA dependent RNA polymerases were found in RNA silencing in plants an lower invertebrates where they can either produce long dsRNA or short RNAs serving as an initiator or amplifier of the response (reviewed in Maida and Masutomi, 2011). Importantly, these independently discovered RdRPs that act in RNA silencing in plants, fungi and nematodes are homologs. Furthermore, homologs of these RdRPs exist in metazoan phyla, including 
Nematoda (e.g. C. elegans), Cnidaria (hydra), Chelicerata (tick), Hemichordata (acorn worm), Urochordata (sea squirt), and Cephalochordata (lancelet) but appear absent in others, including Platyhelminthes (planaria), Hexapoda (Drosophila), and Craniata (vertebrates). Phylogenetic analysis suggests that RdRPs in RNA silencing pathways have a monophyletic origin, i.e. evolved once from an ancestral RdRP presumably acting in a common ancestral RNA silencing mechanism (Cerutti and Casas-Mollano, 2006; Murphy et al., 2008). At the same time, mammalian genomes do not contain a homolog of these RdRPs, suggesting that it was lost during evolution and is absent in RNA silencing in mammals. Whether RdRP activity completely disappeared from RNAi in mammals is unclear. One could speculate that RdRP orthologs in RNA silencing in vertebrates could be replaced by another RdRP, for example by exaptation of some viral RdRP.

Our literature search for mammalian RdRPs related to RNA silencing yielded ten original research papers related to mammalian RdRPs that could be put in some context of RNA silencing. Of these, four articles actually dealt with viral RdRPs and silencing of viruses by exogenous siRNAs and not with an RdRP possibly involved in RNAi (Lee et al., 2002; Meng et al., 2006; Moon et al., 2016; Nygardas et al., 2009).

One article reported that ectopic expression of plant RdRP has no effect on RNAi in the mouse model (De Wit et al., 2002). Another report described Aquarius (AQR), a murine protein of unknown function with a weak homology to viral RdRPs expressed in embryos and ESCs (Sam et al., 1998). A follow up search for AQR-related data (28 papers citing the original one and text search by gene name) suggested that Aquarius is an RNA/DNA helicase involved in R-loop processing (Sollier et al., 2014).

An important contribution to RdRP function in mammalian RNAi was provided by a report demonstrating the lack of RdRP activity in RNAi in mouse oocytes (Stein et al., 2003). This report is of a particular importance because mouse oocytes are the only mammalian cell type, with strong endogenous RNAi pathway (reviewed in Svoboda, 2014).

Two reports proposed that two other RdRP activities could generate dsRNA that could be processed by Dicer. An indirect support for a putative RdRP emerged from Drosophila, where it was discovered ELP1, a non-canonical RdRP conserved in all eukaryotes, which associates with DCR-2 and its loss results in reduction of endo-siRNAs and upregulation of transposon transcripts (Lipardi and Paterson, 2009). However, a follow up search revealed the lack of data supporting that notion in mammals.

The second proposed mammalian RdRP acting in RNAi is a ribonucleoprotein complex of the human telomerase reverse transcriptase (TERT) and the RNA component of mitochondrial RNA processing endoribonuclease (RMRP). RMRP shows a strong preference for substrates that have 3' fold-back structures and produces dsRNA that can be processed by Dicer yielding self-targeting endogenous siRNAs (Maida et al., 2009). A follow-up analysis of these siRNAs revealed that these off-target effects of these endo-siRNAs would mimic miRNA activities (Maida et al., 2013). The latest report shows that TERT RdRP generates short RNAs that are complementary to template RNAs and have 5'-triphosphorylated ends, which indicates de novo synthesis of the RNAs (Maida et al., 2016).

Taken together, all available data suggest that mammalian RNAi does not employ an RdRP in a canonical way known from plants or nematodes. There are two candidate mechanisms that might involve RdRP in RNA silencing - one of them is based just on existence 
of a homolog of ELP1, so further experimental evidence is needed to confirm whether mammalian ELP1 homologs participate in RNAi at all. This would include analysis of small RNAs in mammalian cells lacking Elpl and further characterization of complexes containing ELP1. The second candidate mechanism, the TERT RdRP system, seems to produce a unique small population self-targeting endo-siRNAs in a highly localized manner, i.e. does not appear to be an RdRP acting in RNAi in trans.

\section{Argonaute proteins}

Once a small RNA duplex is produced by Dicer, one of the strands of the duplex is selected and loaded onto an Argonaute protein. However, before summarizing AGO loading and RISC assembly, I will review structure of AGO proteins, their covalent modifications and binding partners.

\section{AGO2 structure and function}

Argonaute proteins are the key components of miRNA and RNAi pathways as they bridge the small RNA, its target and the silencing effect. According to the model (Yuan et al., 2005), AGO proteins exist in four basic states according to the bound RNAs: apo (no substrate), pre-RISC (with a passenger and a guide), activated RISC (with a guide), and RISC targeting (with a guide and a target). Transitions between these four states correspond to RISC loading, RISC activation and target recognition, which will be discussed later.

Argonaute proteins are divided into two subfamilies: AGO proteins, which accommodate miRNAs and siRNAs (and are in the focus of this section), and PIWI proteins, which accommodate piRNAs. As piRNAs are produced in the germline in a Dicer-independent manner from single-stranded RNAs, hence they are not systematically covered in this report and neither are PIWI proteins.

Mammalian genomes encode four AGO proteins, where AGO1, 3, and 4 are encoded within one locus on the human chromosome 1 (chromosome 4 in mice) and AGO2 is encoded separately on chromosome 8 (chromosome 15 in mice). independent studies have revealed that mammalian Argonautes were originally discovered through screening of antibodies generated against intracellular membrane fractions from rat pancreas, which yielded a 95-kDa protein that localizing to the Golgi complex or the endoplasmic reticulum (Cikaluk et al., 1999). This initial observation was later refined by analysis of compartmentalization of miRNA- and siRNA-loaded AGO2 populations that co-sediment almost exclusively with the rough endoplasmic reticulum membranes, together with Dicer, TARBP2, and PACT (Stalder et al., 2013). The identified protein of unknown function was originally named GERp95 (Golgi ER protein $95 \mathrm{kDa}$ ) and was identified as a member of a family of highly conserved proteins in metazoans which function in the germline (Cikaluk et al., 1999). Apart from the original GERp95 reference, there are three others identified with the GERp95 keyword search. In one of them, GERP95 was found to bind Hsp90 chaperon (Tahbaz et al., 2001) before it was finally linked to RNAi and RISC complex and became classified as an Argonaute family member (Martinez et al., 2002; Thonberg et al., 2004). 
AGO2 is the catalytical engine of RNAi and all four AGO proteins operate in the miRNA pathway. The key for understanding functional significance of AGO proteins for RNA silencing and for summarizing the molecular mechanisms mediated by AGO proteins are their structural analyses.

Unlike Dicer, mammalian AGO proteins were successfully crystalized. The core of the structural reports is thus formed by six articles reporting crystal structures of human Argonaute proteins (Elkayam et al., 2012; Faehnle et al., 2013; Nakanishi et al., 2013; Schirle and MacRae, 2012; Schirle et al., 2015; Schirle et al., 2014). Four articles describe crystal structures of AGO2 (Elkayam et al., 2012; Schirle and MacRae, 2012; Schirle et al., 2015; Schirle et al., 2014), which is capable of cleaving cognate transcripts and two describe miRNA-pathway dedicated AGO1 (Faehnle et al., 2013; Nakanishi et al., 2013).

There is a number of additional articles, which provided partial insights into AGO functional structure. These could be divided, as in the case of Dicer, into:

(I) Biochemical studies of recombinant human AGO proteins and individual domains (Deleavey et al., 2013; Kalia et al., 2016; Lima et al., 2009; Liu et al., 2004; Meister et al., 2004; Rivas et al., 2005; Tan et al., 2009).

(II) The pioneering AGO crystal structures from archaea Pyrococcus furiosus (Song et al., 2004) and Aquifex aeolicus (Yuan et al., 2005) that served as a comparative scaffold.

(III) Crystallographic studies on individual domains of human AGO proteins (PAZ of human AGO 1 (Ma et al., 2004), or MID domain of human AGO2 (Frank et al., 2011; Frank et al., 2010)) or modelling (Deerberg et al., 2013; Gan and Gunsalus, 2015; Jiang et al., 2015; Kandeel and Kitade, 2013; Kinch and Grishin, 2009).

\section{Human AGO1 and AGO2 structures}

Among the four mammalian AGO proteins associating with 21-23 nt long small RNAs, only AGO2 was identified to have has the ,slicer“ activity (Liu et al., 2004; Meister et al., 2004). The crystal structure of human AGO2 revealed a bilobed molecule with a central cleft for binding guide and target RNAs (Elkayam et al., 2012; Schirle and MacRae, 2012; Schirle et al., 2015; Schirle et al., 2014) (Fig. 4). This domain organization is consistent with that found in archaeal AGO proteins (Song et al., 2004; Yuan et al., 2005). The 3' end of a short RNA is anchored by the PAZ domain in a sequence-independent manner (Ma et al., 2004). The 5' phosphate of the siRNA is buried in a pocket at the interface between the MID domain and the PIWI domain. Structural studies of archaeal AGO homologs showed that the PIWI domain has an RNase H-like fold and possess the endonucleolytic "slicer" activity (Song et al., 2004; Yuan et al., 2005). The 5' end of the base pairing cognate mRNA enters between the N-terminal and PAZ domains and its 3' end exits between the PAZ and MID domains.

Structural analysis provided a model for recognition of target RNAs, including the first nucleotide in the cognate site, which does not base pair with the loaded RNA. Yet, interaction with the cognate site is enhanced by adenosine in the position 1 of miRNA binding site; the structural analysis revealed that it is recognized indirectly by AGO2 (Schirle et al., 2015). Importantly, N6 adenosine methylation blocks recognition of the adenosine, which 

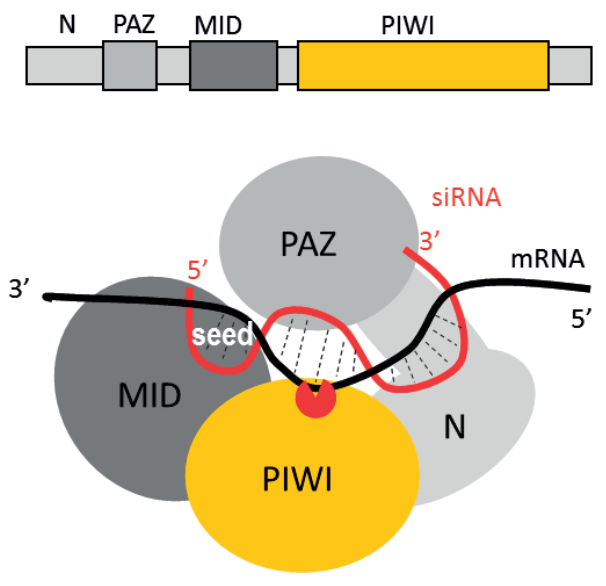

Figure 4 AGO2 structure

The figure shows domain composition and structure of human AGO2. Different colours indicate positions of the central PAZ domain and the c-terminal PIWI domain, which has an RNase $\mathrm{H}$ fold (Song et al., 2004). Two additional domains are recognized, the N-terminal domain and the MID domain between PAZ and PIWI domains. The ribbon model was taken from (Schirle et al., 2014).

might reflect a possible mechanism for regulating of miRNA binding through covalent modification of the binding site (Schirle et al., 2015). Nucleotides 2 to 6 of a heterogeneous mixture of guide RNAs are positioned in an A-form conformation for base pairing with target messenger RNAs. Between nucleotides 6 and 7, there is a kink that may function in miRNA target recognition or release of sliced RNA products. (Schirle and MacRae, 2012). Crystallization of loaded human AGO2 in the presence of target RNA sequences suggested a stepwise mechanism for interaction with cognate RNAs. First, AGO2 exposes guide nucleotides (nt) 2 to 5 for initial target pairing, which then promotes conformational changes that expose nt 2 to 8 and 13 to 16 for further target recognition (Schirle et al., 2014). miRNA binding seem to lock otherwise flexible AGO2 enzyme in a stable conformation (Elkayam et al., 2012). The structure of human Ago2 bound to miRNA-20a implies that the miRNA is anchored at both ends by the MID and PAZ domains with several kinks and turns along the binding groove (Elkayam et al., 2012). Spurious slicing of miRNA targets is avoided through an inhibitory coordination of one catalytic magnesium ion (Schirle et al., 2014). Importantly, the PIWI domain contains tandem tryptophan-binding pockets, which function in recruitment of glycine-tryptophan-182 (GW182) or other tryptophan-rich cofactors (Schirle and MacRae, 2012).

Structures of human AGO1 bound to endogenous co-purified RNAs or loaded with let-7 miRNA are strikingly similar to the structures of AGO2 (Faehnle et al., 2013; Nakanishi et al., 2013). Evolutionary changes that rendered hAGO1 inactive included a mutation of a catalytic tetrad residue and mutations on a loop near the actives site (Faehnle et al., 2013; Nakanishi et al., 2013).

Taken together, crystal structures of $\mathrm{AGO} 2$ explained the nucleotide-pairing patterns that emerged during previous studies of miRNA sequences, namely analyses of 
conservations of miRNA binding sites and biochemical analyses of target recognition, which are discussed later.

\section{Post-translational modifications of AGO proteins}

Annotation of Argonaute-related literature yielded a number of reports describing covalent modifications of AGO proteins implicated in post-translational regulations, namely phosphorylation (Horman et al., 2013; Lopez-Orozco et al., 2015; Mazumder et al., 2013; Patranabis and Bhattacharyya, 2016; Rudel et al., 2011; Shen et al., 2013; Zeng et al., 2008), prolyl 4-hydroxylation (Qi et al., 2008; Wu et al., 2011), sumoylation (Josa-Prado et al., 2015), ubiquitination (Bronevetsky et al., 2013; Johnston et al., 2010; Rybak et al., 2009; Smibert et al., 2013), and poly-ADP-ribosylation (Leung et al., 2011; Seo et al., 2013).

\section{Phosphorylation}

Phosphorylation of AGO occurs at multiple positions and published data imply that phosphorylation operates through multiple different mechanisms. Phosphorylation has been detected on AGO1 and AGO2 in the PAZ domain (pS253, pT303, pT307), the PIWI domain (pS798), in the L2 linker region (pS387, pY393) and in the MID domain (pY529) (Rudel et al., 2011).

S387 phosphorylation - Serine-387 (S387) was found to be the major Ago2 phosphorylation site in vivo (Zeng et al., 2008). Phosphorylation of Ago2 at S387 was significantly induced upon stress in a mitogen-activated protein kinase (MAPK)-dependent manner but, apparently independently of JNK and MEK kinases (Zeng et al., 2008). Another kinase implicated in S387 phosphorylation was AKT3 (Horman et al., 2013). S387A mutation or treatment with a p38 MAPK inhibitor reduced the localization of Ago2 to cytoplasmic P-bodies suggesting a potential regulatory mechanism (Zeng et al., 2008). Conversely, S387 phosphorylation downregulated RNAi-like cleavage and increased miRNA-mediated translational repression (Horman et al., 2013). Furthermore, AGO2 phosphorylation at S387 facilitated interaction with GW182 and localization to P bodies (Horman et al., 2013).

S798 phosphorylation - Serine-798 (S798) phosphorylation has been also associated with P-body localization as mutation analysis of phospho-residues within AGO2 revealed that S798D completely abrogated association of Ago2 with P-bodies and stress granules (Lopez-Orozco et al., 2015).

Y529 phosphorylation - Tyrosine 529 (Y529), which is conserved in all other species that have been analyzed. is located in the small RNA 5'-end-binding pocket of Ago proteins was found to be phosphorylated in vivo (Rudel et al., 2011). Y529E phosphomimicking mutation strongly reduced small RNA binding suggesting a potential regulatory role (Rudel et al., 2011). Y529 phosphorylation causing impaired binding miRNAs was subsequently implicated as a mechanisms transiently relieving miRNA repression during macrophage function (Mazumder et al., 2013). Y529 phosphorylation was also proposed as a mechanism relieving Let-7-mediated repression during neuronal differentiation (Patranabis and Bhattacharyya, 2016) 
Y393 phosphorylation - Tyrosine 393 (Y393) was implicated in EGFR-mediated repression of miRNA biogenesis during hypoxia (Shen et al., 2013). According to the model, Y393 negatively impacts the interaction between AGO2 and Dicer and inhibits maturation of long-loop pre-miRNAs carrying tumour-suppressor-like miRNAs (Shen et al., 2013).

\section{Prolyl 4-hydroxylation}

Prolyl 4- hydroxylation has been implicated in AGO stabilization and increased RNAi. Mass spectrometry analysis hydroxylation of the endogenous AGO2 at proline 700 (P700) and P700A mutation resulted in destabilization of AGO2 (Qi et al., 2008). Prolyl hydroxylation was observed under hypoxic conditions, where it lead to increased AGO2 stability (Wu et al., 2011). AGO2 hydroxylation correlated with increased miRNA levels as well as the endonuclease activity of AGO2 (Wu et al., 2011). Conversely, human cells depleted and mouse embryonic fibroblast cells depleted of a specific prolyl-4-hydroxylase showed reduced stability of AGO2 and impaired RISC activity (Qi et al., 2008). Hydroxylation of AGO2 was required for its association with HSP90 (see further below), which is implicated in the RISC loading with miRNAs and translocation to stress granules (Wu et al., 2011).

\section{SUMOylation}

The small ubiquitin-like modifier (SUMO) regulates various cellular processes. AGO2 was identified as a substrate for SUMO E3 ligase PIAS3. AGO2 was SUMOylated in mammalian cells by both SUMO1 and SUMO2 primarily at lysine 402. Mutation of the SUMO consensus site reduced RNAi activity of AGO2, suggesting that SUMOylation might regulate endonucleolytic activity of AGO2 (Josa-Prado et al., 2015)

\section{Ubiquitination}

Ubiquitin-proteasome apparently tunes AGO levels to adjust miRNA, AGO and Dicer stoichiometry (Smibert et al., 2013). It was found that levels of AGO1 are adjusted according to miRNA expression in a ubiquitin-proteasome-dependent manner (Smibert et al., 2013). Similarly, lower stability of AGO2 in Dicer-knockout cells could be rescued by proteasome inhibition or Dicer expression (Smibert et al., 2013). AGO and GW182 protein levels also depend on HSP90 availability (Johnston et al., 2010). Two studies show examples of developmentally regulated ubiquitination, which is apparently used to suppress AGO activities during developmental transitions. First, the let-7 target $\mathrm{Lin}-41$ gene in mice is a stem cell specific E3 ubiquitin ligase targeting AGO1, AGO2, and AGO4 proteins (Rybak et al., 2009). Second, AGO proteins are downregulated in a proteasome-dependent manner during $\mathrm{T}$ cell differentiation, presumably as a part of gene expression reprogramming (Bronevetsky et al., 2013).

\section{Poly-ADP-ribosylation}

This AGO modifications seems to be linked to suppression of RNA silencing. Poly(ADP-ribose) has been associated with the assembly of stress granules, which accumulate 
RNA-binding proteins regulating mRNAs stability and translation upon stress. Stress granule proteins modified by poly(ADP-ribose) include AGO1 - 4 (Leung et al., 2011). Interestingly, poly-ADP-ribosylation of RISC associated with reduced RISC activity has been observed upon viral infection (Seo et al., 2013). According to the model, poly-ADP-ribosylation after viral infection releases miRNA-mediated repression of interferon-stimulated genes, hence boosting innate antiviral pathways (Seo et al., 2013).

\section{Other Dicer and AGO interacting proteins}

Apart from the RISC-loading complex and miRISC components such as GW182/TNRC6 or DDX6 and others mentioned above and elsewhere, a large number of AGO-interacting partners has been identified in the past and reported individually (see further below) or comprehensively (Meister et al., 2005). Here, I provide an overview of those interacting partners.

DDX3 - DEAD-box helicase 3 is one of the helicases sensing viral double-stranded RNAs. DDX3 was also among the P-body components recruited to the West Nile virus replication sites and regulating viral replication (Chahar et al., 2013). DDX3 was also identified by an RNAi screen as an essential factor involved in RNAi pathway (Kasim et al., 2013). DDX3 is co-localized with AGO2 and a dominant negative mutant of DDX3 affected the RNAi activity (Kasim et al., 2013).

CLIMP-63 - The cytoskeleton-linking endoplasmic reticulum (ER) membrane protein of $63 \mathrm{kDa}$ (CLIMP-63) was identified as a novel Dicer-interacting protein through a yeast two-hybrid screening. CLIMP-63 interacts with Dicer to form a high molecular weight complex, which is catalytically active in pre-miRNA processing (Pepin et al., 2012). These results are consistent with analysis of Dicer compartmentalization, which showed that loading of small RNAs into RISC, cognate mRNA binding, and Ago2-mediated mRNA slicing in mammalian cells are nucleated at the rough endoplasmic reticulum (Stalder et al., 2013). While the major RNAi pathway proteins are found in most subcellular compartments, the miRNA- and siRNA-loaded AGO2 populations co-sediment almost exclusively with the rough endoplasmic reticulum membranes, together with Dicer, TARBP2, and PACT (Stalder et al., 2013).

NUP153 - The nuclear pore complex protein NUP1 was found to associate with human Dicer protein. The association was detected mainly in the cytoplasm but was also apparent at the nuclear periphery. Accordingly, it has been suggested that NUP153 plays a role in the nuclear localization of Dicer (Ando et al., 2011b)

FMRP - X mental retardation protein (FMRP) is included in the list despite its questionable role in mammalian RNA silencing. In any case, our literature search revealed a number of articles dealing with mammalian FMRPs because FMMRP is a highly conserved protein and its Drosophila ortholog dFXR was implicated in RNAi (Caudy et al., 2002; Ishizuka et al., 2002). According to the available data, FMRP is associated with RNA silencing factors. FMRP co-localized with AGO2 (Goodier et al., 2007) and immunoprecipitation suggested that a portion of Dicer and AGO were associated with each other and with FMRP (Lugli et al., 2005). In vitro data using recombinant proteins, suggested 
that human FMRP can act as a miRNA acceptor protein for Dicer and facilitate the assembly of miRNAs on specific target RNA sequences (Plante et al., 2006). The requirement of FMRP for efficient RNAi was also supported in vivo by reporter assays supporting the role of FMRP in the mammalian RISC (Plante et al., 2006). However, the loss of mammalian FMRP did not reveal any apparent direct impact on RISC function (Didiot et al., 2009; Madsen et al., 2009).

Huntingtin - AGO2 was found as one of the Huntingtin associated proteins by co-immunprecipitation. Furthermore, Huntingtin and AGO2 co-localized in P-bodies and, importantly, depletion of Huntingtin compromised RNA-mediated gene silencing (Savas et al., 2008). However, the molecular mechanism by which Huntingtin would influence RNA silencing remain unknown.

14-3-3 - Cell cycle regulating 14-3-3 proteins were reported to bind the amino terminus of AGO1 and AGO2 (Stoica et al., 2006). Overexpression of the Ago1 amino terminus in yeast resulted in cell cycle delay at the $\mathrm{G}(2) / \mathrm{M}$ boundary prompting a hypothesis that 14-3-3 proteins contribute to Argonaute protein functions in cell cycle and/or gene-silencing pathways (Stoica et al., 2006).

UPF1 - mRNA surveillance protein appears to provide a nexus between three different mechanisms of RNA metabolism: adenosine deamination, mRNA surveillance (non-sense-mediated decay) and RNA silencing. Both, human ADAR1 and UPF1 were found associated within nuclear RNA-splicing complexes (Agranat et al., 2008). At the same time, UPF1 was connected to RNA silencing (Jin et al., 2009). UPF1 interacts with human AGO1 and AGO2 and co-localizes with them into P-bodies. UPF knockdown yielded upregulation of miRNA targets while its overexpression resulted in their downregulation (Jin et al., 2009). This would suggest that UPF may contribute to RNA silencing, maybe at the level of RISC binding to its targets and accelerating their decay (Jin et al., 2009).

RBM4 - The RNA-binding motif protein 4 (RBM4) plays multiple roles in mRNA metabolism.RBM4 was found during proteomic analysis of AGO-containing miRNPs (i.e. miRISC) and RBM4 knockdown showed that it is required for miRNA-guided gene regulation (Hock et al., 2007). It was also found to co-localize with AGO2 during muscle cell differentiation (Lin and Tarn, 2009). RBM4 interacts directly with AGO2 and may selectively enhance miRISC association with target mRNAs (Lin and Tarn, 2009). RBM4 was also implicated it miRNA-mediated repression in inflammation where inflammation-induced miRNA-146 promotes a feed-forward loop that modifies through phosphorylation the subcellular localization RBM4 and promotes its interaction with AGO2 and, subsequently, tames an excessive acute inflammatory response (Brudecki et al., 2013)

TRIM32 - TRIM-NHL 32 protein regulates protein degradation and miRNA activity in neural progenitor cells to control the balance between differentiating neurons and daughter cells retaining the progenitor fate. TRIM32 was shown to bind AGO1 and increase the activity of specific miRNAs, such as Let-7 (Schwamborn et al., 2009)

QKI-6 - QKI-6 is one of the protein isoforms encoded by the qkI gene in mice. QKI-6 was found to interact with $\mathrm{AGO} 2$ and to co-localize with $\mathrm{AGO} 2$ into stress granules (Wang et al., 2010). At the same time QKI-6 depletion lead to increased miR-7 expression while QKI-6 presence inhibits processing of pri-miR-7 into miR-7 in glioblastoma cells (Wang 
et al., 2013). It has been suggested that OKI-6 mediates selective nuclear retention of primiR-7, hence preventing its processing (Wang et al., 2013). Further research is needed to clarify these two seemingly distant activities of OKI-6.

RACK - receptor for activated protein kinase C (RACK1), a constituent of the eukaryotic $40 \mathrm{~S}$ subunit, was reported to be important for miRNA-mediated gene regulation in C. elegans and humans, essentially linking miRISC with the ribosome (Jannot et al., 2011). RACK1 was also identified as a gene necessary for full miRNA function a screen for genes regulating miRNA function (Otsuka et al., 2011). RACK1 interacts with components of the miRISC in nematodes and mammals; the alteration of RACK1 expression alters miRNA function and impairs the association of the miRNA complex with the translating ribosomes (Jannot et al., 2011). Another study found that RACK1 binds to KH-type splicing regulatory protein (KSRP) and is required for the recruitment of mature miRNAs to RISC (Otsuka et al., 2011)

PTB - Polypyrimidine Tract Binding Protein (hnRNP I) was found during a search for proteins involved in let-7 mediated gene regulation. (Engels et al., 2012). PTB interacts with miRNAs and human AGO2 through RNA and there is a population of cellular targets that are co-regulated by PTB and AGO2 (Engels et al., 2012).

LRRK2 - leucine-rich repeat kinase 2 (LRRK2) gain-of-function mutations cause age-dependent degeneration of dopaminergic neurons. the analysis of the molecular mechanism of pathogenesis in Drosophila and humans revealed that LRRK2 associates with Drosophila AGO1 or human AGO2 (Gehrke et al., 2010) and that the gain-of-function LRRK2 mutant antagonizes let-7, causing derepression of Let-7 targets (Gehrke et al., 2010)

APOBEC3G - the apolipoprotein-B-mRNA-editing enzyme catalytic polypeptide-like $3 \mathrm{G}$ (APOBEC3G or $\mathrm{A} 3 \mathrm{G}$ ) is cytidine deaminase. APOBEC3G is an antiviral factor is found in P-bodies (Izumi et al., 2013; Wichroski et al., 2006). APOBEC3H also inhibits miRNA-mediated repression of translation (Huang et al., 2007) by competitively inhibiting binding of MOV10 to AGO2, causing either abnormal assembly or abnormal maturation of miRISC (Liu et al., 2012a).

\section{AGO loading and RISC formation}

The next important step is formation of RISC, the effector complex of miRNA and RNAi pathways. It involves formation of the RISC Loading Complex (RLC), transfer of a small RNA on an AGO-protein, and RISC activation.

\section{RISC Loading Complex (RLC)}

RISC assembly was so far explored more in Drosophila (Iwasaki et al., 2010; Pham et al., 2004; Tomari et al., 2004a; Tomari et al., 2004b) than in mammals (Bernard et al., 2015; Gregory et al., 2005; MacRae et al., 2008) perhaps because of the robust in vitro system of Drosophila embryo lysate. Mammals differ from Drosophila because they do not use different Dicer and Argonaute proteins dedicated to RNAi and miRNA pathway although 
it is assumed that both pathways use a similar if not the same RLC. Our knowledge of the mammalian RLC comes mainly from cells where RLC normally loads miRNAs or from in vitro reconstitution of the RLC with purified proteins. The minimal RLC is composed of Dicer, TARBP2 and AGO2 (Gregory et al., 2005; MacRae et al., 2008). In vitro reconstituted mammalian RLC contains one copy of each protein and has dicing, guide-strand selection, loading, and slicing activities (Bernard et al., 2015; Gregory et al., 2005; MacRae et al., 2008; Martinez et al., 2002).

AGO interacts with Dicer through a subregion of the PIWI domain (the PIWI-box), which binds directly to the Dicer RNase III domain. (Tahbaz et al., 2004). Single-particle EM analysis suggested that Dicer's N-terminal DExH/D domain interacts with TARBP2, whereas its C-terminal catalytic domains in the main body are proximal to AGO2 (Wang et al., 2009). Interestingly, binding of AGO to Dicer inhibits dicing activity in vitro (Tahbaz et al., 2004). Analysis of individual siRNA positions revealed that RNA sequences at positions 9-12 and 15-18 were associated with TARBP2 while positions 19-21 with AGO. AGO binding was enhanced by positions 15-18 (Takahashi et al., 2014). AGO2 was reported to binds primarily to the 5'- and alternatively, to the 3'-end of pre-miRNAs. (Tan et al., 2011). All four human AGO proteins show remarkably similar structural preferences for small-RNA duplexes: central mismatches promote RISC loading, and seed or 3'-mid (guide position 12-15) mismatches facilitate unwinding. All these features of human AGO proteins are highly reminiscent of fly AGO1 but not fly AGO2. (Yoda et al., 2010). Biochemical and structural analysis suggests that TARBP2 is flexibly bound to the Dicer DExH/D domain (Daniels et al., 2009; Wang et al., 2009). TARBP2 seems to bridge release of the siRNA by Dicer and loading of the duplex onto AGO2. Binding by TARBP2 may allow the siRNA intermediate to stay associated with the RLC after release from Dicer and may also help in orientation of the siRNA for AGO2 loading. Just as in flies, human RISC assembly is uncoupled from dicing (Yoda et al., 2010).

Analysis of miRNA-carrying RISC (miRISC) yielded a similar picture. Since loading of miRNA duplexes to AGO proteins is assisted by HSP70/ HSP90 chaperones (Maniataki and Mourelatos, 2005b; Yoda et al., 2010), HSP90 is sometimes also included as the component of miRLC (Liu et al., 2012b). At the same time, AGO2 and Dicer are sufficient for processing and loading of miRNAs into RISC (Tan et al., 2011).

Combination of in vivo studies in Dicer ${ }^{--}$cells reconstituted with wild-type or catalytically inactive Dicer showed that the miRNA loading complex (miRLC) is the primary machinery linking pre-miRNA processing to miRNA loading and lead also to definition of a miRNA Precursor Deposit Complex (miPDC) for Dicer-independent RISC loading exemplified by miR-451 (Liu et al., 2012b). miPDC is formed of AGO, pre-miRNA, and HSP chaperone. It functions in Dicer-independent miRNA biogenesis (e.g. miR-451) and also promotes miRNP assembly of certain Dicer-dependent miRNAs (Liu et al., 2012b).

Earlier studies suggested a difference between fly and human systems because human RISC assembly using immunopurified or reconstituted human RLC containing AGO2, Dicer and TARBP2 did not require ATP hydrolysis, (Gregory et al., 2005; MacRae et al., 2008; Maniataki and Mourelatos, 2005b). Recent data suggest that ATP facilitates also human RISC loading while it is dispensable for unwinding (Yoda et al., 2010). 


\section{Accessory RLC factors}

Apart from the three established RLC components, several proteins emerged as RLC cofactors, among which stand out HSP70/90 chaperones. Heat shock protein 90 was actually the first identified AGO-associated protein even before AGO was associated with RNA silencing (Tahbaz et al., 2001). Inhibition of HSP90 reduces AGO levels (Johnston et al., 2010; Martinez and Gregory, 2013; Tahbaz et al., 2001) as well as GW182 protein levels and abolishes P-bodies (Johnston et al., 2010). In addition, stable binding between AGO and Dicer is dependent on the activity of Hsp90 (Tahbaz et al., 2004) and association of AGO2 with HSP90 involves prolyl-hydroxylation of AGO2 (Wu et al., 2011). HSP90 activity is not required for association of AGO with intracellular membrane (Tahbaz et al., 2001) but appears to chaperon AGO proteins before binding RNA and may facilitate loading of small RNAs (Johnston et al., 2010). Interestingly, miRNA*s (miRNA* is an equivalent of the passenger strand) with fast turnover exhibited different sensitivity to HSP90 inhibition suggesting differential HSP90 requirements for different miRNA*s (Guo et al., 2015). HSP90 is also a negative regulator of PKR; it is able to bind and inhibit PKR phosphorylation and prevent apoptosis (Donze et al., 2001). Thus, HSP90 provides a factor bridging RNA silencing and innate immunity.

Furthermore, HSP90 co-chaperones FKBP4/5 control AGO2 expression and facilitate RISC assembly (Martinez et al., 2013). FKBP4/5 were identified as AGO2-associated proteins in mouse embryonic stem cells. Inhibition of FKBP4/5 lead to decreased Ago2 protein levels while overexpression stabilized AGO2 expression (Martinez et al., 2013). Another study has found that FKBP4 forms a stable complex with human AGO2 before small RNA loading in the cytoplasm and is required for efficient RNAi (Pare et al., 2013).

Another component reported to function as an RISC-loading factor is RNA helicase A (RHA, also known as DHX9) Dicer (Robb and Rana, 2007). RHA is a conserved protein with two dsRBDs (Nagata et al., 2012) with multiple roles in the gene expression of cellular and viral mRNAs. RHA recognizes highly structured nucleotides and catalytically rearranges the various interactions between RNA, DNA, and protein molecules to provide a platform for the ribonucleoprotein complex. RHA was shown in human cells to function in the RNAi pathway and interact with siRNA, AGO2, TARBP2, and Dicer (Robb and Rana, 2007). RHA-depleted cells, showed reduced RNAi, apparently as a consequence of lower active RISC suggesting that RHA functions in RISC as an siRNA-loading factor (Robb and Rana, 2007). A later structural analysis of dsRBDs showed that both dsRBDs are required for RISC association, and such association is mediated by dsRNA (Fu and Yuan, 2013).

\section{Are mammalian miRNAs sorted?}

As mentioned above, of the four AGO proteins that can be loaded with small RNAs equally well (Meister et al., 2004). All four mouse AGO proteins seem to be functionally redundant in the miRNA pathway as shown by rescue experiments in ESCs lacking all four Argonaute genes (Su et al., 2009). Consistent with this, all four AGOs are functionally equivalent when accommodating bulged miRNA duplexes, whereas AGO1 and AGO2 appear to be more effective at utilizing perfectly matched siRNAs (Su et al., 2009). Furthermore, AGO2 can 
execute endonucleolytic cleavage of cognate RNAs while all four can mediate translational repression. This raises a question whether small RNAs may undergo some kind of sorting that would result in preferential loading onto specific AGO homologs.

Structural analysis showed that all four human AGO proteins showed similar structural preferences for small-RNA duplexes, which were highly reminiscent of Drosophila AGO1 but not of AGO2 (Yoda et al., 2010). Human AGO2 and AGO3 immunoprecipitation and subsequent sequencing of small RNAs revealed that both AGOs were associated with 21-23 nt RNAs, majority of which were miRNAs (Azuma-Mukai et al., 2008). While fifteen miRNAs showed more than 2-fold significant difference in loading onto AGO2 or AGO3, it is not clear whether this discrimination occurs also in vivo (Azuma-Mukai et al., 2008).

A detailed analysis of small RNAs associated with all four human AGO proteins revealed approximately equivalent amounts of sequence tags derived from miRNA loci associated with individual AGOs with some exceptions that could be coupled to specific AGOs (Burroughs et al., 2011). However, further analysis suggested existence of some sorting mechanism affecting a subset of distinct isomiRs that seemed to be differentially associated with distinct AGO proteins (Burroughs et al., 2011). This observation contrasts with another cloning and deep sequencing experiment addressing distribution of endogenous miRNAs associated with AGO1-3, which did not find evidence for miRNA sorting in human cells. (Dueck et al., 2012).

It is possible that sorting of small RNAs on AGO proteins may not be a general phenomenon while differential presence of small RNAs on AGO proteins can also emerge from selective mechanisms operating after loading. This can be illustrated on selective progressive 3' shortening of AGO2-bound miRNAs observed in the brain (Juvvuna et al., 2012). Furthermore, Dueck et al also reported that AGO identity appears to influence the length of some miRNAs, while others remain unaffected (Dueck et al., 2012). Taken together, it seems that miRNAs are generally not sorted for loading onto AGO proteins. Notable exceptions include miRNAs with unique biogenesis such as miR-451 whose biogenesis requires AGO2 slicing activity (Dueck et al., 2012).

\section{Loading asymmetry}

While both siRNA strands can guide post-transcriptional silencing in mammals (Wei et al., 2009), selection of the loaded strand exhibits a clear and long-known thermodynamic bias where the strand whose $5^{\prime}$-end is less thermodynamically stable is preferentially loaded onto AGO as the guide strand (Khvorova et al., 2003; Schwarz et al., 2003). Selection of the guide strand involves multiple sensors - this includes AGO2 strand selection capability (Noland and Doudna, 2013; Suzuki et al., 2015), which is enhanced in complex with Dicer and TARBP2 or PACT. In addition, strand selection for some miRNAs is enhanced in complexes containing PACT but not TARBP2 (Noland and Doudna, 2013). Notably, TARBP2 was predicted to be a sensor of the thermodynamic stability of 5' siRNA in strand selection during RISC loading, similarly to DCR-2 and R2D2 (a TARBP2 homolog) in Drosophila (Wang et al., 2009). However, the supporting evidence is inconclusive (Haase et al., 2005) although some argue that TARBP2 can indeed acts as a sensor (Gredell et al., 2010). Furthermore, while TARBP2 function is similar to that of R2D2, TARBP2 sequence 
is more closely related to Loquacious than R2D2 (Murphy et al., 2008). Finally, quantitative analysis of RISC assembly and target silencing activity in the presence or absence of Dicer suggest that the mammalian Dicer is nonessential for asymmetric RISC loading in vivo and in vitro. (Betancur and Tomari, 2012).

\section{RISC activation}

The next step after AGO loading is removal of the passenger strand from the loaded duplex RNA. In some cases, the passenger strand can be eliminated by the slicer activity where the RISC complex uses the guide siRNA to cleave the passenger strand. In other words the first cleavage actually targets the passenger strand of a loaded siRNA duplex to free the guiding strand, so it can base pair to cognate mRNAs (Matranga et al., 2005). The cleavage-assisted mechanism is typical for AGO2-loaded fly and human siRNAs in the RNAi pathway while passenger strand cleavage is not important for loading miRNAs (Matranga et al., 2005).

Slicer-independent mechanism is needed to remove the passenger strands from non-slicing AGO proteins and from miRNA duplexes bound to AGO2 where the passenger strand cannot be cleaved. As slicer-deficient hAGO1, hAGO3, and hAGO4 are able to eject the passenger strand of siRNA duplexes at $37^{\circ} \mathrm{C}$, it is apparent that $\mathrm{AGO1}, 3$, and 4 can be readily programmed with siRNAs at the physiological temperature (Park and Shin, 2015). This implies that a slicer-independent mechanism, which relies on the thermal dynamics of the PAZ domain (Gu et al., 2012a; Park and Shin, 2015), is likely a common feature of human AGOs.

Importantly, RISC activation has been associated with additional factors. One of them is $\mathrm{C} 3 \mathrm{PO}$, an endonuclease that activates RISC (Ye et al., 2011). According to the model of RISC activation that integrates the $\mathrm{C} 3 \mathrm{PO}$ crystal structure, Ago2 directly binds duplex siRNA and nicks the passenger strand, and then C3PO activates RISC by degrading the Ago2-nicked passenger strand (Ye et al., 2011) > Another factors is La, Sjogren's syndrome antigen B (SSB)/autoantigen, which is acting as an activator of the RISC-mediated mRNA cleavage activity. (Liu et al., 2011). Thus, similarly to C3PO, La is a regulatory factor helping to remove AGO2-cleaved products in order to promote active RISC formation (Liu et al., 2011).

\section{Additional small RNAs associated with AGO proteins}

Our literature search revealed a heterogeneous group of publications describing small RNAs loaded on AGO proteins that were clearly distinct from canonical miRNAs - small RNAs generated by the mechanism described above. A canonical miRNA is transcribed by polymerase II, the primary transcript contains a $\sim 70 \mathrm{nt}$ short hairpin precursor pre-miRNA, which is released by the Microprocessor complex, transported to the cytoplasm where Dicer cleaves of the loop and one of the strands of the miRNA duplex is loaded onto miRISC. However, next generation sequencing revealed existence of AGO-loaded small RNAs that were apparently generated from different substrates and by molecular mechanisms, which deviated from the canonical pathway. Below is an overview of diversity of AGO-bound RNAs, which emerged from the literature search. 
Non-canonical miRNAs can be divided across two axes - (I) according to the RNA precursor and (II) according to the proteins involved in (or omitted from) their biogenesis. Non-canonical miRNAs were discovered during systematic analyses of small RNAs in different model systems, such as disease models (e.g. (Xia et al., 2013) or cultured cells (Babiarz et al., 2011; Babiarz et al., 2008). A good experimental strategy to identify non-canonical miRNAs is a high throughput sequencing analysis of genetic models lacking some of the components of RNA silencing such as Dicer or DGCR8 (Babiarz et al., 2011; Babiarz et al., 2008). Phenotypic difference and differential expression of distinct miRNA-like sequences can indicate biological roles of non-canonical miRNAs while knock-out data offer an insight into the non-canonical biogenesis mechanism

Perhaps the best known non-canonical miRNA class, which comes from unique, Microprocessor-independent precursors, are mirtrons, miRNA-like molecules arising from spliced-out introns, which are Microprocessor-independent (Babiarz et al., 2011; Berezikov et al., 2007; Ladewig et al., 2012; Schamberger et al., 2012; Sibley et al., 2012; Westholm et al., 2012). Interestingly, some predicted mirtron-like miRNAs (miR-1225 and miR-1228) are splicing-independent (simtrons) and their biogenesis involves Drosha but neither DGCR8 nor Dicer (Havens et al., 2012). Other non-canonical substrates can be, for example, 5'-Capped RNAs (Xie et al., 2013), SINE repeat-derived, (Babiarz et al., 2008; Castellano and Stebbing, 2013), small vault RNA (svtRNA2-1a) (Minones-Moyano et al., 2013), or RNase III transcripts (Maurin et al., 2012) including annotated RNAs such as snoRNAs (Burroughs et al., 2011; Ender et al., 2008; Li et al., 2012), 7SL RNA (Ren et al., 2012), tRNA fragments (Burroughs et al., 2011; Haussecker et al., 2010; Kumar et al., 2014; Li et al., 2012; Maniataki and Mourelatos, 2005a; Maute et al., 2013; Venkatesh et al., 2016). Non-canonical miRNAs can be also produced from viral RNAs (Bogerd et al., 2010; Kincaid et al., 2014; Li et al., 2009; Xu et al., 2009). A non-canonical small RNA class of unclear significance are semi-microRNAs (smiRNAs), which are $\sim 12$ nt short RNA fragments apparently emerging from other miRNAs, such as let-7 or miR-223 (Plante et al., 2012).

Non-canonical miRNAs can be also classified by their biogenesis as Microprocessor, DGCR8-, or Dicer-independent. For instance, the above-mention mirtrons do not require the Microprocessor complex while simtrons require Drosha but neither DGCR8 nor Dicer. Non-canonical miRNAs can be also produced from bona-fide miRNA precursors, which give a rise to a small RNA in a non-canonical way, for example by a dual role of AGO protein (Diederichs and Haber, 2007). A classic example is miR-451, a Dicer-independent miRNA biogenesis pathway that requires Ago catalysis (Cheloufi et al., 2010). A unique type of non-canonical miRNAs are loop-miRs, which are released from the loop region of a pre-miRNA (Okamura et al., 2013; Winter et al., 2013).

\section{Target recognition and modes of silencing}

\section{Target recognition}

Target recognition by RISC is mediated by base pairing between RISC-loaded small RNAs and cognate RNAs. Consistently with the structural analysis of AGO proteins, target 
recognition by siRNAs exhibits a distinct 5 ' bias. Analysis of miRNA-targeted mRNAs in Drosophila and mammals revealed that miRNA bases 2-8 form a distinct ,seed“, which base pairs perfectly to the target transcript (Enright et al., 2003; Lewis et al., 2003). This is consistent with the fact that the 5 ' half of a small RNA provides most of the binding energy that tethers RISC to a target RNA (Doench et al., 2003; Haley and Zamore, 2004). Structural features of the target site are only important for RISC binding, while sequence features such as the A/U content of the 3' UTR are important for mRNA degradation. (Hausser et al., 2009). According to analyses of RISC kinetics, small RNAs loaded onto AGO proteins are actually composed of five distinct domains (Fig. 5): the anchor, seed, central, 3' supplementary, and tail (Wee et al., 2012).

Biochemical analysis of target recognition by mammalian RISC showed that the RISC is apparently not systematically scanning transcripts. RISC is unable to unfold structured RNA. Thus, RISC randomly transiently contacts single-stranded RNA and promotes siRNA-target base pairing where the 5'end of the loaded siRNA creates a thermodynamic threshold for stable association of RISC with its target (Ameres et al., 2007).

The fact that 5' and 3' ends of a siRNA are bound by distinct binding pockets and that both ends contribute differently to binding to the target lead to a ,two-state model of Argonaute function proposed based on the Drosophila model (Tomari et al., 2004b). In this model, the 3' end is bound in the PAZ domain and the 5' end to in a pocket at the interface between the MID and the PIWI domains. The 5' end is pre-organized to interact with the cognate mRNA and, upon binding, the 3 ' end is dislodged from the binding pocket to allow for base pairing of the 3' end.

Importantly, kinetics of silencing is critical for understanding target recognition and silencing by of small RNAs. A kinetic study of Drosophila and mouse AGO2 found that mouse AGO2, which mainly mediates miRNA-directed repression in vivo, dissociates rapidly and with similar rates for fully paired and seed-matched targets (Wee et al., 2012). An important conclusion from this study is that low-abundant miRNAs are unlikely to contribute much biologically meaningful regulation because they are present at a concentration less than their KD for seed-matching targets (Wee et al., 2012).

These results were subsequently corroborated by single molecule analysis. Single-molecule fluorescence experiments using a minimal RISC (a small RNA and AGO2) showed that target binding starts at the seed region of the guide RNA (Chandradoss et al., 2015; Jo et al., 2015a; Jo et al., 2015b). AGO2 initially scans for complementarity to nucleotides 2-4 of the miRNA and this interaction propagates into a stable association when target complementarity extends across the seed (Chandradoss et al., 2015). Stable RISC binding is thus efficiently established with the seed match only, providing a potential explanation for the seed-match rule of miRNA target selection (Chandradoss et al., 2015; Jo et al., 2015a; Jo et al., 2015b). Remarkably, mouse AGO2 binds tighter to miRNA targets than its RNAi cleavage product, even though the cleaved product contains more base pairs (Salomon et al., 2015). In contrast, target cleavage required extensive sequence complementarity and accelerated core-RISC dissociation for recycling (Jo et al., 2015b) and sensitively depended on the sequence (Jo et al., 2015a). RISC thus utilizes short RNAs as specificity determinants with thermodynamic and kinetic properties more typical of RNA-binding proteins while a small RNA loaded on AGO no longer follows rules by which sole oligonucleotides 


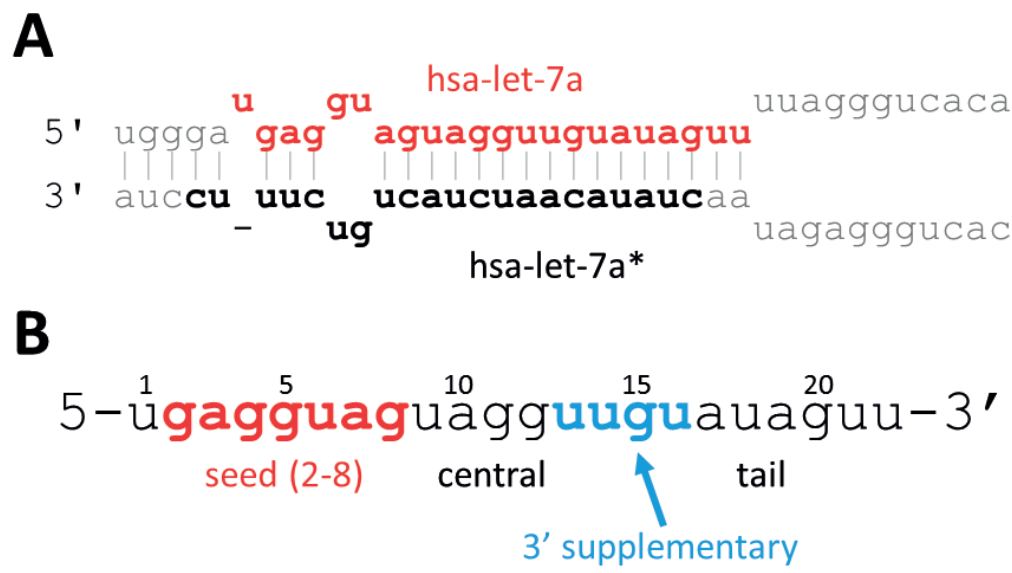

Figure 5 miRNA/siRNA functional domain (A) miRNA precursor stem loop (exemplified by human Let-7a from miRBase). In red is highlighted sequence of mature miRNA, in black is depicted the passenger (miRNA-star) strand. (B) Mature miRNA can be divided into several functionally distinct regions (Wee et al., 2012).

find, bind, and dissociate from complementary nucleic acid sequences (Salomon et al., 2015). Importantly, target site recognition is coupled to lateral diffusion of RISC along the target RNA, which facilitates recognition of target sites within the cellular transcriptome space (Chandradoss et al., 2015).

To put the aforementioned kinetic data into comparison with laboratory practice, the table below shows on ten randomly selected examples of RNAi experiments that an effective repression by exo-RNAi in cultured cells usually employs siRNA transfection in $20-50 \mathrm{nM}$ range (v 1). Thus, a robust biological effect of a specifically designed perfectly complementary siRNA acting through the AGO2-mediated endonucleolytic cleavage is usually observed with a nanomolar concentration of siRNA. Silencing by direct endonucleolytic cleavage - RNAi-like silencing

Base pairing in the middle of siRNA results in correct positioning of the cognate strand and its cleavage in the active site of the PIWI domain of AGO2 - out of four mammalian AGO proteins, which bind small RNAs, only AGO2 has the „slicer" activity (Liu et al., 2004; Meister et al., 2004). These simple facts have several important consequences. First, siRNAs loaded on AGO1, AGO3, and AGO4 will repress their perfectly complementary targets by other means (translational repression/deadenylation/decapping) than by cleavage. Conversely, AGO2 loaded miRNA binding perfectly complementary targets will behave as siRNAs and mediate RNAi-like endonucleolytic cleavage. Third, imperfect complementarity in the middle of the base pairing site of AGO-loaded siRNAs will result in miRNA-like effects (Doench et al., 2003). Thus, RISC-loaded siRNAs have additional silencing effects due to 2-8 nucleotide seed matches to other RNAs (which is the molecular foundation of the off-targeting phenomenon). 
Table 1 Random selection of RNAi experiments in cultured cells

\begin{tabular}{|l|l|l|l|l|l|}
\hline concentration & method & cells & $\begin{array}{l}\text { exposure } \\
\text { time }\end{array}$ & $\begin{array}{l}\text { knockdown } \\
\text { efficiency }\end{array}$ & reference - doi \\
\hline $5 \mathrm{nM}$ & transfection & $\begin{array}{l}\text { mouse preadipocytes } \\
3 \mathrm{~T} 3-\mathrm{L} 1\end{array}$ & $48 \mathrm{~h}$ & $70 \%$ & $\begin{array}{l}10.1128 / \\
\text { MCB.01856-08 }\end{array}$ \\
\hline $20 \mathrm{nM}$ & transfection & human HUVEC & $0-5 \mathrm{~d}$ & $60-80 \%$ & $\begin{array}{l}10.1016 / \\
\text { j.bone.2014.12.060 }\end{array}$ \\
\hline $20 \mu \mathrm{M}$ & transfection & $\begin{array}{l}\text { human breast } \\
\text { carcinoma cell lines }\end{array}$ & $48 \mathrm{~h}$ & $60 \%$ & $10.1038 /$ onc.2014.421 \\
\hline $20 \mu \mathrm{M}$ & transfection & $\begin{array}{l}\text { murine breast } \\
\text { epithelial cell }\end{array}$ & $48 \mathrm{~h}$ & $60 \%$ & $10.1038 /$ onc.2014.421 \\
\hline $25 \mathrm{nM}$ & transfection & mouse kidney & $7 \mathrm{~d}$ & $20 \%$ & $\begin{array}{l}10.1152 / \\
\text { ajprenal.00052.2014 }\end{array}$ \\
\hline $20-40 \mathrm{nM}$ & transfection & human LA4 & $24 \mathrm{~h}$ & $60-80 \%$ & $10.1155 / 2015 / 473742$ \\
\hline $50 \mathrm{nM}$ & transfection & mouse cardiomyocyte & $48 \mathrm{~h}$ & $60 \%$ & $\begin{array}{l}10.5582 / \\
\text { bst.2015.01159. }\end{array}$ \\
\hline $80 \mathrm{nM}$ & transfection & mouse osteoblasts & $0-5 \mathrm{~d}$ & $40-50 \%$ & $\begin{array}{l}10.1016 / \\
\text { j.bone.2014.12.060 }\end{array}$ \\
\hline $100 \mathrm{nM}$ & transfection & $\begin{array}{l}\text { human endothelial } \\
\text { cells }\end{array}$ & $72 \mathrm{~h}$ & $80 \%$ & $\begin{array}{l}10.1152 / \\
\text { ajplung.00263.2009 }\end{array}$ \\
\hline $500 \mathrm{nM}$ & electroporation & human monocytic cell & $24 \mathrm{~h}$ & $60-90 \%$ & $10.1111 /$ jdi.12434 \\
\hline
\end{tabular}

\section{Silencing by translational repression and RNA degradation - common miRNA silencing}

In order to understand the molecular mechanism of repression mediated by small RNA-loaded AGO, many AGO-associated proteins were identified in mammalian cells (reviewed in detail in Peters and Meister, 2007). These include MOV10, DDX6 (Rck/p54), DDX20 (Gemin3), TNRC6A (GW182), and many others (Hauptmann et al., 2015; Meister et al., 2005). Over a decade of research focused on understanding how miRNA-guided RISC suppresses gene expression. it became clear that miRNAs provide the guide while the repression is mediated through AGO and associated proteins as shown by suppression occurring upon tethering AGO proteins (Pillai et al., 2004). Building a model of silencing, was a lengthy and convoluted process (which is not over yet) from which emerged GW182 as a key docking factor integrating miRNA-mediated silencing.

\section{GW182 proteins}

Mammalian GW182 proteins carry at the N-terminus multiple glycine-tryptophan (GW) repeats, a central ubiquitin-associated (UBA) domain, a glutamine-rich (Q-rich) domain, and an RNA recognition motif (RRM) (Fig. 6). Interestingly, GW182 seems to be largely unstructured protein, including functionally essential domains, such as the N-terminus, which is interacting with AGO2 (Behm-Ansmant et al., 2006; Lazzaretti et al., 2009; Lian 


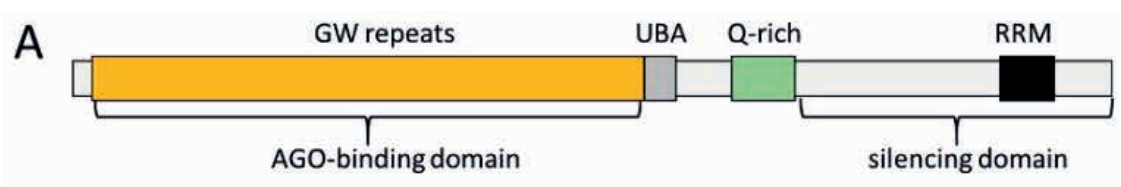

B

\section{CCR4-NOT deadenylation}

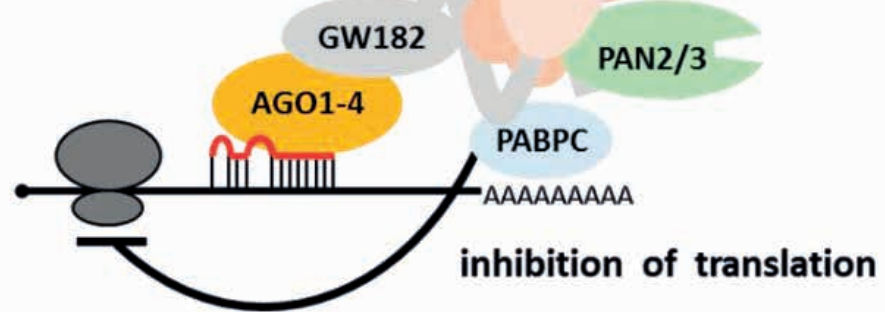

Figure 6 GW182 domain structure and function.

(A) Domain composition: UBA, ubiquitin-associated motif; RRM, RNA recognition motif. (B) A schematic depiction of GW182-mediated bridging of AGO and translational repression and mRNA degradation. See the text below for details.

et al., 2009; Takimoto et al., 2009). Similarly, the silencing domain is also predicted to be disordered (Huntzinger et al., 2010; Lazzaretti et al., 2009; Zipprich et al., 2009).

The N-terminal part of GW182 can interact with any human (and presumably all mammalian) AGO proteins through multiple GW repeats, which bind tryptophan binding pockets on AGO and contribute to the interaction in an additive manner (El-Shami et al., 2007; Jakymiw et al., 2005; Lazzaretti et al., 2009; Lian et al., 2009; Liu et al., 2005a; Schirle and MacRae, 2012; Takimoto et al., 2009; Yao et al., 2011; Zipprich et al., 2009). The role of AGO is most likely to anchor through miRNA-mediated interaction GW182 to the silenced RNA as tethering of GW182 alone or even of (its silencing domain) is sufficient to mediate repression (Chekulaeva et al., 2011). The silencing domain directly binds PolyA Binding protein (PABP) as well as with PAN3 and NOT1 components of PAN2/PAN3 and CCR4NOT deadenylase complexes, respectively. (Chekulaeva et al., 2011; Fabian et al., 2009; Huntzinger et al., 2010; Jinek et al., 2010; Zekri et al., 2009).

The general, somewhat simplistic model of miRNA-mediated silencing proposes that GW182 interacts with PABP of the cognate RNA and recruit PAN2/3 and CCR4-NOT deadenylases, which would result in translational repression likely occurring at the level of initiation and the repressed RNA would be deadenylated (Braun et al., 2011; Chekulaeva et al., 2011; Christie et al., 2013; Fabian et al., 2011a; Fabian et al., 2011b; Huntzinger et al., 2013; Kuzuoglu-Ozturk et al., 2016; Zekri et al., 2013). Deadenylated RNAs in mammalian cells are generally decapped and degraded by XRN1 exonuclease (Schoenberg and Maquat, 2012).

Several mechanisms have been proposed how the GW182-PABPC interaction contributes to silencing (Fabian et al., 2009; Jinek et al., 2010; Zekri et al., 2009) although it is not 
clear how critical this role is as mRNAs lacking polyA tail (i.e. neither circularize nor are deadenylated) are silenced nonetheless (Chekulaeva et al., 2011; Pillai et al., 2005; Zekri et al., 2013). This would suggest for a non-essential role of PABPC/GW182 interaction.

There are additional RISC interactions, which are important for miRNA-mediated silencing, such as DDX6/RCKp54 (Chu and Rana, 2006; Kuzuoglu-Ozturk et al., 2016; Mathys et al., 2014) or LIM domain proteins LIMD1, Ajuba, and WTIP, which are required for miRNA-mediated, but not siRNA-mediated gene silencing (James et al., 2010). According to the model, the LIM proteins facilitate miRNA-mediated gene silencing by creating an inhibitory closed-loop complex where they bridge the translationally inhibited cap structure and AGO1/2 within the miRISC complex bound to the 3'-UTR (James et al., 2010). The list of proteins associated with RISC or the target repression is longer, and includes, for example, also Pumilio/FBF , a miRNA targeting cofactor (Friend et al., 2012) or eIF4GI (Ryu et al., 2013). However, for understanding the miRNA mechanism, their detailed review is would be overreaching.

\section{P-bodies}

Studies on localization of miRNA pathways components revealed their presence in distinct cytoplasmic foci, known as P-bodies or GW-bodies (Liu et al., 2005a; Liu et al., 2005b; Pillai et al., 2005; Yu et al., 2005). P-bodies (reviewed in detail by Jain and Parker, 2013) are distinct cytoplasmic foci, which contain proteins associated miRNA-mediated repression (Behm-Ansmant et al., 2006; Chahar et al., 2013; Chu and Rana, 2006; James et al., 2010; Johnston et al., 2010; Liu et al., 2005b; Ozgur and Stoecklin, 2013; Pare et al., 2011; Rehwinkel et al., 2005; Yu et al., 2005; Zhou et al., 2009). P-body association has been observed for the mature RISC components and RNA degradation pathway proteins but not for Dicer or TARBP2, indicating that P-bodies are associated with miRNA-mediated suppression but not biogenesis. This is supported by the presence of miRNA-targeted mRNAs in P-bodies (Liu et al., 2005b; Shih et al., 2011). P-bodies are highly dynamic (Aizer et al., 2014; Kedersha et al., 2005) but not an essential structure for miRNA-mediated repression (Eulalio et al., 2007). P-bodies probably should be seen as aggregating foci, which may facilitate miRNA-mediated repression. Since inhibition of the miRNA pathway prevents P-body formation, it was proposed that aggregation of miRNA pathway factors to P-bodies is not required for miRNA function and mRNA degradation per se but rather is a consequence of miRNA activity (Eulalio et al., 2007).

\section{Acknowledgement}

I would like to thank my colleagues Jan Paces, Miloslav Nic, and Tomas Novotny for help with collecting literature for the review. The review content was produced under a contract OC/EFSA/GMO/2015/01-CT 01 with European Food Safety Authority (EFSA); the opinions expressed are those of the contractor only and do not represent EFSA's official position. Publication of the review was funded by LO1220 and LM2015063 by the Ministry of Education, Youth and Sports. 


\section{References}

Agranat, L., Raitskin, O., Sperling, J., and Sperling, R. (2008). The editing enzyme ADAR1 and the mRNA surveillance protein hUpf1 interact in the cell nucleus. Proc Natl Acad Sci U S A 105, 5028-5033.

Aizer, A., Kalo, A., Kafri, P., Shraga, A., Ben-Yishay, R., Jacob, A., Kinor, N., and Shav-Tal, Y. (2014). Quantifying mRNA targeting to P-bodies in living human cells reveals their dual role in mRNA decay and storage. Journal of Cell Science 127, 4443-4456.

Alarcon, C.R., Lee, H., Goodarzi, H., Halberg, N., and Tavazoie, S.F. (2015). N-6-methyladenosine marks primary microRNAs for processing. Nature 519, 482-+.

Alon, S., Mor, E., Vigneault, F., Church, G.M., Locatelli, F., Galeano, F., Gallo, A., Shomron, N., and Eisenberg, E. (2012). Systematic identification of edited microRNAs in the human brain. Genome Research 22, 1533-1540.

Ameres, S.L., Martinez, J., and Schroeder, R. (2007). Molecular basis for target RNA recognition and cleavage by human RISC. Cell 130, 101-112.

Ando, Y., Maida, Y., Morinaga, A., Burroughs, A.M., Kimura, R., Chiba, J., Suzuki, H., Masutomi, K., and Hayashizaki, Y. (2011a). Two-step cleavage of hairpin RNA with 5 ' overhangs by human DICER. BMC Molecular Biology 12, 6-6.

Ando, Y., Tomaru, Y., Morinaga, A., Burroughs, A.M., Kawaji, H., Kubosaki, A., Kimura, R., Tagata, M., Ino, Y., Hirano, H., et al. (2011b). Nuclear Pore Complex Protein Mediated Nuclear Localization of Dicer Protein in Human Cells. Plos One 6, e23385-e23385.

Azuma-Mukai, A., Oguri, H., Mituyama, T., Qian, Z.R., Asai, K., Siomi, H., and Siomi, M.C. (2008). Characterization of endogenous human Argonautes and their miRNA partners in RNA silencing. Proc Natl Acad Sci U S A 105, 7964-7969.

Babiarz, J.E., Hsu, R., Melton, C., Thomas, M., Ullian, E.M., and Blelloch, R. (2011). A role for noncanonical microRNAs in the mammalian brain revealed by phenotypic differences in Dgcr8 versus Dicer1 knockouts and small RNA sequencing. RNA 17, 1489-1501.

Babiarz, J.E., Ruby, J.G., Wang, Y.M., Bartel, D.P., and Blelloch, R. (2008). Mouse ES cells express endogenous shRNAs, siRNAs, and other Microprocessor-independent, Dicer-dependent small RNAs. Genes \& Development 22, 2773-2785.

Bahn, J.H., Ahn, J., Lin, X.Z., Zhang, Q., Lee, J.H., Civelek, M., and Xiao, X.S. (2015). Genomic analysis of ADAR1 binding and its involvement in multiple RNA processing pathways. Nature Communications 6, 6355-6355.

Bannwarth, S., Talakoub, L., Letourneur, F., Duarte, M., Purcell, D.F., Hiscott, J., and Gatignol, A. (2001). Organization of the human tarbp2 gene reveals two promoters that are repressed in an astrocytic cell line. Journal of Biological Chemistry 276, 48803-48813.

Barad, O., Mann, M., Chapnik, E., Shenoy, A., Blelloch, R., Barkai, N., and Hornstein, E. (2012). Efficiency and specificity in microRNA biogenesis. Nature Structural \& Molecular Biology 19, 650-652.

Barr, I., and Guo, F. (2014). Primary microRNA processing assay reconstituted using recombinant drosha and DGCR8. Methods in Molecular Biology 1095, 73-86.

Barr, I., Weitz, S.H., Atkin, T., Hsu, P.K., Karayiorgou, M., Gogos, J.A., Weiss, S., and Guo, F. (2015). Cobalt(III) Protoporphyrin Activates the DGCR8 Protein and Can Compensate microRNA Processing Deficiency. Chemistry \& Biology 22, 793-802. 
Behm-Ansmant, I., Rehwinkel, J., Doerks, T., Stark, A., Bork, P., and Izaurralde, E. (2006). MRNA degradation by miRNAs and GW182 requires both CCR4 : NOT deadenylase and DCP1 : DCP2 decapping complexes. Genes \& Development 20, 1885-1898.

Bellemer, C., Bortolin-Cavaille, M.L., Schmidt, U., Jensen, S.M.R., Kjems, J., Bertrand, E., and Cavaille, J. (2012). Microprocessor dynamics and interactions at endogenous imprinted C19MC microRNA genes. Journal of Cell Science 125, 2709-2720.

Benoit, M., and Plevin, M.J. (2013). Backbone resonance assignments of the micro-RNA precursor binding region of human TRBP. Biomolecular Nmr Assignments 7, 229-233.

Berezikov, E., Chung, W.J., Willis, J., Cuppen, E., and Lai, E.C. (2007). Mammalian mirtron genes. Molecular Cell 28, 328-336.

Bernard, M.A., Wang, L.Y., and Tachado, S.D. (2015). DICER-ARGONAUTE2 Complex in Continuous Fluorogenic Assays of RNA Interference Enzymes. Plos One 10, e0120614-e0120614.

Bernstein, E., Caudy, A.A., Hammond, S.M., and Hannon, G.J. (2001). Role for a bidentate ribonuclease in the initiation step of RNA interference. Nature 409, 363-366.

Betancur, J.G., and Tomari, Y. (2012). Dicer is dispensable for asymmetric RISC loading in mammals. RNA 18, 24-30.

Bogerd, H.P., Karnowski, H.W., Cai, X.Z., Shin, J., Pohlers, M., and Cullen, B.R. (2010). A Mammalian Herpesvirus Uses Noncanonical Expression and Processing Mechanisms to Generate Viral MicroRNAs. Molecular Cell 37, 135-142.

Borchert, G.M., Lanier, W., and Davidson, B.L. (2006). RNA polymerase III transcribes human microRNAs. Nat Struct Mol Biol 13, 1097-1101.

Braun, J.E., Huntzinger, E., Fauser, M., and Izaurralde, E. (2011). GW182 Proteins Directly Recruit Cytoplasmic Deadenylase Complexes to miRNA Targets. Molecular Cell 44, 120-133.

Bronevetsky, Y., Villarino, A.V., Eisley, C.J., Barbeau, R., Barczak, A.J., Heinz, G.A., Kremmer, E., Heissmeyer, V., McManus, M.T., Erle, D.J., et al. (2013). T cell activation induces proteasomal degradation of Argonaute and rapid remodeling of the microRNA repertoire. Journal of Experimental Medicine 210, 417-432.

Brudecki, L., Ferguson, D.A., McCall, C.E., and El Gazzar, M. (2013). MicroRNA-146a and RBM4 form a negative feed-forward loop that disrupts cytokine mRNA translation following TLR4 responses in human THP-1 monocytes. Immunology and Cell Biology 91, 532-540.

Burroughs, A.M., Ando, Y., de Hoon, M.J.L., Tomaru, Y., Suzuki, H., Hayashizaki, Y., and Daub, C.O. (2011). Deep-sequencing of human argonaute-associated small RNAs provides insight into miRNA sorting and reveals argonaute association with RNA fragments of diverse origin. RNA Biology 8, 158-177.

Canella, D., Praz, V., Reina, J.H., Cousin, P., and Hernandez, N. (2010). Defining the RNA polymerase III transcriptome: Genome-wide localization of the RNA polymerase III transcription machinery in human cells. Genome Res 20, 710-721.

Castellano, L., and Stebbing, J. (2013). Deep sequencing of small RNAs identifies canonical and non-canonical miRNA and endogenous siRNAs in mammalian somatic tissues. Nucleic Acids Research 41, 3339-3351.

Caudy, A.A., Myers, M., Hannon, G.J., and Hammond, S.M. (2002). Fragile X-related protein and VIG associate with the RNA interference machinery. Genes \& Development 16, 2491-2496.

Cerutti, H., and Casas-Mollano, J.A. (2006). On the origin and functions of RNA-mediated silencing: from protists to man. Current genetics 50, 81-99. 
Chahar, H.S., Chen, S.P., and Manjunath, N. (2013). P-body components LSM1, GW182, DDX3, DDX6 and XRN1 are recruited to WNV replication sites and positively regulate viral replication. Virology 436, 1-7.

Chakravarthy, S., Sternberg, S.H., Kellenberger, C.A., and Doudna, J.A. (2010). Substrate-Specific Kinetics of Dicer-Catalyzed RNA Processing. Journal of Molecular Biology 404, 392-402.

Chandradoss, S.D., Schirle, N.T., Szczepaniak, M., MacRae, I.J., and Joo, C. (2015). A Dynamic Search Process Underlies MicroRNA Targeting. Cell 162, 96-107.

Chekulaeva, M., Mathys, H., Zipprich, J.T., Attig, J., Colic, M., Parker, R., and Filipowicz, W. (2011). miRNA repression involves GW182-mediated recruitment of CCR4-NOT through conserved W-containing motifs. Nature Structural \& Molecular Biology 18, 1218-U1262.

Cheloufi, S., Dos Santos, C.O., Chong, M.M.W., and Hannon, G.J. (2010). A Dicer-independent miRNA biogenesis pathway that requires Ago catalysis. Nature 465, 584-U576.

Chen, T., Xiang, J.F., Zhu, S.S., Chen, S.Y., Yin, Q.F., Zhang, X.O., Zhang, J., Feng, H., Dong, R., Li, X.J., et al. (2015). ADAR1 is required for differentiation and neural induction by regulating microRNA processing in a catalytically independent manner. Cell Research 25, 459-476.

Chendrimada, T.P., Gregory, R.I., Kumaraswamy, E., Norman, J., Cooch, N., Nishikura, K., and Shiekhattar, R. (2005). TRBP recruits the Dicer complex to Ago2 for microRNA processing and gene silencing. Nature 436, 740-744.

Cheng, T.L., Wang, Z.Z., Liao, Q.M., Zhu, Y., Zhou, W.H., Xu, W.Q., and Qiu, Z.L. (2014). MeCP2 Suppresses Nuclear MicroRNA Processing and Dendritic Growth by Regulating the DGCR8/Drosha Complex. Developmental Cell 28, 547-560.

Chong, M.M.W., Zhang, G.A., Cheloufi, S., Neubert, T.A., Hannon, G.J., and Littman, D.R. (2010). Canonical and alternate functions of the microRNA biogenesis machinery. Genes \& Development 24, 1951-1960.

Christie, M., Boland, A., Huntzinger, E., Weichenrieder, O., and Izaurralde, E. (2013). Structure of the PAN3 Pseudokinase Reveals the Basis for Interactions with the PAN2 Deadenylase and the GW182 Proteins. Molecular Cell 51, 360-373.

Chu, C.Y., and Rana, T.M. (2006). Translation repression in human cells by microRNA-induced gene silencing requires RCK/p54. Plos Biology 4, 1122-1136.

Cikaluk, D.E., Tahbaz, N., Hendricks, L.C., DiMattia, G.E., Hansen, D., Pilgrim, D., and Hobman, T.C. (1999). GERp95, a membrane-associated protein that belongs to a family of proteins involved in stem cell differentiation. Molecular Biology of the Cell 10,3357-3372.

Cosentino, G.P., Venkatesan, S., Serluca, F.C., Green, S.R., Mathews, M.B., and Sonenberg, N. (1995). Double-stranded-RNA-dependent protein kinase and TAR RNA-binding protein form homo- and heterodimers in vivo. Proc Natl Acad Sci U S A 92, 9445-9449.

Daher, A., Laraki, G., Singh, M., Melendez-Pena, C.E., Bannwarth, S., Peters, A., Meurs, E.F., Braun, R.E., Patel, R.C., and Gatignol, A. (2009). TRBP Control of PACT-Induced Phosphorylation of Protein Kinase R Is Reversed by Stress. Molecular and Cellular Biology 29, 254-265.

Daniels, S.M., and Gatignol, A. (2012). The Multiple Functions of TRBP, at the Hub of Cell Responses to Viruses, Stress, and Cancer. Microbiology and Molecular Biology Reviews 76, 652-+.

Daniels, S.M., Melendez-Pena, C.E., Scarborough, R.J., Daher, A., Christensen, H.S., El Far, M., Purcell, D.F.J., Laine, S., and Gatignol, A. (2009). Characterization of the TRBP domain required for Dicer interaction and function in RNA interference. BMC Molecular Biology 10, 38-38. 
De Wit, T., Grosveld, F., and Drabek, D. (2002). The tomato RNA-directed RNA polymerase has no effect on gene silencing by RNA interference in transgenic mice. Transgenic Research 11, 305-310.

Deerberg, A., Willkomm, S., and Restle, T. (2013). Minimal mechanistic model of siRNA-dependent target RNA slicing by recombinant human Argonaute 2 protein. Proc Natl Acad Sci U S A 110, $17850-17855$.

Deleavey, G.F., Frank, F., Hassler, M., Wisnovsky, S., Nagar, B., and Damha, M.J. (2013). The 5 ‘ Binding MID Domain of Human Argonaute2 Tolerates Chemically Modified Nucleotide Analogues. Nucleic Acid Therapeutics 23, 81-87.

Didiot, M.C., Subramanian, M., Flatter, E., Mandel, J.L., and Moine, H. (2009). Cells lacking the fragile $\mathrm{X}$ mental retardation protein (FMRP) have normal RISC activity but exhibit altered stress granule assembly. Molecular Biology of the Cell 20, 428-437.

Diederichs, S., and Haber, D.A. (2007). Dual role for argonautes in microRNA processing and posttranscriptional regulation of microRNA expression. Cell 131, 1097-1108.

Doench, J.G., Petersen, C.P., and Sharp, P.A. (2003). siRNAs can function as miRNAs. Genes \& Development 17, 438-442.

Donze, O., Abbas-Terki, T., and Picard, D. (2001). The Hsp90 chaperone complex is both a facilitator and a repressor of the dsRNA-dependent kinase PKR. EMBO J 20, 3771-3780.

Du, Z., Lee, J.K., Tjhen, R., Strould, R.M., and James, T.L. (2008). Structural and biochemical insights into the dicing mechanism of mouse Dicer: A conserved lysine is critical for dsRNA cleavage. Proc Natl Acad Sci U S A 105, 2391-2396.

Dueck, A., Ziegler, C., Eichner, A., Berezikov, E., and Meister, G. (2012). microRNAs associated with the different human Argonaute proteins. Nucleic Acids Research 40, 9850-9862.

El-Shami, M., Pontier, D., Lahmy, S., Braun, L., Picart, C., Vega, D., Hakimi, M.-A., Jacobsen, S.E., Cooke, R., and Lagrange, T. (2007). Reiterated WG/GW motifs form functionally and evolutionarily conserved ARGONAUTE-binding platforms in RNAi-related components. Genes \& Development 21, 2539-2544.

Elkayam, E., Kuhn, C.D., Tocilj, A., Haase, A.D., Greene, E.M., Hannon, G.J., and Joshua-Tor, L. (2012). The Structure of Human Argonaute-2 in Complex with miR-20a. Cell 150, 100-110.

Ender, C., Krek, A., Friedlander, M.R., Beitzinger, M., Weinmann, L., Chen, W., Pfeffer, S., Rajewsky, N., and Meister, G. (2008). A Human snoRNA with MicroRNA-Like Functions. Molecular Cell 32, 519-528.

Engels, B., Jannot, G., Remenyi, J., Simard, M.J., and Hutvagner, G. (2012). Polypyrimidine Tract Binding Protein (hnRNP I) Is Possibly a Conserved Modulator of miRNA-Mediated Gene Regulation. Plos One 7, e33144-e33144.

Enright, A.J., John, B., Gaul, U., Tuschl, T., Sander, C., and Marks, D.S. (2003). MicroRNA targets in Drosophila. Genome Biology 5, R1.

Eulalio, A., Behm-Ansmant, I., Schweizer, D., and Izaurralde, E. (2007). P-body formation is a consequence, not the cause, of RNA-mediated gene silencing. Molecular and Cellular Biology 27, 3970-3981.

Fabian, M.R., Cieplak, M.K., Frank, F., Morita, M., Green, J., Srikumar, T., Nagar, B., Yamamoto, T., Raught, B., Duchaine, T.F., et al. (2011a). miRNA-mediated deadenylation is orchestrated by GW182 through two conserved motifs that interact with CCR4-NOT. Nature Structural \& Molecular Biology 18, 1211-U1252. 
Fabian, M.R., Mathonnet, G., Sundermeier, T., Mathys, H., Zipprich, J.T., Svitkin, Y.V., Rivas, F., Jinek, M., Wohischlegel, J., Doudna, J.A., et al. (2009). Mammalian miRNA RISC Recruits CAF1 and PABP to Affect PABP-Dependent Deadenylation. Molecular Cell 35, 868-880.

Fabian, M.R., Svitkin, Y.V., and Sonenberg, N. (2011b). An Efficient System for Let-7 MicroRNA and GW182 Protein-Mediated Deadenylation In Vitro. In Argonaute Proteins: Methods and Protocols, pp. 207-217.

Faehnle, C.R., Elkayam, E., Haase, A.D., Hannon, G.J., and Joshua-Tor, L. (2013). The Making of a Slicer: Activation of Human Argonaute-1. Cell Reports 3, 1901-1909.

Feng, Y., Zhang, X.X., Graves, P., and Zeng, Y. (2012). A comprehensive analysis of precursor microRNA cleavage by human Dicer. RNA 18, 2083-2092.

Filippov, V., Solovyev, V., Filippova, M., and Gill, S.S. (2000). A novel type of RNase III family proteins in eukaryotes. Gene 245, 213-221.

Flemr, M., Malik, R., Franke, V., Nejepinska, J., Sedlacek, R., Vlahovicek, K., and Svoboda, P. (2013). A Retrotransposon-Driven Dicer Isoform Directs Endogenous Small Interfering RNA Production in Mouse Oocytes. Cell 155, 807-816.

Flores-Jasso, C.F., Arenas-Huertero, C., Reyes, J.L., Contreras-Cubas, C., Covarrubias, A., and Vaca, L. (2009). First step in pre-miRNAs processing by human Dicer. Acta Pharmacologica Sinica 30, $1177-1185$.

Fortin, K.R., Nicholson, R.H., and Nicholson, A.W. (2002). Mouse ribonuclease III. cDNA structure, expression analysis, and chromosomal location. BMC Genomics 3.

Frank, F., Fabian, M.R., Stepinski, J., Jemielity, J., Darzynkiewicz, E., Sonenberg, N., and Nagar, B. (2011). Structural analysis of 5 '-mRNA-cap interactions with the human AGO2 MID domain. EMBO Rep 12, 415-420.

Frank, F., Sonenberg, N., and Nagar, B. (2010). Structural basis for 5 '-nucleotide base-specific recognition of guide RNA by human AGO2. Nature 465, 818-822.

Friend, K., Campbell, Z.T., Cooke, A., Kroll-Conner, P., Wickens, M.P., and Kimble, J. (2012). A conserved PUF-Ago-eEF1A complex attenuates translation elongation. Nature Structural \& Molecular Biology 19, 176-183.

Fu, Q.Q., and Yuan, Y.A. (2013). Structural insights into RISC assembly facilitated by dsRNA-binding domains of human RNA helicase A (DHX9). Nucleic Acids Research 41, 3457-3470.

Gan, H.H., and Gunsalus, K.C. (2015). Assembly and analysis of eukaryotic Argonaute-RNA complexes in microRNA-target recognition. Nucleic Acids Research 43, 9613-9625.

Ganesan, G., and Rao, S.M.R. (2008). A novel noncoding RNA processed by Drosha is restricted to nucleus in mouse. RNA 14, 1399-1410.

Gantier, M.P., and Williams, B.R. (2007). The response of mammalian cells to double-stranded RNA. Cytokine Growth Factor Rev 18, 363-371.

Garcia-Lopez, J., Hourcade, J.D., and del Mazo, J. (2013). Reprogramming of microRNAs by adenosine-to-inosine editing and the selective elimination of edited microRNA precursors in mouse oocytes and preimplantation embryos. Nucleic Acids Research 41, 5483-5493.

Gehrke, S., Imai, Y., Sokol, N., and Lu, B.W. (2010). Pathogenic LRRK2 negatively regulates microRNA-mediated translational repression. Nature 466, 637-U639.

Goodarzi, H., Zhang, S., Buss, C.G., Fish, L., Tavazoie, S., and Tavazoie, S.F. (2014). Metastasis-suppressor transcript destabilization through TARBP2 binding of mRNA hairpins. Nature 513, 256-+. 
Goodier, J.L., Zhang, L., Vetter, M.R., and Kazazian, H.H. (2007). LINE-1 ORF1 protein localizes in stress granules with other RNA-Binding proteins, including components of RNA interference RNA-induced silencing complex. Molecular and Cellular Biology 27, 6469-6483.

Gredell, J.A., Dittmer, M.J., Wu, M., Chan, C., and Walton, S.P. (2010). Recognition of siRNAAsymmetry by TAR RNA Binding Protein. Biochemistry 49, 3148-3155.

Gregory, R.I., Chendrimada, T.P., Cooch, N., and Shiekhattar, R. (2005). Human RISC couples microRNA biogenesis and posttranscriptional gene silencing. Cell 123, 631-640.

Gregory, R.I., Chendrimada, T.P., and Shiekhattar, R. (2006). MicroRNA biogenesis - Isolation and characterization of the microprocessor complex. In Methods in Molecular Biology, pp. 33-47.

Gregory, R.I., Yan, K.P., Amuthan, G., Chendrimada, T., Doratotaj, B., Cooch, N., and Shiekhattar, R. (2004). The Microprocessor complex mediates the genesis of microRNAs. Nature 432, 235-240.

Gu, S., Jin, L., Huang, Y., Zhang, F.J., and Kay, M.A. (2012a). Slicing-Independent RISC Activation Requires the Argonaute PAZ Domain. Current Biology 22, 1536-1542.

Gu, S., Jin, L., Zhang, Y., Huang, Y., Zhang, F.J., Valdmanis, P.N., and Kay, M.A. (2012b). The Loop Position of shRNAs and Pre-miRNAs Is Critical for the Accuracy of Dicer Processing In Vivo. Cell 151, 900-911.

Guo, Y.W., Liu, J., Elfenbein, S.J., Ma, Y.H., Zhong, M., Qiu, C.H., Ding, Y., and Lu, J. (2015). Characterization of the mammalian miRNA turnover landscape. Nucleic Acids Research 43, 2326-2341.

Gupta, V., Huang, X., and Patel, R.C. (2003). The carboxy-terminal, M3 motifs of PACT and TRBP have opposite effects on PKR activity. Virology 315, 283-291.

Gurtan, A.M., Lu, V., Bhutkar, A., and Sharp, P.A. (2012). In vivo structure-function analysis of human Dicer reveals directional processing of precursor miRNAs. RNA 18, 1116-1122.

Haase, A.D., Jaskiewicz, L., Zhang, H.D., Laine, S., Sack, R., Gatignol, A., and Filipowicz, W. (2005). TRBP, a regulator of cellular PKR and HIV-1 virus expression, interacts with Dicer and functions in RNA silencing. EMBO Rep 6, 961-967.

Haley, B., and Zamore, P.D. (2004). Kinetic analysis of the RNAi enzyme complex. Nature Structural \& Molecular Biology 11, 599-606.

Han, C., Liu, Y.H., Wan, G.H., Choi, H.J., Zhao, L.Q., Ivan, C., He, X.M., Sood, A.K., Zhang, X.N., and Lu, X.B. (2014). The RNA-Binding Protein DDX1 Promotes Primary MicroRNA Maturation and Inhibits Ovarian Tumor Progression. Cell Reports 8, 1447-1460.

Han, J.J., Lee, Y., Yeom, K.H., Kim, Y.K., Jin, H., and Kim, V.N. (2004). The Drosha-DGCR8 complex in primary microRNA processing. Genes \& Development 18, 3016-3027.

Hauptmann, J., Schraivogel, D., Bruckmann, A., Manickavel, S., Jakob, L., Eichner, N., Pfaff, J., Urban, M., Sprunck, S., Hafner, M., et al. (2015). Biochemical isolation of Argonaute protein complexes by Ago-APP. Proc Natl Acad Sci U S A 112, 11841-11845.

Haussecker, D., Huang, Y., Lau, A., Parameswaran, P., Fire, A.Z., and Kay, M.A. (2010). Human tRNA-derived small RNAs in the global regulation of RNA silencing. RNA 16, 673-695.

Hausser, J., Landthaler, M., Jaskiewicz, L., Gaidatzis, D., and Zavolan, M. (2009). Relative contribution of sequence and structure features to the mRNA binding of Argonaute/EIF2C-miRNA complexes and the degradation of miRNA targets. Genome Research 19, 2009-2020.

Havens, M.A., Reich, A.A., Duelli, D.M., and Hastings, M.L. (2012). Biogenesis of mammalian microRNAs by a non-canonical processing pathway. Nucleic Acids Research 40, 4626-4640. 
Herbert, K.M., Pimienta, G., DeGregorio, S.J., Alexandrov, A., and Steitz, J.A. (2013). Phosphorylation of DGCR8 Increases Its Intracellular Stability and Induces a Progrowth miRNA Profile. Cell Reports 5, 1070-1081.

Herbert, K.M., Sarkar, S.K., Mills, M., De la Herran, H.C.D., Neuman, K.C., and Steitz, J.A. (2016). A heterotrimer model of the complete Microprocessor complex revealed by single-molecule subunit counting. RNA 22, 175-183.

Hock, J., Weinmann, L., Ender, C., Rudel, S., Kremmer, E., Raabe, M., Urlaub, H., and Meister, G. (2007). Proteomic and functional analysis of Argonaute-containing mRNA-protein complexes in human cells. EMBO Rep 8, 1052-1060.

Horman, S.R., Janas, M.M., Litterst, C., Wang, B., MacRae, I.J., Sever, M.J., Morrissey, D.V., Graves, P., Luo, B., Umesalma, S., et al. (2013). Akt-mediated phosphorylation of argonaute 2 downregulates cleavage and upregulates translational repression of MicroRNA targets. Molecular Cell 50, 356-367.

Huang, J., Liang, Z., Yang, B., Tian, H., Ma, J., and Zhang, H. (2007). Derepression of microRNA-mediated protein translation inhibition by apolipoprotein B mRNA-editing enzyme catalytic polypeptide-like 3G (APOBEC3G) and its family members. Journal of Biological Chemistry 282, $33632-33640$.

Huang, X., Hutchins, B., and Patel, R.C. (2002). The C-terminal, third conserved motif of the protein activator PACT plays an essential role in the activation of double-stranded-RNA-dependent protein kinase (PKR). Biochemical Journal 366, 175-186.

Huntzinger, E., Braun, J.E., Heimstaedt, S., Zekri, L., and Izaurralde, E. (2010). Two PABPC1-binding sites in GW182 proteins promote miRNA-mediated gene silencing. EMBO J 29, 4146-4160.

Huntzinger, E., Kuzuoglu-Oeztuerk, D., Braun, J.E., Eulalio, A., Wohlbold, L., and Izaurralde, E. (2013). The interactions of GW182 proteins with PABP and deadenylases are required for both translational repression and degradation of miRNA targets. Nucleic Acids Research 41, 978-994.

Ishizuka, A., Siomi, M.C., and Siomi, H. (2002). A Drosophila fragile X protein interacts with components of RNAi and ribosomal proteins. Genes \& Development 16, 2497-2508.

Iwasaki, S., Kobayashi, M., Yoda, M., Sakaguchi, Y., Katsuma, S., Suzuki, T., and Tomari, Y. (2010). Hsc70/Hsp90 chaperone machinery mediates ATP-dependent RISC loading of small RNA duplexes. Molecular Cell 39, 292-299.

Izumi, T., Burdick, R., Shigemi, M., Plisov, S., Hu, W.S., and Pathak, V.K. (2013). Mov10 and APOBEC3G Localization to Processing Bodies Is Not Required for Virion Incorporation and Antiviral Activity. Journal of Virology 87, 11047-11062.

Jain, S., and Parker, R. (2013). The discovery and analysis of P Bodies. Adv Exp Med Biol 768, $23-43$.

Jakymiw, A., Lian, S.L., Eystathioy, T., Li, S.Q., Satoh, M., Hamel, J.C., Fritzler, M.J., and Chan, E.K.L. (2005). Disruption of GW bodies impairs mammalian RNA interference. Nature Cell Biology $7,1267-1274$.

James, V., Zhang, Y., Foxler, D.E., de Moor, C.H., Kong, Y.W., Webb, T.M., Self, T.J., Feng, Y., Lagos, D., Chu, C.-Y., et al. (2010). LIM-domain proteins, LIMD1, Ajuba, and WTIP are required for microRNA-mediated gene silencing. Proc Natl Acad Sci U S A 107, 12499-12504.

Jannot, G., Bajan, S., Giguere, N.J., Bouasker, S., Banville, I.H., Piquet, S., Hutvagner, G., and Simard, M.J. (2011). The ribosomal protein RACK1 is required for microRNA function in both C. elegans and humans. EMBO Rep 12, 581-586. 
Jaskiewicz, L., and Filipowicz, W. (2008). Role of Dicer in posttranscriptional RNA silencing. Current topics in microbiology and immunology 320, 77-97.

Jiang, H.L., Sheong, F.K., Zhu, L.Z., Gao, X., Bernauer, J., and Huang, X.H. (2015). Markov State Models Reveal a Two-Step Mechanism of miRNA Loading into the Human Argonaute Protein: Selective Binding followed by Structural Re-arrangement. Plos Computational Biology 11, e1004404-e1004404.

Jin, H., Suh, M.R., Han, J., Yeom, K.H., Lee, Y., Heo, I., Ha, M., Hyun, S., and Kim, V.N. (2009). Human UPF1 Participates in Small RNA-Induced mRNA Downregulation. Molecular and Cellular Biology 29, 5789-5799.

Jinek, M., Fabian, M.R., Coyle, S.M., Sonenberg, N., and Doudna, J.A. (2010). Structural insights into the human GW182-PABC interaction in microRNA-mediated deadenylation. Nature Structural \& Molecular Biology 17, 238-240.

Jo, M.H., Shin, S., Jung, S.R., Kim, E., Song, J.J., and Hohng, S. (2015a). Human Argonaute 2 Has Diverse Reaction Pathways on Target RNAs. Molecular Cell 59, 117-124.

Jo, M.H., Song, J.-J., and Hohng, S. (2015b). Single-molecule fluorescence measurements reveal the reaction mechanisms of the core-RISC, composed of human Argonaute 2 and a guide RNA. BMB Reports 48, 643-644.

Johanson, T.M., Lew, A.M., and Chong, M.M. (2013). MicroRNA-independent roles of the RNase III enzymes Drosha and Dicer. Open biology 3, 130144.

Johnston, M., Geoffroy, M.-C., Sobala, A., Hay, R., and Hutvagner, G. (2010). HSP90 Protein Stabilizes Unloaded Argonaute Complexes and Microscopic P-bodies in Human Cells. Molecular Biology of the Cell 21, 1462-1469.

Josa-Prado, F., Henley, J.M., and Wilkinson, K.A. (2015). SUMOylation of Argonaute-2 regulates RNA interference activity. Biochem Biophys Res Commun 464, 1066-1071.

Juvvuna, P.K., Khandelia, P., Lee, L.M., and Makeyev, E.V. (2012). Argonaute identity defines the length of mature mammalian microRNAs. Nucleic Acids Research 40, 6808-6820.

Kalia, M., Willkomm, S., Claussen, J.C., Restle, T., and Bonvin, A.M. (2016). Novel Insights into Guide RNA 5'-Nucleoside/Tide Binding by Human Argonaute 2. Int J Mol Sci 17.

Kandeel, M., and Kitade, Y. (2013). In silico molecular docking analysis of the human Argonaute 2 PAZ domain reveals insights into RNA interference. Journal of Computer-Aided Molecular Design 27, 605-614.

Kasim, V., Wu, S.R., Taira, K., and Miyagishi, M. (2013). Determination of the Role of DDX3 a Factor Involved in Mammalian RNAi Pathway Using an shRNA-Expression Library. Plos One 8, e59445-e59445.

Kedersha, N., Stoecklin, G., Ayodele, M., Yacono, P., Lykke-Andersen, J., Fritzler, M.J., Scheuner, D., Kaufman, R.J., Golan, D.E., and Anderson, P. (2005). Stress granules and processing bodies are dynamically linked sites of mRNP remodeling. Journal of Cell Biology 169, 871-884.

Kennedy, E.M., Whisnant, A.W., Kornepati, A.V.R., Marshall, J.B., Bogerd, H.P., and Cullen, B.R. (2015). Production of functional small interfering RNAs by an amino-terminal deletion mutant of human Dicer. Proc Natl Acad Sci U S A 112, E6945-E6954.

Ketting, R.F., Fischer, S.E., Bernstein, E., Sijen, T., Hannon, G.J., and Plasterk, R.H. (2001). Dicer functions in RNA interference and in synthesis of small RNA involved in developmental timing in C. elegans. Genes \& Development 15, 2654-2659. 
Khvorova, A., Reynolds, A., and Jayasena, S.D. (2003). Functional siRNAs and miRNAs exhibit strand bias. Cell 115, 209-216.

Kim, D.H., Behlke, M.A., Rose, S.D., Chang, M.S., Choi, S., and Rossi, J.J. (2005). Synthetic dsRNA Dicer substrates enhance RNAi potency and efficacy. Nature Biotechnology 23, 222-226.

Kim, Y., Yeo, J., Lee, J.H., Cho, J., Seo, D., Kim, J.S., and Kim, V.N. (2014). Deletion of Human tarbp2 Reveals Cellular MicroRNA Targets and Cell-Cycle Function of TRBP. Cell Reports 9, 1061-1074.

Kincaid, R.P., Chen, Y.T., Cox, J.E., Rethwilm, A., and Sullivan, C.S. (2014). Noncanonical MicroRNA (miRNA) Biogenesis Gives Rise to Retroviral Mimics of Lymphoproliferative and Immunosuppressive Host miRNAs. MBio 5, e00074-e00074.

Kinch, L.N., and Grishin, N.V. (2009). The human Ago2 MC region does not contain an eIF4E-like mRNA cap binding motif. Biology Direct 4, 2-2.

Kini, H.K., and Walton, S.P. (2007). In vitro binding of single-stranded RNA by human Dicer. FEBS Letters 581, 5611-5616.

Knuckles, P., Vogt, M.A., Lugert, S., Milo, M., Chong, M.M.W., Hautbergue, G.M., Wilson, S.A., Littman, D.R., and Taylor, V. (2012). Drosha regulates neurogenesis by controlling Neurogenin 2 expression independent of microRNAs. Nature Neuroscience 15, 962-969.

Kok, K.H., Ng, M.H.J., Ching, Y.P., and Jin, D.Y. (2007). Human TRBP and PACT directly interact with each other and associate with dicer to facilitate the production of small interfering RNA. Journal of Biological Chemistry 282, 17649-17657.

Kowalinski, E., Lunardi, T., McCarthy, A.A., Louber, J., Brunel, J., Grigorov, B., Gerlier, D., and Cusack, S. (2011). Structural basis for the activation of innate immune pattern-recognition receptor RIG-I by viral RNA. Cell 147, 423-435.

Kumar, P., Anaya, J., Mudunuri, S.B., and Dutta, A. (2014). Meta-analysis of tRNA derived RNA fragments reveals that they are evolutionarily conserved and associate with AGO proteins to recognize specific RNA targets. BMC Biology 12, 78-78.

Kuzuoglu-Ozturk, D., Bhandari, D., Huntzinger, E., Fauser, M., Helms, S., and Izaurralde, E. (2016). miRISC and the CCR4-NOT complex silence mRNA targets independently of 43S ribosomal scanning. EMBO J.

Kwon, S.C., Nguyen, T.A., Choi, Y.G., Jo, M.H., Hohng, S., Kim, V.N., and Woo, J.S. (2016). Structure of Human DROSHA. Cell 164, 81-90.

Ladewig, E., Okamura, K., Flynt, A.S., Westholm, J.O., and Lai, E.C. (2012). Discovery of hundreds of mirtrons in mouse and human small RNA data. Genome Research 22, 1634-1645.

Lamontagne, B., Larose, S., Boulanger, J., and Elela, S.A. (2001). The RNase III family: a conserved structure and expanding functions in eukaryotic dsRNA metabolism. Current issues in molecular biology 3, 71-78.

Landthaler, M., Yalcin, A., and Tuschl, T. (2004). The human DiGeorge syndrome critical region gene 8 and its D-melanogaster homolog are required for miRNA biogenesis. Current Biology 14, 2162-2167.

Laraki, G., Clerzius, G., Daher, A., Melendez-Pena, C., Daniels, S., and Gatignol, A. (2008). Interactions between the double-stranded RNA-binding proteins TRBP and PACT define the Medipal domain that mediates protein-protein interactions. RNA Biology 5, 92-103.

Lau, P.W., Guiley, K.Z., De, N., Potter, C.S., Carragher, B., and MacRae, I.J. (2012). The molecular architecture of human Dicer. Nature Structural \& Molecular Biology 19, 436-440. 
Lau, P.W., Potter, C.S., Carragher, B., and MacRae, I.J. (2009). Structure of the Human Dicer-TRBP Complex by Electron Microscopy. Structure 17, 1326-1332.

Lazzaretti, D., Tournier, I., and Izaurralde, E. (2009). The C-terminal domains of human TNRC6A, TNRC6B, and TNRC6C silence bound transcripts independently of Argonaute proteins. RNA 15, 1059-1066.

Lee, H.Y., Zhou, K., Smith, A.M., Noland, C.L., and Doudna, J.A. (2013). Differential roles of human Dicer-binding proteins TRBP and PACT in small RNA processing. Nucleic Acids Research 41, 6568-6576.

Lee, N.S., Dohjima, T., Bauer, G., Li, H.T., Li, M.J., Ehsani, A., Salvaterra, P., and Rossi, J. (2002). Expression of small interfering RNAs targeted against HIV-1 rev transcripts in human cells. Nature Biotechnology 20, 500-505.

Lee, Y., Ahn, C., Han, J.J., Choi, H., Kim, J., Yim, J., Lee, J., Provost, P., Radmark, O., Kim, S., et al. (2003). The nuclear RNase III Drosha initiates microRNA processing. Nature 425, 415-419.

Leung, A.K., Vyas, S., Rood, J.E., Bhutkar, A., Sharp, P.A., and Chang, P. (2011). Poly(ADP-ribose) regulates stress responses and microRNA activity in the cytoplasm. Molecular Cell 42, 489-499.

Lewis, B.P., Shih, I.H., Jones-Rhoades, M.W., Bartel, D.P., and Burge, C.B. (2003). Prediction of mammalian microRNA targets. Cell 115, 787-798.

Li, Z.H., Ender, C., Meister, G., Moore, P.S., Chang, Y., and John, B. (2012). Extensive terminal and asymmetric processing of small RNAs from rRNAs, snoRNAs, snRNAs, and tRNAs. Nucleic Acids Research 40, 6787-6799.

Li, Z.H., Kim, S.W., Lin, Y.F., Moore, P.S., Chang, Y., and John, B. (2009). Characterization of Viral and Human RNAs Smaller than Canonical MicroRNAs. Journal of Virology 83, 12751-12758.

Lian, S.L., Li, S.Q., Abadal, G.X., Pauley, B.A., Fritzler, M.J., and Chan, E.K.L. (2009). The C-terminal half of human Ago2 binds to multiple GW-rich regions of GW182 and requires GW182 to mediate silencing. RNA 15, 804-813.

Liang, X.H., and Crooke, S.T. (2011). Depletion of key protein components of the RISC pathway impairs pre-ribosomal RNA processing. Nucleic Acids Research 39, 4875-4889.

Lima, W.F., Wu, H.J., Nichols, J.G., Sun, H., Murray, H.M., and Crooke, S.T. (2009). Binding and Cleavage Specificities of Human Argonaute2. Journal of Biological Chemistry 284, 26017-26028.

Lin, J.C., and Tarn, W.Y. (2009). RNA-binding Motif Protein 4 Translocates to Cytoplasmic Granules and Suppresses Translation via Argonaute2 during Muscle Cell Differentiation. Journal of Biological Chemistry 284, 34658-34665.

Lipardi, C., and Paterson, B.M. (2009). Identification of an RNA-dependent RNA polymerase in Drosophila involved in RNAi and transposon suppression. Proc Natl Acad Sci U S A 106, 15645-15650.

Liu, C., Zhang, X., Huang, F., Yang, B., Li, J., Liu, B.F., Luo, H.H., Zhang, P., and Zhang, H. (2012a). APOBEC3G Inhibits MicroRNA-mediated Repression of Translation by Interfering with the Interaction between Argonaute-2 and MOV10. Journal of Biological Chemistry 287, 29373-29383.

Liu, J., Rivas, F.V., Wohlschlegel, J., Yates, J.R., 3rd, Parker, R., and Hannon, G.J. (2005a). A role for the P-body component GW182 in microRNA function. Nature Cell Biology 7, 1261-1266.

Liu, J.D., Carmell, M.A., Rivas, F.V., Marsden, C.G., Thomson, J.M., Song, J.J., Hammond, S.M., Joshua-Tor, L., and Hannon, G.J. (2004). Argonaute2 is the catalytic engine of mammalian RNAi. Science 305, 1437-1441. 
Liu, J.D., Valencia-Sanchez, M.A., Hannon, G.J., and Parker, R. (2005b). MicroRNA-dependent localization of targeted mRNAs to mammalian P-bodies. Nature Cell Biology 7, 719-U118.

Liu, X.H., Jin, D.Y., McManus, M.T., and Mourelatos, Z. (2012b). Precursor MicroRNA-Programmed Silencing Complex Assembly Pathways in Mammals. Molecular Cell 46, 507-517.

Liu, Y., Tan, H.L., Tian, H., Liang, C.Y., Chen, S., and Liu, Q.H. (2011). Autoantigen La Promotes Efficient RNAi, Antiviral Response, and Transposon Silencing by Facilitating Multiple-Turnover RISC Catalysis. Molecular Cell 44, 502-508.

Lopez-Orozco, J., Pare, J.M., Holme, A.L., Chaulk, S.G., Fahlman, R.P., and Hobman, T.C. (2015). Functional analyses of phosphorylation events in human Argonaute 2. RNA 21, 2030-2038.

Lugli, G., Larson, J., Martone, M.E., Jones, Y., and Smalheiser, N.R. (2005). Dicer and eIF2c are enriched at postsynaptic densities in adult mouse brain and are modified by neuronal activity in a calpain-dependent manner. Journal of Neurochemistry 94, 896-905.

Ma, E., MacRae, I.J., Kirsch, J.F., and Doudna, J.A. (2008). Autoinhibition of human dicer by its internal helicase domain. Journal of Molecular Biology 380, 237-243.

Ma, E.B., Zhou, K.H., Kidwell, M.A., and Doudna, J.A. (2012). Coordinated Activities of Human Dicer Domains in Regulatory RNA Processing. Journal of Molecular Biology 422, 466-476.

Ma, H.M., Wu, Y.G., Choi, J.G., and Wu, H.Q. (2013). Lower and upper stem-single-stranded RNA junctions together determine the Drosha cleavage site. Proc Natl Acad Sci U S A 110, 20687-20692.

Ma, J.B., Ye, K., and Patel, D.J. (2004). Structural basis for overhang-specific small interfering RNA recognition by the PAZ domain. Nature 429, 318-322.

Macias, S., Cordiner, R.A., Gautier, P., Plass, M., and Caceres, J.F. (2015). DGCR8 Acts as an Adaptor for the Exosome Complex to Degrade Double-Stranded Structured RNAs. Molecular Cell 60, 873-885.

Macias, S., Plass, M., Stajuda, A., Michlewski, G., Eyras, E., and Caceres, J.F. (2012). DGCR8 HITSCLIP reveals novel functions for the Microprocessor. Nature Structural \& Molecular Biology 19, 760-766.

MacRae, I.J., Li, F., Zhou, K., Cande, W.Z., and Doudna, J.A. (2006a). Structure of Dicer and mechanistic implications for RNAi. Cold Spring Harbor Symposia on Quantitative Biology 71, 73-80.

MacRae, I.J., Ma, E., Zhou, M., Robinson, C.V., and Doudna, J.A. (2008). In vitro reconstitution of the human RISC-loading complex. Proc Natl Acad Sci U S A 105, 512-517.

MacRae, I.J., Zhou, K., and Doudna, J.A. (2007). Structural determinants of RNA recognition and cleavage by Dicer. Nature Structural \& Molecular Biology 14, 934-940.

MacRae, I.J., Zhou, K., Li, F., Repic, A., Brooks, A.N., Cande, W.Z., Adams, P.D., and Doudna, J.A. (2006b). Structural basis for double-stranded RNA processing by Dicer. Science 311, 195-198.

Madsen, C., Gronskov, K., Brondum-Nielsen, K., and Jensen, T.G. (2009). Normal RNAi response in human fragile x fibroblasts. BMC research notes 2, 177-177.

Maida, Y., Kyo, S., Lassmann, T., Hayashizaki, Y., and Masutomi, K. (2013). Off-Target Effect of Endogenous siRNA Derived from RMRP in Human Cells. International Journal of Molecular Sciences 14, 9305-9318.

Maida, Y., and Masutomi, K. (2011). RNA-dependent RNA polymerases in RNA silencing. Biological Chemistry 392, 299-304.

Maida, Y., Yasukawa, M., Furuuchi, M., Lassmann, T., Possemato, R., Okamoto, N., Kasim, V., Hayashizaki, Y., Hahn, W.C., and Masutomi, K. (2009). An RNA-dependent RNA polymerase formed by TERT and the RMRP RNA. Nature 461, 230-U104. 
Maida, Y., Yasukawa, M., and Masutomi, K. (2016). De Novo RNA Synthesis by RNA-Dependent RNA Polymerase Activity of Telomerase Reverse Transcriptase. Molecular and cellular biology $36,1248-1259$.

Maniataki, E., and Mourelatos, Z. (2005a). Human mitochondrial tRNA(Met) is exported to the cytoplasm and associates with the Argonaute 2 protein. RNA 11, 849-852.

Maniataki, E., and Mourelatos, Z. (2005b). A human, ATP-independent, RISC assembly machine fueled by pre-miRNA. Genes \& Development 19, 2979-2990.

Martinez, J., Patkaniowska, A., Urlaub, H., Luhrmann, R., and Tuschl, T. (2002). Single-stranded antisense siRNAs guide target RNA cleavage in RNAi. Cell 110, 563-574.

Martinez, N.J., Chang, H.M., Borrajo, J.D., and Gregory, R.I. (2013). The co-chaperones Fkbp4/5 control Argonaute2 expression and facilitate RISC assembly. RNA 19, 1583-1593.

Martinez, N.J., and Gregory, R.I. (2013). Argonaute2 expression is post-transcriptionally coupled to microRNA abundance. RNA 19, 605-612.

Mathys, H., Basquin, J., Ozgur, S., Czarnocki-Cieciura, M., Bonneau, F., Aartse, A., Dziembowski, A., Nowotny, M., Conti, E., and Filipowicz, W. (2014). Structural and biochemical insights to the role of the CCR4-NOT complex and DDX6 ATPase in microRNA repression. Molecular Cell 54, $751-765$.

Matranga, C., Tomari, Y., Shin, C., Bartel, D.P., and Zamore, P.D. (2005). Passenger-strand cleavage facilitates assembly of siRNA into Ago2-containing RNAi enzyme complexes. Cell 123, 607-620.

Maurin, T., Cazalla, D., Yang, J.S., Bortolamiol-Becet, D., and Lai, E.C. (2012). RNase III-independent microRNA biogenesis in mammalian cells. RNA 18, 2166-2173.

Maute, R.L., Schneider, C., Sumazin, P., Holmes, A., Califano, A., Basso, K., and Dalla-Favera, R. (2013). tRNA-derived microRNA modulates proliferation and the DNA damage response and is down-regulated in B cell lymphoma. Proc Natl Acad Sci U S A 110, 1404-1409.

Mazumder, A., Bose, M., Chakraborty, A., Chakrabarti, S., and Bhattacharyya, S.N. (2013). A transient reversal of miRNA-mediated repression controls macrophage activation. EMBO Rep 14, 1008-1016.

Meister, G., Landthaler, M., Patkaniowska, A., Dorsett, Y., Teng, G., and Tuschl, T. (2004). Human Argonaute 2 mediates RNA cleavage targeted by miRNAs and siRNAs. Molecular Cell 15, 185-197.

Meister, G., Landthaler, M., Peters, L., Chen, P.Y., Urlaub, H., Luhrmann, R., and Tuschl, T. (2005). Identification of novel argonaute-associated proteins. Current Biology 15, 2149-2155.

Meng, B., Lui, Y.W., Meng, S., Ca, C., and Hu, Y. (2006). Identification of effective siRNA blocking the expression of SARS viral envelope e and RDRP genes. Molecular Biotechnology 33, 141-148.

Minones-Moyano, E., Friedländer, M.R., Pallares, J., Kagerbauer, B., Porta, S., Escaramis, G., Ferrer, I., Estivill, X., and Marti, E. (2013). Upregulation of a small vault RNA (svtRNA2-1a) is an early event in parkinson disease and induces neuronal dysfunction. RNA Biology 10, 1093-1106.

Moon, J.S., Lee, S.H., Han, S.H., Kim, E.J., Cho, H., Lee, W., Kim, M.K., Kim, T.E., Park, H.J., Rhee, J.K., et al. (2016). Inhibition of hepatitis $C$ virus in mouse models by lipidoid nanoparticle-mediated systemic delivery of siRNA against PRK2. Nanomedicine : nanotechnology, biology, and medicine.

Murphy, D., Dancis, B., and Brown, J.R. (2008). The evolution of core proteins involved in microRNA biogenesis. BMC Evolutionary Biology 8, 92-92. 
Nagata, T., Tsuda, K., Kobayashi, N., Shirouzu, M., Kigawa, T., Guntert, P., Yokoyama, S., and Muto, Y. (2012). Solution structures of the double-stranded RNA-binding domains from RNA helicase A. Proteins-Structure Function and Bioinformatics 80, 1699-1706.

Nakanishi, K., Ascano, M., Gogakos, T., Ishibe-Murakami, S., Serganov, A.A., Briskin, D., Morozov, P., Tuschl, T., and Patel, D.J. (2013). Eukaryote-Specific Insertion Elements Control Human ARGONAUTE Slicer Activity. Cell Reports 3, 1893-1900.

Nejepinska, J., Flemr, M., and Svoboda, P. (2012a). The Canonical RNA Interference Pathway in Animals. In Regulatory RNAs, B. Mallick, and Z. Ghosh, eds. (Springer Berlin Heidelberg), pp. 111-149.

Nejepinska, J., Malik, R., Filkowski, J., Flemr, M., Filipowicz, W., and Svoboda, P. (2012b). dsRNA expression in the mouse elicits RNAi in oocytes and low adenosine deamination in somatic cells. Nucleic Acids Research 40, 399-413.

Nguyen, T.A., Jo, M.H., Choi, Y.G., Park, J., Kwon, S.C., Hohng, S., Kim, V.N., and Woo, J.S. (2015). Functional Anatomy of the Human Microprocessor. Cell 161, 1374-1387.

Nicholson, R.H., and Nicholson, A.W. (2002). Molecular characterization of a mouse cDNA encoding Dicer, a ribonuclease III ortholog involved in RNA interference. Mammalian Genome 13, 67-73.

Noland, C.L., and Doudna, J.A. (2013). Multiple sensors ensure guide strand selection in human RNAi pathways. RNA 19, 639-648.

Nygardas, M., Vuorinen, T., Aalto, A.P., Bamford, D.H., and Hukkanen, V. (2009). Inhibition of coxsackievirus B3 and related enteroviruses by antiviral short interfering RNA pools produced using phi 6 RNA-dependent RNA polymerase. Journal of General Virology 90, 2468-2473.

Nykanen, A., Haley, B., and Zamore, P.D. (2001). ATP requirements and small interfering RNA structure in the RNA interference pathway. Cell 107, 309-321.

Okamura, K., Ladewig, E., Zhou, L., and Lai, E.C. (2013). Functional small RNAs are generated from select miRNA hairpin loops in flies and mammals. Genes \& Development 27, 778-792.

Otsuka, M., Takata, A., Yoshikawa, T., Kojima, K., Kishikawa, T., Shibata, C., Takekawa, M., Yoshida, H., Omata, M., and Koike, K. (2011). Receptor for Activated Protein Kinase C: Requirement for Efficient MicroRNA Function and Reduced Expression in Hepatocellular Carcinoma. Plos One 6, e24359-e24359.

Ozgur, S., and Stoecklin, G. (2013). Role of Rck-Pat1b binding in assembly of processing-bodies. RNA Biology 10, 528-539.

Pare, J.M., LaPointe, P., and Hobman, T.C. (2013). Hsp90 cochaperones p23 and FKBP4 physically interact with hAgo 2 and activate RNA interference-mediated silencing in mammalian cells. Molecular Biology of the Cell 24, 2303-2310.

Pare, J.M., Lopez-Orozco, J., and Hobman, T.C. (2011). MicroRNA-binding is required for recruitment of human Argonaute 2 to stress granules and P-bodies. Biochem Biophys Res Commun 414, 259-264.

Park, H.S., Davies, M.V., Langland, J.O., Chang, H.W., Nam, Y.S., Tartaglia, J., Paoletti, E., Jacobs, B.L., Kaufman, R.J., and Venkatesan, S. (1994). TAR RNA-binding protein is an inhibitor of the interferon-induced protein kinase PKR. Proc Natl Acad Sci U S A 91, 4713-4717.

Park, J.E., Heo, I., Tian, Y., Simanshu, D.K., Chang, H., Jee, D., Patel, D.J., and Kim, V.N. (2011). Dicer recognizes the 5 ' end of RNA for efficient and accurate processing. Nature 475, 201-U107.

Park, J.H., and Shin, C. (2015). Slicer-independent mechanism drives small-RNA strand separation during human RISC assembly. Nucleic Acids Research 43, 9418-9433. 
Patranabis, S., and Bhattacharyya, S.N. (2016). Phosphorylation of Ago2 and Subsequent Inactivation of let-7a RNP-Specific MicroRNAs Control Differentiation of Mammalian Sympathetic Neurons. Molecular and Cellular Biology 36, 1260-1271.

Pawlicki, J.M., and Steitz, J.A. (2008). Primary microRNA transcript retention at sites of transcription leads to enhanced microRNA production. Journal of Cell Biology 182, 61-76.

Pawlicki, J.M., and Steitz, J.A. (2009). Subnuclear compartmentalization of transiently expressed polyadenylated pri-microRNAs Processing at transcription sites or accumulation in SC35 foci. Cell Cycle 8, 345-356.

Peng, Z.Y., Cheng, Y.B., Tan, B.C.M., Kang, L., Tian, Z.J., Zhu, Y.K., Zhang, W.W., Liang, Y., Hu, X.D., Tan, X.M., et al. (2012). Comprehensive analysis of RNA-Seq data reveals extensive RNA editing in a human transcriptome. Nature Biotechnology 30, 253-+.

Pepin, G., Perron, M.P., and Provost, P. (2012). Regulation of human Dicer by the resident ER membrane protein CLIMP-63. Nucleic Acids Research 40, 11603-11617.

Peters, L., and Meister, G. (2007). Argonaute proteins: Mediators of RNA silencing. Molecular Cell 26, 611-623.

Pham, J.W., Pellino, J.L., Lee, Y.S., Carthew, R.W., and Sontheimer, E.J. (2004). A Dicer-2-dependent 80s complex cleaves targeted mRNAs during RNAi in Drosophila. Cell 117, 83-94.

Phua, S.L.C., Sivakamasundari, V., Shao, Y., Cai, X.H., Zhang, L.F., Lufkin, T., and Featherstone, M. (2011). Nuclear Accumulation of an Uncapped RNA Produced by Drosha Cleavage of a Transcript Encoding miR-10b and HOXD4. Plos One 6, e25689-e25689.

Pillai, R.S., Artus, C.G., and Filipowicz, W. (2004). Tethering of human Ago proteins to mRNA mimics the miRNA-mediated repression of protein synthesis. RNA 10, 1518-1525.

Pillai, R.S., Bhattacharyya, S.N., Artus, C.G., Zoller, T., Cougot, N., Basyuk, E., Bertrand, E., and Filipowicz, W. (2005). Inhibition of translational initiation by Let-7 microRNA in human cells. Science 309, 1573-1576.

Plante, I., Davidovic, L., Ouellet, D.L., Gobeil, L.A., Tremblay, S., Khandjian, E.W., and Provost, P. (2006). Dicer-derived MicroRNAs are utilized by the fragile X mental retardation protein for assembly on target RNAs. Journal of Biomedicine and Biotechnology 2006, 64347-64347.

Plante, I., Ple, H., Landry, P., Gunaratne, P.H., and Provost, P. (2012). Modulation of microRNA activity by semi-nnicroRNAs. Frontiers in Genetics 3, 99-Article No.: 99.

Provost, P., Dishart, D., Doucet, J., Frendewey, D., Samuelsson, B., and Radmark, O. (2002). Ribonuclease activity and RNA binding of recombinant human Dicer. EMBO J 21, 5864-5874.

Qi, H.H., Ongusaha, P.P., Myllyharju, J., Cheng, D.M., Pakkanen, O., Shi, Y.J., Lee, S.W., Peng, J.M., and Shi, Y. (2008). Prolyl 4-hydroxylation regulates Argonaute 2 stability. Nature 455, 421-U478.

Rehwinkel, J., Behm-Ansmant, I., Gatfield, D., and Izaurralde, E. (2005). A crucial role for GW182 and the DCP1:DCP2 decapping complex in miRNA-mediated gene silencing. RNA 11, 1640-1647.

Ren, Y.F., Li, G.L., Wu, J.M., Xue, Y.F., Song, Y.J., Lv, L., Zhang, X.J., and Tang, K.F. (2012). Dicer-Dependent Biogenesis of Small RNAs Derived from 7SL RNA. Plos One 7, e40705-e40705.

Rivas, F.V., Tolia, N.H., Song, J.J., Aragon, J.P., Liu, J.D., Hannon, G.J., and Joshua-Tor, L. (2005). Purified Argonaute 2 and an siRNA form recombinant human RISC. Nature Structural \& Molecular Biology 12, 340-349.

Robb, G.B., and Rana, T.M. (2007). RNA helicase A interacts with RISC in human cells and functions in RISC loading. Molecular Cell 26, 523-537. 
Rudel, S., Wang, Y.L., Lenobel, R., Korner, R., Hsiao, H.H., Urlaub, H., Patel, D., and Meister, G. (2011). Phosphorylation of human Argonaute proteins affects small RNA binding. Nucleic Acids Research 39, 2330-2343.

Rybak, A., Fuchs, H., Hadian, K., Smirnova, L., Wulczyn, E.A., Michel, G., Nitsch, R., Krappmann, D., and Wulczyn, F.G. (2009). The let-7 target gene mouse lin-41 is a stem cell specific E3 ubiquitin ligase for the miRNA pathway protein Ago2. Nature Cell Biology 11, 1411-U1458.

Ryu, I., Park, J.H., An, S., Kwon, O.S., and Jang, S.K. (2013). eIF4GI Facilitates the MicroRNA-Mediated Gene Silencing. Plos One 8, e55725-e55725.

Salomon, W.E., Jolly, S.M., Moore, M.J., Zamore, P.D., and Serebrov, V. (2015). Single-Molecule Imaging Reveals that Argonaute Reshapes the Binding Properties of Its Nucleic Acid Guides. Cell 162, 84-95.

Sam, M., Wurst, W., Kluppel, M., Jin, O., Heng, H., and Bernstein, A. (1998). Aquarius, a novel gene isolated by gene trapping with an RNA-dependent RNA polymerase motif. Developmental Dynamics 212, 304-317.

Savas, J.N., Makusky, A., Ottosen, S., Baillat, D., Then, F., Krainc, D., Shiekhattar, R., Markey, S.P., and Tanese, N. (2008). Huntington's disease protein contributes to RNA-mediated gene silencing through association with Argonaute and P bodies. Proc Natl Acad Sci U S A 105, 10820-10825.

Schamberger, A., Sarkadi, B., and Orban, T.I. (2012). Human mirtrons can express functional microRNAs simultaneously from both arms in a flanking exon-independent manner. RNA Biology 9, 1177-1185.

Schirle, N.T., and MacRae, I.J. (2012). The Crystal Structure of Human Argonaute2. Science 336, 1037-1040.

Schirle, N.T., Sheu-Gruttadauria, J., Chandradoss, S.D., Joo, C., and MacRae, I.J. (2015). Water-mediated recognition of t1-adenosine anchors Argonaute2 to microRNA targets. Elife 4.

Schirle, N.T., Sheu-Gruttadauria, J., and MacRae, I.J. (2014). Structural basis for microRNA targeting. Science 346, 608-613.

Schoenberg, D.R., and Maquat, L.E. (2012). Regulation of cytoplasmic mRNA decay. Nature Reviews Genetics 13, 246-259.

Schwamborn, J.C., Berezikov, E., and Knoblich, J.A. (2009). The TRIM-NHL Protein TRIM32 Activates MicroRNAs and Prevents Self-Renewal in Mouse Neural Progenitors. Cell 136, 913-925.

Schwarz, D.S., Hutvagner, G., Du, T., Xu, Z., Aronin, N., and Zamore, P.D. (2003). Asymmetry in the assembly of the RNAi enzyme complex. Cell 115, 199-208.

Seo, G.J., Kincaid, R.P., Phanaksri, T., Burke, J.M., Pare, J.M., Cox, J.E., Hsiang, T.Y., Krug, R.M., and Sullivan, C.S. (2013). Reciprocal Inhibition between Intracellular Antiviral Signaling and the RNAi Machinery in Mammalian Cells. Cell Host \& Microbe 14, 435-445.

Seong, Y., Lim, D.H., Kim, A., Seo, J.H., Lee, Y.S., Song, H., and Kwon, Y.S. (2014). Global identification of target recognition and cleavage by the Microprocessor in human ES cells. Nucleic Acids Research 42, 12806-12821.

Shapiro, J.S., Schmid, S., Aguado, L.C., Sabin, L.R., Yasunaga, A., Shim, J.V., Sachs, D., Cherry, S., and Tenoever, B.R. (2014). Drosha as an interferon-independent antiviral factor. Proc Natl Acad Sci U S A 111, 7108-7113.

Shen, J., Xia, W.Y., Khotskaya, Y.B., Huo, L.F., Nakanishi, K., Lim, S.O., Du, Y., Wang, Y., Chang, W.C., Chen, C.H., et al. (2013). EGFR modulates microRNA maturation in response to hypoxia through phosphorylation of AGO2. Nature 497, 383-387. 
Shih, J.D., Waks, Z., Kedersha, N., and Silver, P.A. (2011). Visualization of single mRNAs reveals temporal association of proteins with microRNA-regulated mRNA. Nucleic Acids Research 39, 7740-7749.

Sibley, C.R., Seow, Y., Saayman, S., Dijkstra, K.K., El Andaloussi, S., Weinberg, M.S., and Wood, M.J.A. (2012). The biogenesis and characterization of mammalian microRNAs of mirtron origin. Nucleic Acids Research 40, 438-448.

Smibert, P., Yang, J.-S., Azzam, G., Liu, J.-L., and Lai, E.C. (2013). Homeostatic control of Argonaute stability by microRNA availability. Nature Structural \& Molecular Biology 20, 789-+.

Sohn, S.Y., Bae, W.J., Kim, J.J., Yeom, K.H., Kim, V.N., and Cho, Y. (2007). Crystal structure of human DGCR8 core. Nature Structural \& Molecular Biology 14, 847-853.

Soifer, H.S., Sano, M., Sakurai, K., Chomchan, P., Saetrom, P., Sherman, M.A., Collingwood, M.A., Behlke, M.A., and Rossi, J.J. (2008). A role for the Dicer helicase domain in the processing of thermodynamically unstable hairpin RNAs. Nucleic Acids Research 36, 6511-6522.

Sollier, J., Stork, C.T., Garcia-Rubio, M.L., Paulsen, R.D., Aguilera, A., and Cimprich, K.A. (2014). Transcription-coupled nucleotide excision repair factors promote R-loop-induced genome instability. Molecular Cell 56, 777-785.

Song, J.J., Smith, S.K., Hannon, G.J., and Joshua-Tor, L. (2004). Crystal structure of Argonaute and its implications for RISC slicer activity. Science 305, 1434-1437.

Stalder, L., Heusermann, W., Sokol, L., Trojer, D., Wirz, J., Hean, J., Fritzsche, A., Aeschimann, F., Pfanzagl, V., Basselet, P., et al. (2013). The rough endoplasmatic reticulum is a central nucleation site of siRNA-mediated RNA silencing. EMBO J 32, 1115-1127.

Stein, P., Svoboda, P., Anger, M., and Schultz, R.M. (2003). RNAi: Mammalian oocytes do it without RNA-dependent RNA polymerase. RNA 9, 187-192.

Stoica, C., Carmichael, J.B., Parker, H., Pare, J., and Hobman, T.C. (2006). Interactions between the RNA interference effector protein Agol and 14-3-3 proteins - Consequences for cell cycle progression. Journal of Biological Chemistry 281, 37646-37651.

Su, H., Trombly, M.I., Chen, J., and Wang, X.Z. (2009). Essential and overlapping functions for mammalian Argonautes in microRNA silencing. Genes \& Development 23, 304-317.

Suzuki, H.I., Katsura, A., Yasuda, T., Ueno, T., Mano, H., Sugimoto, K., and Miyazono, K. (2015). Small-RNA asymmetry is directly driven by mammalian Argonautes. Nature Structural \& Molecular Biology 22, 512-+.

Svoboda, P. (2014). Renaissance of mammalian endogenous RNAi. FEBS Letters 588, 2550-2556.

Tahbaz, N., Carmichael, J.B., and Hobman, T.C. (2001). GERp95 belongs to a family of signal-transducing proteins and requires Hsp90 activity for stability and Golgi localization. Journal of Biological Chemistry 276, 43294-43299.

Tahbaz, N., Kolb, F.A., Zhang, H.D., Jaronczyk, K., Filipowicz, W., and Hobman, T.C. (2004). Characterization of the interactions between mammalian PAZ PIWI domain proteins and Dicer. EMBO Rep 5, 189-194.

Takahashi, T., Zenno, S., Ishibashi, O., Takizawa, T., Saigo, K., and Ui-Tei, K. (2014). Interactions between the non-seed region of siRNA and RNA-binding RLC/RISC proteins, Ago and TRBP, in mammalian cells. Nucleic Acids Research 42, 5256-5269.

Takeshita, D., Zenno, S., Lee, W.C., Nagata, K., Saigo, K., and Tanokura, M. (2007). Homodimeric structure and double-stranded RNA cleavage activity of the c-terminal RNase III domain of human Dicer. Journal of Molecular Biology 374, 106-120. 
Takimoto, K., Wakiyama, M., and Yokoyama, S. (2009). Mammalian GW182 contains multiple Argonaute-binding sites and functions in microRNA-mediated translational repression. RNA 15, 1078-1089.

Tan, G.S., Garchow, B.G., Liu, X.H., Metzler, D., and Kiriakidou, M. (2011). Clarifying mammalian RISC assembly in vitro. BMC Molecular Biology 12, 19-19.

Tan, G.S., Garchow, B.G., Liu, X.H., Yeung, J., Morris, J.P., Cuellar, T.L., McManus, M.T., and Kiriakidou, M. (2009). Expanded RNA-binding activities of mammalian Argonaute 2. Nucleic Acids Research 37, 7533-7545.

Taylor, D.W., Ma, E.B., Shigematsu, H., Cianfrocco, M.A., Noland, C.L., Nagayama, K., Nogales, E., Doudna, J.A., and Wang, H.W. (2013). Substrate-specific structural rearrangements of human Dicer. Nature Structural \& Molecular Biology 20, 662-+.

Thonberg, H., Scheele, C.C., Dahlgren, C., and Wahlestedt, C. (2004). Characterization of RNA interference in rat PC12 cells: requirement of GERp95. Biochem Biophys Res Commun 318, 927-934.

Tian, Y., Simanshu, D.K., Ma, J.B., Park, J.E., Heo, I., Kim, V.N., and Patel, D.J. (2014). A Phosphate-Binding Pocket within the Platform-PAZ-Connector Helix Cassette of Human Dicer. Molecular Cell 53, 606-616.

Tomari, Y., Du, T., Haley, B., Schwarz, D.S., Bennett, R., Cook, H.A., Koppetsch, B.S., Theurkauf, W.E., and Zamore, P.D. (2004a). RISC assembly defects in the Drosophila RNAi mutant armitage. Cell 116, 831-841.

Tomari, Y., Matranga, C., Haley, B., Martinez, N., and Zamore, P.D. (2004b). A protein sensor for siRNA asymmetry. Science 306, 1377-1380.

Tomaselli, S., Galeano, F., Alon, S., Raho, S., Galardi, S., Polito, V.A., Presutti, C., Vincenti, S., Eisenberg, E., Locatelli, F., et al. (2015). Modulation of microRNA editing, expression and processing by ADAR2 deaminase in glioblastoma. Genome Biology 16, 5-5.

Tsujimura, K., Irie, K., Nakashima, H., Egashira, Y., Fukao, Y., Fujiwara, M., Itoh, M., Uesaka, M., Imamura, T., Nakahata, Y., et al. (2015). miR-199a Links MeCP2 with mTOR Signaling and Its Dysregulation Leads to Rett Syndrome Phenotypes. Cell Reports 12, 1887-1901.

Tu, C.C., Zhong, Y., Nguyen, L., Tsai, A., Sridevi, P., Tarn, W.Y., and Wang, J.Y.J. (2015). The kinase ABL phosphorylates the microprocessor subunit DGCR8 to stimulate primary microRNA processing in response to DNA damage. Science Signaling 8, ra64-ra64.

Vance, V., and Vaucheret, H. (2001). RNA silencing in plants--defense and counterdefense. Science 292, 2277-2280.

Venkatesh, T., Suresh, P.S., and Tsutsumi, R. (2016). tRFs: miRNAs in disguise. Gene 579, 133-138. Vesely, C., Tauber, S., Sedlazeck, F.J., Tajaddod, M., von Haeseler, A., and Jantsch, M.F. (2014). ADAR2 induces reproducible changes in sequence and abundance of mature microRNAs in the mouse brain. Nucleic Acids Research 42, 12155-12168.

Vesely, C., Tauber, S., Sedlazeck, F.J., von Haeseler, A., and Jantsch, M.F. (2012). Adenosine deaminases that act on RNA induce reproducible changes in abundance and sequence of embryonic miRNAs. Genome Research 22, 1468-1476.

Wang, H.W., Noland, C., Siridechadilok, B., Taylor, D.W., Ma, E.B., Felderer, K., Doudna, J.A., and Nogales, E. (2009). Structural insights into RNA processing by the human RISC-loading complex. Nature Structural \& Molecular Biology 16, 1148-U1144. 
Wang, X.H., Aliyari, R., Li, W.X., Li, H.W., Kim, K., Carthew, R., Atkinson, P., and Ding, S.W. (2006). RNA interference directs innate immunity against viruses in adult Drosophila. Science 312, 452-454.

Wang, Y., Lacroix, G., Haines, J., Doukhanine, E., Almazan, G., and Richard, S. (2010). The QKI-6 RNA binding protein localizes with the MBP mRNAs in stress granules of glial cells. Plos One $5,1-10$.

Wang, Y., Vogel, G., Yu, Z., and Richard, S. (2013). The QKI-5 and QKI-6 RNA binding proteins regulate the expression of microRNA 7 in glial cells. Molecular and Cellular Biology 33, 1233-1243.

Wee, L.M., Flores-Jasso, C.F., Salomon, W.E., and Zamore, P.D. (2012). Argonaute Divides Its RNA Guide into Domains with Distinct Functions and RNA-Binding Properties. Cell 151, 1055-1067.

Wei, J.X., Yang, J., Sun, J.F., Jia, L.T., Zhang, Y., Zhang, H.Z., Li, X., Meng, Y.L., Yao, L.B., and Yang, A.G. (2009). Both Strands of siRNA Have Potential to Guide Posttranscriptional Gene Silencing in Mammalian Cells. Plos One 4, e5382-e5382.

Weitz, S.H., Gong, M., Barr, I., Weiss, S., and Guo, F. (2014). Processing of microRNA primary transcripts requires heme in mammalian cells. Proc Natl Acad Sci U S A 111, 1861-1866.

Wen, J.Y., Ladewig, E., Shenker, S., Mohammed, J., and Lai, E.C. (2015). Analysis of Nearly One Thousand Mammalian Mirtrons Reveals Novel Features of Dicer Substrates. Plos Computational Biology 11, e1004441-e1004441.

Westholm, J.O., Ladewig, E., Okamura, K., Robine, N., and Lai, E.C. (2012). Common and distinct patterns of terminal modifications to mirtrons and canonical microRNAs. RNA 18, 177-192.

Wichroski, M.J., Robb, G.B., and Rana, T.M. (2006). Human retroviral host restriction factors APOBEC3G and APOBEC3F localize to mRNA processing bodies. Plos Pathogens 2, 374-383.

Wilkins, C., Dishongh, R., Moore, S.C., Whitt, M.A., Chow, M., and Machaca, K. (2005). RNA interference is an antiviral defence mechanism in Caenorhabditis elegans. Nature 436, 1044-1047.

Wilson, R.C., Tambe, A., Kidwell, M.A., Noland, C.L., Schneider, C.P., and Doudna, J.A. (2015). Dicer-TRBP Complex Formation Ensures Accurate Mammalian MicroRNA Biogenesis. Molecular Cell 57, 397-407.

Winter, J., Link, S., Witzigmann, D., Hildenbrand, C., Previti, C., and Diederichs, S. (2013). LoopmiRs: active microRNAs generated from single-stranded loop regions. Nucleic Acids Research $41,5503-5512$.

Wu, C., So, J., Davis-Dusenbery, B.N., Qi, H.H., Bloch, D.B., Shi, Y., Lagna, G., and Hata, A. (2011). Hypoxia Potentiates MicroRNA-Mediated Gene Silencing through Posttranslational Modification of Argonaute2. Molecular and Cellular Biology 31, 4760-4774.

Xia, J., Joyce, C.E., Bowcock, A.M., and Zhang, W.X. (2013). Noncanonical microRNAs and endogenous siRNAs in normal and psoriatic human skin. Human Molecular Genetics 22, 737-748.

Xie, M.Y., Li, M.F., Vilborg, A., Lee, N., Shu, M.D., Yartseva, V., Sestan, N., and Steitz, J.A. (2013). Mammalian 5 '-Capped MicroRNA Precursors that Generate a SingleMicroRNA. Cell 155, $1568-1580$.

$\mathrm{Xu}$, N., Gkountela, S., Saeed, K., and Akusjarvi, G. (2009). The 5'-end heterogeneity of adenovirus virus-associated RNAI contributes to the asymmetric guide strand incorporation into the RNA-induced silencing complex. Nucleic Acids Research 37, 6950-6959.

Yang, W.D., Chendrimada, T.P., Wang, Q.D., Higuchi, M., Seeburg, P.H., Shiekhattar, R., and Nishikura, K. (2006). Modulation of microRNA processing and expression through RNA editing by ADAR deaminases. Nature Structural \& Molecular Biology 13, 13-21. 
Yao, B., Li, S.Q., Lian, S.L., Fritzler, M.J., and Chan, E.K.L. (2011). Mapping of Ago2-GW182 Functional Interactions. In Argonaute Proteins: Methods and Protocols, pp. 45-62.

Ye, X.C., Huang, N.A., Liu, Y., Paroo, Z., Huerta, C., Li, P., Chen, S., Liu, Q.H., and Zhang, H. (2011). Structure of C3PO and mechanism of human RISC activation. Nature Structural \& Molecular Biology 18, 650-U643.

Yoda, M., Kawamata, T., Paroo, Z., Ye, X.C., Iwasaki, S., Liu, Q.H., and Tomari, Y. (2010). ATP-dependent human RISC assembly pathways. Nature Structural \& Molecular Biology 17, 17-U29.

Yu, J.H., Yang, W.H., Gulick, T., Bloch, K.D., and Bloch, D.B. (2005). Ge-1 is a central component of the mammalian cytoplasmic mRNA processing body. RNA 11, 1795-1802.

Yuan, Y.R., Pei, Y., Ma, J.B., Kuryavyi, V., Zhadina, M., Meister, G., Chen, H.Y., Dauter, Z., Tuschl, T., and Patel, D.J. (2005). Crystal structure of A-aeolicus Argonaute, a site-specific DNA-guided endoribonuclease, provides insights into RISC-mediated mRNA cleavage. Molecular Cell 19, 405-419.

Zamore, P.D., Tuschl, T., Sharp, P.A., and Bartel, D.P. (2000). RNAi: double-stranded RNA directs the ATP-dependent cleavage of mRNA at 21 to 23 nucleotide intervals. Cell 101, 25-33.

Zekri, L., Huntzinger, E., Heimstadt, S., and Izaurralde, E. (2009). The silencing domain of GW182 interacts with PABPC1 to promote translational repression and degradation of microRNA targets and is required for target release. Molecular and Cellular Biology 29, 6220-6231.

Zekri, L., Kuzuoglu-Ozturk, D., and Izaurralde, E. (2013). GW182 proteins cause PABP dissociation from silenced miRNA targets in the absence of deadenylation. EMBO J 32, 1052-1065.

Zeng, Y., Sankala, H., Zhang, X.X., and Graves, P.R. (2008). Phosphorylation of Argonaute 2 at serine-387 facilitates its localization to processing bodies. Biochemical Journal 413, 429-436.

Zhang, H.D., Kolb, F.A., Brondani, V., Billy, E., and Filipowicz, W. (2002). Human Dicer preferentially cleaves dsRNAs at their termini without a requirement for ATP. EMBO J 21, 5875-5885.

Zhang, H.D., Kolb, F.A., Jaskiewicz, L., Westhof, E., and Filipowicz, W. (2004). Single processing center models for human dicer and bacterial RNase III. Cell 118, 57-68.

Zhou, H.M., Yang, L., Li, H.J., Li, L.J., and Chen, J.M. (2009). Residues that affect human Argonaute2 concentration in cytoplasmic processing bodies. Biochem Biophys Res Commun 378, 620-624.

Zipprich, J.T., Bhattacharyya, S., Mathys, H., and Filipowicz, W. (2009). Importance of the C-terminal domain of the human GW182 protein TNRC6C for translational repression. RNA 15, 781-793.

Zou, J., Chang, M., Nie, P., and Secombes, C.J. (2009). Origin and evolution of the RIG-I like RNA helicase gene family. BMC Evolutionary Biology 9, 85. 


\title{
RNAi AND mIRNA PATHWAYS IN MAMMALS II - BIOLOGICAL ROLES
}

Keywords: dsRNA, siRNA, miRNA, Dicer, TARBP2, PACT, Argonaute

\section{PETR SVOBODA}

Institute of Molecular Genetics, Academy of Sciences of the Czech Republic, Videnska 1083, 14220 Prague 4, Czech Republic

Correspondence to: Petr Svoboda, Institute of Molecular Genetics ASCR, Videnska 1083, 14220 Prague 4, Czech Republic, tel. \#+420 241063147, e-mail: svobodap@img.cas.cz.

\begin{abstract}
RNA silencing denotes sequence-specific repression mediated by small RNAs. In mammals, there are two closely related pathways, which share several protein factors: RNA interference (RNAi) and microRNA (miRNA) pathway. The miRNA pathway regulates endogenous protein-coding gene expression. It has been implicated in many biological processes and majority of mammalian genes appear to be directly or indirectly exposed to miRNA-mediated regulations. RNAi generally serves as a form of innate immunity targeting viruses and mobile elements, although it occasionally also acquired function in protein-coding gene regulation. The function of RNAi in mammals is still poorly understood but it is clear that proteins supporting RNAi are also involved in miRNA biogenesis and function. Because of the large volume of the existing literature, the review of mammalian miRNA and RNAi pathways was divided into two parts, where first one reviewed components of the pathways and the second one, presented here, reviews roles and significance of the pathways.
\end{abstract}

\section{Introduction}

In the first part of the review off mammalian RNAi and miRNA pathways, I focused on mechanistic description of the pathways. Here, I will provide an overview of biological roles and biological phenomena associated with mammalian RNAi and miRNA pathways (Fig. 1).

\section{miRNA-mediated control of gene expression - important functional aspects}

The current miRBase (Kozomara and Griffiths-Jones, 2014) edition 22.1 annotates 1917 human miRNA loci that give rise to 2654 annotated miRNAs. There are 1234 precursors and 


\section{mammalian somatic cells}

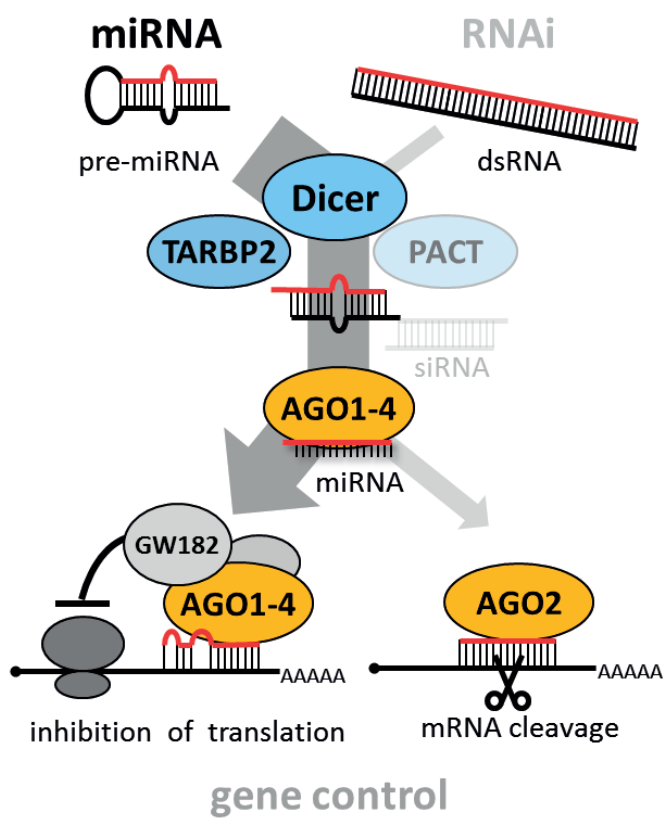

Figure 1 Mechanistical merging of miRNA and RNAi pathways in mammals

1978 mature miRNAs annotated in mouse. A simple connection of these counts with the fact that only nucleotides $2-8$ of a miRNA are sufficient for target recognition and suppression implies that miRNA-mediated repression is a widespread and extremely evolvable regulatory system for gene expression. At the same time, one should not forget the above-mentioned stoichiometry between miRNAs and their target sites that is needed for efficient silencing.

Evolution of miRNAs is fast - there are only a few miRNAs conserved between Drosophila and mammals. Given the diversity of canonical and non-canonical miRNAs, it is conceivable that miRNAs to emerge from random formation of Drosha/Dicer substrates. Newly evolving miRNAs likely form a considerable portion of annotated miRNAs, especially in species where miRNAs are deeply sequenced and low-abundant miRNAs are identified.

According to the evolutionary theory, new miRNAs would either acquire function and become fixed during evolution or they would be lost. In addition, the target repertoire of existing miRNAs can also rapidly evolve since a single point mutation can weaken an existing regulation or create a new one. This idea is consistent with the data showing that mammalian mRNAs are under selective pressure to maintain and/or avoid specific 7-nucleotide seeding regions (Farh et al., 2005). It can be nicely exemplified on the Texel sheep phenotype where a single mutation creating a novel miRNA target site in myostatin causes the exceptional meatiness of this breed (Clop et al., 2006). 
The set of miRNAs in each cell type forms a combinatorial post-transcriptional regulation system stabilizing gene expression pattern. miRNAs have widespread impact on expression and evolution of protein-coding genes (Farh et al., 2005). The number of mRNAs that have functionally important interaction with miRNAs (i.e. suppression of this interaction yields a phenotype) in a studied model system is presumably small and certainly difficult to discern among the possible interactions. Thus, every search for functionally important interactions between miRNAs and their targets has to face the fact that miRNAs represent a dynamically evolving system with countless random interactions, which are not biologically relevant.

\section{Extracellular microRNAs}

An interesting research field developed around the release of miRNAs from cells, detection of extracellular miRNAs, and transfer between cells. Importantly, the vast majority of the references provided descriptive and correlative data documenting presence of circulating miRNAs under different conditions (e.g. (Arroyo et al., 2011; Bellingham et al., 2012; Huang et al., 2013b; Luo et al., 2009; Novellino et al., 2012; Turchinovich et al., 2011). I will not review the bulk of the circulating RNA literature, which provides data concerning biomarker potential of circulating miRNAs, undoubtedly of extreme clinical relevance but of minimal relevance for this review. Below, I summarize results, which admittedly raise more questions than provide satisfactory answers.

Small RNAs can be transmitted from one cell to another under physiological conditions, as evidenced, for example, by systemic RNAi in arthropods or plants. Small RNAs can utilize dedicated transporters, common communication channels, or secretory vehicles. It was also reported that Gap junctions can serve for miRNA transfer from microvascular endothelial cells to colon cancer cells (Thuringer et al., 2016). Circulating mammalian miRNAs were reported 2008 when they were found in serum of lymphoma patients; they were immediately recognized as potential non-invasive biomarkers for cancer diagnostics and treatment (Lawrie et al., 2008). The same year, placental miRNAs were found circulating in maternal plasma (Chim et al., 2008), which was one of the discoveries leading to the notion that miRNAs could be a mobile regulating molecule (Iguchi et al., 2010) and that could even mediate transgenerational epigenetic heritance (Sharma, 2015) or be transmitted across species (Buck et al., 2014; Zhang et al., 2012). Since then, extracellular miRNAs were identified in a broad range of biological fluids, including plasma, aqueous humour, cerebrospinal fluid, nasal mucus, or milk (Baglio et al., 2015; Dismuke et al., 2015; Huang et al., 2013b; Izumi et al., 2015; Kropp et al., 2014; Pegtel et al., 2011; Wu et al., 2015a). miRNAs were identified in the cargo of exosomes, membranous vesicles 40 to $100 \mathrm{~nm}$ in diameter, which are constitutively released by almost all cell type and are found essentially in every biological fluid (reviewed, for example, in Rak, 2013; Yoon et al., 2014)

However, extracellular miRNAs do not need to be necessarily encapsulated in extracellular vesicles, as two studies showed that $95-99 \%$ of extracellular miRNA are not in extracellular vesicles but associated with AGO proteins in serum and cell culture media (Arroyo et al., 2011; Turchinovich et al., 2011). Furthermore, most individual exosomes in standard 
preparations do not seem to contain biologically significant numbers of miRNAs (Chevillet et al., 2014). The molecular mechanism of miRNA release, either as a cargo in a vesicle or free, is poorly understood and the current knowledge does not allow for building a coherent model as the literature is scarce. Non-templated nucleotide additions were found to distinguish between cellular miRNAs, which were 3' end adenylated in cells whereas 3' end uridylated isoforms appeared overrepresented in exosomes suggesting a possible role of 3 ' terminal modifications in sorting miRNAs into extracellular vesicles (Koppers-Lalic et al., 2014). Recently, ALIX, an accessory protein of the endosomal sorting complex, it has been implicated in sorting miRNAs into extracellular vesicles based on its interaction with $\mathrm{AGO} 2$ and reduced miRNAs levels in extracellular vesicles upon Alix knock-down (Iavello et al., 2016).

Importantly, any model where miRNAs would be carried over to regulate gene expression by the canonical miRNA activity must face the kinetic data mentioned above (Wee et al., 2012). While one cannot exclude a non-canonical signalling function of circulating miRNAs (which has not been conclusively demonstrated yet), the literature on circulating RNAs may include misleading statements, which are unsupported by experimental evidence.

Taken together, while existence of circulating miRNAs has been demonstrated beyond a doubt, experimental evidence for their function (if any) is not conclusive. Exosomal vesicles can carry miRNAs and siRNAs - in the latter case, exosomes were adapted for a delivery tool for siRNAs, which has a good potential for further development of siRNA therapy (El-Andaloussi et al., 2012; Kumar et al., 2015; Lasser, 2012; Lee et al., 2012; Nguyen and Szoka, 2012; Shtam et al., 2013; Wahlgren et al., 2012; Wahlgren et al., 2016).

\section{RNAi pathway in mammals- important functional aspects}

It should reiterated that the, "so-called" RNAi knock-down with siRNAs in mammalian cells is essentially using the miRNA pathway with retained the ability to cleave perfectly complementary targets by AGO2. The mammalian canonical RNAi (i.e. long dsRNA-driven) is a dormant pathway, at best. By that is meant that the protein factors present in every mammalian cells (Dicer, TARBP2, and AGO2) are competent to support RNAi but long dsRNA does not efficiently induce RNAi in most mammalian cells (Nejepinska et al., 2012). This notion is supported by the reconstitution of human RNA interference in budding yeast demonstrates that Dicer, TARBP2, and AGO2 are sufficient to functionally reconstitute RNAi (Suk et al., 2011). This demonstrates that these three proteins constitute the essential core of RNAi mechanism although RNAi is not properly reconstituted when bona fide RNAi precursors were co-expressed (Wang et al., 2013). The problem is apparently at the level of Dicer processing as the human slicer AGO2 RNAi role is so conserved that it could function in RNAi in the early divergent protozoan Trypanosoma brucei, demonstrating conservation of basic features of the RNAi mechanism (Shi et al., 2006). In an analogous experiment, human AGO2 could not replace Arabidopsis thaliana AGO1 in the miRNA pathway (Deveson et al., 2013). In a sense, these different results are not that surprising considering the minimal requirements for RNAi and the complexity of the miRNA pathway, which provides a larger space for evolution of incompatible adaptations. 
However, there are some cases indicating that RNAi is still active in mammals and, under unique circumstances, may be even an essential pathway.

The main bottleneck for canonical RNAi in mammals is efficient production of siRNAs from long dsRNA, which is poor in most mammalian cells (Flemr et al., 2013; Nejepinska et al., 2012). However, several reports showed that induction of RNAi with intracellular expression of long dsRNA can be achieved in transformed and primary somatic cells (Diallo et al., 2003; Elbashir et al., 2001; Gan et al., 2002; Shinagawa and Ishii, 2003; Tran et al., 2004; Yi et al., 2003). These data imply that RNAi can occur if there is a sufficient amount of long dsRNA, which is directed preferentially to RNAi but not into other dsRNA pathways. Under these circumstances, the limiting factor is just Dicer's ability to produce siRNA (Flemr et al., 2013).

\section{Endogenous RNAi in the germline}

\section{Retrotransposon repression in mouse oocytes}

RNAi-mediated mobile element silencing has also been documented in the mouse germline (Tam et al., 2008; Watanabe et al., 2006; Watanabe et al., 2008). Mutations in the piRNA pathway components are detrimental to sperm development, suggesting that piRNAs are the dominant class of small RNAs controlling mobile element activity in the male germline (reviewed in Toth et al., 2016). In contrast, female mice lacking functional piRNA pathway are fertile with no obvious defects in oocytes (Carmell et al., 2007). Endo-siRNAs suppress TEs silencing in mammalian oocytes as documented by derepression of some retrotransposons in oocytes depleted of Dicer or AGO2 (Murchison et al., 2007; Watanabe et al., 2008). As already proposed for invertebrates, the piRNA and endo-siRNA pathways likely cooperate in creating a complex silencing network against mobile elements in the mammalian germline. Long terminal repeat MT elements and SINE elements are strongly upregulated in Dicer ${ }^{-/}$oocytes, while the levels of IAP transposon are elevated in the absence of MILI protein but not in Dicer/- oocytes (Murchison et al., 2007; Watanabe et al., 2008). Still many loci composed of other types of TEs, e.g. LINE retrotransposons, give rise to both piRNAs and endo-siRNAs, again suggesting that the biogenesis of these small RNAs is interdependent. The role of endogenous RNAi in TE silencing extends from germ cells to preimplantation embryo stages. Apart from maternally derived piRNAs and endo-siRNAs, which persist in the embryos for a large part of preimplantation development, zygotic endo-siRNAs are generated de novo mainly to control the activity of zygotically activated MuERV-L retrotransposon (Ohnishi et al., 2010; Svoboda et al., 2004). SINE-derived endo-siRNAs also increase in abundance in early embryo stages, which is consistent with the observation that B1/Alu SINE endo-siRNAs account for a vast majority of endo-siRNAs sequenced from mouse ES cells (mESCs) (Babiarz et al., 2008). Whether these SINE endo-siRNAs play an active role in TE silencing in mESCs similarly to other TE-derived endo-siRNAs in oocytes remains to be determined. RNAi-dependent silencing of LINE transposons has also been described in cultured HeLa cells, where endo-siRNAs derived from bidirectional transcripts of sense and antisense L1 promoter were proposed to control L1 activity (Yang and Kazazian, 2006). Although some evidence for retrotransposon-derived endo-siRNAs 
from mammalian somatic cells was obtained from deep sequencing data (Kawaji et al., 2008), a convincing support for the function of endo-siRNAs in TE silencing in mammalian somatic tissues, has yet to be provided.

\section{Control of endogenous genes in mouse oocytes}

In mice, perturbation of the endo-siRNA pathway in oocytes is responsible for severe meiotic defects and resulting female infertility. Targeted oocyte-specific knockout of both Dicer and $A g o 2$ lead to similar phenotypes including chromosome misalignment and defective spindle (Kaneda et al., 2009; Murchison et al., 2007; Tang et al., 2007). These effects were originally attributed to the loss of maternal miRNAs. However, miRNA pathway is suppressed in mouse oocytes and oocytes lacking $D g c r 8$, which is required for canonical miRNA biogenesis, can be fertilized and do not show any significant disturbance of the transcriptome (Ma et al., 2010; Suh et al., 2010). This means that the canonical miRNA pathway is non-essential and largely inactive in mouse oocytes despite intact biogenesis of miRNAs (Fig. 2). In fact, the spindle phenotype is caused by the loss of a highly active RNAi pathway in mouse oocytes. High-throughput analysis of small RNAs in mouse oocytes revealed a unique class of endo-siRNAs derived from processed pseudogenes (Tam et al., 2008; Watanabe et al., 2008). Transcriptomes of oocytes lacking Dicer and Ago2 (including oocytes expressing catalytically-dead AGO2) are similarly affected (Kaneda et al., 2009; Stein et al., 2015). At the same time, genes matching pseudogene-derived endo-siRNAs are enriched in the group of upregulated genes in both knockouts (Kaneda et al., 2009; Stein et al., 2015; Tam et al., 2008; Watanabe et al., 2008).

In addition, putative endo-siRNA targets are enriched in cell cycle regulators and genes involved in microtubule organization and dynamics (Tam et al., 2008). These findings suggest that regulation of protein-coding genes by endo-siRNAs controls the equilibrium of protein factors required for proper spindle formation, chromosome segregation and meiosis progression in mouse oocytes. As pseudogenes are rapidly evolving source of dsRNA for endo-siRNA production, it will be interesting to investigate whether the role of RNAi in spindle formation during meiotic maturation of oocytes is conserved in mammals.

The reason for high levels of endo-siRNAs and the high RNAi activity in mouse oocytes is the aforementioned truncated Dicer isoform that lacks the N-terminal helicase domain (Flemr et al., 2013) (Fig. 2). It efficiently generates siRNAs from long dsRNAs, and is sufficient for enhancing RNAi in cultured cells while its loss in mouse oocytes yields the same phenotype as conditional knock-outs of Dicer or Ago2 (Flemr et al., 2013).

Endo-siRNAs have also been proposed to contribute to the self-renewal and proliferation of mouse embryonic stem cells (mESCs), since the proliferation and differentiation defects observed in Dicer-/- mESCs are more dramatic than in $\mathrm{Dgcr}$ 8-/ $^{-/}$mESCs (Kanellopoulou et al., 2005; Murchison et al., 2005; Wang et al., 2007). A population of endo-siRNAs derived mostly from hairpin forming B1/Alu subclass of SINE elements was identified in mESCs (Babiarz et al., 2008). Fragments of SINE elements are commonly present in untranslated regions of protein-coding transcripts and it is therefore possible that SINE-derived endo-siRNAs participate in posttranscriptional gene silencing in mESCs. However, this hypothesis has not been tested experimentally. 


\section{mouse oocytes}

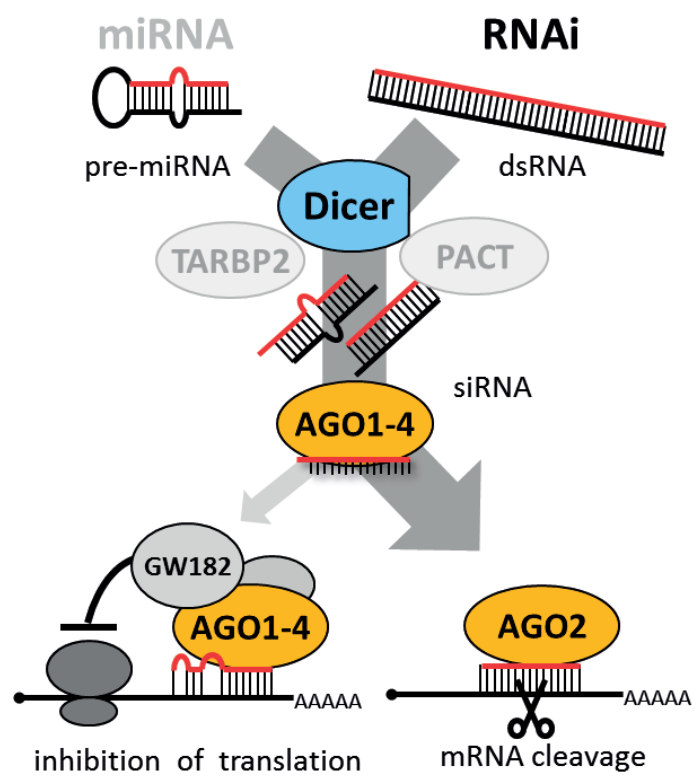

gene control \& defense

Figure 2 miRNA \& RNA arrangement in mouse oocytes

\section{Endogenous RNAi in the soma}

Little evidence is available for potential role of endo-siRNAs in the regulation of protein-coding mRNAs in mammalian somatic tissues. The natural antisense transcription in somatic cells, which has a potential to generate dsRNA, yields low levels of endo-siRNAs, whose biological relevance is questionable. At the same time, endo-siRNAs derived from natural antisense transcripts of Slc34a gene were identified in mouse kidney, where $\mathrm{Na}$ /phosphate cotransporter exerts its physiological function (Carlile et al., 2009). However, changes in expression levels of Slc34a upon suppression of the endo-siRNA pathway have not been addressed. In mouse hippocampus, deep sequencing revealed a set of potential endo-siRNAs generated from overlapping sense/antisense transcripts and from hairpin structures within introns of protein-coding genes (Smalheiser et al., 2011) . The most abundant endo-siRNAs from SynGAPl gene locus were also found in complexes with AGO proteins and FMRP in vivo. Interestingly, a large part of potential hippocampal endo-siRNA targets encode for proteins involved in the control of synaptic plasticity and the number of endo-siRNAs derived from these gene loci increased significantly during olfactory discrimination training (Smalheiser et al., 2011). Given the fact that vast majority of identified endo-siRNA sequences mapped to intronic regions, the endo-siRNAs could 
act co-transcriptionally on nuclear pre-mRNAs, perhaps similarly to the mechanism of RNAi-mediated inhibition of RNA Pol II elongation described in C. elegans (Guang et al., 2010). Alternatively, endo-siRNAs could control correct distribution of target mRNAs as unspliced pre-mRNA can be exported from the neuronal nucleus and transported to dendrites for processing (Glanzer et al., 2005). In any case, these findings open an attractive hypothesis that endo-siRNAs participate in synaptic plasticity during learning process and the neuronal endo-siRNA pathway might be also linked to various neurodegenerative disorders (Smalheiser et al., 2011).

\section{Antiviral RNAi}

In contrast to nematodes and insects, data supporting involvement of mammalian RNAi in antiviral defense is weak (reviewed in detail in Cullen, 2006; Cullen et al., 2013). It is unlikely that RNAi substantially acts as an antiviral mechanism in mammals where long dsRNA induces a complex sequence-independent antiviral response, commonly known as the interferon response (reviewed in Gantier and Williams, 2007). Consistent with this, no siRNAs of viral origin have been found in human cells infected with a wide range of viruses (Pfeffer et al., 2005). Occasional observations, such as detection of a single siRNA in HIV-1 infected cells (Bennasser et al., 2005) does not provide any conclusive evidence that RNAi is processing viral dsRNA and suppresses viruses under physiological conditions in vivo.

It must be stressed that circumstantial evidence suggesting the role of RNAi in viral suppression must be critically examined and interpreted. One has to keep in mind, for example, that data, which appear as evidence for viral suppression by RNAi, could reflect miRNA-mediated effects. Since viruses co-evolve with different hosts and explore all possible strategies to maintain and increase their fitness, it is not surprising that viral reproductive strategies come into contact with mammalian RNA silencing pathways, particularly the miRNA pathway, which shares components with the RNAi pathway. For example, EpsteinBarr virus (EBV) and several other viruses encode their own miRNAs (Parameswaran et al., 2010; Pfeffer et al., 2005; Pfeffer et al., 2004; Sullivan et al., 2005) or take advantage of host cell miRNAs to enhance their replication (Jopling et al., 2005).

Another evidence for an interaction between viruses and RNA silencing is the presence of putative suppressors of RNA silencing (SRS) in various viruses. As viral genomes rapidly evolve, SRS should be functionally relevant. For example, B2 protein in Nodaviruses (e.g. FHV) is essential for replication, inhibits Dicer function, and B2-deficient FHV can be rescued by artificial inhibition of RNAi response ( $\mathrm{Li}$ et al., 2002). B2 protein also enhances the accumulation of Nodaviral RNA in infected mammalian cells (Fenner et al., 2006; Johnson et al., 2004). Other potential SRS molecules have been identified in viruses infecting vertebrates, such as Adenovirus VA1 noncoding RNA (Lu and Cullen, 2004), Influenza NS1 protein (Li et al., 2004), Vaccinia virus E3L protein (Li et al., 2004), Ebola virus VP35 protein (Haasnoot et al., 2007), TAS protein in primate foamy virus (Lecellier et al., 2005), or HIV-1 TAT protein (Bennasser et al., 2005).

The existence of SRS in viruses infecting mammals does not prove that these viruses are targeted by mammalian RNAi. First, viruses may have a broader range of hosts (or vectors), 
including, e.g. blood sucking insects. Thus, a virus can be targeted by RNAi in one host and by another defense mechanism in another one. For example, the Dengue virus, whose life cycle takes place in humans and mosquitoes, is targeted by RNAi in mosquitoes and it likely evolved an adaptation to circumvent RNAi (Sanchez-Vargas et al., 2009). Second, viral SRS in mammalian cells may have other purpose than counteracting viral suppression by RNAi. Since biogenesis and mechanism of action of mammalian miRNAs overlaps with RNAi, it is possible that the role of such SRS is to modify cellular gene expression by suppressing the activity of miRNAs. Third, the main effect of SRS may be aimed at other defense mechanisms recognizing and responding to dsRNA and, as a consequence, SRS effects on RNAi are observed.

\section{Systemic RNAi in mammals}

Non-cell autonomous RNA with an extent similar to that of C. elegans or in some insects is highly unlikely to function in vertebrates. However, a limited environmental or systemic RNAi may exist there as the homologs of sid- 1 have been found in all sequenced vertebrate genomes (Jose and Hunter, 2007). Two sid-1 homologs (SidT1 and SidT2) are present in mice and humans with a documented role for SidT1 in dsRNA uptake in humans (Duxbury et al., 2005; Wolfrum et al., 2007). Furthermore, experimental overexpression of human SidT1 significantly facilitated cellular uptake of siRNAs and resulted in increased RNAi efficacy (Duxbury et al., 2005). As it will be discussed later, the mammalian immune system employs a number of proteins responding to dsRNA independently of RNAi (Gantier and Williams, 2007), while RNAi does not seem to participate in the innate immunity (Cullen, 2006; Cullen et al., 2013). Thus, the primary role of a dsRNA uptake mechanism in mammals is likely not involving RNAi even though it could have served such a role in an ancestral organism.

\section{Nuclear function of small RNAs}

The literature search yielded a large heterogeneous group of publications concerning nuclear localization of Dicer and AGO proteins as well as nuclear effects, including transcriptional gene silencing. Some of these observations might come from physiologically relevant nuclear silencing mechanisms. However, when critically evaluating published studies, not enough evidence was found, to establish a model for transcriptional silencing in mammals; except of the PIWI-induced transcriptional silencing in the germline (REF). Here, I will provide an overview of nuclear aspects of RNA silencing and highlight those observations which might be related to the miRNA pathway or long dsRNA response.

Homology-dependent phenomena and observations that may reflect nuclear mechanisms involving small RNAs can be sorted into several areas, which will be discussed further below:

Indirect effects of miRNAs on chromatin

Nuclear RNAi (nuclear post-transcriptional silencing)

Transcriptional regulations (stimulation/repression) by exogenous small RNAs 
Transcriptional regulations (stimulation/repression) by miRNAs

27-nt RNA-mediated regulation of endothelial nitric oxide synthase gene

Splicing regulation by small $R N A$ s

$D N A$ repair-associated small $R N A s$

First, it is necessary to discuss nuclear localization of miRNA and RNAi factors, since their nuclear localization is a pre-requisite for nuclear silencing. Pioneering RNAi work indicated that silencing occurs in the cytoplasm because dsRNA against intronic sequences had no silencing effect (Fire et al., 1998) and the RISC complex co-purified with ribosomes (Hammond et al., 2000). Early studies in mammalian cells also suggested that RNAi is cytoplasmic (Billy et al., 2001; Zeng et al., 2002). However this notion was subsequently challenged by a series of studies reporting nuclear RNAi and small RNA-induced transcriptional silencing (Morris et al., 2004; Robb et al., 2005; Ting et al., 2005).

\section{Nuclear localization of Dicer}

A number of works directly or indirectly implies nuclear localization of Dicer (Doyle et al., 2013; Drake et al., 2014; Gagnon et al., 2014a; Gullerova and Proudfoot, 2012; Haussecker and Proudfoot, 2005; Neve et al., 2016; Ohrt et al., 2012; Sinkkonen et al., 2010; White et al., 2014), which contrasts with a recent in vivo study on mouse Dicer where tagging of an endogenous Dicer gene with an antibody epitope yielded exclusively cytoplasmic localization in all analyzed tissues with an extremely sensitive detection limit for nuclear Dicer (Much et al., 2016). Thus, despite the collection of the reports below, one should still approach the nuclear aspect cautiously. One of the earlier implications for nuclear localization of Dicer was the reported Dicer-dependent turnover of intergenic transcripts from the human beta-globin gene cluster (Haussecker and Proudfoot, 2005). However, this study showed mostly correlation of abundance of nuclear transcripts. Nuclear Dicer processing was also implicated by several other studies (Flemr et al., 2013; Neve et al., 2016; Valen et al., 2011; White et al., 2014). In terms of function, nuclear Dicer was thought to be involved in nuclear dsRNA processing (?) (White et al., 2014), selection of alternative polyadenylation sites (Neve et al., 2016) or rRNA processing (Liang and Crooke, 2011). Several studies documented Dicer nuclear localization by microscopy. Dicer was detected in cultured mammalian cells with several different antibodies in the chromatin where it resided in rDNA clusters on acrocentric human chromosomes (Sinkkonen et al., 2010). Finer mapping using chromatin immunoprecipitation suggested that Dicer localizes in the proximity of the rRNA transcribed region (Sinkkonen et al., 2010). However, this study failed to reveal any functional significance of Dicer localization and it is not clear if the localization is related to the later reported role of Dicer in pre-rRNA processing (Liang and Crooke, 2011) or to rDNA-derived small RNAs (Wei et al., 2013).

Furthermore, localization of Dicer to rDNA is distinct from the nuclear localization of Dicer detected by fluorescence correlation/cross-correlation spectroscopy (FCS/ FCCS) (Ohrt et al., 2012), since FCS/ FCCS can detect diffusing and not rDNA chromatin-bound Dicer. FCS/FCCS identified Dicer in the nucleus in HeLa cells and suggested that nuclear Dicer is alone while the cytoplasmic Dicer exists in a large complex with AGO2 (presumably RLC) (Ohrt et al., 2012). This would imply that while Dicer could process nuclear 
substrates, they would not be loaded on AGO proteins in the nucleus. However, a later study suggested that Dicer, TARBP2, AGO2 and GW182 associate into a large complex in the nucleus although, consistently with FCS/FCCS data, loading of nuclear small RNA duplexes was not detected (Gagnon et al., 2014a). Regarding the nuclear localization mechanism of Dicer, it does not employ a canonical nuclear localization signal (NLS). A pyruvate kinase fusion system suggested that dsRBP of Dicer could function as an NLS similarly to ADAR dsRBD (Doyle et al., 2013). However, this study did not prove nuclear localization of full-length Dicer under physiological conditions. Interestingly, an additional report implied phosphorylation of Dicer in nuclear localization in nematodes, humans and mice (Drake et al., 2014). Remarkably, one of the two reported phosphorylation sites for ERK was in the dsRBD, providing a hypothetical link to analysis of the study implying dsRBD in nuclear localization of Dicer. Yet, as mentioned above, EGFP tagging of Dicer in mice does not support nuclear localization of Dicer (Much et al., 2016). Taken together, nuclear localization/function of Dicer is still poorly understood and further research is needed to build a more coherent picture from the contradictory observations.

\section{Nuclear AG01-4}

A similarly unclear situation exists for nuclear AGO1-4 proteins. AGO1-4 proteins were observed in the nucleus under different circumstances including immunofluorescent staining, such as, for example (Ahlenstiel et al., 2012; Allo et al., 2014; Aporntewan et al., 2011; Bai et al., 2014; Berezhna et al., 2006; Gagnon et al., 2014a; Huang et al., 2013a; Jang et al., 2012; Janowski et al., 2006; Kim et al., 2012; Kim et al., 2006; Liang and Crooke, 2011; Nishi et al., 2013; Ohrt et al., 2008; Ohrt et al., 2012; Robb et al., 2005; Rudel et al., 2008; Sharma et al., 2016; Tan et al., 2009a). Notably, one should be cautious about nuclear localization detected by antibodies and cellular fractionation in the absence of appropriate controls. While antibody cross-reactivity often cannot be excluded, biochemical fractionations suffer from impurities and endoplasmic reticulum contamination is frequently not examined. In any case, if there would be any consensus about nuclear AGO localization, it seems that a small fraction of AGO proteins is indeed in the nucleus and can engage complementary RNAs (Berezhna et al., 2006; Gagnon et al., 2014b; Ohrt et al., 2008; Robb et al., 2005). The mechanism of nuclear import of AGO proteins is unclear. AGO proteins do not carry a canonical NLS. It has been proposed that AGO proteins could be imported into the nucleus by GW182 (Nishi et al., 2013) or via Importin 8 (Weinmann et al., 2009).

The next section reviews effects of small RNA mechanisms in the nucleus, starting with indirect ones.

\section{Indirect effects of miRNAs on chromatin}

Studies of miRNA targets suggest that 10 to $30 \%$ of human genes are potential miRNA targets (John et al., 2004; Lewis et al., 2003). Also, experiments with delivering miRNAs into different cell types suggest that individual miRNAs can down-regulate a large number of genes (Lim et al., 2005). Thus, it is not surprising that many genes regulating chromatin structure are directly or indirectly regulated by miRNAs. One of the recent examples of 
such a connection is the regulation of DNA methylation in murine embryonic stem cells (ESCs). Phenotype analysis of Dicer ${ }^{-/}$cells revealed that the loss of Dicer in ES cells leads to defects in differentiation and it may (Kanellopoulou et al., 2005) or may not (Murchison et al., 2005) lead to aberrant changes in centromeric chromatin. It has been speculated that Dicer functions in a pathway similar to that of Schizosaccharomyces pombe (Kanellopoulou et al., 2005). This interpretation was consistent with previous analysis of chicken-human hybrid DT40 cell line lacking Dicer, where defects in heterochromatin were also observed (Fukagawa et al., 2004). However, cloning of small RNAs from WT and Dicer ${ }^{-/}$ES cells suggests that ES cells do not naturally produce endogenous siRNAs and that Dicer exclusively produces miRNAs (Calabrese et al., 2007).

Detailed analysis of the transcriptome of murine Dicer-/ ES cells (Sinkkonen et al., 2008) correlated changes of gene expression with the presence of binding sites for AAGUGC-seeded miRNAs (miR-290 cluster and other miRNAs), which were previously found in human and murine ES cells (Houbaviy et al., 2005; Suh et al., 2004). The loss of Dicer and miRNAs resulted in down-regulation of de novo DNA methyltransferases and defects in de novo DNA methylation during differentiation. This defect could be rescued by over-expressing de novo DNA methyltransferases or by transfection of the miR-290 cluster miRNAs (Sinkkonen et al., 2008). These data were complemented by the study of Benetti et al., who showed that the loss of Dicer leads to decreased DNA methylation, concomitant with increased telomere recombination and telomere elongation (Benetti et al., 2008).

Regulation of de novo DNA methyltransferases by miRNAs is likely much more complex than described above because other miRNAs were also implicated in their direct regulation in other cell types (Duursma et al., 2008; Fabbri et al., 2007). Importantly, the genetic background or culturing conditions can also influence epigenetic changes in studied cells, which might explain why a third study of Dicer-deficient ESCs observed normal DNA methylation dynamics (Ip et al., 2012).

In any case, it is very likely that miRNAs play a similar role in other aspects of chromatin formation. Considering that up to $60 \%$ of the genes are possibly regulated by miRNAs, data from experiments which directly or indirectly affect the miRNA pathway (including siRNA off-targeting), should be handled with open mind and great caution.

\section{Post-transcriptional regulations by small RNAs in the nucleus - nuclear RNAi}

In mammalian cells, Robb et al. showed that nuclear RNAs can be targeted by RNAi (Robb et al., 2005). In addition they also provided biochemical data showing that AGO1 and AGO2 localize into the nuclear RISC (Robb et al., 2005). While these data did not make a conclusive evidence as contamination of the nuclear fraction with AGO proteins associated with outer nuclear envelope could not be excluded, nuclear localization of AGOs was later backed up by other data (Berezhna et al., 2006; Gagnon et al., 2014b; Ohrt et al., 2008). Berezhna et al. showed that siRNAs accumulate in the nucleus in a cognate mRNA dependent manner (Berezhna et al., 2006). Ohrt et al. reported that siRNAs against firefly luciferase microinjected into HeLa cells enter nucleus but are actively excluded from non-nucleolar space in Exportin-5 dependent manner (Ohrt et al., 2006). 
Taken together, loaded AGO2 seems to be able to engage nuclear RNAs and, in case of perfect complementarity, it can cleave its targets. How nuclear RNAi incorporates nuclear and cytoplasmic small RNA precursors and how it is working under physiological conditions on perfectly complementary and partially complementary targets remains largely unknown.

\section{Transcriptional regulations (stimulation/repression) by exo-siRNAs}

In plants and fungi, RNA silencing mechanisms also mediate transcriptional silencing. Similar transcriptional silencing phenomena were intensely searched also in mammals. The first two reports of transcriptional silencing in mammals were published in 2004 when two groups reported siRNA-mediated transcriptional silencing coupled with DNA methylation (Kawasaki and Taira, 2004; Morris et al., 2004) and laid problematic foundations of transcriptional silencing research in mammals. The reason is that reproducibility of both reports was quickly questioned and one of them was later forced retracted, formally because "a proper data notebook is not available as evidence to support our findings, which constitutes non-adherence to ethical standards in scientific research. In accordance with the recommendations from the National Institute of Advanced Industrial Science and Technology, K.T. therefore wishes to retract this paper." (Taira, 2006). While the second report has not been retracted, DNA methylation has been doubted as a key silencing effect (Ting et al., 2005). Furthermore, the report did not analyse induction of DNA methylation by informative bisulfite sequencing but relied on suboptimal methylation-sensitive restriction digest. Another troubling aspect of the second report is a technically impossible transcriptional silencing experiment shown in Fig. 1B, which raises a question how careful was the peer reviewing process. In any case, transcriptional misregulation by exogenous oligonucleotides complementary to promoter sequences has been reported by different laboratories for different promoters in different cells arguing that some complementarity-based transcriptional regulation by small RNAs functions in mammalian cells. Importantly, the underlying mechanism was not conclusively revealed after a decade and is still a matter of debates.

The siRNA-induced transcriptional silencing involves changes in the chromatin structure such as loss of "active" histone modifications (H3K4 methylation, histone acetylation) (Janowski et al., 2006; Morris et al., 2004), appearance of "inactive" histone modifications (H3K9 and H3K27 methylation) (Castanotto et al., 2005; Janowski et al., 2006; Jiang et al., 2012; Kim et al., 2006; Kim et al., 2007; Weinberg et al., 2006), and occasionally DNA methylation (Morris et al., 2004). These are all common features of transcriptional repression and could be either directly induced by siRNA-containing complexes or they be a consequence of transcriptional silencing. DNA methylation is apparently a secondary effect (Ting et al., 2005) although a systematic analysis revealed a group of gene promoters whose methylation was dependent on Dicer (Ting et al., 2008).

Small RNAs used for silencing were either "classical" synthesized siRNAs (agRNAs) (Ahlenstiel et al., 2012; Castanotto et al., 2005; Hawkins et al., 2009; Janowski et al., 2005a; Jiang et al., 2012; Kim et al., 2007; Morris et al., 2004; Napoli et al., 2009; Ting et al., 2005) or shRNAs expressed from a plasmid (Castanotto et al., 2005; Kim et al., 2007). Importantly, transcriptional silencing could be induced by a variety of antisense oligomers 
targeting promoter sequences such as single-stranded antigene (ag) peptide nucleic acid (PNA) (Janowski et al., 2005b), PNA-peptide conjugates (Hu and Corey, 2007), locked nucleic acid (LNA) (Beane et al., 2007) or duplex RNA (siRNA) oligos (Janowski et al., 2005a). These results represent a remarkably comprehensive set of data concerning inhibition of human progesterone receptor A (hPR-A) and B (hPR-B) isoforms. Despite all approaches achieved inhibition of gene expression, these silencing oligonucleotides have radically different properties and it is questionable whether they would all operate loaded on an AGO protein in a RISC-like complex.

Furthermore, studies of oligonucleotides targeting promoter sequences revealed that some oligonucleotides have a positive effect on transcription (Janowski et al., 2007; Li et al., 2006). The activating effect of small RNAs also appeared later in other reports (Hu et al., 2012; Wang et al., 2015a; Zhang et al., 2014). "Scanning" a promoter with oligonucleotides revealed the existence of sites whose targeting results in transcriptional repression as well as sites whose targeting promotes expression (Janowski et al., 2005a; Janowski et al., 2007). According to these data, a shift by several nucleotides could have an opposite effect on gene expression that would correlate with changes in histone modifications. These data would imply existence of a still-unknown RISC-like nuclear complex acting as a switch or siRNA strand-selection onto a single RISC-like complex, where the opposing effects would stem from targeting sense and antisense RNAs in the locus. Alternatively, the observed silencing stems from the oligonucleotide binding and is not mechanistically involving AGO proteins.

To date, the active agent of the silencing has not been conclusively identified and critically assessed. It still remains a question whether there is a dedicated transcriptional silencing machinery in mammalian cells involving AGO proteins and some binding partners as proposed by some reports (Cho et al., 2014; Hawkins et al., 2009; Hu et al., 2012; Janowski et al., 2006; Kim et al., 2006; Suzuki et al., 2008), which are partially contradicting themselves and are not supported by proteomic analysis of AGO complexes (Hauptmann et al., 2015; Hock et al., 2007; Meister et al., 2005), or various artificial disturbances of lncRNAs generate the observed effects.

\section{Transcriptional regulations (stimulation/repression) by miRNAs}

A peculiar phenomenon of miRNA-associated transcriptional activation was reported from cultured mammalian cells for the E-cadherin and cold-shock domain-containing protein $\mathrm{C} 2$ (CSDC2) promoters and the miR-373 (Place et al., 2008). The initial observation leading to discovery of this phenomenon was that transfection of siRNAs homologous to E-cadherin, $\mathrm{p} 21 \mathrm{WAF} 1 / \mathrm{CIP} 1$, and VEGF promoters lead to unexpected transcriptional activation (Li et al., 2006). Subsequent sequence analysis of the E-cadherin promoter revealed high complementarity between the miR-373 and the sequence at position -645 relative to the transcription start site (Place et al., 2008). Delivery of miR-373, pre-miR373 and a synthetic siRNA sequence targeting -640 position could stimulate E-cadherin expression by 5-7-fold in PC-3 cells. Interestingly, intact miRNA pathway was required for the E-cadherin stimulation as partial down-regulation of Dicer protein by morpholino oligonucleotides abolished the stimulatory effect of pre-miR-373. Since then, several other reports of 
miRNA-mediated transcriptional silencing appeared, including transcriptional inhibition of HOXD4 expression by miRNA-10a (Tan et al., 2009b), miR-320-mediated transcriptional silencing of POLR3D (Kim et al., 2008), and others (Adilakshmi et al., 2012; Benhamed et al., 2012; Kim et al., 2011)

It remains an open question whether effects of exogenous siRNAs represent the same molecular mechanism as those attributed to nuclear activities of miRNAs. It would be supported by non-cleaving AGO1 implicated in transcriptional regulation (Huang et al., 2013a; Janowski et al., 2006; Kim et al., 2006) and transcriptional silencing by miRNA mimics targeting gene promoters (Younger and Corey, 2011). In terms of the possible miRNA-mediated nuclear silencing, it was reported that POLR3D silencing involves miRNA-mediated promoter association with a complex including AGO1 and EZH2 (H3K27 histone methyltransferase) (Kim et al., 2008). It was also suggested that AGO1 interacts with polymerase II (Huang et al., 2013a; Kim et al., 2006). At the same time, others implicated GW182 as the factor important for nuclear localization and function of nuclear AGO proteins (Nishi et al., 2013; Nishi et al., 2015). However, the mechanistic link between AGO-GW182 and histone modifications still remains elusive.

\section{7nt RNAs - mediated regulation of endothelial nitric oxide synthase gene}

This phenomenon has been observed in endothelial nitric oxide synthase (eNOS) where a $27 \mathrm{nt}$ repeat polymorphism in intron 4 was a source of predominantly nuclear $27 \mathrm{nt}$ small RNAs, which could be either a novel class of small RNAs, or atypical miRNAs (Zhang et al., 2008b). In any case, these $27 \mathrm{nt}$ RNAs were implicated in eNOS suppression by altering histone acetylation and DNA methylation in regions adjacent to the $27 \mathrm{nt}$ repeat element and core promoter (Zhang et al., 2008a). Whether this phenomenon is related to other ones described here remains unclear.

\section{Regulation of splicing}

In addition to transcriptional and post-transcriptional silencing, one of the reported effects of small RNAs transfected into mammalian cells was also an impact on alternative splicing (Allo et al., 2009). A mechanism was proposed, which involves AGO1 recruitment to transcriptional enhancers (Allo et al., 2014), while other reports implicated AGO2 (Liu et al., 2012; Liu et al., 2015) or both (Ameyar-Zazoua et al., 2012).

\section{Small RNAs associated with DNA repair}

The last nuclear phenomenon associated with small RNAs is their emergence upon DNA damage. It was reported that DICER and DROSHA-dependent small RNAs emerge as DNA-damage response and are functionally associated with it through the MRE11 complex (Francia et al., 2012). In addition, knock-down of Dicer or AGO2 in human cells reduced double-stranded break repair (Wei et al., 2012). It was proposed that small RNAs emerging from DNA -damage loci may function as guide molecules directing chromatin modifications or the recruitment of protein complexes to facilitate repair (Francia et al., 
2012; Wei et al., 2012). What is somewhat confusing in DNA-repair associated small RNAs is the role of miRNA-specific factors Drosha (Francia et al., 2012) or DGCR8 (Swahari et al., 2016).

Despite the heterogeneity of the nuclear effects and many unknowns, some common themes emerged, allowing for formulating testable hypotheses that could be critically evaluated. First, nuclear effects can be mediated by small RNAs provided in trans. Second, small RNAs recruit AGO proteins in a sequence-specific manner, most likely recognizing a local transcript (perhaps an ncRNA). Third, the effect involves a change in the chromatin structure. Thus, by carefully examining essential exogenous siRNA properties in previously reported nuclear effects, one should be able to demonstrate that the silencing phenomenon truly involves an AGO-loaded small RNA engaging another nuclear RNA and whether the effect requires the "slicer" activity. Detailed examination of the seed sequence would also discern between specific nuclear effects and off-targeting. Furthermore, should the effect involve small RNA loaded AGO protein, the kinetics of the phenomenon should be in agreement with known RISC kinetics discussed above. Finally, if the aforementioned phenomena rely on localized recruitment of AGO-loaded small RNAs, one should be able to mimic those effects by tethering AGO proteins through sequence-specific DNA binding modules such as those employed by TALEN or CRISPR nucleases. These research directions should be combined with validated antibodies for chromatin immunoprecipitation and immunofluorescence (or epitope knock-in into candidate genes), more extensive use of mutants defective in RNA silencing, detailed quantitative analysis of cellular fractionation and identifications of interacting partner, studies of putative nuclear import and export signals of Dicer and AGO proteins, and advanced imaging techniques.

\section{Other dsRNA-associated mechanisms I - dsRNA sensing in the interferon pathway}

Long dsRNA is not a usual RNA molecule in eukaryotic cells while RNA viruses produce dsRNA during replication. A common mechanism repressing viruses in non-vertebrate species is RNA silencing (Wang et al., 2006; Wilkins et al., 2005). However, response to foreign long dsRNA in mammals is much more complex and involves a set of sequence-independent sensors triggering expression of a defined set of genes known as interferon-stimulated genes (ISGs). The interferon pathway is the most ubiquitous sequence-independent pathway induced by dsRNA in mammalian cells (reviewed in detail in de Veer et al., 2005). Among the relevant sensors recognizing cytoplasmic dsRNA are protein kinase R (PKR), the helicase RIG-I, MDA5, 2', 5' -oligoadenylate synthetase (2',5'-OAS), or Toll-like receptors (TLR3, 7, 8) (reviewed in Gantier and Williams, 2007; Sadler and Williams, 2007). Notably, there are also dsRNA-independent mechanisms that can activate interferons in mammalian cells. Altogether, different stimuli are being sensed and converge on activation of overlapping but distinct sets of ISGs (Geiss et al., 2001). The situation is even more convoluted by cellular diversity as some cell types, particularly immune cells, can elicit the interferon response by additional, cell-type-specific pathways (reviewed in Schlee and Hartmann, 2010). 


\section{PKR}

PKR is the oldest known mammalian dsRNA sensing protein. A pioneering work by Hunter et al. showed that different types of dsRNA can block translation in reticulocyte lysates (Hunter et al., 1975). Analysis of the phenomenon identified PKR that is activated upon binding to dsRNA and blocks translation by phosphorylating the alpha subunit of eukaryotic initiation factor 2 (eIF-2 $\alpha$ ) (Meurs et al., 1990). Activation of PKR also includes activation of the $\mathrm{NF} \kappa \mathrm{B}$ transcription factor and a large number of interferon-stimulated genes (ISGs) (Geiss et al., 2001). PKR response to viral dsRNA can be coordinated with other dsRNA sensors, such as RIG-I and MDA5 (Sen et al., 2011). PKR can also respond to endogenous RNAs in unique physiological regulations (Bevilacqua et al., 1998; Bommer et al., 2002). However, endogenously expressed long dsRNA does not necessarily induce canonical PKR response with interferon activation, although PKR binding to dsRNA and restricted translational repression can be observed (Nejepinska et al., 2012; Nejepinska et al., 2014). It was believed that dsRNA $<30$-bp in length does not induce PKR. However, Marques et al. reported that, siRNAs can bind and activate PKR in vitro regardless of siRNA termini (Marques et al., 2006) arguing against the long-established 30-bp length as the minimal size-limit for PKR activation. There are also other data indicating sensitivity of PKR to dsRNA motifs shorter than 30-bp (Puthenveetil et al., 2006; Reynolds et al., 2006; Zheng and Bevilacqua, 2004).

\section{RIG-I-like receptors (RIG-I, MDA5, LGP2)}

Mammalian somatic cells can respond to dsRNA in a sequence-independent manner.. In addition to PKR, several other proteins recognizing dsRNA are integrated to the interferon response, including helicases RIG-I (retinoic-acid-inducible gene-I, also known as DDX58), MDA5 (IFIH1), and LGP2 (DHX58), which sense cytoplasmic dsRNA and activate interferon expression.

RIG-I is a cytoplasmic sensor differentiating between endogenous and foreign RNAs structures. In particular, RIG-I is activated by blunt-ended dsRNAs with or without a 5'-triphosphate, by single-stranded RNA marked by a 5'- triphosphate, and by polyuridine sequences. RIG-I domains organize into a ring around dsRNA, capping one end, while contacting both strands; the structure is consistent with dsRNA translocation without unwinding and cooperative binding to RNA (Jiang et al., 2011a; Jiang et al., 2011b). Like RIG-I and LGP2, MDA5 preferentially binds dsRNA with blunt ends (Li et al., 2009a). RIG-I, MDA5, and LGP2 exhibit differences in recognizing specific RNA structures and different types of viruses providing a broader range of coordinated sensitivity do different potential threats (Kato et al., 2006; Li et al., 2009b; McCartney et al., 2008; Sen et al., 2011; Slater et al., 2010; Wu et al., 2015b). Interestingly, RIG-I can become activated also with siRNAs lacking 2-nt 3' overhangs (Marques et al., 2006). These data imply that 2-nt 3' overhangs generated by Dicer are the structural basis for discriminating between Dicer products and other short dsRNA. Roles of MDA5 and LGP2 in siRNA-mediated interferon response remains to be addressed. Furthermore, recognition 5' triphosphate RNA ends RIG-I (Hornung et al., 2006; Pichlmair et al., 2006) highlights importance of appropriate 
processing of 5' termini of RNAs produced by phage polymerases when such RNAs are used in mammalian cells.

It is not clear how PKR and RIG-I pathways are integrated. RIG-I binds siRNAs (with or without 2-nt 3' overhangs) in vitro and it shows greater unwinding of blunt-ended siRNAs. Unwinding is then translated into the interferon activation mediated via IRF-3.

\section{Toll-like Receptor 3 (TLR3)}

TLR3 is a member of the Toll-like receptor (TLR) family and functions as a sensor of extracellular, intracellular and viral dsRNAs (Amarante et al., 2011; Seo et al., 2013; Wang et al., 2015b; Wu et al., 2015b; Yang et al., 2006b). TLR3 has distinct or complementary roles to RIG-I and related helicases in sensing foreign molecules and activating downstream responses (Livengood et al., 2007; McCartney et al., 2009; Slater et al., 2010; Wu et al., 2015b).

\section{Oligoadenylate Synthetase (OAS)}

Interferon and dsRNA also activate $2^{\prime}, 5^{\prime}$-oligoadenylate synthetase $\left(2^{\prime}, 5^{\prime}-\mathrm{OAS}\right)$ that produces 2',5' oligoadenylates with 5'-terminal triphosphate residues that subsequently induce activation of RNAse L; a protein responsible for general RNA degradation (de Veer et al., 2005).

\section{TARBP2 and PACT}

Interactions between RNAi, miRNA, and interferon response are poorly understood. There are two clear mechanistic connections between these two pathways. First, TARBP2 and PACT, two dsRNA binding proteins, which were mentioned earlier as Dicer-interacting proteins, interact also with PKR. Notably, while TARBP2 inhibits PKR (Cosentino et al., 1995; Park et al., 1994), PACT has the opposite role (Patel and Sen, 1998). While cytoplasmic long dsRNA in somatic cells apparently triggers the interferon response, it is not clear if the same dsRNA is also routed into the RNAi pathways. Experiments in oocytes and undifferentiated embryonic stem cells (Stein et al., 2005; Yang et al., 2001) suggest that RNAi dominates response to cytoplasmic long dsRNA in the absence of a strong interferon response and that the interferon pathway dominates when its relevant components are present. On the other hand, this view may be too simplistic as it does not explain the lack of both, RNAi and interferon response, in somatic cells expressing long dsRNA (Nejepinska et al., 2012; Nejepinska et al., 2014). In any case, understanding the role of TARBP2 and PACT isoforms in routing long dsRNA into RNAi and interferon pathways requires further studies.

There is a clear evolutionary connection between RNAi and interferon response. The above-mentioned mammalian RNA helicases RIG-I, LGP2 and MDA5 are the closest homologs of helicases involved in processing of long dsRNA during RNAi in C. elegans. Notably, RIG-I is an established component of the interferon response to long dsRNA (Yoneyama et al., 2004). This suggests that the interferon response, which has a common 
trigger and evolved after the RNAi pathway, adopted several components from the latter pathway. It remains to be determined whether these and other components of RNAi lost their function in RNAi entirely or mediate some form of a cross-talk between RNAi and interferon response.

Finally, there is also a complex relationship between miRNA and interferon pathways (Ingle et al., 2015; Ostermann et al., 2012; Shapiro et al., 2014; Xu et al., 2011). One connection is exemplified by viral miRNAs, which viruses use to regulate the host response, in particular factors of the interferon pathway (Ostermann et al., 2012) or other cellular signalling (Xu et al., 2011). However endogenous cellular miRNAs may also act to suppress the interferon response factors, such as the case mir-485, which has a dual role in targeting RIG-I as well as the influenza virus H5N1 (Ingle et al., 2015).

\section{Other dsRNA-associated mechanisms II - Adenosine deamination}

A-to-I editing is a covalent RNA modification system of broad significance (reviewed in Nishikura, 2016). It is mediated by adenosine deaminases acting on RNA (ADARs), enzymes that carry two or three dsRBD and recognize both inter- and intramolecular dsRNAs longer than 20-30 bp (Nishikura et al., 1991). ADARs convert adenosines to inosines, which base pair with cytosines, which are interpreted as guanosines during translation. Thus, RNA editing affects coding potential, fidelity of RNA replication reverse transcription, or formation/stability of RNA secondary structures where a change of a single base in a sequence may result either in dsRNA destabilization (inosine-uridine pair) or stabilization (inosine-cytidine pair) (Nishikura, 2010). Such transition in the local and global stability of dsRNA structure can influence further processing of dsRNA, such as the selection of the effective miRNA strand (Bartel, 2004; Meister and Tuschl, 2004).

Mammals (and vertebrates in general) have three ADAR genes (reviewed in Nishikura, 2016) (Fig. 3). Two encode proteins carrying deaminase activity: ADAR1, which is interferon-inducible, and ADAR2, which is constitutively expressed. ADAR3 is mostly expressed in the brain but its editing activity has not been shown yet. The specificity of the ADAR1 and ADAR2 deaminases ranges from highly site-selective to non-selective, dependent on the duplex structure of the substrate RNA.

The complete ADAR structure has not been solved yet but structure of several domains is known - the $\mathrm{Z}$ alpha domain of the human editing enzyme ADAR1 (Schwartz et al., 1999) and dsRBDs of ADAR2 (Stefl et al., 2010). The analysis of dsRBDs provided an insight into editing of a specific substrate and revealed that dsRBDs of ADAR not only recognize the shape but also the sequence of the dsRNA (Stefl et al., 2010). The unexpected direct readout of the RNA primary sequence by dsRBDs is achieved via the minor groove of the dsRNA and this recognition is critical for both editing and binding affinity of edited RNA (Stefl et al., 2010). It was also shown that ADAR2 forms dimers in vivo and that dsRBDs are necessary and sufficient for dimerization of the enzyme (Poulsen et al., 2006).

ADARs exhibit complex regulation of localization. For example, it was shown that mouse ADAR1 isoforms are differentially localized in cellular compartments and that their 


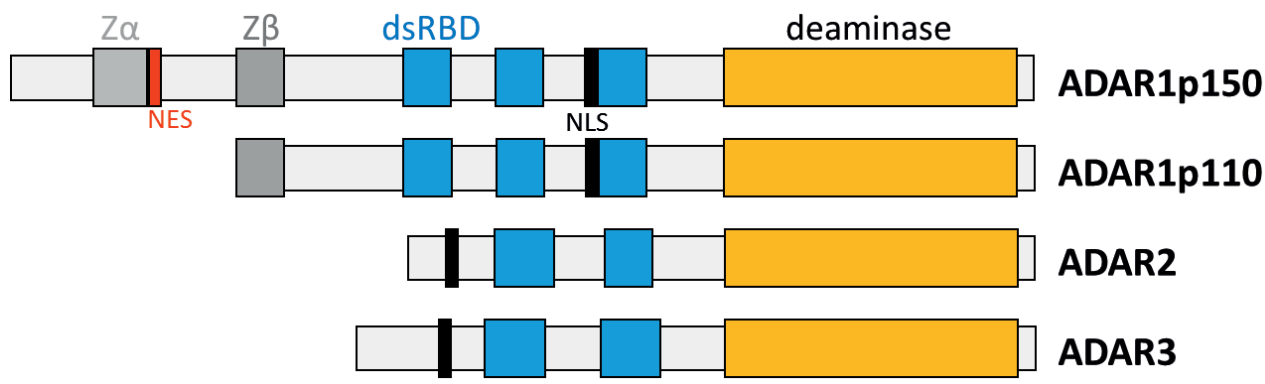

Figure 3 Domain composition of mammalian ADAR proteins

NES, nuclear export signal, NLS, nuclear localization signal; dsRBD, dsRNA binding domain.

localization is controlled by several independent signals, which include a nuclear localization signal (NLS), the nucleolar localization signal (NoLS), the nuclear exporter signal (NES) near the $\mathrm{N}$ terminus (Nie et al., 2004). ADAR1 interacts with TUDOR-SN nuclease (Nishikura, 2010; Scadden, 2005; Weissbach and Scadden, 2012; Yang et al., 2006a) and localizes to stress granules upon stress induction (Weissbach and Scadden, 2012) while tudor-SN degrades hyperedited dsRNA (Scadden, 2005).

RNA editing concerns a broad range of RNAs including viral and cellular RNAs. Many long perfect dsRNAs (>100 bp) undergo extensive editing with a conversion of approximately $50 \%$ of adenosines to inosines (Nishikura et al., 1991; Polson and Bass, 1994). Extensive editing (hyperediting) is linked with nuclear retention (reviewed in DeCerbo and Carmichael, 2005). On the other hand, short RNAs ( 20-30 bp) or imperfect long dsRNAs are edited selectively; usually only a few adenines at specific sites are deaminated (Lehmann and Bass, 1999). High throughput analyses revealed the extent of RNA editing of mammalian RNAs in terms of substrate diversity and frequency of editing in the transcriptome (Carmi et al., 2011; Peng et al., 2012). Edited endogenous RNAs (Dawson et al., 2004; Hundley et al., 2008; Morse et al., 2002; Salameh et al., 2015) include mRNAs, repetitive sequences (mainly Alu (Athanasiadis et al., 2004)), and miRNAs. It was predicted that more than $85 \%$ of pre-mRNAs may be edited, predominantly in the non-coding regions (Athanasiadis et al., 2004).

Several pri-miRNAs (e.g. miR-142) are known to undergo editing, which inhibits Drosha cleavage or even causes degradation of pri-miRNA by Tudor SN (Nishikura, 2010; Scadden, 2005; Yang et al., 2006a). In other cases, pri-miRNA editing does not influence Drosha activity but inhibits processing of pre-miRNA by Dicer (e.g. miR-151) (Kawahara et al., 2007a). Last but not least, RNA editing might also inhibit export of miRNAs from the nucleus (Nishikura, 2010). A systematic analysis of edited miRNAs in the human brain showed that editing of miRNAs affects several miRNAs but it is not widespread (Alon et al., 2012). A similar picture was obtained from analysis of embryonic miRNAs (Garcia-Lopez et al., 2013; Vesely et al., 2012)

One of the roles of ADARs in immunity is to prevent innate immune sensing of self-RNA (Heraud-Farlow and Walkley, 2016). ADARs also affect viral RNAs in various ways ADARs are both antiviral and proviral; the effect on virus growth and persistence depends 
upon the specific virus. (Samuel, 2011). Viruses targeted by ADARs in mammals include HIV (Clerzius et al., 2009), herpesvirus (Gandy et al., 2007), HRSV (Martinez and Melero, 2002), HCMV (Nachmani et al., 2014), VSV (Nie et al., 2007), and HDV (Wong and Lazinski, 2002).

\section{Crosstalk between RNA editing and other dsRNA pathways.}

ADARs affect other dsRNA pathways in several ways. In RNA silencing, ADARs can compete with RNAi for dsRNA substrates (including siRNAs). The ADAR1 isoform (ADAR1p150) strongly binds siRNA and reduces thus the availability of dsRNA for RNAi, resulting in less efficient RNAi in normal cells compared to Adar $1^{-/-}$cells (Yang et al., 2005). Interestingly, injection of high doses of siRNAs enhances ADAR1 expression, suggesting a role of ADAR1 in a cellular feedback mechanism in response to siRNA (Hong et al., 2005).

Editing affects base pairing quality of dsRNA substrates as well as target recognition since a single nucleotide mismatch between siRNA and target mRNA can reduce RNAi efficacy (Scadden and Smith, 2001) or modify target specificity, especially when occurring in the seed sequence (Kawahara et al., 2007b). MiRNAs would be affected in a similar way. A moderate deamination (one I-U pair per siRNA) does not prevent Dicer processing to siRNAs (Zamore et al., 2000$)$ but, hyperediting ( $\sim 50 \%$ of deaminated adenosines) can make dsRNA resistant to Dicer processing (Scadden and Smith, 2001).

Thus, ADARs are factors conferring to formation of RNAi resistance (Hong et al., 2005), which may be one of the viral strategies to avoid being targeted through a dsRNA-responding pathway (Zheng et al., 2005). ADARs influence the innate immunity either indirectly by preventing sensing of self-RNA (Heraud-Farlow and Walkley, 2016) or by interacting with innate immunity factors, such as PKR (Clerzius et al., 2009). The immunosuppressive role of ADAR1 could explain the phenotype of the Aicardi-Goutieres syndrome (AGS, OMIM\# 225750), an autoimmune disorder caused by ADAR1 mutations (Rice et al., 2012). It has been proposed that in the absence of ADAR1, accumulation of cytoplasmic dsRNA may provoke interferon signalling and cause upregulation of interferon-stimulated genes, which is observed in AGS (Rice et al., 2012).

\section{Summary}

In mammals (Fig. 4), the miRNA pathway seems to be the dominant small RNA pathway in the soma while the existence and functionality of endogenous RNAi remains unclear. The only cell type with well documented robust and mechanistically explained endo-RNAi is the mouse oocyte. Somatic cells typically respond to long dsRNA with a sequence-independent interferon response, which is employing multiple dsRNA sensors, which trigger a complex interferon response. 


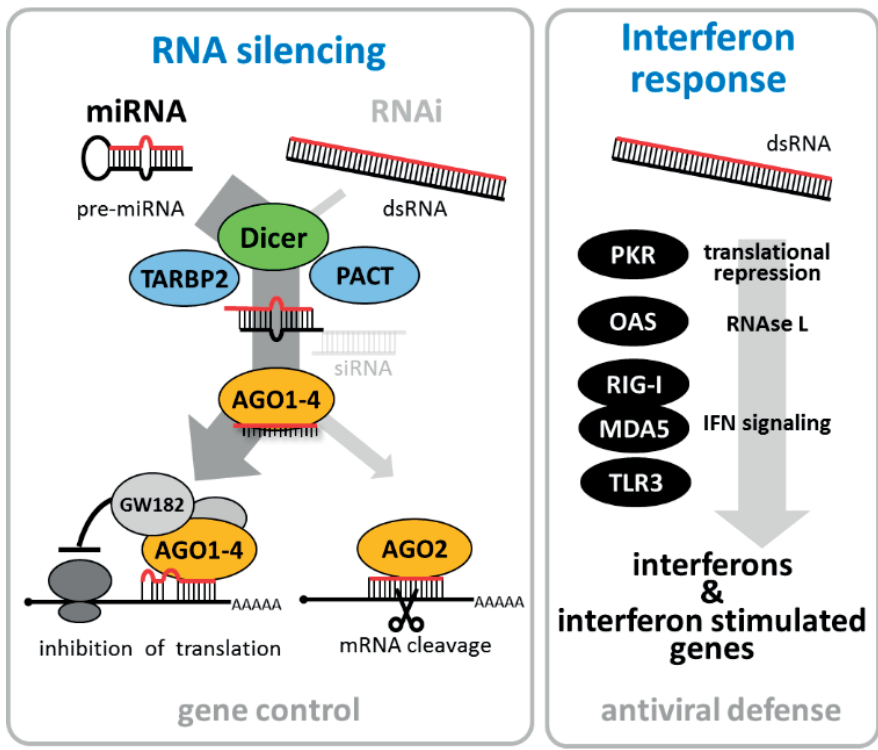

Figure 4 Summary of mammalian RNA silencing and dsRNA response pathways. On the right are schematically depicted dsRNA sensors in the sequence-independent interferon response.

\section{Acknowledgement}

I would like to thank my colleagues Jan Paces, Miloslav Nic, and Tomas Novotny for help with collecting literature for the review. The review content was produced under a contract OC/EFSA/GMO/2015/01-CT 01 with European Food Safety Authority (EFSA); the opinions expressed are those of the contractor only and do not represent EFSA's official position. Publication of the review was funded by LO1220 and LM2015063 by the Ministry of Education, Youth and Sports.

\section{References}

Adilakshmi, T., Sudol, I., and Tapinos, N. (2012). Combinatorial Action of miRNAs Regulates Transcriptional and Post-Transcriptional Gene Silencing following in vivo PNS Injury. Plos One 7, e39674-e39674.

Ahlenstiel, C.L., Lim, H.G.W., Cooper, D.A., Ishida, T., Kelleher, A.D., and Suzuki, K. (2012). Direct evidence of nuclear Argonaute distribution during transcriptional silencing links the actin cytoskeleton to nuclear RNAi machinery in human cells. Nucleic Acids Research 40, 1579-1595.

Allo, M., Agirre, E., Bessonov, S., Bertucci, P., Acuna, L.G., Buggiano, V., Bellora, N., Singh, B., Petrillo, E., Blaustein, M., et al. (2014). Argonaute-1 binds transcriptional enhancers and controls constitutive and alternative splicing in human cells. Proc Natl Acad Sci U S A 111, $15622-15629$. 
Allo, M., Buggiano, V., Fededa, J.P., Petrillo, E., Schor, I., de la Mata, M., Agirre, E., Plass, M., Eyras, E., Elela, S.A., et al. (2009). Control of alternative splicing through siRNA-mediated transcriptional gene silencing. Nature Structural \& Molecular Biology 16, 717-724.

Alon, S., Mor, E., Vigneault, F., Church, G.M., Locatelli, F., Galeano, F., Gallo, A., Shomron, N., and Eisenberg, E. (2012). Systematic identification of edited microRNAs in the human brain. Genome Research 22, 1533-1540.

Amarante, M.K., Oda, J.M.M., Reiche, E.M.V., Morimoto, H.K., Aoki, M.N., and Watanabe, M.A.E. (2011). Human endogenous RNAs: Implications for the immunomodulation of Toll-like receptor 3. Experimental and Therapeutic Medicine 2, 925-929.

Ameyar-Zazoua, M., Rachez, C., Souidi, M., Robin, P., Fritsch, L., Young, R., Morozova, N., Fenouil, R., Descostes, N., Andrau, J.-C., et al. (2012). Argonaute proteins couple chromatin silencing to alternative splicing. Nature Structural \& Molecular Biology 19, 998-U946.

Aporntewan, C., Phokaew, C., Piriyapongsa, J., Ngamphiw, C., Ittiwut, C., Tongsima, S., and Mutirangura, A. (2011). Hypomethylation of Intragenic LINE-1 Represses Transcription in Cancer Cells through AGO2. Plos One 6, e17934-e17934.

Arroyo, J.D., Chevillet, J.R., Kroh, E.M., Ruf, I.K., Pritchard, C.C., Gibson, D.F., Mitchell, P.S., Bennett, C.F., Pogosova-Agadjanyan, E.L., Stirewalt, D.L., et al. (2011). Argonaute2 complexes carry a population of circulating microRNAs independent of vesicles in human plasma. Proc Natl Acad Sci U S A 108, 5003-5008.

Athanasiadis, A., Rich, A., and Maas, S. (2004). Widespread A-to-I RNA editing of Alu-containing mRNAs in the human transcriptome. Plos Biology 2, e391.

Babiarz, J.E., Ruby, J.G., Wang, Y.M., Bartel, D.P., and Blelloch, R. (2008). Mouse ES cells express endogenous shRNAs, siRNAs, and other Microprocessor-independent, Dicer-dependent small RNAs. Genes \& Development 22, 2773-2785.

Baglio, S.R., Rooijers, K., Koppers-Lalic, D., Verweij, F.J., Pérez Lanzón, M., Zini, N., Naaijkens, B., Perut, F., Niessen, H.W.M., Baldini, N., et al. (2015). Human bone marrow- and adipose-mesenchymal stem cells secrete exosomes enriched in distinctive miRNA and tRNA species. Stem Cell Research and Therapy 6.

Bai, B.Y., Liu, H., and Laiho, M. (2014). Small RNA expression and deep sequencing analyses of the nucleolus reveal the presence of nucleolus-associated microRNAs. FEBS Open Bio 4, 441-449.

Bartel, D.P. (2004). MicroRNAs: genomics, biogenesis, mechanism, and function. Cell 116, 281-297.

Beane, R.L., Ram, R., Gabillet, S., Arar, K., Monia, B.P., and Corey, D.R. (2007). Inhibiting gene expression with locked nucleic acids (LNAs) that target chromosomal DNA. Biochemistry 46, 7572-7580.

Bellingham, S.A., Coleman, B.M., and Hill, A.F. (2012). Small RNA deep sequencing reveals a distinct miRNA signature released in exosomes from prion-infected neuronal cells. Nucleic Acids Research 40, 10937-10949.

Benetti, R., Gonzalo, S., Jaco, I., Munoz, P., Gonzalez, S., Schoeftner, S., Murchison, E., Andl, T., Chen, T., Klatt, P., et al. (2008). A mammalian microRNA cluster controls DNA methylation and telomere recombination via Rbl2-dependent regulation of DNA methyltransferases. Nature Structural \& Molecular Biology 15, 268-279.

Benhamed, M., Herbig, U., Ye, T., Dejean, A., and Bischof, O. (2012). Senescence is an endogenous trigger for microRNA-directed transcriptional gene silencing in human cells. Nature Cell Biology 14, 266-+. 
Bennasser, Y., Le, S.Y., Benkirane, M., and Jeang, K.T. (2005). Evidence that HIV-1 encodes an siRNA and a suppressor of RNA silencing. Immunity 22, 607-619.

Berezhna, S.Y., Supekova, L., Supek, F., Schultz, P.G., and Deniz, A.A. (2006). siRNA in human cells selectively localizes to target RNA sites. Proc Natl Acad Sci U S A 103, 7682-7687.

Bevilacqua, P.C., George, C.X., Samuel, C.E., and Cech, T.R. (1998). Binding of the protein kinase PKR to RNAs with secondary structure defects: Role of the tandem A-G mismatch and noncontiguous helixes. Biochemistry 37, 6303-6316.

Billy, E., Brondani, V., Zhang, H.D., Muller, U., and Filipowicz, W. (2001). Specific interference with gene expression induced by long, double-stranded RNA in mouse embryonal teratocarcinoma cell lines. Proc Natl Acad Sci U S A 98, 14428-14433.

Bommer, U.A., Borovjagin, A.V., Greagg, M.A., Jeffrey, I.W., Russell, P., Laing, K.G., Lee, M., and Clemens, M.J. (2002). The mRNA of the translationally controlled tumor protein P23/TCTP is a highly structured RNA, which activates the dsRNA-dependent protein kinase PKR. RNA 8 , 478-496.

Buck, A.H., Coakley, G., Simbari, F., McSorley, H.J., Quintana, J.F., Le Bihan, T., Kumar, S., Abreu-Goodger, C., Lear, M., Harcus, Y., et al. (2014). Exosomes secreted by nematode parasites transfer small RNAs to mammalian cells and modulate innate immunity. Nature Communications $5,5488-5488$.

Calabrese, J.M., Seila, A.C., Yeo, G.W., and Sharp, P.A. (2007). RNA sequence analysis defines Dicer's role in mouse embryonic stem cells. Proc Natl Acad Sci U S A 104, 18097-18102.

Carlile, M., Swan, D., Jackson, K., Preston-Fayers, K., Ballester, B., Flicek, P., and Werner, A. (2009). Strand selective generation of endo-siRNAs from the Na/phosphate transporter gene Slc34a1 in murine tissues. Nucleic Acids Research 37, 2274-2282.

Carmell, M.A., Girard, A., van de Kant, H.J.G., Bourc'his, D., Bestor, T.H., de Rooij, D.G., and Hannon, G.J. (2007). MIWI2 is essential for spermatogenesis and repression of transposons in the mouse male germline. Developmental Cell 12, 503-514.

Carmi, S., Borukhov, I., and Levanon, E.Y. (2011). Identification of Widespread Ultra-Edited Human RNAs. Plos Genetics 7, e1002317-e1002317.

Castanotto, D., Tommasi, S., Li, M., Li, H., Yanow, S., Pfeifer, G.P., and Rossi, J.J. (2005). Short hairpin RNA-directed cytosine $(\mathrm{CpG})$ methylation of the RASSF1A gene promoter in HeLa cells. Molecular Therapy 12, 179-183.

Chevillet, J.R., Kang, Q., Ruf, I.K., Briggs, H.A., Vojtech, L.N., Hughes, S.M., Cheng, H.H., Arroyo, J.D., Meredith, E.K., Gallichotte, E.N., et al. (2014). Quantitative and stoichiometric analysis of the microRNA content of exosomes. Proc Natl Acad Sci U S A 111, 14888-14893.

Chim, S.S., Shing, T.K., Hung, E.C., Leung, T.Y., Lau, T.K., Chiu, R.W., and Lo, Y.M. (2008). Detection and characterization of placental microRNAs in maternal plasma. Clinical chemistry 54, 482-490.

Cho, S., Park, J.S., and Kang, Y.K. (2014). AGO2 and SETDB1 cooperate in promoter-targeted transcriptional silencing of the androgen receptor gene. Nucleic Acids Research 42, 13545-13556.

Clerzius, G., Gelinas, J.F., Daher, A., Bonnet, M., Meurs, E.F., and Gatignol, A. (2009). ADAR1 Interacts with PKR during Human Immunodeficiency Virus Infection of Lymphocytes and Contributes to Viral Replication. Journal of Virology 83, 10119-10128.

Clop, A., Marcq, F., Takeda, H., Pirottin, D., Tordoir, X., Bibe, B., Bouix, J., Caiment, F., Elsen, J.M., Eychenne, F., et al. (2006). A mutation creating a potential illegitimate microRNA target site in the myostatin gene affects muscularity in sheep. Nature Genetics $38,813-818$. 
Cosentino, G.P., Venkatesan, S., Serluca, F.C., Green, S.R., Mathews, M.B., and Sonenberg, N. (1995). Double-stranded-RNA-dependent protein kinase and TAR RNA-binding protein form homo- and heterodimers in vivo. Proc Natl Acad Sci U S A 92, 9445-9449.

Cullen, B.R. (2006). Is RNA interference involved in intrinsic antiviral immunity in mammals? Nature Immunology 7, 563-567.

Cullen, B.R., Cherry, S., and tenOever, B.R. (2013). Is RNA interference a physiologically relevant innate antiviral immune response in mammals? Cell Host Microbe 14, 374-378.

Dawson, T.R., Sansam, C.L., and Emeson, R.B. (2004). Structure and sequence determinants required for the RNA editing of ADAR2 substrates. Journal of Biological Chemistry 279, 4941-4951.

de Veer, M.J., Sledz, C.A., and Williams, B.R. (2005). Detection of foreign RNA: implications for RNAi. Immunol Cell Biol 83, 224-228.

DeCerbo, J., and Carmichael, G.G. (2005). Retention and repression: fates of hyperedited RNAs in the nucleus. Current Opinion in Cell Biology 17, 302-308.

Deveson, I., Li, J.Y., and Millar, A.A. (2013). Expression of human ARGONAUTE 2 inhibits endogenous microRNA activity in Arabidopsis. Frontiers in Plant Science 4, 96-96.

Diallo, M., Arenz, C., Schmitz, K., Sandhoff, K., and Schepers, U. (2003). Long endogenous dsRNAs can induce complete gene silencing in mammalian cells and primary cultures. Oligonucleotides 13, 381-392.

Dismuke, W.M., Challa, P., Navarro, I., Stamer, W.D., and Liu, Y.T. (2015). Human aqueous humor exosomes. Experimental Eye Research 132, 73-77.

Doyle, M., Badertscher, L., Jaskiewicz, L., Guttinger, S., Jurado, S., Hugenschmidt, T., Kutay, U., and Filipowicz, W. (2013). The double-stranded RNA binding domain of human Dicer functions as a nuclear localization signal. RNA 19, 1238-1252.

Drake, M., Furuta, T., Suen, K.M., Gonzalez, G., Liu, B., Kalia, A., Ladbury, J.E., Fire, A.Z., Skeath, J.B., and Arur, S. (2014). A requirement for ERK-dependent Dicer phosphorylation in coordinating oocyte-to-embryo transition in C. elegans. Developmental Cell 31, 614-628.

Duursma, A.M., Kedde, M., Schrier, M., le Sage, C., and Agami, R. (2008). miR-148 targets human DNMT3b protein coding region. RNA 14, 872-877.

Duxbury, M.S., Ashley, S.W., and Whang, E.E. (2005). RNA interference: a mammalian SID-1 homologue enhances siRNA uptake and gene silencing efficacy in human cells. Biochem Biophys Res Commun 331, 459-463.

El-Andaloussi, S., Lee, Y., Lakhal-Littleton, S., Li, J., Seow, Y., Gardiner, C., Alvarez-Erviti, L., Sargent, I.L., and Wood, M.J.A. (2012). Exosome-mediated delivery of siRNA in vitro and in vivo. Nature Protocols 7, 2112-2126.

Elbashir, S.M., Harborth, J., Lendeckel, W., Yalcin, A., Weber, K., and Tuschl, T. (2001). Duplexes of 21-nucleotide RNAs mediate RNA interference in cultured mammalian cells. Nature 411, 494-498.

Fabbri, M., Garzon, R., Cimmino, A., Liu, Z., Zanesi, N., Callegari, E., Liu, S., Alder, H., Costinean, S., Fernandez-Cymering, C., et al. (2007). MicroRNA-29 family reverts aberrant methylation in lung cancer by targeting DNA methyltransferases 3A and 3B. Proc Natl Acad Sci U S A 104, $15805-15810$.

Farh, K.K., Grimson, A., Jan, C., Lewis, B.P., Johnston, W.K., Lim, L.P., Burge, C.B., and Bartel, D.P. (2005). The widespread impact of mammalian MicroRNAs on mRNA repression and evolution. Science 310, 1817-1821. 
Fenner, B.J., Thiagarajan, R., Chua, H.K., and Kwang, J. (2006). Betanodavirus B2 is an RNA interference antagonist that facilitates intracellular viral RNA accumulation. Journal of Virology 80 , 85-94.

Fire, A., Xu, S., Montgomery, M.K., Kostas, S.A., Driver, S.E., and Mello, C.C. (1998). Potent and specific genetic interference by double-stranded RNA in Caenorhabditis elegans. Nature 391, 806-811.

Flemr, M., Malik, R., Franke, V., Nejepinska, J., Sedlacek, R., Vlahovicek, K., and Svoboda, P. (2013). A Retrotransposon-Driven Dicer Isoform Directs Endogenous Small Interfering RNA Production in Mouse Oocytes. Cell 155, 807-816.

Francia, S., Michelini, F., Saxena, A., Tang, D., de Hoon, M., Anelli, V., Mione, M., Carninci, P., and di Fagagna, F.D. (2012). Site-specific DICER and DROSHA RNA products control the DNA-damage response. Nature 488, 231-+.

Fukagawa, T., Nogami, M., Yoshikawa, M., Ikeno, M., Okazaki, T., Takami, Y., Nakayama, T., and Oshimura, M. (2004). Dicer is essential for formation of the heterochromatin structure in vertebrate cells. Nature Cell Biology 6, 784-791.

Gagnon, K.T., Li, L.D., Chu, Y.J., Janowski, B.A., and Corey, D.R. (2014a). RNAi Factors Are Present and Active in Human Cell Nuclei. Cell Reports 6, 211-221.

Gagnon, K.T., Li, L.D., Janowski, B.A., and Corey, D.R. (2014b). Analysis of nuclear RNA interference in human cells by subcellular fractionation and Argonaute loading. Nature Protocols 9 , 2045-2060.

Gan, L., Anton, K.E., Masterson, B.A., Vincent, V.A., Ye, S., and Gonzalez-Zulueta, M. (2002). Specific interference with gene expression and gene function mediated by long dsRNA in neural cells. J Neurosci Methods 121, 151-157.

Gandy, S.Z., Linnstaedt, S.D., Muralidhar, S., Cashman, K.A., Rosenthal, L.J., and Casey, J.L. (2007). RNA editing of the human herpesvirus 8 kaposin transcript eliminates its transforming activity and is induced during lytic replication. Journal of Virology 81, 13544-13551.

Gantier, M.P., and Williams, B.R. (2007). The response of mammalian cells to double-stranded RNA. Cytokine Growth Factor Rev 18, 363-371.

Garcia-Lopez, J., Hourcade, J.D., and del Mazo, J. (2013). Reprogramming of microRNAs by adenosine-to-inosine editing and the selective elimination of edited microRNA precursors in mouse oocytes and preimplantation embryos. Nucleic Acids Research 41, 5483-5493.

Geiss, G., Jin, G., Guo, J., Bumgarner, R., Katze, M.G., and Sen, G.C. (2001). A comprehensive view of regulation of gene expression by double-stranded RNA-mediated cell signaling. Journal of Biological Chemistry 276, 30178-30182.

Glanzer, J., Miyashiro, K.Y., Sul, J.Y., Barrett, L., Belt, B., Haydon, P., and Eberwine, J. (2005). RNA splicing capability of live neuronal dendrites. Proc Natl Acad Sci U S A 102, 16859-16864.

Guang, S., Bochner, A.F., Burkhart, K.B., Burton, N., Pavelec, D.M., and Kennedy, S. (2010). Small regulatory RNAs inhibit RNA polymerase II during the elongation phase of transcription. Nature 465, 1097-1101.

Gullerova, M., and Proudfoot, N.J. (2012). Convergent transcription induces transcriptional gene silencing in fission yeast and mammalian cells. Nature Structural \& Molecular Biology 19, 1193-1201.

Haasnoot, J., de Vries, W., Geutjes, E.J., Prins, M., de Haan, P., and Berkhout, B. (2007). The Ebola virus VP35 protein is a suppressor of RNA silencing. Plos Pathogens 3, e86. 
Hammond, S.M., Bernstein, E., Beach, D., and Hannon, G.J. (2000). An RNA-directed nuclease mediates post-transcriptional gene silencing in Drosophila cells. Nature 404, 293-296.

Hauptmann, J., Schraivogel, D., Bruckmann, A., Manickavel, S., Jakob, L., Eichner, N., Pfaff, J., Urban, M., Sprunck, S., Hafner, M., et al. (2015). Biochemical isolation of Argonaute protein complexes by Ago-APP. Proc Natl Acad Sci U S A 112, 11841-11845.

Haussecker, D., and Proudfoot, N.J. (2005). Dicer-dependent turnover of intergenic transcripts from the human beta-globin gene cluster. Molecular and Cellular Biology 25, 9724-9733.

Hawkins, P.G., Santoso, S., Adams, C., Anest, V., and Morris, K.V. (2009). Promoter targeted small RNAs induce long-term transcriptional gene silencing in human cells. Nucleic Acids Research 37, 2984-2995.

Heraud-Farlow, J.E., and Walkley, C.R. (2016). The role of RNA editing by ADAR1 in prevention of innate immune sensing of self-RNA. J Mol Med (Berl).

Hock, J., Weinmann, L., Ender, C., Rudel, S., Kremmer, E., Raabe, M., Urlaub, H., and Meister, G. (2007). Proteomic and functional analysis of Argonaute-containing mRNA-protein complexes in human cells. EMBO Rep 8, 1052-1060.

Hong, J., Qian, Z.K., Shen, S.Y., Min, T.S., Tan, C., Xu, J.F., Zhao, Y.C., and Huang, W.D. (2005). High doses of siRNAs induce eri-1 and adar-1 gene expression and reduce the efficiency of RNA interference in the mouse. Biochemical Journal 390, 675-679.

Hornung, V., Ellegast, J., Kim, S., Brzozka, K., Jung, A., Kato, H., Poeck, H., Akira, S., Conzelmann, K.K., Schlee, M., et al. (2006). 5'-Triphosphate RNA is the ligand for RIG-I. Science 314, 994-997.

Houbaviy, H.B., Dennis, L., Jaenisch, R., and Sharp, P.A. (2005). Characterization of a highly variable eutherian rnicroRNA gene. RNA $11,1245-1257$.

Hu, J., Chen, Z., Xia, D., Wu, J., Xu, H., and Ye, Z.Q. (2012). Promoter-associated small double-stranded RNA interacts with heterogeneous nuclear ribonucleoprotein A2/B1 to induce transcriptional activation. Biochemical Journal 447, 407-416.

Hu, J., and Corey, D.R. (2007). Inhibiting gene expression with peptide nucleic acid (PNA)--peptide conjugates that target chromosomal DNA. Biochemistry 46, 7581-7589.

Huang, V., Zheng, J.S., Qi, Z.X., Wang, J., Place, R.F., Yu, J.W., Li, H., and Li, L.C. (2013a). Ago1 Interacts with RNA Polymerase II and Binds to the Promoters of Actively Transcribed Genes in Human Cancer Cells. Plos Genetics 9, e1003821-e1003821.

Huang, X.Y., Yuan, T.Z., Tschannen, M., Sun, Z.F., Jacob, H., Du, M.J., Liang, M.H., Dittmar, R.L., Liu, Y., Liang, M.Y., et al. (2013b). Characterization of human plasma-derived exosomal RNAs by deep sequencing. BMC Genomics 14, 319-319.

Hundley, H.A., Krauchuk, A.A., and Bass, B.L. (2008). C-elegans and H-sapiens mRNAs with edited 3 'UTRs are present on polysomes. RNA 14, 2050-2060.

Hunter, T., Hunt, T., Jackson, R.J., and Robertson, H.D. (1975). The characteristics of inhibition of protein synthesis by double-stranded ribonucleic acid in reticulocyte lysates. Journal of Biological Chemistry 250, 409-417.

Iavello, A., Frech, V.S.L., Gai, C., Deregibus, M.C., Quesenberry, P.J., and Camussi, G. (2016). Role of Alix in miRNA packaging during extracellular vesicle biogenesis. International Journal of Molecular Medicine 37, 958-966.

Iguchi, H., Kosaka, N., and Ochiya, T. (2010). Secretory microRNAs as a versatile communication tool. Communicative \& integrative biology 3, 478-481. 
Ingle, H., Kumar, S., Raut, A.A., Mishra, A., Kulkarni, D.D., Kameyama, T., Takaoka, A., Akira, S., and Kumar, H. (2015). The microRNA miR-485 targets host and influenza virus transcripts to regulate antiviral immunity and restrict viral replication. Science Signaling 8, ra126-ra126.

Ip, J., Canham, P., Choo, K.H.A., Inaba, Y., Jacobs, S.A., Kalitsis, P., Mattiske, D.M., Ng, J., Saffery, R., Wong, N.C., et al. (2012). Normal DNA Methylation Dynamics in DICER1-Deficient Mouse Embryonic Stem Cells. Plos Genetics 8, e1002919-e1002919.

Izumi, H., Tsuda, M., Sato, Y., Kosaka, N., Ochiya, T., Iwamoto, H., Namba, K., and Takeda, Y. (2015). Bovine milk exosomes contain microRNA and mRNA and are taken up by human macrophages. Journal of Dairy Science 98, 2920-2933.

Jang, J.H., Jung, J.S., Choi, J.I., and Kang, S.K. (2012). Nuclear Ago2/HSP60 Contributes to Broad Spectrum of hATSCs Function via Oct4 Regulation. Antioxidants \& Redox Signaling 16, 383-399.

Janowski, B.A., Huffman, K.E., Schwartz, J.C., Ram, R., Hardy, D., Shames, D.S., Minna, J.D., and Corey, D.R. (2005a). Inhibiting gene expression at transcription start sites in chromosomal DNA with antigene RNAs. Nature chemical biology 1, 216-222.

Janowski, B.A., Huffman, K.E., Schwartz, J.C., Ram, R., Nordsell, R., Shames, D.S., Minna, J.D., and Corey, D.R. (2006). Involvement of AGO1 and AGO2 in mammalian transcriptional silencing. Nature Structural \& Molecular Biology 13, 787-792.

Janowski, B.A., Kaihatsu, K., Huffman, K.E., Schwartz, J.C., Ram, R., Hardy, D., Mendelson, C.R., and Corey, D.R. (2005b). Inhibiting transcription of chromosomal DNA with antigene peptide nucleic acids. Nature chemical biology 1, 210-215.

Janowski, B.A., Younger, S.T., Hardy, D.B., Ram, R., Huffman, K.E., and Corey, D.R. (2007). Activating gene expression in mammalian cells with promoter-targeted duplex RNAs. Nature chemical biology 3, 166-173.

Jiang, F., Miller, M.T., and Marcotrigiano, J. (2011a). Structural Basis for RNA Recognition and Activation of RIG-I. Nucleic Acid Database.

Jiang, F.G., Ramanathan, A., Miller, M.T., Tang, G.Q., Gale, M., Patel, S.S., and Marcotrigiano, J. (2011b). Structural basis of RNA recognition and activation by innate immune receptor RIG-I. Nature 479, 423-U184.

Jiang, G., Zheng, L., Pu, J., Mei, H., Zhao, J., Huang, K., Zeng, F., and Tong, Q. (2012). Small RNAs Targeting Transcription Start Site Induce Heparanase Silencing through Interference with Transcription Initiation in Human Cancer Cells. Plos One 7, e31379-e31379.

John, B., Enright, A.J., Aravin, A., Tuschl, T., Sander, C., and Marks, D.S. (2004). Human MicroRNA targets. Plos Biology 2, e363.

Johnson, K.L., Price, B.D., Eckerle, L.D., and Ball, L.A. (2004). Nodamura virus nonstructural protein B2 can enhance viral RNA accumulation in both mammalian and insect cells. Journal of Virology 78, 6698-6704.

Jopling, C.L., Yi, M., Lancaster, A.M., Lemon, S.M., and Sarnow, P. (2005). Modulation of hepatitis C virus RNA abundance by a liver-specific MicroRNA. Science 309, 1577-1581.

Jose, A.M., and Hunter, C.P. (2007). Transport of sequence-specific RNA interference information between cells. Annu Rev Genet 41, 305-330.

Kaneda, M., Tang, F.C., O'Carroll, D., Lao, K.Q., and Surani, M.A. (2009). Essential role for Argonaute2 protein in mouse oogenesis. Epigenetics \& Chromatin 2, 9-9. 
Kanellopoulou, C., Muljo, S.A., Kung, A.L., Ganesan, S., Drapkin, R., Jenuwein, T., Livingston, D.M., and Rajewsky, K. (2005). Dicer-deficient mouse embryonic stem cells are defective in differentiation and centromeric silencing. Genes \& Development 19, 489-501.

Kato, H., Takeuchi, O., Sato, S., Yoneyama, M., Yamamoto, M., Matsui, K., Uematsu, S., Jung, A., Kawai, T., Ishii, K.J., et al. (2006). Differential roles of MDA5 and RIG-I helicases in the recognition of RNA viruses. Nature 441, 101-105.

Kawahara, Y., Zinshteyn, B., Chendrimada, T.P., Shiekhattar, R., and Nishikura, K. (2007a). RNA editing of the microRNA-151 precursor blocks cleavage by the Dicer-TRBP complex. EMBO Rep 8, 763-769.

Kawahara, Y., Zinshteyn, B., Sethupathy, P., Iizasa, H., Hatzigeorgiou, A.G., and Nishikura, K. (2007b). Redirection of silencing targets by adenosine-to-inosine editing of miRNAs. Science 315, 1137-1140.

Kawaji, H., Nakamura, M., Takahashi, Y., Sandelin, A., Katayama, S., Fukuda, S., Daub, C.O., Kai, C., Kawai, J., Yasuda, J., et al. (2008). Hidden layers of human small RNAs. BMC Genomics 9, 157.

Kawasaki, H., and Taira, K. (2004). Induction of DNA methylation and gene silencing by short interfering RNAs in human cells. Nature 431, 211-217.

Kim, B.S., Im, Y.B., Jung, S.J., Park, C.H., and Kang, S.K. (2012). Argonaute2 Regulation for K+ Channel-Mediated Human Adipose Tissue-Derived Stromal Cells Self-Renewal and Survival in Nucleus. Stem Cells and Development 21, 1736-1748.

Kim, B.S., Jung, J.S., Jang, J.H., Kang, K.S., and Kang, S.K. (2011). Nuclear Argonaute 2 regulates adipose tissue-derived stem cell survival through direct control of miR10b and selenoprotein N1 expression. Aging Cell 10, 277-291.

Kim, D.H., Saetrom, P., Snove, O., and Rossi, J.J. (2008). MicroRNA-directed transcriptional gene silencing in mammalian cells. Proc Natl Acad Sci U S A 105, 16230-16235.

Kim, D.H., Villeneuve, L.M., Morris, K.V., and Rossi, J.J. (2006). Argonaute-1 directs siRNA-mediated transcriptional gene silencing in human cells. Nature Structural \& Molecular Biology 13, 793-797.

Kim, J.W., Zhang, Y.H., Zern, M.A., Rossi, J.J., and Wu, J. (2007). Short hairpin RNA causes the methylation of transforming growth factor-beta receptor II promoter and silencing of the target gene in rat hepatic stellate cells. Biochem Biophys Res Commun 359, 292-297.

Koppers-Lalic, D., Hackenberg, M., Bijnsdorp, I.V., van Eijndhoven, M.A.J., Sadek, P., Sie, D., Zini, N., Middeldorp, J.M., Ylstra, B., de Menezes, R.X., et al. (2014). Nontemplated Nucleotide Additions Distinguish the Small RNA Composition in Cells from Exosomes. Cell Reports 8, 1649-1658.

Kozomara, A., and Griffiths-Jones, S. (2014). miRBase: annotating high confidence microRNAs using deep sequencing data. Nucleic Acids Res 42, D68-73.

Kropp, J., Salih, S.M., and Khatib, H. (2014). Expression of microRNAs in bovine and human pre-implantation embryo culture media. Frontiers in Genetics 5, 91-91.

Kumar, L., Verma, S., Vaidya, B., and Gupta, V. (2015). Exosomes: Natural Carriers for siRNA Delivery. Current Pharmaceutical Design 21, 4556-4565.

Lasser, C. (2012). Exosomal RNA as biomarkers and the therapeutic potential of exosome vectors. Expert Opinion on Biological Therapy 12, S189-S197.

Lawrie, C.H., Gal, S., Dunlop, H.M., Pushkaran, B., Liggins, A.P., Pulford, K., Banham, A.H., Pezzella, F., Boultwood, J., Wainscoat, J.S., et al. (2008). Detection of elevated levels of 
tumour-associated microRNAs in serum of patients with diffuse large B-cell lymphoma. British journal of haematology 141, 672-675.

Lecellier, C.H., Dunoyer, P., Arar, K., Lehmann-Che, J., Eyquem, S., Himber, C., Saib, A., and Voinnet, O. (2005). A cellular microRNA mediates antiviral defense in human cells. Science 308, 557-560.

Lee, Y., El Andaloussi, S., and Wood, M.J.A. (2012). Exosomes and microvesicles: extracellular vesicles for genetic information transfer and gene therapy. Human Molecular Genetics 21, R125-R134.

Lehmann, K.A., and Bass, B.L. (1999). The importance of internal loops within RNA substrates of ADAR1. Journal of Molecular Biology 291, 1-13.

Lewis, B.P., Shih, I.H., Jones-Rhoades, M.W., Bartel, D.P., and Burge, C.B. (2003). Prediction of mammalian microRNA targets. Cell 115, 787-798.

Li, H., Li, W.X., and Ding, S.W. (2002). Induction and suppression of RNA silencing by an animal virus. Science 296, 1319-1321.

Li, L.C., Okino, S.T., Zhao, H., Pookot, D., Place, R.F., Urakami, S., Enokida, H., and Dahiya, R. (2006). Small dsRNAs induce transcriptional activation in human cells. Proc Natl Acad Sci U S A 103, 17337-17342.

Li, W.X., Li, H., Lu, R., Li, F., Dus, M., Atkinson, P., Brydon, E.W., Johnson, K.L., Garcia-Sastre, A., Ball, L.A., et al. (2004). Interferon antagonist proteins of influenza and vaccinia viruses are suppressors of RNA silencing. Proc Natl Acad Sci U S A 101, 1350-1355.

Li, X.J., Lu, C., Stewart, M., Xu, H.Y., Strong, R.K., Igumenova, T., and Li, P.W. (2009a). Structural basis of double-stranded RNA recognition by the RIG-I like receptor MDA5. Archives of Biochemistry and Biophysics 488, 23-33.

Li, X.J., Ranjith-Kumar, C.T., Brooks, M.T., Dharmaiah, S., Herr, A.B., Kao, C., and Li, P.W. (2009b). The RIG-I-like Receptor LGP2 Recognizes the Termini of Double-stranded RNA. Journal of Biological Chemistry 284, 13881-13891.

Liang, X.H., and Crooke, S.T. (2011). Depletion of key protein components of the RISC pathway impairs pre-ribosomal RNA processing. Nucleic Acids Research 39, 4875-4889.

Lim, L.P., Lau, N.C., Garrett-Engele, P., Grimson, A., Schelter, J.M., Castle, J., Bartel, D.P., Linsley, P.S., and Johnson, J.M. (2005). Microarray analysis shows that some microRNAs downregulate large numbers of target mRNAs. Nature 433, 769-773.

Liu, J., Hu, J.X., and Corey, D.R. (2012). Expanding the action of duplex RNAs into the nucleus: redirecting alternative splicing. Nucleic Acids Research 40, 1240-1250.

Liu, J., Hu, J.X., Hicks, J.A., Prakash, T.P., and Corey, D.R. (2015). Modulation of Splicing by Single-Stranded Silencing RNAs. Nucleic Acid Therapeutics 25, 113-120.

Livengood, A.J., Wu, C.C.N., and Carson, D.A. (2007). Opposing roles of RNA receptors TLR3 and RIG-I in the inflammatory response to double-stranded RNA in a Kaposi's sarcoma cell line. Cellular Immunology 249, 55-62.

Lu, S.H., and Cullen, B.R. (2004). Adenovirus VA1 noncoding RNA can inhibit small interfering RNA and microRNA biogenesis. Journal of Virology 78, 12868-12876.

Luo, S.S., Ishibashi, O., Ishikawa, G., Ishikawa, T., Katayama, A., Mishima, T., Takizawa, T., Shigihara, T., Goto, T., Izumi, A., et al. (2009). Human Villous Trophoblasts Express and Secrete Placenta-Specific MicroRNAs into Maternal Circulation via Exosomes. Biology of Reproduction 81, 717-729.

Ma, J., Flemr, M., Stein, P., Berninger, P., Malik, R., Zavolan, M., Svoboda, P., and Schultz, R.M. (2010). MicroRNA Activity Is Suppressed in Mouse Oocytes. Current Biology 20, 265-270. 
Marques, J.T., Devosse, T., Wang, D., Zamanian-Daryoush, M., Serbinowski, P., Hartmann, R., Fujita, T., Behlke, M.A., and Williams, B.R.G. (2006). A structural basis for discriminating between self and nonself double-stranded RNAs in mammalian cells. Nature Biotechnology 24, 559-565.

Martinez, I., and Melero, J.A. (2002). A model for the generation of multiple A to G transitions in the human respiratory syncytial virus genome: predicted RNA secondary structures as substrates for adenosine cleaminases that act on RNA. Journal of General Virology 83, 1445-1455.

McCartney, S., Vermi, W., Gilfillan, S., Cella, M., Murphy, T.L., Schreiber, R.D., Murphy, K.M., and Colonna, M. (2009). Distinct and complementary functions of MDA5 and TLR3 in poly(I:C)-mediated activation of mouse NK cells. Journal of Experimental Medicine 206, 2967-2976.

McCartney, S.A., Thackray, L.B., Gitlin, L., Gilfillan, S., Virgin, H.W., and Colonna, M. (2008). MDA-5 recognition of a murine norovirus. Plos Pathogens 4, e1000108-e1000108.

Meister, G., Landthaler, M., Peters, L., Chen, P.Y., Urlaub, H., Luhrmann, R., and Tuschl, T. (2005). Identification of novel argonaute-associated proteins. Current Biology 15, 2149-2155.

Meister, G., and Tuschl, T. (2004). Mechanisms of gene silencing by double-stranded RNA. Nature $431,343-349$.

Meurs, E., Chong, K., Galabru, J., Thomas, N.S., Kerr, I.M., Williams, B.R., and Hovanessian, A.G. (1990). Molecular cloning and characterization of the human double-stranded RNA-activated protein kinase induced by interferon. Cell 62, 379-390.

Morris, K.V., Chan, S.W., Jacobsen, S.E., and Looney, D.J. (2004). Small interfering RNA-induced transcriptional gene silencing in human cells. Science 305, 1289-1292.

Morse, D.P., Aruscavage, P.J., and Bass, B.L. (2002). RNA hairpins in noncoding regions of human brain and Caenorhabditis elegans mRNA are edited by adenosine deaminases that act on RNA. Proc Natl Acad Sci U S A 99, 7906-7911.

Much, C., Auchynnikava, T., Pavlinic, D., Buness, A., Rappsilber, J., Benes, V., Allshire, R., and O'Carroll, D. (2016). Endogenous Mouse Dicer Is an Exclusively Cytoplasmic Protein. Plos Genetics 12, e1006095.

Murchison, E.P., Partridge, J.F., Tam, O.H., Cheloufi, S., and Hannon, G.J. (2005). Characterization of Dicer-deficient murine embryonic stem cells. Proc Natl Acad Sci U S A 102, 12135-12140.

Murchison, E.P., Stein, P., Xuan, Z., Pan, H., Zhang, M.Q., Schultz, R.M., and Hannon, G.J. (2007). Critical roles for Dicer in the female germline. Genes \& Development 21, 682-693.

Nachmani, D., Zimmermann, A., Djian, E., Weisblum, Y., Livneh, Y., Le, V.T.K., Galun, E., Horejsi, V., Isakov, O., Shomron, N., et al. (2014). MicroRNA Editing Facilitates Immune Elimination of HCMV Infected Cells. Plos Pathogens 10, e1003963-e1003963.

Napoli, S., Pastori, C., Magistri, M., Carbone, G.M., and Catapano, C.V. (2009). Promoter-specific transcriptional interference and c-myc gene silencing by siRNAs in human cells. EMBO J 28, 1708-1719.

Nejepinska, J., Malik, R., Filkowski, J., Flemr, M., Filipowicz, W., and Svoboda, P. (2012). dsRNA expression in the mouse elicits RNAi in oocytes and low adenosine deamination in somatic cells. Nucleic Acids Research 40, 399-413.

Nejepinska, J., Malik, R., Wagner, S., and Svoboda, P. (2014). Reporters transiently transfected into mammalian cells are highly sensitive to translational repression induced by dsRNA expression. Plos One 9, e87517.

Neve, J., Burger, K., Li, W.C., Hoque, M., Patel, R., Tian, B., Gullerova, M., and Furger, A. (2016). Subcellular RNA profiling links splicing and nuclear DICER1 to alternative cleavage and polyadenylation. Genome Research 26, 24-35. 
Nguyen, J., and Szoka, F.C. (2012). Nucleic Acid Delivery: The Missing Pieces of the Puzzle? Accounts of Chemical Research 45, 1153-1162.

Nie, Y.Z., Hammond, G.L., and Yang, J.H. (2007). Double-stranded RNA deaminase ADAR1 increases host susceptibility to virus infection. Journal of Virology 81, 917-923.

Nie, Y.Z., Zhao, Q.C., Su, Y.J., and Yang, J.H. (2004). Subcellular distribution of ADAR1 isoforms is synergistically determined by three nuclear discrimination signals and a regulatory motif. Journal of Biological Chemistry 279, 13249-13255.

Nishi, K., Nishi, A., Nagasawa, T., and Ui-Tei, K. (2013). Human TNRC6A is an Argonaute-navigator protein for microRNA-mediated gene silencing in the nucleus. RNA 19, 17-35.

Nishi, K., Takahashi, T., Suzawa, M., Miyakawa, T., Nagasawa, T., Ming, Y., Tanokura, M., and Ui-Tei, K. (2015). Control of the localization and function of a miRNA silencing component TNRC6A by Argonaute protein. Nucleic Acids Research 43, 9856-9873.

Nishikura, K. (2010). Functions and Regulation of RNA Editing by ADAR Deaminases. In Annual Review of Biochemistry, Vol 79, pp. 321-349.

Nishikura, K. (2016). A-to-I editing of coding and non-coding RNAs by ADARs. Nature Reviews Molecular Cell Biology 17, 83-96.

Nishikura, K., Yoo, C., Kim, U., Murray, J.M., Estes, P.A., Cash, F.E., and Liebhaber, S.A. (1991). Substrate specificity of the dsRNA unwinding/modifying activity. EMBO J 10, 3523-3532.

Novellino, L., Rossi, R.L., Bonino, F., Cavallone, D., Abrignani, S., Pagani, M., and Brunetto, M.R. (2012). Circulating Hepatitis B Surface Antigen Particles Carry Hepatocellular microRNAs. Plos One 7, e31952-e31952.

Ohnishi, Y., Totoki, Y., Toyoda, A., Watanabe, T., Yamamoto, Y., Tokunaga, K., Sakaki, Y., Sasaki, H., and Hohjoh, H. (2010). Small RNA class transition from siRNA/piRNA to miRNA during pre-implantation mouse development. Nucleic Acids Research 38, 5141-5151.

Ohrt, T., Merkle, D., Birkenfeld, K., Echeverri, C.J., and Schwille, P. (2006). In situ fluorescence analysis demonstrates active siRNA exclusion from the nucleus by Exportin 5. Nucleic Acids Research 34, 1369-1380.

Ohrt, T., Muetze, J., Staroske, W., Weinmann, L., Hock, J., Crell, K., Meister, G., and Schwille, P. (2008). Fluorescence correlation spectroscopy and fluorescence cross-correlation spectroscopy reveal the cytoplasmic origination of loaded nuclear RISC in vivo in human cells. Nucleic Acids Research 36, 6439-6449.

Ohrt, T., Muetze, J., Svoboda, P., and Schwille, P. (2012). Intracellular Localization and Routing of miRNA and RNAi Pathway Components. Curr Top Med Chem 12, 79-88.

Ostermann, E., Tuddenham, L., Macquin, C., Alsaleh, G., Schreiber-Becker, J., Tanguy, M., Bahram, S., Pfeffer, S., and Georgel, P. (2012). Deregulation of Type I IFN-Dependent Genes Correlates with Increased Susceptibility to Cytomegalovirus Acute Infection of Dicer Mutant Mice. Plos One 7, e43744-e43744.

Parameswaran, P., Sklan, E., Wilkins, C., Burgon, T., Samuel, M.A., Lu, R., Ansel, K.M., Heissmeyer, V., Einav, S., Jackson, W., et al. (2010). Six RNA Viruses and Forty-One Hosts: Viral Small RNAs and Modulation of Small RNA Repertoires in Vertebrate and Invertebrate Systems. Plos Pathogens 6, e1000764-e1000764.

Park, H.S., Davies, M.V., Langland, J.O., Chang, H.W., Nam, Y.S., Tartaglia, J., Paoletti, E., Jacobs, B.L., Kaufman, R.J., and Venkatesan, S. (1994). TAR RNA-binding protein is an inhibitor of the interferon-induced protein kinase PKR. Proc Natl Acad Sci U S A 91, 4713-4717. 
Patel, R.C., and Sen, G.C. (1998). PACT, a protein activator of the interferon-induced protein kinase, PKR. EMBO J 17, 4379-4390.

Pegtel, D.M., van de Garde, M.D.B., and Middeldorp, J.M. (2011). Viral miRNAs exploiting the endosomal-exosomal pathway for intercellular cross-talk and immune evasion. Biochimica Et Biophysica Acta-Gene Regulatory Mechanisms 1809, 715-721.

Peng, Z.Y., Cheng, Y.B., Tan, B.C.M., Kang, L., Tian, Z.J., Zhu, Y.K., Zhang, W.W., Liang, Y., Hu, X.D., Tan, X.M., et al. (2012). Comprehensive analysis of RNA-Seq data reveals extensive RNA editing in a human transcriptome. Nature Biotechnology 30, 253-+.

Pfeffer, S., Sewer, A., Lagos-Quintana, M., Sheridan, R., Sander, C., Grasser, F.A., van Dyk, L.F., Ho, C.K., Shuman, S., Chien, M., et al. (2005). Identification of microRNAs of the herpesvirus family. Nature Methods 2, 269-276.

Pfeffer, S., Zavolan, M., Grasser, F.A., Chien, M., Russo, J.J., Ju, J., John, B., Enright, A.J., Marks, D., Sander, C., et al. (2004). Identification of virus-encoded microRNAs. Science 304, 734-736.

Pichlmair, A., Schulz, O., Tan, C.P., Naslund, T.I., Liljestrom, P., Weber, F., and Reis e Sousa, C. (2006). RIG-I-mediated antiviral responses to single-stranded RNA bearing 5'-phosphates. Science 314, 997-1001.

Place, R.F., Li, L.C., Pookot, D., Noonan, E.J., and Dahiya, R. (2008). MicroRNA-373 induces expression of genes with complementary promoter sequences. Proc Natl Acad Sci U S A 105, 1608-1613.

Polson, A.G., and Bass, B.L. (1994). Preferential selection of adenosines for modification by double-stranded RNA adenosine deaminase. EMBO J 13, 5701-5711.

Poulsen, H., Jorgensen, R., Heding, A., Nielsen, F.C., Bonven, B., and Egebjerg, J. (2006). Dimerization of ADAR2 is mediated by the double-stranded RNA binding domain. RNA 12, 1350-1360.

Puthenveetil, S., Whitby, L., Ren, J., Kelnar, K., Krebs, J.F., and Beal, P.A. (2006). Controlling activation of the RNA-dependent protein kinase by siRNAs using site-specific chemical modification. Nucleic Acids Research 34, 4900-4911.

Rak, J. (2013). Extracellular vesicles - biomarkers and effectors of the cellular interactome in cancer. Frontiers in Pharmacology 4, 21-21.

Reynolds, A., Anderson, E.M., Vermeulen, A., Fedorov, Y., Robinson, K., Leake, D., Karpilow, J., Marshall, W.S., and Khvorova, A. (2006). Induction of the interferon response by siRNA is cell type- and duplex length-dependent. RNA 12, 988-993.

Rice, G.I., Kasher, P.R., Forte, G.M.A., Mannion, N.M., Greenwood, S.M., Szynkiewicz, M., Dickerson, J.E., Bhaskar, S.S., Zampini, M., Briggs, T.A., et al. (2012). Mutations in ADAR1 cause Aicardi-Goutieres syndrome associated with a type I interferon signature. Nature Genetics 44 , 1243-1248.

Robb, G.B., Brown, K.M., Khurana, J., and Rana, T.M. (2005). Specific and potent RNAi in the nucleus of human cells. Nature Structural \& Molecular Biology 12, 133-137.

Rudel, S., Flatley, A., Weinmann, L., Kremmer, E., and Meister, G. (2008). A multifunctional human Argonaute2-specific monoclonal antibody. RNA 14, 1244-1253.

Sadler, A.J., and Williams, B.R. (2007). Structure and function of the protein kinase R. Current topics in microbiology and immunology 316, 253-292.

Salameh, A., Lee, A.K., Cardo-Vila, M., Nunes, D.N., Efstathiou, E., Staquicini, F.I., Dobroff, A.S., Marchio, S., Navone, N.M., Hosoya, H., et al. (2015). PRUNE2 is a human prostate cancer suppressor regulated by the intronic long noncoding RNA PCA3. Proc Natl Acad Sci U S A 112, 8403-8408. 
Samuel, C.E. (2011). Adenosine deaminases acting on RNA (ADARs) are both antiviral and proviral. Virology 411, 180-193.

Sanchez-Vargas, I., Scott, J.C., Poole-Smith, B.K., Franz, A.W., Barbosa-Solomieu, V., Wilusz, J., Olson, K.E., and Blair, C.D. (2009). Dengue virus type 2 infections of Aedes aegypti are modulated by the mosquito's RNA interference pathway. Plos Pathogens 5, e1000299.

Scadden, A.D. (2005). The RISC subunit Tudor-SN binds to hyper-edited double-stranded RNA and promotes its cleavage. Nature Structural \& Molecular Biology 12, 489-496.

Scadden, A.D., and Smith, C.W. (2001). RNAi is antagonized by A-->I hyper-editing. EMBO Rep 2, 1107-1111.

Schlee, M., and Hartmann, G. (2010). The Chase for the RIG-I Ligand-Recent Advances. Molecular Therapy $18,1254-1262$.

Schwartz, T., Rould, M.A., Lowenhaupt, K., Herbert, A., and Rich, A. (1999). Crystal structure of the $\mathrm{Z}$ alpha domain of the human editing enzyme ADAR1 bound to left-handed Z-DNA. Science 284, 1841-1845.

Sen, A., Pruijssers, A.J., Dermody, T.S., Garcia-Sastre, A., and Greenberg, H.B. (2011). The Early Interferon Response to Rotavirus Is Regulated by PKR and Depends on MAVS/IPS-1, RIG-I, MDA-5, and IRF3. Journal of Virology 85, 3717-3732.

Seo, G.J., Kincaid, R.P., Phanaksri, T., Burke, J.M., Pare, J.M., Cox, J.E., Hsiang, T.Y., Krug, R.M., and Sullivan, C.S. (2013). Reciprocal Inhibition between Intracellular Antiviral Signaling and the RNAi Machinery in Mammalian Cells. Cell Host \& Microbe 14, 435-445.

Shapiro, J.S., Schmid, S., Aguado, L.C., Sabin, L.R., Yasunaga, A., Shim, J.V., Sachs, D., Cherry, S., and Tenoever, B.R. (2014). Drosha as an interferon-independent antiviral factor. Proc Natl Acad Sci U S A 111, 7108-7113.

Sharma, A. (2015). Transgenerational epigenetic inheritance: resolving uncertainty and evolving biology. Biomolecular concepts 6, 87-103.

Sharma, N.R., Wang, X.H., Majerciak, V., Ajiro, M., Kruhlak, M., Meyers, C., and Zheng, Z.M. (2016). Cell Type- and Tissue Context-dependent Nuclear Distribution of Human Ago2. Journal of Biological Chemistry 291, 2302-2309.

Shi, H., Tschudi, C., and Ullu, E. (2006). Functional replacement of Trypanosoma brucei Argonaute by the human slicer Argonaute2. RNA 12, 943-947.

Shinagawa, T., and Ishii, S. (2003). Generation of Ski-knockdown mice by expressing a long double-strand RNA from an RNA polymerase II promoter. Genes \& Development 17, 1340-1345.

Shtam, T.A., Kovalev, R.A., Varfolomeeva, E.Y., Makarov, E.M., Kil, Y.V., and Filatov, M.V. (2013). Exosomes are natural carriers of exogenous siRNA to human cells in vitro. Cell Communication and Signaling 11, 88-88.

Sinkkonen, L., Hugenschmidt, T., Berninger, P., Gaidatzis, D., Mohn, F., Artus-Revel, C.G., Zavolan, M., Svoboda, P., and Filipowicz, W. (2008). MicroRNAs control de novo DNA methylation through regulation of transcriptional repressors in mouse embryonic stem cells. Nature Structural \& Molecular Biology 15, 259-267.

Sinkkonen, L., Hugenschmidt, T., Filipowicz, W., and Svoboda, P. (2010). Dicer Is Associated with Ribosomal DNA Chromatin in Mammalian Cells. Plos One 5, e12175-e12175.

Slater, L., Bartlett, N.W., Haas, J.J., Zhu, J., Message, S.D., Walton, R.P., Sykes, A., Dahdaleh, S., Clarke, D.L., Belvisi, M.G., et al. (2010). Co-ordinated Role of TLR3, RIG-I and MDA5 in the Innate Response to Rhinovirus in Bronchial Epithelium. Plos Pathogens 6, e1001178-e1001178. 
Smalheiser, N.R., Lugli, G., Thimmapuram, J., Cook, E.H., and Larson, J. (2011). Endogenous siRNAs and noncoding RNA-derived small RNAs are expressed in adult mouse hippocampus and are up-regulated in olfactory discrimination training. RNA 17, 166-181.

Stefl, R., Oberstrass, F.C., Hood, J.L., Jourdan, M., Zimmermann, M., Skrisovska, L., Maris, C., Peng, L., Hofr, C., Emeson, R.B., et al. (2010). The solution structure of the ADAR2 dsRBM-RNA complex reveals a sequence-specific readout of the minor groove. Cell 143, 225-237.

Stein, P., Rozhkov, N.V., Li, F., Cardenas, F.L., Davydenk, O., Vandivier, L.E., Gregory, B.D., Hannon, G.J., and Schultz, R.M. (2015). Essential Role for Endogenous siRNAs during Meiosis in Mouse Oocytes. Plos Genetics 11.

Stein, P., Zeng, F., Pan, H., and Schultz, R.M. (2005). Absence of non-specific effects of RNA interference triggered by long double-stranded RNA in mouse oocytes. Developmental Biology 286, 464-471.

Suh, M.R., Lee, Y., Kim, J.Y., Kim, S.K., Moon, S.H., Lee, J.Y., Cha, K.Y., Chung, H.M., Yoon, H.S., Moon, S.Y., et al. (2004). Human embryonic stem cells express a unique set of microRNAs. Developmental Biology 270, 488-498.

Suh, N., Baehner, L., Moltzahn, F., Melton, C., Shenoy, A., Chen, J., and Blelloch, R. (2010). MicroRNA Function Is Globally Suppressed in Mouse Oocytes and Early Embryos. Current Biology 20, 271-277.

Suk, K., Choi, J., Suzuki, Y., Ozturk, S.B., Mellor, J.C., Wong, K.H., MacKay, J.L., Gregory, R.I., and Roth, F.P. (2011). Reconstitution of human RNA interference in budding yeast. Nucleic Acids Research 39, E43-U59.

Sullivan, C.S., Grundhoff, A.T., Tevethia, S., Pipas, J.M., and Ganem, D. (2005). SV40-encoded microRNAs regulate viral gene expression and reduce susceptibility to cytotoxic $\mathrm{T}$ cells. Nature 435, 682-686.

Suzuki, K., Juelich, T., Lim, H., Ishida, T., Watanebe, T., Cooper, D.A., Rao, S., and Kelleher, A.D. (2008). Closed chromatin architecture is induced by an RNA duplex targeting the HIV-1 promoter region. Journal of Biological Chemistry 283, 23353-23363.

Svoboda, P., Stein, P., Anger, M., Bernstein, E., Hannon, G.J., and Schultz, R.M. (2004). RNAi and expression of retrotransposons MuERV-L and IAP in preimplantation mouse embryos. Developmental Biology 269, 276-285.

Swahari, V., Nakamura, A., Baran-Gale, J., Garcia, I., Crowther, A.J., Sons, R., Gershon, T.R., Hammond, S., Sethupathy, P., and Deshmukh, M. (2016). Essential Function of Dicer in Resolving DNA Damage in the Rapidly Dividing Cells of the Developing and Malignant Cerebellum. Cell Reports 14, 216-224.

Taira, K. (2006). Induction of DNA methylation and gene silencing by short interfering RNAs in human cells. Nature 441, 1176.

Tam, O.H., Aravin, A.A., Stein, P., Girard, A., Murchison, E.P., Cheloufi, S., Hodges, E., Anger, M., Sachidanandam, R., Schultz, R.M., et al. (2008). Pseudogene-derived small interfering RNAs regulate gene expression in mouse oocytes. Nature 453, 534-U538.

Tan, G.S., Garchow, B.G., Liu, X.H., Yeung, J., Morris, J.P., Cuellar, T.L., McManus, M.T., and Kiriakidou, M. (2009a). Expanded RNA-binding activities of mammalian Argonaute 2. Nucleic Acids Research 37, 7533-7545. 
Tan, Y.L., Zhang, B., Wu, T., Skogerbo, G., Zhu, X.P., Guo, X.Q., He, S.M., and Chen, R.S. (2009b). Transcriptional inhibiton of Hoxd4 expression by miRNA-10a in human breast cancer cells. BMC Molecular Biology 10, 12-12.

Tang, F., Kaneda, M., O’Carroll, D., Hajkova, P., Barton, S.C., Sun, Y.A., Lee, C., Tarakhovsky, A., Lao, K.Q., and Surani, M.A. (2007). Maternal microRNAs are essential for mouse zygotic development. Genes \& Development 21, 644-648.

Thuringer, D., Jego, G., Berthenet, K., Hammann, A., Solary, E., and Garrido, C. (2016). Gap junction-mediated transfer of miR-145-5p from microvascular endothelial cells to colon cancer cells inhibits angiogenesis. Oncotarget.

Ting, A.H., Schuebel, K.E., Herman, J.G., and Baylin, S.B. (2005). Short double-stranded RNA induces transcriptional gene silencing in human cancer cells in the absence of DNA methylation. Nature Genetics 37, 906-910.

Ting, A.H., Suzuki, H., Cope, L., Schuebel, K.E., Lee, B.H., Toyota, M., Imai, K., Shinomura, Y., Tokino, T., and Baylin, S.B. (2008). A requirement for DICER to maintain full promoter CpG island hypermethylation in human cancer cells. Cancer Research 68, 2570-2575.

Toth, K.F., Pezic, D., Stuwe, E., and Webster, A. (2016). The piRNA Pathway Guards the Germline Genome Against Transposable Elements. In Non-Coding Rna and the Reproductive System, pp. 51-77.

Tran, N., Raponi, M., Dawes, I.W., and Arndt, G.M. (2004). Control of specific gene expression in mammalian cells by co-expression of long complementary RNAs. FEBS Letters 573, 127-134.

Turchinovich, A., Weiz, L., Langheinz, A., and Burwinkel, B. (2011). Characterization of extracellular circulating microRNA. Nucleic Acids Research 39, 7223-7233.

Valen, E., Preker, P., Andersen, P.R., Zhao, X., Chen, Y., Ender, C., Dueck, A., Meister, G., Sandelin, A., and Jensen, T.H. (2011). Biogenic mechanisms and utilization of small RNAs derived from human protein-coding genes. Nature Structural \& Molecular Biology 18, 1075-1082.

Vesely, C., Tauber, S., Sedlazeck, F.J., von Haeseler, A., and Jantsch, M.F. (2012). Adenosine deaminases that act on RNA induce reproducible changes in abundance and sequence of embryonic miRNAs. Genome Research 22, 1468-1476.

Wahlgren, J., Karlson, T.D., Brisslert, M., Sani, F.V., Telemo, E., Sunnerhagen, P., and Valadi, H. (2012). Plasma exosomes can deliver exogenous short interfering RNA to monocytes and lymphocytes. Nucleic Acids Research 40, e130-e130.

Wahlgren, J., Statello, L., Skogberg, G., Telemo, E., and Valadi, H. (2016). Delivery of Small Interfering RNAs to Cells via Exosomes. In Sirna Delivery Methods: Methods and Protocols, pp. 105-125.

Wang, J., Huang, V., Ye, L., Barcena, A., Lin, G., Lue, T.F., and Li, L.-C. (2015a). Identification of Small Activating RNAs that Enhance Endogenous OCT4 Expression in Human Mesenchymal Stem Cells. Stem Cells and Development 24, 345-353.

Wang, S.Q., Liu, D.Y., Jin, R., Zhu, Y.P., and Xu, A.E. (2015b). Differential Responses of Normal Human Melanocytes to Intra- and Extracellular dsRNA. DNA and Cell Biology 34, 391-399.

Wang, X.H., Aliyari, R., Li, W.X., Li, H.W., Kim, K., Carthew, R., Atkinson, P., and Ding, S.W. (2006). RNA interference directs innate immunity against viruses in adult Drosophila. Science $312,452-454$.

Wang, Y., Mercier, R., Hobman, T.C., and LaPointe, P. (2013). Regulation of RNA interference by Hsp90 is an evolutionarily conserved process. Biochimica Et Biophysica Acta-Molecular Cell Research 1833, 2673-2681. 
Wang, Y.M., Medvid, R., Melton, C., Jaenisch, R., and Blelloch, R. (2007). DGCR8 is essential for microRNA biogenesis and silencing of embryonic stem cell self-renewal. Nature Genetics 39, 380-385. Watanabe, T., Takeda, A., Tsukiyama, T., Mise, K., Okuno, T., Sasaki, H., Minami, N., and Imai, H. (2006). Identification and characterization of two novel classes of small RNAs in the mouse germline: retrotransposon-derived siRNAs in oocytes and germline small RNAs in testes. Genes \& Development 20, 1732-1743.

Watanabe, T., Totoki, Y., Toyoda, A., Kaneda, M., Kuramochi-Miyagawa, S., Obata, Y., Chiba, H., Kohara, Y., Kono, T., Nakano, T., et al. (2008). Endogenous siRNAs from naturally formed dsRNAs regulate transcripts in mouse oocytes. Nature 453, 539-U539.

Wee, L.M., Flores-Jasso, C.F., Salomon, W.E., and Zamore, P.D. (2012). Argonaute Divides Its RNA Guide into Domains with Distinct Functions and RNA-Binding Properties. Cell 151, 1055-1067.

Wei, H.B., Zhou, B., Zhang, F., Tu, Y.Y., Hu, Y.N., Zhang, B.G., and Zhai, Q.W. (2013). Profiling and Identification of Small rDNA-Derived RNAs and Their Potential Biological Functions. Plos One 8, e56842-e56842.

Wei, W., Ba, Z.Q., Gao, M., Wu, Y., Ma, Y.T., Amiard, S., White, C.I., Danielsen, J.M.R., Yang, Y.G., and Qi, Y.J. (2012). A Role for Small RNAs in DNA Double-Strand Break Repair. Cell 149, 101-112.

Weinberg, M.S., Villeneuve, L.M., Ehsani, A., Amarzguioui, M., Aagaard, L., Chen, Z.X., Riggs, A.D., Rossi, J.J., and Morris, K.V. (2006). The antisense strand of small interfering RNAs directs histone methylation and transcriptional gene silencing in human cells. RNA 12, 256-262.

Weinmann, L., Hock, J., Ivacevic, T., Ohrt, T., Mutze, J., Schwille, P., Kremmer, E., Benes, V., Urlaub, H., and Meister, G. (2009). Importin 8 is a gene silencing factor that targets argonaute proteins to distinct mRNAs. Cell 136, 496-507.

Weissbach, R., and Scadden, A.D.J. (2012). Tudor-SN and ADAR1 are components of cytoplasmic stress granules. RNA 18, 462-471.

White, E., Schlackow, M., Kamieniarz-Gdula, K., Proudfoot, N.J., and Gullerova, M. (2014). Human nuclear Dicer restricts the deleterious accumulation of endogenous double-stranded RNA. Nature Structural \& Molecular Biology 21, 552-559.

Wilkins, C., Dishongh, R., Moore, S.C., Whitt, M.A., Chow, M., and Machaca, K. (2005). RNA interference is an antiviral defence mechanism in Caenorhabditis elegans. Nature 436, 1044-1047.

Wolfrum, C., Shi, S., Jayaprakash, K.N., Jayaraman, M., Wang, G., Pandey, R.K., Rajeev, K.G., Nakayama, T., Charrise, K., Ndungo, E.M., et al. (2007). Mechanisms and optimization of in vivo delivery of lipophilic siRNAs. Nature Biotechnology 25, 1149-1157.

Wong, S.K., and Lazinski, D.W. (2002). Replicating hepatitis delta virus RNA is edited in the nucleus by the small form of ADAR1. Proc Natl Acad Sci U S A 99, 15118-15123.

Wu, G.P., Yang, G.H., Zhang, R.X., Xu, G.Y., Zhang, L., Wen, W., Lu, J.B., Liu, J.Y., and Yu, Y. (2015a). Altered microRNA Expression Profiles of Extracellular Vesicles in Nasal Mucus From Patients With Allergic Rhinitis. Allergy Asthma \& Immunology Research 7, 449-457.

Wu, W.X., Zhang, W., Duggan, E.S., Booth, J.L., Zou, M.H., and Metcalf, J.P. (2015b). RIG-I and TLR3 are both required for maximum interferon induction by influenza virus in human lung alveolar epithelial cells. Virology 482, 181-188.

Xu, S., Xue, C.Y., Li, J.P., Bi, Y.Z., and Cao, Y.C. (2011). Marek's Disease Virus Type 1 MicroRNA miR-M3 Suppresses Cisplatin-Induced Apoptosis by Targeting SMAD2 of the Transforming Growth Factor Beta Signal Pathway. Journal of Virology 85, 276-285. 
Yang, N., and Kazazian, H.H., Jr. (2006). L1 retrotransposition is suppressed by endogenously encoded small interfering RNAs in human cultured cells. Nature Structural \& Molecular Biology 13, $763-771$.

Yang, S., Tutton, S., Pierce, E., and Yoon, K. (2001). Specific double-stranded RNA interference in undifferentiated mouse embryonic stem cells. Molecular and Cellular Biology 21, 7807-7816.

Yang, W.D., Chendrimada, T.P., Wang, Q.D., Higuchi, M., Seeburg, P.H., Shiekhattar, R., and Nishikura, K. (2006a). Modulation of microRNA processing and expression through RNA editing by ADAR deaminases. Nature Structural \& Molecular Biology 13, 13-21.

Yang, W.D., Wang, Q.D., Howell, K.L., Lee, J.T., Cho, D.S.C., Murray, J.M., and Nishikura, K. (2005). ADAR1 RNA deaminase limits short interfering RNA efficacy in mammalian cells. Journal of Biological Chemistry 280, 3946-3953.

Yang, X., Murthy, V., Schultz, K., Tatro, J.B., Fitzgerald, K.A., and Beasley, D. (2006b). Toll-like receptor 3 signaling evokes a proinflammatory and proliferative phenotype in human vascular smooth muscle cells. American Journal of Physiology-Heart and Circulatory Physiology 291, H2334-H2343.

Yi, C.E., Bekker, J.M., Miller, G., Hill, K.L., and Crosbie, R.H. (2003). Specific and potent RNA interference in terminally differentiated myotubes. Journal of Biological Chemistry 278, 934-939.

Yoneyama, M., Kikuchi, M., Natsukawa, T., Shinobu, N., Imaizumi, T., Miyagishi, M., Taira, K., Akira, S., and Fujita, T. (2004). The RNA helicase RIG-I has an essential function in double-stranded RNA-induced innate antiviral responses. Nature Immunology 5, 730-737.

Yoon, Y.J., Kim, O.Y., and Gho, Y.S. (2014). Extracellular vesicles as emerging intercellular communicasomes. BMB Reports 47, 531-539.

Younger, S.T., and Corey, D.R. (2011). Transcriptional gene silencing in mammalian cells by miRNA mimics that target gene promoters. Nucleic Acids Research 39, 5682-5691.

Zamore, P.D., Tuschl, T., Sharp, P.A., and Bartel, D.P. (2000). RNAi: double-stranded RNA directs the ATP-dependent cleavage of mRNA at 21 to 23 nucleotide intervals. Cell 101, 25-33.

Zeng, Y., Wagner, E.J., and Cullen, B.R. (2002). Both natural and designed micro RNAs can inhibit the expression of cognate mRNAs when expressed in human cells. Molecular Cell 9, 1327-1333.

Zhang, L., Hou, D., Chen, X., Li, D., Zhu, L., Zhang, Y., Li, J., Bian, Z., Liang, X., Cai, X., et al. (2012). Exogenous plant MIR168a specifically targets mammalian LDLRAP1: evidence of cross-kingdom regulation by microRNA. Cell Research 22, 107-126.

Zhang, M.X., Zhang, C., Shen, Y.H., Wang, J., Li, X.N., Chen, L., Zhang, Y., Coselli, J.S., and Wang, X.L. (2008a). Effect of 27nt small RNA on endothelial nitric-oxide synthase expression. Molecular Biology of the Cell 19, 3997-4005.

Zhang, M.X., Zhang, C., Shen, Y.H., Wang, J., Li, X.N., Zhang, Y., Coselli, J., and Wang, X.L. (2008b). Biogenesis of short intronic repeat 27nt small RNA from endothelial nitric oxide synthase gene. Journal of Biological Chemistry.

Zhang, X.Z., Li, H.T., Burnett, J.C., and Rossi, J.J. (2014). The role of antisense long noncoding RNA in small RNA-triggered gene activation. RNA 20, 1916-1928.

Zheng, X.F., and Bevilacqua, P.C. (2004). Activation of the protein kinase PKR by short double-stranded RNAs with single-stranded tails. RNA 10, 1934-1945.

Zheng, Z.M., Tang, S.A., and Tao, M.F. (2005). Development of resistance to RNAi in mammalian cells. In Strategies for Silencing Gene Expression, pp. 105-118. 


\title{
RNAi AND miRNA PATHWAYS IN BIRDS
}

Keywords: dsRNA, siRNA, miRNA, Dicer, TARBP2, PACT, Argonaute

\section{PETR SVOBODA}

Institute of Molecular Genetics, Academy of Sciences of the Czech Republic, Videnska 1083, 14220 Prague 4, Czech Republic

Correspondence to: Petr Svoboda, Institute of Molecular Genetics ASCR, Videnska 1083, 14220 Prague 4, Czech Republic, tel. \#+420 241063147, e-mail: svobodap@img.cas.cz.

\begin{abstract}
RNA silencing denotes sequence-specific repression mediated by small RNAs. In vertebrates, there are two closely related pathways, which share several protein factors: RNA interference (RNAi) and microRNA (miRNA) pathway. The miRNA pathway regulates endogenous protein-coding gene expression and has been implicated in many biological processes. RNAi generally serves as a form of innate immunity targeting viruses and mobile elements. This text reviews miRNA and RNAi pathways in birds. Although the available literature on RNA silencing in birds is very limited, many features can be deduced from the genomic data in the public domain. miRNA, RNAi and other dsRNA-responding pathways in birds appear very much like those in mammals, important bird-specific features of RNA silencing pathways are yet to be identified. The miRNA pathway is likely the dominant small RNA pathway while the existence and functionality of endogenous RNAi remains unclear. Some variations may be present in the main bird antiviral interferon system.
\end{abstract}

\section{Introduction}

Birds (Aves) belong together with mammals and fishes to the group Craniata within chordates. Some of the birds are of high economic importance (food industry) or medical relevance (viral vectors causing zoonoses). Bird ancestors branched of mammalian ancestors over 300 MYA when the synapsid lineage leading to mammals branched of the sauropsid lineage leading to dinosaurs and birds. There are $\sim 9000$ extant bird species (Margulis and Schwartz, 1998). During their evolution, birds evolved numerous physiological adaptations in which they differ from mammals, including feathers, shelled eggs with external development, or different sex chromosome system, to name a few. At the same time, they are the closest mammal-related group covered in this series, in terms of synteny and sequence similarity. This is useful for assessing features of dsRNA and miRNA pathways because the available literature on RNA silencing in birds is very limited. However, many features can be deduced from the genomic data in the public domain. miRNA, RNAi and other 
dsRNA-responding pathways in birds are very much like those in mammals and the literature does not report an important bird-specific feature in RNA silencing pathways. Since mechanistical principles of vertebrate miRNA and RNAi pathways were introduced in the first two reviews of this series (Svoboda, 2019a, b) and in further detail elsewhere (Bartel, 2018; Svoboda, 2014), I will focus here directly on features of these pathways described for birds.

\section{Dicer}

According to the complete genome sequences of chicken and Zebra Finch, birds have one Dicer protein. Chicken Dicer has been assigned to the chromosome 5 according to the radiation hybrid mapping (Tian et al., 2007) which is in agreement with the current chicken genome map. There is no detailed analysis of avian Dicer specificity and activity, which have to be inferred indirectly from other results. Chicken Dicer can process both, long dsRNA and miRNA precursors, as evidenced by induction of RNAi with long dsRNA (Mauti et al., 2008; Pekarik et al., 2003) and hundreds of avian miRNAs in the miRBase.

The common Dicer product size seems to be 21-23nt with a typical size of 22nt. This information can be inferred from available miRBase data (Fig. 1). Thus, the avian Dicer produces small RNAs with the same sizes as the mammalian Dicer (Fig. 1). Another possible substrate of Dicer in birds might be snoRNAs, although the biological significance of this observation remains unclear (Taft et al., 2009).

It is unclear if there are functionally different avian Dicer isoforms as is the case in murine oocytes and somatic cells (Flemr et al., 2013). There is one report of different Dicer splice variant in goose (Anser cygnoides) where one variant lacks a linker between DEAD box and helicase $\mathrm{C}$ domains at the $\mathrm{N}$-terminus (gDicer-b) (Hu et al., 2014). The shorter isoform gDicer-b is present in multiple tissues, however its functional significance is unclear. The truncation is found in the N-terminus, which is associated with substrate selectivity and efficient processing. Therefore, one might speculate about some functional divergence in substrate processing between the two isoforms. However, there is no experimental evidence at the moment. The only available data, so far, concern cloning of the short isoform and expression analysis of several tissues and follicular stages by RT-PCR (Hu et al., 2014).

\section{dsRBPs}

dsRBP binding partners of Dicer have not been studied, so far. Interestingly, the chicken genome contains a dsRBP, which is related to TARBP2 and PACT, suggesting a more ancestral vertebrate state and a reduced crosstalk between RNAi and the interferon pathway.

\section{Argonaute proteins}

Argonaute family proteins are effectors of RNA silencing mechanisms. They are divided into two subfamilies: AGO proteins, which accommodate miRNAs and siRNAs, and PIWI 
proteins, which accommodate piRNAs. Avian AGO proteins have not been characterized in a published report but public chicken genome data show that the setup is the same as in mammals: Studies in chicken revealed four AGO proteins, where AGO1, 3, and 4 are encoded within one locus on chromosome 23 and AGO2 is encoded separately on chromosome 2. This arrangement appears to be shared within mammals and birds (Zhou et al., 2010). Additional information about avian AGOs can be inferred indirectly from the existence of functional RNAi and miRNA pathways (discussed below), which implies that at least one AGO protein is a "slicer" (presumably AGO2, given its conserved role as a slicer from Drosophila to mammals). Avian AGO proteins can also mediate post-transcriptional silencing guided by imperfectly base paired miRNAs.

In addition, there were two publications found, which mention avian PIWI proteins, which primarily control genome integrity in the germline and are not within the scope of this report (Kim et al., 2012; Lim et al., 2013).

\section{Other factors}

Birds have additional proteins involved in other dsRNA responses, which are either associated with adenosine deamination (Herbert et al., 1995) or interferon response. Interferon response factors, which recognize some form of dsRNA and are also found in mammals, include MDA5 (Hayashi et al., 2014; Lee et al., 2012, 2014), RIG-I (Chen et al., 2015; Li et al., 2014a; Xu et al., 2015), and PKR (Gonzalez-Lopez et al., 2003; Lostale-Seijo et al., 2016; Zhang et al., 2014). Interestingly, chicken lack the RHA/DHX9 homolog (Sato et al., 2015). The antiviral response to dsRNA will be discussed further below.

\section{miRNA pathway}

According to miRBase (Kozomara and Griffiths-Jones, 2014), bird genomes encode hundreds of miRNAs (Table 1) During the systematic literature review, miRNA-related publications lacking a mechanistic molecular insight into the miRNA pathway were the most common class of annotated publications for birds ( $\sim 50 \%$ of all selected publications). These publications fall into four basic categories:

a) annotations of novel miRNAs, including high-throughput expression analyses (for example (Godnic et al., 2013; Luo et al., 2012; Taft et al., 2009) and many others). This category also includes the original chicken and Zebra Finch genome annotation papers (International Chicken Genome Sequencing, 2004; Warren et al., 2010).

b) studies of miRNAs in different biological contexts, including reproduction (Lee et al., 2015; Lee et al., 2011), skeletomuscular apparatus (Chen et al., 2009a), bird song physiology (Gunaratne et al., 2011), growth/weight gain (Li et al., 2013), and many others; their comprehensive listing would be beyond the scope of this report.

c) studies of relationship between miRNAs and the immune system, especially antiviral - these will be discussed further below in the section 3.1.2.7. Other dsRNA response pathways 
miRNA size distribution in Gallus gallus

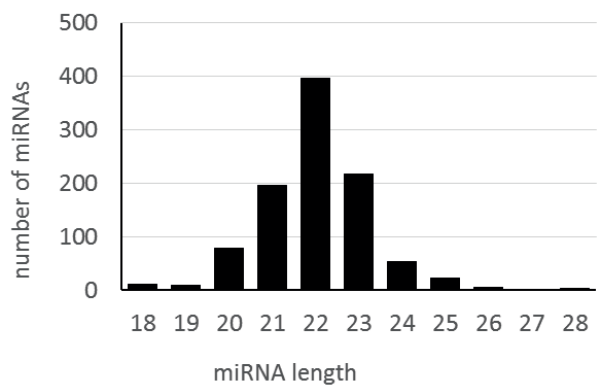

miRNA size distribution in Mus musculus

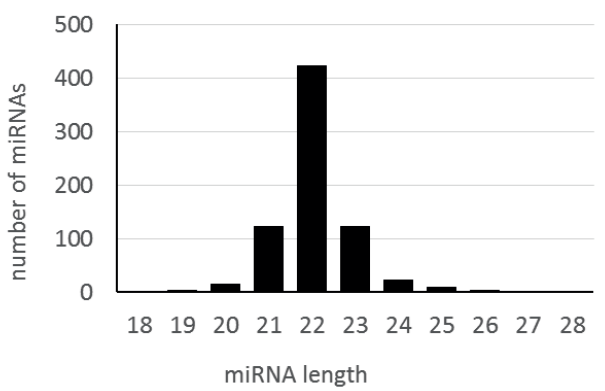

Figure 1 Avian miRNA lengths

The left graph depicts size distribution of all 994 chicken miRNAs deposited in the miRBase (release 21). For comparison, the right graph shows size distribution of 721 high-confidence murine miRNAs.

d) false positives of the search- reports describing mRNA knock-down through short hairpin RNAs adopting miRNA-like appearance. There is a series of nearly identical methodological papers, apparently published twice in 2006 and 2013, which fall in this category (Deng et al., 2015; Lin et al., 2006a; Lin et al., 2006b; Lin and Ying, 2006; Lin et al., 2013a; Lin et al., 2013b; Lin and Ying, 2013; Ying and Lin, 2009; Ying et al., 2010) and several other publications concerning development and adaptations of shRNA systems (e.g. (Andermatt et al., 2014; Chen et al., 2011; Das et al., 2006). These articles actually belong to the RNAi section below but due to the confusing use of nomenclature, they would also fall into the miRNA category.

Taken together, essentially all miRNA-related publications dealt with miRNA annotation, analysis of biological functions of miRNAs, and adoption of miRNAs for artificial knock-down systems allowing for suppressing any gene of interest. Avian miRNA-related publications did not reveal any avian-specific mechanistic insight into miRNA biogenesis, in which birds would differ from the general consensus for mammals, or other vertebrates in general. The complete list of all miRNA-related publications is available in a library accompanying this section.

Table 1 Bird miRNAs in the miRBase (release 22.1 (Kozomara and Griffiths-Jones, 2014)):

\begin{tabular}{|l|c|c|}
\hline species [genome annotation] & miRNA precursors & mature miRNA \\
\hline Gallus gallus [Gallus-gallus-4.0] & 882 & 1232 \\
\hline Taeniopygia guttata [taeGlu3.2.4] & 247 & 334 \\
\hline
\end{tabular}

\section{RNAi}

Avian RNAi-related literature deals mainly with experimental knock-down of gene expression, which does not reveal much about the physiological role of RNAi pathway in birds. These studies cannot all be included in the report due to the high number, but they are 
available in the reference library accompanying this section). What can be inferred from those studies is that birds have the complete molecular mechanisms for canonical RNAi and can efficiently execute it. This is evidenced by efficient knock-downs with long dsRNA (Mauti et al., 2008; Pekarik et al., 2003).

Published exogenous RNAi data provide insights into possible routes nucleic acids can become biologically active in birds and concern areas of EFSA main interests as various forms of RNAi technology (siRNAs or transgenic) were considered a way for preventing virulent strain circulation in poultry (O'Neill, 2007) although results of these efforts were relatively modest, being typically developed in cultured cells (Hutcheson et al., 2015; Sahare et al., 2015; Stewart et al., 2011; Yin et al., 2010). Exogenous RNAi in vivo required non-physiological manipulations such as 1) plasmid or siRNA electroporation (Andermatt et al., 2014; Baeriswyl et al., 2008; Mauti et al., 2008; Pekarik et al., 2003; Sato et al., 2004; Wilson and Stoeckli, 2011, 2012), 2) transfection (Dai et al., 2005; Lin et al., 2006a; Lin et al., 2013a; Wei et al., 2015), 3) recombinant virus (Lambeth et al., 2009b), or 4) recombinant lentivirus delivery (Chen et al., 2009b; Haesler et al., 2007). Altogether, these data suggest that exogenous RNAi would not be achieved by just exposing birds to small RNAs or their precursors in the environment or food.

Regarding the endogenous RNAi, it remains what its physiological role is. There are three possible roles for endogenous RNAi: antiviral defense, genome defense against retrotransposons and control of gene expression. These roles would be associated with production of viral siRNAs, retrotransposon siRNAs and mRNA-targeting siRNAs in vivo. However, an unequivocal evidence for existence of these classes and their function was not provided yet.

One report attempted to examine the role of Dicer in retrotransposon repression. It was shown that the loss of Dicer in chicken cells does not result in accumulation of chicken CR1 retrotransposon while introduction of a human L1 element into cells lacking Dicer results in accumulation of L1 transcripts and increased retrotransposition (Lee et al., 2009). However, these data are difficult to interpret as different scenarios could lead to the same observations, especially downstream effects of a perturbed miRNA pathway and chromatin-mediated silencing of CR1.

\section{Other dsRNA response pathways}

\section{Chromatin regulation by small RNAs}

Two studies involving bird models brought up a possible nuclear function of Dicer and its link to chromatin regulation, which is of the unsettled issues in vertebrate models. Despite a decade of research, there is still no proposed molecular mechanism explaining these phenomena while the literature contains a number of contradicting observations.

Fukagawa et al. produced a conditional loss-of-function Dicer mutant in a chicken-human hybrid DT40 cell line that contains human chromosome 21. The loss of Dicer resulted in cell death and accumulation of premature sister chromatid separation. Furthermore, aberrant accumulation of transcripts from human centromeric repeats was also found suggesting 
loss of heterochromatin at centromeres. While localization of two heterochromatin proteins $(\operatorname{Rad} 21$ and BubR1) was abnormal, localization of core centromeric heterochromatin proteins CENP-A and -C was normal (Fukagawa et al., 2004). Although the article is highly cited (335 times up to date according to WOS core collection), the molecular mechanism of the effect remains elusive. It is possible that the phenomenon is an indirect consequence of perturbing the miRNA pathway. Furthermore, the model system is unique and human heterochromatin sequences might exhibit unusual behaviour in the chicken nuclear environment.

Giles et al. examined a 16 kilobase $(\mathrm{kb})$ heterochromatin domain in the chicken erythroid progenitor cell line $6 \mathrm{C} 2$. RNAi-mediated downregulation of the enzyme Dicer resulted in increased histone acetylation and transcript levels from the heterochromatin locus while compact chromatin structure became more accessible to restriction endonucleases. It was also shown that chicken AGO2 homolog binds the $16 \mathrm{~kb}$ region in a Dicer-dependent manner and is necessary for a condensed chromatin structure (Giles et al., 2010). The article has been cited 26 times up to date according to WOS (core collection), yet there was no follow up providing any mechanistic explanation of the phenomenon. It is possible that the observed effects could be an indirect effect of suppression of the miRNA pathway or even an experimental artefact. Additional controls and experiments would be needed to address these concerns and clarify inconsistencies with other reports. Therefore, this report should be considered an interesting observation without a clear mechanistic explanation.

Taken together, small RNA-mediated chromatin changes in birds remain an open question. Without knowing the molecular mechanism, especially that of biogenesis of small RNAs regulating chromatin and their mode of action, there is simply not enough information for qualified conclusions.

\section{Antiviral defense - interferon response and crosstalk with RNA silencing}

Many studies deal with various aspects of viral infections in birds or avian cells. The most studied model for viral infections in birds is Marek's disease, which is a consequence of a Herpesvirus infection in poultry. Publications linked to Marek's disease addressed virus encoded miRNAs (Coupeau et al., 2012; Luo et al., 2011; Morgan et al., 2008; Muylkens et al., 2010; Strassheim et al., 2012; Xu et al., 2011; Yao et al., 2008; Zhao et al., 2011; Zhao et al., 2009), changes in host miRNA expression during infection (Dinh et al., 2014; Han et al., 2016; Lambeth et al., 2009a; Li et al., 2014b; Li et al., 2014c; Lian et al., 2015a; Lian et al., 2015b; Stik et al., 2013; Tian et al., 2012; Xu et al., 2010; Yao et al., 2008), or attempts to block the virus with RNAi (Chen et al., 2009b; Chen et al., 2008; Lambeth et al., 2009b). A similar set of articles has been found for other studied viruses infecting birds e.g. avian influenza virus H5N1 and H9N2, bursal disease virus, subgroup J avian leucosis virus. The complete list is available in the library accompanying this section.

Reports concerning host and virus-encoded miRNAs generally represent adaptations manipulating the miRNA pathway for the benefit of the pathogen. At the same time, these articles did not reveal some unique adaptation of the chicken miRNA pathway, which would differ from molecular mechanisms and principles described in the previous section. 

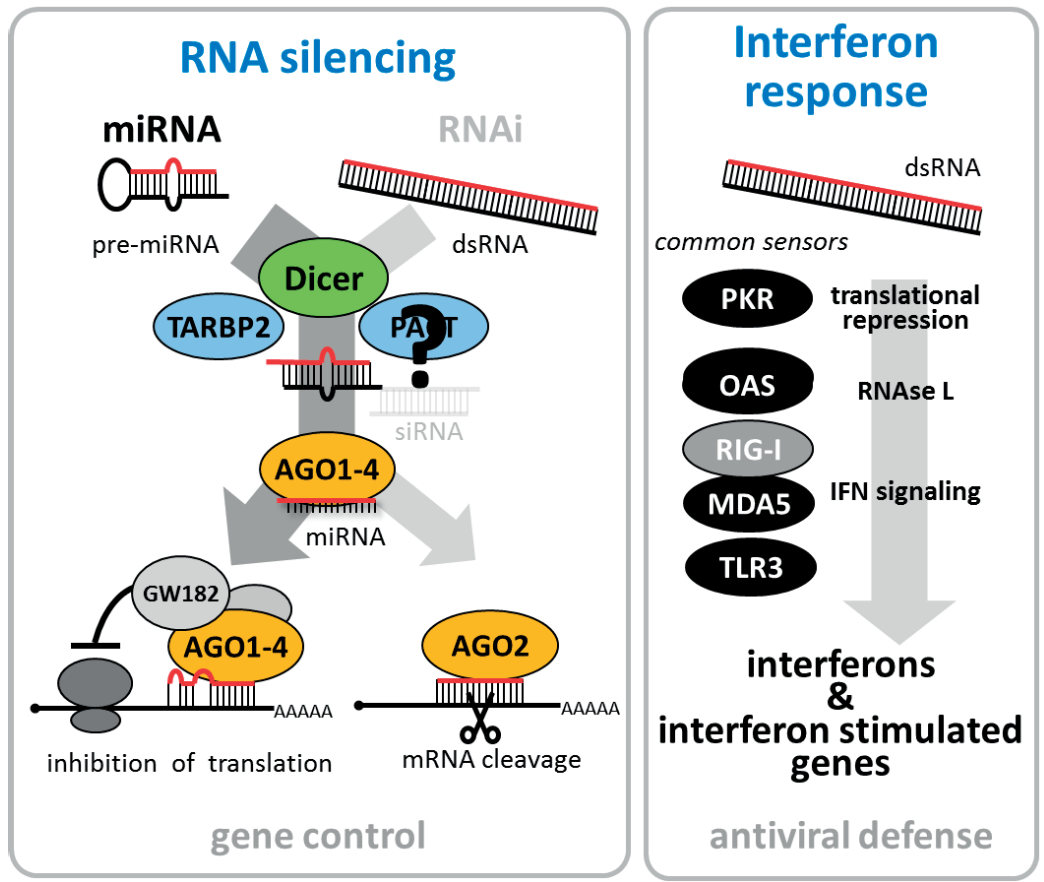

Figure 2 Overview of avian pathways

dsRNA and miRNA pathways in birds are very much similar to the mammalian ones with some minor exceptions. Birds have only a single dsRBP homologous to TARBP2, and lack PACT ortholog.

The last group of articles reviewed here represent publications covering the interferon system, the common antiviral system induced by dsRNA and other RNA species (Karpala et al., 2008; Kint et al., 2015; Lostale-Seijo et al., 2016). Birds generally utilize the same antiviral interferon system including its key dsRNA sensing proteins: PKR (Gonzalez-Lopez et al., 2003; Lostale-Seijo et al., 2016; Zhang et al., 2014), RIG-I (Chen et al., 2015; Li et al., 2014a; Xu et al., 2015), and MDA5 (Hayashi et al., 2014; Lee et al., 2012, 2014), 2',5'-OAS (Lee et al., 2014; Villanueva et al., 2011). However, there seem to be some species-specific variations. For example, RIG-I is found in some birds, such as ducks or pigeons (Chen et al., 2015; Xu et al., 2015) but not in chicken, which lack RIG-I and the RNA sensing RHA/DHX9 helicase homolog (Sato et al., 2015). Although the lack of RIG-I is partially compensated by chicken MDA5 activity (Hayashi et al., 2014; Karpala et al., 2011) the absence of RIG-I-like function may contribute to the chicken's susceptibility to highly pathogenic influenza (Karpala et al., 2011; Li et al., 2014a).

\section{Adenosine deamination}

Birds have also adenosine deaminases that act on RNA (Herbert et al., 1995) but their physiological significance in birds is unknown at the moment. 


\section{Summary}

In terms of the mode-of-action of dsRNA and miRNA pathways, birds are closely resembling mammals despite over 300 millions of years of separate evolution. The molecular mechanism of RNAi and miRNA pathways seems to be essentially identical to that of mammals except of a single dsRBD instead of two different ones. The significance of this difference is unclear. The miRNA pathway seems to be the dominant small RNA pathway while the existence and functionality of endogenous RNAi remains unclear. Some variations were found in the interferon system (lack of RIG-I in chicken), which appears to be the main antiviral system in birds.

\section{Acknowledgement}

I would like to thank my colleagues Jan Paces, Miloslav Nic, and Tomas Novotny for help with collecting literature for the review. The review content was produced under a contract OC/EFSA/GMO/2015/01-CT 01 with European Food Safety Authority (EFSA); the opinions expressed are those of the contractor only and do not represent EFSA's official position. Publication of the review was funded by LO1220 and LM2015063 by the Ministry of Education, Youth and Sports.

\section{References}

Andermatt, I., Wilson, N., and Stoeckli, E.T. (2014). In ovo electroporation of miRNA-based-plasmids to investigate gene function in the developing neural tube. Methods in Molecular Biology $1101,353-368$.

Baeriswyl, T., Mauti, O., and Stoeckli, E.T. (2008). Temporal control of gene silencing by in ovo electroporation. Methods in Molecular Biology 442, 231-244.

Bartel, D.P. (2018). Metazoan MicroRNAs. Cell 173, 20-51.

Chen, C.H., Zhou, Y.L., Wu, Y.F., Cao, Y., Gao, J.S., and Tang, J.B. (2009a). Effectiveness of MicroRNA in Down-Regulation of TGF-beta Gene Expression in Digital Flexor Tendons of Chickens: In Vitro and In Vivo Study. Journal of Hand Surgery-American Volume 34A, 1777-1784.

Chen, M., Payne, W.S., Dunn, J.R., Chang, S., Zhang, H.M., Hunt, H.D., and Dodgson, J.B. (2009b). Retroviral delivery of RNA interference against Marek's disease virus in vivo. Poultry Science 88 , 1373-1380.

Chen, M., Payne, W.S., Hunt, H., Zhang, H.M., Holmen, S.L., and Dodgson, J.B. (2008). Inhibition of Marek's disease virus replication by retroviral vector-based RNA interference. Virology 377, $265-272$.

Chen, S.C.Y., Stern, P., Guo, Z.Y., and Chen, J.Z. (2011). Expression of Multiple Artificial MicroRNAs from a Chicken miRNA126-Based Lentiviral Vector. Plos One 6, e22437-e22437.

Chen, Y., Huang, Z.Y., Wang, B., Yu, Q.M., Liu, R., Xu, Q., Chang, G.B., Ding, J.T., and Chen, G.H. (2015). Duck RIG-I CARD Domain Induces the Chicken IFN-beta by Activating NF-kappa B. Biomed Research International 2015, 348792-348792. 
Coupeau, D., Dambrine, G., and Rasschaert, D. (2012). Kinetic expression analysis of the cluster mdv1-mir-M9-M4, genes meq and vIL-8 differs between the lytic and latent phases of Marek's disease virus infection. Journal of General Virology 93, 1519-1529.

Dai, F.P., Yusuf, F., Farjah, G.H., and Brand-Saberi, B. (2005). RNAi-induced targeted silencing of developmental control genes during chicken embryogenesis. Developmental Biology 285, 80-90.

Das, R.M., Van Hateren, N.J., Howell, G.R., Farrell, E.R., Bangs, F.K., Porteous, V.C., Manning, E.M., McGrew, M.J., Ohyama, K., Sacco, M.A., et al. (2006). A robust system for RNA interference in the chicken using a modified microRNA operon. Developmental Biology 294, 554-563.

Deng, J.H., Deng, P., Lin, S.L., and Ying, S.Y. (2015). Gene Silencing In Vitro and In Vivo Using Intronic MicroRNAs. In RNA Interference: Challenges and Therapeutic Opportunities, pp. 321-340.

Dinh, H., Hong, Y.H., and Lillehoj, H.S. (2014). Modulation of microRNAs in two genetically disparate chicken lines showing different necrotic enteritis disease susceptibility. Veterinary Immunology and Immunopathology 159, 74-82.

Flemr, M., Malik, R., Franke, V., Nejepinska, J., Sedlacek, R., Vlahovicek, K., and Svoboda, P. (2013). A retrotransposon-driven dicer isoform directs endogenous small interfering RNA production in mouse oocytes. Cell 155, 807-816.

Fukagawa, T., Nogami, M., Yoshikawa, M., Ikeno, M., Okazaki, T., Takami, Y., Nakayama, T., and Oshimura, M. (2004). Dicer is essential for formation of the heterochromatin structure in vertebrate cells. Nature Cell Biology 6, 784-791.

Giles, K.E., Ghirlando, R., and Felsenfeld, G. (2010). Maintenance of a constitutive heterochromatin domain in vertebrates by a Dicer-dependent mechanism. Nature Cell Biology 12, 94-U246.

Godnic, I., Zorc, M., Skok, D.J., Calin, G.A., Horvat, S., Dovc, P., Kovac, M., and Kunej, T. (2013). Genome-Wide and Species-Wide In Silico Screening for Intragenic MicroRNAs in Human, Mouse and Chicken. Plos One 8, e65165-e65165.

Gonzalez-Lopez, C., Martinez-Costas, J., Esteban, M., and Benavente, J. (2003). Evidence that avian reovirus sigma A protein is an inhibitor of the double-stranded RNA-dependent protein kinase. Journal of General Virology 84, 1629-1639.

Gunaratne, P.H., Lin, Y.C., Benham, A.L., Drnevich, J., Coarfa, C., Tennakoon, J.B., Creighton, C.J., Kim, J.H., Milosavljevic, A., Watson, M., et al. (2011). Song exposure regulates known and novel microRNAs in the zebra finch auditory forebrain. BMC Genomics 12, 277-277.

Haesler, S., Rochefort, C., Georgi, B., Licznerski, P., Osten, P., and Scharff, C. (2007). Incomplete and inaccurate vocal imitation after knockdown of FoxP2 in songbird basal ganglia nucleus area X. Plos Biology 5, 2885-2897.

Han, B., Lian, L., Li, X., Zhao, C., Qu, L., Liu, C., Song, J., and Yang, N. (2016). Chicken ggamiR-103-3p Targets CCNE1 and TFDP2 and Inhibits MDCC-MSB1 Cell Migration. G3-Genes Genomes Genetics.

Hayashi, T., Watanabe, C., Suzuki, Y., Tanikawa, T., Uchida, Y., and Saito, T. (2014). Chicken MDA5 Senses Short Double-Stranded RNA with Implications for Antiviral Response against Avian Influenza Viruses in Chicken. Journal of Innate Immunity 6, 58-71.

Herbert, A., Lowenhaupt, K., Spitzner, J., and Rich, A. (1995). Double-stranded RNA adenosine deaminase binds Z-DNA in vitro. Nucleic Acids Symp Ser, 16-19. 
Hu, S.Q., Cao, W., Yang, M.J., Liu, H.H., Li, L., and Wang, J.W. (2014). Molecular characterization, tissue distribution, and expression of two ovarian Dicer isoforms during follicle development in goose (Anser cygnoides). Biology 170, 33-41.

Hutcheson, J.M., Susta, L., Stice, S.L., Afonso, C.L., and West, F.D. (2015). Delayed Newcastle disease virus replication using RNA interference to target the nucleoprotein. Biologicals 43, 274-280.

International Chicken Genome Sequencing, C. (2004). Sequence and comparative analysis of the chicken genome provide unique perspectives on vertebrate evolution. Nature 432, 695-716.

Karpala, A.J., Lowenthal, J.W., and Bean, A.G. (2008). Activation of the TLR3 pathway regulates IFN beta production in chickens. Developmental and Comparative Immunology 32, 435-444.

Karpala, A.J., Stewart, C., McKay, J., Lowenthal, J.W., and Bean, A.G. (2011). Characterization of chicken Mda5 activity: regulation of IFN-beta in the absence of RIG-I functionality. Journal of immunology 186, 5397-5405.

Kim, T.H., Yun, T.W., Rengaraj, D., Lee, S.I., Lim, S.M., Seo, H.W., Park, T.S., and Han, J.Y. (2012). Conserved functional characteristics of the PIWI family members in chicken germ cell lineage. Theriogenology 78, 1948-1959.

Kint, J., Fernandez-Gutierrez, M., Maier, H.J., Britton, P., Langereis, M.A., Koumans, J., Wiegertjes, G.F., and Forlenza, M. (2015). Activation of the chicken type I interferon response by infectious bronchitis coronavirus. Journal of Virology 89, 1156-1167.

Kozomara, A., and Griffiths-Jones, S. (2014). miRBase: annotating high confidence microRNAs using deep sequencing data. Nucleic Acids Res 42, D68-73.

Lambeth, L.S., Yao, Y.X., Smith, L.P., Zhao, Y.G., and Nair, V. (2009a). MicroRNAs 221 and 222 target p27(Kip1) in Marek's disease virus-transformed tumour cell line MSB-1. Journal of General Virology 90, 1164-1171.

Lambeth, L.S., Zhao, Y.G., Smith, L.P., Kgosana, L., and Nair, V. (2009b). Targeting Marek’s disease virus by RNA interference delivered from a herpesvirus vaccine. Vaccine 27, 298-306.

Lee, C.C., Wu, C.C., and Lin, T.L. (2012). Characterization of chicken melanoma differentiation-associated gene 5 (MDA5) from alternative translation initiation. Comparative Immunology Microbiology and Infectious Diseases 35, 335-343.

Lee, C.C., Wu, C.C., and Lin, T.L. (2014). Chicken melanoma differentiation-associated gene 5 (MDA5) recognizes infectious bursal disease virus infection and triggers MDA5-related innate immunity. Archives of Virology 159, 1671-1686.

Lee, S.H., Eldi, P., Cho, S.Y., and Rangasamy, D. (2009). Control of chicken CR1 retrotransposons is independent of Dicer-mediated RNA interference pathway. BMC Biology 7, 53-53.

Lee, S.I., Ji, M.R., Jang, Y.J., Jeon, M.H., Kim, J.S., Park, J.K., Jeon, I.S., and Byun, S.J. (2015). Characterization and miRNA-mediated posttranscriptional regulation of vitelline membrane outer layer protein I in the adult chicken oviduct. In Vitro Cellular \& Developmental Biology-Animal $51,222-229$.

Lee, S.I., Lee, B.R., Hwang, Y.S., Lee, H.C., Rengaraj, D., Song, G., Park, T.S., and Han, J.Y. (2011). MicroRNA-mediated posttranscriptional regulation is required for maintaining undifferentiated properties of blastoderm and primordial germ cells in chickens. Proc Natl Acad Sci U S A 108, 10426-10431.

Li, H., Sun, G.R., Tian, Y.D., Han, R.L., Li, G.X., and Kang, X.T. (2013). MicroRNAs-1614-3p gene seed region polymorphisms and association analysis with chicken production traits. Journal of Applied Genetics 54, 209-213. 
Li, W.Z., Chen, H.J., Sutton, T., Obadan, A., and Perez, D.R. (2014a). Interactions between the Influenza A Virus RNA Polymerase Components and Retinoic Acid-Inducible Gene I. Journal of Virology $88,10432-10447$.

Li, X., Lian, L., Zhang, D.X., Qu, L.J., and Yang, N. (2014b). gga-miR-26a targets NEK6 and suppresses Marek's disease lymphoma cell proliferation. Poultry Science 93, 1097-1105.

Li, Z.J., Zhang, Y.P., Li, Y., Zheng, H.W., Zheng, Y.S., and Liu, C.J. (2014c). Distinct expression pattern of miRNAs in Marek's disease virus infected-chicken splenic tumors and non-tumorous spleen tissues. Research in Veterinary Science 97, 156-161.

Lian, L., Li, X., Zhao, C.F., Han, B., Qu, L.J., Song, J.Z., Liu, C.J., and Yang, N. (2015a). Chicken gga-miR-181a targets MYBL1 and shows an inhibitory effect on proliferation of Marek's disease virus-transformed lymphoid cell line. Poultry Science 94, 2616-2621.

Lian, L., Zhang, D.X., Wang, Q., Yang, N., and Qu, L.J. (2015b). The inhibitory effects of ggamiR-199-3p, gga-miR-140-3p, and gga-miR-221-5p in Marek's disease tumorigenesis. Poultry Science 94, 2131-2135.

Lim, S.L., Tsend-Ayush, E., Kortschak, R.D., Jacob, R., Ricciardelli, C., Oehler, M.K., and Grutzner, F. (2013). Conservation and Expression of PIWI-Interacting RNA Pathway Genes in Male and Female Adult Gonad of Amniotes. Biology of Reproduction 89, 136-136.

Lin, S.-L., Chang, D.C., and Ying, S.-Y. (2006a). Isolation and identification of gene-specific microRNAs. In Methods in Molecular Biology, pp. 313-320.

Lin, S.-L., Chang, S.-J.E., and Ying, S.-Y. (2006b). Transgene-like animal models using intronic microRNAs. In Methods in Molecular Biology, pp. 321-334.

Lin, S.-L., and Ying, S.-Y. (2006). Gene silencing in vitro and in vivo using intronic microRNAs. In Methods in Molecular Biology, pp. 295-312.

Lin, S.L., Chang, D.C., and Ying, S.Y. (2013a). Isolation and identification of gene-specific microRNAs. Methods in Molecular Biology 936, 271-278.

Lin, S.L., Chang, S.J., and Ying, S.Y. (2013b). Transgene-like animal models using intronic microRNAs. Methods in Molecular Biology 936, 279-294.

Lin, S.L., and Ying, S.Y. (2013). Gene silencing in vitro and in vivo using intronic microRNAs. Methods in Molecular Biology 936, 209-229.

Lostale-Seijo, I., Martinez-Costas, J., and Benavente, J. (2016). Interferon induction by avian reovirus. Virology 487, 104-111.

Luo, G.Z., Hafner, M., Shi, Z., Brown, M., Feng, G.H., Tuschl, T., Wang, X.J., and Li, X. (2012). Genome-wide annotation and analysis of zebra finch microRNA repertoire reveal sex-biased expression. BMC Genomics 13, 727.

Luo, J., Sun, A.J., Teng, M., Zhou, H., Cui, Z.Z., Qu, L.H., and Zhang, G.P. (2011). Expression profiles of microRNAs encoded by the oncogenic Marek's disease virus reveal two distinct expression patterns in vivo during different phases of disease. Journal of General Virology 92, 608-620.

Margulis, L., and Schwartz, K.V. (1998). Five kingdoms : an illustrated guide to the phyla of life on earth, 3rd edn (New York: W.H. Freeman).

Mauti, O., Baeriswyl, T., and Stoeckli, E.T. (2008). Gene Silencing by Injection and Electroporation of dsRNA in Avian Embryos. Cold Spring Harbor Protocols 2008, pdb.prot5094-pdb.prot5094.

Morgan, R., Anderson, A., Bernberg, E., Kamboj, S., Huang, E., Lagasse, G., Isaacs, G., Parcells, M., Meyers, B.C., Green, P.J., et al. (2008). Sequence Conservation and Differential Expression of Marek's Disease Virus MicroRNAs. Journal of Virology 82, 12213-12220. 
Muylkens, B., Coupeau, D., Dambrine, G., Trapp, S., and Rasschaert, D. (2010). Marek's disease virus microRNA designated Mdv1-pre-miR-M4 targets both cellular and viral genes. Archives of Virology 155, 1823-1837.

O’Neill, G. (2007). Australia tackles bird flu using RNAi. Nature Biotechnology 25, 605-606.

Pekarik, V., Bourikas, D., Miglino, N., Joset, P., Preiswerk, S., and Stoeckli, E.T. (2003). Screening for gene function in chicken embryo using RNAi and electroporation. Nature Biotechnology 21, 93-96.

Sahare, A.A., Bedekar, M.K., Jain, S.K., Singh, A., Singh, S., and Sarkhel, B.C. (2015). Inhibition of Infectious Bursal Disease Virus by Vector Delivered SiRNA in Cell Culture. Animal Biotechnology 26, 58-64.

Sato, F., Nakagawa, T., Ito, M., Kitagawa, Y., and Hattori, M.A. (2004). Application of RNA interference to chicken embryos using small interferfing RNA. Journal of Experimental Zoology Part a-Comparative Experimental Biology 301A, 820-827.

Sato, H., Oshiumi, H., Takaki, H., Hikono, H., and Seya, T. (2015). Evolution of the DEAD box helicase family in chicken: Chickens have no DHX9 ortholog. Microbiology and Immunology 59, 633-640.

Stewart, C.R., Karpala, A.J., Lowther, S., Lowenthal, J.W., and Bean, A.G. (2011). Immunostimulatory Motifs Enhance Antiviral siRNAs Targeting Highly Pathogenic Avian Influenza H5N1. Plos One 6, e21552-e21552.

Stik, G., Dambrine, G., Pfeffer, S., and Rasschaert, D. (2013). The Oncogenic MicroRNA OncomiR-21 Overexpressed during Marek's Disease Lymphomagenesis Is Transactivated by the Viral Oncoprotein Meq. Journal of Virology 87, 80-93.

Strassheim, S., Stik, G., Rasschaert, D., and Laurent, S. (2012). mdv1-miR-M7-5p, located in the newly identified first intron of the latency-associated transcript of Marek's disease virus, targets the immediate-early genes ICP4 and ICP27. Journal of General Virology 93, 1731-1742.

Svoboda, P. (2014). Renaissance of mammalian endogenous RNAi. FEBS Lett 588, 2550-2556.

Svoboda, P. (2019a). Introduction to RNAi and miRNA pathways.

Svoboda, P. (2019b). RNAi and miRNA pathways in mammals I-molecular mechanisms.

Taft, R.J., Glazov, E.A., Lassmann, T., Hayashizaki, Y., Carninci, P., and Mattick, J.S. (2009). Small RNAs derived from snoRNAs. RNA 15, 1233-1240.

Tian, F., Luo, J., Zhang, H.M., Chang, S., and Song, J.Z. (2012). MiRNA expression signatures induced by Marek's disease virus infection in chickens. Genomics 99, 152-159.

Tian, Y., Lu, L.Z., Fu, Y., Zhao, J., Zhang, C., Yuan, Q.Y., and Shen, J.D. (2007). Assignment of Dicer gene to chicken chromosome 5 by radiation hybrid panel mapping. Biochemical Genetics $45,239-243$.

Villanueva, A.I., Kulkarni, R.R., and Sharif, S. (2011). Synthetic double-stranded RNA oligonucleotides are immunostimulatory for chicken spleen cells. Developmental and Comparative Immunology $35,28-34$.

Warren, W.C., Clayton, D.F., Ellegren, H., Arnold, A.P., Hillier, L.W., Kunstner, A., Searle, S., White, S., Vilella, A.J., Fairley, S., et al. (2010). The genome of a songbird. Nature 464, 757-762.

Wei, R.R., Ma, X.Q., Wang, G.H., Guo, H.J., Liu, J.Z., Fan, L.X., and Cheng, Z.Q. (2015). Synergistic inhibition of avian leukosis virus subgroup J replication by miRNA-embedded siRNA interference of double-target. Virology Journal 12, 45-45. 
Wilson, N.H., and Stoeckli, E.T. (2011). Cell type specific, traceable gene silencing for functional gene analysis during vertebrate neural development. Nucleic Acids Research 39, e133-e133.

Wilson, N.H., and Stoeckli, E.T. (2012). In ovo Electroporation of miRNA-based Plasmids in the Developing Neural Tube and Assessment of Phenotypes by DiI Injection in Open-book Preparations. Jove-Journal of Visualized Experiments.

Xu, H.T., Yao, Y.X., Smith, L.P., and Nair, V. (2010). MicroRNA-26a-mediated regulation of interleukin-2 expression in transformed avian lymphocyte lines. Cancer Cell International 10, 15-15.

Xu, S., Xue, C.Y., Li, J.P., Bi, Y.Z., and Cao, Y.C. (2011). Marek's Disease Virus Type 1 MicroRNA miR-M3 Suppresses Cisplatin-Induced Apoptosis by Targeting SMAD2 of the Transforming Growth Factor Beta Signal Pathway. Journal of Virology 85, 276-285.

Xu, W.P., Shao, Q., Zang, Y.L., Guo, Q., Zhang, Y.C., and Li, Z.D. (2015). Pigeon RIG-I Function in Innate Immunity against H9N2 IAV and IBDV. Viruses-Basel 7, 4131-4151.

Yao, Y.X., Zhao, Y.G., Xu, H.T., Smith, L.P., Lawrie, C.H., Watson, M., and Nair, V. (2008). MicroRNA profile of Marek's disease virus-transformed T-cell line MSB-1: Predominance of virus-encoded microRNAs. Journal of Virology 82, 4007-4015.

Yin, R.F., Ding, Z.A., Liu, X.X., Mu, L.Z., Cong, Y.L., and Stoeger, T. (2010). Inhibition of Newcastle disease virus replication by RNA interference targeting the matrix protein gene in chicken embryo fibroblasts. Journal of Virological Methods 167, 107-111.

Ying, S.-Y., and Lin, S.-L. (2009). Intron-Mediated RNA Interference and microRNA Biogenesis. In Methods in Molecular Biology, pp. 387-413.

Ying, S.Y., Chang, C.P., and Lin, S.L. (2010). Intron-Mediated RNA Interference, Intronic MicroRNAs, and Applications. In RNA Therapeutics: Function, Design, and Delivery, pp. 203-235.

Zhang, S.L., Sun, Y.J., Chen, H.J., Dai, Y.B., Zhan, Y., Yu, S.Q., Qiu, X.S., Tan, L., Song, C.P., and Ding, C. (2014). Activation of the PKR/eIF2 alpha signaling cascade inhibits replication of Newcastle disease virus. Virology Journal 11, 62-62.

Zhao, Y.G., Xu, H.T., Yao, Y.X., Smith, L.P., Kgosana, L., Green, J., Petherbridge, L., Baigent, S.J., and Nair, V. (2011). Critical Role of the Virus-Encoded MicroRNA-155 Ortholog in the Induction of Marek's Disease Lymphomas. Plos Pathogens 7, e1001305-e1001305.

Zhao, Y.G., Yao, Y.X., Xu, H.T., Lambeth, L., Smith, L.P., Kgosana, L., Wang, X.W., and Nair, V. (2009). A Functional MicroRNA-155 Ortholog Encoded by the Oncogenic Marek's Disease Virus. Journal of Virology 83, 489-492.

Zhou, X., Guo, H., Chen, K., Cheng, H.H., and Zhou, R.J. (2010). Identification, chromosomal mapping and conserved synteny of porcine Argonaute family of genes. Genetica 138, 805-812. 



\title{
RNAi AND miRNA PATHWAYS IN FISH
}

Keywords: dsRNA, siRNA, miRNA, Dicer, TARBP2, PACT, Argonaute

\section{PETR SVOBODA}

Institute of Molecular Genetics, Academy of Sciences of the Czech Republic, Videnska 1083, 14220 Prague 4, Czech Republic

Correspondence to: Petr Svoboda, Institute of Molecular Genetics ASCR, Videnska 1083, 14220 Prague 4, Czech Republic, tel. \#+420 241063147, e-mail: svobodap@img.cas.cz.

\begin{abstract}
RNA silencing denotes sequence-specific repression mediated by small RNAs. In vertebrates, there are two closely related pathways, which share several protein factors: RNA interference (RNAi) and microRNA (miRNA) pathway. The miRNA pathway regulates endogenous protein-coding gene expression and has been implicated in many biological processes. RNAi generally serves as a form of innate immunity targeting viruses and mobile elements. This text reviews miRNA and RNAi pathways in fish. RNAi and other dsRNA-responding pathways in fish are very much like those in mammals, which is remarkable considering the mammalian and fish lineages separated in Paleozoicum some 400 million years ago. The miRNA pathway is likely the dominant small RNA pathway while, similarly to other vertebrates, the existence and functionality of endogenous RNAi remains unclear.
\end{abstract}

\section{Introduction}

Fish are an aquatic heterogeneous paraphyletic group with the majority of the species belonging to the bony fish class (Osteichthyes) group, which has 25000 species, the highest species diversity than any other vertebrate group (Margulis and Schwartz, 1998). Overall, the organization of small RNA pathways in fish is very similar to that of mammals (Fig. 1), which is notable considering the common ancestor of fish and mammals existed 3602-4500 million years ago (Volff, 2005).

Since mechanistical principles of vertebrate miRNA and RNAi pathways were introduced in the first two reviews of this series (Svoboda, 2019a, b) and in further detail elsewhere (Bartel, 2018; Svoboda, 2014), I will focus here directly on features of these pathways reported from fish models. Next generation sequencing analysis of zebrafish small RNAs identified miRNAs and germline piRNAs as common small RNAs. The dominant small RNA pathway in fish is the miRNA pathway. Studies of roles of miRNAs account for the vast majority of the literature on small RNA in fish. At the same time, the molecular 
mechanism of miRNA and RNAi pathways was seldom drectly studied in the fish model. The second most studied studied small RNA pathway in fish is the piRNA pathway, which protects the germline from mobile elements (Houwing et al., 2007; Huang et al., 2011; Kamminga et al., 2010) and differs from miRNA and RNAi pathways in small RNA biogenesis, which does not require Dicer.

\section{Dicer}

Fish genomes carry a single gene for Dicer, which is an ortholog of the mammalian Dicer and Dicer-1 in Drosophila (Murphy et al., 2008). This notion is supported by annotated fish genome data in the UCSC genome browser (https://genome.ucsc.edu/), and tblastn search of fish sequences at NCBI (e.g. Salmo salar, Danio rerio, Takifugu rubripes, Gasterosteus aculeatus (stickleback), Oryzias latipes (medaka)). Existence of a single Dicer gene in fish genomes is remarkable in teleost fish species, which underwent genome duplication (Howe et al., 2013; Meyer and Schartl, 1999). This suggests selective pressure could exist against Dicer gene duplication. Zebrafish Dicer is essential for development and its primary role seems to be miRNA biogenesis (Wienholds et al., 2003). The role of Dicer in endogenous RNAi in fish has not been addressed in much detail. A study of Dicer in grass carp Ctenopharyngodon idellar revealed a CDS encoding Dicer protein carrying all known functional domains found typically in other Dicers (Shen et al., 2013). Ctenopharyngodon idellar Dicer is abundantly expressed in brain, gill, head kidney, liver, spleen, heart, muscle and intestine. A positive correlation was found between Ctenopharyngodon idellar Dicer mRNA expression and infection with grass carp reovirus (GCRV) infection in cultured kidney cells and in the liver (8.46-fold, $\mathrm{P}<0.01,12 \mathrm{~h}$ post-infection) and spleen in vivo (Shen et al., 2013).This suggests that Ctenopharyngodon idellar Dicer is an inducible gene responding to viral infection although evidence for virus-derived endogenous siRNAs has not been provided.

\section{dsRBPs}

Fish genomes typically contain orthologs of TRBP2 and PACT, which are mammalian Dicer-interacting dsRBPs (Murphy et al., 2008). However, their function in small RNA silencing was not studied in the fish model, so far.

\section{Argonaute proteins}

Fish AGO proteins are orthologs of AGO proteins in other vertebrates (Murphy et al., 2008). However, teleost fish clade contains an additional AGO paralog, which emerged from a fish-specific genome duplication event that occurred approximately 350 million years ago (McFarlane et al., 2011). All five Ago genomic loci in teleosts contain specific, conserved sequence elements in non-coding regions indicating that the teleost AGO 
paralogs are differentially regulated, which is consistent with expression analysis in the zebrafish model. Multiple sequence alignments show that teleost homologs possess critical aminoacid residues for AGO function as well as ortholog-specific features retained throughout the vertebrate lineage (McFarlane et al., 2011).

\section{miRNA pathway}

In terms of small RNA research, fish models are typically used for exploring miRNA populations and studying biological roles of different miRNAs. Articles annotating miRNAs and analyzing their expression and function represent the bulk of the fish-related references. We have found 348 articles, which annotated and/or analyzed expression and function of miRNAs in fish. However, almost none of these articles brought any specific mechanistic insight into the molecular mechanism of miRNA in fish.

According to the miRBase (release 22.1), miRNA population in fish appears less complex than in mammals - fish models have less annotated miRNAs (Table 1) although there are dozens of reports on next generation sequencing analysis and miRNA annotation. However, zebrafish is an experimentally easily accessible model for exploring conserved roles of miRNAs in different tissues, which is also reflected in the number of references.

Table 1 Numbers of annotated miRNAs in selected fishes in miRBase 22.1

\begin{tabular}{|l|c|c|}
\hline species & miRNA precursors & mature miRNA \\
\hline Cyprinus carpio & 134 & 146 \\
\hline Danio rerio & 355 & 373 \\
\hline Fugu rubripes & 131 & 108 \\
\hline Ictalurus punctatus & 281 & 205 \\
\hline Oryzias latipes & 168 & 146 \\
\hline Salmo salar & 371 & 498 \\
\hline Tetraodon nigroviridis & 132 & 109 \\
\hline
\end{tabular}

In terms of the molecular mechanism of RNA silencing, studies in the zebrafish and other fish models brought several interesting discoveries concerning specific miRNA functions and unique adaptations in fish. Several examples have relevance for the research of the molecular mechanism of miRNA pathway:

The first example is the biology of the miR-430 family of miRNAs. While miRNAs in mice are essentially irrelevant for the oocyte-to-embryo transition (Suh et al., 2010), the zebrafish oocyte-to-embryo transition incorporates zygotically-expressed miR-430 family in maternal mRNA degradation (Giraldez et al., 2006; Mishima et al., 2006). Furthermore, the onset of miR-430 activity in the zebrafish zygote allowed for addressing the relationship between miRNA-induced translational repression and mRNA degradation (Bazzini et al., 2012; Mishima et al., 2012). Using ribosome profiling of zygotic stages, it was showed that miR-430 reduces translation before causing mRNA decay (Bazzini et al., 
2012). A significant technological outcome of these studies was development of target protectors, morpholino oligonucleotides specifically disrupting miRNA-mediated repression via hybridizing to and masking miRNA-binding sites (Choi et al., 2007).

Another contribution to small RNA biology coming from fish model was discovery of one of the non-canonical miRNA biogenesis mechanisms, namely Dicer-independent miRNA biogenesis of miR-451, which uses AGO2 slicing activity followed by uridylation and trimming (Cifuentes et al., 2010). The process also employs translation initiation factor eIF1A, which directly interacts with AGO2 and promotes miR-451 biogenesis (Yi et al., 2015).

Data from zebrafish also contributed to understanding other miRNA regulations. Two related terminal uridyl transferases (TUTases), Zcchc6 (TUT7) and Zcchc11 (TUT4), selectively 3 ' monouridylate a subset of miRNAs (Thornton et al., 2014). TUTase inhibition in zebrafish embryos causes developmental defects and aberrant Hox gene expression (Thornton et al., 2014).

Another miRNA regulator discovered in the zebrafish is dead end 1 (DND1), which is negatively regulating miRNA targeting. DND1 is an evolutionary conserved RNA-binding protein (RBP) that counteracts the function of several miRNAs in zebrafish primordial germ cells as well as in human cells. DND1 binds mRNAs and prohibits miRNAs from binding cognate mRNAs. DND1 effects involve uridine-rich regions present in the miRNA-targeted mRNAs (Kedde et al., 2007)

Taken together studies of molecular mechanism of miRNA-mediated repression in fish did not reveal any notable deviation from what has been observed in mammals. As the protein machinery appears to be well-conserved, miRNA pathways among vertebrate taxons primarily differ in sets of miRNAs and their targets, which dynamically evolve over time. This was for example demonstrated for zebrafish miR-430 and murine miR-290-295 miRNA clusters, which share common ancestry, both are associated with early development but do not regulate the same genes although some targets seem to be conserved (Svoboda and Flemr, 2010).

\section{RNAi}

The presence of RNAi response was examined in zebrafish at the same as in other animal models during the turn of the century. However, unlike specific RNAi observed in mouse oocytes and early embryos (Svoboda et al., 2000; Wianny and Zernicka-Goetz, 2000), long dsRNA injection into zebrafish had been yielding inconsistent results (Li et al., 2000; Mangos et al., 2001; Oates et al., 2000; Zhao et al., 2001). While some reported specific knockdown effects (Li et al., 2000; Mangos et al., 2001), others observed non-specific effects (Oates et al., 2000; Zhao et al., 2001). Non-specific effects remained a recurring theme also in later studies (Wang et al., 2010; Zhao et al., 2008) although some authors were able to achieve specific RNAi effects (De Rienzo et al., 2012; Dong et al., 2013; Ying et al., 2010).Non-specific effects in zebrafish embryos were not remedied by the use of siRNAs and it was later shown that the basis of the non-specific effects is interference with miRNA function (Zhao et al., 2008). Injection of zebrafish zygotes with siRNA caused a significant reduction in miR-430 levels leading to unspecific developmental defects (Zhao et al., 2008). 
Interestingly, literature survey revealed that experiments with the parasitic sea lamprey (Petromyzon marinus), a jawless fish relative, showed that uptake of free siRNA at $5 \mu \mathrm{g} / \mathrm{ml}$ did not trigger an RNAi response (Heath et al., 2014). In any case, RNAi did not become a favourite knock-down strategy to study genes during zebrafish development; microinjection of morpholino oligonucleotides (Blum et al., 2015; Eisen and Smith, 2008) became the preferred approach instead.

Taken together, successful RNAi experiments with long dsRNA demonstrate that zebrafish holds the molecular machinery for executing RNAi: Dicer, TARBP2, and AGO2. However, its capacity for mediating specific knock-down effects is limited because the same machinery is being simultaneously utilized by the miRNA pathway. Importantly, the availability of the machinery above the minimum capacity sustaining the miRNA pathway functionality likely differs during development and among different cell types.

While zebrafish holds the molecular machinery for executing RNAi, the question remains whether the endogenous RNAi has any significant role in fish. Available data do not provide unequivocal evidence for significant endogenous RNAi in fish. Next generation sequencing of small RNAs contains fractions of non-miRNA small RNAs of endo-siRNA size, yet it is not clear if these fragments truly represent bona fide endo-siRNAs. Some other data indirectly point to a possible antiviral role, namely GCRV-induced transient upregulation of Ago2 in rare minnow (Gobiocypris rarus) and Dicer upregulation in grass carp (Ctenopharyngodon idella) (Guo et al., 2012; Shen et al., 2013). Interaction of GCRV with the small RNA machinery (the miRNA pathway should not be excluded) has been suggested based on the observation in grass carp kidney cells that GCRV dsRNA could be processed into siRNAs but GCRV infection did not yield GCRV-derived siRNAs while Dicer upregulation occurred (Gotesman et al., 2014). It has been thus proposed that an unidentified RNAi suppressor might contribute to the survival of the viral genome and efficient viral replication (Gotesman et al., 2014). The presence of a virus-derived inhibitor of RNA silencing in a fish RNA virus would be indicative of an existing antiviral role of small RNAs, which is being suppressed. However, an alternative scenario that should be considered as well is that dsRNA formed during viral replication is not accessible for Dicer-mediated cleavage. Therefore, further research is needed to address this issue.

\section{Other notable silencing phenomena}

There are two phenomena, which clearly overlap with RNA silencing but their underlying molecular mechanism remains unclear and will require further investigation.

Andrews et al found that introduction of transgenes containing convergent transcription units in zebrafish embryos induced stable transcriptional gene silencing in cis and trans. The silencing was suppressed upon Dicer knockdown, indicating processing of double stranded RNA. ChIP revealed that silencing was accompanied by enrichment of the constitutive heterochromatin mark H3K9me3 (Andrews et al., 2014). While small RNA-induced transcriptional silencing is well established in fungi and plants (and seems to be a product of convergent evolution), the molecular mechanism underlying seemingly related observations in vertebrates is unclear. 
The second phenomenon deals with the role of small RNAs during DNA-damage response, which has been discussed above in the mammalian section (Francia et al., 2012). It was shown in zebrafish that DICER and DROSHA, but not downstream elements of the RNAi pathway, are necessary to activate the DDR upon exogenous DNA damage and oncogene-induced genotoxic stress, as studied by DDR foci formation and by checkpoint assays. It was also reported that formation of DDR foci requires site-specific DICER- and DROSHA-dependent small RNAs, which are sufficient to restore the DDR in RNase-Atreated cells (Francia et al., 2012).

\section{Summary}

The molecular mechanism of RNAi and miRNA pathways in fish seems to be essentially identical to that of mammals (Fig. 1). While there are two dsRBPs, the role of PACT in RNA silencing remains to be determined. miRNA pathway is the dominant small RNA pathway while the existence and functionality of endogenous RNAi are unclear. Some variations (DNA-binding PKR homologs (Rothenburg et al., 2008)) were found in the interferon system, which is the main fish antiviral system.
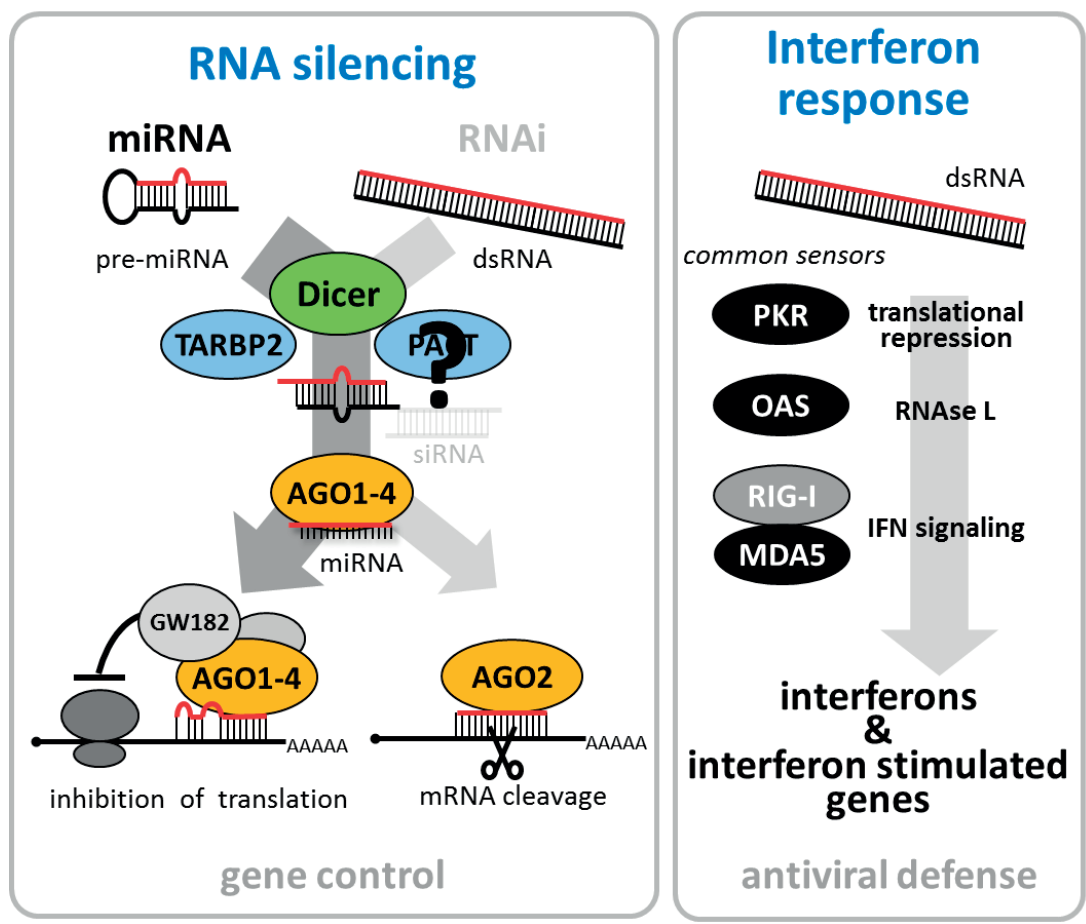

Figure 1 Overview of RNA silencing and dsRNA response in FISH dsRNA and miRNA pathways in bony fishes are very much similar to the mammalian ones. 


\section{Acknowledgement}

I would like to thank my colleagues Jan Paces, Miloslav Nic, and Tomas Novotny for help with collecting literature for the review. The review content was produced under a contract OC/EFSA/GMO/2015/01-CT 01 with European Food Safety Authority (EFSA); the opinions expressed are those of the contractor only and do not represent EFSA's official position. Publication of the review was funded by LO1220 and LM2015063 by the Ministry of Education, Youth and Sports.

\section{References}

Andrews, O.E., Cha, D.J., Wei, C.Y., and Patton, J.G. (2014). RNAi-Mediated Gene silencing in Zebrafish Triggered by Convergent Transcription. Scientific Reports 4, 5222-5222.

Bartel, D.P. (2018). Metazoan MicroRNAs. Cell 173, 20-51.

Bazzini, A.A., Lee, M.T., and Giraldez, A.J. (2012). Ribosome Profiling Shows That miR-430 Reduces Translation Before Causing mRNA Decay in Zebrafish. Science 336, 233-237.

Blum, M., De Robertis, E.M., Wallingford, J.B., and Niehrs, C. (2015). Morpholinos: Antisense and Sensibility. Developmental Cell 35, 145-149.

Choi, W.Y., Giraldez, A.J., and Schier, A.F. (2007). Target protectors reveal dampening and balancing of nodal agonist and antagonist by miR-430. Science 318, 271-274.

Cifuentes, D., Xue, H., Taylor, D.W., Patnode, H., Mishima, Y., Cheloufi, S., Ma, E., Mane, S., Hannon, G.J., Lawson, N.D., et al. (2010). A novel miRNA processing pathway independent of dicer requires argonaute2 catalytic activity. Science 328, 1694-1698.

De Rienzo, G., Gutzman, J.H., and Sive, H. (2012). Efficient shRNA-Mediated Inhibition of Gene Expression in Zebrafish. Zebrafish 9, 97-107.

Dong, Z.Q., Peng, J.S., and Guo, S. (2013). Stable Gene Silencing in Zebrafish with Spatiotemporally Targetable RNA Interference. Genetics 193, 1065-+.

Eisen, J.S., and Smith, J.C. (2008). Controlling morpholino experiments: don’t stop making antisense. Development 135, 1735-1743.

Francia, S., Michelini, F., Saxena, A., Tang, D., de Hoon, M., Anelli, V., Mione, M., Carninci, P., and d'Adda di Fagagna, F. (2012). Site-specific DICER and DROSHA RNA products control the DNA-damage response. Nature 488, 231-235.

Giraldez, A.J., Mishima, Y., Rihel, J., Grocock, R.J., Van Dongen, S., Inoue, K., Enright, A.J., and Schier, A.F. (2006). Zebrafish MiR-430 promotes deadenylation and clearance of maternal mRNAs. Science 312, 75-79.

Gotesman, M., Soliman, H., Besch, R., and El-Matbouli, M. (2014). In vitro inhibition of Cyprinid herpesvirus-3 replication by RNAi. J Virol Methods 206, 63-66.

Guo, S., Xu, D., Xu, H.-X., Wang, T., Li, J.-1., and Lu, L.-q. (2012). Suppression of RNA interference pathway in vitro by Grass carp reovirus. Virologica Sinica 27, 109-119.

Heath, G., Childs, D., Docker, M.F., McCauley, D.W., and Whyard, S. (2014). RNA Interference Technology to Control Pest Sea Lampreys - A Proof-of-Concept. Plos One 9, e88387-e88387. 
Houwing, S., Kamminga, L.M., Berezikov, E., Cronembold, D., Girard, A., van den Elst, H., Filippov, D.V., Blaser, H., Raz, E., Moens, C.B., et al. (2007). A role for Piwi and piRNAs in germ cell maintenance and transposon silencing in zebrafish. Cell 129, 69-82.

Howe, K., Clark, M.D., Torroja, C.F., Torrance, J., Berthelot, C., Muffato, M., Collins, J.E., Humphray, S., McLaren, K., Matthews, L., et al. (2013). The zebrafish reference genome sequence and its relationship to the human genome. Nature 496, 498-503.

Huang, H.Y., Houwing, S., Kaaij, L.J.T., Meppelink, A., Redl, S., Gauci, S., Vos, H., Draper, B.W., Moens, C.B., Burgering, B.M., et al. (2011). Tdrd1 acts as a molecular scaffold for Piwi proteins and piRNA targets in zebrafish. EMBO J 30, 3298-3308.

Kamminga, L.M., Luteijn, M.J., den Broeder, M.J., Redl, S., Kaaij, L.J.T., Roovers, E.F., Ladurner, P., Berezikov, E., and Ketting, R.F. (2010). Hen1 is required for oocyte development and piRNA stability in zebrafish. EMBO J 29, 3688-3700.

Kedde, M., Strasser, M.J., Boldajipour, B., Vrielink, J., Le Sage, C., Nagel, R., Voorhoeve, P.M., Van Duijse, J., Orom, U.A., Lund, A.H., et al. (2007). RNA-binding protein Dnd1 inhibits microRNA access to target mRNA. Cell 131, 1273-1286.

Li, Y.X., Farrell, M.J., Liu, R.P., Mohanty, N., and Kirby, M.L. (2000). Double-stranded RNA injection produces null phenotypes in zebrafish. Developmental Biology 217, 394-405.

Mangos, S., Vanderbeld, B., Krawetz, R., Sudol, K., and Kelly, G.M. (2001). Ran binding protein RanBP1 in zebrafish embryonic development. Molecular Reproduction and Development 59, 235-248.

Margulis, L., and Schwartz, K.V. (1998). Five kingdoms : an illustrated guide to the phyla of life on earth, 3rd edn (New York: W.H. Freeman).

McFarlane, L., Svingen, T., Braasch, I., Koopman, P., Schartl, M., and Wilhelm, D. (2011). Expansion of the Ago gene family in the teleost clade. Development Genes and Evolution 221, 95-104.

Meyer, A., and Schartl, M. (1999). Gene and genome duplications in vertebrates: the one-tofour (-to-eight in fish) rule and the evolution of novel gene functions. Curr Opin Cell Biol 11, 699-704.

Mishima, Y., Fukao, A., Kishimoto, T., Sakamoto, H., Fujiwara, T., and Inoue, K. (2012). Translational inhibition by deadenylation-independent mechanisms is central to microRNA-mediated silencing in zebrafish. Proc Natl Acad Sci U S A 109, 1104-1109.

Mishima, Y., Giraldez, A.J., Takeda, Y., Fujiwara, T., Sakamoto, H., Schier, A.F., and Inoue, K. (2006). Differential regulation of germline mRNAs in soma and germ cells by zebrafish miR-430. Current Biology 16, 2135-2142.

Murphy, D., Dancis, B., and Brown, J.R. (2008). The evolution of core proteins involved in microRNA biogenesis. BMC Evolutionary Biology 8, 92-92.

Oates, A.C., Bruce, A.E.E., and Ho, R.K. (2000). Too much interference: Injection of double-stranded RNA has nonspecific effects in the zebrafish embryo. Developmental Biology 224, 20-28.

Rothenburg, S., Deigendesch, N., Dey, M., Dever, T.E., and Tazi, L. (2008). Double-stranded RNA-activated protein kinase PKR of fishes and amphibians: Varying the number of double-stranded RNA binding domains and lineage-specific duplications. BMC Biology 6, 12-12.

Shen, X.B., Xu, D., Li, J.L., and Lu, L.Q. (2013). Molecular cloning and immune responsive expression of a ribonuclease III orthologue involved in RNA interference, dicer, in grass carp Ctenopharyngodon idella. Journal of Fish Biology 83, 1234-1248. 
Suh, N., Baehner, L., Moltzahn, F., Melton, C., Shenoy, A., Chen, J., and Blelloch, R. (2010). MicroRNA Function Is Globally Suppressed in Mouse Oocytes and Early Embryos. Current Biology 20, 271-277.

Svoboda, P. (2014). Renaissance of mammalian endogenous RNAi. FEBS Lett 588, 2550-2556.

Svoboda, P. (2019a). Introduction to RNAi and miRNA pathways.

Svoboda, P. (2019b). RNAi and miRNA pathways in mammals I - molecular mechanisms.

Svoboda, P., and Flemr, M. (2010). The role of miRNAs and endogenous siRNAs in maternal-to-zygotic reprogramming and the establishment of pluripotency. EMBO reports 11, 590-597.

Svoboda, P., Stein, P., Hayashi, H., and Schultz, R.M. (2000). Selective reduction of dormant maternal mRNAs in mouse oocytes by RNA interference. Development 127, 4147-4156.

Thornton, J.E., Du, P., Jing, L.L., Sjekloca, L., Lin, S.B., Grossi, E., Sliz, P., Zon, L.I., and Gregory, R.I. (2014). Selective microRNA uridylation by Zcchc6 (TUT7) and Zcchc11 (TUT4). Nucleic Acids Research 42, 11777-11791.

Volff, J.N. (2005). Genome evolution and biodiversity in teleost fish. Heredity (Edinb) 94, 280-294.

Wang, L., Zhou, J.Y., Yao, J.H., Lu, D.R., Qiao, X.J., and Jia, W. (2010). U6 promoter-driven siRNA injection has nonspecific effects in zebrafish. Biochem Biophys Res Commun 391, 1363-1368.

Wianny, F., and Zernicka-Goetz, M. (2000). Specific interference with gene function by double-stranded RNA in early mouse development. Nature Cell Biology 2, 70-75.

Wienholds, E., Koudijs, M.J., van Eeden, F.J.M., Cuppen, E., and Plasterk, R.H.A. (2003). The microRNA-producing enzyme Dicer1 is essential for zebrafish development. Nature Genetics 35, $217-218$.

Yi, T.F., Arthanari, H., Akabayov, B., Song, H.D., Papadopoulos, E., Qi, H.H., Jedrychowski, M., Guttler, T., Guo, C.C., Luna, R.E., et al. (2015). eIF1A augments Ago2-mediated Dicer-independent miRNA biogenesis and RNA interference. Nature Communications 6, 7194-7194.

Ying, S.Y., Chang, C.P., and Lin, S.L. (2010). Intron-Mediated RNA Interference, Intronic MicroRNAs, and Applications. In Rna Therapeutics: Function, Design, and Delivery, pp. 203-235.

Zhao, X.F., Fjose, A., Larsen, N., Helvik, J.V., and Drivenes, O. (2008). Treatment with small interfering RNA affects the microRNA pathway and causes unspecific defects in zebrafish embryos. FEBS Journal 275, 2177-2184.

Zhao, Z.X., Cao, Y., Li, M., and Meng, A.M. (2001). Double-stranded RNA injection produces nonspecific defects in zebrafish. Developmental Biology 229, 215-223. 



\title{
RNAi AND mIRNA PATHWAYS IN ARTHROPODS
}

Keywords: dsRNA, PKR, Dicer, R2D2, LOQS, AGO1, AGO2

\author{
PETR SVOBODA \\ Institute of Molecular Genetics, Academy of Sciences of the Czech Republic, \\ Videnska 1083, 14220 Prague 4, Czech Republic \\ Correspondence to: Petr Svoboda, Institute of Molecular Genetics ASCR, \\ Videnska 1083, 14220 Prague 4, Czech Republic, tel. \# +420 241063147, \\ e-mail: svobodap@img.cas.cz.
}

\begin{abstract}
RNA silencing denotes sequence-specific repression mediated by small RNAs. In vertebrates, there are two closely related pathways, which share several protein factors: RNA interference (RNAi) and microRNA (miRNA) pathway. The miRNA pathway regulates endogenous protein-coding gene expression and has been implicated in many biological processes. RNAi generally serves as a form of innate immunity targeting viruses and mobile elements. While Arthropoda are an extremely large and diverse phylum, research on microRNA (miRNA) and RNA interference (RNAi) pathway in this phylum primarily used the Drosophila melanogaster model system and related species. Notably, both pathways are genetically separated; they utilize dedicated Dicer proteins to produce miRNAs and small interfering RNAs (siRNAs), which are sorted onto different Argonaute effector proteins. This review focuses on the miRNA pathway and pathways initiated by long dsRNA in arthropods. The first part introduces the key molecular players of RNA silencing. The second discussed biological roles of miRNA and dsRNA-induced pathways in Arthropods.
\end{abstract}

\section{Introduction}

Arthropoda are an extremely large and diverse group of animals. In fact, they are the largest animal group on Earth with a million of named species ( $~ 80 \%$ of described animal species!) and estimated tens of millions of species (Margulis and Schwartz, 1998). Arthropoda are typically classified into five subphyla (Fig. 1). (Regier et al., 2010). Trilobita are a famous extinct group of marine animals that declined in the Late Devonian extinction and completely disappeared in the Permian-Triassic extinction. Chelicerata include living fossil horseshoe crabs, spiders, mites, ticks, scorpions and related organisms. Their characteristic features are chelicerae appendages, which appear in scorpions and horseshoe crabs as claws while spiders use them to inject venom. Myriapoda have repetitive body segments carrying one or two pairs of legs and include centipedes, millipedes, and their relatives. Crustacea 


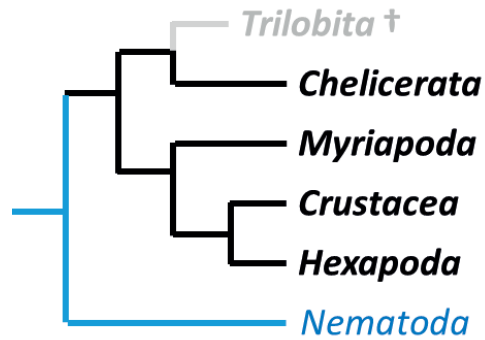

Figure 1 Simplified division of Arthropoda used in the text

The scheme reflects the Mandibulata model of arthropod phylogenetics described in (Regier et al., 2010)

are with, some exceptions (e.g. armadillo bug an relatives known as woodlice), aquatic and have differentiated segmented body and biramous appendages. They include shrimp, crayfish, lobsters, crabs, barnacles, prawns and others. Hexapoda comprise insects and insect-like animals with six thoracic legs.

The key model organism for arthropods is Drosophila, which has been a workhorse of biology for over hundred years. miRNA, RNAi and other dsRNA pathways in Drosophila are well understood and will serve as benchmarks for the entire phylum. Drosophila evolved an extensive genetic separation of miRNA and RNAi pathways where each pathway has a dedicated Dicer, dsRBP, and Argonaute protein. Given the complexity of the phylum and evolutionary time, one could question how representative of arthropods is the Drosophila model. However, analysis of Dicer and AGO indicates that Drosophila is a more-or-less acceptable model for most arthropods as the "two Dicer system" can be recognized within phylogeny of Dicer and AGOs also in Chelicerata (whose common ancestors with Drosophila branched in the most distant past), Myriapoda, and Crustacea (Palmer and Jiggins, 2015). However, it should be kept in mind that some variability could emerge during half a billion years of arthropod evolution.

Since mechanistical principles of vertebrate miRNA and RNAi pathways were introduced in the first review of this series (Svoboda, 2019) and in further detail elsewhere (Bartel, 2018), I will focus here directly on features of these pathways discovered in Arthropods. The formal structure of the report will be as in other animal taxons - upon miRNA dna RNAi molecular features of key individual components of reviewed mechanisms, I will discuss the silencing mechanisms and their biological roles. Importantly, to provide an overview of miRNA and dsRNA mechanisms in arthropods, I will focus on description of molecular mechanisms identified in Drosophila and will highlight and discuss significant deviations observed elsewhere in arthropods, especially in more studied organisms, such as mosquitos, flower beetle, silk moth, and shrimps.

\section{The Microprocessor complex}

Drosophila utilizes the same Microprocessor complex as the earlier discussed Metazoa, i.e. a complex of Drosha and DGCR8 homologs, the latter being named Pasha (partner of 
Drosha) (Denli et al., 2004; Filippov et al., 2000; Landthaler et al., 2004). The complex cleaves the pri-miRNA into pre-miRNA in the nucleus. Suppression of Pasha in Drosophila interferes with pri-miRNA processing, leading to an accumulation of pri-miRNAs and a reduction in mature miRNAs (Denli et al., 2004; Landthaler et al., 2004). Like in other animals, Pasha is essential for processing of canonical miRNAs but is dispensable for mirtrons (Flynt et al., 2010; Martin et al., 2009; Smibert et al., 2011). Drosophila Pasha is possibly phosphorylated by ERK/MAPK, as suggested by phosphorylation of human DGCR8 in insect cells; the phosphorylation appears to increase protein stability without altering miRNA processing activity (Herbert et al., 2013). miRNA biogenesis in Drosophila also involves SmD1, a component of the Drosophila small nuclear ribonucleoprotein particle (snRNP), which interacts with both the microprocessor component Pasha and pri-miRNAs, and is indispensable for optimal miRNA biogenesis (Xiong et al., 2015).

Analysis of transcriptome changes upon Drosha knock-down in S2 cells identified 137 Drosha-regulated RNAs, including 11 relatively long $(>10 \mathrm{~kb}$ ) pri-miRNAs (Kadener et al., 2009). Interestingly, $>100$ RNAs not annotated as miRNAs could be direct targets of Drosha action (Kadener et al., 2009), which is consistent with other model systems where Drosha is having roles beyond miRNA biogenesis. Drosha-regulated RNAs contain conserved hairpins similar to those recognized by the Drosha-Pasha/DGCR8 complex in pri-miRNAs, one of such hairpins is found also in Pasha suggesting a negative feedback loop regulating miRNA-biogenesis (Kadener et al., 2009). miRNA-independent roles of the Microprocessor complex components seem to be reflected in phenotypes of some of their mutants (Luhur et al., 2014).

In terms of evolutionary diversity of the Microprocessor complex in arthropods, the miRNA pathway seemed to expand in the pea aphid (insect, Hemiptera), whose genome carries four expressed copies of Pasha (Jaubert-Possamai et al., 2010). At the same time, the brown planthopper (insect, Hemiptera), the fall armyworm (insect, Lepidoptera) or the desert locust (insect, Orthoptera) all have a single Pasha (Ghosh et al., 2014; Wynant et al., 2015; Xu et al., 2013), which appears the common case among arthropods when browsing available genome databases. Analysis of Pasha in Litopenaeus vannamei (shrimp) revealed high sequence conservation and nuclear localization, suggesting a well-conserved role in miRNA biogenesis (Chen et al., 2012). Conservation of miRNA pathway in shrimps is further supported by requirement for Drosha, Dicer1 and Agol for production of viral RNAs in infected shrimps (He and Zhang, 2012; Huang et al., 2012).

\section{Dicer}

Drosophila utilizes two Dicer proteins (Fig. 2), Dicer-1 (DCR-1) and Dicer-2 (DCR-2), which are dedicated to miRNA and RNAi pathways, respectively (Lee et al., 2004). This makes Drosophila (and arthropods in general) unique among the reviewed metazoan model systems (Fig. 3), which employ a single Dicer protein producing multiple classes of small RNAs (miRNAs, endo-siRNAs, exo-siRNAs). Separation of miRNA and RNAi at Dicer level could have an advantage in terms of uncoupling antagonistic evolutionary forces acting on Dicer, i.e. (i) selective pressure on conservation of the miRNA pathway machinery 


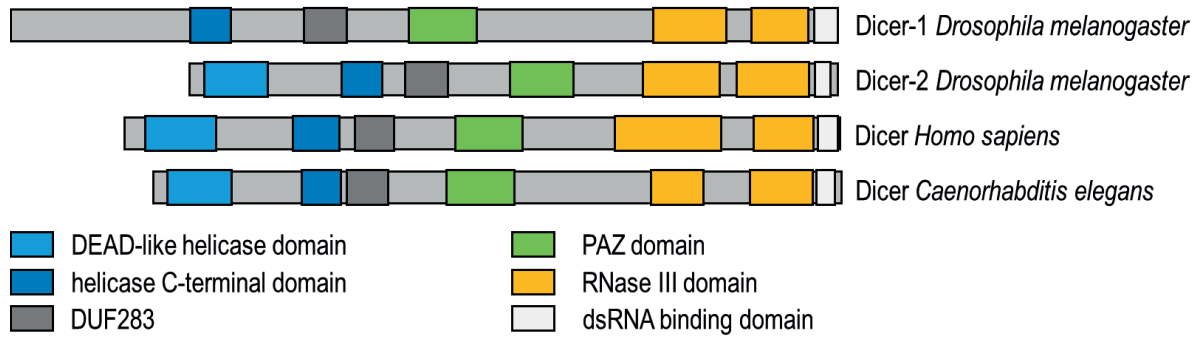

Figure 2 Comparison of $C$. elegans, human and Drosophila Dicer proteins Domain composition was adopted from (Jaskiewicz and Filipowicz, 2008).

and (ii) host-pathogen arms race where Dicer evolves to avoid viral proteins interfering with its function.

The domain organization of Drosophila Dicer proteins is generally the same as in other metazoan Dicer proteins - they are composed of domains ordered from the N- to the C-terminus as follows: N-terminal helicase domains, a domain of unknown function DUF283, PAZ domain, RNase IIIa and RNase IIIb domains, and the C-terminal dsRBD (Fig. 2). As for other metazoan Dicers, Drosophila Dicer proteins have not been crystallized yet but their structure can be inferred from biochemical studies of recombinant Dicer and individual domains (Tsutsumi et al., 2011; Ye et al., 2007), the crystal structure of Giardia intestinalis Dicer (MacRae et al., 2007; MacRae et al., 2006), domain modelling or cryo-EM studies (Lau et al., 2012).

\section{Dicer-1}

Dicer-1 was originally identified as one of two homologs in Drosophila, which was able to produce siRNAs in vitro and participated in RNAi (Bernstein et al., 2001). Subsequent analysis of Dicer mutants showed that mutation in dicer-1 blocked processing of miRNA precursors while dicer-2 mutants were defective for processing siRNA precursors (Lee et al., 2004). However, consistent with the initial study, Dicer-1 was also implicated in RNAi (Lee et al., 2004). Biochemical analysis of Dicer-1 showed that its functional core consists of a DUF283 domain, a PAZ domain, and two RIII domains (Ye et al., 2007). With respect to the size of cleavage products, Dicer-1 apparently does not differ from other metazoan Dicers, as the typical product size is $22 \mathrm{nt}$ long (Fig. 4). DCR-1 also functions in biogenesis of mirtron class of miRNAs (Okamura et al., 2007).

Dicer-1 differs from Dicer-2 in substrate specificities and ATP requirements (Jiang et al., 2005). Like human Dicer, Dicer-1 generates small RNAs in an ATP-independent manner (Jiang et al., 2005), whereas Dicer-2 or Dicer-2/R2D2 required ATP hydrolysis for efficient siRNA production (Liu et al., 2003). Dicer-1 shows a preference for pre-miRNAs (Jiang et al., 2005; Tsutsumi et al., 2011). It recognizes the single-stranded terminal loop structure of pre-miRNAs through its N-terminal helicase domain, checks the loop size and measures the distance between the $3^{\prime}$ overhang and the terminal loop - this allows Dicer-1 to inspect the authenticity of pre-miRNA structures (Tsutsumi et al., 2011). 
In terms of evolutionary diversity of Dicer-1 in arthropods, as mentioned above, the miRNA pathway seemed to expand in pea aphid (insect, Hemiptera) which utilizes two active copies of Dicer 1 (Jaubert-Possamai et al., 2010; Ortiz-Rivas et al., 2012). However, this duplication is a relatively recent event while single Dicer-1 was also identified elsewhere among arthropods (Jaubert-Possamai et al., 2010; Ortiz-Rivas et al., 2012), including shrimp (Su et al., 2008), mosquito (Bernhardt et al., 2012), cockroach (Gomez-Orte and Belles, 2009) or locust (Wynant et al., 2015) species.

\section{Dicer-2}

Dicer-2 in Drosophila is mainly producing siRNAs from long dsRNA and functions in RNAi and antiviral defense (Galiana-Arnoux et al., 2006; Kim et al., 2006). Dicer-2 has actually a dual role in antiviral defense - apart from RNAi, it has an RNAi-independent role in promoting Toll signalling (Wang et al., 2015b), but biological aspects of Dicer-2 role will be covered later in the text.

Unlike mammalian Dicer or Dicer-1 paralog, Dicer-2 requires ATP for processive cleavage of dsRNA (Liu et al., 2003; Nykanen et al., 2001; Provost et al., 2002; Welker et al., 2011; Zhang et al., 2002). Remarkably, analysis of shapes of a mammalian Dicer and Dicer-2 by cryo-EM yielded an L-shaped reconstruction with dimensions strikingly similar to those of the human enzyme (Lau et al., 2012). Therefore, despite striking functional differences in ATP requirement and substrate preference, the overall three-dimensional architecture of Dicer is well conserved (Lau et al., 2012).

Dicer-2 contains an N-terminal helicase motif and hydrolyzes ATP; ATP hydrolysis is required for Dicer-2 to process long dsRNA, but not pre-miRNA (Cenik et al., 2011). Dicer-2 works as a dsRNA-stimulated ATPase that hydrolyzes ATP to ADP; and it was suggested that Dicer-2 helicase domain uses ATP to generate many siRNAs from a single molecule of dsRNA before dissociating from its substrate. (Cenik et al., 2011).

The helicase domain of Dicer-2 also governs substrate recognition and cleavage efficiency through discriminating among dsRNA ends. First, it was shown that the helicase domain is essential for cleaving dsRNA with blunt or 5'-overhanging termini, but not those with 3' overhangs, as in pre-miRNAs (Welker et al., 2011). Subsequently, it was found that the discrimination of termini takes place during initial binding (Sinha et al., 2015). In the absence of ATP, Dicer-2 binds 3' overhanging (pre-miRNA-like), but not blunt termini. in the presence of ATP, Dicer-2 binds both types of termini, with highest-affinity binding observed with blunt dsRNA (Sinha et al., 2015).

An important factor in substrate discrimination and processing is inorganic phosphate, which inhibits Dicer-2 cleavage of pre-miRNAs, but not long dsRNAs (Cenik et al., 2011). It was proposed that the inorganic phosphate occupies a PAZ domain 5' phosphate binding pocket required to bind the 5 ' terminal phosphate of short substrates, blocking their use and restricting pre-miRNA processing in flies to Dicer-1 (Fukunaga et al., 2014). Binding of long dsRNA is not inhibited when the inorganic phosphate occupies the PAZ domain binding pocket because it also involves the helicase domain and/or the central dsRNA-binding domain, which might be combined with displacement of the inorganic phosphate from its binding pocket (Fukunaga et al., 2014) 


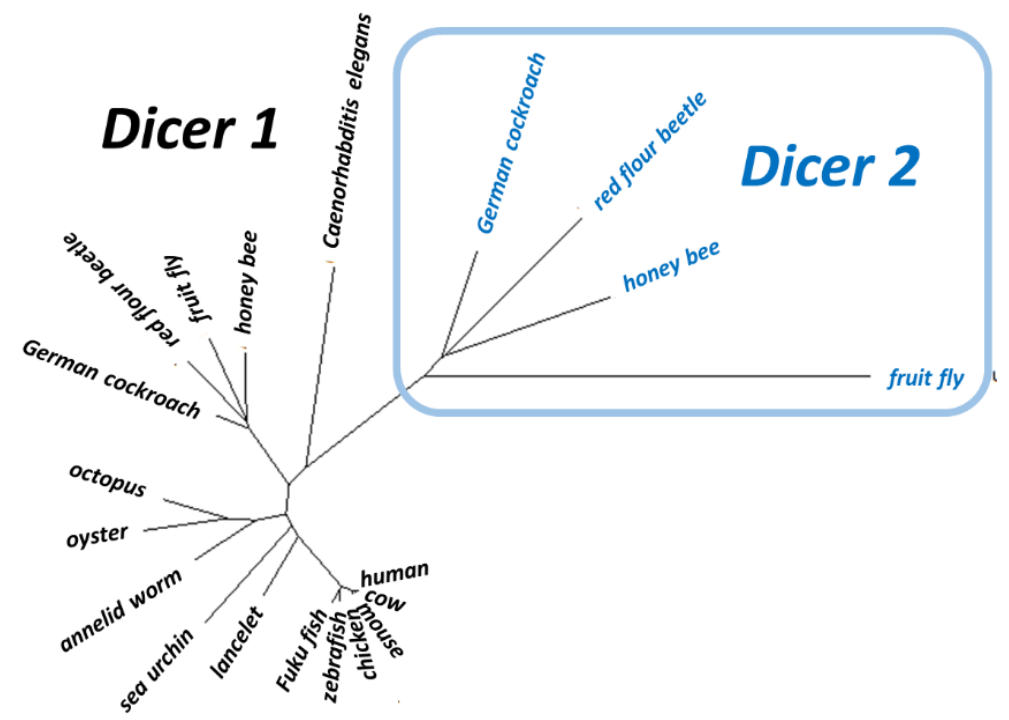

Figure 3 Metazoan Dicer phylogeny

The unrooted tree shows phylogenetic relationships of Dicer proteins in Metazoa. The blue frame depicts Dicer2 homologs in arthropods. As the length of each branch indicates evolutionary distance (or sequence divergence), it is apparent that arthropod's Dicer 2 proteins acting in RNAi are evolving at much faster pace than Dicer 1 protein, which function in the miRNA pathway. This is consistent with the above-mentioned notion of antagonistic evolutionary forces acting on Dicer where the miRNA pathway functionality is being conserved while the RNAi functionality is evolving during the host-pathogen arms race where Dicer evolves to avoid viral proteins interfering with its function.

In terms of evolutionary diversity of Dicer-2 in arthropods, most species seem to use only one Dicer-2 but some underwent duplication, such as Daphnia (Crustacea, two Dicer-2 paralogs) or Metaseiulus (Chelicerata, five Dicer-5 paralogs) (Palmer and Jiggins, 2015). Among the experimentally approached species, one Dicer-2 was reported in experimental results from silk moth (Kolliopoulou and Swevers, 2013), mosquito (Leger et al., 2013), cockroach (Lozano et al., 2012), Hessian fly (Kolliopoulou and Swevers, 2013), planthopper (Zhang et al., 2013), emerald ash borer (Zhao et al., 2015), mite (Hoy et al., 2016), bumble bee (Niu et al., 2016), or shrimp (Niu et al., 2016).

\section{dsRBPs in arthropods - R2D2 and LOQS homologs}

Drosophila also utilizes Dicer partner dsRBPs with tandemly arranged dsRBDs - Loquacious (LOQS) and R2D2. The first Dicer partner dsRBP in Drosophila is Loquacious, which was found to associate with Dicer-1, suggesting that the miRNA pathway in Drosophila employs a distinct dsRBP in substrate routing (Forstemann et al., 2005; Saito et al., 2005). However, it was also found that Dicer-2-generated siRNAs in the endogenous RNAi pathway depend preferentially on Loquacious and not on R2D2, the canonical Dicer-2 partner 
(Czech et al., 2008). it turned out that Loquacious gene actually produces three protein isoforms, which associate with Dicer-1 and miRNA pathway (LOQS-PA and LOQS-PB isoform) and Dicer-2 and RNAi (LOQS-PD isoform) (Fukunaga et al., 2012; Hartig et al., 2009; Miyoshi et al., 2010a; Zhou et al., 2009).

LOQS-PB uses the second dsRNA-binding domain to bind pre-miRNA and the third dsRNA-binding domain to interact with Dcr-1. Both domains of LOQS-PB are required for efficient miRNA production by enhancing the affinity of Dcr-1 for pre-miRNA (Ye et al., 2007).

LOQS-PD and R2D2 function sequentially and non-redundantly in the endogenous RNAi pathway. LOQS-PD stimulates DCR-2-mediated processing of dsRNA whereas R2D2 acts downstream during RISC loading (Hartig and Forstemann, 2011; Marques et al., 2010; Miyoshi et al., 2010a). Taken together, LOQS and R2D2 contribute to the profound mechanistic separation of miRNA and RNAi pathways, which evolved in Drosophila (and presumably in arthropods in general).

R2D2 associates with Dicer-2 and acts in RNAi; it was co-purified with Dicer-2 during purifying siRNA-generating activity from Drosophila S2 cell lysates (Liu et al., 2003). Although R2D2 bears 33\% similarity to RDE-4 (see the section Nematoda) its role is different. R2D2 does not influence DCR-2 enzymatic activity (Liu et al., 2003) but restricts Dicer-2 function to processing of long dsRNAs (Cenik et al., 2011; Fukunaga et al., 2014). It also facilitates passing the cleavage product to $\mathrm{AGO} 2$ excluding miRNA-like duplexes with imperfect base pairing (Tomari et al., 2004a). R2D2 has two roles - it is sensing siRNA thermodynamic asymmetry for strand selection and it is a licensing factor for entry of authentic siRNAs into the RNAi pathway (Nishida et al., 2013; Tomari et al., 2004b).

Unlike the Microprocessor complex, Dicer or Argonautes, dsRBPs seem undergo various functional adaptations between different taxons (compare, for example TARBP2, RDE-4, R2D2 or LOQS). This possibly also happens among arthropods. An example is the lack of the RNAi-associated LOQS-PD isoform outside Drosophila (Haac et al., 2015). Analysis of dsRBPs in the mosquito Aedes aegypti revealed absence of LOQS-PD isoform, conserved roles of R2D2 and LOQS-PB, and LOQS-PA role in biogenesis of both, miRNAs and endo-siRNAs (Haac et al., 2015).

\section{Argonaute proteins}

AGO proteins of arthropods are conserved, i.e. their domain composition is the same as that of mammalian proteins, which was discussed in detail (Peters and Meister, 2007). Arthropods have varying number of Argonaute proteins of the AGO and PIWI clade but it seems that their archetypal state is two AGO proteins, each being associated with one Dicer and one type of small RNA (Palmer and Jiggins, 2015).

\section{AGO1 and its loading with miRNAs}

AGO1 RISC loading is similar to that of human RISC assembly described earlier (Yoda et al., 2010). Dicer-1/LOQS-PB heterodimer functions in assembling AGO1 RISC, which 
is preferentially loaded with miRNA/miRNA* duplexes while siRNAs are being excluded from the assembly (Tomari et al., 2007).

AGO1 requires ATP for miRISC loading, presumably to trigger the dynamic conformational opening of AGO proteins so that they can accept small-RNA duplexes (Kawamata et al., 2009). Unwinding of miRNA-miRNA* duplexes is a passive process that does not require ATP or slicer activity of Ago 1 (Kawamata et al., 2009).

Two distinct AGO complexes were identified (Miyoshi et al., 2009): (i) AGO1-Dicer-1 complex with pre-miRNA processing activity where the resultant mature RNA was loaded onto AGO1 within the complex - this complex corresponds to miRLC (miRISC loading complex) (ii) the AGO1-GW182 complex with excluded DCR-1, containing mature miRNA no pre-miRNA processing activity - this complex corresponds to miRISC. AGO1 loading also involves R3D1-L, a dsRBP that functions as a cofactor interacting with Dicer-1 and AGO1 (Jiang et al., 2005).

AGO1 might also have miRNA-independent role in translational repression where AGO1 is recruited to mRNA via an RNA-binding protein SMAUG and not through miRNA:mRNA interaction (Pinder and Smibert, 2013).

\section{AGO2 and its loading with siRNAs}

The model of RNAi RISC loading in Drosophila suggests that RISC assembly occurs in several steps, which involve a several complexes (Tomari and Zamore, 2005). The first complex is formed by siRNA, R2D2 and DCR-2, also known as R1 or R2/D2/DCR-2 initiator (RDI) complex (Kim et al., 2007; Pham et al., 2004), which develops into a mature form of the RISC loading complex RLC (Tomari and Zamore, 2005). The RLC determines strand selection and recruits AGO2 (and other proteins) to form pre-RISC (Kim et al., 2007), which contains duplex siRNA. Finally, the release of the passenger strand from the duplex produces holo-RISC, which can base pair with complementary mRNA substrates. The loading is assisted by Hsc70/Hsp90 chaperones (Iwasaki et al., 2015; Miyoshi et al., 2010b).

The coupling of dsRNA cleavage and RISC assembly is a matter of debate. It was suggested that, after cleavage, small-RNA duplexes need to dissociate from Dicer and then rebind to a sensor of the thermodynamic asymmetry of the duplex, because the guide strand of an siRNA will be at random orientation (Tomari et al., 2004b).

AGO2 requires ATP for RISC loading (Kawamata et al., 2009; Pham et al., 2004; Tomari et al., 2004a). ATP is presumably used to trigger the dynamic conformational opening of AGO proteins so that they can accept small-RNA duplexes (Kawamata et al., 2009).

Strand selection in fly RLC is controlled by R2D2. Analysis of the interaction of DCR2/R2D2 complex with siRNA duplexes showed that R2D2 orients the complex according to thermodynamic stabilities of siRNA strands and binds the 5' phosphate of the passenger strand at the thermodynamically more stable end (Tomari et al., 2004b). Thus, R2D2 functions as a licensing factor for routing siRNAs into the RNAi pathway. Interestingly, a thorough analysis of AGO2 complexes revealed that, unlike mature miRNAs, which are loaded on AGO1, complementary strands of mature miRNAs (miRNA*) are efficiently loaded on AGO2 in DCR2/R2D2-dependent manner (Ghildiyal et al., 2010; Okamura et al., 
2011). Thus, the role of R2D2 in sorting small RNAs is wider and extends into the miRNA pathway.

The final step in assembly of an active RISC is the release of the passenger strand from the siRNA duplex. Drosophila is Armitage helicase is a candidate for a mechanism separating the two siRNA strands while the guide remains bound to AGO2 (Tomari et al., 2004a). However, experimental data support a simple solution where passenger strand cleavage by AGO2 slicer activity liberates the single-stranded guide siRNA strand from the pre-RISC complex (Kim et al., 2007; Matranga et al., 2005; Miyoshi et al., 2005). Removal of siRNA passenger strand cleavage products is assisted by $\mathrm{C} 3 \mathrm{PO}$ endoribonuclease, which was identified as a RISC-enhancing factor that promotes RISC activation (Liu et al., 2009). The cleavage-assisted mechanism is typical for AGO2-loaded fly and human siRNAs in the RNAi pathway while passenger strand cleavage is not important for loading miRNAs (Matranga et al., 2005).

\section{Small RNA sorting and mRNA targeting by AGO1 and AGO2}

Drosophila sorts Dicer-produced small RNAs onto functionally distinct AGO proteins where AGO1 is dedicated to the miRNA pathway while AGO2 served for RNAi. Small RNA sorting is initiated by substrate recognition and continues through sorted loading onto the AGO proteins. Small-RNA duplexes are actively sorted into AGO-containing complexes according to their intrinsic structures (Forstemann et al., 2007; Tomari et al., 2007). Importantly, separation of miRNA and RNAi at the level of small RNA sorting onto AGO1 and AGO2 is not completely pre-determined by small RNA origins (Tomari et al., 2007). It was found that miRNA*s are often loaded as functional species into AGO2 (Czech et al., 2009; Ghildiyal et al., 2010; Okamura et al., 2009). Furthermore, miRNAs produced by Dicer-1 and LOQS can be loaded by Dicer-2 and R2D2 into an AGO2 RISC (Forstemann et al., 2007). Finally, siRNAs derived from long hairpin RNA genes (hpRNA) also show a hybrid biogenesis combining RNAi factors DIcer-2 and AGO2 and Loquacious isoform (Okamura et al., 2008c).

Subsequently, AGO2-RISC mediates RNAi while only AGO1 is able to repress mRNAs with central mismatches in miRNA-binding sites (Forstemann et al., 2007). At the same time, AGO1 cannot mediate RNAi, because it is an inefficient nuclease with a catalytic rate limited by the dissociation of reaction products (Forstemann et al., 2007). AGO1 and AGO2 RISCs also differ in mechanisms of translational repression - AGO1-RISC represses translation primarily by ATP-dependent deadenylation while Ago2-RISC competitively blocks the interaction of eIF4E with eIF4G and inhibits the cap function (Fukaya et al., 2014; Iwasaki et al., 2009). AGO1-mediated translational repression involves GW182 in the same manner as in mammals (GW182 is separately described further below). miRNA-mediated silencing involves recruitment of PABP, CCR4-NOT deadenylase and decapping complex to RISC (Behm-Ansmant et al., 2006; Chekulaeva et al., 2011; Eulalio et al., 2008; Fukaya and Tomari, 2011; Huntzinger et al., 2010; Huntzinger et al., 2013; Moretti et al., 2012; Rehwinkel et al., 2005). miRNA-mediated repression occurs on ribosome complexes but is independent of ribosomal scanning(Antic et al., 2015; Kuzuoglu-Ozturk et al., 2016). 


\section{Evolutionary perspective}

As mentioned above, the archetypal state arthropod state is two AGO proteins, AGO1 and AGO2. Apart from Drosophila, such a situation is found in Daphnia (Crustacea) and Metaseiulus (mite, Chelicerata) (Palmer and Jiggins, 2015). However, upon detailed inspection, one frequently finds variability in the number of AGO proteins across the phylum or even across smaller taxonomic units. The miRNA pathway seemed to expand in pea aphid (insect, Hemiptera), whose genome two expressed copies of ago1, one of which (agolb) shows signs of positive selection (Jaubert-Possamai et al., 2010). At the same time, a single AGO1 but duplications of AGO2 were found Ixodes (tick, Chelicerata, three AGO2 paralogs), Strigamia (centipede, Myriapoda, two AGO2 paralogs), Mesobuthus (scorpion, Chelicerata, six AGO2 paralogs) or Tetranychus (spider mite, Chelicerata, six AGO2 paralogs) (Palmer and Jiggins, 2015). Penaeus monodon (black tiger shrimp) has four functionally diversified AGO paralogs (Dechklar et al., 2008; Leebonoi et al., 2015; Phetrungnapha et al., 2013; Yang et al., 2014b). Analysis of Argonaute genes across 86 Dipteran species showed that variation in copy number can occur rapidly, and that there is constant flux in some RNAi mechanisms; this suggests that Argonautes undergo frequent evolutionary expansions that facilitate functional divergence (Lewis et al., 2016).

\section{Additional miRNA and RNAi factors}

There is a large number of accessory factors beyond those described above. For example, a systematic screen of $40 \%$ of the genome for genes acting in the miRNA pathway yielded 45 mutations in 24 genes and an estimate of $\sim 100$ genes are required to execute the miRNA program (Pressman et al., 2012). Here, we will describe several additional factors, which have been associated with miRNA or RNAi pathways.

Nibbler - Nibbler is a 3'-5; exoribonuclease involved in trimming 3' ends of miRNAs and piRNAs (Feltzin et al., 2015; Han et al., 2011; Liu et al., 2011; Wang et al., 2016; Yang et al., 2014a). In the miRNA pathway, Nibbler shortens distinct longer miRNAs during RISC assembly, yielding miRNA isoforms that are compatible with the preferred length of AGO1-bound small RNAs (Han et al., 2011; Liu et al., 2011). It has been estimated that about a quarter of miRNAs undergoes such a trimming (Han et al., 2011).

HEN1 - HEN1 (Pimet, Dmhen1)is an enzyme catalyzing addition of a 2'-O-methyl group at the 3' end of small RNAs (Horwich et al., 2007; Saito et al., 2007). While this modification is predominantly found on piRNAs in Drosophila, it was also found on siRNAs and miRNAs (Abe et al., 2014; Horwich et al., 2007; Yang et al., 2014a). Functionally, 2'-O-methylation of siRNAs loaded on $\mathrm{AGO} 2$ prevents tailing and trimming of siRNAs (Ameres et al., 2010). Generally HEN1 and Nibbler thus have antagonistic activities at the 3 ' end of small RNAs where Nibbler promotes small RNA trimming while Hen1 prevents it (Ameres et al., 2010; Yang et al., 2014a). 2'-O-methylation is also found on select miRNA isoforms and appeared to increase with age while its reduction was associated with neurodegeneration and shorter life span (Abe et al., 2014). 
nucleotidyltransferases - tailing of short RNAs is mediated by terminal nucleotidyltransferases, which produce 3' uridylation or adenylation. PAPD4 has been identified as a primary miRNA adenylating enzyme in Drosophila, adenylation did not appear to affect miRNA stability on a genome-wide scale (Burroughs et al., 2010). Another non-canonical adenylase is Wispy, which is responsible for adenylation of miRNAs and biologically it may facilitate clearance of maternal miRNAs in the embryo (Lee et al., 2014). Uridylation is mediated by Tailor, which is a uridylyltransferase that is required for the majority of 3' end modifications of microRNAs in Drosophila and predominantly targets mirtron hairpins (Reimao-Pinto et al., 2015; Westholm et al., 2012).

GW182 - GW182 is the key co-factor of AGO1 in miRISC. Its role has been described in detail in the mammalian section, he we will briefly note its key features with respect to arthropods. GW182 and its interaction with AGO1 were found to be required for miRNA-mediated repression in Drosophila cells (Behm-Ansmant et al., 2006; Eulalio et al., 2008; Rehwinkel et al., 2005). miRNA-mediated repression also required the decapping complex DCP1:DCP2 and CCR4-NOT deadenylase (Behm-Ansmant et al., 2006; Rehwinkel et al., 2005). Multiple domains of GW182 contribute to miRNA-mediated repression (Chekulaeva et al., 2009; Chekulaeva et al., 2011; Chekulaeva et al., 2010; Eulalio et al., 2009). Similarly to mammals, Drosophila GW182 directly interacts with PABP and CCR4-NOT (Chekulaeva et al., 2011; Fukaya and Tomari, 2011; Huntzinger et al., 2010; Huntzinger et al., 2013; Moretti et al., 2012).

Armitage - RNA helicase, which was identified as a maternal effect gene required for RNAi (Tomari et al., 2004a). Armitage is probably not required for RISC activity. Instead, it was proposed to facilitate removal of the passenger strand during RISC formation (Tomari et al., 2004a). Armitage was also implicated in piRNA biogenesis (Huang et al., 2014; Murota et al., 2014; Nagao et al., 2010; Qi et al., 2011; Saito et al., 2010).

dFMR1 - Drosophila ortholog of human fragile X mental retardation protein (FMRP) was identified as a RISC component (Caudy et al., 2002; Ishizuka et al., 2002; Pham et al., 2004). dFMR1 is associated with ribosomes through interaction with ribosomal proteins L5 and L1 and with complexes containing miRNAs (Ishizuka et al., 2002). dFMR1 is not a conserved RISC component involved in RNAi as depletion of dFMR1 reduces RNAi efficiency in Drosophila S2 cells but not in mammals (Caudy et al., 2002). dFMR has been also implicated in the piRNA pathway (Bozzetti et al., 2015; Jiang et al., 2016).

VIG - Vasa Intronic Gene (Caudy et al., 2002; Pham et al., 2004). VIG is a conserved protein, which encodes a putative RNA binding protein, whose depletion reduces RNAi efficiency (Caudy et al., 2002). Vig mutants are more susceptible to viral infections in Drosophila (Zambon et al., 2006). Whether this role of VIG is coupled with its presence in the RISC complex is not known. There is no evidence that SERBP1, the closest mammalian VIG homolog, would be associated with RISC. VIG was also implicated in heterochromatin formation (Gracheva et al., 2009).

Tudor-SN - Tudor Staphylococcal Nuclease is a protein containing five staphylococ$\mathrm{cal} /$ micrococcal nuclease domains and a Tudor domain. It is a component of the RISC in C. elegans, Drosophila and mammals (Caudy et al., 2003; Pham et al., 2004). The role of Tudor-SN in RISC RNAi remains enigmatic. TSN is not the „slicer“ (Schwarz et al., 2004) and its knock-down in silk moth cells had not effect on RNAi efficiency (Zhu et al., 
2012). In Drosophila, Tudor-SN has also been linked to piRNA pathway regulation (Ku et al., 2016).

DMP68 (RM62) - this conserved helicase was co-purified with AGO1 and dFMR1 (Ishizuka et al., 2002). This helicase seems to be required for RNAi in S2 cells where depletion of DMP68 results in inhibition of RNAi (Ishizuka et al., 2002). Whether DMP68 is needed for RISC formation or for RISC activity/stability is not known. Later publications on RM62 linked it to antiviral response (Zambon et al., 2006) and to other mechanisms than RNAi.

CRIF1 - the Drosophila homolog of the mammalian CR6-interacting factor 1 (CRIF1), was identified as a potential new regulator of the RNAi pathway during a screen for genetic mutations in Drosophila that alter the efficiency of RNAi. CRIF1 loss-of-function mutant flies are deficient in exo-RNAi, in siRNA biogenesis and in antiviral immunity. (Lim et al., 2014)

\section{RdRPs in arthropods}

One of the less understood areas of RNA silencing in arthropods is utilization of RdRPs. A phylogenetic analysis suggests that RdRPs in RNA silencing pathways have a monophyletic origin, i.e. evolved from a single ancestral RdRP (Cerutti and Casas-Mollano, 2006; Murphy et al., 2008). A simple TBLASTN search with C. elegans RRF-1 proteins sequence reveals RdRPs in species across Metazoa, including Cnidaria (hydra), Nematoda (RdRPs in C. elegans will be discussed later), Mollusca (oyster), Hemichordata (acorn worm), or Urochordata (sea squirt). At the same time, RdRPs seem to be absent in other groups including Platyhelminthes, Annelida, and vertebrates. What this implies for arthropods is that the common ancestors of protostomes and deuterostomes still had RdRPs and that RdRPs were repeatedly lost in different taxons. We performed a diagnostic TBLASTN search with C. elegans RRF-1 proteins sequence also for the major groups of arthropods and we have identified RdRP homologs in diverse representatives of the subphylum Chelicerata (spiders, horseshoe crab, ticks, mites) but none in the remaining subphyla - Myriapoda, Crustacea, and Hexapoda. This would suggest that RdRP was lost early in the arthropod evolution, being retained only in Chelicerata. Thus, one could assume that except of Chelicerata, RNA silencing does not employ RdRP-generated secondary siRNAs like those found in C. elegans.

Whether RdRP activity completely disappeared from RNAi in Drosophila (and those arthropods lacking an RdRP ortholog) is not completely understood but available data suggest that it is could the case. One should consider also the option that a missing RdRP ortholog in RNA silencing could be replaced by another RdRP, for example by horizontal transfer of some viral RdRP. In fact, there is a report of RdRP activity in Drosophila (Lipardi et al., 2001) but, was contradicted by experiments demonstrating the absence of transitive RNAi generating secondary sequences upstream of the region targeted by siRNAs (Roignant et al., 2003; Schwarz et al., 2002), so the issue remained unresolved. Later, dELP1, a non-canonical RdRP conserved in all eukaryotes, was suggested to associate with Dicer-2 and function as an RdRP (Lipardi and Paterson, 2009). However, the article was later retracted because the measured biochemical activity did not seem to be an authentic 
RdRP (Lipardi and Paterson, 2011). Taken together, as of now, there is no evidence for RdRP activity yielding secondary siRNAs and transitive RNAi in arthropod species lacking orthologs of ancestral RNA silencing-related RdRPs.

\section{miRNA-mediated control of gene expression}

miRNAs play physiologically important roles in arthropods, as suggested by phenotypes of mutants of miRNA pathway. The loss of Dicer-1 or Ago1 causes embryonic lethality (Lee et al., 2004; Pressman et al., 2012). Dicer-1 is also needed to maintain ovarian stem cells in Drosophila (Jin and Xie, 2007). Similarly, Ago1 is essential for oocyte formation and maintenance of germline stem cells in Drosophila (Azzam et al., 2012; Yang et al., 2007) and for locust oogenesis (Song et al., 2013). The miRNA pathway can also participate in response to physiological cues (Dekanty et al., 2010) or in regulation of immunity in arthropods, as shown for the Plasmodium infection mosquito (Winter et al., 2007). The miRNA pathway apparently expanded in pea aphid (insect, Hemiptera), whose genome contains four pasha, two dcr-1 and two ago2 paralogs, all of which are expressed and where one of the ago 1 paralogs shows signs of positive selection (Jaubert-Possamai et al., 2010). Notably, these expansions occurred concomitantly within a brief evolutionary period. it has been speculated that the miRNA pathway diversity could contribute to adapted phenotypes, which the pea aphid is able to produce from a single genotype (Jaubert-Possamai et al., 2010).

Drosophila's miRNA annotation in mirBase (Kozomara and Griffiths-Jones, 2014) is likely the most thoroughly done; it includes meta-analysis of $>10^{9}$ raw reads from 187 RNA-seq libraries comprising diverse developmental stages, specific tissue- and cell-types, mutant conditions, and/or Argonaute immunoprecipitations yielded a thorough annotation of miRNA loci, including definition of multiple phased by-products of cropping and dicing, abundant alternative 5' termini of certain miRNAs, frequent 3' untemplated additions, and potential editing events (Berezikov et al., 2011). Considering incomplete miRNA annotations in other species, miRBase numbers indicate that arthropods might have about one order of magnitude less miRNAs than mammals suggesting somewhat less expanded miRNA-mediated control of gene expression. In addition, there are only a few miRNAs conserved between Drosophila and mammals (the best known is Let-7 (Pasquinelli et al., 2000)).

Arthropods also employ non-canonical miRNAs. These include mirtrons that bypass Drosha processing (Martin et al., 2009; Okamura et al., 2007; Ruby et al., 2007). In addition, specific miRNA loops may accumulate as non-canonical miRNAs on AGO1 and mediate miRNA-type repression (Okamura et al., 2013). Another non-canonical miRNA was identified in rDNA arrays. Its processing requires Dicer-1 but not the Microprocessor complex and it is conserved among Diptera (Chak et al., 2015).

\section{RNAi pathway in arthropods}

Although Drosophila Dcr-2 or Ago-2 are nonessential and mutants can develop to adults with no strong phenotypes under standard laboratory conditions (Deshpande et al., 2005; 
miRNA size distribution in Drosophila.

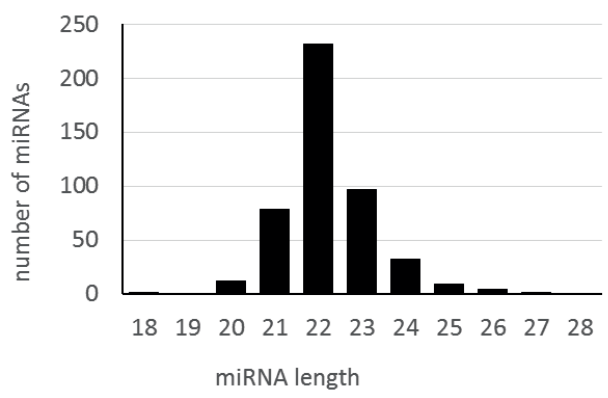

miRNA size distribution in Mus musculus

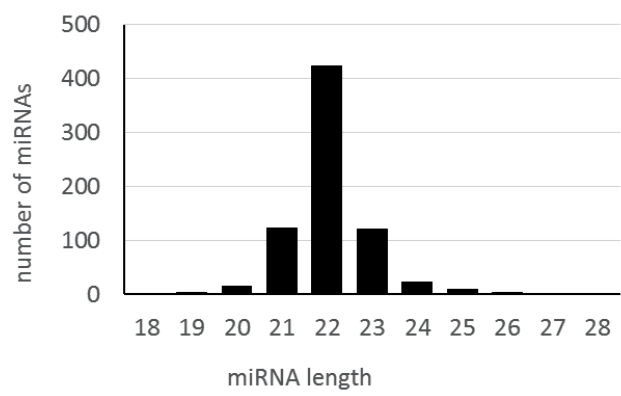

Figure 4 D. melanogaster miRNA lengths

The left graph depicts size distribution of all 466 miRNAs of a Drosophila melanogaster deposited in the miRBase (version 21). For comparison, the right graph shows size distribution of 721 high-confidence murine miRNAs.

Lee et al., 2004; Li et al., 2013; Xu et al., 2004), some minor phenotypes appear and severe defects in embryonic development have been noted in these mutants upon exposure to temperature perturbations (Deshpande et al., 2005; Li et al., 2013; Lucchetta et al., 2009).

RNAi is functional across other arthropods subphyla, including Chelicerata (ticks and mites (Hoy et al., 2016; Kurscheid et al., 2009; Schnettler et al., 2014)) and Crustacea (shrimps (Chen et al., 2011; Hoy et al., 2016; Huang and Zhang, 2013; Jariyapong et al., 2015; Maralit et al., 2015; Sabin and Cherry, 2013; Yang et al., 2014b). We did not find published functional RNAi data for Myriapoda but genomic analyses show that they have the necessary machinery (Palmer and Jiggins, 2015). In this section, we will discuss the role of antiviral RNAi and endo-siRNAs in Drosophila and arthropods in general.

\section{Antiviral RNAi}

RNAi plays a key role in innate immunity in arthropods and a large volume of the reviewed literature across the taxon dealt with antiviral role of RNAi. As it could be expected, most of the mechanistic data came from the Drosophila model.

The first evidence for antiviral RNAi in arthropods emerged in 2002 from a study that used flock house virus (FHV) is inducing RNAi (and is being targeted by RNAi) Drosophila host cells (Li et al., 2002). Infection of 14 different Drosophila RNA silencing mutants with a dsRNA X virus (DXV) showed that all but three lines were significantly more susceptible to viral infection (reduced survival and elevated viral titers) than normal flies. Moreover, replication of DXV was sequence-specifically inhibited (but not absolutely blocked) by „,immunizing“ Drosophila S2 cells with dsRNA from the coding region of DXV before infection (Zambon et al., 2006). Remarkably, increased susceptibility was observed not only for mutants of the RNAi pathway, such as $r 2 d 2$, armi, or ago2, but also for mutants of the piRNA pathway (aubergine and piwi), suggesting that RNAi is not the only RNA silencing pathway in Drosophila that can respond to a viral infection (Morazzani et al., 2012). The engagement of the piRNA pathway also extends to mosquitos (Leger et al., 
2013; Schnettler et al., 2013a; Vodovar et al., 2012). A number of studies provided ample mechanistic evidence that RNAi plays an essential role in antiviral response in Drosophila (Galiana-Arnoux et al., 2006; Nayak et al., 2010; van Rij et al., 2006; Wang et al., 2006).

The antiviral role of RNAi is conserved across the entire phylum. here, we will just list a few representative examples of taxons across arthropods with documented antiviral role of RNAi.

RNAi is an antiviral system in mosquitos, where RNAi also comes into contact also with viruses that infect humans, such as Dengue Virus Type 2 (Sanchez-Vargas et al., 2009), O'nyong-nyong virus (Keene et al., 2004), Sindbis virus (Adelman et al., 2012; Campbell et al., 2008), West Nile virus (Paradkar et al., 2012), Rift Valley Fever virus (Leger et al., 2013), Arbovirus (Schnettler et al., 2013b; van Cleef et al., 2014), Mosinovirus (Schuster et al., 2014), Culex Y virus (van Cleef et al., 2014). Importantly, viruses facing RNAi-based innate immunity evolve different RNAi inhibitors, proteins, which interfere with various stages of siRNA biogenesis. Such proteins have been identified in most of the aforementioned viruses. Needless to say that antiviral RNAi was reported also from other hexapods, such as silk moth (Liu et al., 2015; Zografidis et al., 2015). Antiviral RNAi was also reported for Chelicerata (ticks and mites (Hoy et al., 2016; Schnettler et al., 2014)) or Crustacea (shrimps (Hoy et al., 2016; Huang and Zhang, 2013; Jariyapong et al., 2015; Maralit et al., 2015; Sabin and Cherry, 2013; Yang et al., 2014b).

\section{Endogenous RNAi in the germline and soma}

Severe defects in embryonic development have been noted in Drosophila mutants lacking $D c r-2$ or $A g o 2$, exposed to temperature perturbations while these mutants otherwise develop under standard laboratory conditions to normal adults with no specific phenotype (Lucchetta et al., 2009). This indicated that one of the functions of endo-siRNA pathway is to stabilize embryonic development under environmental stress (Lucchetta et al., 2009). Subsequent analysis of the distinct phenotypes in RNAi-defective mutants (Deshpande et al., 2005; Li et al., 2013) and RNA-seq data lead to recognition of diversity of the endogenous RNAi pathway and various types of endo-siRNAs.

A substantial source of Drosophila endo-siRNAs comes from mobile elements (Chung et al., 2008; Czech et al., 2008; Ghildiyal et al., 2008; Kawamura et al., 2008; Okamura et al., 2008a). Endo-siRNAs in somatic tissues and cultured cells thus complement piRNAs that are responsible for genome surveillance predominantly in the germline. Importantly, as endo-siRNAs and piRNAs were found that originate from the same loci, it is possible that piRNA and endo-siRNA pathways might be interdependent in repression of mobile elements in Drosophila (Ghildiyal et al., 2008). The notion of interdependence in mobile element repression also resonates with above-mentioned piRNA contribution to antiviral defense.

Other endo-siRNAs map to protein-coding genes and potentially contribute to control of gene expression. Among them a significant portion maps to protein-coding regions (Czech et al., 2008; Ghildiyal et al., 2008; Kawamura et al., 2008; Okamura et al., 2008a; Okamura et al., 2008b). However, only endo-siRNAs derived from a small number of loci are produced in sufficient amount to reduce target mRNA levels, as exemplified by the esi-2 locus-derived endo-siRNAs targeting DNA damage-response gene Mus-308 (Czech et al., 2008). 
Table 1 Numbers of annotated miRNAs in selected arthropods in miRBase 22.1. Note: only species with >100 annotated miRNA precursors are shown for Hexapoda

\begin{tabular}{|l|l|c|c|}
\hline subphylum & species & $\begin{array}{c}\text { miRNA } \\
\text { precursors }\end{array}$ & $\begin{array}{c}\text { mature } \\
\text { miRNA }\end{array}$ \\
\hline Chelicerata & Ixodes scapularis & 49 & 49 \\
\hline & Rhipicephalus microplus & 24 & 24 \\
\hline & Tetranychus urticae & 52 & 92 \\
\hline Myriapoda & Strigamia maritima & 3 & 4 \\
\hline Crustacea & Daphnia pulex & 44 & 45 \\
\hline & Triops canciformis & 148 & 160 \\
\hline & Aedes aegypti & 122 & 164 \\
\hline & Apis mellifera & 254 & 262 \\
\hline & Acyrthosiphon pisum & 123 & 97 \\
\hline & Bombyx mori & 487 & 469 \\
\hline & Drosophila melanogaster & 258 & 213 \\
\hline & Drosophila simulans & 148 & 328 \\
\hline & Drosophila virilis & 180 & 127 \\
\hline & Plutella xylostella & 133 & 590 \\
\hline & Tribolium castaneum & 342 & \\
\hline
\end{tabular}

Another type of Drosophila endo-siRNAs arises from overlapping antisense transcripts observed in hundreds of protein-coding loci (Okamura et al., 2008a). Abundance of such endo-siRNAs is generally low. Potential mRNA targets of such endo-siRNAs are not upregulated in Ago2-deficient flies, suggesting that these endo-siRNAs are not involved in posttranscriptional control of mRNA levels under physiological conditions (Czech et al., 2008). Interestingly, a dsRNA/endo-siRNA-binding protein Blanks, which associates with DCR-2 and forms an alternative Argonaute-independent functional RISC complex, has a role in spermatogenesis (Gerbasi et al., 2011). As Blanks deletion does not affect transposon activity, this finding suggests a role for endo-siRNAs in regulation of protein-coding mRNAs in Drosophila sperm development.

Yet another distinct type of endo-siRNAs are those derived from hairpin RNAs, whose biogenesis involves HEN1, canonical RNAi factors Dicer-2 and AGO2 plus miRNA factor Loquacious (Okamura et al., 2008c), specifically the LOQS-PD isoform(Zhou et al., 2009). One of the roles of hairpin RNA-derived endo-siRNAs is regulation of gene expression (Wen et al., 2015).

\section{Systemic RNAi}

Some insects, such as red flour beetle Tribalism, have efficient systemic RNAi where injection of adults causes RNAi effects in the progeny (Bucher et al., 2002; Tomoyasu et al., 2008). One of the well-known systemic RNAi factors is SID-1, a transmembrane protein transporting dsRNA across cell boundaries, which was first identified in C. elegans 
(Feinberg and Hunter, 2003). Ectopic expression of SID-1 is sufficient for permitting a systemic RNAi through dsRNA soaking in insect cells (Feinberg and Hunter, 2003; Mon et al., 2013; Shih and Hunter, 2011).

Non-cell autonomous RNAi exists across arthropods where different taxa have different numbers of sid-1 homologs. Importantly, some taxons lack systemic RNAi, which is not necessarily accompanied with the absence of sid-1 homologs; systemic RNAi can also be developmentally restricted or simply inefficient despite the expression of sid-1 homologs (Tomoyasu et al., 2008). Importantly, SID-1 is not the only system of systemic RNAi as was demonstrated in the locust model (Luo et al., 2012) or in Tribalism (Bucher et al., 2002; Tomoyasu et al., 2008).

There is a number of insect species, in which was found systemic RNAi or at least sid-1 homologs, include the aforementioned red flour beetle, Colorado potato beetle (Cappelle et al., 2016), juvenile grasshopper (Dong and Friedrich, 2005), brown planthopper (Xu et al., 2013), oriental leaf worm moth (Gong et al., 2015), diamondback moth (Wang et al., 2014), silk moth (Tomoyasu et al., 2008), honeybee (Honeybee Genome Sequencing, 2006), soybean aphid (Bansal and Michel, 2013), cotton/melon aphid (Xu and Han, 2008), grain aphid (Xu and Han, 2008). Beyond Hexapoda, there was also one report of sid-1 homolog in Crustacea (shrimp) (Labreuche et al., 2010; Maralit et al., 2015).

Sid-like genes were not found in Drosophila (Roignant et al., 2003), Hessian fly (Shreve et al., 2013), or mosquito Anopheles gambiae (Blandin et al., 2002). However, it should be kept in mind that this is not a conclusive evidence for absence of non-cell autonomous RNAi. For example, non-cell autonomous RNAi could be experimentally achieved also in Drosophila (Dzitoyeva et al., 2003) and it has been proposed that it would have a natural role in conjunction with antiviral RNAi in adult flies (Saleh et al., 2009).

\section{Dicer-dependent nuclear silencing}

Small RNAs in plants and fungi can mediate transcriptional silencing via chromatin remodelling/DNA methylation. A nuclear role and chromatin remodelling has been also attributed to the PIWI-loaded piRNA class of small RNAs protecting genome integrity in the germline. The role of Dicer-dependent small RNAs in transcriptional silencing in Metazoa is poorly understood and, in some cases, controversial. In any case, studies in Drosophila yielded some, albeit heterogeneous, evidence connecting Dicer-dependent small RNAs with transcriptional silencing and chromatin changes.

The best known small RNA-dependent transcriptional silencing mechanism is the piRNA pathway, which controls transcriptional silencing of retrotransposons (reviewed in $\mathrm{Fu}$ and Wang, 2014; Haase, 2016; Han and Zamore, 2014; Sato and Siomi, 2013). We do not cover the piRNA pathway as it is neither miRNA nor RNAi; piRNAs are not produced from a dsRNA but from complementary single-stranded transcripts through a concerted action of multiple factors. In any case, the piRNA pathway occasionally intersects with RNAi and miRNA pathways. For instance, it was shown that a functional miRNA pathway is required for the piRNA-mediated transcriptional silencing of mobile elements (Mugat et al., 2015). The mechanistic link seems to be provided by two specific miRNAs, miR-14 and miR-34 
(Mugat et al., 2015). This highlights the issue of discerning miRNA-mediated effects on chromatin and direct chromatin regulation by small RNAs.

A possible existence of transcriptional silencing mediated by Dicer-dependent small RNAs emerged from several analyses. First, it was found that AGO1 is found in the nucleus and cytosol in early embryos and that repeat induced silencing and transcriptional co-suppression were disrupted by Ago-1 mutation (Pushpavalli et al., 2012). The effect was accompanied by reduced H3K9me2 and H3K27me3 histone modifications (Pushpavalli et al., 2012). However, it is not clear whether this phenomenon is caused by a direct endo-siRNA-mediated heterochromatin induction or an indirect effect of miRNAs (similarly to the situation mentioned in the previous paragraph). Another possible bridge between the miRNA pathway and transcriptional regulation is Glioma amplified sequence41 (Gas41), a chromatin remodeler, implicated in repeat-induced transgene silencing, which also interacts with Dicer-1 (Gandhi et al., 2015). Others proposed that AGO1 (and Dicer-2) interacts with RNA pol II and contribute to heterochromatin formation (Kavi and Birchler, 2009).

AGO2 has been implicated in alternative splicing and transcriptional silencing, which included Polycomb group complex (associated with H3K27 methylation) (Taliaferro et al., 2013). Again, it is not clear how direct and indirect mechanism is responsible for the observed effects. RNAi machinery is not certainly an essential component of polycomb-mediated silencing as it was demonstrated that RNAi pathway is dispensable for the polycomb-mediated silencing of the homeotic Bithorax Complex (Cernilogar et al., 2013). $\mathrm{AGO} 2$ was also implicated in chromatin insulator function that would be independent of RNAi (Moshkovich et al., 2011). AGO2 was localized by chromatin immunoprecipitation to euchromatin but not heterochromatin and co-localized and physically interacted with CTCF/CP190 chromatin insulators (Moshkovich et al., 2011). AGO2, together with Dicer-2 and R2D2 was implicated in H3K9 methylation, suggesting that endo-siRNAs might regulate heterochromatin (Fagegaltier et al., 2009). A possible bridge between RNA silencing and chromatin could be VIG, the aforementioned RISC component (Gracheva et al., 2009). Chromatin-related factors also emerged from screens for RNAi and miRNA genes (Ghosh et al., 2014; Pressman et al., 2012).

Taken together, despite a relatively large volume of evidence, there is still not a consensus and a validated model explaining how would miRNAs or endo-siRNAs guide chromatin remodelling in Drosophila (or in arthropods in general).

\section{Other dsRNA response pathways in arthropods}

While RNAi is an important antiviral innate immunity mechanism in arthropods, it should be pointed out that it is not the only one and that the innate immunity of arthropods is much more complex. A study of five chelicerates, a myriapod, and a crustacean revealed traces of an ancient origin of innate immunity, with some arthropods having Toll-like receptors and $\mathrm{C} 3$-complement factors that are more closely related in sequence or structure to vertebrates than other arthropods (Palmer and Jiggins, 2015). Thus, apart from a robust and sequence-specific RNAi, arthropods also have a largely unexplored potential 
to mount a sequence-independent response to dsRNA (reviewed in Wang et al., 2015a). Such sequence-independent response would, for example, explain increased expression of apoptosis-related genes 24 hours upon exposing shrimps to encapsulated dsRNA (Jariyapong et al., 2015).In fact, shrimp has several interferon system-related genes such as dsRNA-dependent protein kinase PKR and Toll-like receptor 3, which are induced upon dsRNA exposure (Wang et al., 2013). Furthermore, Crustacea have many genes homologous to genes of the vertebrate interferon response suggesting that they might combine sequence-specific and sequence-independent innate immunity response to nucleic acids (Wang et al., 2013).

\section{$A D A R$}

Similarly to all previously discussed model systems, Drosophila (and presumably all arthropods) have A-to-I editing system. Drosophila has a single ADAR related to vertebrate ADAR2 (Barraud et al., 2012). ADAR is developmentally regulated and essential gene (Palladino et al., 2000). Drosophila ADAR edits convergent transcripts (Peters et al., 2003), antisense read-through transcripts of KP elements (Peters et al., 2003) as well as miRNAs (Chawla and Sokol, 2014). Drosophila ADAR edit primary microRNA (pri-miRNA) transcripts to alter the structural conformation of these precursors resulting in positive or negative modulation of miRNA expression or its activity (Chawla and Sokol, 2014; Cui et al., 2015). Despite its biochemical activity would imply it, there is no good evidence to show that ADAR antagonizes RNAi in Drosophila (Paro et al., 2012).

\section{Summary}

Taken together, arthropods are an extremely large and diverse taxon, characterized by an extended genetic separation of miRNA and RNAi pathways (Fig. 5). The separation is not complete and structure of small RNAs appearing in the system strongly influences their sorting onto AGO proteins. The main arthropod model system - Drosophila - lost the RdRP component of RNA silencing, which seems to be also the case for Hexapoda, Crustacea and Myriapoda but not Chelicerata.

\section{Acknowledgement}

I would like to thank my colleagues Jan Paces, Miloslav Nic, and Tomas Novotny for help with collecting literature for the review and Eliska Svobodova for help with phylogenetic analysis of Dicer. The review content was produced under a contract OC/ EFSA/GMO/2015/01-CT 01 with European Food Safety Authority (EFSA); the opinions expressed are those of the contractor only and do not represent EFSA's official position. Publication of the review was funded by LO1220 and LM2015063 by the Ministry of Education, Youth and Sports. 

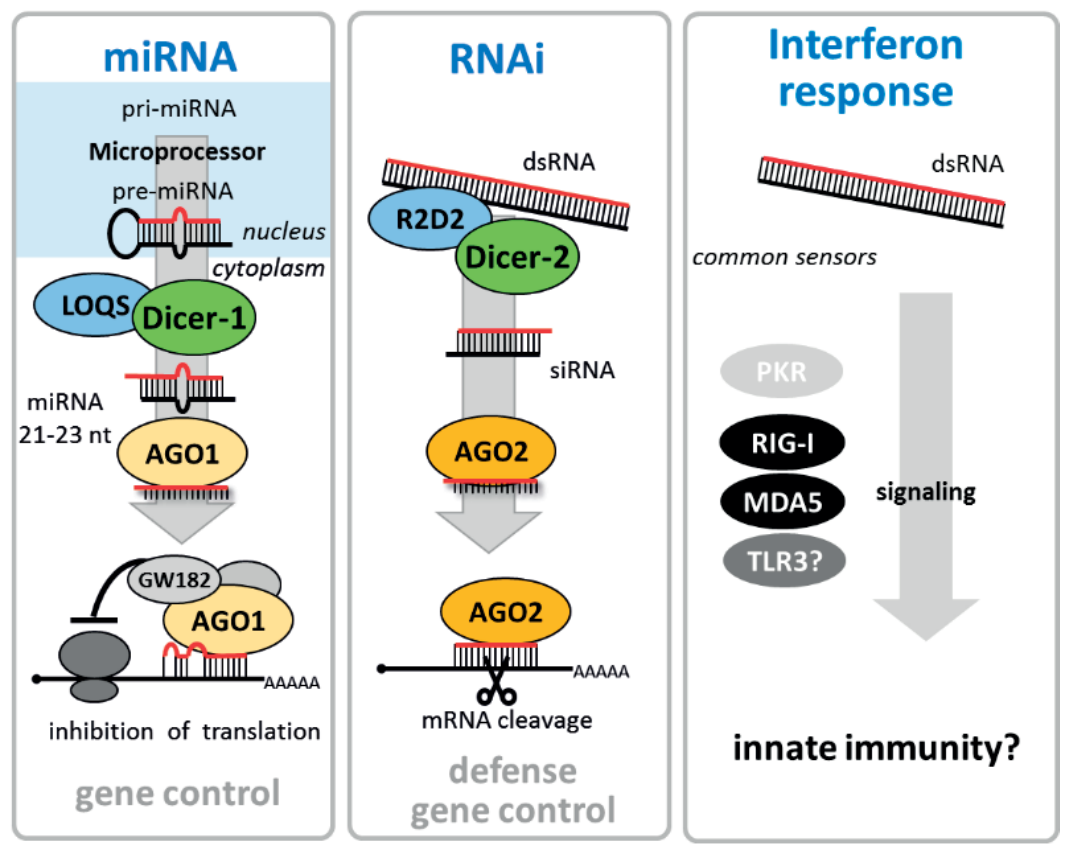

Figure 5 Overview of discussed arthropod pathways dsRNA and miRNA pathways in Arthropoda show separation of miRNA and RNAi pathways, each utilizing a more-or-less dedicated set of proteins for small RNA biogenesis and effector complex.

\section{References}

Abe, M., Naqvi, A., Hendriks, G.J., Feltzin, V., Zhu, Y.Q., Grigoriev, A., and Bonini, N.M. (2014). Impact of age-associated increase in 2 '-O-methylation of miRNAs on aging and neurodegeneration in Drosophila. Genes \& Development 28, 44-57.

Adelman, Z.N., Anderson, M.A.E., Liu, M., Zhang, L., and Myles, K.M. (2012). Sindbis virus induces the production of a novel class of endogenous siRNAs in Aedes aegypti mosquitoes. Insect Molecular Biology 21, 357-368.

Ameres, S.L., Horwich, M.D., Hung, J.H., Xu, J., Ghildiyal, M., Weng, Z.P., and Zamore, P.D. (2010). Target RNA-Directed Trimming and Tailing of Small Silencing RNAs. Science 328, 1534-1539.

Antic, S., Wolfinger, M.T., Skucha, A., Hosiner, S., and Dorner, S. (2015). General and MicroRNA-Mediated mRNA Degradation Occurs on Ribosome Complexes in Drosophila Cells. Molecular and Cellular Biology 35, 2309-2320.

Azzam, G., Smibert, P., Lai, E.C., and Liu, J.L. (2012). Drosophila Argonaute 1 and its miRNA biogenesis partners are required for oocyte formation and germline cell division. Developmental Biology 365, 384-394.

Bansal, R., and Michel, A.P. (2013). Core RNAi Machinery and Sid1, a Component for Systemic RNAi, in the Hemipteran Insect, Aphis glycines. International Journal of Molecular Sciences 14, 3786-3801. 
Barraud, P., Heale, B.S.E., O’Connell, M.A., and Allain, F.H.T. (2012). Solution structure of the N-terminal dsRBD of Drosophila ADAR and interaction studies with RNA. Biochimie 94, 1499-1509. Bartel, D.P. (2018). Metazoan MicroRNAs. Cell 173, 20-51.

Behm-Ansmant, I., Rehwinkel, J., Doerks, T., Stark, A., Bork, P., and Izaurralde, E. (2006). MRNA degradation by miRNAs and GW182 requires both CCR4 : NOT deadenylase and DCP1 : DCP2 decapping complexes. Genes \& Development 20, 1885-1898.

Berezikov, E., Robine, N., Samsonova, A., Westholm, J.O., Naqvi, A., Hung, J.H., Okamura, K., Dai, Q., Bortolamiol-Becet, D., Martin, R., et al. (2011). Deep annotation of Drosophila melanogaster microRNAs yields insights into their processing, modification, and emergence. Genome Research 21, 203-215.

Bernhardt, S.A., Simmons, M.P., Olson, K.E., Beaty, B.J., Blair, C.D., and Black, W.C. (2012). Rapid Intraspecific Evolution of miRNA and siRNA Genes in the Mosquito Aedes aegypti. Plos One 7, e44198-e44198.

Bernstein, E., Caudy, A.A., Hammond, S.M., and Hannon, G.J. (2001). Role for a bidentate ribonuclease in the initiation step of RNA interference. Nature 409, 363-366.

Blandin, S., Moita, L.F., Kocher, T., Wilm, M., Kafatos, F.C., and Levashina, E.A. (2002). Reverse genetics in the mosquito Anopheles gambiae: targeted disruption of the Defensin gene. EMBO Rep 3, 852-856.

Bozzetti, M.P., Specchia, V., Cattenoz, P.B., Laneve, P., Geusa, A., Sahin, H.B., Di Tommaso, S., Friscini, A., Massari, S., Diebold, C., et al. (2015). The Drosophila fragile X mental retardation protein participates in the piRNA pathway. Journal of Cell Science 128, 2070-2084.

Bucher, G., Scholten, J., and Klingler, M. (2002). Parental RNAi in Tribolium (Coleoptera). Curr Biol 12, R85-86.

Burroughs, A.M., Ando, Y., de Hoon, M.J.L., Tomaru, Y., Nishibu, T., Ukekawa, R., Funakoshi, T., Kurokawa, T., Suzuki, H., Hayashizaki, Y., et al. (2010). A comprehensive survey of 3 ' animal miRNA modification events and a possible role for 3 ' adenylation in modulating miRNA targeting effectiveness. Genome Research 20, 1398-1410.

Campbell, C.L., Keene, K.M., Brackney, D.E., Olson, K.E., Blair, C.D., Wilusz, J., and Foy, B.D. (2008). Aedes aegypti uses RNA interference in defense against Sindbis virus infection. BMC Microbiology 8, 47-47.

Cappelle, K., de Oliveira, C.F., Van Eynde, B., Christiaens, O., and Smagghe, G. (2016). The involvement of clathrin-mediated endocytosis and two Sid-1-like transmembrane proteins in double-stranded RNA uptake in the Colorado potato beetle midgut. Insect molecular biology.

Caudy, A.A., Ketting, R.F., Hammond, S.M., Denli, A.M., Bathoorn, A.M.P., Tops, B.B.J., Silva, J.M., Myers, M.M., Hannon, G.J., and Plasterk, R.H.A. (2003). A micrococcal nuclease homologue in RNAi effector complexes. Nature 425, 411-414.

Caudy, A.A., Myers, M., Hannon, G.J., and Hammond, S.M. (2002). Fragile X-related protein and VIG associate with the RNA interference machinery. Genes \& Development 16, 2491-2496.

Cenik, E.S., Fukunaga, R., Lu, G., Dutcher, R., Wang, Y.M., Hall, T.M.T., and Zamore, P.D. (2011). Phosphate and R2D2 Restrict the Substrate Specificity of Dicer-2, an ATP-Driven Ribonuclease. Molecular Cell 42, 172-184.

Cernilogar, F.M., Burroughs, A.M., Lanzuolo, C., Breiling, A., Imhof, A., and Orlando, V. (2013). RNA-Interference Components Are Dispensable for Transcriptional Silencing of the Drosophila Bithorax-Complex. Plos One 8, e65740-e65740. 
Cerutti, H., and Casas-Mollano, J.A. (2006). On the origin and functions of RNA-mediated silencing: from protists to man. Current genetics 50, 81-99.

Chak, L.L., Mohammed, J., Lai, E.C., Tucker-Kellogg, G., and Okamura, K. (2015). A deeply conserved, noncanonical miRNA hosted by ribosomal DNA. RNA 21, 375-384.

Chawla, G., and Sokol, N.S. (2014). ADAR mediates differential expression of polycistronic microRNAs. Nucleic Acids Research 42, 5245-5255.

Chekulaeva, M., Filipowicz, W., and Parker, R. (2009). Multiple independent domains of dGW182 function in miRNA-mediated repression in Drosophila. RNA 15, 794-803.

Chekulaeva, M., Mathys, H., Zipprich, J.T., Attig, J., Colic, M., Parker, R., and Filipowicz, W. (2011). miRNA repression involves GW182-mediated recruitment of CCR4-NOT through conserved W-containing motifs. Nature Structural \& Molecular Biology 18, 1218-U1262.

Chekulaeva, M., Parker, R., and Filipowicz, W. (2010). The GW/WG repeats of Drosophila GW182 function as effector motifs for miRNA-mediated repression. Nucleic Acids Research 38, 6673-6683.

Chen, Y.H., Jia, X.T., Zhao, L., Li, C.Z., Zhang, S.A., Chen, Y.G., Weng, S.P., and He, J.G. (2011). Identification and functional characterization of Dicer2 and five single VWC domain proteins of Litopenaeus vannamei. Developmental and Comparative Immunology 35, 661-671.

Chen, Y.H., Zhao, L., Jia, X.T., Li, X.Y., Li, C.Z., Yan, H., Weng, S.P., and He, J.G. (2012). Isolation and characterization of cDNAs encoding Ars2 and Pasha homologues, two components of the RNA interference pathway in Litopenaeus vannamei. Fish \& Shellfish Immunology 32, 373-380.

Chung, W.J., Okamura, K., Martin, R., and Lai, E.C. (2008). Endogenous RNA interference provides a somatic Defense against Drosophila transposons. Current Biology 18, 795-802.

Cui, Y.L., Huang, T.Z., and Zhang, X.B. (2015). RNA editing of microRNA prevents RNA-induced silencing complex recognition of target mRNA. Open Biology 5, 150126-150126.

Czech, B., Malone, C.D., Zhou, R., Stark, A., Schlingeheyde, C., Dus, M., Perrimon, N., Kellis, M., Wohlschlegel, J.A., Sachidanandam, R., et al. (2008). An endogenous small interfering RNA pathway in Drosophila. Nature 453, 798-U797.

Czech, B., Zhou, R., Erlich, Y., Brennecke, J., Binari, R., Villalta, C., Gordon, A., Perrimon, N., and Hannon, G.J. (2009). Hierarchical Rules for Argonaute Loading in Drosophila. Molecular Cell 36, 445-456.

Dechklar, M., Udomkit, A., and Panyim, S. (2008). Characterization of Argonaute cDNA from Penaeus monodon and implication of its role in RNA interference. Biochem Biophys Res Commun 367, 768-774.

Dekanty, A., Romero, N.M., Bertolin, A.P., Thomas, M.G., Leishman, C.C., Perez-Perri, J.I., Boccaccio, G.L., and Wappner, P. (2010). Drosophila Genome-Wide RNAi Screen Identifies Multiple Regulators of HIF-Dependent Transcription in Hypoxia. Plos Genetics 6, e1000994-e1000994.

Denli, A.M., Tops, B.B.J., Plasterk, R.H.A., Ketting, R.F., and Hannon, G.J. (2004). Processing of primary microRNAs by the Microprocessor complex. Nature 432, 231-235.

Deshpande, G., Calhoun, G., and Schedl, P. (2005). Drosophila argonaute-2 is required early in embryogenesis for the assembly of centric/centromeric heterochromatin, nuclear division, nuclear migration, and germ-cell formation. Genes \& Development 19, 1680-1685.

Dong, Y., and Friedrich, M. (2005). Nymphal RNAi: systemic RNAi mediated gene knockdown in juvenile grasshopper. BMC Biotechnology 5, 25-25.

Dzitoyeva, S., Dimitrijevic, N., and Manev, H. (2003). Gamma-aminobutyric acid B receptor 1 mediates behavior-impairing actions of alcohol in Drosophila: adult RNA interference and 
pharmacological evidence. Proceedings of the National Academy of Sciences of the United States of America 100, 5485-5490.

Eulalio, A., Huntzinger, E., and Izaurralde, E. (2008). GW182 interaction with Argonaute is essential for miRNA-mediated translational repression and mRNA decay. Nature Structural \& Molecular Biology 15, 346-353.

Eulalio, A., Tritschler, F., Buettner, R., Weichenrieder, O., Izaurralde, E., and Truffault, V. (2009). The RRM domain in GW182 proteins contributes to miRNA-mediated gene silencing. Nucleic Acids Research 37, 2974-2983.

Fagegaltier, D., Bouge, A.L., Berry, B., Poisot, E., Sismeiro, O., Coppee, J.Y., Theodore, L., Voinnet, O., and Antoniewski, C. (2009). The endogenous siRNA pathway is involved in heterochromatin formation in Drosophila. Proceedings of the National Academy of Sciences of the United States of America 106, 21258-21263.

Feinberg, E.H., and Hunter, C.P. (2003). Transport of dsRNA into cells by the transmembrane protein SID-1. Science 301, 1545-1547.

Feltzin, V.L., Khaladkar, M., Abe, M., Parisi, M., Hendriks, G.J., Kim, J., and Bonini, N.M. (2015). The exonuclease Nibbler regulates age-associated traits and modulates piRNA length in Drosophila. Aging Cell 14, 443-452.

Filippov, V., Solovyev, V., Filippova, M., and Gill, S.S. (2000). A novel type of RNase III family proteins in eukaryotes. Gene 245, 213-221.

Flynt, A.S., Greimann, J.C., Chung, W.J., Lima, C.D., and Lai, E.C. (2010). MicroRNA Biogenesis via Splicing and Exosome-Mediated Trimming in Drosophila. Molecular Cell 38, 900-907.

Forstemann, K., Horwich, M.D., Wee, L., Tomari, Y., and Zamore, P.D. (2007). Drosophila microRNAs are sorted into functionally distinct argonaute complexes after production by Dicer-1. Cell 130, 287-297.

Forstemann, K., Tomari, Y., Du, T.T., Vagin, V.V., Denli, A.M., Bratu, D.P., Klattenhoff, C., Theurkauf, W.E., and Zamore, P.D. (2005). Normal microRNA maturation and germ-line stem cell maintenance requires loquacious, a double-stranded RNA-binding domain protein. Plos Biology 3, 1187-1201.

$\mathrm{Fu}$, Q., and Wang, P.J. (2014). Mammalian piRNAs: Biogenesis, function, and mysteries. Spermatogenesis 4, e27889-e27889.

Fukaya, T., Iwakawa, H.-o., and Tomari, Y. (2014). MicroRNAs Block Assembly of eIF4F Translation Initiation Complex in Drosophila. Molecular Cell 56, 67-78.

Fukaya, T., and Tomari, Y. (2011). PABP is not essential for microRNA-mediated translational repression and deadenylation in vitro. EMBO J 30, 4998-5009.

Fukunaga, R., Colpan, C., Han, B.W., and Zamore, P.D. (2014). Inorganic phosphate blocks binding of pre-miRNA to Dicer-2 via its PAZ domain. EMBO J 33, 371-384.

Fukunaga, R., Han, B.W., Hung, J.H., Xu, J., Weng, Z.P., and Zamore, P.D. (2012). Dicer Partner Proteins Tune the Length of Mature miRNAs in Flies and Mammals. Cell 151, 533-546.

Galiana-Arnoux, D., Dostert, C., Schneemann, A., Hoffmann, J.A., and Imler, J.L. (2006). Essential function in vivo for Dicer-2 in host defense against RNA viruses in drosophila. Nature Immunology 7, 590-597.

Gandhi, S.G., Bag, I., Sengupta, S., Pal-Bhadra, M., and Bhadra, U. (2015). Drosophila oncogene Gas41 is an RNA interference modulator that intersects heterochromatin and the small interfering RNA pathway. Febs Journal 282, 153-173. 
Gerbasi, V.R., Preall, J.B., Golden, D.E., Powell, D.W., Cummins, T.D., and Sontheimer, E.J. (2011). Blanks, a nuclear siRNA/dsRNA-binding complex component, is required for Drosophila spermiogenesis. Proceedings of the National Academy of Sciences of the United States of America 108, 3204-3209.

Ghildiyal, M., Seitz, H., Horwich, M.D., Li, C.J., Du, T.T., Lee, S., Xu, J., Kittler, E.L.W., Zapp, M.L., Weng, Z.P., et al. (2008). Endogenous siRNAs derived from transposons and mRNAs in Drosophila somatic cells. Science 320, 1077-1081.

Ghildiyal, M., Xu, J., Seitz, H., Weng, Z.P., and Zamore, P.D. (2010). Sorting of Drosophila small silencing RNAs partitions microRNA* strands into the RNA interference pathway. RNA 16, 43-56.

Ghosh, S., Kakumani, P.K., Kumar, A., Malhotra, P., Mukherjee, S.K., and Bhatnagar, R.K. (2014). Genome wide screening of RNAi factors of Sf21 cells reveal several novel pathway associated proteins. BMC Genomics 15, 775-775.

Gomez-Orte, E., and Belles, X. (2009). MicroRNA-dependent metamorphosis in hemimetabolan insects. Proceedings of the National Academy of Sciences of the United States of America 106, 21678-21682.

Gong, L., Wang, Z., Wang, H., Qi, J., Hu, M., and Hu, Q. (2015). Core RNAi machinery and three Sid-1 related genes in Spodoptera litura (Fabricius). International Journal of Agriculture and Biology 17, 937-944.

Gracheva, E., Dus, M., and Elgin, S.C.R. (2009). Drosophila RISC Component VIG and Its Homolog Vig2 Impact Heterochromatin Formation. Plos One 4, e6182-e6182.

Haac, M.E., Anderson, M.A.E., Eggleston, H., Myles, K.M., and Adelman, Z.N. (2015). The hub protein loquacious connects the microRNA and short interfering RNA pathways in mosquitoes. Nucleic Acids Research 43, 3688-3700.

Haase, A.D. (2016). A Small RNA-Based Immune System Defends Germ Cells against Mobile Genetic Elements. Stem Cells International 2016, 7595791-7595791.

Han, B.W., Hung, J.H., Weng, Z.P., Zamore, P.D., and Ameres, S.L. (2011). The 3 '-to-5 ' Exoribonuclease Nibbler Shapes the 3 ' Ends of MicroRNAs Bound to Drosophila Argonaute1. Current Biology 21, 1878-1887.

Han, B.W., and Zamore, P.D. (2014). PiRNAs. Current Biology 24, R730-R733.

Hartig, J.V., Esslinger, S., Bottcher, R., Saito, K., and Forstemann, K. (2009). Endo-siRNAs depend on a new isoform of loquacious and target artificially introduced, high-copy sequences. EMBO J $28,2932-2944$.

Hartig, J.V., and Forstemann, K. (2011). Loqs-PD and R2D2 define independent pathways for RISC generation in Drosophila. Nucleic Acids Research 39, 3836-3851.

He, Y.D., and Zhang, X.B. (2012). Comprehensive characterization of viral miRNAs involved in white spot syndrome virus (WSSV) infection. RNA Biology 9, 1019-1029.

Herbert, K.M., Pimienta, G., DeGregorio, S.J., Alexandrov, A., and Steitz, J.A. (2013). Phosphorylation of DGCR8 Increases Its Intracellular Stability and Induces a Progrowth miRNA Profile. Cell Reports 5, 1070-1081.

Honeybee Genome Sequencing, C. (2006). Insights into social insects from the genome of the honeybee Apis mellifera. Nature 443, 931-949.

Horwich, M.D., Li, C.J., Matranga, C., Vagin, V., Farley, G., Wang, P., and Zamore, P.D. (2007). The Drosophila RNA methyltransferase, DmHen1, modifies germline piRNAs and single-stranded siRNAs in RISC. Current Biology 17, 1265-1272. 
Hoy, M.A., Waterhouse, R.M., Wu, K., Estep, A.S., Ioannidis, P., Palmer, W.J., Pomerantz, A.F., Simao, F.A., Thomas, J., Jiggins, F.M., et al. (2016). Genome sequencing of the phytoseiid predatory mite Metaseiulus occidentalis reveals completely atomised Hox genes and super-dynamic intron evolution. Genome biology and evolution.

Huang, H.D., Li, Y.J., Szulwach, K.E., Zhang, G.Q., Jin, P., and Chen, D.H. (2014). AGO3 Slicer activity regulates mitochondria-nuage localization of Armitage and piRNA amplification. Journal of Cell Biology 206, 217-230.

Huang, T.Z., Xu, D.D., and Zhang, X.B. (2012). Characterization of shrimp Drosha in virus infection. Fish \& Shellfish Immunology 33, 575-581.

Huang, T.Z., and Zhang, X.B. (2013). Host defense against DNA virus infection in shrimp is mediated by the siRNA pathway. European Journal of Immunology 43, 137-146.

Huntzinger, E., Braun, J.E., Heimstaedt, S., Zekri, L., and Izaurralde, E. (2010). Two PABPC1-binding sites in GW182 proteins promote miRNA-mediated gene silencing. EMBO J 29, 4146-4160.

Huntzinger, E., Kuzuoglu-Oeztuerk, D., Braun, J.E., Eulalio, A., Wohlbold, L., and Izaurralde, E. (2013). The interactions of GW182 proteins with PABP and deadenylases are required for both translational repression and degradation of miRNA targets. Nucleic Acids Research 41, 978-994.

Ishizuka, A., Siomi, M.C., and Siomi, H. (2002). A Drosophila fragile X protein interacts with components of RNAi and ribosomal proteins. Genes \& Development 16, 2497-2508.

Iwasaki, S., Kawamata, T., and Tomari, Y. (2009). Drosophila Argonaute1 and Argonaute2 Employ Distinct Mechanisms for Translational Repression. Molecular Cell 34, 58-67.

Iwasaki, S., Sasaki, H.M., Sakaguchi, Y., Suzuki, T., Tadakuma, H., and Tomari, Y. (2015). Defining fundamental steps in the assembly of the Drosophila RNAi enzyme complex. Nature 521, 533-U274.

Jariyapong, P., Weerachatyanukul, W., Direkbusarakom, S., Hirono, I., Wuthisuthimethavee, S., and Chotwiwatthanakun, C. (2015). Enhancement of shrimp immunity against white spot syndrome virus by Macrobrachium rosenbergii nodavirus-like particle encapsulated VP28 double-stranded RNA. Aquaculture 446, 325-332.

Jaskiewicz, L., and Filipowicz, W. (2008). Role of Dicer in posttranscriptional RNA silencing. Current topics in microbiology and immunology 320, 77-97.

Jaubert-Possamai, S., Rispe, C., Tanguy, S., Gordon, K., Walsh, T., Edwards, O., and Tagu, D. (2010). Expansion of the miRNA Pathway in the Hemipteran Insect Acyrthosiphon pisum. Molecular Biology and Evolution 27, 979-987.

Jiang, F., Ye, X.C., Liu, X., Fincher, L., McKearin, D., and Liu, Q.H. (2005). Dicer-1 and R3D1-L catalyze microRNA maturation in Drosophila. Genes \& Development 19, 1674-1679.

Jiang, F.F., Lu, F.L., Li, P.X., Liu, W., Zhao, L., Wang, Q.F., Cao, X.F., Zhang, L., and Zhang, Y.Q. (2016). Drosophila Homolog of FMRP Maintains Genome Integrity by Interacting with Piwi. Journal of Genetics and Genomics 43, 11-24.

Jin, Z.G., and Xie, T. (2007). Dcr-1 maintains Drosophila ovarian stem cells. Current Biology 17, 539-544.

Kadener, S., Rodriguez, J., Abruzzi, K.C., Khodor, Y.L., Sugino, K., Marr, M.T., Nelson, S., and Rosbash, M. (2009). Genome-wide identification of targets of the drosha-pasha/DGCR8 complex. RNA 15, 537-545.

Kavi, H.H., and Birchler, J.A. (2009). Interaction of RNA polymerase II and the small RNA machinery affects heterochromatic silencing in Drosophila. Epigenetics \& Chromatin 2, 15-15. 
Kawamata, T., Seitz, H., and Tomari, Y. (2009). Structural determinants of miRNAs for RISC loading and slicer-independent unwinding. Nature Structural \& Molecular Biology 16, 953-U977.

Kawamura, Y., Saito, K., Kin, T., Ono, Y., Asai, K., Sunohara, T., Okada, T.N., Siomi, M.C., and Siomi, H. (2008). Drosophila endogenous small RNAs bind to Argonaute 2 in somatic cells. Nature 453, 793-U795.

Keene, K.M., Foy, B.D., Sanchez-Vargas, I., Beaty, B.J., Blair, C.D., and Olson, K.E. (2004). RNA interference acts as a natural antiviral response to O'nyong-nyong virus (Alphavirus; Togaviridae) infection of Anopheles gambiae. Proceedings of the National Academy of Sciences of the United States of America 101, 17240-17245.

Kim, K., Lee, Y.S., and Carthew, R.W. (2007). Conversion of pre-RISC to holo-RISC by Ago2 during assembly of RNAi complexes. RNA 13, 22-29.

Kim, K., Lee, Y.S., Harris, D., Nakahara, K., and Carthew, R.W. (2006). The RNAi pathway initiated by Dicer-2 in Drosophila. Cold Spring Harbor Symposia on Quantitative Biology 71, 39-44.

Kolliopoulou, A., and Swevers, L. (2013). Functional analysis of the RNAi response in ovary-derived silkmoth Bm5 cells. Insect Biochemistry and Molecular Biology 43, 654-663.

Kozomara, A., and Griffiths-Jones, S. (2014). miRBase: annotating high confidence microRNAs using deep sequencing data. Nucleic Acids Res 42, D68-73.

Ku, H.Y., Gangaraju, V.K., Qi, H.Y., Liu, N., and Lin, H.F. (2016). Tudor-SN Interacts with Piwi Antagonistically in Regulating Spermatogenesis but Synergistically in Silencing Transposons in Drosophila. Plos Genetics 12, e1005813-e1005813.

Kurscheid, S., Lew-Tabor, A.E., Valle, M.R., Bruyeres, A.G., Doogan, V.J., Munderloh, U.G., Guerrero, F.D., Barrero, R.A., and Bellgard, M.I. (2009). Evidence of a tick RNAi pathway by comparative genomics and reverse genetics screen of targets with known loss-of-function phenotypes in Drosophila. BMC Molecular Biology 10, 26-26.

Kuzuoglu-Ozturk, D., Bhandari, D., Huntzinger, E., Fauser, M., Helms, S., and Izaurralde, E. (2016). miRISC and the CCR4-NOT complex silence mRNA targets independently of 43S ribosomal scanning. EMBO J.

Labreuche, Y., Veloso, A., de la Vega, E., Gross, P.S., Chapman, R.W., Browdy, C.L., and Warr, G.W. (2010). Non-specific activation of antiviral immunity and induction of RNA interference may engage the same pathway in the Pacific white leg shrimp Litopenaeus vannamei. Developmental and Comparative Immunology 34, 1209-1218.

Landthaler, M., Yalcin, A., and Tuschl, T. (2004). The human DiGeorge syndrome critical region gene 8 and its D-melanogaster homolog are required for miRNA biogenesis. Current Biology 14, 2162-2167.

Lau, P.W., Guiley, K.Z., De, N., Potter, C.S., Carragher, B., and MacRae, I.J. (2012). The molecular architecture of human Dicer. Nature Structural \& Molecular Biology 19, 436-440.

Lee, M., Choi, Y., Kim, K., Jin, H., Lim, J., Nguyen, T.A., Yang, J., Jeong, M., Giraldez, A.J., Yang, H., et al. (2014). Adenylation of maternally inherited microRNAs by Wispy. Molecular Cell 56, 696-707.

Lee, Y.S., Nakahara, K., Pham, J.W., Kim, K., He, Z.Y., Sontheimer, E.J., and Carthew, R.W. (2004). Distinct roles for Drosophila Dicer-1 and Dicer-2 in the siRNA/miRNA silencing pathways. Cell 117, 69-81.

Leebonoi, W., Sukthaworn, S., Panyim, S., and Udomkit, A. (2015). A novel gonad-specific Argonaute 4 serves as a defense against transposons in the black tiger shrimp Penaeus monodon. Fish \& Shellfish Immunology 42, 280-288. 
Leger, P., Lara, E., Jagla, B., Sismeiro, O., Mansuroglu, Z., Coppee, J.Y., Bonnefoy, E., and Bouloy, M. (2013). Dicer-2-and Piwi-Mediated RNA Interference in Rift Valley Fever Virus-Infected Mosquito Cells. Journal of Virology 87, 1631-1648.

Lewis, S.H., Salmela, H., and Obbard, D.J. (2016). Duplication and Diversification of Dipteran Argonaute Genes, and the Evolutionary Divergence of Piwi and Aubergine. Genome biology and evolution 8, 507-518.

Li, H., Li, W.X., and Ding, S.W. (2002). Induction and suppression of RNA silencing by an animal virus. Science 296, 1319-1321.

Li, W.H., Prazak, L., Chatterjee, N., Gruninger, S., Krug, L., Theodorou, D., and Dubnau, J. (2013). Activation of transposable elements during aging and neuronal decline in Drosophila. Nature Neuroscience 16, 529-+.

Lim, S.J., Scott, A., Xiong, X.P., Vahidpour, S., Karijolich, J., Guo, D.D., Pei, S.S., Yu, Y.T., Zhou, R., and Li, W.X. (2014). Requirement for CRIF1 in RNA interference and Dicer-2 stability. RNA Biology 11, 1171-1179.

Lipardi, C., and Paterson, B.M. (2009). Identification of an RNA-dependent RNA polymerase in Drosophila involved in RNAi and transposon suppression (Retracted article. See vol. 108, pg. 15010, 2011). Proceedings of the National Academy of Sciences of the United States of America $106,15645-15650$.

Lipardi, C., and Paterson, B.M. (2011). Retraction for Lipardi and Paterson, "Identification of an RNA-dependent RNA polymerase in Drosophila involved in RNAi and transposon suppression". Proceedings of the National Academy of Sciences of the United States of America 108, 15010.

Lipardi, C., Wei, Q., and Paterson, B.M. (2001). RNAi as random degradative PCR: siRNA primers convert mRNA into dsRNAs that are degraded to generate new siRNAs. Cell 107, 297-307.

Liu, N., Abe, M., Sabin, L.R., Hendriks, G.J., Naqvi, A.S., Yu, Z.M., Cherry, S., and Bonini, N.M. (2011). The Exoribonuclease Nibbler Controls 3 ' End Processing of MicroRNAs in Drosophila. Current Biology 21, 1888-1893.

Liu, Q.H., Rand, T.A., Kalidas, S., Du, F.H., Kim, H.E., Smith, D.P., and Wang, X.D. (2003). R2D2, a bridge between the initiation and effector steps of the Drosophila RNAi pathway. Science 301, 1921-1925.

Liu, W., Liu, J.B., Lu, Y.H., Gong, Y.C., Zhu, M., Chen, F., Liang, Z., Zhu, L.Y., Kuang, S.L., Hu, X.L., et al. (2015). Immune signaling pathways activated in response to different pathogenic micro-organisms in Bombyx mori. Molecular Immunology 65, 391-397.

Liu, Y., Ye, X.C., Jiang, F., Liang, C.Y., Chen, D.M., Peng, J.M., Kinch, L.N., Grishin, N.V., and Liu, Q.H. (2009). C3PO, an Endoribonuclease That Promotes RNAi by Facilitating RISC Activation. Science $325,750-753$.

Lozano, J., Gomez-Orte, E., Lee, H.J., and Belles, X. (2012). Super-induction of Dicer-2 expression by alien double-stranded RNAs: an evolutionary ancient response to viral infection? Development Genes and Evolution 222, 229-235.

Lucchetta, E.M., Carthew, R.W., and Ismagilov, R.F. (2009). The Endo-siRNA Pathway Is Essential for Robust Development of the Drosophila Embryo. Plos One 4, e7576-e7576.

Luhur, A., Chawla, G., Wu, Y.C., Li, J., and Sokol, N.S. (2014). Drosha-independent DGCR8/Pasha pathway regulates neuronal morphogenesis. Proceedings of the National Academy of Sciences of the United States of America 111, 1421-1426. 
Luo, Y., Wang, X.H., Yu, D., and Kang, L. (2012). The SID-1 double-stranded RNA transporter is not required for systemic RNAi in the migratory locust. RNA Biology 9, 663-671.

MacRae, I.J., Zhou, K., and Doudna, J.A. (2007). Structural determinants of RNA recognition and cleavage by Dicer. Nature Structural and Molecular Biology 14, 934-940.

MacRae, I.J., Zhou, K., Li, F., Repic, A., Brooks, A.N., Cande, W.Z., Adams, P.D., and Doudna, J.A. (2006). Structural basis for double-stranded RNA processing by Dicer. Science 311, 195-198.

Maralit, B.A., Komatsu, M., Hipolito, S.G., Hirono, I., and Kondo, H. (2015). Microarray Analysis of Immunity Against WSSV in Response to Injection of Non-specific Long dsRNA in Kuruma Shrimp, Marsupenaeus japonicus. Marine Biotechnology 17, 493-501.

Margulis, L., and Schwartz, K.V. (1998). Five kingdoms : an illustrated guide to the phyla of life on earth, 3rd edn (New York: W.H. Freeman).

Marques, J.T., Kim, K., Wu, P.H., Alleyne, T.M., Jafari, N., and Carthew, R.W. (2010). Loqs and R2D2 act sequentially in the siRNA pathway in Drosophila. Nature Structural \& Molecular Biology 17, 24-U37.

Martin, R., Smibert, P., Yalcin, A., Tyler, D.M., Schafer, U., Tuschl, T., and Lai, E.C. (2009). A Drosophila pasha Mutant Distinguishes the Canonical MicroRNA and Mirtron Pathways. Molecular and Cellular Biology 29, 861-870.

Matranga, C., Tomari, Y., Shin, C., Bartel, D.P., and Zamore, P.D. (2005). Passenger-strand cleavage facilitates assembly of siRNA into Ago2-containing RNAi enzyme complexes. Cell 123, 607-620.

Miyoshi, K., Miyoshi, T., Hartig, J.V., Siomi, H., and Siomi, M.C. (2010a). Molecular mechanisms that funnel RNA precursors into endogenous small-interfering RNA and microRNA biogenesis pathways in Drosophila. RNA 16, 506-515.

Miyoshi, K., Okada, T.N., Siomi, H., and Siomi, M.C. (2009). Characterization of the miRNA-RISC loading complex and miRNA-RISC formed in the Drosophila miRNA pathway. RNA 15, 1282-1291.

Miyoshi, K., Tsukumo, H., Nagami, T., Siomi, H., and Siomi, M.C. (2005). Slicer function of Drosophila Argonautes and its involvement in RISC formation. Genes \& Development 19, 2837-2848.

Miyoshi, T., Takeuchi, A., Siomi, H., and Siomi, M.C. (2010b). A direct role for Hsp90 in pre-RISC formation in Drosophila. Nature Structural \& Molecular Biology 17, 1024-1026.

Mon, H., Li, Z., Kobayashi, I., Tomita, S., Lee, J., Sezutsu, H., Tamura, T., and Kusakabe, T. (2013). Soaking RNAi in Bombyx mori BmN4-SID1 cells arrests cell cycle progression. Journal of Insect Science 13, 155-155.

Morazzani, E.M., Wiley, M.R., Murreddu, M.G., Adelman, Z.N., and Myles, K.M. (2012). Production of Virus-Derived Ping-Pong-Dependent piRNA-like Small RNAs in the Mosquito Soma. Plos Pathogens 8, e1002470-e1002470.

Moretti, F., Kaiser, C., Zdanowicz-Specht, A., and Hentze, M.W. (2012). PABP and the poly(A) tail augment microRNA repression by facilitated miRISC binding. Nature Structural \& Molecular Biology 19, 603-+.

Moshkovich, N., Nisha, P., Boyle, P.J., Thompson, B.A., Dale, R.K., and Lei, E.P. (2011). RNAi-independent role for Argonaute2 in CTCF/CP190 chromatin insulator function. Genes \& Development $25,1686-1701$.

Mugat, B., Akkouche, A., Serrano, V., Armenise, C., Li, B., Brun, C., Fulga, T.A., Van Vactor, D., Pelisson, A., and Chambeyron, S. (2015). MicroRNA-Dependent Transcriptional Silencing of Transposable Elements in Drosophila Follicle Cells. Plos Genetics 11, e1005194-e1005194. 
Murota, Y., Ishizu, H., Nakagawa, S., Iwasaki, Y.W., Shibata, S., Kamatani, M.K., Saito, K., Okano, H., Siomi, H., and Siomi, M.C. (2014). Yb Integrates piRNA Intermediates and Processing Factors into Perinuclear Bodies to Enhance piRISC Assembly. Cell Reports 8, 103-113.

Murphy, D., Dancis, B., and Brown, J.R. (2008). The evolution of core proteins involved in microRNA biogenesis. BMC Evolutionary Biology 8, 92-92.

Nagao, A., Mituyama, T., Huang, H.D., Chen, D.H., Siomi, M.C., and Siomi, H. (2010). Biogenesis pathways of piRNAs loaded onto AGO3 in the Drosophila testis. RNA 16, 2503-2515.

Nayak, A., Berry, B., Tassetto, M., Kunitomi, M., Acevedo, A., Deng, C.H., Krutchinsky, A., Gross, J., Antoniewski, C., and Andino, R. (2010). Cricket paralysis virus antagonizes Argonaute 2 to modulate antiviral defense in Drosophila. Nature Structural \& Molecular Biology 17, 547-U541.

Nishida, K.M., Miyoshi, K., Ogino, A., Miyoshi, T., Siomi, H., and Siomi, M.C. (2013). Roles of R2D2, a Cytoplasmic D2 Body Component, in the Endogenous siRNA Pathway in Drosophila. Molecular Cell 49, 680-691.

Niu, J.Z., Smagghe, G., De Coninck, D.I.M., Van Nieuwerburgh, F., Deforce, D., and Meeus, I. (2016). In vivo study of Dicer-2-mediated immune response of the small interfering RNA pathway upon systemic infections of virulent and avirulent viruses in Bombus terrestris. Insect Biochemistry and Molecular Biology 70, 127-137.

Nykanen, A., Haley, B., and Zamore, P.D. (2001). ATP requirements and small interfering RNA structure in the RNA interference pathway. Cell 107, 309-321.

Okamura, K., Balla, S., Martin, R., Liu, N., and Lai, E.C. (2008a). Two distinct mechanisms generate endogenous siRNAs from bidirectional transcription in Drosophila melanogaster. Nature Structural \& Molecular Biology 15, 581-590.

Okamura, K., Chung, W.J., and Lai, E.C. (2008b). The long and short of inverted repeat genes in animals - microRNAs, mirtrons and hairpin RNAs. Cell Cycle 7, 2840-2845.

Okamura, K., Chung, W.J., Ruby, J.G., Guo, H.L., Bartel, D.P., and Lai, E.C. (2008c). The Drosophila hairpin RNA pathway generates endogenous short interfering RNAs. Nature 453, 803-U808.

Okamura, K., Hagen, J.W., Duan, H., Tyler, D.M., and Lai, E.C. (2007). The mirtron pathway generates microRNA-class regulatory RNAs in Drosophila. Cell 130, 89-100.

Okamura, K., Ladewig, E., Zhou, L., and Lai, E.C. (2013). Functional small RNAs are generated from select miRNA hairpin loops in flies and mammals. Genes \& Development 27, 778-792.

Okamura, K., Liu, N., and Lai, E.C. (2009). Distinct Mechanisms for MicroRNA Strand Selection by Drosophila Argonautes. Molecular Cell 36, 431-444.

Okamura, K., Robine, N., Liu, Y., Liu, Q.H., and Lai, E.C. (2011). R2D2 Organizes Small Regulatory RNA Pathways in Drosophila. Molecular and Cellular Biology 31, 884-896.

Ortiz-Rivas, B., Jaubert-Possamai, S., Tanguy, S., Gauthier, J.P., Tagu, D., and Claude, R. (2012). Evolutionary study of duplications of the miRNA machinery in aphids associated with striking rate acceleration and changes in expression profiles. BMC Evolutionary Biology 12, 216-216.

Palladino, M.J., Keegan, L.P., O’Connell, M.A., and Reenan, R.A. (2000). dADAR, a Drosophila double-stranded RNA-specific adenosine deaminase is highly developmentally regulated and is itself a target for RNA editing. RNA 6, 1004-1018.

Palmer, W.J., and Jiggins, F.M. (2015). Comparative Genomics Reveals the Origins and Diversity of Arthropod Immune Systems. Molecular Biology and Evolution 32, 2111-2129.

Paradkar, P.N., Trinidad, L., Voysey, R., Duchemin, J.B., and Walker, P.J. (2012). Secreted Vago restricts West Nile virus infection in Culex mosquito cells by activating the Jak-STAT 
pathway. Proceedings of the National Academy of Sciences of the United States of America 109, 18915-18920.

Paro, S., Li, X.H., O’Connell, M.A., and Keegan, L.P. (2012). Regulation and Functions of ADAR in Drosophila. In Adenosine Deaminases Acting on Rna, pp. 221-236.

Pasquinelli, A.E., Reinhart, B.J., Slack, F., Martindale, M.Q., Kuroda, M.I., Maller, B., Hayward, D.C., Ball, E.E., Degnan, B., Muller, P., et al. (2000). Conservation of the sequence and temporal expression of let-7 heterochronic regulatory RNA. Nature 408, 86-89.

Peters, L., and Meister, G. (2007). Argonaute proteins: mediators of RNA silencing. Molecular Cell 26, 611-623.

Peters, N.T., Rohrbach, J.A., Zalewski, B.A., Byrkett, C.M., and Vaughn, J.C. (2003). RNA editing and regulation of Drosophila $4 \mathrm{f}-\mathrm{rnp}$ expression by sas-10 antisense readthrough mRNA transcripts. RNA 9, 698-710.

Pham, J.W., Pellino, J.L., Lee, Y.S., Carthew, R.W., and Sontheimer, E.J. (2004). A Dicer-2-dependent 80S complex cleaves targeted mRNAs during RNAi in Drosophila. Cell 117, 83-94.

Phetrungnapha, A., Ho, T., Udomkit, A., Panyim, S., and Ongvarrasopone, C. (2013). Molecular cloning and functional characterization of Argonaute-3 gene from Penaeus monodon. Fish \& Shellfish Immunology 35, 874-882.

Pinder, B.D., and Smibert, C.A. (2013). microRNA-independent recruitment of Argonaute 1 to nanos mRNA through the Smaug RNA-binding protein. EMBO Rep 14, 80-86.

Pressman, S., Reinke, C.A., Wang, X.H., and Carthew, R.W. (2012). A Systematic Genetic Screen to Dissect the MicroRNA Pathway in Drosophila. G3-Genes Genomes Genetics 2, 437-448.

Provost, P., Dishart, D., Doucet, J., Frendewey, D., Samuelsson, B., and Radmark, O. (2002). Ribonuclease activity and RNA binding of recombinant human Dicer. EMBO J 21, 5864-5874.

Pushpavalli, S., Bag, I., Pal-Bhadra, M., and Bhadra, U. (2012). Drosophila Argonaute-1 is critical for transcriptional cosuppression and heterochromatin formation. Chromosome Research 20, 333-351.

Qi, H., Watanabe, T., Ku, H.-Y., Liu, N., Zhong, M., and Lin, H. (2011). The Yb Body, a Major Site for Piwi-associated RNA Biogenesis and a Gateway for Piwi Expression and Transport to the Nucleus in Somatic Cells. Journal of Biological Chemistry 286, 3789-3797.

Regier, J.C., Shultz, J.W., Zwick, A., Hussey, A., Ball, B., Wetzer, R., Martin, J.W., and Cunningham, C.W. (2010). Arthropod relationships revealed by phylogenomic analysis of nuclear protein-coding sequences. Nature 463, 1079-1083.

Rehwinkel, J., Behm-Ansmant, I., Gatfield, D., and Izaurralde, E. (2005). A crucial role for GW182 and the DCP1 : DCP2 decapping complex in miRNA-mediated gene silencing. RNA 11, 1640-1647.

Reimao-Pinto, M.M., Ignatova, V., Burkard, T.R., Hung, J.H., Manzenreither, R.A., Sowemimo, I., Herzog, V.A., Reichholf, B., Farina-Lopez, S., and Ameres, S.L. (2015). Uridylation of RNA Hairpins by Tailor Confines the Emergence of MicroRNAs in Drosophila. Molecular Cell 59, 203-216.

Roignant, J.Y., Carre, C., Mugat, B., Szymczak, D., Lepesant, J.A., and Antoniewski, C. (2003). Absence of transitive and systemic pathways allows cell-specific and isoform-specific RNAi in Drosophila. RNA 9, 299-308.

Ruby, J.G., Jan, C.H., and Bartel, D.P. (2007). Intronic microRNA precursors that bypass Drosha processing. Nature $448,83-86$. 
Sabin, L.R., and Cherry, S. (2013). Small creatures use small RNAs to direct antiviral defenses. European Journal of Immunology 43, 27-33.

Saito, K., Ishizu, H., Komai, M., Kotani, H., Kawamura, Y., Nishida, K.M., Siomi, H., and Siomi, M.C. (2010). Roles for the $\mathrm{Yb}$ body components Armitage and $\mathrm{Yb}$ in primary piRNA biogenesis in Drosophila. Genes \& Development 24, 2493-2498.

Saito, K., Ishizuka, A., Siomi, H., and Siomi, M.C. (2005). Processing of pre-microRNAs by the Dicer-1-Loquacious complex in Drosophila cells. Plos Biology 3, 1202-1212.

Saito, K., Sakaguchi, Y., Suzuki, T., Suzuki, T., Siomi, H., and Siomi, M.C. (2007). Pimet, the Drosophila homolog of HEN1, mediates 2 '-O-methylation of PIWI-interacting RNAs at their 3 ' ends. Genes \& Development 21, 1603-1608.

Saleh, M.C., Tassetto, M., van Rij, R.P., Goic, B., Gausson, V., Berry, B., Jacquier, C., Antoniewski, C., and Andino, R. (2009). Antiviral immunity in Drosophila requires systemic RNA interference spread. Nature 458, 346-350.

Sanchez-Vargas, I., Scott, J.C., Poole-Smith, B.K., Franz, A.W.E., Barbosa-Solomieu, V., Wilusz, J., Olson, K.E., and Blair, C.D. (2009). Dengue Virus Type 2 Infections of Aedes aegypti Are Modulated by the Mosquito's RNA Interference Pathway. Plos Pathogens 5, e1000299-e1000299.

Sato, K., and Siomi, M.C. (2013). Piwi-interacting RNAs: biological functions and biogenesis. Essays in biochemistry $54,39-52$.

Schnettler, E., Donald, C.L., Human, S., Watson, M., Siu, R.W.C., McFarlane, M., Fazakerley, J.K., Kohl, A., and Fragkoudis, R. (2013a). Knockdown of piRNA pathway proteins results in enhanced Semliki Forest virus production in mosquito cells. Journal of General Virology 94, 1680-1689.

Schnettler, E., Ratinier, M., Watson, M., Shaw, A.E., McFarlane, M., Varela, M., Elliott, R.M., Palmarini, M., and Kohl, A. (2013b). RNA Interference Targets Arbovirus Replication in Culicoides Cells. Journal of Virology 87, 2441-2454.

Schnettler, E., Tykalova, H., Watson, M., Sharma, M., Sterken, M.G., Obbard, D.J., Lewis, S.H., McFarlane, M., Bell-Sakyi, L., Barry, G., et al. (2014). Induction and suppression of tick cell antiviral RNAi responses by tick-borne flaviviruses. Nucleic Acids Research 42, 9436-9446.

Schuster, S., Zirkel, F., Kurth, A., van Cleef, K.W.R., Drosten, C., van Rij, R.P., and Junglen, S. (2014). A Unique Nodavirus with Novel Features: Mosinovirus Expresses Two Subgenomic RNAs, a Capsid Gene of Unknown Origin, and a Suppressor of the Antiviral RNA Interference Pathway. Journal of Virology 88, 13447-13459.

Schwarz, D.S., Hutvagner, G., Haley, B., and Zamore, P.D. (2002). Evidence that siRNAs function as guides, not primers, in the Drosophila and human RNAi pathways. Molecular Cell 10, 537-548.

Schwarz, D.S., Tomari, Y., and Zamore, P.D. (2004). The RNA-induced silencing complex is a Mg2+-dependent endonuclease. Current Biology 14, 787-791.

Shih, J.D., and Hunter, C.P. (2011). SID-1 is a dsRNA-selective dsRNA-gated channel. RNA 17, 1057-1065.

Shreve, J.T., Shukle, R.H., Subramanyam, S., Johnson, A.J., Schemerhorn, B.J., Williams, C.E., and Stuart, J.J. (2013). A genome-wide survey of small interfering RNA and microRNA pathway genes in a galling insect. Journal of Insect Physiology 59, 367-376.

Sinha, N.K., Trettin, K.D., Aruscavage, P.J., and Bass, B.L. (2015). Drosophila Dicer-2 Cleavage Is Mediated by Helicase- and dsRNA Termini-Dependent States that Are Modulated by Loquacious-PD. Molecular Cell 58, 406-417. 
Smibert, P., Bejarano, F., Wang, D., Garaulet, D.L., Yang, J.S., Martin, R., Bortolamiol-Becet, D., Robine, N., Hiesinger, P.R., and Lai, E.C. (2011). A Drosophila genetic screen yields allelic series of core microRNA biogenesis factors and reveals post-developmental roles for microRNAs. RNA 17, 1997-2010.

Song, J.S., Guo, W., Jiang, F., Kang, L., and Zhou, S.T. (2013). Argonaute 1 is indispensable for juvenile hormone mediated oogenesis in the migratory locust, Locusta migratoria. Insect Biochemistry and Molecular Biology 43, 879-887.

Su, J.S., Oanh, D.T.H., Lyons, R.E., Leeton, L., van Hulten, M.C.W., Tan, S.H., Song, L., Rajendran, K.V., and Walker, P.J. (2008). A key gene of the RNA interference pathway in the black tiger shrimp, Penaeus monodon: Identification and functional characterisation of Dicer-1. Fish \& Shellfish Immunology 24, 223-233.

Svoboda, P. (2019). Introduction to RNAi and miRNA pathways.

Taliaferro, J.M., Aspden, J.L., Bradley, T., Marwha, D., Blanchette, M., and Rio, D.C. (2013). Two new and distinct roles for Drosophila Argonaute-2 in the nucleus: alternative pre-mRNA splicing and transcriptional repression. Genes \& Development 27, 378-389.

Tomari, Y., Du, T., and Zamore, P.D. (2007). Sorting of Drosophila small silencing RNAs. Cell 130, 299-308.

Tomari, Y., Du, T.T., Haley, B., Schwarz, D.S., Bennett, R., Cook, H.A., Koppetsch, B.S., Theurkauf, W.E., and Zamore, P.D. (2004a). RISC assembly defects in the Drosophila RNAi mutant armitage. Cell 116, 831-841.

Tomari, Y., Matranga, C., Haley, B., Martinez, N., and Zamore, P.D. (2004b). A protein sensor for siRNA asymmetry. Science 306, 1377-1380.

Tomari, Y., and Zamore, P.D. (2005). Perspective: machines for RNAi. Genes Dev 19, 517-529.

Tomoyasu, Y., Miller, S.C., Tomita, S., Schoppmeier, M., Grossmann, D., and Bucher, G. (2008). Exploring systemic RNA interference in insects: a genome-wide survey for RNAi genes in Tribolium. Genome Biology 9, R10-R10.

Tsutsumi, A., Kawamata, T., Izumi, N., Seitz, H., and Tomari, Y. (2011). Recognition of the pre-miRNA structure by Drosophila Dicer-1. Nature Structural \& Molecular Biology 18, 1153-U1189. van Cleef, K.W.R., van Mierlo, J.T., Miesen, P., Overheul, G.J., Fros, J.J., Schuster, S., Marklewitz, M., Pijlman, G.P., Junglen, S., and van Rij, R.P. (2014). Mosquito and Drosophila entomobirnaviruses suppress dsRNA- and siRNA-induced RNAi. Nucleic Acids Research 42, 8732-8744.

van Rij, R.P., Saleh, M.C., Berry, B., Foo, C., Houk, A., Antoniewski, C., and Andino, R. (2006). The RNA silencing endonuclease Argonaute 2 mediates specific antiviral immunity in Drosophila melanogaster. Genes \& Development 20, 2985-2995.

Vodovar, N., Bronkhorst, A.W., van Cleef, K.W.R., Miesen, P., Blanc, H., van Rij, R.P., and Saleh, M.C. (2012). Arbovirus-Derived piRNAs Exhibit a Ping-Pong Signature in Mosquito Cells. Plos One 7, e30861-e30861.

Wang, H., Ma, Z.J., Niu, K.Y., Xiao, Y., Wu, X.F., Pan, C.Y., Zhao, Y., Wang, K., Zhang, Y.Y., and Liu, N. (2016). Antagonistic roles of Nibbler and Hen1 in modulating piRNA 3 ' ends in Drosophila. Development 143, 530-539.

Wang, H.D., Gong, L., Qi, J.W., Hu, M.Y., Zhong, G.H., and Gong, L. (2014). Molecular cloning and characterization of a SID-1-like gene in Plutella xylostella. Archives of Insect Biochemistry and Physiology 87, 164-176. 
Wang, P.H., Weng, S.P., and He, J.G. (2015a). Nucleic acid-induced antiviral immunity in invertebrates: An evolutionary perspective. Developmental and Comparative Immunology 48, 291-296.

Wang, P.H., Yang, L.S., Gu, Z.H., Weng, S.P., Yu, X.Q., and He, J.G. (2013). Nucleic acid-induced antiviral immunity in shrimp. Antiviral Research 99, 270-280.

Wang, X.H., Aliyari, R., Li, W.X., Li, H.W., Kim, K., Carthew, R., Atkinson, P., and Ding, S.W. (2006). RNA interference directs innate immunity against viruses in adult Drosophila. Science $312,452-454$.

Wang, Z., Wu, D., Liu, Y., Xia, X., Gong, W., Qiu, Y., Yang, J., Zheng, Y., Li, J., Wang, Y.F., et al. (2015b). Drosophila Dicer-2 has an RNA interference-independent function that modulates Toll immune signaling. Science advances 1, e1500228-e1500228.

Welker, N.C., Maity, T.S., Ye, X.C., Aruscavage, P.J., Krauchuk, A.A., Liu, Q.H., and Bass, B.L. (2011). Dicer's Helicase Domain Discriminates dsRNA Termini to Promote an Altered Reaction Mode. Molecular Cell 41, 589-599.

Wen, J.Y., Duan, H., Bejarano, F., Okamura, K., Fabian, L., Brill, J.A., Bortolamiol-Becet, D., Martin, R., Ruby, J.G., and Lai, E.C. (2015). Adaptive Regulation of Testis Gene Expression and Control of Male Fertility by the Drosophila Harpin RNA Pathway. Molecular Cell 57, 165-178.

Westholm, J.O., Ladewig, E., Okamura, K., Robine, N., and Lai, E.C. (2012). Common and distinct patterns of terminal modifications to mirtrons and canonical microRNAs. RNA 18, 177-192.

Winter, F., Edaye, S., Huttenhofer, A., and Brunel, C. (2007). Anopheles gambiae miRNAs as actors of defence reaction against Plasmodium invasion. Nucleic Acids Research 35, 6953-6962.

Wynant, N., Santos, D., Subramanyam, S.H., Verlinden, H., and Broeck, J.V. (2015). Drosha, Dicer-1 and Argonaute-1 in the desert locust: Phylogenetic analyses, transcript profiling and regulation during phase transition and feeding. Journal of Insect Physiology 75, 20-29.

Xiong, X.P., Vogler, G., Kurthkoti, K., Samsonova, A., and Zhou, R. (2015). SmD1 Modulates the miRNA Pathway Independently of Its Pre-mRNA Splicing Function. Plos Genetics 11, e1005475-e1005475.

Xu, H.J., Chen, T., Ma, X.F., Xue, J., Pan, P.L., Zhang, X.C., Cheng, J.A., and Zhang, C.X. (2013). Genome-wide screening for components of small interfering RNA (siRNA) and micro-RNA (miRNA) pathways in the brown planthopper, Nilaparvata lugens (Hemiptera: Delphacidae). Insect Molecular Biology 22, 635-647.

Xu, K.Y., Bogert, B.A., Li, W.J., Su, K., Lee, A., and Gao, F.B. (2004). The fragile X-related gene affects the crawling behavior of Drosophila larvae by regulating the mRNA level of the DEG/ ENaC protein pickpocket1. Current Biology 14, 1025-1034.

$\mathrm{Xu}, \mathrm{W}$., and Han, Z. (2008). Cloning and phylogenetic analysis of sid-1-like genes from aphids. Journal of insect science (Online) 8, 1-6.

Yang, J.S., Smibert, P., Westholm, J.O., Jee, D., Maurin, T., and Lai, E.C. (2014a). Intertwined pathways for Argonaute-mediated microRNA biogenesis in Drosophila. Nucleic Acids Research 42, 1987-2002.

Yang, L.L., Chen, D.S., Duan, R.H., Xia, L.X., Wang, J., Qurashi, A., Jin, P., and Chen, D.H. (2007). Argonaute 1 regulates the fate of germline stem cells in Drosophila. Development 134, 4265-4272.

Yang, L.S., Li, X.L., Jiang, S., Qiu, L.H., Zhou, F.L., Liu, W.J., and Jiang, S.G. (2014b). Characterization of Argonaute2 gene from black tiger shrimp (Penaeus monodon) and its responses to immune challenges. Fish \& Shellfish Immunology 36, 261-269. 
Ye, X.C., Paroo, Z., and Liu, Q.H. (2007). Functional anatomy of the Drosophila MicroRNA- generating enzyme. Journal of Biological Chemistry 282, 28373-28378.

Yoda, M., Kawamata, T., Paroo, Z., Ye, X.C., Iwasaki, S., Liu, Q.H., and Tomari, Y. (2010). ATP-dependent human RISC assembly pathways. Nature Structural \& Molecular Biology 17, 17-U29.

Zambon, R.A., Vakharia, V.N., and Wu, L.P. (2006). RNAi is an antiviral immune response against a dsRNA virus in Drosophila melanogaster. Cellular Microbiology 8, 880-889.

Zhang, H., Kolb, F.A., Brondani, V., Billy, E., and Filipowicz, W. (2002). Human Dicer preferentially cleaves dsRNAs at their termini without a requirement for ATP. EMBO J 21, 5875-5885.

Zhang, X.Y., Lu, K., Zhou, J.L., and Zhou, Q. (2013). Molecular characterization and gene functional analysis of Dicer-2 gene from Nilaparvata lugens (Hemiptera: Geometroidea). Insect Science 20, $61-68$.

Zhao, C.Y., Gonzales, M.A.A., Poland, T.M., and Mittapalli, O. (2015). Core RNAi machinery and gene knockdown in the emerald ash borer (Agrilus planipennis). Journal of Insect Physiology 72, 70-78.

Zhou, R., Czech, B., Brennecke, J., Sachidanandam, R., Wohlschlegel, J.A., Perrimon, N., and Hannon, G.J. (2009). Processing of Drosophila endo-siRNAs depends on a specific Loquacious isoform. RNA 15, 1886-1895.

Zhu, L., Tatsuke, T., Li, Z.Q., Mon, H., Xu, J., Lee, J.M., and Kusakabe, T. (2012). Molecular cloning of BmTUDOR-SN and analysis of its role in the RNAi pathway in the silkworm, Bombyx mori (Lepidoptera: Bombycidae). Applied Entomology and Zoology 47, 207-215.

Zografidis, A., Van Nieuwerburgh, F., Kolliopoulou, A., Apostolou-Karampelis, K., Head, S.R., Deforce, D., Smagghe, G., and Swevers, L. (2015). Viral Small-RNA Analysis of Bombyx mori Larval Midgut during Persistent and Pathogenic Cytoplasmic Polyhedrosis Virus Infection. Journal of Virology 89, 11473-11486. 


\title{
RNAi AND mIRNA PATHWAYS IN ANNELIDS AND MOLLUSCS
}

Keywords: dsRNA, PKR, Dicer, TARBP2, PACT

\author{
PETR SVOBODA \\ Institute of Molecular Genetics, Academy of Sciences of the Czech Republic, \\ Videnska 1083, 14220 Prague 4, Czech Republic \\ Correspondence to: Petr Svoboda, Institute of Molecular Genetics ASCR, \\ Videnska 1083, 14220 Prague 4, Czech Republic, tel. \# +420 241063147, \\ e-mail: svobodap@img.cas.cz.
}

\begin{abstract}
RNA silencing denotes sequence-specific repression mediated by small RNAs. In metazoa, there are two mechanistically closely related pathways: RNA interference (RNAi) and microRNA (miRNA) pathway. The miRNA pathway regulates endogenous protein-coding gene expression and has been implicated in many biological processes. RNAi generally serves as a form of innate immunity targeting viruses and mobile elements. This review is summarizing information about RNAi and miRNA pathways in protostome phyla: Annelida and Mollusca. The molecular mechanisms of dsRNA and miRNA pathways in annelids remain largely unexplored. The available information points towards coexistence of miRNA and RNAi pathways, however, their integration or genetic separation remain unclear. Molluscs are an interesting taxon, which appears to have a unique setup of RNA silencing possibly employing a single Argonaute protein while it also employs interferon-like pathway elements.
\end{abstract}

\section{Introduction}

The mechanistical principles of vertebrate miRNA and RNAi pathways were introduced in the first review of this series (Svoboda, 2019) and in further detail elsewhere (Bartel, 2018), I will focus here directly on features of these pathways reported from Annelid and Molluscs taxons, which do not have common model species, which would be investigated in depth like C. elegans or Drosophila. The review is divided into Annelid and Molluscs parts. In each of them, I'll review published data concerning components of miRNA and RNAi pathways and discuss biological roles of the two pathways. 


\section{Annelids}

Annelids are coelomate protostome worms whose body is organized into a series of ringshaped repetitive segments. $\sim 15000$ annelid species are grouped into three classes: Polychaeta (bristle worms, e.g. Platynereis), Oligochaeta (earthworms, e.g. Lumbricus), and Hirudinea (leeches, e.g. Hirudo). Literature on RNA silencing and dsRNA pathways is extremely limited. There were under twenty publications dealing with small RNAs, most of which was related to detection of miRNAs.

\section{Dicer}

There was no publication concerning Dicer structure or function in annelids, thus all information provided here had to be extracted from genomic databases. Blast search of annelid entries in Genbank (query: murine Dicer protein, algorithm: tblastn, database: Nucleotide collection $\mathrm{nr} / \mathrm{nt}$, results restricted to Annelida (taxid:6340)) identified a single mRNA XM_009031272 from the leech Helobdella robusta encoding a 1316 aa Dicer protein which lacked $\sim 400$ aminoacids at the N-terminus. The protein contained HELICc domain at the N-terminus but lacked the N-terminal DExD domain. Thus, this Dicer is structurally reminiscent of the N-terminally truncated Dicer capable of producing endo-siRNAs (Flemr et al., 2013). Importantly, analysis of Helobdella robusta genomic sequence identified a single Dicer gene on a contig ref|NW_008705401.1|, which also carried the entire N-terminus, which was lacking in the identified mRNA. This would imply a similar scenario as observed in mouse oocytes - Dicer encodes two protein isoforms, where the longer one is adapted for the production of miRNAs, while the shorter can produce both, miRNAs and siRNAs. However, this information would need to be validated experimentally and it needs to be also tested how common would be this scenario for annelids in general. Finally, annelid Dicer also produces miRNAs with median length of 22 nucleotides as estimated from the miRBase data (Kozomara and Griffiths-Jones, 2014) (Fig. 1).

\section{dsRBPs}

There is no literature concerning dsRBP proteins participating in RNA silencing, Rosani et al. suggest that annelids employ a single TARBP2 homolog (Rosani et al., 2016).

\section{Argonaute proteins}

Likewise, there is essentially no information regarding the AGO subfamily of Argonaute proteins. There is a study of protein components in molluscs, which included two annelid species and found that annelids have three (Capitella) and four (Helobdella) Argonaute proteins from both, PIWI and AGO clades (Rosani et al., 2016). There are five papers concerning Argonaute, however of the PIWI clade, which is functioning in the germline (Giani et al., 2011; Kozin and Kostyuchenko, 2015; Ozpolat and Bely, 2015; Sugio et al., 2008; Weigert et al., 2013). Blast search of Helobdella genome identified two AGO paralogs (XM_009021176.1 and XM_009031816.1) and two possible PIWI proteins 

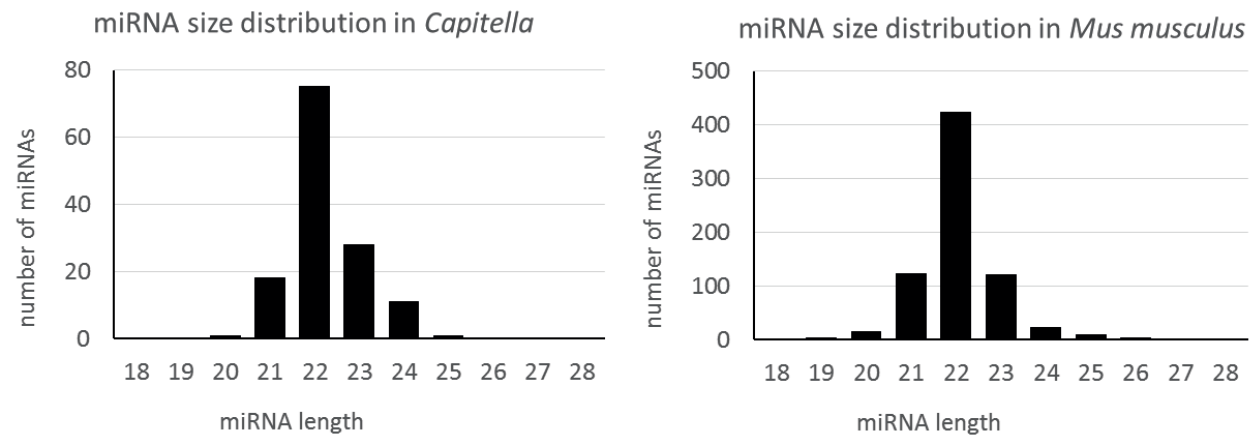

Figure 1 Annelid miRNA lengths

The left graph depicts size distribution of all 134 miRNAs of a polychaete worm Capitella teleta deposited in the miRBase (version 21). For comparison, the right graph shows size distribution of 721 high-confidence murine miRNAs.

(XM_009015681.1 and XM_009021522.1). The two paralogs of PIWI proteins would be consistent with the aforementioned analysis of Myzostoma cirriferum PIWI proteins (Weigert et al., 2013). Whether the two AGO paralogs are functionally dedicated to RNAi and miRNA pathways like AGO proteins in Drosophila is unknown. Furthermore, if annelids would have two PIWI proteins, it is possible that Capitella might have only one AGO protein serving in RNAi and miRNA pathways.

\section{Other factors}

No other proteins factors from miRNA or RNAi pathway have been specifically reported. Data from Rosani et al. show that annelids employ microprocessor complex (Rosani et al., 2016)

\section{miRNA}

There is one annelid species, which has annotated miRNAs in the miRBase: Capitella teleta -129 precursor miRNAs and 134 mature miRNAs. Literature search revealed seven publications reporting annelid miRNA identification and or expression (Christodoulou et al., 2010; Gong et al., 2010; Helm et al., 2012; Huang et al., 2012; Kenny et al., 2015; Sperling et al., 2009; Tessmar-Raible et al., 2007). As shown above, annelid miRNAs have an average length 22 nucleotides like other animal miRNAs.

\section{RNAi}

The only information available concerning RNAi is that it is functional (Takeo et al., 2010; Yoshida-Noro and Tochinai, 2010). The only one published experimental paper reporting RNAi employed long dsRNA that was injected into the coelom $(1 \mu \mathrm{g} / \mu \mathrm{l}, 100 \mathrm{nl}$ per worm, i.e. $100 \mathrm{ng}$ of dsRNA/worm). 


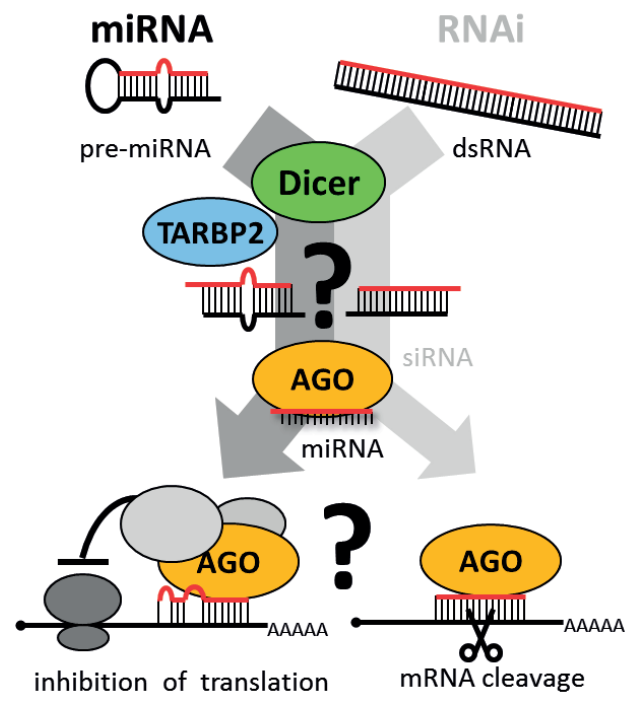

Figure 2 Overview of annelid pathways

dsRNA and miRNA pathways in annelids are poorly understood. Among the missing pieces of information are: 1) degree of separation of miRNA and RNAi pathways (dedicated AGO proteins, co-existence of Dicer isoforms). Proteins from other dsRNA pathways related to innate immunity include OAS, most likely RIG-I and MDA5 homologs but probably not PKR.

\section{Other dsRNA responding pathways}

Only one reference mentioned another protein involved in dsRNA response - OAS (Kjaer et al., 2009). Blast search for murine PKR, RIG-I, and MDA5 (algorithm: tblastn, database: Nucleotide collection $\mathrm{nr} / \mathrm{nt}$, results restricted to Annelida (taxid:6340)) revealed many sequences of Helobdella, and six of Platynereis, which were similar only to the second half of PKR, suggesting they were not orthologs. RIG-I and MDA5 searches identified two hypothetical proteins in Helobdella (ref|XM_009026626.1| and XM_009014668.1) with $\sim 35 \%$ identity and $\sim 50 \%$ similarity, which could be orthologs.

\section{Summary}

In terms of the molecular mechanism of dsRNA and miRNA pathways, annelids remain largely unexplored. While, the available information points towards coexistence of miRNA and RNAi pathways, their integration or genetic separation remain unclear.

\section{Molluscs}

Molluscs are a large and extremely diverse group of coelomate protostomes, which have an unsegmented soft body, internal or external shell, and a muscular foot. There are $\sim 50000$ 
described species, which makes molluscs the second largest phylum after Arthropoda (third if Chelicerata and Hexapoda would be considered separate phyla). Apart from the complex classification of molluscs into 7-10 classes, three groups of molluscs are commonly recognized: Cephalopoda (squid, octopus), Gastropoda (snails and slugs), and Bivalvia (clams, mussels, scallops, oysters). Literature on RNA silencing and dsRNA pathways is limited. There were 92 publications dealing with small RNAs, most of which was related to use of RNAi as an experimental tool for suppressing gene expression.

\section{Dicer}

There was no specific functional analysis of Dicer in molluscs. There is one study from 2016, which identified and bioinformatically analyzed Dicer and other components of miRNA and RNAi pathways in marine bivalves with a focus on a mussel Mytillus galloprovincialis and oyster Cassostrea gigas (Rosani et al., 2016). Their results show that all examined molluscs ( $>30$ species of cephalopods, gastropods, and bivalves) have a single Dicer protein, which participates in both, RNAi and miRNA pathways and that Dicer of Mytillus galloprovincialis and Cassostrea gigas has a common structure found in Metazoa.

Additional information regarding Dicer structure was extracted from genomic databases. Blast search of molluscs entries in Genbank (query: murine Dicer protein, algorithm: tblastn, database: Nucleotide collection $\mathrm{nr} / \mathrm{nt}$, results restricted to molluscs (taxid:6447)) identified transcripts from Mytilus, Crasostrea, Lottia, Aplysia, Miomphalaria, and Octopus that apparently encoded full-length Dicer orthologs. Molluscs Dicer also produces miRNAs with median length of 22 nucleotides as estimated from the miRBase data (Kozomara and Griffiths-Jones, 2014). Interestingly, the incidence of $23 \mathrm{nt}$ long miRNAs seems to be higher in molluscs (Fig. 3). However, given the low number of miRNAs (64) and unexplored diversity of molluses, it should not be considered a significant feature.

\section{dsRBPs}

There is no literature concerning dsRBP proteins participating in RNA silencing in molluscs. The above-mentioned analysis of miRNA and RNAi pathway components identified only a single dsRBP (TARBP2) homolog (Rosani et al., 2016).

\section{Argonaute proteins}

Likewise, there is essentially no information regarding the AGO subfamily of Argonaute proteins. The above-mentioned analysis of miRNA and RNAi pathway components identified one to four Argonaute proteins from both, AGO and PIWI clades (Rosani et al., 2016). However, general and derived roles of AGO proteins in molluscs remain unknown at the moment. Data from Mytillus galloprovincialis indicate presence of one AGO and two PIWI proteins (Rosani et al., 2016). This is remarkable because this AGO protein would act in both, RNAi and miRNA pathways like $\mathrm{AGO} 2$ in mammals.

In addition, there were two articles concerning the PIWI clade, which is acting in the piRNA pathway in the germline. In one of them, authors reported differential proteomic 

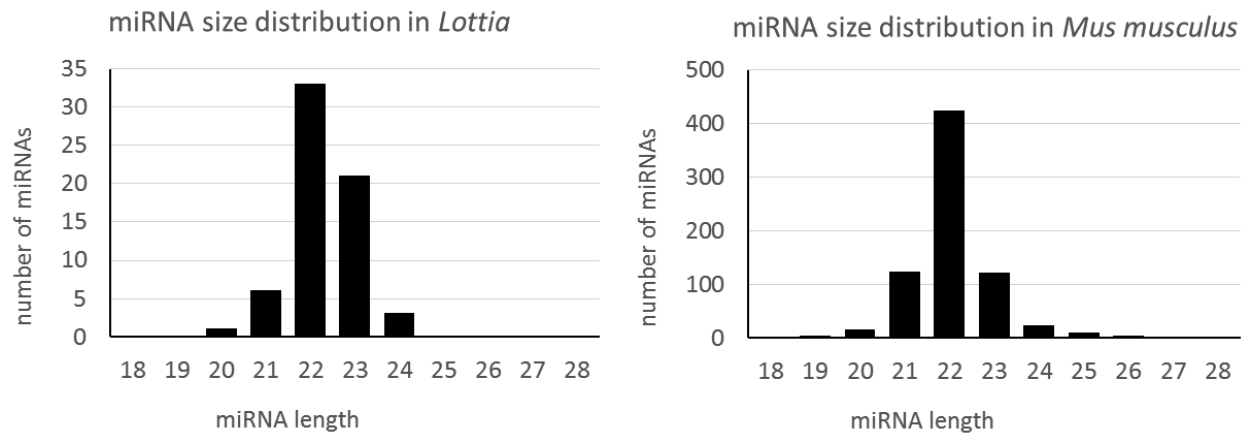

Figure 3 Molluscs miRNA lengths

The left graph depicts size distribution of all 64 miRNAs of sea snail Lottia gigantea deposited in the miRBase (version 21). For comparison, the right graph shows size distribution of 721 high-confidence murine miRNAs.

responses to generic dsRNA (poly I:C and poly A:U) in two oyster species (Saccostrea glomerata and Crassostrea gigas), which have differential susceptibility to ostreid herpesvirus infection. Interestingly, Saccostrea glomerata, which is not susceptible, showed production of proteins implicated in the TLR signalling pathway and PIWI protein was also found in Saccostrea glomerata but not in Crassostrea gigas when challenged with dsRNA (Masood et al., 2016). Although it is unclear whether PIWI could be mistaken for AGO, it is possible that piRNAs might have acquired additional roles in molluscs, perhaps including also immunity. This notion would be supported by the second report, which identified $28 \mathrm{nt}$ piRNAs in brain (while piRNAs are generally restricted to gonads if not into germline cells only). These piRNAs had unique biogenesis patterns, nuclear localization, sensitivity to serotonin, and were implicated in stable long-term changes in neurons associated with memory (Rajasethupathy et al., 2012).

\section{Other factors}

According to the genome analysis of Mytillus galloprovincialis, Precambrian molluscs/ mammalian ancestors must have shared all ancestral proteins in the miRNA pathway, including DROSHA, DGCR8 and GW182 (Rosani et al., 2016). There are no published mechanistic data suggesting that there would be any difference in activity of any of these factors in molluscs.

Importantly, we examined if molluscs genomes also contain an RdRP, which is found in RNA silencing in plants and Nematodes but not in insects or mammals: query: C. elegans RRF-1 NP_001250555, algorithm: tblastn, database: Nucleotide collection nr/nt, results restricted to molluscs (taxid:6447)) identified transcripts six different transcripts from Crassostrea gigas (XM_011450789, XM_011427600, XR_900019, XR_902698, XM_011450791, XR_900018) suggesting that molluscs might indeed employ RdRPs in RNAi. This would be a significant observation, making molluscs RNA silencing an intermediate type between those found in nematodes, arthropods, and mammals. 


\section{miRNA}

There is three molluscs species, which have annotated miRNAs in the miRBase (Kozomara and Griffiths-Jones, 2014): Haliotis rufescens (red abalone sea snail), Lottia gigantean (owl limpet sea snai)l, and Melibe leonine (lion nudibranch sea slug). Unfortunately, there are no other representatives of other main mollusk groups such as Cephalopodes or Bivalves. Annelid miRNAs have are $\sim 22 \mathrm{nt}$ long like other animal miRNAs (Fig. 4). There is a small number of reports concerning miRNA identification/annotation and/or analysis of expression/function in molluscs (Biggar et al., 2012; Bitel et al., 2012; Chen et al., 2014; Jiao et al., 2014; Jiao et al., 2015; Kenny et al., 2015; Martin-Gomez et al., 2014; Millan, 2011; Rajasethupathy et al., 2009; Tian et al., 2015; Xu et al., 2014; Zhao et al., 2016; Zheng et al., 2016a; Zheng et al., 2016b; Zhou et al., 2014).

\section{RNAi}

RNAi is functional in molluses as evidenced by 18 reports, which employed RNAi in different molluscs species (see the table below). The canonical RNAi (i.e. using long dsRNA) has been observed in the following species upon various forms of delivery including larva soaking, animal injection (adductor muscle, gonad, brain, larva, post-renal sinus etc.), polyethyleneimin-mediated delivery, or cell injection. Collectively, these data imply that different molluscs have an intact machinery to execute RNAi. Effects of injection into body cavity would suggest that molluscs might have some cellular uptake mechanism for dsRNA or systemic RNAi but the direct evidence for any of that is lacking at the moment. Other dsRNA responding pathways

Molluscs seem to have a complex dsRNA response, which includes interferon-like response functioning in antiviral response (reviewed in Green et al., 2015a; Wang et al., 2015b). 21 articles dealt with dsRNA-induced interferon-like response. Several studies in Oyster have reported that dsRNA mimic poly(I:C) can strongly induce non-specific antiviral immune responses (De Zoysa et al., 2007; Green and Barnes, 2009; Green and Montagnani, 2013; Green et al., 2015b; Masood et al., 2016; Wang et al., 2016b; Wang et al., 2016c).

Molluscs have 2'5'- oligoadenylate synthetases (Kjaer et al., 2009; Pari et al., 2014), RIG-I-like protein (Zhang et al., 2014), MDA5 (Green et al., 2014), and PKR (Green et al., 2014; Green and Montagnani, 2013; Green et al., 2015b). Poly I:C can be also bound by Leucine-rich repeat (LRR)-only protein found in scallop Chlamys farreri (Wang et al., 2016b; Wang et al., 2016c). Another gene, which is induced by poly I:C or sodium alginate is Myxovirus resistance (Mx) protein, which has been found in Abalone (Cheng et al., 2012; De Zoysa et al., 2007). Mx is one of intensely studied antiviral proteins, which is induced by the type I interferon system (IFN alpha/beta).

However, it is important to recognize that molluscs are a heterogeneous group with distinct antiviral adaptations. For example, proteomic profiling of two oyster species with differential susceptibility to ostreid herpesviruses showed that the resistant species has a stronger manifestation of the interferon-like response in the proteome upon induction with poly I:C (Masood et al., 2016). 


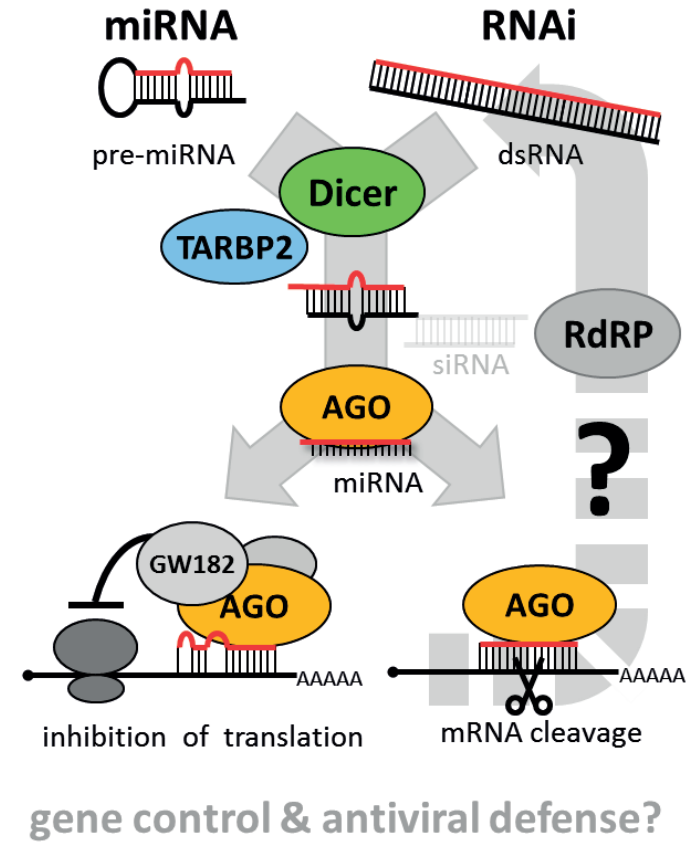

Figure 4 Overview of mollusc pathways

dsRNA and miRNA pathways in molluscs are poorly understood. Among the missing pieces of information are: 1) degree of separation of miRNA and RNAi pathways (dedicated AGO proteins, co-existence of Dicer isoforms). 2) existence and functionality of RdRPs in molluscs, 3) derived roles of piRNAs in gene regulation and antiviral defense. Proteins from other dsRNA pathways related to innate immunity include PKR, OAS, RIG-I and MDA5 homolog, Mx protein and others.

\section{Summary}

Taken together, molluscs are an interesting taxon, which appears to have a unique setup of RNA silencing (Fig. 4) and its nexus with antiviral responses, which warrant further investigation.

\section{Acknowledgement}

I would like to thank my colleagues Jan Paces, Miloslav Nic and Tomas Novotny for help with collecting literature for the review. The review content was produced under a contract OC/EFSA/GMO/2015/01-CT 01 with European Food Safety Authority (EFSA); the opinions expressed are those of the contractor only and do not represent EFSA's official position. Publication of the review was funded by LO1220 and LM2015063 by the Ministry of Education, Youth and Sports. 
Table 1 RNAi in molluscs induced with short dsRNA molecules

\begin{tabular}{|l|l|l|l|}
\hline species & siRNA amount & delivery method \& effect & reference \\
\hline Chlamys farreri & $1 \mu \mathrm{g} / \mathrm{g}$ & $\begin{array}{l}\text { adductor muscle siRNA injection } \\
70 \% \mathrm{KD} \text { at } 72 \mathrm{~h}\end{array}$ & (Miao et al., 2016) \\
\hline $\begin{array}{l}\text { Biomphalaria } \\
\text { glabrata }\end{array}$ & $772 \mathrm{ng} / 250 \mu \mathrm{l}$ & $\begin{array}{l}\text { polyethyleneimine-mediated delivery } \\
>50-90 \% \mathrm{KD} \text { at } 72 \mathrm{~h} \text { by ELISA }\end{array}$ & (Knight et al., 2011) \\
\hline Lymnaea stagnalis & $5 \mu \mathrm{l}$ of $200 \mathrm{ng} / \mu \mathrm{l}$ & $\begin{array}{l}\text { gonad injection, 27-mer } \\
30-50 \% \mathrm{KD}\end{array}$ & (Fei et al., 2007) \\
\hline Lymnaea stagnalis & $2 \mu \mathrm{l}$ of $20 \mu \mathrm{M}$ & $\begin{array}{l}\text { head injection above central ganglia, 27-mer, } \\
\text { (Hui et al., 2007) }\end{array}$ \\
\hline
\end{tabular}

Table 2 RNAi in molluscs induced with long dsRNA molecules

\begin{tabular}{|c|c|c|c|c|}
\hline \multirow[t]{2}{*}{ species } & \multicolumn{2}{|l|}{ long dsRNA } & \multirow{2}{*}{ delivery method } & \multirow{2}{*}{ reference } \\
\hline & concentration & length & & \\
\hline $\begin{array}{l}\text { Haliotis } \\
\text { diversicolor }\end{array}$ & $5 \mu \mathrm{g} / \mathrm{ml}$ & $136-819 \mathrm{bp}$ & larva soaking & (Wang et al., 2016a) \\
\hline $\begin{array}{l}\text { Haliotis } \\
\text { diversicolor }\end{array}$ & $5 \mu \mathrm{g} / \mathrm{ml}$ & $136-819 \mathrm{bp}$ & larva soaking & (Wang et al., 2015a) \\
\hline $\begin{array}{l}\text { Crassostrea } \\
\text { gigas }\end{array}$ & $\begin{array}{l}150-750 \mu \mathrm{g} / \mathrm{ml} \\
10 \mu \mathrm{g} / \mathrm{g}\end{array}$ & $652 \mathrm{bp}$ & $\begin{array}{l}2 \times 100 \mu l \text { injection } \\
\sim 50 \% \mathrm{KD} \text { at } 48 \mathrm{~h} \\
\end{array}$ & (Huvet et al., 2015) \\
\hline $\begin{array}{l}\text { Crassostrea } \\
\text { gigas }\end{array}$ & $50 \mu \mathrm{g} /$ oyster & $723 \mathrm{bp}$ & $\begin{array}{l}\text { adductor muscle inject. } \\
1-7 \text { days, good effect }\end{array}$ & (Choi et al., 2013) \\
\hline $\begin{array}{l}\text { Crassostrea } \\
\text { gigas }\end{array}$ & $\begin{array}{l}530 \mu \mathrm{g} / \mathrm{ml} \\
53 \mu \mathrm{g} / \text { oyster }\end{array}$ & $425 \mathrm{bp}$ & $\begin{array}{l}\text { gonad injection } \\
77.52 \% \mathrm{KD}\end{array}$ & (Huvet et al., 2012) \\
\hline $\begin{array}{l}\text { Nipponacmea } \\
\text { fuscoviridis }\end{array}$ & $5 \mu \mathrm{g} / \mathrm{ml}$ & $\begin{array}{l}947 \mathrm{bp} \\
667 \mathrm{bp}\end{array}$ & larva injection & $\begin{array}{l}\text { (Hashimoto } \\
\text { et al., 2012) }\end{array}$ \\
\hline Aplysia & $500 \mathrm{ng} / \mu \mathrm{l}$ & 316 bp & $\begin{array}{l}\text { sensory cell injection } \\
\text { protein not decreased }\end{array}$ & (Lyles et al., 2006) \\
\hline $\begin{array}{l}\text { Lymnaea } \\
\text { stagnalis }\end{array}$ & $\begin{array}{l}500 \mathrm{ng} / \mu \mathrm{l} \\
2 \mu \mathrm{g} / \text { oyster }\end{array}$ & $\sim 300$ bp & $\begin{array}{l}\text { central ring ganglia } \\
60 \% \mathrm{KD} \text { at } 24 \mathrm{~h}\end{array}$ & (Guo et al., 2010) \\
\hline $\begin{array}{l}\text { Lymnaea } \\
\text { stagnalis }\end{array}$ & $5 \mu \mathrm{l}$ of $200 \mathrm{ng} / \mu \mathrm{l}$ & $321 \mathrm{bp}$ & $\begin{array}{l}\text { snail ganglia injection } \\
30-50 \% \mathrm{KD}\end{array}$ & (Fei et al., 2007) \\
\hline $\begin{array}{l}\text { Crassostrea } \\
\text { gigas. }\end{array}$ & 20 or $100 \mu \mathrm{g} /$ oyster & $\begin{array}{l}525 \mathrm{bp} \\
877 \mathrm{bp}\end{array}$ & $\begin{array}{l}\text { gonad injection } \\
39 \% \text { \& } 87 \% \mathrm{KD}\end{array}$ & (Fabioux et al., 2009) \\
\hline $\begin{array}{l}\text { Biomphalaria } \\
\text { glabrata }\end{array}$ & $0.1,1.0$ and $5.0 \mu \mathrm{g} / \mathrm{snail}$ & $\begin{array}{l}537 \mathrm{bp} \\
541 \mathrm{bp}\end{array}$ & $\begin{array}{l}\text { post-renal sinus inj. } \\
70-80 \% \mathrm{KD}\end{array}$ & (Jiang et al., 2006) \\
\hline $\begin{array}{l}\text { Biomphalaria } \\
\text { glabrata }\end{array}$ & $120 \mathrm{ng} / 250 \mu \mathrm{l}$ & $397 \mathrm{bp}$ & $\begin{array}{l}\text { polyethyleneimine-mediated } \\
\text { delivery } \\
>50 \% \mathrm{KD} \text { at } 72 \mathrm{~h} \text { by ELISA. }\end{array}$ & (Knight et al., 2011) \\
\hline Aplysia & $500 \mathrm{ng} / \mu \mathrm{l}$ & $316 \mathrm{bp}$ & $\begin{array}{l}\text { sensory cell injection } \\
\text { protein not decreased }\end{array}$ & (Lyles et al., 2006) \\
\hline Aplysia & up to $700 \mu \mathrm{g} / \mathrm{mL}$ & N.A. & $\begin{array}{l}\sim 20 \text { giant neurons of the } \\
\text { abdominal ganglion inj., } \\
80-95 \% \mathrm{KD}\end{array}$ & (Lee et al., 2001) \\
\hline Aplysia & $500 \mu \mathrm{g} / \mathrm{mL}$ & $800 \mathrm{bp}$ & $\begin{array}{l}\text { sensory neuron injection } \\
\text { decrease by microscopy }\end{array}$ & (Ormond et al., 2004) \\
\hline
\end{tabular}




\section{References}

Bartel, D.P. (2018). Metazoan MicroRNAs. Cell 173, 20-51.

Biggar, K.K., Kornfeld, S.F., Maistrovski, Y., and Storey, K.B. (2012). MicroRNA Regulation in Extreme Environments: Differential Expression of MicroRNAs in the Intertidal Snail Littorina littorea During Extended Periods of Freezing and Anoxia. Genomics Proteomics \& Bioinformatics 10, 302-309.

Bitel, C.L., Singh, V., and Frederikse, P.H. (2012). miR-124, miR-125b, let-7 and Vesicle Transport Proteins in Squid Lenses in L. pealei. Current Eye Research 37, 388-394.

Chen, G.F., Zhang, C.Y., Jiang, F.J., Wang, Y.Y., Xu, Z., and Wang, C.M. (2014). Bioinformatics analysis of hemocyte miRNAs of scallop Chlamys farreri against acute viral necrobiotic virus (AVNV). Fish \& Shellfish Immunology 37, 75-86.

Cheng, W., Tsai, R.T., and Chang, C.C. (2012). Dietary sodium alginate administration enhances Mx gene expression of the tiger grouper, Epinephelus fuscoguttatus receiving poly I:C. Aquaculture 324, 201-208.

Choi, S.H., Jee, B.Y., Lee, S.J., Cho, M.Y., Lee, S.J., Kim, J.W., Do Jeong, H., and Kim, K.H. (2013). Effects of RNA interference-mediated knock-down of hypoxia-inducible factor-alpha on respiratory burst activity of the Pacific oyster Crassostrea gigas hemocytes. Fish \& Shellfish Immunology 35, 476-479.

Christodoulou, F., Raible, F., Tomer, R., Simakov, O., Trachana, K., Klaus, S., Snyman, H., Hannon, G.J., Bork, P., and Arendt, D. (2010). Ancient animal microRNAs and the evolution of tissue identity. Nature 463, 1084-U1105.

De Zoysa, M., Kang, H.S., Song, Y.B., Jee, Y., Lee, Y.D., and Lee, J. (2007). First report of invertebrate Mx: Cloning, characterization and expression analysis of Mx cDNA in disk abalone (Haliotis discus discus). Fish \& Shellfish Immunology 23, 86-96.

Fabioux, C., Corporeau, C., Quillien, V., Favrel, P., and Huvet, A. (2009). In vivo RNA interference in oyster-vasa silencing inhibits germ cell development. Febs Journal 276, 2566-2573.

Fei, G.H., Guo, C.H., Sun, H.S., and Feng, Z.P. (2007). Chronic hypoxia stress-induced differential modulation of heat-shock protein 70 and presynaptic proteins. Journal of Neurochemistry 100, 50-61.

Flemr, M., Malik, R., Franke, V., Nejepinska, J., Sedlacek, R., Vlahovicek, K., and Svoboda, P. (2013). A Retrotransposon-Driven Dicer Isoform Directs Endogenous Small Interfering RNA Production in Mouse Oocytes. Cell 155, 807-816.

Giani, V.C., Yamaguchi, E., Boyle, M.J., and Seaver, E.C. (2011). Somatic and germline expression of piwi during development and regeneration in the marine polychaete annelid Capitella teleta. Evodevo 2, 10-10.

Gong, P., Xie, F.L., Zhang, B.H., and Perkins, E.J. (2010). In silico identification of conserved microRNAs and their target transcripts from expressed sequence tags of three earthworm species. Computational Biology and Chemistry 34, 313-319.

Green, T.J., and Barnes, A.C. (2009). Inhibitor of REL/NF-B-K is regulated in Sydney rock oysters in response to specific double-stranded RNA and Vibrio alginolyticus, but the major immune anti-oxidants EcSOD and Prx6 are non-inducible. Fish \& Shellfish Immunology 27, 260-265.

Green, T.J., Benkendorff, K., Robinson, N., Raftos, D., and Speck, P. (2014). Anti-viral gene induction is absent upon secondary challenge with double-stranded RNA in the Pacific oyster, Crassostrea gigas. Fish \& Shellfish Immunology 39, 492-497. 
Green, T.J., and Montagnani, C. (2013). Poly I:C induces a protective antiviral immune response in the Pacific oyster (Crassostrea gigas) against subsequent challenge with Ostreid herpesvirus (OsHV-1 mu var). Fish \& Shellfish Immunology 35, 382-388.

Green, T.J., Raftos, D., Speck, P., and Montagnani, C. (2015a). Antiviral immunity in marine molluscs. Journal of General Virology 96, 2471-2482.

Green, T.J., Rolland, J.L., Vergnes, A., Raftos, D., and Montagnani, C. (2015b). OsHV-1 countermeasures to the Pacific oyster's anti-viral response. Fish \& Shellfish Immunology 47, 435-443.

Guo, C.H., Senzel, A., Li, K., and Feng, Z.P. (2010). De Novo Protein Synthesis of Syntaxin-1 and Dynamin-1 in Long-Term Memory Formation Requires CREB1 Gene Transcription in Lymnaea stagnalis. Behavior Genetics 40, 680-693.

Hashimoto, N., Kurita, Y., and Wada, H. (2012). Developmental role of dpp in the gastropod shell plate and co-option of the dpp signaling pathway in the evolution of the operculum. Developmental Biology 366, 367-373.

Helm, C., Bernhart, S.H., Siederdissen, C.H.Z., Nickel, B., and Bleidorn, C. (2012). Deep sequencing of small RNAs confirms an annelid affinity of Myzostomida. Molecular Phylogenetics and Evolution 64, 198-203.

Huang, X.M., Tian, Q.N., Bao, Z.X., Qin, Y.F., Chen, S.J., Lu, P., Zhang, X.L., Zhang, Y.Z., and Zhang, S.T. (2012). Cloning and Identification of MicroRNAs in Earthworm (Eisenia fetida). Biochemical Genetics 50,1-11.

Hui, K., Fei, G.H., Saab, B.J., Su, J., Roder, J.C., and Feng, Z.P. (2007). Neuronal calcium sensor-1 modulation of optimal calcium level for neurite outgrowth. Development 134, 4479-4489.

Huvet, A., Beguel, J.P., Cavaleiro, N.P., Thomas, Y., Quillien, V., Boudry, P., Alunno-Bruscia, M., and Fabioux, C. (2015). Disruption of amylase genes by RNA interference affects reproduction in the Pacific oyster Crassostrea gigas. Journal of Experimental Biology 218, 1740-1747.

Huvet, A., Fleury, E., Corporeau, C., Quillien, V., Daniel, J.Y., Riviere, G., Boudry, P., and Fabioux, C. (2012). In Vivo RNA Interference of a Gonad-Specific Transforming Growth Factor-beta in the Pacific Oyster Crassostrea gigas. Marine Biotechnology 14, 402-410.

Jiang, Y.G., Loker, E.S., and Zhang, S.M. (2006). In vivo and in vitro knockdown of FREP2 gene expression in the snail Biomphalaria glabrata using RNA interference. Developmental and Comparative Immunology 30, 855-866.

Jiao, Y., Zheng, Z., Du, X.D., Wang, Q.H., Huang, R.L., Deng, Y.W., Shi, S.L., and Zhao, X.X. (2014). Identification and Characterization of MicroRNAs in Pearl Oyster Pinctada martensii by Solexa Deep Sequencing. Marine Biotechnology 16, 54-62.

Jiao, Y., Zheng, Z., Tian, R.R., Du, X.D., Wang, Q.H., and Huang, R.L. (2015). MicroRNA, Pm-miR-2305, Participates in Nacre Formation by Targeting Pearlin in Pearl Oyster Pinctada martensii. International Journal of Molecular Sciences 16, 21442-21453.

Kenny, N.J., Namigai, E.K.O., Marletaz, F., Hui, J.H.L., and Shimeld, S.M. (2015). Draft genome assemblies and predicted microRNA complements of the intertidal lophotrochozoans Patella vulgata (Mollusca, Patellogastropoda) and Spirobranchus (Pomatoceros) lamarcki (Annelida, Serpulida). Marine Genomics 24, 139-146.

Kjaer, K.H., Poulsen, J.B., Reintamm, T., Saby, E., Martensen, P.M., Kelve, M., and Justesen, J. (2009). Evolution of the 2'-5'-Oligoadenylate Synthetase Family in Eukaryotes and Bacteria. Journal of Molecular Evolution 69, 612-624. 
Knight, M., Miller, A., Liu, Y.J., Scaria, P., Woodle, M., and Ittiprasert, W. (2011). Polyethyleneimine (PEI) Mediated siRNA Gene Silencing in the Schistosoma mansoni Snail Host, Biomphalaria glabrata. Plos Neglected Tropical Diseases 5, e1212-e1212.

Kozin, V.V., and Kostyuchenko, R.P. (2015). Vasa, PL10, and Piwi gene expression during caudal regeneration of the polychaete annelid Alitta virens. Development Genes and Evolution 225, $129-138$.

Kozomara, A., and Griffiths-Jones, S. (2014). miRBase: annotating high confidence microRNAs using deep sequencing data. Nucleic Acids Res 42, D68-73.

Lee, J.A., Kim, H.K., Kim, K.H., Han, J.H., Lee, Y.S., Lim, C.S., Chang, D.J., Kubo, T., and Kaang, B.K. (2001). Overexpression of and RNA interference with the CCAAT enhancer-binding protein on long-term facilitation of Aplysia sensory to motor synapses. Learning \& Memory 8, 220-226.

Lyles, V., Zhao, Y.L., and Martin, K.C. (2006). Synapse formation and mRNA localization in cultured Aplysia neurons. Neuron 49, 349-356.

Martin-Gomez, L., Villalba, A., Kerkhoven, R.H., and Abollo, E. (2014). Role of microRNAs in the immunity process of the flat oyster Ostrea edulis against bonamiosis. Infection Genetics and Evolution 27, 40-50.

Masood, M., Raftos, D.A., and Nair, S.V. (2016). Two oyster species that show differential susceptibility to virus infection also show differential proteomic responses to generic dsRNA. Journal of proteome research.

Miao, G., Qi, H., Li, L., Que, H., and Zhang, G. (2016). Characterization and functional analysis of two inhibitor of apoptosis genes in Zhikong scallop Chlamys farreri. Developmental and comparative immunology $60,1-11$.

Millan, M.J. (2011). MicroRNA in the regulation and expression of serotonergic transmission in the brain and other tissues. Current Opinion in Pharmacology 11, 11-22.

Ormond, J., Hislop, J., Zhao, Y.L., Webb, N., Vaillaincourt, F., Dyer, J.R., Ferraro, G., Barker, P., Martin, K.C., and Sossin, W.S. (2004). ApTrkl, a Trk-like receptor, mediates serotonin-dependent ERK activation and long-term facilitation in Aplysia sensory neurons. Neuron 44, 715-728.

Ozpolat, B.D., and Bely, A.E. (2015). Gonad establishment during asexual reproduction in the annelid Pristina leidyi. Developmental Biology 405, 123-136.

Pari, M., Kuusksalu, A., Lopp, A., Kjaer, K.H., Justesen, J., and Kelve, M. (2014). Enzymatically active 2 ', 5 ' -oligoadenylate synthetases are widely distributed among Metazoa, including protostome lineage. Biochimie 97, 200-209.

Rajasethupathy, P., Antonov, I., Sheridan, R., Frey, S., Sander, C., Tuschl, T., and Kandel, E.R. (2012). A Role for Neuronal piRNAs in the Epigenetic Control of Memory-Related Synaptic Plasticity. Cell 149, 693-707.

Rajasethupathy, P., Fiumara, F., Sheridan, R., Betel, D., Puthanveettil, S.V., Russo, J.J., Sander, C., Tuschl, T., and Kandel, E. (2009). Characterization of Small RNAs in Aplysia Reveals a Role for miR-124 in Constraining Synaptic Plasticity through CREB. Neuron 63, 803-817.

Rosani, U., Pallavicini, A., and Venice, P. (2016). The miRNA biogenesis in marine bivalves. Peerj 4, e1763-e1763.

Sperling, E.A., Vinther, J., Moy, V.N., Wheeler, B.M., Semon, M., Briggs, D.E.G., and Peterson, K.J. (2009). MicroRNAs resolve an apparent conflict between annelid systematics and their fossil record. Proceedings of the Royal Society B-Biological Sciences 276, 4315-4322. 
Sugio, M., Takeuchi, K., Kutsuna, J., Tadokoro, R., Takahashi, Y., Yoshida-Noro, C., and Tochinai, S. (2008). Exploration of embryonic origins of germline stem cells and neoblasts in Enchytraeus japonensis (Oligochaeta, Annelida). Gene Expression Patterns 8, 227-236.

Svoboda, P. (2019). Introduction to RNAi and miRNA pathways.

Takeo, M., Yoshida-Noro, C., and Tochinai, S. (2010). Functional analysis of grimp, a novel gene required for mesodermal cell proliferation at an initial stage of regeneration in Enchytraeus japonensis (Enchytraeidae, Oligochaete). International Journal of Developmental Biology 54, 151-160.

Tessmar-Raible, K., Raible, F., Christodoulou, F., Guy, K., Rembold, M., Hausen, H., and Arendt, D. (2007). Conserved sensory-neurosecretory cell types in annelid and fish forebrain: Insights into hypothalamus evolution. Cell 129, 1389-1400.

Tian, R.R., Zheng, Z., Huang, R.L., Jiao, Y., and Du, X.D. (2015). miR-29a Participated in Nacre Formation and Immune Response by Targeting Y2R in Pinctada martensii. International Journal of Molecular Sciences 16, 29436-29445.

Wang, G.D., Li, N., Zhang, L.L., Zhang, L.H., Zhang, Z.P., and Wang, Y.L. (2015a). IGFBP7 promotes hemocyte proliferation in small abalone Haliotis diversicolor, proved by dsRNA and cap mRNA exposure. Gene 571, 65-70.

Wang, G.D., Li, N., Zhang, L.L., Zhang, L.H., Zhang, Z.P., and Wang, Y.L. (2016a). IGFBP7 is involved in abalone metamorphosis. Aquaculture 451, 377-384.

Wang, M., Wang, L., Xin, L., Wang, X., Wang, L., Xu, J., Jia, Z., Yue, F., Wang, H., and Song, L. (2016b). Two novel LRR-only proteins in Chlamys farreri: Similar in structure, yet different in expression profile and pattern recognition. Developmental and Comparative Immunology 59, 99-109.

Wang, M.Q., Wang, L.L., Guo, Y., Yi, Q.L., and Song, L.S. (2016c). An LRR-only protein representing a new type of pattern recognition receptor in Chlamys farreri. Developmental and Comparative Immunology 54, 145-155.

Wang, P.H., Weng, S.P., and He, J.G. (2015b). Nucleic acid-induced antiviral immunity in invertebrates: An evolutionary perspective. Developmental and Comparative Immunology 48, 291-296.

Weigert, A., Helm, C., Hausen, H., Zakrzewski, A.C., and Bleidorn, C. (2013). Expression pattern of Piwi-like genes in adult Myzostoma cirriferum (Annelida). Development Genes and Evolution 223, 329-334.

Xu, F., Wang, X.T., Feng, Y., Huang, W., Wang, W., Li, L., Fang, X.D., Que, H.Y., and Zhang, G.F. (2014). Identification of Conserved and Novel MicroRNAs in the Pacific Oyster Crassostrea gigas by Deep Sequencing. Plos One 9, e104371-e104371.

Yoshida-Noro, C., and Tochinai, S. (2010). Stem cell system in asexual and sexual reproduction of Enchytraeus japonensis (Oligochaeta, Annelida). Development Growth \& Differentiation 52, $43-55$.

Zhang, Y., Yu, F., Li, J., Tong, Y., Zhang, Y.H., and Yu, Z.N. (2014). The first invertebrate RIG-I-like receptor (RLR) homolog gene in the pacific oyster Crassostrea gigas. Fish \& Shellfish Immunology 40, 466-471.

Zhao, X.L., Yu, H., Kong, L.F., Liu, S.K., and Li, Q. (2016). High throughput sequencing of small RNAs transcriptomes in two Crassostrea oysters identifies microRNAs involved in osmotic stress response. Scientific Reports 6, 22687-22687. 
Zheng, Z., Jiao, Y., Du, X.D., Tian, Q.L., Wang, Q.H., Huang, R.L., and Deng, Y.W. (2016a). Computational prediction of candidate miRNAs and their potential functions in biomineralization in pearl oyster Pinctada martensii. Saudi Journal of Biological Sciences 23, 372-378.

Zheng, Z., Liang, J., Huang, R., Du, X., Wang, Q., Deng, Y., and Jiao, Y. (2016b). Identification of a novel miR-146a from Pinctada martensii involved in the regulation of the inflammatory response. Fish and Shellfish Immunology 54, 40-45.

Zhou, Z., Wang, L.L., Song, L.S., Liu, R., Zhang, H., Huang, M.M., and Chen, H. (2014). The Identification and Characteristics of Immune-Related MicroRNAs in Haemocytes of Oyster Crassostrea gigas. Plos One 9, e88397-e88397. 


\title{
RNAi AND mIRNA PATHWAYS IN NEMATODES
}

Keywords: dsRNA, siRNA, miRNA, Dicer, Argonaute

\author{
PETR SVOBODA \\ Institute of Molecular Genetics, Academy of Sciences of the Czech Republic, \\ Videnska 1083, 14220 Prague 4, Czech Republic \\ Correspondence to: Petr Svoboda, Institute of Molecular Genetics ASCR, \\ Videnska 1083, 14220 Prague 4, Czech Republic, tel. \#+420 241063147, \\ e-mail: svobodap@img.cas.cz.
}

\begin{abstract}
RNA silencing denotes sequence-specific repression mediated by small RNAs. In metazoa, there are two mechanistically closely related pathways: RNA interference (RNAi) and microRNA (miRNA) pathway. The miRNA pathway regulates endogenous protein-coding gene expression and has been implicated in many biological processes. RNAi generally serves as a form of innate immunity targeting viruses and mobile elements. This review is summarizing information about RNAi and miRNA pathways in the phylum Nematoda. Research on miRNA and RNAi pathways in the phylum Nematoda has been primarily using Caenorhabditis elegans model system and related species. In fact, miRNA and RNAi pathways were both discovered in this model system. Studies in Caenorhabditis elegans revealed highly complex organization of small RNA pathways despite there is just a single Dicer gene. The complexity of small RNA pathways in Caenorhabditis elegans stems from a large number of Argonaute proteins and three RNA-dependent RNA polymerases, which establish an intricate system of primary and secondary small RNAs with different biological functions in the cytoplasm and nucleus. This review focuses on the miRNA pathway and pathways initiated by long dsRNA. The first part introduces the key molecular players of RNA silencing in nematodes. The second summarizes miRNA and dsRNA-induced mechanisms, in this case RNAi (exogenous $=$ exo-RNAi and endogenous $=$ endo-RNAi) and adenosine deamination (nematodes do not have the interferon system like vertebrate groups).
\end{abstract}

\section{Introduction}

Nematodes are free living or parasitic unsegmented pseudocoelomate worms. There is $\sim 80000$ described species, estimates of the total number of species are reaching 1 million (Margulis and Schwartz, 1998). Parasitic nematodes cause disease in animals and plants, thus are of high medical and economical significance. In addition, a free-living soil nematode Caenorhabditis elegans (C. elegans) became a laboratory workhorse for the last half a century that contributed to our understanding of every major molecular mechanism in multicellular organisms. In fact, miRNA and RNAi pathways were both first discovered in C. elegans. 

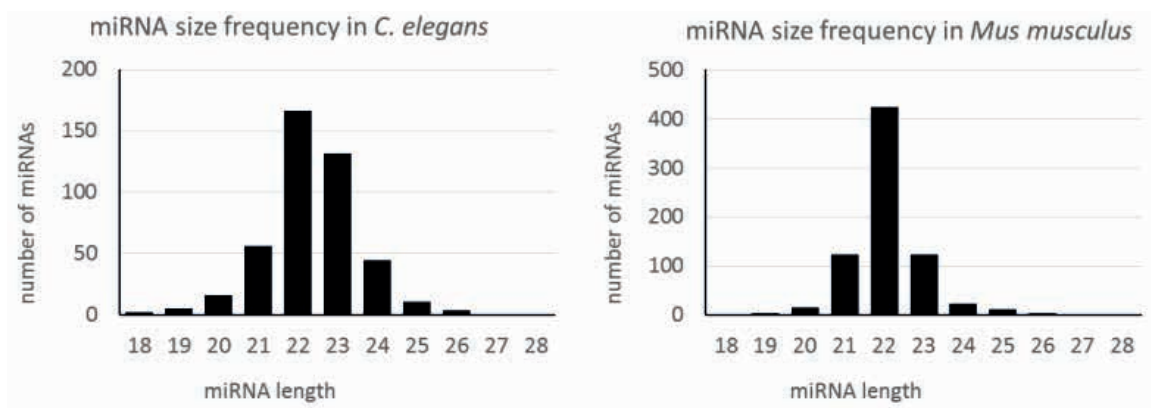

Figure 1 Nematode miRNA lengths

The left graph depicts size distribution of all 334 miRNAs of a C. elegans deposited in the miRBase (version 21). For comparison, the right graph shows size distribution of 721 high-confidence murine miRNAs.

RNA silencing in C. elegans is very complex despite it has only one Dicer gene. The complexity is created by the extreme diversity of downstream pathways, which employ miRNAs as well as primary and secondary siRNA and other small RNAs that are loaded on 26 Argonaute proteins of three subfamilies, which have different biological functions in the cytoplasm and nucleus. For the purpose of the report, I focus on the miRNA pathway and pathways initiated by long dsRNA. This part of the report is organized similarly to the other taxon-oriented reports - first I introduce the key molecular players of RNA silencing in nematodes and then I summarize miRNA and dsRNA-induced mechanisms, in this case RNAi (exogenous $=$ exo-RNAi and endogenous $=$ endo-RNAi) and adenosine deamination (nematodes do not have the interferon system like vertebrate groups).

\section{The Microprocessor complex}

C. elegans utilizes the same Microprocessor complex as the earlier discussed Metazoa, i.e. a complex of Drosha (drsh-1) and DGCR8 homologs, the latter being named Pasha (partner of Drosha, pash-1) (Denli et al., 2004). The complex cleaves the pri-miRNA into pre-miRNA in the nucleus. Suppression of Pasha expression in C. elegans interferes with pri-miRNA processing, leading to an accumulation of pri-miRNAs and a reduction in mature miRNAs (Denli et al., 2004). Furthermore, pash-1 mutation relieves let-7-mediated repression and the phenotype overlaps with those observed in $d c r-1$ or $d r s h-1$ mutants (Denli et al., 2004). NGS of small RNAs showed that Drosha cleavage sites were enriched with specific nucleotides (mainly uridine) and structural distortions resulting in reduced stability on the 5' arm (Warf et al., 2011).

\section{Dicer}

C. elegans genome contains a single Dicer gene (dcr-1), which was discovered as a miRNA and RNAi biogenesis factor in 2001 (Grishok et al., 2001; Ketting et al., 2001; Knight 
and Bass, 2001). Dicer is the central component in biogenesis miRNAs, endo-siRNAs and exo-siRNA. The loss of Dicer results in sterility, abnormal oocytes, and heterochronic phenotypes (Grishok et al., 2001; Ketting et al., 2001; Knight and Bass, 2001).

Most nematodes possessed only one Dicer gene (Gao et al., 2014). Dicer cleaves long dsRNA into $23 \mathrm{bp}$ siRNAs in vitro in the presence of ATP (Ketting et al., 2001). However the length of the primary cleave product is retained only in miRNAs, where C. elegans miRNAs deposited in the miRBase are 22-23 nt long (Fig. 1) and in RDE-1-bound siRNAs in the exo-RNAi. The endogenous siRNAs are longer and belong to the class of 26G RNAs whose biogenesis requires additional factors beyond Dicer.

There is neither crystal structure of C. elegans Dicer nor a cryo-EM analysis of the shape of the molecule. However, the general domain composition of C. elegans Dicer is very similar to that of human Dicer (Fig. 2). Thus, some information can be inferred from sequence comparison as well as from biochemical analyses of isolated domains.

Analysis of the PAZ domain showed that the 5' and 3' pockets are conserved across members of the Dicer1 subfamily, with the exception of the 5' pocket in nematode Bursaphelenchus xylophilus. The helicase domain of Dicer is not necessary for miRNA processing, or exo-RNAi. Comparisons of wild-type and helicase-defective strains showed that the helicase domain is required by a subset of annotated endo-siRNAs, in particular, 26G RNAs (Welker et al., 2010). Consistently with these observations, the helicase domain is essential for cleaving dsRNA with blunt or 5'-overhangs but not with 3' overhangs, such as miRNA precursors (Welker et al., 2011). Further, blunt termini, but not 3' overhangs yield siRNAs from internal regions of dsRNA (Welker et al., 2011). RNAse IIIb and dsRNA binding domains contain two conserved phosphorylation sites, which are phosphorylated by ERK. The phosphorylation is necessary and sufficient to trigger Dicer's nuclear translocation (Drake et al., 2014) and plays a role during oocyte-to-embryo transition (Drake et al., 2014).

C. elegans evolved a system where Dicer processes substrates for different pathways (endogenous RNAi, exogenous RNAi, antiviral defense) into 22 nt small RNAs. Thus, needs to DCR-1 function as a hub for several small RNA pathways where it contributes to sorting of small RNAs into different pathways. This contrasts with mammals, where there is minimal, if any, sorting during loading of Dicer-produced small RNAs, and with Drosophila, which employs two dedicated Dicer proteins for RNAi and miRNA pathways. Sorting involves substrate structure and distinct protein factors (Jannot et al., 2008). Pre-miRNAs have unique structures that facilitate loading onto ALG-1/2 Argonaute proteins. Pre-miRNA analysis showed structural distortions adjacent to Dicer cleavage sites (Warf et al., 2011) and common internal bulges/mismatches. Remarkably, nucleotide changes in the pre-let-7 miRNA precursor that create a perfectly complementary stem cause loading of resulting small RNAs on RDE-1, which is the Argonaute protein acting in the exogenous RNAi pathway (Steiner et al., 2007). Reintroducing mismatches into the stem restores loading onto ALG-1 (Steiner et al., 2007). The second sorting system relies of proteins recognizing Dicer substrates and presenting it to Dicer, such as RDE-4 discussed in the next section, or Dicer associated proteins, which modify DCR-1 products and direct them on distinct Argonaute proteins (see further below).

DCR-1 also binds RNAs without generating small RNAs. Analysis of Dicer-associated RNAs in C. elegans by PAR-CLIP (Photoactivatable Ribonucleoside-Enhanced 


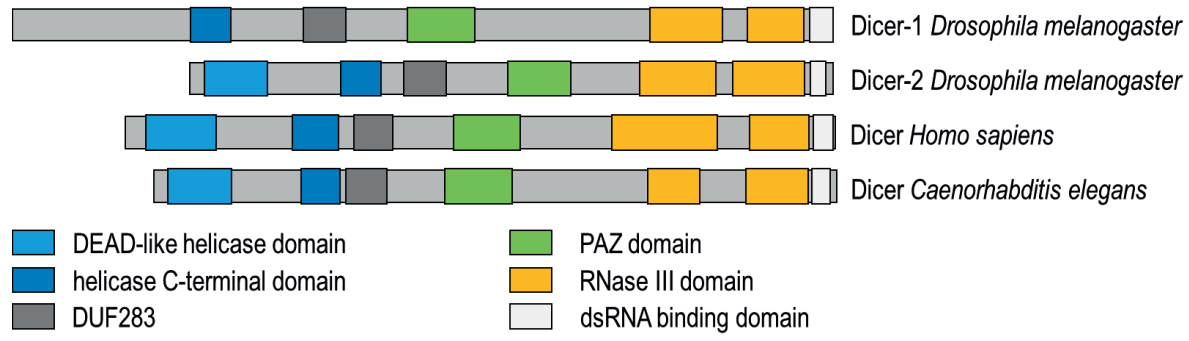

Figure 2 Comparison of $C$. elegans, human and Drosophila Dicer proteins Domain composition was adopted from (Jaskiewicz and Filipowicz, 2008).

Crosslinking and Immunoprecipitation) revealed 2500 Dicer binding sites in the $C$. elegans transcriptome (Rybak-Wolf et al., 2014). Apart from miRNA precursors, DCR-1 also binds a variety of RNA molecules (structural RNAs, promoter RNAs, and mitochondrial transcript). However, Dicer-binding sites beyond miRNAs mostly reside on mRNAs/ lncRNAs that are not significantly processed into small RNAs. These passive binding (i.e. without dicing) typically involves small, Dicer-bound hairpins within intact transcripts and generally stabilizes target expression (Rybak-Wolf et al., 2014). It was proposed that passive binding might be one of the mechanisms balancing siRNA/miRNA production since it was shown that passive binding sites can sequester Dicer and reduce microRNA expression (Rybak-Wolf et al., 2014). For example, a noncoding RNA rncs-1 is a non-cleaved Dicer substrate, which appears to reduce Dicer activity in hypodermis and intestine (Hellwig and Bass, 2008)

Remarkably, two distinct truncated Dicer isoforms produced by proteolytic cleavage were reported from C. elegans. While both isoforms are C-terminal fragments created by proteolytic cleavage, they show profound functional differences. The first one is created by cleavage of DCR-1 by CED-3 caspase. The released $43 \mathrm{kDa}$ C-terminal fragment changes its RNase activity into DNase, which fragments chromosomal DNA during apoptosis (Nakagawa et al., 2010). Subsequent molecular analysis proposed a model where the N-terminal helicase domain is suppressing DNase activity and release of the C-terminal part upon cleavage at Asp1472 (within the RNAse IIIa domain) by CED-3 activates a DNA-binding activity and enables DNA cleavage (Ge et al., 2014). The second truncated form of Dicer exists as a $\sim 95 \mathrm{kDa} C$-terminal Dicer fragment generated by proteolytic cleavage upstream of RNase IIIa domain. It is abundant in adult $C$. elegans cells where it might enhance exogenous and antiviral RNAi while negatively regulating miRNA biogenesis (Sawh and Duchaine, 2013). The $95 \mathrm{kDa}$ Dicer fragment does not interact with RDE-4 nor with the ERI complex but it was found to bind miRNA-associated AGO proteins ALG-1/2 suggesting that it sequesters ALG-1/2 from the full-length DCR-1, thereby acting as a competitive inhibitor of miRNA processing (Sawh and Duchaine, 2013).

There has been a number of identified Dicer binding partners, which form different complexes, which act in different types of RNAi pathways (Duchaine et al., 2006; Lee et al., 2006). One of them is the exo-RNAi Dicer complex composed of RDE-4 (dsRBP), DCR-1, RDE-1 (Argonaute protein), and DRH-1/2 (Dicer-related helicases) (Tabara et al., 
2002). Another complex is ERI/Dicer complex, which was reported for endo-RNAi during sperm development (Pavelec et al., 2009). It is composed of ERI-1/3/5, DCR-1, and RRF-3 (RdRP) and produces 26G primary endo-siRNAs, which then drive production of secondary 22G RNAs (Duchaine et al., 2006; Gent et al., 2009; Han et al., 2009). ERI-1 is an exoribonuclease that degrades siRNAs in vitro (Kennedy et al., 2004) and acts in 26G biogenesis in vivo (Duchaine et al., 2006; Gent et al., 2009; Han et al., 2009), ERI-3 has no known domain (Duchaine et al., 2006), and ERI-5 is a tudor domain protein, which tethers the RdRP to Dicer (Thivierge et al., 2012). An additional genetic screen identified ERI-9 as an additional component of ERI/DCR complex acting in the endo-RNAi pathway (Pavelec et al., 2009). The miRNA pathway-dedicated complex involves interaction between Dicer and ALG-1/2 and, eventually, LIN-41 regulating let-7 miRNA (Duchaine et al., 2006). The list of Dicer-binding proteins in C. elegans is even longer and involves proteins with roles in different aspects of silencing or with yet undetermined functions (Beshore et al., 2009; Beshore et al., 2011; Duchaine et al., 2006; Racen et al., 2008).

\section{dsRBPs - RDE-4 and homologs}

RDE-4 is a dsRBP partner of Dicer in C. elegans. It is a 385-amino acid protein carrying two N-terminal dsRBDs and a third degenerate dsRBD at the C-terminus. A similar organization is found in other dsRBDs mentioned above - R2D2 and Loquacious in Drosophila and TRBP and PACT in mammals. RDE-4 was identified by a systematic screen for C. elegans RNAi-deficient mutants (Tabara et al., 1999). The $r d e-4$ mutant was completely deficient in RNAi but failed to show any discernible phenotype, including the absence of transposon activation, which was observed in some other $r d e$ mutants (Tabara et al., 1999). The loss of RDE-4 function can be compensated with injection of synthetic siRNA (Parrish and Fire, 2001) or with high amounts of dsRNA and siRNAs (Habig et al., 2008).

Mutants and biochemical analyses support a model where RDE-4 dimerizes through the C-terminal domain; dimers cooperatively bind long dsRNA, interacts with Dicer through the linker region, and forms a complex initiating the RNAi together with Dicer, RDE-1, and DRH-1/2 (Blanchard et al., 2011; Chiliveri and Deshmukh, 2014; Parker et al., 2006; Parker et al., 2008; Parrish and Fire, 2001; Tabara et al., 2002). The presence of an Argonaute protein in the complex suggests that in exo-RNAi, dsRNA recognition, processing into siRNA and loading of the Argonaute-containing effector complex is integrated in a one complex. Importantly, while RDE-4 is involved in siRNA production from exogenous and endogenous dsRNAs, RDE4, Dicer, RDE-1 and DRH-1/2 act in the exo-RNAi (Lee et al., 2006). The endogenous RNAi pathway (endo-RNAi), which targets endogenous genes, employs a distinct mechanism of siRNA production involving DCR-1 and RDE-4 but not RDE-1 and DRH-1/2 (Gent et al., 2010; Lee et al., 2006).

Importantly, RDE-4 differs from TARBP2 role in RNAi. While RDE-4 is involved in siRNA production from dsRNA but is not essential for later steps of RNAi because RDE4 immunoprecipitates with trigger dsRNA but not siRNA (Tabara et al., 2002), TARBP2 functions during RISC formation and AGO loading. Binding properties in vitro correspond to different roles of the two proteins in vivo. RDE-4 preferentially binds long dsRNA, while 
TRBP binds siRNA with an affinity that is independent of dsRNA length (Parker et al., 2008). This stems from the fact that RDE-4 binds cooperatively, via contributions from multiple domains, while TRBP binds non-cooperatively (Parker et al., 2008).

\section{Argonaute proteins}

Caenorhabditis has the largest diversity of Argonaute proteins of all studied model systems $-25-27$ Argonaute family members (Table 1 ) were described to carry primary and secondary small RNAs that act in soma and germline in multiple inter-related pathways (Buck and Blaxter, 2013; Yigit et al., 2006) (Arabidopsis, which has complex RNA silencing has 10 Argonaute proteins (reviewed in Buck and Blaxter, 2013). In addition to the above-described AGO and PIWI clades of the Argonaute family, nematodes utilize a unique Argonaute subfamily termed WAGO, which are "Worm-specific AGO" proteins, which bind secondary 22G small RNAs (summarized in (Buck and Blaxter, 2013). The complete description of small RNA pathways in C. elegans is beyond the scope of this report. Here, I will mainly focus on Argonaute proteins acting as the primary recipients of Dicer-generated small RNAs and will include the downstream pathways when directly related to the miRNA pathway or dsRNA response.

\section{ALG-1/2 - miRNA-binding Argonaute proteins}

There are two Argonaute proteins carrying canonical miRNAs in C. elegans: ALG-1 and ALG-2 (collectively designated ALG-1/2). They were identified as RDE-1 homologs, which cause heterochronic phenotypes similar to lin-4 and let-7 mutations and which are (together with $d c r-1$ ) necessary for the maturation and activity of the lin-4 and let-7 miRNAs. (Grishok et al., 2001). ALG-1/2 are required for the miRNA function, but not for the siRNA-directed gene silencing (Jannot et al., 2008). Although ALG-1 and ALG-2 are almost identical and highly redundant (only loss of both genes leads to embryonic lethality), several non-redundant functions have been observed in processing pre-miRNA processing/ miRISC formation (Kuzuoglu-Oeztuerk et al., 2012; Steiner et al., 2007; Tops et al., 2006; Vasquez-Rifo et al., 2012). Furthermore, while ALG-1/2 spatio-temporal expression profiles overlap, there is variability in dominating ALG expression in various cells and differential association to specific miRNAs (Vasquez-Rifo et al., 2012).

Interestingly, ALG- $1 / 2$ have conserved key residues for the slicing activity suggesting that they might function as active slicers despite the general lack of miRNA-mediated site-specific cleavage of cognate mRNAs. In any case, the slicing activity of ALG-1/2 has been demonstrated in vitro and in vivo (Bouasker and Simard, 2012). The loss of the slicing activity results in the accumulation of truncated miRNA precursors and altered miRISC formation suggesting that the slicing activity is necessary during miRISC formation (Bouasker and Simard, 2012). This notion is further supported by mutations in ALG-1, which separate miRISC loading and repression of cognate RNAs (Zinovyeva et al., 2014)

Canonical miRNA-mediated repression involves AIN-1 (ALG-1 INteracting protein), GW182 homolog, which interacts with miRISC and has been implicated in targeting 
Table 1 Argonaute proteins in Caenorhabditis elegans

The table was compiled form the following literature (Batista et al., 2008; Buckley et al., 2012; Fischer et al., 2011; Fischer et al., 2013; Tijsterman et al., 2002a; Tijsterman et al., 2002b; Vasale et al., 2010; Wang and Reinke, 2008; Yigit et al., 2006). Slicer activity "+" indicates that a given Argonaute protein has potential to act as a slicer, not that slicing is its primary mode of action. In some case, slicing potential has been inferred from the sequence, i.e. it is not supported with experimental evidence

\begin{tabular}{|c|c|c|c|c|c|c|c|}
\hline \multirow{2}{*}{ common name(s) } & \multirow{2}{*}{ subfamily } & \multirow{2}{*}{ slicer } & \multicolumn{5}{|c|}{ associated small RNA } \\
\hline & & & type & length & 5'nt & 5' end & 3 ' end \\
\hline ALG-1, F48F7.1 & $\mathrm{AGO}$ & + & miRNA & $22-23$ & & mono-P & $-\mathrm{OH}$ \\
\hline ALG-2 & $\mathrm{AGO}$ & + & miRNA & $22-23$ & & mono-P & $-\mathrm{OH}$ \\
\hline ALG-3, T22B3.2 & $\mathrm{AGO}$ & $+?$ & 26G siRNA & 26 & G & mono-P & $-\mathrm{OH}$ \\
\hline ALG-4, ZK757.3 & $\mathrm{AGO}$ & $+?$ & 26G siRNA & 26 & G & mono-P & $-\mathrm{OH}$ \\
\hline RDE-1, K08H10.7 & $\mathrm{AGO}$ & + & primary siRNA & $22-23$ & & mono-P & $-\mathrm{OH}$ \\
\hline ERGO-1, R09A1.1 & $\mathrm{AGO}$ & + & 26G siRNA & 26 & G & mono-P & 2'-O-met \\
\hline CSR-1, F20D12.1 & $\mathrm{AGO}$ & + & $22 \mathrm{G}$ siRNA & 22 & G & tri-P & $-\mathrm{OH}$ \\
\hline C06A1.4 & AGO? & - & & & & & \\
\hline H10D12.2, M03D4.6 & AGO? & - & & & & & \\
\hline C14B1.7 & PIWI? & - & & & & & \\
\hline T23D8.7, HPO-24 & PIWI? & - & & & & & \\
\hline C04F12.1 & PIWI? & $+?$ & & & & & \\
\hline PRG-1, D2030.6 & PIWI & + & $21 \mathrm{U}$ piRNA & 21 & $\mathrm{U}$ & mono-P & 2'-O-met \\
\hline PRG-2, C01G5.2 & PIWI & + & $21 \mathrm{U}$ piRNA & 21 & $\mathrm{U}$ & & \\
\hline WAGO-1, R06C7.1 & WAGO branch1 & - & 22G siRNA & 22 & G & tri-P & $-\mathrm{OH}$ \\
\hline WAGO-2, F55A12.1 & WAGO branch1 & - & 22G siRNA & 22 & G & tri-P & $-\mathrm{OH}$ \\
\hline $\begin{array}{l}\text { PPW-2, WAGO-3, } \\
\text { Y110A7A.18 }\end{array}$ & WAGO branch1 & - & 22G siRNA & 22 & G & tri-P & $-\mathrm{OH}$ \\
\hline WAGO-4, F58G1.1 & WAGO branch1 & - & 22G siRNA & 22 & G & tri-P & $-\mathrm{OH}$ \\
\hline WAGO-5, ZK1248.7 & WAGO branch1 & - & 22G siRNA & 22 & G & tri-P & $-\mathrm{OH}$ \\
\hline $\begin{array}{l}\text { SAGO-2, WAGO-6/8, } \\
\text { F56A6.1 }\end{array}$ & WAGO branch2 & - & 22G siRNA & 22 & G & tri-P & $-\mathrm{OH}$ \\
\hline $\begin{array}{l}\text { PPW-1, WAGO-7, } \\
\text { C18E3.7 }\end{array}$ & WAGO branch2 & - & 22G siRNA & 22 & G & tri-P & $-\mathrm{OH}$ \\
\hline $\begin{array}{l}\text { SAGO-1, WAGO-8/6, } \\
\text { K12B6.1 }\end{array}$ & WAGO branch2 & - & $22 \mathrm{G}$ siRNA & 22 & G & tri-P & $-\mathrm{OH}$ \\
\hline $\begin{array}{l}\text { HRDE-1, WAGO-9, } \\
\text { C16C10.3 }\end{array}$ & WAGO branch 3 & - & 22G siRNA & 22 & G & tri-P & $-\mathrm{OH}$ \\
\hline WAGO-10, T22H9.3 & WAGO branch 3 & - & 22G siRNA & 22 & G & tri-P & $-\mathrm{OH}$ \\
\hline WAGO-11, Y49F6A.1 & WAGO branch 3 & - & $22 \mathrm{G}$ siRNA & 22 & G & tri-P & $-\mathrm{OH}$ \\
\hline $\begin{array}{l}\text { NRDE-3, WAGO-12, } \\
\text { R04A9.2 }\end{array}$ & WAGO branch 3 & - & 22G siRNA & 22 & G & tri-P & $-\mathrm{OH}$ \\
\hline
\end{tabular}


ALG-1 to P-bodies (Ding et al., 2005; Ding and Grosshans, 2009). It was reported that has lin-4-mediated repression acts at the level of translation initiation and frequently but not always coincides with transcript degradation (Ding and Grosshans, 2009). Remarkably, despite AIN-1/2 (two redundant GW182 homologs (Zhang et al., 2007)) lack most of the domains found in vertebrate proteins, they still interact with ALG-1/2 (Kuzuoglu-Oeztuerk et al., 2012). The interaction is mediated by GW repeats in the central part of AIN-1/2 but only AIN-1 interacts with PABPC1, PAN3, NOT1 and NOT2 (Kuzuoglu-Oeztuerk et al., 2012).This implies that the key principle of miRNA mediated repression, GW182-mediated tethering of PABPC1 and deadenylase complexes to AGO, is broadly conserved across metazoa.

The key determinant of target recognition is the seed binding as described in the mammalian section. Direct probing of the RNA backbone in isolated native C. elegans miRISC that the seed region is uniquely constrained, while the rest of the microRNA structure is conformationally flexible. Probing the Watson-Crick edges of the bases shows that bases 2-4 are largely inaccessible to solvent, while seed region bases $5-8$ are readily modified (Lambert et al., 2011). These data are consistent with structural analyses of AGO proteins in mammals and elsewhere.

\section{RDE-1 - primary siRNA-binding Argonaute in exo-RNAi and antiviral RNAi}

RDE-1 has the central role in RNAi. It was identified through a screen for RNA-deficient mutants (Tabara et al., 1999). Rde-1 mutants are strongly resistant to RNAi but without obvious defects in growth or development (Tabara et al., 1999). As mentioned above, RDE-1 associates with RDE-4, Dicer and DRH1/2 acting in the exo-RNAi and antiviral RNAi (Lu et al., 2005; Parrish and Fire, 2001; Tabara et al., 2002; Wilkins et al., 2005). As mentioned above, RDE-1 accommodates perfect siRNA duplexes, which includes artificially modified miRNA precursors (Steiner et al., 2007). It was proposed that RDE-1 functions as a scavenger taking up small RNAs from different sources (unlike ALG-1/2), which exclusively bind miRNAs (Correa et al., 2010).

Interestingly, the bulk of the slicing activity in C. elegans extracts in vitro comes from CSR-1 loaded with secondary 22G RNAs and not from RDE-1 loaded with primary siRNA (Aoki et al., 2007). While RDE-1 is a "slicer", it uses its endonucleolytic activity for removal of the passenger strand of the siRNA duplex and not for cleaving cognate RNAs, suggesting uncoupled roles of the RDE-1 slicer activity in siRNA maturation from and cognate RNA cleavage (Steiner et al., 2009). It was found that RDE-1 recruits RDE8 endoribonuclease to target RNA (Tsai et al., 2015). According to the model, RDE-8 cleaves cognate RNAs and is needed for the production of 3' uridylated fragments of target mRNA, which provide templates for RdRP-mediated production of secondary siRNAs (Tsai et al., 2015).

Taken together, RDE-1 loaded with primary siRNAs stands at the beginning of an amplification cascade, while other Argonaute proteins loaded with secondary siRNAs mediate downstream silencing (Yigit et al., 2006). A remarkable feature of the downstream AGOs loaded with $22 \mathrm{G}$ siRNAs is the lack the slicer activity. RDE-1 does not initiate only post-transcriptional repression; it has also been implicated in initiation (but not 
maintenance) of long-term silencing ( $>25$ generations) induced by long dsRNA in neurons (Buckley et al., 2012).

\section{G Argonaute effectors in RNAi - ERGO-1 - primary 26G siRNA-binding Argonaute in endo-RNAi}

ERGO-1 is a PIWI-clade effector that binds 26G RNAs generated in the endo-RNAi pathway in female germline and embryo (Gent et al., 2010; Pavelec et al., 2009; Vasale et al., 2010). ERGO-1 targets exhibit a non-random distribution in the genome and appear to include many gene duplications, suggesting that this pathway may control overexpression resulting from gene expansion (Vasale et al., 2010). In endo-RNAi in the soma, ERGO-1 accommodates $26 \mathrm{G}$ small RNAs in a process involving the ERI/Dicer complex and leading to production of more abundant secondary 22G small RNAs (Duchaine et al., 2006; Gent et al., 2009; Han et al., 2009). The biogenesis of primary 26G RNAs involves RdRP RRF-3 and RDE-4 while the biogenesis of secondary $22 \mathrm{G}$ siRNAs involves a second RdRP (RRF1 or EGO-1) and loading on WAGOs. Additional proteins implicated in ERGO-1-mediated repression include the helicase ERI-6/7 (Armitage helicase homolog) (Fischer et al., 2011), MUT-2, MUT-7, MUT-16 (Zhang et al., 2011), and RDE10/RDE-11 complex (Zhang et al., 2012), and RDE-12 (Shirayama et al., 2014).

\section{CSR-1 - secondary 22G siRNA-binding Argonautes}

The "executive arm" of RNAi in C. elegans are secondary 22G RNAs. One of the proteins binding 22G RNAs is CSR-1, which was identified in in vitro experiments with $C$. elegans lysates as a dominant slicer activity. However, CSR-1 was shown to rather associate with chromatin to promote proper organization or assembly of targets within the holocentric chromosomes (Campbell and Updike, 2015; Cecere et al., 2014; Claycomb et al., 2009; Seth et al., 2013; Tu et al., 2015; Wedeles et al., 2013a, b). CSR-1 22G RNAs are produced in the germline by a complex of DRH-3 (helicase), EKL-1 (tudor protein), and EGO-1 (RdRP) similarly to WAGO 22G RNAs, which utilize RRF-1 RdRP (Claycomb et al., 2009).

\section{WAGO proteins - secondary 22G siRNA-binding Argonautes}

WAGO proteins are loaded with $22 \mathrm{G}$ RNAs, which were produced by RdRPs. They represent secondary effectors, which execute silencing in slicer-independent fashion in the soma and/or germline in the cytoplasm and the nucleus. NRDE-3 and HRDE-1 were identified as WAGO proteins mediating nuclear silencing (Allo and Kornblihtt, 2010; Buckley et al., 2012; Burton et al., 2011; Gent et al., 2010). HRDE-1 has been implicated in initiation and maintenance of long-term silencing ( $>25$ generations) induced by long dsRNA in neurons (Buckley et al., 2012; Burton et al., 2011). For example, WAGO-1 silences genes, transposons, pseudogenes, and cryptic loci at the chromatin level by directing H3K27me3 through the NRDE pathway (Mao et al., 2015) and possibly in some association with components of the nonsense-mediated decay (NMD) (Gu et al., 2009). A C. elegans isolate from Hawaii 
had a natural defect in RNAi that was specific to the germline and was a result of multiple mutations the WAGO protein PPW-1 (Tijsterman et al., 2002b).

\section{RdRPs}

C. elegans is the main animal model for studying RdRPs. While an earlier study of RdRP in C. elegans suggested that dsRNA synthesis can be primed by primary siRNAs (a model of "degradative PCR") (Sijen et al., 2001), later studies demonstrated that RdRPs do not require the priming by primary siRNAs and produce short RNAs using RISC-targeted mRNAs as templates (Pak and Fire, 2007; Sijen et al., 2007). Surprisingly, sequencing of small RNAs associated with ongoing RNAi in C. elegans showed that Dicer-independent secondary siRNAs constitute the majority of cloned siRNAs (Pak and Fire, 2007). These secondary siRNAs are only antisense, carry 5' di- or triphosphates, and are not bound by RDE-1 but by other Argonaute proteins (Pak and Fire, 2007; Sijen et al., 2007). C. elegans genome four putative RdRPs, three of which were implicated in RNA silencing (Duchaine et al., 2006; Lee et al., 2006; Sijen et al., 2001; Smardon et al., 2000).

Of the three RdRPs, RRF-3 was implicated in the upstream part of RNAi, i.e. production of primary 26G siRNAs associated with ERGO-1 or ALG-3/4 Argonaute proteins (Conine et al., 2010; Conine et al., 2013; Duchaine et al., 2006; Gent et al., 2010; Gent et al., 2009; Han et al., 2009; Pavelec et al., 2009; Vasale et al., 2010) while EGO-1 and RRF-1 produce 22 G RNAs (Duchaine et al., 2006; Gent et al., 2010; Gent et al., 2009; Han et al., 2009; Jose et al., 2011; Pavelec et al., 2009; Vasale et al., 2010).

\section{miRNA pathway-specific features in nematodes}

C. elegans was the first organism, in which the first miRNA (lin-4) was identified (Lee et al., 1993) and which revealed that a conserved let-7 miRNA is conserved up to humans (Pasquinelli et al., 2000; Reinhart et al., 2000). The first two miRNAs were identified as regulators of developmental timing Remarkably, phenotype of $d c r-1$ mutants is similar to those of lin-4 and let-7 mutants (Grishok et al., 2001; Ketting et al., 2001; Knight and Bass, 2001) suggesting that miRNAs are the main Dicer product in C. elegans and that lin-4 and let-7 are functionally among the most significant miRNAs of the 434 annotated C. elegans mature miRNAs. C. elegans also produces non-canonical miRNAs, such as mirtrons (Ruby et al., 2007). High throughput analyses provided estimates of $\sim 3500-5000$ of binding sites bound by miRNAs in C. elegans (Zhang et al., 2007; Zisoulis et al., 2010).

C. elegans miRNAs are exposed to different regulations, some of which are also found in other model systems - this includes regulation of let-7 by LIN-28 and sequence uridylation (Lehrbach et al., 2009; Morita and Han, 2006; Newman et al., 2008; Ouchi et al., 2014; Stefani et al., 2015; Vadla et al., 2012; Van Wynsberghe et al., 2011), regulation of let-7 by RACK1 (Chu et al., 2014), and miRNA turnover by XRN-2 (Chatterjee and Grosshans, 2009) and DCS-1-XRN-1 complex (Bossé et al, 2013). miRNA-mediated repression is further regulated at the cellular level by autophagy (Zhang and Zhang, 2013) and 
other regulatory factors, such as the Golgi-associated retrograde protein (GARP) complex (Vasquez-Rifo et al., 2013). At the same time, specific miRNAs influence other RNA silencing pathways - they may interfere with RNAi (Massirer and Pasquinelli, 2013) or direct siRNA biogenesis (Correa et al., 2010).

\section{RNAi pathway-specific features in nematodes}

As it could be expected from diversity of factors mentioned above, RNAi in C. elegans is complex - different variants of RNAi operate in the germline and soma, RNAi recognizes different substrates and has a sequential production of small RNAs, which are the main executors of RNAi. RNAi in C. elegans has two "flavors" - endo-RNAi and exo-RNAi.

\section{Endogenous RNAi in the germline and soma}

The role of endogenous RNAi in shaping the transcriptomes of protein-coding genes during development has been challenged by mutant worms and flies lacking essential components of the RNAi pathway, which were viable and produced healthy offspring (Lee et al., 2004; Okamura et al., 2004; Tabara et al., 1999). Because of that, RNAi had been viewed as a defense mechanism against invasive nucleic acids. However, deep sequencing analyses revealed that endo-siRNAs with sequence complementarity to hundreds of protein-coding mRNAs are present in C. elegans (Ambros et al., 2003; Ruby et al., 2006). Endo-RNAi contributes to control of gene expression. This was revealed by microarray analysis of mutant worms lacking various RNAi-related factors, which found non-overlapping sets of differentially expressed genes, supporting the idea of multiple functionally distinct RNAi pathways in nematodes (Lee et al., 2006). At the same time, the multiple functionality also brings competition between small RNA pathways, which are manifested in different mutants - e.g. endo-siRNA mutants have increased expression of miRNA-regulated stage-specific developmental genes (Zhuang and Hunter, 2012).

Endo-RNAi pathway is initiated by endogenous dsRNAs, which are processed into primary siRNAs (26G RNAs associated with ERGO-1). Their biogenesis involves RDE-4, ERI-1/3/5/9, and RdRP RRF-3. Primary 26G RNAs recognize their targets, leading to production of secondary $22 \mathrm{G}$ RNAs, which will be antisense to the target and represent the main repressive force of endo-RNAi. 22G RNA biogenesis involves EGO-1 and RRF-1 RdRPs, RNAs are loaded mainly on CSR-1 or WAGO proteins. Secondary siRNAs are generated by RdRPs thus serve as amplifiers of the endogenous RNAi in both soma and germline.

A number of endo-siRNA genes were identified in a genetic screen for factors silencing a multicopy transgene. They included genes required for siRNA biogenesis or stability in the oocyte-specific ERGO-1 pathway, including eri-12, encoding an interactor of the RNAi-defective protein RDE-10, and ntl-9/CNOT9, one of several identified CCR4-NOT complex genes, and a conserved ARF-like small Cell ARL-8 required for primary siRNA biogenesis or stability in the sperm-specific ALG-3/4 endogenous RNAi pathway (Fischer et al., 2013). Additional studies revealed that mutations in RNAi-related genes, result in 
defects in meiotic chromosome disjunction, spindle formation or microtubule organization during sperm development and ultimately lead to male sterility or embryonic lethality of the offspring (Gent et al., 2009; Han et al., 2009; Han et al., 2008; Pavelec et al., 2009).

In search for the cellular and developmental processes, which might be controlled by endo-siRNAs in C. elegans, spermatogenesis-associated genes were found enriched in the group of transcripts matching endo-siRNAs (Ruby et al., 2006). Analysis of endo-siRNA target suggested a difference between primary and secondary endo-siRNA targets as 18to 22-mer siRNAs associated with genes required for embryonic development, 23-mers associated uniquely with post-embryonic development, and 24-26-mers associated with phosphorus metabolism or protein modification (Asikainen et al., 2008). It was also shown that in oocytes and embryos, ERGO-1-associated 26G siRNAs and NRDE-3-associated $22 \mathrm{G}$ siRNAs silence recently duplicated genes (Fischer et al., 2011; Vasale et al., 2010).

\section{Exogenous RNAi-systemic and environmental}

Exo-RNAi is triggered by exogenous dsRNA, which can come in many forms; RNAi in C. elegans can be triggered by injecting dsRNA essentially anywhere in the animal, soaking the animal in dsRNA solution or even by feeding it bacteria expressing long dsRNA (Tabara et al., 1998). This allows not only experimental manipulations in the lab but also target parasitic nematodes by creating nematode resistant plants (Yang et al., 2013). However, there is a heterogeneity in the nature in the presence of non-cell autonomous RNAi. For example, only one of eight tested Caenorhabditis species showed efficient environmental RNAi (Winston et al., 2007). Remarkably, RNAi also operates in zero gravity in space (Etheridge et al., 2011). It has zero significance for the report, but worth of mentioning.

The spreading of the silencing signal among cells is controlled by dsRNA-transporting channels encoded by sid-1 and sid-2 genes, which were identified in forward genetic screen to be responsible for systemic RNAi in C. elegans (Winston et al., 2002). SID-1 (systemic RNAi deficient-1) is a conserved transmembrane protein that forms a dsRNA channel (Feinberg and Hunter, 2003; Shih et al., 2009; Shih and Hunter, 2011). Sid-1 has homologs in a wide range of animals, including mammals. Sid-1 mutants have intact cell autonomous RNAi, but cannot perform neither systemic RNAi nor environmental RNAi in response to feeding, soaking, or injection of dsRNA (Winston et al., 2002). SID-2 is a transmembrane protein localized to an apical membrane of intestinal cells. It is necessary for the initial import of dsRNA from gut lumen, but not for the systemic spread of silencing signals among cells (McEwan et al., 2012). Sid-2 homologs have been identified only in two other Caenorhabditis species (Winston et al., 2007). SID-3 is a conserved tyrosine kinase required for the efficient import of dsRNA. Without SID-3, cells perform RNA silencing well but import dsRNA poorly (Jose et al., 2012). The next and distinct systemic RNAi SID factor is SID-5 (SID-4 does not exist), an endosome-associated protein, which promotes transport of RNA silencing signals between cells and act differently than SID1-3 (Hinas et al., 2012). Remarkably, SID-1 sensitizes Drosophila and silkworm cells to RNAi induced by soaking by enabling concentration-dependent cellular uptake of dsRNA (Feinberg and Hunter, 2003; Shih et al., 2009) (Mon et al., 2012; Mon et al., 2013; Shih and Hunter, 2011; Xu et al., 2013a; Xu et al., 2013b). 
In cells, exo-RNAi involves the above-mentioned RDE-4/Dicer/DRH-1 complex in processing dsRNA into primary siRNAs, which are loaded on RDE-1 Argonaute protein. RDE-1 itself does not cleave cognate RNAs. Instead, it recruits RDE-ribonuclease, which is involved in production of templates for RdRP-mediated production of secondary siRNAs (Tsai et al., 2015). In addition, genetic, proteomic, and biochemical data suggested that downstream of RDE-1 operates RDE-10/RDE-11 complex, which is recruited for degradation of target mRNA and RRF-1-dependent secondary siRNA synthesis (Zhang et al., 2012). The link between the RDE-10/RDE-11 complex and the aforementioned RDE- 8 nuclease is unknown at the moment. Finally, several negative regulators of exo-RNAi were identified in genetic screens, including the ERI-1 nuclease (Kennedy et al., 2004), the ERI2/RRF-3 RdRP (Simmer et al., 2002), ERI-3 (Duchaine et al., 2006), ERI-9, and the Argonaute ERI-8/ERGO-1 (Pavelec et al., 2009).

\section{Antiviral RNAi}

RNAi functions as an antiviral defense in C. elegans. This role of RNAi was experimentally addressed even though endogenous viral pathogens of $C$. elegans were unknown. The problem of absenting endogenous C. elegans viruses was bypassed by using an "artificial" infection with viruses, which had a broad host range and could infect $C$. elegans under laboratory conditions. Model viral infections were based on the $(+)$ ssRNA flock house virus (FHV) (Lu et al., 2005) or the (-)ssRNA vesicular stomatitis virus (VSV) (Schott et al., 2005; Wilkins et al., 2005). Infection with the recombinant VSV was augmented in strong RNAi mutant animals ( $r d e-1$ and $r d e-4)$, which produced higher viral titers. Furthermore, VSV infection was attenuated in $r r f-3$ and eri-1 mutants that are hypersensitive to RNAi (Wilkins et al., 2005). Similar results were obtained from infected cultured cells (Schott et al., 2005) and FHV infection of rde-1 mutants (Lu et al., 2005). Not surprisingly, genes misregulated in C. elegans deficient in Dicer, RDE-4, or RDE-1 are enriched for innate immunity genes (Welker et al., 2007). Antiviral RNAi involves also other genes, such as $r s d-2$ (RNAi spreading defective 2), which was implicated in secondary viral siRNA biogenesis in RDE-4 independent manner (Guo et al., 2013b).

The antiviral role of exo-RNAi was subsequently confirmed in a natural viral infection of C. elegans and C. briggsae by Orsay virus (Felix et al., 2011). Analysis of gene expression in $C$. elegans infected with Orsay virus revealed competition of the antiviral response with endogenous small RNA pathways, which was manifested as redirection of RDE-1 from its endogenous small RNA cofactors, leading to loss of repression of endogenous RDE-1 targets and reduction of miRNA levels and an up-regulation of their target genes (Sarkies et al., 2013). Interestingly, genome-wide association study in C. elegans wild populations identified as a major determinant of viral sensitivity DRH-1, the aforementioned RIG-1 homolog (Ashe et al., 2013). Similarly, a genetic analysis revealed an essential role for both DRH-1 and DRH-3 in antiviral RNAi (Guo et al., 2013a). DRH-3 is another Dicer-RIG-I family protein that is essential for RNA silencing and germline development (Nakamura et al., 2007). DRH-3 binds both single-stranded and double-stranded RNAs with high affinity. However, the ATPase activity of DRH-3 is stimulated only by double-stranded RNA (Matranga and Pyle, 2010). Analysis of DRH-1 and DRH-3 in infected 


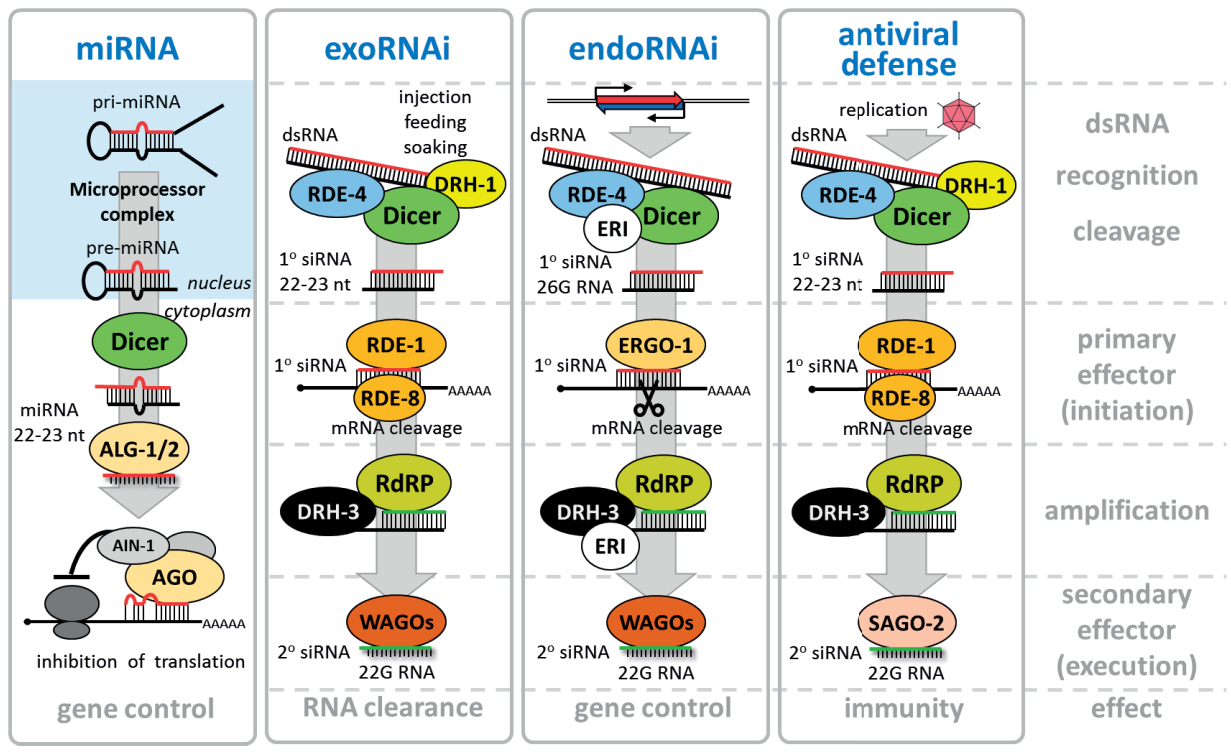

Figure 3 Schematic overview of reviewed small RNA pathways in C. elegans

worms suggested distinct roles where DRH-1 was involved in production of viral primary siRNAs, whereas DRH-3 is involved in biogenesis of 22 nt secondary siRNAs (Fitzgerald et al., 2014; Guo et al., 2013a) (Fig. 3). Taken together, RIG-I homologs function in innate immunity through evolution of Metazoa where they adapting to specific molecular mechanisms - primary and secondary siRNA biogenesis in RNAi in nematodes and stimulation of the interferon response in mammals.

Importantly, innate immunity operates beyond viruses and RNAi; Bacillus thuringiensis strain DB27 is virulent bacterium for C. elegans but not another nematode species. An analysis of virulence factors revealed that $d c r-1$ mutant alleles deficient in microRNA (miRNA) processing, but not those deficient only in RNAi, are resistant to $B$. thuringiensis DB27 (Iatsenko et al., 2013). This suggests that Dicer is a part of a system balancing small RNA pathways and antiviral immunity. This model is further extended with the miR-3541 family, regulates the efficiency of RNAi (Massirer and Pasquinelli, 2013). The loss of miR-35-41 results in enhanced exo-RNAi sensitivity and reduced endo-RNAi effectiveness suggesting that these miRNAs normally help balance the RNAi pathways (Massirer and Pasquinelli, 2013).

\section{Adenosine deamination}

A connection between A-to-I editing and RNAi has been revealed in C. elegans. A transcriptome-wide analysis of A-to-I editing sites identified as many as 664 editing-enriched regions, which represent the core of A-to-I editing substrates in C. elegans. Among the 
known substrates are mRNAs with hairpin structures in 3' UTRs; editing however does not necessarily prevent nuclear export and presence on polysomes (Hundley et al., 2008; Morse et al., 2002; Morse and Bass, 1999). Approximately 1.7\% of C. elegans mRNAs contained such editing-enriched regions (Whipple et al., 2015). A-to-I editing also affects small RNAs.

In contrast to mice, where the loss of A-to-I editing is lethal, in Drosophila and C. elegans Adar null phenotype causes only weak phenotypic alterations (Palladino et al., 2000; Tonkin and Bass, 2003; Tonkin et al., 2002). Importantly, $a d r-1$ or $a d r-2$ mutant worms exhibit a defective chemotaxis but the phenotype is reverted when worms lacking Adar are crossed with RNAi-defective strains (Tonkin and Bass, 2003). Analysis of ADAR mutants showed that A-to-I editing affects microRNAs as well as 26G endo-siRNAs (Warf et al., 2012). That ADAR and RNAi pathways are competing for common targets is supported by common loci from which originate edited transcripts and in which are found RNAi-dependent short RNAs upregulated in ADAR mutants (Wu et al., 2011).

\section{Summary}

The principles of primary and secondary small RNA production adopted during C. elegans evolution provide an explanation how such a complex of small RNA pathway can rely on a single Dicer protein. The key apparently lies in structural differences among Dicer substrates, Dicer co-factors facilitating loading onto correct Argonaute proteins, and cognate RNAs (targeted by primary small RNA), which serve as templates for secondary small RNAs. Given the complexity of the Nematode phylum, one could ask how representative is C. elegans model of the phylum. There has been a survey for orthologs of 77 C. elegans RNAi pathway proteins in 13 nematode species, which revealed that while proteins responsible for uptake and spread of exogenously applied dsRNA are absent from parasitic species. Furthermore, Argonaute proteins regulating gene expression but not exo-RNAi Argonautes are broadly conserved; secondary Argonautes (SAGO/WAGO) are poorly conserved, and the nuclear AGO NRDE-3 was not identified in any parasite (Dalzell et al., 2011). Taken together, Caenorhabditis possess an expanded RNAi effector repertoire relative to the parasitic nematodes while all nematode species displayed qualitatively similar coverage of functional protein groups (Dalzell et al., 2011).

\section{Acknowledgement}

I would like to thank my colleagues Jan Paces, Miloslav Nic and Tomas Novotny for help with collecting literature for the review. The review content was produced under a contract OC/EFSA/GMO/2015/01-CT 01 with European Food Safety Authority (EFSA); the opinions expressed are those of the contractor only and do not represent EFSA's official position. Publication of the review was funded by LO1220 and LM2015063 by the Ministry of Education, Youth and Sports. 


\section{References}

Allo, M., and Kornblihtt, A.R. (2010). Gene Silencing: Small RNAs Control RNA Polymerase II Elongation. Current Biology 20, R704-R707.

Ambros, V., Lee, R.C., Lavanway, A., Williams, P.T., and Jewell, D. (2003). MicroRNAs and other tiny endogenous RNAs in C-elegans. Current Biology 13, 807-818.

Aoki, K., Moriguchi, H., Yoshioka, T., Okawa, K., and Tabara, H. (2007). In vitro analyses of the production and activity of secondary small interfering RNAs in C-elegans. EMBO J 26, 5007-5019.

Ashe, A., Bélicard, T., Le Pen, J., Sarkies, P., Frézal, L., Lehrbach, N.J., Félix, M.A., and Miska, E.A. (2013). A deletion polymorphism in the Caenorhabditis elegans RIG-I homolog disables viral RNA dicing and antiviral immunity. Elife 2013.

Asikainen, S., Heikkinen, L., Wong, G., and Storvik, M. (2008). Functional characterization of endogenous siRNA target genes in Caenorhabditis elegans. BMC Genomics 9, 270-270.

Batista, P.J., Ruby, J.G., Claycomb, J.M., Chiang, R., Fahlgren, N., Kasschau, K.D., Chaves, D.A., Gu, W., Vasale, J.J., Duan, S., et al. (2008). PRG-1 and 21U-RNAs interact to form the piRNA complex required for fertility in C-elegans. Molecular Cell 31, 67-78.

Beshore, E.L., McEwen, T., Marshall, J., and Bennett, K. (2009). Activating interactions between GLH-1, microRNAs and Dicer in C. elegans. Developmental Biology 331, 418-418.

Beshore, E.L., McEwen, T.J., Jud, M.C., Marshall, J.K., Schisa, J.A., and Bennett, K.L. (2011). C. elegans Dicer interacts with the P-granule component GLH-1 and both regulate germline RNPs. Developmental Biology 350, 370-381.

Blanchard, D., Parameswaran, P., Lopez-Molina, J., Gent, J., Saynuk, J.F., and Fire, A. (2011). On the nature of in vivo requirements for rde-4 in RNAi and developmental pathways in C-elegans. RNA Biology 8, 458-467.

Bouasker, S., and Simard, M.J. (2012). The slicing activity of miRNA-specific Argonautes is essential for the miRNA pathway in C. elegans. Nucleic Acids Research 40, 10452-10462.

Buck, A.H., and Blaxter, M. (2013). Functional diversification of Argonautes in nematodes: an expanding universe. Biochemical Society Transactions 41, 881-886.

Buckley, B.A., Burkhart, K.B., Gu, S.G., Spracklin, G., Kershner, A., Fritz, H., Kimble, J., Fire, A., and Kennedy, S. (2012). A nuclear Argonaute promotes multigenerational epigenetic inheritance and germline immortality. Nature 489, 447-451.

Burton, N.O., Burkhart, K.B., and Kennedy, S. (2011). Nuclear RNAi maintains heritable gene silencing in Caenorhabditis elegans. Proceedings of the National Academy of Sciences of the United States of America 108, 19683-19688.

Campbell, A.C., and Updike, D.L. (2015). CSR-1 and P granules suppress sperm-specific transcription in the C. elegans germline. Development 142, 1745-1755.

Cecere, G., Hoersch, S., O’Keeffe, S., Sachidanandam, R., and Grishok, A. (2014). Global effects of the CSR-1 RNA interference pathway on the transcriptional landscape. Nature Structural \& Molecular Biology 21, 358-U394.

Chatterjee, S., and Grosshans, H. (2009). Active turnover modulates mature microRNA activity in Caenorhabditis elegans. Nature 461, 546-U120.

Chiliveri, S.C., and Deshmukh, M.V. (2014). Structure of RDE-4 dsRBDs and mutational studies provide insights into dsRNA recognition in the Caenorhabditis elegans RNAi pathway. Biochemical Journal 458, 119-130. 
Chu, Y.D., Wang, W.C., Chen, S.A.A., Hsu, Y.T., Yeh, M.W., Slack, F.J., and Chan, S.P. (2014). RACK-1 regulates let-7 microRNA expression and terminal cell differentiation in Caenorhabditis elegans. Cell Cycle 13, 1995-2009.

Claycomb, J.M., Batista, P.J., Pang, K.M., Gu, W.F., Vasale, J.J., van Wolfswinkel, J.C., Chaves, D.A., Shirayama, M., Mitani, S., Ketting, R.F., et al. (2009). The Argonaute CSR-1 and Its 22G-RNA Cofactors Are Required for Holocentric Chromosome Segregation. Cell 139, 123-134.

Conine, C.C., Batista, P.J., Gu, W.F., Claycomb, J.M., Chaves, D.A., Shirayama, M., and Mello, C.C. (2010). Argonautes ALG-3 and ALG-4 are required for spermatogenesis-specific 26G-RNAs and thermotolerant sperm in Caenorhabditis elegans. Proceedings of the National Academy of Sciences of the United States of America 107, 3588-3593.

Conine, C.C., Moresco, J.J., Gu, W., Shirayama, M., Conte, D., Jr., Yates, J.R., III, and Mello, C.C. (2013). Argonautes Promote Male Fertility and Provide a Paternal Memory of Germline Gene Expression in C. elegans. Cell 155, 1532-1544.

Correa, R.L., Steiner, F.A., Berezikov, E., and Ketting, R.F. (2010). MicroRNA-Directed siRNA Biogenesis in Caenorhabditis elegans. Plos Genetics 6, e1000903-e1000903.

Dalzell, J.J., McVeigh, P., Warnock, N.D., Mitreva, M., Bird, D.M., Abad, P., Fleming, C.C., Day, T.A., Mousley, A., Marks, N.J., et al. (2011). RNAi Effector Diversity in Nematodes. Plos Neglected Tropical Diseases 5, e1176-e1176.

Denli, A.M., Tops, B.B.J., Plasterk, R.H.A., Ketting, R.F., and Hannon, G.J. (2004). Processing of primary microRNAs by the Microprocessor complex. Nature 432, 231-235.

Ding, L., Spencer, A., Morita, K., and Han, M. (2005). The developmental timing regulator AIN-1 interacts with miRISCs and may target the argonaute protein ALG-1 to cytoplasmic P bodies in C. elegans. Molecular Cell 19, 437-447.

Ding, X.C., and Grosshans, H. (2009). Repression of C-elegans microRNA targets at the initiation level of translation requires GW182 proteins. EMBO J 28, 213-222.

Drake, M., Furuta, T., Suen, K.M., Gonzalez, G., Liu, B., Kalia, A., Ladbury, J.E., Fire, A.Z., Skeath, J.B., and Arur, S. (2014). A Requirement for ERK-Dependent Dicer Phosphorylation in Coordinating Oocyte-to-Embryo Transition in C. elegans. Developmental Cell 31, 614-628.

Duchaine, T.F., Wohlschlegel, J.A., Kennedy, S., Bei, Y.X., Conte, D., Pang, K.M., Brownell, D.R., Harding, S., Mitani, S., Ruvkun, G., et al. (2006). Functional proteomics reveals the biochemical niche of C-elegans DCR-1 in multiple small-RNA-mediated pathways. Cell 124, 343-354.

Etheridge, T., Nemoto, K., Hashizume, T., Mori, C., Sugimoto, T., Suzuki, H., Fukui, K., Yamazaki, T., Higashibata, A., Szewczyk, N.J., et al. (2011). The Effectiveness of RNAi in Caenorhabditis elegans Is Maintained during Spaceflight. Plos One 6, e20459-e20459.

Feinberg, E.H., and Hunter, C.P. (2003). Transport of dsRNA into cells by the transmembrane protein SID-1. Science 301, 1545-1547.

Felix, M.A., Ashe, A., Piffaretti, J., Wu, G., Nuez, I., Belicard, T., Jiang, Y., Zhao, G., Franz, C.J., Goldstein, L.D., et al. (2011). Natural and experimental infection of Caenorhabditis nematodes by novel viruses related to nodaviruses. Plos Biology 9, e1000586.

Fischer, S.E.J., Montgomery, T.A., Zhang, C., Fahlgren, N., Breen, P.C., Hwang, A., Sullivan, C.M., Carrington, J.C., and Ruvkun, G. (2011). The ERI-6/7 Helicase Acts at the First Stage of an siRNA Amplification Pathway That Targets Recent Gene Duplications. Plos Genetics 7, e1002369-e1002369. 
Fischer, S.E.J., Pan, Q., Breen, P.C., Qi, Y., Shi, Z., Zhang, C., and Ruvkun, G. (2013). Multiple small RNA pathways regulate the silencing of repeated and foreign genes in C. elegans. Genes \& Development 27, 2678-2695.

Fitzgerald, M.E., Vela, A., and Pyle, A.M. (2014). Dicer-related helicase 3 forms an obligate dimer for recognizing 22G-RNA. Nucleic Acids Research 42, 3919-3930.

Frezal, L., and Felix, M.A. (2015). C. elegans outside the Petri dish. Elife 4.

Gao, Z.Q., Wang, M., Blair, D., Zheng, Y.D., and Dou, Y.X. (2014). Phylogenetic Analysis of the Endoribonuclease Dicer Family. Plos One 9, e95350-e95350.

Ge, X., Zhao, X., Nakagawa, A., Gong, X.Q., Skeen-Gaar, R.R., Shi, Y., Gong, H.P., Wang, X.Q., and Xue, D. (2014). A novel mechanism underlies caspase-dependent conversion of the dicer ribonuclease into a deoxyribonuclease during apoptosis. Cell Research 24, 218-232.

Gent, J.I., Lamm, A.T., Pavelec, D.M., Maniar, J.M., Parameswaran, P., Tao, L., Kennedy, S., and Fire, A.Z. (2010). Distinct Phases of siRNA Synthesis in an Endogenous RNAi Pathway in C. elegans Soma. Molecular Cell 37, 679-689.

Gent, J.I., Schvarzstein, M., Villeneuve, A.M., Gu, S.G., Jantsch, V., Fire, A.Z., and Baudrimont, A. (2009). A Caenorhabditis elegans RNA-Directed RNA Polymerase in Sperm Development and Endogenous RNA Interference. Genetics 183, 1297-1314.

Grishok, A., Pasquinelli, A.E., Conte, D., Li, N., Parrish, S., Ha, I., Baillie, D.L., Fire, A., Ruvkun, G., and Mello, C.C. (2001). Genes and mechanisms related to RNA interference regulate expression of the small temporal RNAs that control C-elegans developmental timing. Cell 106, 23-34.

Gu, W.F., Shirayama, M., Conte, D., Vasale, J., Batista, P.J., Claycomb, J.M., Moresco, J.J., Youngman, E.M., Keys, J., Stoltz, M.J., et al. (2009). Distinct Argonaute-Mediated 22G-RNA Pathways Direct Genome Surveillance in the C. elegans Germline. Molecular Cell 36, 231-244.

Guo, X.Y., Zhang, R., Wang, J., Ding, S.W., and Lu, R. (2013a). Homologous RIG-I-like helicase proteins direct RNAi-mediated antiviral immunity in C. elegans by distinct mechanisms. Proceedings of the National Academy of Sciences of the United States of America 110, 16085-16090.

Guo, X.Y., Zhang, R., Wang, J., and Lu, R. (2013b). Antiviral RNA Silencing Initiated in the Absence of RDE-4, a Double-Stranded RNA Binding Protein, in Caenorhabditis elegans. Journal of Virology $87,10721-10729$.

Habig, J.W., Aruscavage, P.J., and Bass, B.L. (2008). In C. elegans, High Levels of dsRNA Allow RNAi in the Absence of RDE-4. Plos One 3, e4052-e4052.

Han, T., Manoharan, A.P., Harkins, T.T., Bouffard, P., Fitzpatrick, C., Chu, D.S., Thierry-Mieg, D., Thierry-Mieg, J., and Kim, J.K. (2009). 26G endo-siRNAs regulate spermatogenic and zygotic gene expression in Caenorhabditis elegans. Proceedings of the National Academy of Sciences of the United States of America 106, 18674-18679.

Han, W., Sundaram, P., Kenjale, H., Grantham, J., and Timmons, L. (2008). The Caenorhabditis elegans rsd-2 and rsd-6 genes are required for chromosome functions during exposure to unfavorable environments. Genetics 178, 1875-1893.

Hellwig, S., and Bass, B.L. (2008). A starvation-induced noncoding RNA modulates expression of Dicer-regulated genes. Proceedings of the National Academy of Sciences of the United States of America 105, 12897-12902.

Hinas, A., Wright, A.J., and Hunter, C.P. (2012). SID-5 Is an Endosome-Associated Protein Required for Efficient Systemic RNAi in C. elegans. Current Biology 22, 1938-1943. 
Hundley, H.A., Krauchuk, A.A., and Bass, B.L. (2008). C-elegans and H-sapiens mRNAs with edited 3 'UTRs are present on polysomes. RNA 14, 2050-2060.

Iatsenko, I., Sinha, A., Rodelsperger, C., and Sommer, R.J. (2013). New Role for DCR-1/Dicer in Caenorhabditis elegans Innate Immunity against the Highly Virulent Bacterium Bacillus thuringiensis DB27. Infection and Immunity 81, 3942-3957.

Jannot, G., Boisvert, M.E.L., Banville, I.H., and Simard, M.J. (2008). Two molecular features contribute to the Argonaute specificity for the microRNA and RNAi pathways in C-elegans. RNA 14, 829-835.

Jaskiewicz, L., and Filipowicz, W. (2008). Role of Dicer in posttranscriptional RNA silencing. Current topics in microbiology and immunology 320, 77-97.

Jose, A.M., Garcia, G.A., and Hunter, C.P. (2011). Two classes of silencing RNAs move between Caenorhabditis elegans tissues. Nature Structural \& Molecular Biology 18, 1183-U1111.

Jose, A.M., Kim, Y.A., Leal-Ekman, S., and Hunter, C.P. (2012). Conserved tyrosine kinase promotes the import of silencing RNA into Caenorhabditis elegans cells. Proceedings of the National Academy of Sciences of the United States of America 109, 14520-14525.

Kennedy, S., Wang, D., and Ruvkun, G. (2004). A conserved siRNA-degrading RNase negatively regulates RNA interference in C. elegans. Nature 427, 645-649.

Ketting, R.F., Fischer, S.E.J., Bernstein, E., Sijen, T., Hannon, G.J., and Plasterk, R.H.A. (2001). Dicer functions in RNA interference and in synthesis of small RNA involved in developmental timing in C-elegans. Genes \& Development 15, 2654-2659.

Knight, S.W., and Bass, B.L. (2001). A role for the RNase III enzyme DCR-1 in RNA interference and germ line development in Caenorhabditis elegans. Science 293, 2269-2271.

Kuzuoglu-Oeztuerk, D., Huntzinger, E., Schmidt, S., and Izaurralde, E. (2012). The Caenorhabditis elegans GW182 protein AIN-1 interacts with PAB-1 and subunits of the PAN2-PAN3 and CCR4NOT deadenylase complexes. Nucleic Acids Research 40, 5651-5665.

Lambert, N.J., Gu, S.G., and Zahler, A.M. (2011). The conformation of microRNA seed regions in native microRNPs is prearranged for presentation to mRNA targets. Nucleic Acids Research 39, 4827-4835.

Lee, R.C., Feinbaum, R.L., and Ambros, V. (1993). The C. elegans heterochronic gene lin-4 encodes small RNAs with antisense complementarity to lin-14. Cell 75, 843-854.

Lee, R.C., Hammell, C.M., and Ambros, V. (2006). Interacting endogenous and exogenous RNAi pathways in Caenorhabditis elegans. RNA 12, 589-597.

Lee, Y.S., Nakahara, K., Pham, J.W., Kim, K., He, Z., Sontheimer, E.J., and Carthew, R.W. (2004). Distinct roles for Drosophila Dicer-1 and Dicer-2 in the siRNA/miRNA silencing pathways. Cell 117, 69-81.

Lehrbach, N.J., Armisen, J., Lightfoot, H.L., Murfitt, K.J., Bugaut, A., Balasubramanian, S., and Miska, E.A. (2009). LIN-28 and the poly(U) polymerase PUP-2 regulate let-7 microRNA processing in Caenorhabditis elegans. Nature Structural \& Molecular Biology 16, 1016-U1027.

Lu, R., Maduro, M., Li, F., Li, H.W., Broitman-Maduro, G., Li, W.X., and Ding, S.W. (2005). Animal virus replication and RNAi-mediated antiviral silencing in Caenorhabditis elegans. Nature 436, 1040-1043.

Mao, H., Zhu, C.M., Zong, D.D., Weng, C.C., Yang, X.W., Huang, H., Liu, D., Feng, X.Z., and Guang, S.H. (2015). The Nrde Pathway Mediates Small-RNA-Directed Histone H3 Lysine 27 Trimethylation in Caenorhabditis elegans. Current Biology 25, 2398-2403. 
Margulis, L., and Schwartz, K.V. (1998). Five kingdoms : an illustrated guide to the phyla of life on earth, 3rd edn (New York: W.H. Freeman).

Massirer, K.B., and Pasquinelli, A.E. (2013). MicroRNAs that interfere with RNAi. Worm 2, e21835-e21835.

Matranga, C., and Pyle, A.M. (2010). Double-stranded RNA-dependent ATPase DRH-3 INSIGHT INTO ITS ROLE IN RNA SILENCING IN CAENORHABDITIS ELEGANS. Journal of Biological Chemistry 285, 25363-25371.

McEwan, D.L., Weisman, A.S., and Huntert, C.P. (2012). Uptake of Extracellular Double-Stranded RNA by SID-2. Molecular Cell 47, 746-754.

Mon, H., Kobayashi, I., Ohkubo, S., Tomita, S., Lee, J.M., Sezutsu, H., Tamura, T., and Kusakabe, T. (2012). Effective RNA interference in cultured silkworm cells mediated by overexpression of Caenorhabditis elegans SID-1. RNA Biology 9, 40-46.

Mon, H., Li, Z., Kobayashi, I., Tomita, S., Lee, J., Sezutsu, H., Tamura, T., and Kusakabe, T. (2013). Soaking RNAi in Bombyx mori BmN4-SID1 cells arrests cell cycle progression. Journal of Insect Science 13, 155-155.

Morita, K., and Han, M. (2006). Multiple mechanisms are involved in regulating the expression of the developmental timing regulator lin-28 in Caenorhabditis elegans. EMBO J 25, 5794-5804.

Morse, D.P., Aruscavage, P.J., and Bass, B.L. (2002). RNA hairpins in noncoding regions of human brain and Caenorhabditis elegans mRNA are edited by adenosine deaminases that act on RNA. Proceedings of the National Academy of Sciences of the United States of America 99, 7906-7911.

Morse, D.P., and Bass, B.L. (1999). Long RNA hairpins that contain inosine are present in Caenorhabditis elegans poly $(\mathrm{A})(+)$ RNA. Proceedings of the National Academy of Sciences of the United States of America 96, 6048-6053.

Nakagawa, A., Shi, Y., Kage-Nakadai, E., Mitani, S., and Xue, D. (2010). Caspase-Dependent Conversion of Dicer Ribonuclease into a Death-Promoting Deoxyribonuclease. Science 328, 327-334.

Nakamura, M., Ando, R., Nakazawa, T., Yudazono, T., Tsutsumi, N., Hatanaka, N., Ohgake, T., Hanaoka, F., and Eki, T. (2007). Dicer-related drh-3 gene functions in germ-line development by maintenance of chromosomal integrity in Caenorhabditis elegans. Genes to Cells 12, 997-1010.

Newman, M.A., Thomson, J.M., and Hammond, S.M. (2008). Lin-28 interaction with the Let-7 precursor loop mediates regulated microRNA processing. RNA 14, 1539-1549.

Okamura, K., Ishizuka, A., Siomi, H., and Siomi, M.C. (2004). Distinct roles for Argonaute proteins in small RNA-directed RNA cleavage pathways. Genes Dev 18, 1655-1666.

Ouchi, Y., Yamamoto, J., and Iwamoto, T. (2014). The Heterochronic Genes lin-28a and lin-28b Play an Essential and Evolutionarily Conserved Role in Early Zebrafish Development. Plos One 9, e88086-e88086.

Pak, J., and Fire, A. (2007). Distinct populations of primary and secondary effectors during RNAi in C-elegans. Science 315, 241-244.

Palladino, M.J., Keegan, L.P., O’Connell, M.A., and Reenan, R.A. (2000). A-to-I pre-mRNA editing in Drosophila is primarily involved in adult nervous system function and integrity. Cell 102, 437-449.

Parker, G.S., Eckert, D.M., and Bass, B.L. (2006). RDE-4 preferentially binds long dsRNA and its dimerization is necessary for cleavage of dsRNA to siRNA. RNA 12, 807-818.

Parker, G.S., Maity, T.S., and Bass, B.L. (2008). dsRNA Binding Properties of RDE-4 and TRBP Reflect Their Distinct Roles in RNAi. Journal of Molecular Biology 384, 967-979. 
Parrish, S., and Fire, A. (2001). Distinct roles for RDE-1 and RDE-4 during RNA interference in Caenorhabditis elegans. RNA 7, 1397-1402.

Pasquinelli, A.E., Reinhart, B.J., Slack, F., Martindale, M.Q., Kuroda, M.I., Maller, B., Hayward, D.C., Ball, E.E., Degnan, B., Muller, P., et al. (2000). Conservation of the sequence and temporal expression of let-7 heterochronic regulatory RNA. Nature 408, 86-89.

Pavelec, D.M., Lachowiec, J., Duchaine, T.F., Smith, H.E., and Kennedy, S. (2009). Requirement for the ERI/DICER Complex in Endogenous RNA Interference and Sperm Development in Caenorhabditis elegans. Genetics 183, 1283-1295.

Racen, E.L., McEwen, T.J., and Bennett, K.L. (2008). The intriguing interaction of Dicer (DCR1) with GLH-1, a P granule component in Caenorhabditis elegans. Developmental Biology 319 , 547-547.

Reinhart, B.J., Slack, F.J., Basson, M., Pasquinelli, A.E., Bettinger, J.C., Rougvie, A.E., Horvitz, H.R., and Ruvkun, G. (2000). The 21-nucleotide let-7 RNA regulates developmental timing in Caenorhabditis elegans. Nature 403, 901-906.

Ruby, J.G., Jan, C., Player, C., Axtell, M.J., Lee, W., Nusbaum, C., Ge, H., and Bartel, D.P. (2006). Large-scale sequencing reveals $21 \mathrm{U}-\mathrm{RNAs}$ and additional microRNAs and endogenous siRNAs in C. elegans. Cell 127, 1193-1207.

Ruby, J.G., Jan, C.H., and Bartel, D.P. (2007). Intronic microRNA precursors that bypass Drosha processing. Nature 448, 83-86.

Rybak-Wolf, A., Jens, M., Murakawa, Y., Herzog, M., Landthaler, M., and Rajewsky, N. (2014). A Variety of Dicer Substrates in Human and C-elegans. Cell 159, 1153-1167.

Sarkies, P., Ashe, A., Le Pen, J., McKie, M.A., and Miska, E.A. (2013). Competition between virus-derived and endogenous small RNAs regulates gene expression in Caenorhabditis elegans. Genome Research 23, 1258-1270.

Sawh, A.N., and Duchaine, T.F. (2013). A Truncated Form of Dicer Tilts the Balance of RNA Interference Pathways. Cell Reports 4, 454-463.

Schott, D.H., Cureton, D.K., Whelan, S.P., and Hunter, C.P. (2005). An antiviral role for the RNA interference machinery in Caenorhabditis elegans. Proceedings of the National Academy of Sciences of the United States of America 102, 18420-18424.

Seth, M., Shirayama, M., Gu, W.F., Ishidate, T., Conte, D., and Mello, C.C. (2013). The C. elegans CSR-1 Argonaute Pathway Counteracts Epigenetic Silencing to Promote Germline Gene Expression. Developmental Cell 27, 656-663.

Shih, J.D., Fitzgerald, M.C., Sutherlin, M., and Hunter, C.P. (2009). The SID-1 double-stranded RNA transporter is not selective for dsRNA length. RNA 15, 384-390.

Shih, J.D., and Hunter, C.P. (2011). SID-1 is a dsRNA-selective dsRNA-gated channel. RNA 17, 1057-1065.

Shirayama, M., Stanney, W., Gu, W.F., Seth, M., and Mello, C.C. (2014). The Vasa Homolog RDE-12 Engages Target mRNA and Multiple Argonaute Proteins to Promote RNAi in C. elegans. Current Biology 24, 845-851.

Sijen, T., Fleenor, J., Simmer, F., Thijssen, K.L., Parrish, S., Timmons, L., Plasterk, R.H.A., and Fire, A. (2001). On the role of RNA amplification in dsRNA-triggered gene silencing. Cell 107, 465-476.

Sijen, T., Steiner, F.A., Thijssen, K.L., and Plasterk, R.H.A. (2007). Secondary siRNAs result from unprimed RNA synthesis and form a distinct class. Science 315, 244-247. 
Simmer, F., Tijsterman, M., Parrish, S., Koushika, S.P., Nonet, M.L., Fire, A., Ahringer, J., and Plasterk, R.H.A. (2002). Loss of the putative RNA-directed RNA polymerase RRF-3 makes C. elegans hypersensitive to RNAi. Current Biology 12, 1317-1319.

Smardon, A., Spoerke, J.M., Stacey, S.C., Klein, M.E., Mackin, N., and Maine, E.M. (2000). EGO-1 is related to RNA-directed RNA polymerase and functions in germ-line development and RNA interference in C-elegans. Current Biology 10, 169-178.

Stefani, G., Chen, X.W., Zhao, H.Y., and Slack, F.J. (2015). A novel mechanism of LIN-28 regulation of let-7 microRNA expression revealed by in vivo HITS-CLIP in C. elegans. RNA 21, 985-996.

Steiner, F.A., Hoogstrate, S.W., Okihara, K.L., Thijssen, K.L., Ketting, R.F., Plasterk, R.H.A., and Sijen, T. (2007). Structural features of small RNA precursors determine Argonaute loading in Caenorhabditis elegans. Nature Structural \& Molecular Biology 14, 927-933.

Steiner, F.A., Okihara, K.L., Hoogstrate, S.W., Sijen, T., and Ketting, R.F. (2009). RDE-1 slicer activity is required only for passenger-strand cleavage during RNAi in Caenorhabditis elegans. Nature Structural \& Molecular Biology 16, 207-211.

Tabara, H., Grishok, A., and Mello, C.C. (1998). RNAi in C. elegans: soaking in the genome sequence. Science 282, 430-431.

Tabara, H., Sarkissian, M., Kelly, W.G., Fleenor, J., Grishok, A., Timmons, L., Fire, A., and Mello, C.C. (1999). The rde-1 gene, RNA interference, and transposon silencing in C-elegans. Cell 99, 123-132.

Tabara, H., Yigit, E., Siomi, H., and Mello, C.C. (2002). The dsRNA binding protein RDE-4 interacts with RDE-1, DCR-1, and a DExX-box helicase to direct RNAi in C-elegans. Cell 109, 861-871.

Thivierge, C., Makil, N., Flamand, M., Vasale, J.J., Mello, C.C., Wohlschlegel, J., Conte, D., and Duchaine, T.F. (2012). Tudor domain ERI-5 tethers an RNA-dependent RNA polymerase to DCR1 to potentiate endo-RNAi. Nature Structural \& Molecular Biology 19, 90-U114.

Tijsterman, M., Ketting, R.F., Okihara, K.L., Sijen, T., and Plasterk, R.H.A. (2002a). RNA helicase MUT-14-dependent gene silencing triggered in C-elegans by short antisense RNAs. Science 295, 694-697.

Tijsterman, M., Okihara, K.L., Thijssen, K., and Plasterk, R.H.A. (2002b). PPW-1, a PAZ/PIWI protein required for efficient germline RNAi, is defective in a natural isolate of C-elegans. Current Biology 12, 1535-1540.

Tonkin, L.A., and Bass, B.L. (2003). Mutations in RNAi rescue aberrant chemotaxis of ADAR mutants. Science 302, 1725.

Tonkin, L.A., Saccomanno, L., Morse, D.P., Brodigan, T., Krause, M., and Bass, B.L. (2002). RNA editing by ADARs is important for normal behavior in Caenorhabditis elegans. EMBO J 21, 6025-6035.

Tops, B.B.J., Plasterk, R.H.A., and Ketting, R.F. (2006). The Caenorhabditis elegans argonautes ALG-1 and ALG-2: Almost identical yet different. Cold Spring Harbor Symposia on Quantitative Biology 71, 189-194.

Tsai, H.Y., Chen, C.C.G., Conte, D., Moresco, J.J., Chaves, D.A., Mitani, S., Yates, J.R., Tsai, M.D., and Mello, C.C. (2015). A Ribonuclease Coordinates siRNA Amplification and mRNA Cleavage during RNAi. Cell 160, 407-419.

Tu, S.K., Wu, M.Z., Wang, J., Cutter, A.D., Weng, Z.P., and Claycomb, J.M. (2015). Comparative functional characterization of the CSR-1 22G-RNA pathway in Caenorhabditis nematodes. Nucleic Acids Research 43, 208-224. 
Vadla, B., Kemper, K., Alaimo, J., Heine, C., and Moss, E.G. (2012). lin-28 Controls the Succession of Cell Fate Choices via Two Distinct Activities. Plos Genetics 8, e1002588-e1002588.

Van Wynsberghe, P.M., Kai, Z.S., Massirer, K.B., Burton, V.H., Yeo, G.W., and Pasquinelli, A.E. (2011). LIN-28 co-transcriptionally binds primary let-7 to regulate miRNA maturation in Caenorhabditis elegans. Nature Structural \& Molecular Biology 18, 302-U386.

Vasale, J.J., Gu, W.F., Thivierge, C., Batista, P.J., Claycomb, J.M., Youngman, E.M., Duchaine, T.F., Mello, C.C., and Conte, D. (2010). Sequential rounds of RNA-dependent RNA transcription drive endogenous small-RNA biogenesis in the ERGO-1/Argonaute pathway. Proceedings of the National Academy of Sciences of the United States of America 107, 3582-3587.

Vasquez-Rifo, A., Bosse, G.D., Rondeau, E.L., Jannot, G., Dallaire, A., and Simard, M.J. (2013). A New Role for the GARP Complex in MicroRNA-Mediated Gene Regulation. Plos Genetics 9, e1003961-e1003961.

Vasquez-Rifo, A., Jannot, G., Armisen, J., Labouesse, M., Irfan, S., Bukhari, A., Rondeau, E.L., Miska, E.A., and Simard, M.J. (2012). Developmental Characterization of the MicroRNA-Specific C. elegans Argonautes alg-1 and alg-2. Plos One 7, e33750-e33750.

Wang, G., and Reinke, V. (2008). A C. elegans Piwi, PRG-1, regulates 21U-RNAs during spermatogenesis. Current Biology 18, 861-867.

Warf, M.B., Johnson, W.E., and Bass, B.L. (2011). Improved annotation of C. elegans microRNAs by deep sequencing reveals structures associated with processing by Drosha and Dicer. RNA 17, 563-577.

Warf, M.B., Shepherd, B.A., Johnson, W.E., and Bass, B.L. (2012). Effects of ADARs on small RNA processing pathways in C. elegans. Genome Research 22, 1488-1498.

Wedeles, C.J., Wu, M.Z., and Claycomb, J.M. (2013a). A multitasking Argonaute: exploring the many facets of C-elegans CSR-1. Chromosome Research 21, 573-586.

Wedeles, C.J., Wu, M.Z., and Claycomb, J.M. (2013b). Protection of Germline Gene Expression by the C. elegans Argonaute CSR-1. Developmental Cell 27, 664-671.

Welker, N.C., Habig, J.W., and Bass, B.L. (2007). Genes misregulated in C. elegans deficient in Dicer, RDE-4, or RDE-1 are enriched for innate immunity genes. RNA 13, 1090-1102.

Welker, N.C., Maity, T.S., Ye, X.C., Aruscavage, P.J., Krauchuk, A.A., Liu, Q.H., and Bass, B.L. (2011). Dicer's Helicase Domain Discriminates dsRNA Termini to Promote an Altered Reaction Mode. Molecular Cell 41, 589-599.

Welker, N.C., Pavelec, D.M., Nix, D.A., Duchaine, T.F., Kennedy, S., and Bass, B.L. (2010). Dicer's helicase domain is required for accumulation of some, but not all, C-elegans endogenous siRNAs. RNA 16, 893-903.

Whipple, J.M., Youssef, O.A., Aruscavage, P.J., Nix, D.A., Hong, C.J., Johnson, W.E., and Bass, B.L. (2015). Genome-wide profiling of the C. elegans dsRNAome. RNA 21, 786-800.

Wilkins, C., Dishongh, R., Moore, S.C., Whitt, M.A., Chow, M., and Machaca, K. (2005). RNA interference is an antiviral defence mechanism in Caenorhabditis elegans. Nature 436, 1044-1047.

Winston, W.M., Molodowitch, C., and Hunter, C.P. (2002). Systemic RNAi in C-elegans requires the putative transmembrane protein SID-1. Science 295, 2456-2459.

Winston, W.M., Sutherlin, M., Wright, A.J., Feinberg, E.H., and Hunter, C.P. (2007). Caenorhabditis elegans SID-2 is required for environmental RNA interference. Proceedings of the National Academy of Sciences of the United States of America 104, 10565-10570. 
Wu, D., Lamm, A.T., and Fire, A.Z. (2011). Competition between ADAR and RNAi pathways for an extensive class of RNA targets. Nature Structural \& Molecular Biology 18, 1094-U1024.

Xu, J., Mon, H., Kusakabe, T., Li, Z.Q., Zhu, L., Iiyama, K., Masuda, A., Mitsudome, T., and Lee, J.M. (2013a). Establishment of a soaking RNA interference and Bombyx mori nucleopolyhedrovirus (BmNPV)-hypersensitive cell line using Bme21 cell. Applied Microbiology and Biotechnology 97, 10435-10444.

Xu, J., Nagata, Y., Mon, H., Li, Z.Q., Zhu, L., Iiyama, K., Kusakabe, T., and Lee, J.M. (2013b). Soaking RNAi-mediated modification of Sf9 cells for baculovirus expression system by ectopic expression of Caenorhabditis elegans SID-1. Applied Microbiology and Biotechnology 97, 5921-5931.

Yang, Y.Z., Jittayasothorn, Y., Chronis, D., Wang, X.H., Cousins, P., and Zhong, G.Y. (2013). Molecular Characteristics and Efficacy of 16D10 siRNAs in Inhibiting Root-Knot Nematode Infection in Transgenic Grape Hairy Roots. Plos One 8, e69463-e69463.

Yigit, E., Batista, P.J., Bei, Y.X., Pang, K.M., Chen, C.C.G., Tolia, N.H., Joshua-Tor, L., Mitani, S., Simard, M.J., and Mello, C.C. (2006). Analysis of the C-elegans argonaute family reveals that distinct argonautes act sequentially during RNAi. Cell 127, 747-757.

Zhang, C., Montgomery, T.A., Fischer, S.E.J., Garcia, S., Riedel, C.G., Fahlgren, N., Sullivan, C.M., Carrington, J.C., and Ruvkun, G. (2012). The Caenorhabditis elegans RDE-10/RDE-11 Complex Regulates RNAi by Promoting Secondary siRNA Amplification. Current Biology 22, 881-890.

Zhang, C., Montgomery, T.A., Gabel, H.W., Fischer, S.E.J., Phillips, C.M., Fahlgren, N., Sullivan, C.M., Carrington, J.C., and Ruvkun, G. (2011). mut-16 and other mutator class genes modulate $22 \mathrm{G}$ and $26 \mathrm{G}$ siRNA pathways in Caenorhabditis elegans. Proceedings of the National Academy of Sciences of the United States of America 108, 1201-1208.

Zhang, L., Ding, L., Cheung, T.H., Dong, M.Q., Chen, J., Sewell, A.K., Liu, X., Yates, J.R., and Han, M. (2007). Systematic identification of C. elegans miRISC proteins, miRNAs, and mRNA targets by their interactions with GW182 proteins AIN-1 and AIN-2. Molecular Cell 28, 598-613.

Zhang, P.P., and Zhang, H. (2013). Autophagy modulates miRNA-mediated gene silencing and selectively degrades AIN-1/GW182 in C-elegans. EMBO Rep 14, 568-576.

Zhuang, J.J., and Hunter, C.P. (2012). The Influence of Competition Among C. elegans Small RNA Pathways on Development. Genes 3, 671-685.

Zinovyeva, A.Y., Bouasker, S., Simard, M.J., Hammell, C.M., and Ambros, V. (2014). Mutations in Conserved Residues of the C. elegans microRNA Argonaute ALG-1 Identify Separable Functions in ALG-1 miRISC Loading and Target Repression. Plos Genetics 10, e1004286-e1004286.

Zisoulis, D.G., Lovci, M.T., Wilbert, M.L., Hutt, K.R., Liang, T.Y., Pasquinelli, A.E., and Yeo, G.W. (2010). Comprehensive discovery of endogenous Argonaute binding sites in Caenorhabditis elegans. Nature Structural \& Molecular Biology 17, 173-U176. 


\title{
RNAi AND mIRNA PATHWAYS IN PLANTS I - MOLECULAR MECHANISMS
}

Keywords: dsRNA, miRNA, siRNA, Dicer, Argonaute, RdRP, RNAi, PTGS, DNA methylation

\section{PETR SVOBODA}

Institute of Molecular Genetics, Academy of Sciences of the Czech Republic, Videnska 1083, 14220 Prague 4, Czech Republic

Correspondence to: Petr Svoboda, Institute of Molecular Genetics ASCR, Videnska 1083, 14220 Prague 4, Czech Republic, tel. \#+420 241063147, e-mail: svobodap@img.cas.cz.

\begin{abstract}
RNA silencing denotes sequence-specific repression mediated by small RNAs. It includes post-transcriptional silencing mechanisms such as RNA interference (RNAi) and microRNA (miRNA) pathway and transcriptional silencing. RNA silencing regulates endogenous protein-coding gene expression, serves as a form of innate immunity targeting viruses, and protects genome integrity by repression of retrotransposons. This review provides overview of RNA silencing in flowering plants, from which comes the bulk of published data. Plants have arguably the most complex RNA silencing system among eukaryots because of existence of many paralogs of key protein factors, which form an intricate network of primary and secondary small RNAs, which mediate transcriptional and post-transcriptional effects. The first half of the review will provide a detailed catalogue of components of RNA silencing in plants while the second half will discuss the specific silencing mechanisms themselves and their biological roles, with a particular focus on the miRNA pathway, PTGS/VIG (plant's equivalent of RNAi), and transcriptional gene silencing (TGS), which is a unique RNA silencing adaptation existing in plants.
\end{abstract}

\section{Introduction}

Plants are extremely large and diverse group of multicellular organisms. Among their defining features are cell walls are made of cellulose, the ability to perform photosynthesis in chloroplasts, double membrane organelles containing chlorophyll $\mathrm{a} / \mathrm{b}$, and use of starch to store photosynthetic products. There are $\sim 500000$ described species (Margulis and Schwartz, 1998), which are classified in 10-12 phyla by different taxonomical concepts. In this review, I will focus on so-called angiosperms (phylum Magnoliophyta or Anthophyta), which is the most populous plant phylogenetic group (Palmer et al., 2004) and from which are most data concerning RNA silencing in plants. 
RNA silencing is extremely convoluted in plants. It is the most complex RNA silencing among the reviewed taxons thanks to existence of many Dicer, Argonaute, and RNA-dependent RNA polymerase (RdRP) paralogs, which fucntion in an intricate network of primary and secondary small RNAs, which mediate transcriptional and post-transcriptional effects. The nomenclature of small RNAs in plants is complex and arbitrary; small RNAs include miRNAs and various types of siRNAs produced from ds RNA, which are distinguished by their origin (viral siRNA), biogenesis (phased siRNAs) or their effect (heterochromatinizing siRNAs). RNA silencing in plants can be divided in three to four main systems: miRNA, RNAi/ post-transcriptional gene silencing (PTGS), antiviral defense/ virus-induced gene silencing (VIGS), and transcriptional gene silencing (TGS) (Bologna and Voinnet, 2014; Bonnet et al., 2006; Borges and Martienssen, 2015; Carbonell and Carrington, 2015; Chen, 2009; Galun, 2005; Mallory et al., 2008; Van Ex et al., 2011; Vazquez, 2006; Zhang et al., 2015).

Here, I will focus on data from Arabidopsis thaliana and first review the molecular features of key individual components of RNA silencing related to the miRNA pathway and dsRNA response - Dicers, Argonautes, and RdRPs. In the second half of this review, I will discuss the reviewed mechanisms themselves and their biological roles, with a particular focus on the miRNA pathway, PTGS/VIG - plant's equivalent of RNAi, and transcriptional gene silencing (TGS), which is a unique adaptation found in plants. Unless specifically stated, presented information comes from the Arabidopsis thaliana model.

\section{Dicer proteins - DCL1-4 and additional Dicer family members}

Plants have specialized and compartmentalized Dicer (Dicer-like - DCL) proteins that act partially redundantly and hierarchically in small RNA production in different pathways miRNA, antiviral defense, endo-RNAi pathways, or chromatin remodelling (Liu et al., 2009b; Moissiard and Voinnet, 2006; Xie et al., 2005). The "basal" plant state found in Arabidopsis (or, for example, cotton (Silva et al., 2011)) is four Dicers, which presumably evolved through duplications of an ancestral Dicer (Margis et al., 2006). Some plants (such as monocots) have higher counts of Dicer homologs - there are five in poplar and eight in rice; the additional Dicers evolved through duplications of one of the four "basic" plant Dicers (Kapoor et al., 2008; Margis et al., 2006; Mukherjee et al., 2013).

Plant Dicer proteins are large multidomain proteins, which have essentially the same domain organization as animal Dicers (Fig. 1), i.e. they are composed of domains ordered from the $\mathrm{N}$ - to the $\mathrm{C}$-terminus as follows: N-terminal DExD and helicase superfamily C-terminal domains, a domain of unknown function DUF283 (not annotated in DCL-3 of Arabidopsis thaliana but annotated in poplar and rice DCL3), a PAZ domain, RNase IIIa and RNase IIIb domains, and the C-terminal dsRBD. The four plant Dicers differ in size and the number of dsRBD domains - DCL1, DCL3, and DCL4 have an additional dsRBD (Fig. 1). Although none of the plant Dicers has been either crystallized or analyzed by cryo-EM, the conserved domain organization and available biochemical data suggest that plant Dicers operate under the same principles as animal Dicers despite their functional diversification. In general, DCL1 is mainly functioning in the miRNA pathway while 


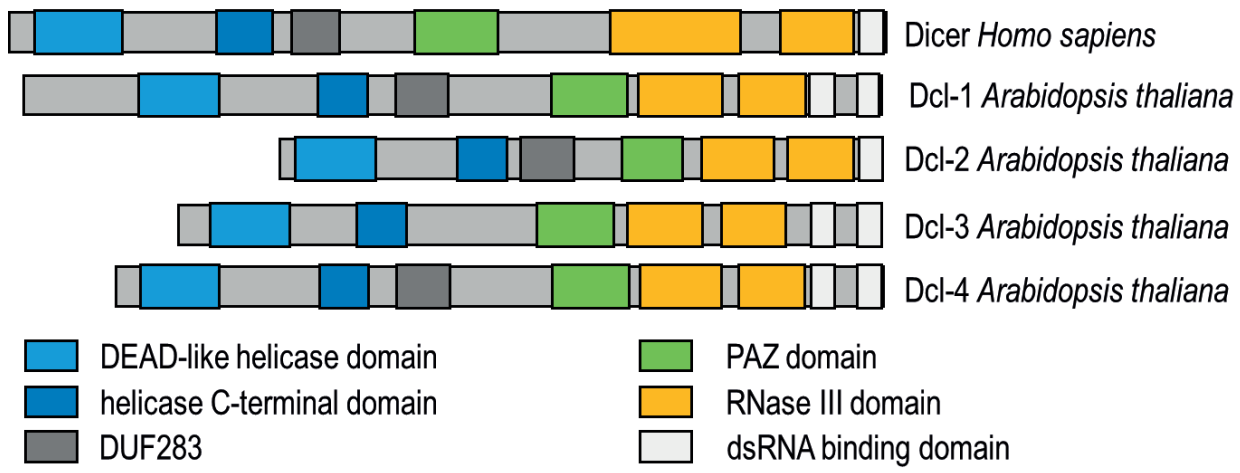

Figure 1 Comparison Dicer protein domain composition among Dicer proteins in A. thaliana, and C. elegans

Domain composition was adopted from (Jaskiewicz and Filipowicz, 2008).

DCL2, 3, 4 produce siRNAs for silencing mobile elements or in antiviral defense where DCL3 represents the nuclear arm of the defense, which is distinguished by production of longer siRNAs (24 nt). Detailed review of individual DCL proteins is provided further below:

\section{DCL1 - miRNA biogenesis \& PTGS}

DCL1 is the oldest known and possibly best characterized member of the DCL gene family in Arabidopsis. DCL1 was repeatedly recovered from mutation screens in plants since early 90's and it has been given several names (EMBRYO DEFECTIVE76 (EMB76), SHORT INTEGUMENTS1 (SIN1), SUSPENSOR1 (SUS1) and CARPEL FACTORY (CAF)) prior recognizing that it is a factor closely related to Dicer proteins acting in RNA silencing in animals (Schauer et al., 2002). DCL1 was first shown to be required for biogenesis of miRNAs but not siRNAs (Park et al., 2002; Reinhart et al., 2002). The size of the cleavage product is $21 \mathrm{nt}$, a nucleotide shorter than average length of animal miRNAs (Fig. 2).

DCL1 contains two putative nuclear localization signals and it localizes to the nucleus where it produces small RNAs (Papp et al., 2003). Furthermore, it was shown that DCL1 is also able to produce $21 \mathrm{nt}$ from a transgenic inverted repeat (Papp et al., 2003).

DCL1 operates with a dsRBD binding partners DRB1 and DRB2 (Curtin et al., 2008; Reis et al., 2016; Reis et al., 2015b). Interestingly, DCL1 represses antiviral RNA silencing through negatively regulating the expression of DCL4 and DCL3 (Qu et al., 2008). DCL1 is an essential gene in Nicotiana attenuata (Bozorov et al., 2012) and its miRNA function is conserved in tomato (Kravchik et al., 2014b).

\section{DCL2}

DCL2 functions in the antiviral response (Curtin et al., 2008; Donaire et al., 2008; Fusaro et al., 2006; Ogwok et al., 2016; Urayama et al., 2010; Zhang et al., 2012) where it produces 
miRNA size distribution in A. thaliana

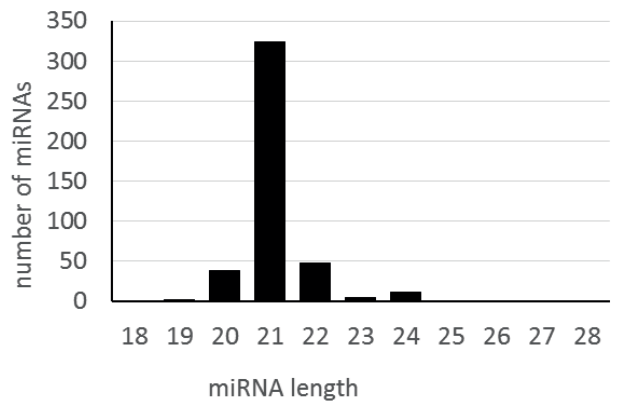

miRNA size distribution in Mus musculus

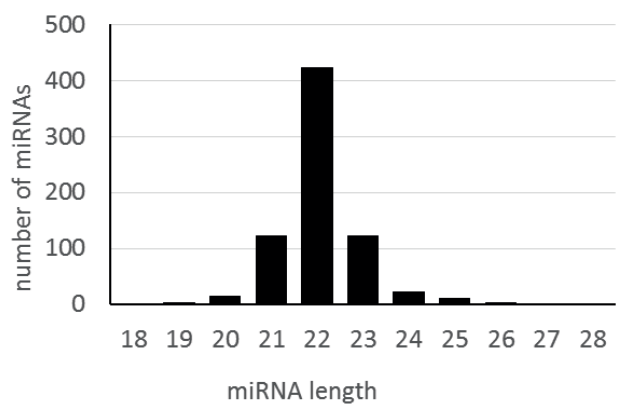

Figure 2 Arabidopsis miRNA size distribution Distribution of Arabidopsis mature miRNA lengths according to miRNA annotations in miRBase (Kozomara and Griffiths-Jones, 2014).

viral siRNAs without requiring assistance from any dsRBP. (Curtin et al., 2008). DCL2 was also implicated in 21/22 nt siRNA production from longer intronic hairpins (sirtrons) (Chen et al., 2011). DCL2 acts hierarchically with DCL4 to produce 22- and 21-nt siRNAs in antiviral resistance and amplification of silencing mediated by RNA-dependent RNA polymerase RDR6 (Brosnan et al., 2007; Deleris et al., 2006; Di Serio et al., 2009; Garcia-Ruiz et al., 2010; Ogwok et al., 2016; Parent et al., 2015). DCL2 can substitute DCL4 and produce 22nt viral siRNAs even in the absence of DCL4, (Bouche et al., 2006; Moissiard et al., 2007; Wang et al., 2011). Under some circumstances, DCL2 can antagonize production of miRNAs and siRNAs by DCL1 (Bouche et al., 2006).

DCL2 is required for transitive cell-autonomous post-transcriptional silencing of transgenes (Mlotshwa et al., 2008) and it was implicated in the transgenerational stress memory (Boyko and Kovalchuk, 2010; Migicovsky and Kovalchuk, 2014; Migicovsky and Kovalchuk, 2015; Migicovsky et al., 2014). Two paralogs of DCL2 were found in Medicago truncatula where are DCL genes differentially expressed during symbiosis with nitrogen fixing bacteria and upon pathogen infection (Tworak et al., 2016). Two paralogs of DCL2 were also found in soybean where DCL2 showed the strongest transcriptional response to stress (Curtin et al., 2012).

\section{DCL3}

DCL3 is producing longer siRNAs (24 nt) than the other three DCL proteins in Arabidopsis and other plants, including tomato, rice, medick, or moss (Coruh et al., 2015; Kravchik et al., 2014a; Tworak et al., 2016; Wei et al., 2014). DCL3 preferentially cleaves dsRNAs with 5' phosphorylated adenosine or uridine and a $1 \mathrm{nt} 3$ ' overhang (Nagano et al., 2014) and produces 24 nt RNA duplexes with 2 nt 3' overhangs; inorganic phosphate, $\mathrm{NaCl}$ and $\mathrm{KCl}$ enhance DCL3 activity (Kravchik et al., 2014a).

DCL3 long dsRNA substrates are typically generated from RNA polymerase IVa/IV and $\mathrm{IVb} / \mathrm{V}$ (Pol IV and Pol V hereafter) transcripts by RDR2 (Daxinger et al., 2009; Zhang 
et al., 2007). DCL3 co-localizes with RDR2, AGO4, NRPD1b (the largest Pol V subunit) and siRNAs within the nucleolus (Pontes et al., 2006).

DCL3 was implicated in $24 \mathrm{nt}$ siRNA production from longer intronic hairpins (sirtrons), which were associated with AGO4 and could mediate RNA-dependent DNA methylation (RdDM) (Chen et al., 2011), and in production of viral 24 nt siRNAs (Akbergenov et al., 2006; Curtin et al., 2008; Diaz-Pendon et al., 2007; Donaire et al., 2008; Fusaro et al., 2006; Raja et al., 2014). DCL3 can produce viral siRNAs without requiring assistance from any dsRBP (Curtin et al., 2008) but, it was also shown that it can function with DRB3 and AGO4 in methylation-mediated antiviral defense (Raja et al., 2014). Interestingly, the loss of DCL3 is partially complemented by DCL4 and DCL2, which produce 21/22 nt small RNAs (Kravchik et al., 2014a) indicating partial functional redundancy of DCL2/3/4 in recognition of dsRNA and silencing.

DCL3 and RDR2 were also implicated in production of a pseudogene-derived $24 \mathrm{nt}$ siRNAs in rice (Guo et al., 2009). DCL3b paralog in rice functions in processing of $24 \mathrm{nt}$ phased small RNAs in miRNA targeted loci suggesting functional divergence of DCL3 paralogs in rice (Song et al., 2012). DCL3 was suggested to participate also in the transgenerational stress memory (Boyko and Kovalchuk, 2010; Migicovsky and Kovalchuk, 2014; Migicovsky and Kovalchuk, 2015; Migicovsky et al., 2014).

Finally, DCL3, RDR2 and Pol IV, also operate in production of 24 nt small RNAs from miRNA loci, which are loaded on AGO4 and mediate RdDM (Chellappan et al., 2010; Wu et al., 2010). DCL3 may be absent in conifers, which radiated from other seed-bearing plants approximately 260 million years ago; there were no significant amounts of $24 \mathrm{nt}$ siRNAs in growing shoot tissue while no evidence for DCL3 was found (Dolgosheina et al., 2008).

\section{DCL4}

DCL4 cleaves long dsRNAs with blunt ends or with a 1 or 2 nt 3' overhang with similar efficiency; inorganic phosphate, $\mathrm{NaCl}$ and $\mathrm{KCl}$ inhibit DCL4 activity (Nagano et al., 2014). DCL4 operates with DRB4 to produce $21 \mathrm{nt}$ trans-acting siRNAs (tasiRNAs, they are $21 \mathrm{nt}$ siRNAs produced from discrete loci (TAS genes). and siRNAs from viral RNA. (Curtin et al., 2008; Fusaro et al., 2006; Nakazawa et al., 2007; Qu et al., 2008). DCL4 alone was sufficient for antiviral silencing in leaves inoculated with Turnip mosaic virus (Garcia-Ruiz et al., 2010). While DCL4 is important for biogenesis of tasiRNA and antiviral response, it does not participate in the miRNA pathway (Xie et al., 2005).

DCL4 acts hierarchically with DCL2 to produce 21- and 22-nt siRNAs and in antiviral resistance and amplification of silencing mediated by RDR6 (Brosnan et al., 2007; Deleris et al., 2006; Di Serio et al., 2009; Garcia-Ruiz et al., 2010; Howell et al., 2007; Liu et al., 2007; Ogwok et al., 2016; Parent et al., 2015; Qu et al., 2008). The dcl4-2 mutants lack each of three families of 21-nt tasiRNAs, have elevated levels of tasiRNA target transcripts, and display heterochronic defects similar to RDR6 mutants (Xie et al., 2005). Furthermore, different double mutant phenotypes also suggested hierarchical redundancy among DCL activities leading to alternative tasiRNA biogenesis in the absence of DCL4 (Xie et al., 2005). 
DCL4 also operates (again, hierarchically with DCL2) in biogenesis of secondary siRNAs in transitive RNAi (Mlotshwa et al., 2008; Moissiard et al., 2007) and it was implicated in 21/22 nt siRNA production from longer intronic hairpins (sirtrons) (Chen et al., 2011)

Two paralogs of DCL4 exist in Medicago truncatula where DCL genes are differentially expressed during symbiosis with nitrogen fixing bacteria and upon pathogen infection (Tworak et al., 2016).

\section{dsRBPs -DRB1-5}

While DCLs act redundantly and hierarchically, there is little if any redundancy or hierarchy among DRBPs in their Dicer-associated functions. Arabidopsis genome encodes five DRBs composed of two types of dsRBDs that form a distinct clade (Clavel et al., 2016). Two additional proteins carrying dsRBDs (At1g80650 and At4g00420, renamed AtDRB7.1 and AtDRB7.2, respectively) were localized in the Arabidopsis genome. They differ from DRB1-5 in terms of dsRBD composition (Clavel et al., 2016). DRB1 and DRB2 associate with DCL1, DRB4 with DCL4, while DCL2 and DCL3 produce viral siRNAs without requiring assistance from any dsRBP. (Curtin et al., 2008).

\section{DRB1/HYL1}

DRB1 has two dsRBDs separated by a linker of $\sim 20$ amino acids (Clavel et al., 2016). DRB1 is a nuclear dsRBP exclusively functioning together with DCL1 and a small RNA methyltransferase HEN1 in miRNA biogenesis (Curtin et al., 2008; Vazquez et al., 2004). This role seems conserved across plants; its homologs were found in all tested plant genomes (Clavel et al., 2016; You et al., 2014). In association with DCL1 it directs the guide strand selection for AGO loading (Eamens et al., 2009) and determines the slicing mode of action of the miRNA-loaded AGO1 (Reis et al., 2015b). It also interacts with a hairpin in short interspersed element SB1 RNA and facilitates DCL1-mediated production of small RNAs from these repetitive elements (Pouch-Pelissier et al., 2008). DRB1 is phosphorylated by mitogen activated protein kinase MPK3 in both rice and Arabidopsis (Raghuram et al., 2015).

\section{DRB2}

DRB2 proteins possess two dsRBDs separated by a linker of 19 amino acids (Clavel et al., 2016). DRB2 is involved in miRNA biogenesis (Eamens et al., 2012a) where it, in association with DCL1, determines the translational repression of miRNA-loaded AGO1 (Reis et al., 2015b). DRB2 is involved in the processing stage of the biogenesis of non-canonical miRNA subsets while DRB3 and DRB5 are somehow required downstream to mediate RNA silencing of DRB2-associated miRNA target genes (Eamens et al., 2012b). The role of DRB2 in the miRNA pathway is distinct from that of DRB1 as shown by proteomic analysis of drb1 and drb2 mutants, which suggested that DRB2-associated translational inhibition appears to be less ubiquitous and specifically aimed toward responses against 
environmental stimuli (Reis et al., 2015c). DRB2 acts redundantly with DRB3 and DRB5 during development and appears unnecessary for other types of plant small RNAs, such as miRNA, tasiRNA, viral siRNA, or heterochromatinising siRNA production (Curtin et al., 2008). The loss of DRB2 protein in Arabidopsis results in increased levels of Pol IV dependent siRNAs, which are involved in RdDM. It was proposed that DRB2 is part of epigenetic regulation suppressing transcription of transposable elements (Clavel et al., 2015; Pelissier et al., 2011).

\section{DRB3}

DRB3 proteins possess two dsRBDs separated by a linker of 19 amino acids (Clavel et al., 2016). DRB3 participates to the RdDM defense against Geminiviruses (Raja et al., 2014). At the same time, it appears unnecessary for miRNA, tasiRNA, viral siRNA, or heterochromatinising siRNA production but acts redundantly DRB2 and DRB5 during development (Curtin et al., 2008). DRB3 is participates in RNA silencing of target genes of DRB2-associated non-canonical miRNAs (Eamens et al., 2012b).

\section{DRB4}

DRB4 protein and its relatives carry three dsRBDs. They are found in all vascular plants but were absent in the tested bryophyte and lycophyte genomes (Clavel et al., 2016). DRB4 operates with DCL4 to produce $21 \mathrm{nt}$ tasiRNAs and 21nt siRNAs from viral RNA (Curtin et al., 2008; Jakubiec et al., 2012; Qu et al., 2008; Shivaprasad et al., 2008). DRB4 expression is regulated by E3 ubiquitin ligase APC/C (Anaphase Promoting Complex or Cyclosome). APC10 interacts with DRB4 through the second dsRBD of DRB4, which is also required for its homodimerization and binding to DCL4 (Marrocco et al., 2012). In contrast to the loss of DRB2 protein in Arabidopsis, the loss of DRB4 results in reduced levels of Pol IV dependent siRNAs, which are involved in RdDM (Pelissier et al., 2011).

\section{DRB5}

DRB5 proteins possess two dsRBDs separated by a linker of 19 amino acids (Clavel et al., 2016). DRB5 appears unnecessary for miRNA, tasiRNA, viral siRNA, or heterochromatinising siRNA production but acts redundantly with DRB2 and DRB3 during development (Curtin et al., 2008). DRB5 is somehow required downstream to mediate RNA silencing of target genes of DRB2-associated non-canonical miRNAs (Eamens et al., 2012b). DRB3 was shown to associate with DCL2 and AGO4 in the RdDM arm of antiviral defense against Geminiviruses (Raja et al., 2014).

\section{DRB6}

DRB6 proteins carry two dsRBDs and are present in all vascular plants except for bryophytes, lycophytes and Brassicaceae species (Clavel et al., 2016). Their biological significance is unknown. 


\section{DRB7}

The DRB7 family has a single dsRBD; there are two members AtDRB7.1 and AtDRB7.2. Their role is largely unknown but it was found that they interact with DRB4 but not with DCL4 in Arabidopsis (Clavel et al., 2016).

\section{Argonaute proteins}

Plants show quite some variability in the AGO homolog pool. For example, Arabidopsis thaliana has 10 AGO genes (Table 1), Oryza sativa has 19, Glycine max (soybean) has 22 (Zhang et al., 2015), Solanum lycopersicum 15 (Bai et al., 2012). A dicot plant Sativa miltiorhiza (red sage) has $10 \mathrm{AGO}$ genes of which AGO1, 2, 3, 7, and 10 were proposed to function similarly to their $A$. thaliana counterparts (Shao and $\mathrm{Lu}, 2013$ ). A systematic survey of 32 plant genomes showed that plants have 6-24 AGO homologs per genome, most often more than 10 (Mirzaei et al., 2014). AGO proteins are phylogenetically divided into three clades, which also reflect different classes of bound small RNAs - clades I and II bind 21-22 nt small RNAs while the clade III accommodates longer ( 24 nt small RNAs) (Zhang et al., 2015). The phylogenetic analysis shows that the varying number of homologs in each species stems from multiple duplication events, which occurred during evolution of different taxons such that some species contain multiple paralogs of an ancestral AGO, which can be present in a single copy in another plant species.

AGO proteins loaded with different types of small RNAs play diverse roles in terms of molecular mode of action mechanisms and biological function. Small RNAs are sorted onto specific AGO proteins based on different factors, which might include subcellular localization or the DCL protein that is producing a particular small RNA. One of the key factors contributing to sorting of small RNAs onto AGO proteins is also the 5' terminal nucleotide. For example, AGO1 favors miRNAs with a 5' terminal uridine, AGO2 and AGO4 prefer small RNAs with a 5' terminal adenosine, AGO5 with a 5' terminal cytosine (Mi et al., 2008; Takeda et al., 2008).

\section{AG01}

Arabidopsis AGO1 gave the name to the entire Argonaute protein family because agol mutants were having a phenotype reminiscent of the tentacles of an Argonauta squid (Bohmert et al., 1998). Arabidopsis has a single AGO1 gene, rice has four paralogs (Wu et al., 2009). AGO1 preferentially binds miRNAs and small RNAs with a 5' uridine (Bohmert et al., 1998; Jeong et al., 2013; Mi et al., 2008; Rogers and Chen, 2013; Vaucheret et al., 2004; Wu et al., 2009). Analysis of miRNAs loaded onto AGO1 paralogs in rice suggested that a subset of miRNAs is specifically incorporated into or excluded from one of these paralogs suggesting they have both redundant and specialized roles in rice (Wu et al., 2009). AGO1 also associates with tasiRNAs. In contrast to miRNAs, tasiRNAs involve RdRP-mediated conversion of cleaved TAS RNA into dsRNA followed by production of phased tasiRNAs (i.e. secondary small RNAs) by DCL-4, which are loaded onto AGO1 (Vaucheret, 2005). 
Table 1 Overview of Argonaute proteins in Arabidopsis thaliana.

\begin{tabular}{|c|c|c|c|c|c|c|c|}
\hline \multirow{2}{*}{$\begin{array}{l}\text { common } \\
\text { name }\end{array}$} & \multirow{2}{*}{ subfamily } & \multirow{2}{*}{ slicer } & \multicolumn{5}{|c|}{ associated small RNA } \\
\hline & & & type & length & 5'nt & 5' end & 3' end \\
\hline AGO1 & plant AGO clade I & + & miRNA & 21 & $\mathrm{U}$ & mono-P & 2'-O-met \\
\hline AGO2 & plant AGO clade II & + & $\begin{array}{c}\text { miRNAs, tasiRNAs, } \\
\text { rasiRNAs }\end{array}$ & 21 & A & mono-P & 2'-O-met \\
\hline AGO3 & plant AGO clade II & + & siRNAs & 24 & A & mono-P & 2'-O-met \\
\hline AGO4 & plant AGO clade III & + & $\begin{array}{l}\text { intergentic siRNAs, } \\
\text { rasiRNAs }\end{array}$ & $23-24$ & A & mono-P & 2'-O-met \\
\hline AGO5 & plant AGO clade I & + & intergenic siRNA & $21,22,24$ & $\mathrm{C}$ & mono-P & 2'-O-met \\
\hline AGO6 & plant AGO clade III & + & siRNAs & 24 & A & mono-P & 2'-O-met \\
\hline AGO7 & plant AGO clade II & + & $\begin{array}{l}\text { miRNA (miR390), } \\
\text { ta-siRNA }\end{array}$ & 21 & A & mono-P & 2'-O-met \\
\hline AGO8 & plant AGO clade III & $(+)$ & - & - & - & - & - \\
\hline AGO9 & plant AGO clade III & $(+)$ & rasiRNAs & 24 & A & mono-P & 2'-O-met \\
\hline AGO10 & plant AGO clade I & + & miRNA (mir165/166) & 21 & $\mathrm{U}$ & mono-P & 2'-O-met \\
\hline
\end{tabular}

\section{AGO2}

AGO2 is a slicing AGO (Carbonell et al., 2012) but can also directly repress translation (Fatyol et al., 2016). It favors small RNAs with a 5' terminal adenosine (Mi et al., 2008; Takeda et al., 2008), which include miR393*, regulating antibacterial innate immunity (Zhang et al., 2011). AGO2 plays a role in the natural cis-antisense (natsiRNA) pathway (Oliver et al., 2014). AGO2 also mediates antiviral defense (Jaubert et al., 2011; Odokonyero et al., 2015) and was implicated (together with a plant-specific GW protein NERD) in nuclear silencing of a set of non-conserved genomic loci (Pontier et al., 2012). In addition, AGO2 was also associated with diRNAs, small RNAs emerging during double-stranded break repair (Oliver et al., 2014; Wei et al., 2012).

\section{AGO3}

Drosophila AGO3 is a close paralog apparently emerging through a genome duplication of the AGO2 locus (Vaucheret, 2008). Interestingly, rice and maize lack the AGO3 ortholog but have a pair of AGO2 genes instead (Kapoor et al., 2008; Zhai et al., 2014).

\section{AGO4}

AGO4 preferentially recruits 24 nt small RNAs with a 5' terminal adenosine (Havecker et al., 2010; Mi et al., 2008) and mediates RNA-directed DNA methylation (Havecker et al., 2010; He et al., 2009; Wu et al., 2010; Xie and Yu, 2015; Zilberman et al., 2004). AGO4-loaded small RNAs are often DCL-3 produced repeat and heterochromatin-associated siRNAs from introns and intergenic regions (Chen et al., 2011; He et al., 2009; Xie 
and Yu, 2015; Zheng et al., 2009; Zilberman et al., 2004) but can also be DCL-4 produced tasiRNAs (Wu et al., 2012). In rice, it was even found that RNA-directed DNA methylation can be guided by a specific miRNA class produced by DCL3 (Wu et al., 2010). AGO4 is also coupled to antibacterial (Agorio and Vera, 2007) and antiviral innate immunity (Jones et al., 2006; Ma et al., 2015; Minoia et al., 2014; Raja et al., 2014).

\section{AGO5}

AGO5 is highly enriched in the germline (Oliver et al., 2014). It binds preferentially $21 \mathrm{nt}$ small siRNAs (phased small RNAs, phasiRNAs) with a 5' terminal cytosine (Komiya et al., 2014; Mi et al., 2008; Takeda et al., 2008). It also binds miRNAs and mediates miRNA-directed target cleavage (Oliver et al., 2014). Arabidopsis AGO5 has been implicated in female gametogenesis (Tucker et al., 2012) and in antiviral defense (Brosseau and Moffett, 2015). MEL1, AGO5 homolog in rice, has specific functions in the development of pre-meiotic germ cells and the progression of meiosis (Komiya et al., 2014).

\section{AGO6}

AGO6 preferentially recruits 24 nt RNAs with a 5' terminal adenosine (Havecker et al., 2010). AGO6 mediates RNA-directed DNA methylation (Eun et al., 2011; Havecker et al., 2010; McCue et al., 2015; Zheng et al., 2007). AGO6 participates in RNA-directed DNA methylation of transcriptionally active transposable elements through incorporation of fragments of PTGS-targeted transcripts of transposable elements onto AGO6 (McCue et al., 2015). AGO6 can also accommodate DCL4-produced produced tasiRNAs (Wu et al., 2012).

\section{AGO7}

AGO7 was originally named ZIPPY and was primarily associated with the regulation of developmental timing and did not have a significant role in transgene silencing (Hunter et al., 2003). It was subsequently found that AGO7 is preferentially loaded with miR390 and triggers production of tasiRNAs from the TAS3 locus (Garcia et al., 2006; Montgomery et al., 2008). TAS3-derived tasiRNA target AUXIN RESPONSE FACTORS and regulate leaf patterning and lateral organ separation (Montgomery et al., 2008; Zhou et al., 2013). This role of AGO7 appears conserved across plants as it has been also reported from rice (Nagasaki et al., 2007; Shi et al., 2007) and maize (Douglas et al., 2010).

\section{AGO8 - seems to be a pseudogene in A. thaliana (Zhang et al., 2015)}

\section{AGO9}

AGO9 is involved in RNA-directed DNA methylation and is highly enriched in the germline (Oliver et al., 2014) where it controls female gamete formation by repressing 
the specification of germ cell fate through epigenetic reprogramming in companion somatic cells (Olmedo-Monfil et al., 2010). Zea mays AGO2 homolog (originally designated ZmAGO104) is also specifically expressed in the somatic cells surrounding future gametes but it had an opposing function - it suppresses the somatic cell fate in germ cells (Singh et al., 2011).

\section{AG010}

AGO10 is the closest homolog of AGO1 and its main role appears to be sequestration of miR156/166 miRNA family from AGO1 (Zhou et al., 2015; Zhu et al., 2011). The targets of miR166/165 are class III HOMEODOMAIN-LEUCINE ZIPPER transcription factors, which determine the shoot apical meristem fate. According to the model, AGO10 uses its higher binding affinity for miR166/165 and functions as a decoy, preventing loading of miR166/165 onto AGO1, hence preventing their suppression and allowing for proper regulation of the shoot apical meristem (Brandt et al., 2013; Ji et al., 2011; Liu et al., 2009a; Roodbarkelari et al., 2015; Tucker et al., 2013; Zhou et al., 2015; Zhu et al., 2011).

\section{Additional relevant homologs}

Monocot genomes encode for AGO18 homologs, which are not found in dicots, such as A. thaliana (Zhang et al., 2015). In rice, AGO18 is important for antiviral defense (Wu et al., 2015).

\section{RdRPs}

The first RdRP homolog found in plants was RdRP from tomato (Schiebel et al., 1993; Schiebel et al., 1998). Arabidopsis genome carries six RdRP genes: RDR1-RDR6 (Yu et al., 2003). There are five RdRPs in Salvia miltiorrhiza (Shao and Lu, 2014) and six in potato Solanum lycopersicum (Lin et al., 2016). Plant RdRPs are homologs of RdRPs acting in RNA silencing in other kingdoms, such as QDE-3 in Neurospora (Salgado et al., 2006; Wassenegger and Krczal, 2006), suggesting that they evolved from a single ancestral RdRP acting in RNA silencing. RdRPs produce dsRNA that can enter the RNA silencing pathway. Hence, they either initiate RNA silencing or function as an amplifier of an already present dsRNA response.

\section{$R D R 1$}

RDR1 (also known as SDE1/SGS2) in Arabidopsis and its homologs in other plant species contribute to RNA silencing-based resistance to virus infection (Blevins et al., 2011; Cao et al., 2014; Diaz-Pendon et al., 2007; Garcia-Ruiz et al., 2010; Leibman et al., 2011; Muangsan et al., 2004; Vaistij et al., 2002; Yang et al., 2004; Yu et al., 2003). It was also implicated in biogenesis of tasiRNAs during juvenile development (Peragine et al., 2004). 


\section{RDR2}

RDR2 has been linked with transcriptional silencing in the nucleus and RdDM of specific loci (Chan et al., 2004; Xie et al., 2004). It participates in biogenesis of endogenous siRNAs (natsiRNAs) (Borges and Martienssen, 2015; Brosnan et al., 2007). RDR2 is not required for production of viral siRNAs from the Cauliflower mosaic pararetrovirus (Blevins et al., 2011), Cabbage leaf curl geminivirus (Aregger et al., 2012). RDR3 converts PolIV transcripts into dsRNA, which is processed by DCL3 into 24 nt siRNAs loaded onto AGO4 (Pontes et al., 2006; Zhang et al., 2007). RDR2 and DCL3 were implicated in production of pseudogene-derived $24 \mathrm{nt}$ siRNAs in rice (Guo et al., 2009).

\section{RDR3 - no functional information available}

\section{RDR4}

RDR4 has been linked to biogenesis of endogenous siRNAs (natsiRNAs) (Borges and Martienssen, 2015).

\section{RDR5 - no functional information obtained}

\section{RDR6}

RDR6 is necessary for sense-transgene mediated silencing and is important in antiviral defense against certain viruses (Beclin et al., 2002; Dalmay et al., 2000; Mourrain et al., 2000). RDR6-dependent antiviral response includes the cucumber mosaic virus in Arabidopsis (Wang et al., 2010) or tobacco mosaic virus in Nicotiana benthamina (Qu et al., 2005) but not the cauliflower mosaic virus in Arabidopsis (Blevins et al., 2011). RDR6 was also implicated in the biogenesis of tasiRNAs and development (Li et al., 2005a; Peragine et al., 2004; Vaucheret, 2005). RDR6-generated dsRNA is being processed by DCL4 (Howell et al., 2007; Qu et al., 2008).

\section{miRNA module}

\section{miRNA biogenesis}

Biogenesis initiates with recognition and cleavage of a primary miRNA (pri-miRNA), which is transcribed by polymerase II (Xie et al., 2010; Zhao et al., 2013). A plant pri-miRNA is a single-stranded RNA carrying a local hairpin structure. Many miRNAs in plants apparently originate from longer inverted repeats carrying sequences of their targets, which were generated by sequence duplications (Allen et al., 2004). These long inverted repeats subsequently eroded during evolution and only a short stem in the pri-miRNA persists as a functional remnant of the original long hairpin. The inverted repeat duplication hypothesis provides an explanation for the evolution of perfectly pairing miRNAs in plants. 
Plant miRNAs differ from animal miRNAs in several aspects. one of them is that they do not use a twostep nuclear-cytoplasmic process employing the nuclear Microprocessor complex with Drosha and cytoplasmic Dicer. Instead, plant miRNAs are produced in the nucleus in a two-step process involving a single Dicer protein - DCL1 (Fig. 3).

DCL1 requires additional cofactors, including DRB1/ HYL1 (a nuclear dsRNA binding protein (Vazquez et al., 2004)), HEN1 (HUA ENHANCER1, a small RNA methyltransferase (Yu et al., 2005)), SE (SERRATE, C2H2type zinc finger, (Lobbes et al., 2006; Yang et al., 2006)). DCL1 resides in a complex, in which physically interacts with DRB1 and HEN1 (Baranauske et al., 2015). DCL1, DRB1 and SE co-localize in the nucleus in so-called dicing bodies (D-bodies) (Fang and Spector, 2007). D-body function and assembly is not fully understood and there is a number of additional components which need to be functionally analyzed to unravel the complex connections between the D-body, signalling cascades, and responses to the environment (Reis et al., 2015a).

In the first step of miRNA biogenesis, DLC1 excises the miRNA/miRNA* duplex processing pri-miRNA from the base of the hairpin toward the loop (base-toloop) (Park et al., 2002; Reinhart et al., 2002). Loopto-base processing occurs in specific cases, such as miR159 and miR319 (Bologna et al., 2009). A unique case of bidirectional processing was observed for miR166, where it seems to play a regulatory role (Zhu et al., 2013). Plants also have non-canonical miRNAs, such as mirtrons, which skip the first cleavage step by

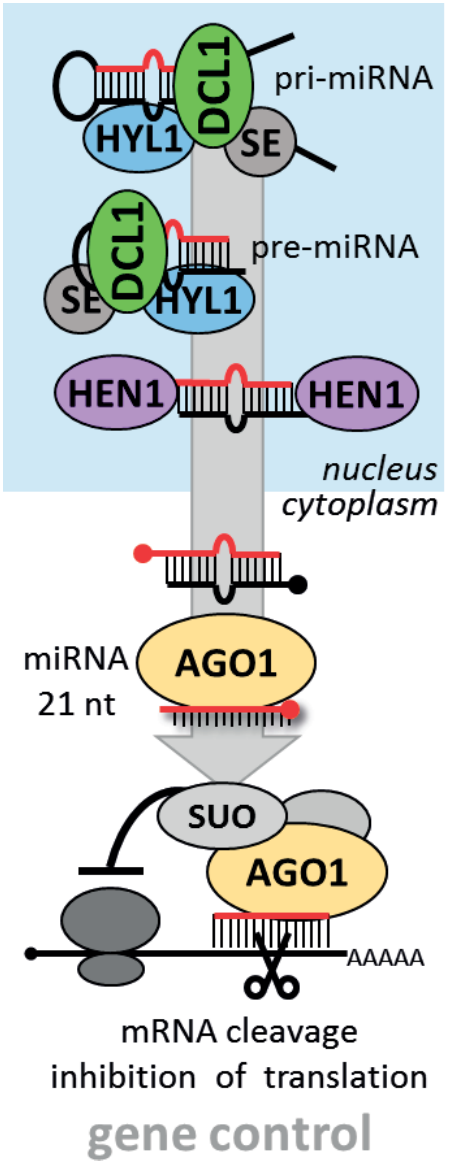

Figure 3 Plant miRNA pathway DCL1 (Meng and Shao, 2012).

The stem loop structure of pri-miRNA is recognized and processed by the DCL1DRB1-SE complex (Finnegan et al., 2003; Lobbes et al., 2006; Vazquez et al., 2004; Yang et al., 2006). Other DRB2 proteins (DRB2, 3, 5) also participate in biogenesis of miRNAs (Eamens et al., 2012a; Eamens et al., 2012b). Some pri-miRNA stems produce a single miRNA, some are longer and two or three additional ones on phase, i.e. require additional DCL1 cuts (Bologna et al., 2009; Zhang et al., 2010). Long hairpins can be processed by a diversity of Dicers to generate either miRNAs or siRNAs (Fig. 4). The subcellular location for dicing by DCL2 and DCL4, and subsequent AGO loading of the resulting siRNAs, is not completely understood (Axtell et al., 2011).

The 3' termini of the miRNA/miRNA* duplex are modified by HEN1 which adds a 2'-O-methyl group to the miRNA (Yu et al., 2005). This modification distinguishes plant miRNAs from animal miRNAs, which are not methylated (perhaps except of some minor 
population of miRNAs in arthropods (Horwich et al., 2007)). HEN1 was first identified genetically as a miRNA biogenesis co-factor, which was shown to localize into the nucleus (Park et al., 2002; Vaucheret et al., 2004; Vazquez et al., 2004). The HEN1 ortholog in rice was identified as WAF (WAVY LEAF), a crucial developmental factor (Abe et al., 2010). Structural analysis of HEN1 and its homologs showed that the catalytic domain of HEN1 is not closely related to any known RNA:2'-OH methyltransferases, but rather to small-molecule methyltransferases (Tkaczuk et al., 2006). One of the functions of the methylation is that it protects miRNAs from uridylation by an AGO1-associated uridylase that uridylates 5' RNA fragments generated by AGO1 cleavage (Li et al., 2005b; Ren et al., 2014).

miRNAs are exported from the nucleus with the assistance of hasty (HST), a plant homolog of Exportin 5 (Park et al., 2005). The subcellular location for dicing by DCL2 and DCL4, and subsequent AGO loading of the resulting siRNAs, is not yet clear. Loading of AGO1 with DCL1 products is assumed to take place in the cytoplasm (Axtell et al., 2011; Park et al., 2005). This loading onto AGO proteins involves sorting miRNAs according to the 5' terminal nucleotide and other factors (Mi et al., 2008; Montgomery et al., 2008; Zhu et al., 2011). Analysis of strand selection suggest that the strand with a lower 5 '-end thermostability is preferentially loaded into AGO1 (Eamens et al., 2009), which shows that plants employ the same loading asymmetry rule as animals. The selective loading of miRNA guide strand is directed by DRB1 (Eamens et al., 2009). DRB1 needs to be dephosphorylated for optimal activity; dephosphorylation is ensured by CPL1 (C-TERMINAL DOMAIN PHOSPHATASE-LIKE 1/FIERY2 (FRY2)) (Manavella et al., 2012). AGO1 miRISC loading also involves cyclophilin40 and HSP90; ATP hydrolysis by HSP helps to release the AGO1-miRNA complex (Earley and Poethig, 2011; Iki et al., 2012; Smith et al., 2009).

Plant miRNAs are mainly loaded onto AGO1, which has an endonuclease activity and is able to suppress gene expression through both target cleavage and translational inhibition (Baumberger and Baulcombe, 2005; Mourrain et al., 2000; Wu et al., 2009). AGO1 shows a preferential loading for miRNAs carrying uridine at their 5 ' end. A change in the 5 ' terminal nucleotide of an miRNA predictably redirects it into a different AGO complex and alters its biological activity (Mi et al., 2008). Remarkably, the DCL1 partnering with DRB1 or DRB2 will determine the mode of action of a loaded miRNA: DRB1 is associated with dicer cleavage while DRB2 with translational repression (Reis et al., 2015b). Interestingly, a subset of miRNAs is only $20 \mathrm{nt}$ long - their length appears to be determined by asymmetric bulges and mismatches at specific positions of the precursor (Lee et al., 2015).

\section{Modes of miRNA action in plant cells}

Similarly to animals, miRNAs loaded on AGO proteins serve as a guide for sequence-specific repression. Similarly to mammalian miRNAs, plant miRNAs can also mediate translational repression and sequence-specific cleavage of cognate mRNAs (e.g. (Beauclair et al., 2010; Brodersen et al., 2008; Li et al., 2014; Mallory and Bouche, 2008; Rhoades et al., 2002). At the same time, activities of plant mRNAs differ from their animal counterparts in several aspects. First, unlike animal miRNAs, many plant miRNAs frequently exhibit 


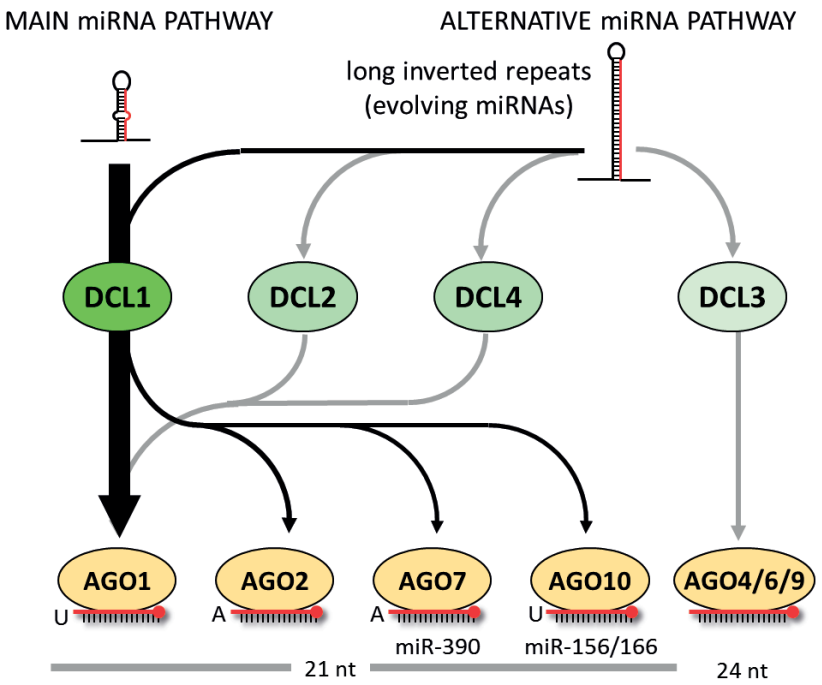

Figure 4 Overview of miRNA biogenesis and AGO loading The scheme summarizes the main and alternative miRNA biogenesis routes in plants; the alternative DCL2/3/4 processing routes are derived from (Axtell et al., 2011).

perfect or nearly perfect complementarity to their substrates resulting in RNAi-like cleavage of their targets (e.g. (Allen et al., 2004; Bowman, 2004; German et al., 2008; Kidner and Martienssen, 2004; Liu et al., 2014; Llave et al., 2002; Mallory et al., 2004; McHale and Koning, 2004; Rhoades et al., 2002; Xie et al., 2003). The high complementarity interactions are also easier to predict and this predictive value is being used for identification of putative plant miRNA targets. (Bonnet et al., 2010; Kumar et al., 2014; Rhoades et al., 2002; Shao et al., 2013). While animal miRNA binding sites are typically localized to 3'UTRs, plant miRNA recognition sites can be found in 5'UTRs, ORFs, or 3'UTRS as well as in non-coding RNAs (e.g. TAS3 RNA (Montgomery et al., 2008)). At the same time, functional features of miRNA binding are still incompletely understood as showed analysis of miR159 sites in MYB33/MYB65 (Li et al., 2014). Importantly, as mentioned above, the mode of miRNA action can be also influenced by a DRB partner during its biogenesis, i.e. independently of the target binding site (Reis et al., 2015b). Finally, plant miRNAs can also mediate transcriptional repression through RNA-dependent DNA methylation. This section will review all three types of miRNA-mediated repression.

\section{miRNA-mediated target cleavage}

miRNA-mediated target cleavage by AGO1 is a functionally important silencing mode as evidenced by the requirement of catalytically active AGO1 in agol mutant complementation experiments (Carbonell et al., 2012). AGO1, guided by a miRNA, cleaves in the middle of the base paired sequence (German et al., 2008; Llave et al., 2002). Similarly to other eukaryotes, exposing a free 5' fragment with a 3' hydroxyl and a 3' fragment with 
a 5' phosphate from a cleaved mRNA leads to decay, which involves a 5'-3' exonuclease (AtXrN4 in Arabidopsis), which attacks the 3' cleaved fragments (Souret et al., 2004). The 5 ' fragments are uridylated at 3 ' ends by HESO1 terminal uridylase; uridylation seem to be coupled with their final demise (Ren et al., 2014).

\section{miRNA-mediated translational inhibition}

Some plant miRNA:mRNA target base pairing could have central mismatches, preventing AGO-mediated cleavage. Other observations also suggest that plant miRNA-target interaction does not always result in AGO-catalyzed slicing but leads to translational repression (Axtell et al., 2006; Brodersen et al., 2008; Franco-Zorrilla et al., 2007; Li et al., 2013; Schwab et al., 2005). The molecular mechanism of miRNA-mediated translational repression in plants is less well understood than in animals. In any case, some similarities emerged. For example, AGO1 localizes to P-bodies, dynamic cytoplasmic foci containing many proteins involved in translational repression and mRNA degradation (Brodersen et al., 2008; Yang et al., 2012).

Another interesting factor, resembling GW182 bridging of target recognition and recruitment of mRNA degrading mechanisms, is SUO, which was identified through a mutation screen for factors contributing to miRNA-mediated repression (Yang et al., 2012). SUO encodes a large protein with N-terminal bromo-adjacent homology and transcription elongation factor S-II domains and, importantly, two C-terminal GW repeats (Yang et al., 2012). The SUO loss-of-function phenotype is a consequence of a defect in miRNA-mediated translational repression and it is reminiscent of plant phenotypes with reduced AGO1 activity (Yang et al., 2012). SUO is present in the nucleus, and co-localizes with DCP1 in the cytoplasm (Yang et al., 2012). An independent study of miRNA-mediated repression showed a functionally important link to decapping through the decapping component VARICOSE (VCS) (Ge-1 homolog), further suggesting that mechanisms underlying miRNA-mediated translational repression in animals and plants are related (Brodersen et al., 2008).

Another line of evidence linking AGO1 and GW-mediated recruitment of downstream repressing factors came from the analysis of viral inhibitors encoded by plant viruses the P1 protein from the Sweet potato mild mottle virus targets AGO1 and inhibits RISC activity through the N-terminal half containing region three WG/GW motifs (Giner et al., 2010).

\section{miRNA-mediated transcriptional silencing/DNA methylation}

In addition to the two usual post-transcriptional modes of action, plant miRNAs can be also plugged into the RNA-dependent DNA methylation mechanism. In this case, miRNA precursors would be processed by DCL3 into longer ( $24 \mathrm{nt})$ species and would be loaded onto AGO4/6/9 system (Axtell et al., 2011). A specific example of such miRNA has been discovered in rice where a class of miRNAs (denoted long miRNA, ImiRNAs) is processed by DCL3, loaded onto AGO4, and directs DNA methylation (Wu et al., 2010) (the molecular mechanism of RNA-dependent DNA methylation is described later). 


\section{Physiological roles of plant miRNAs}

Plant miRNAs have much more "focused" roles than animal miRNAs, in particular mammalian ones. This likely reflects their evolutionary origin, which is connected with their target genes, and the common slicing mode of action (discussed for example in (Svoboda and Cara, 2006)). Briefly, many plant miRNAs seem to originate from inverted repeats, which formed from sequences of their target genes, e.g. through duplication or recombination involving genes and pseudogenes. An interesting aspect of plant miRNA-mediated regulations is the targeting of various transcription factor families, which is translated phenotypic alterations. For example, during miRNA target prediction, of the 49 predicted targets, 34 were members of these transcription factor gene families involved in developmental patterning or cell differentiation (Rhoades et al., 2002). Validated miRNA-targeted transcription factor include the Class III HD-Zip gene family (Bowman, 2004), GRF transcription factors (Debernardi et al., 2014), Scarecrow-like (SCL) family of putative transcription factors (Llave et al., 2002) or MYB33/MYB65 (Li et al., 2014).

Mutants of miRNA factors yielded a whole array of phenotypes suggesting a number of different roles of miRNAs in cell proliferation (Debernardi et al., 2014; Debernardi et al., 2012; Rodriguez et al., 2010), plant development (Abe et al., 2010; Datta and Paul, 2015; Jover-Gil et al., 2012; Schauer et al., 2002), or in response to various physiological conditions, including environmental stress (Huang et al., 2009; Sunkar and Zhu, 2004). One of the notable features of plant miRNAs, which is analogous but distinct from circulating miRNAs in mammals, is that some plant miRNAs can cross cellular boundaries through plasmodesmata to adjacent cells (Marin-Gonzalez and Suarez-Lopez, 2012).

Interestingly, miRNA pathways in Arabidopsis are regulated by a negative feedback loop targeting DCL1 by miR162-guided mRNA cleavage (Xie et al., 2003). An analogous negative feedback loop was observed in Arabidopsis for AGO1, which is targeted by miR168 during development (Vaucheret et al., 2004).

\section{dsRNA-induced post-transcriptional silencing in PTGS \& VIG pathways}

The complexity of RNA silencing in plants comes from multiple layers, which are integrated to provide specific functions of specific small RNA pathway. These layers are (I) structural - e.g. processing of different types of substrates, sorting of small RNAs onto AGO proteins, and molecular effects - endonucleolytic cleavage, translational repression etc. (II) functional/conceptual - e.g. distinguishing between defensive mechanisms and physiological gene regulations, (III) spatiotemporal at multiple levels - including cellular compartmentalization, distinct genomic loci, parts of a plant or its life-cycle, differentiating between somatic and germ cells, leafs, flowering etc. This explains that despite four Dicer proteins, which produce two main classes of small RNAs (21/22 and $24 \mathrm{nt})$, there is over ten different names for AGO-bound small RNAs (Axtell, 2013; Borges and Martienssen, 2015), some of which were already mentioned: miRNA, ImiRNA, hp-siRNA, natsiRNA, cis-natsiRNA, trans-natsiRNA, tasiRNA, phasiRNA, easiRNA, hetsiRNA, diRNAs ... (Fig. 4). 


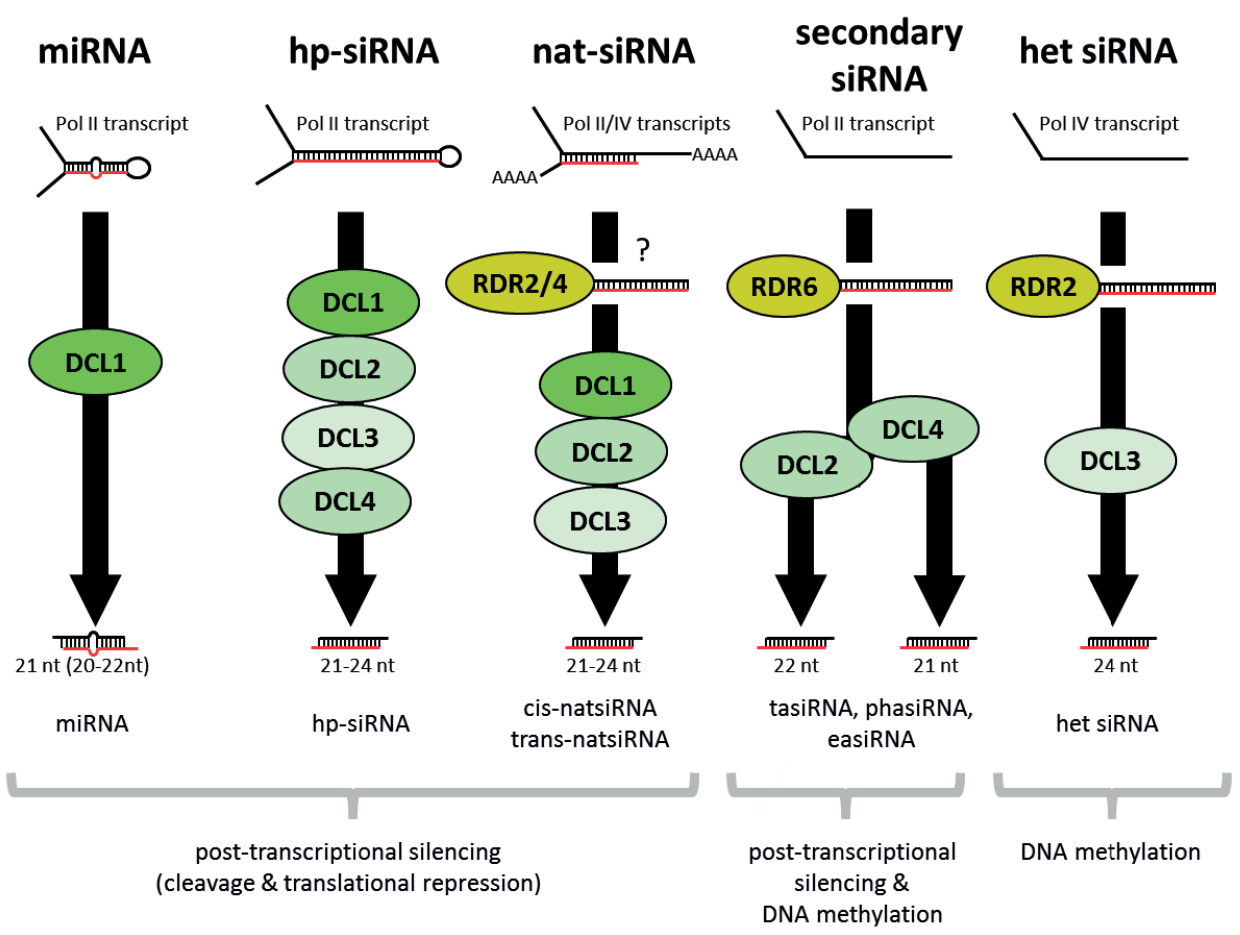

Figure 5 Complexity of small RNAs in plants. The scheme was adapted from (Borges and Martienssen, 2015)

However, while there is aseemingly unpenetrable thicket of substrates, DCLs, AGOs, RDRs, small RNAs, and biological effects, RNA silencing in plants is built from three main mechanistic modules. The three modules are (I) the miRNA module reviewed above, (II) the "RNAi" module, into which can be included dsRNA-induced post-transcriptional silencing in PTGS \& VIG pathways, and the (III) the transcriptional silencing RdDM module, which encompasses $24 \mathrm{nt}$ small RNA-driven de novo DNA methylation and associated chromatin changes and which will be discussed in the next section. This module will thus focus on long dsRNA processing into small RNAs, which is the key step in the dsRNA response.

Many different substrates give a rise to primary and secondary small RNAs that are 21/22 or $24 \mathrm{nt}$ long. Deep sequencing showed that plants have relative to other eukaryotes extraordinarily large and complex populations of small RNAs (Henderson et al., 2006; Howell et al., 2007; Kasschau et al., 2007; Lu et al., 2005; Rajagopalan et al., 2006). More than the half of the small RNAs are $24 \mathrm{nt}$ long "heterochromatic" siRNAs, which map to intergenic regions, particularly to the proximal and distal pericentromeric regions (Rajagopalan et al., 2006). Notably, plant small RNAs are typically methylated at the 2'-hydroxyl group of the 3 ' terminal nucleotide by the methylase HEN1 (Li et al., 2005b; Yu et al., 2005).

Structurally, the three main types of primary substrates for DCL proteins are short, miRNA-like hairpins, long dsRNA hairpins, and long dsRNA (e.g. formed during viral 
replication). In addition, activity of RDRs generates long dsRNA to produce either primary siRNAs (i.e. long dsRNA origin is more or less independent of AGO proteins activity) or secondary siRNAs, where an RDR converts an AGO-targeted transcript into dsRNA. In terms of silencing effects, AGO-loaded small RNAs can induce mRNA cleavage, translational repression, and de novo DNA methylation (RdDM). Biogenesis of small RNAs and the first two modes of action were described above, RdDM will be described in the next section. Thus, the rest of this section will provide an overview of the main routes of dsRNA synthesis and degradation involved in silencing (Fig. 4).

The main Dicer proteins producing small RNAs in the RNAi-like module are DCL2/3/4. All of them participate in antiviral response and in processing various other substrates, which were described above. Importantly, there is a hierarchical and functional separation of the three DCL proteins, such that small RNAs produced by DCL3 can be channelled into the RdDM module. DCL4 and DCL2 act hierarchically on viral and endogenous substrates. DCL4 seems to act earlier while DCL2 later. Both act in a loop involving DRD6, which amplifies the effect (Brosnan et al., 2007; Chen et al., 2011; Deleris et al., 2006; Di Serio et al., 2009; Garcia-Ruiz et al., 2010; Howell et al., 2007; Liu et al., 2007; Mlotshwa et al., 2008; Moissiard et al., 2007; Ogwok et al., 2016; Parent et al., 2015; Qu et al., 2008; Xie et al., 2005).

Small RNAs produced by DCL2/3/4 are sorted onto different AGO proteins, which execute the silencing and, eventually, mark cognate RNAs for RDR6 for amplification. Some of the sorting rules were described above. AGO4/6 accommodate longer $24 \mathrm{nt}$ small RNAs produced by DCL3 and can induce RdDM. Smaller RNAs are loaded onto other AGO proteins depending on the sorting rules and AGO availability - please, refer to the AGO section for more details.

\section{Systemic silencing}

RNAi in higher plants can be non-autonomous (Dunoyer et al., 2005; Himber et al., 2003). It was found that exogenous and endogenous DCL4-dependent $21 \mathrm{nt}$ siRNAs can act as mobile silencing signals between plant cells in a process which likely involves siRNA duplexes rather than loaded AGO1 proteins (Dunoyer et al., 2010). Follow up studies confirmed the core conclusions: graft transmission of endogenous siRNAs inducing silencing (Ali et al., 2013; Liang et al., 2012). The molecular mechanism of systemic RNAi through the vasculature is not completely understood. It seems that small RNAs are transported from cell to cell via plasmodesmata rather than diffusing from their source in the phloem (Liang et al., 2012).

\section{Transcriptional Gene Silencing}

RdDM was actually the first discovered small RNA-controlled epigenetic mechanism. It was originally found in tobacco plants where viroid cDNA, integrated into the genome, became specifically methylated in the presence of autonomous viroid RNA-RNA replication (Wassenegger et al., 1994). It was subsequently shown that as little as $30 \mathrm{bp}$ of targeted 


\section{transgene \& viral silencing}

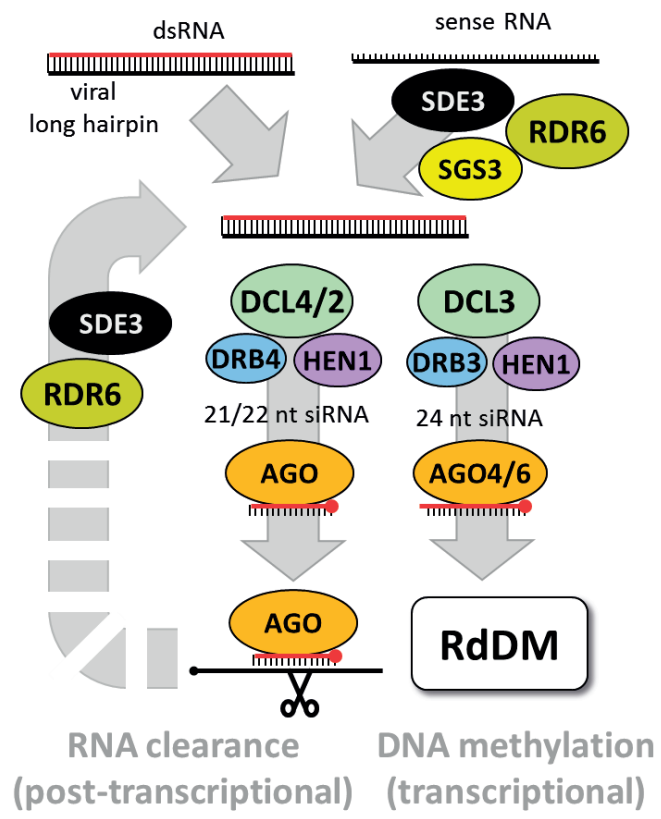

Figure 6 "RNAi module" of RNA silencing in Arabidopsis.

The key step in the RNAi module is conversion of dsRNA into small RNAs by one of the DCL proteins, among which dominate DCL2/3/4. However, DCL1 is also able to produce $21 \mathrm{nt}$ siRNAs from a transgenic inverted repeat (Papp et al., 2003). Various dsRNA substrates can enter the RNAi module. Some of them are produced by RDR6 either as the initial trigger or as an amplification step where AGO-targeted RNAs are converted to dsRNA, which is processed into secondary siRNAs. If the targeting by AGO is precisely defined (e.g. by miRNA), the secondary siRNAs would be phased. DCL3-generated 24 nt siRNAs can induce RdDM.

DNA is sufficient for RdDM and that dsRNA complementary to promoter region can induce promoter methylation and transcriptional silencing (Jones et al., 2001; Mette et al., 2000; Pelissier et al., 1999; Pelissier and Wassenegger, 2000; Thomas et al., 2001).

RdDM not only affects cytosine residues within canonical, symmetrical $\mathrm{CpG}$ dinucleotides, but also $\mathrm{CpNpG}$ and other non-CpG asymmetric targets (Aufsatz et al., 2002a; Pelissier et al., 1999). Since 21-24 nt small RNAs were produced from the original trigger (Mette et al., 2000), RdDM was recognized as one of RNA silencing pathways in plants.

The canonical RdDM pathway (Fig. 7) is initiated by plant-specific RNA polymerase Pol IV that produces single-stranded RNA transcripts from genomic loci to be silenced (Herr et al., 2005; Onodera et al., 2005). RNA transcripts are transported into the nucleolus where they are converted into dsRNA by RNA-dependent RNA polymerase RDR2 and processed by DCL3 into siRNAs, which are then methylated by the methylase HEN1. AGO4, DCL3, RDR2 and 24-nt siRNAs complementary to the heterochromatin regions co-localize in nucleolar processing centers ( $\mathrm{Li}$ et al., 2006; Pontes et al., 2006). Importantly, AGO4 is 
not the only AGO mediating RdDM, RdDM can be induced by AGO4, 6, and 9, which are functionally diverged, largely due to their differential expression (Havecker et al., 2010)

Processing centers are located at a distance from source/target loci and siRNAs trafficking between processing bodies and target regions has to take place. However, mechanisms regulating this process remains unknown at present. Nucleolus-associated so-called Cajal bodies are also centers for AGO1- and DCL1-dependent miRNA processing and are the sites of nonsense-mediated decay indicating closer relationship between RNAi-dependent and other RNA regulating pathways.

Methylated siRNAs associate with AGO4, which interacts with Pol V subunit NRPD1b and the complex moves into the nucleoplasm where it associates with NRPD2a and forms functional Pol V complex. In co-operation with de novo DNA methyltransferase DRM2, and SNF2-like chromatin remodelling protein DEFECTIVE IN RNA-DIRECTED DNA METHYLATION 1 (DRD1), the Pol V complex facilitates de novo DNA methylation of cytosines in all sequence contexts at the targeted locus (Kanno et al., 2005a; Kanno et al., 2005b; Kanno et al., 2004; Li et al., 2006; Pontes et al., 2006).

$\mathrm{CpG}$ methylation in the targeted locus is subsequently maintained during the replication by complexes containing DECREASE IN DNA METHYLATION 1 (DDM1) (Vongs et al., 1993), a maintenance DNA methyltransferase MET1 and histone deacetylase HDA6 (Aufsatz et al., 2004; Aufsatz et al., 2002b; Jones et al., 2001). Methylation at non-CpG nucleotides depends on DNA methyltransferase CHROMOMEHTYLASE3 (CMT3), de novo cytosine methyltransferases DRM1 and DRM2 (DOMAINS REARRANGED METHYLTRANSFERASE), Arabidopsis thaliana homologs of mammalian DNMT3, and a lysine 9 on histone 3 (H3K9) methyltransferase SUVH4/KRYPTONITE (Bartee et al., 2001; Cao and Jacobsen, 2002; Chan et al., 2004; Jackson et al., 2002; Lindroth et al., 2001). Notably, SUVH4 is dispensable for de novo DNA methylation and silencing (Jackson et al., 2002; Malagnac et al., 2002) suggesting that H3K9 methylation in plants does not precede DNA methylation. It has been speculated that the difference in mechanistic relationships between H3K9 methylation and DNA methylation in plants and other model organisms may perhaps reflect dependence and independence of RNA silencing and histone modifications in these models (Matzke and Birchler, 2005).

$\mathrm{RdDM}$ is induced by different types of sequences and has a number of targets. RdDM was studied using two types of dsRNA as inducers of methylation (a) transgenic hairpin constructs (Aufsatz et al., 2002a; Pelissier et al., 1999), and (b) dsRNA viruses (Hall et al., 2002; Vaistij et al., 2002). Notably, these two triggers elicit somewhat different responses. In contrast to the RdDM silencing induced by hairpin constructs, silencing induced by homologous viral transgenes can spread from the region of homology both upstream and downstream (Vaistij et al., 2002). This process depends on RNA-dependent RNA polymerase RDR6, a maintenance methyltransferase MET1, and AGO1 (Jones et al., 2001; Morel et al., 2002). Although histone modifications were not examined in the original paper of Vaistij et al., spreading of silencing resembles RNAi-dependent heterochromatin formation in Schizosaccharomyces pombe (Vaistij et al., 2002). In addition to these exogenous triggers, analysis of endogenous small RNAs and DNA methylation studies revealed that major targets of RdDM are transposons and repeats in constitutive and facultative heterochromatin but not all transposons are repressed by RdDM; only a limited number 


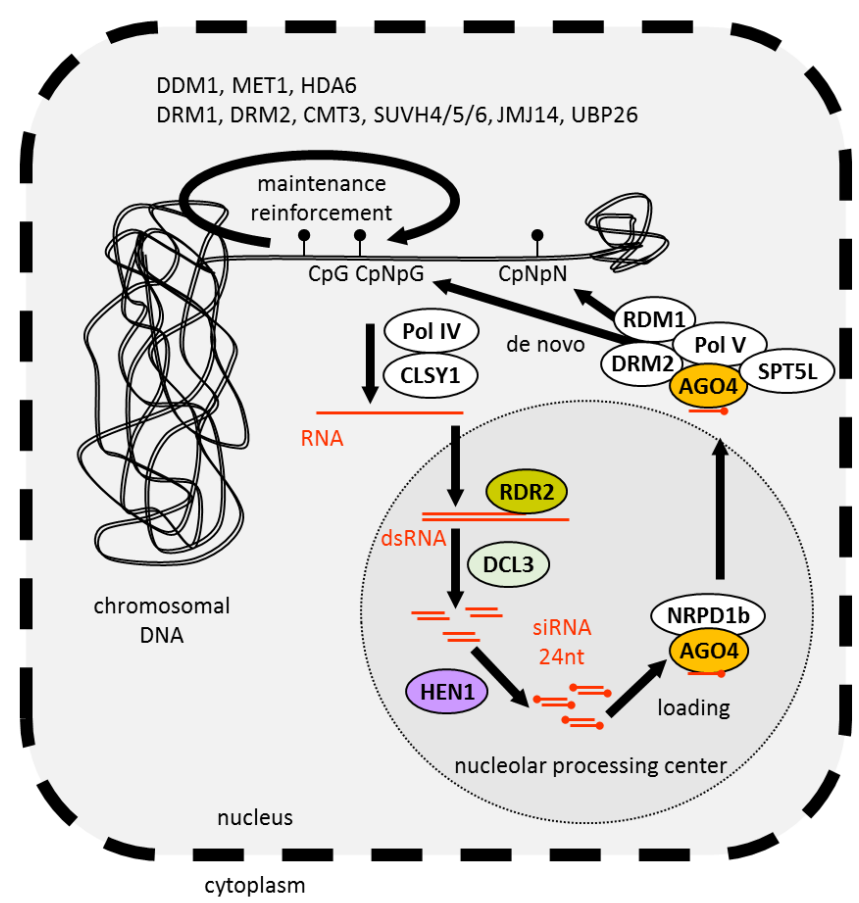

Figure 7 Schematic model of canonical RdDM in A. thaliana

Core components of RNA silencing are colored. Target locus is transcribed by RNA polymerase Pol IV into RNA, which is relocated into a nucleolar processing center where it is converted into dsRNA by RNA-dependent RNA polymerase RDR2 and further processed. Priming of RDR2 may involve cleavage of pol IV transcripts by an AGO protein (AGO4?). dsRNA is cleaved by DCL3 into siRNAs, which are methylated by the methylase HEN1. siRNAs are then loaded onto AGO4, which interacts with Pol $\checkmark$ subunit NRPD1b. The complex moves into the nucleoplasm and forms functional Pol V complex, which, in co-operation with de novo DNA methyltransferase DRM2, and SNF2-like chromatin remodelling protein DRD1, facilitates in a sequence-specific manner de novo DNA methylation of cytosines (black circles on a stalk) in all sequence contexts at homologous loci. Recognition of a target locus probably occurs via binding to an RNA from the silenced locus. CpG methylation is maintained during the replication by complexes containing DDM1, a maintenance DNA methyltransferase MET1, and histone deacetylase HDA6. Methylation at non-CpG nucleotides is dependent on DNA methyltransferase CMT3, de novo cytosine methyltransferases DRM1 and DRM2, and a H3K9 methyltransferase SUVH4/KRYPTONITE.

of targets of RdDM are endogenous genes (Borges and Martienssen, 2015). As RdDM can be reversed by demethylation (Penterman et al., 2007), a picture emerges in which RdDM is not only a repressive mechanism controlling repetitive and viral sequences but also a part of regulatory networks controlling gene expression, which includes other chromatin modifications.

In addition, there is a second RdDM pathway in flowering plants, designated non-canonical RdDM (Matzke et al., 2015), which is initiated by pol II transcripts, which are channeled through DCL3 and AGO4/6 in the "RNAi module" into RdDM (Fig. 8). 


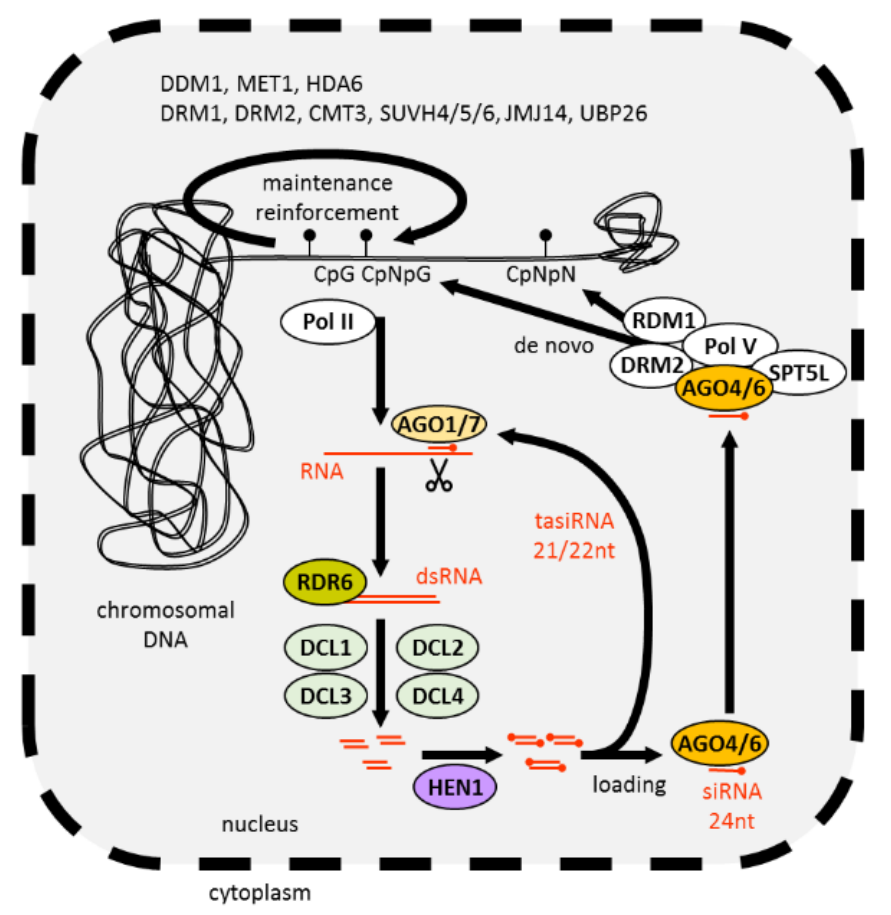

Figure 8 Schematic model of non-canonical RdDM in $A$. thaliana

The target/trigger locus is transcribed by RNA polymerase II into RNA, which is recognized by an AGO-loaded small RNA, is converted into dsRNA by RNA-dependent RNA polymerase RDR6, and it is processed by one of DCL proteins where AGO4/6 bound small RNAs can enter RdDM module, 21/22 nt small RNAs can operate within the RNAi module and amplify the silencing response.

\section{Acknowledgement}

I would like to thank my colleagues Jan Paces, Miloslav Nic, and Tomas Novotny for help with collecting literature for the review. The review content was produced under a contract OC/EFSA/GMO/2015/01-CT 01 with European Food Safety Authority (EFSA); the opinions expressed are those of the contractor only and do not represent EFSA's official position. Publication of the review was funded by LO1220 and LM2015063 by the Ministry of Education, Youth and Sports.

\section{References}

Abe, M., Yoshikawa, T., Nosaka, M., Sakakibara, H., Sato, Y., Nagato, Y., and Itoh, J. (2010). WAVY LEAF1, an Ortholog of Arabidopsis HEN1, Regulates Shoot Development by Maintaining MicroRNA and Trans-Acting Small Interfering RNA Accumulation in Rice. Plant Physiology $154,1335-1346$. 
Agorio, A., and Vera, P. (2007). ARGONAUTE4 is required for resistance to Pseudomonas syringae in Arabidopsis. Plant Cell 19, 3778-3790.

Akbergenov, R., Si-Ammour, A., Blevins, T., Amin, I., Kutter, C., Vanderschuren, H., Zhang, P., Gruissem, W., Meins, F., Hohn, T., et al. (2006). Molecular characterization of geminivirus-derived small RNAs in different plant species. Nucleic Acids Research 34, 462-471.

Ali, E., Kobayashi, K., Yamaoka, N., Ishikawa, M., and Nishiguchi, M. (2013). Graft transmission of RNA silencing to non-transgenic scions for conferring virus resistance in tobacco. Plos One 8 , e63257.

Allen, E., Xie, Z., Gustafson, A.M., Sung, G.H., Spatafora, J.W., and Carrington, J.C. (2004). Evolution of microRNA genes by inverted duplication of target gene sequences in Arabidopsis thaliana. Nature Genetics 36, 1282-1290.

Aregger, M., Borah, B.K., Seguin, J., Rajeswaran, R., Gubaeva, E.G., Zvereva, A.S., Windels, D., Vazquez, F., Blevins, T., Farinelli, L., et al. (2012). Primary and Secondary siRNAs in Geminivirus-induced Gene Silencing. Plos Pathogens 8, e1002941-e1002941.

Aufsatz, W., Mette, M.F., Matzke, A.J., and Matzke, M. (2004). The role of MET1 in RNA-directed de novo and maintenance methylation of CG dinucleotides. Plant Molecular Biology 54, 793-804.

Aufsatz, W., Mette, M.F., van der Winden, J., Matzke, A.J., and Matzke, M. (2002a). RNA-directed DNA methylation in Arabidopsis. Proc Natl Acad Sci U S A 99 Suppl 4, 16499-16506.

Aufsatz, W., Mette, M.F., van der Winden, J., Matzke, M., and Matzke, A.J. (2002b). HDA6, a putative histone deacetylase needed to enhance DNA methylation induced by double-stranded RNA. The EMBO journal 21, 6832-6841.

Axtell, M.J. (2013). Classification and comparison of small RNAs from plants. Annual review of plant biology 64, 137-159.

Axtell, M.J., Jan, C., Rajagopalan, R., and Bartel, D.P. (2006). A two-hit trigger for siRNA biogenesis in plants. Cell 127, 565-577.

Axtell, M.J., Westholm, J.O., and Lai, E.C. (2011). Vive la difference: biogenesis and evolution of microRNAs in plants and animals. Genome Biology 12, 221.

Bai, M., Yang, G.-S., Chen, W.-T., Mao, Z.-C., Kang, H.-X., Chen, G.-H., Yang, Y.-H., and Xie, B.-Y. (2012). Genome-wide identification of Dicer-like, Argonaute and RNA-dependent RNA polymerase gene families and their expression analyses in response to viral infection and abiotic stresses in Solanum lycopersicum. Gene 501, 52-62.

Baranauske, S., Mickute, M., Plotnikova, A., Finke, A., Venclovas, C., Klimasauskas, S., and Vilkaitis, G. (2015). Functional mapping of the plant small RNA methyltransferase: HEN1 physically interacts with HYL1 and DICER-LIKE 1 proteins. Nucleic Acids Research 43, 2802-2812.

Bartee, L., Malagnac, F., and Bender, J. (2001). Arabidopsis cmt3 chromomethylase mutations block non-CG methylation and silencing of an endogenous gene. Genes Dev 15, 1753-1758.

Baumberger, N., and Baulcombe, D.C. (2005). Arabidopsis ARGONAUTE1 is an RNA Slicer that selectively recruits microRNAs and short interfering RNAs. Proc Natl Acad Sci U S A 102, 11928-11933.

Beauclair, L., Yu, A., and Bouche, N. (2010). microRNA-directed cleavage and translational repression of the copper chaperone for superoxide dismutase mRNA in Arabidopsis. Plant Journal 62, 454-462.

Beclin, C., Boutet, S., Waterhouse, P., and Vaucheret, H. (2002). A branched pathway for transgene-induced RNA silencing in plants. Current Biology 12, 684-688. 
Blevins, T., Rajeswaran, R., Aregger, M., Borah, B.K., Schepetilnikov, M., Baerlocher, L., Farinelli, L., Meins, F., Hohn, T., and Pooggin, M.M. (2011). Massive production of small RNAs from a non-coding region of Cauliflower mosaic virus in plant defense and viral counter-defense. Nucleic Acids Research 39, 5003-5014.

Bohmert, K., Camus, I., Bellini, C., Bouchez, D., Caboche, M., and Benning, C. (1998). AGO1 defines a novel locus of Arabidopsis controlling leaf development. The EMBO journal 17, 170-180.

Bologna, N.G., Mateos, J.L., Bresso, E.G., and Palatnik, J.F. (2009). A loop-to-base processing mechanism underlies the biogenesis of plant microRNAs miR319 and miR159. The EMBO journal 28, 3646-3656.

Bologna, N.G., and Voinnet, O. (2014). The Diversity, Biogenesis, and Activities of Endogenous Silencing Small RNAs in Arabidopsis. In Annual Review of Plant Biology, Vol 65, pp. 473-503.

Bonnet, E., He, Y., Billiau, K., and Van de Peer, Y. (2010). TAPIR, a web server for the prediction of plant microRNA targets, including target mimics. Bioinformatics 26, 1566-1568.

Bonnet, E., Van de Peer, Y., and Rouze, P. (2006). The small RNA world of plants. New Phytologist $171,451-468$.

Borges, F., and Martienssen, R.A. (2015). The expanding world of small RNAs in plants. Nature Reviews Molecular Cell Biology 16, 727-741.

Bouche, N., Lauressergues, D., Gasciolli, V., and Vaucheret, H. (2006). An antagonistic function for Arabidopsis DCL2 in development and a new function for DCL4 in generating viral siRNAs. The EMBO journal 25, 3347-3356.

Bowman, J.L. (2004). Class III HD-Zip gene regulation, the golden fleece of ARGONAUTE activity? Bioessays 26, 938-942.

Boyko, A., and Kovalchuk, I. (2010). Transgenerational response to stress in Arabidopsis thaliana. Plant Signaling and Behavior 5, 995-998.

Bozorov, T.A., Pandey, S.P., Dinh, S.T., Kim, S.G., Heinrich, M., Gase, K., and Baldwin, I.T. (2012). DICER-like Proteins and Their Role in Plant-herbivore Interactions in Nicotiana attenuata. Journal of Integrative Plant Biology 54, 189-206.

Brandt, R., Xie, Y.K., Musielak, T., Graeff, M., Stierhof, Y.D., Huang, H., Liu, C.M., and Wenkel, S. (2013). Control of stem cell homeostasis via interlocking microRNA and microProtein feedback loops. Mechanisms of Development 130, 25-33.

Brodersen, P., Sakvarelidze-Achard, L., Bruun-Rasmussen, M., Dunoyer, P., Yamamoto, Y.Y., Sieburth, L., and Voinnet, O. (2008). Widespread translational inhibition by plant miRNAs and siRNAs. Science 320, 1185-1190.

Brosnan, C.A., Mitter, N., Christie, M., Smith, N.A., Waterhouse, P.M., and Carroll, B.J. (2007). Nuclear gene silencing directs reception of long-distance mRNA silencing in Arabidopsis. Proc Natl Acad Sci U S A 104, 14741-14746.

Brosseau, C., and Moffett, P. (2015). Functional and Genetic Analysis Identify a Role for Arabidopsis ARGONAUTE5 in Antiviral RNA Silencing. Plant Cell 27, 1742-1754.

Cao, M.J., Du, P., Wang, X.B., Yu, Y.Q., Qiu, Y.H., Li, W.X., Gal-On, A., Zhou, C.Y., Li, Y., and Ding, S.W. (2014). Virus infection triggers widespread silencing of host genes by a distinct class of endogenous siRNAs in Arabidopsis. Proc Natl Acad Sci U S A 111, 14613-14618.

Cao, X., and Jacobsen, S.E. (2002). Role of the arabidopsis DRM methyltransferases in de novo DNA methylation and gene silencing. Current Biology 12, 1138-1144. 
Carbonell, A., and Carrington, J.C. (2015). Antiviral roles of plant ARGONAUTES. Current Opinion in Plant Biology 27, 111-117.

Carbonell, A., Fahlgren, N., Garcia-Ruiz, H., Gilbert, K.B., Montgomery, T.A., Nguyen, T., Cuperus, J.T., and Carrington, J.C. (2012). Functional Analysis of Three Arabidopsis ARGONAUTES Using Slicer-Defective Mutants. Plant Cell 24, 3613-3629.

Chan, S.W., Zilberman, D., Xie, Z., Johansen, L.K., Carrington, J.C., and Jacobsen, S.E. (2004). RNA silencing genes control de novo DNA methylation. Science 303, 1336.

Chellappan, P., Xia, J., Zhou, X.F., Gao, S., Zhang, X.M., Coutino, G., Vazquez, F., Zhang, W.X., and Jin, H.L. (2010). siRNAs from miRNA sites mediate DNA methylation of target genes. Nucleic Acids Research 38, 6883-6894.

Chen, D.J., Meng, Y.J., Yuan, C.H., Bai, L., Huang, D.L., Lv, S.L., Wu, P., Chen, L.L., and Chen, M. (2011). Plant siRNAs from introns mediate DNA methylation of host genes. RNA 17, 1012-1024.

Chen, X.M. (2009). Small RNAs and Their Roles in Plant Development. In Annual Review of Cell and Developmental Biology, pp. 21-44.

Clavel, M., Pelissier, T., Descombin, J., Jean, V., Picart, C., Charbonel, C., Saez-Vasquez, J., Bousquet-Antonelli, C., and Deragon, J.-M. (2015). Parallel action of AtDRB2 and RdDM in the control of transposable element expression. BMC Plant Biology 15, 455-455.

Clavel, M., Pelissier, T., Montavon, T., Tschopp, M.A., Pouch-Pelissier, M.N., Descombin, J., Jean, V., Dunoyer, P., Bousquet-Antonelli, C., and Deragon, J.M. (2016). Evolutionary history of double-stranded RNA binding proteins in plants: identification of new cofactors involved in easiRNA biogenesis. Plant Molecular Biology 91, 131-147.

Coruh, C., Cho, S.H., Shahid, S., Liu, Q.K., Wierzbicki, A., and Axtella, M.J. (2015). Comprehensive Annotation of Physcomitrella patens Small RNA Loci Reveals That the Heterochromatic Short Interfering RNA Pathway Is Largely Conserved in Land Plants. Plant Cell 27, 2148-2162.

Curtin, S.J., Kantar, M.B., Yoon, H.W., Whaley, A.M., Schlueter, J.A., and Stupar, R.M. (2012). Co-expression of soybean Dicer-like genes in response to stress and development. Functional \& Integrative Genomics 12, 671-682.

Curtin, S.J., Watson, J.M., Smith, N.A., Eamens, A.L., Blanchard, C.L., and Waterhouse, P.M. (2008). The roles of plant dsRNA-binding proteins in RNAi-like pathways. FEBS Letters 582, 2753-2760.

Dalmay, T., Hamilton, A., Rudd, S., Angell, S., and Baulcombe, D.C. (2000). An RNA-dependent RNA polymerase gene in Arabidopsis is required for posttranscriptional gene silencing mediated by a transgene but not by a virus. Cell 101, 543-553.

Datta, R., and Paul, S. (2015). Plant microRNAs: master regulator of gene expression mechanism. Cell Biology International 39, 1185-1190.

Daxinger, L., Kanno, T., Bucher, E., van der Winden, J., Naumann, U., Matzke, A.J.M., and Matzke, M. (2009). A stepwise pathway for biogenesis of 24-nt secondary siRNAs and spreading of DNA methylation. The EMBO journal 28, 48-57.

Debernardi, J.M., Mecchia, M.A., Vercruyssen, L., Smaczniak, C., Kaufmann, K., Inze, D., Rodriguez, R.E., and Palatnik, J.F. (2014). Post-transcriptional control of GRF transcription factors by microRNA miR396 and GIF co-activator affects leaf size and longevity. Plant Journal 79, 413-426.

Debernardi, J.M., Rodriguez, R.E., Mecchia, M.A., and Palatnik, J.F. (2012). Functional specialization of the plant miR396 regulatory network through distinct microRNA-target interactions. Plos Genetics 8, e1002419. 
Deleris, A., Gallego-Bartolome, J., Bao, J.S., Kasschau, K.D., Carrington, J.C., and Voinnet, O. (2006). Hierarchical action and inhibition of plant Dicer-like proteins in antiviral defense. Science 313, 68-71.

Di Serio, F., Gisel, A., Navarro, B., Delgado, S., de Alba, A.-E.M., Donvito, G., and Flores, R. (2009). Deep Sequencing of the Small RNAs Derived from Two Symptomatic Variants of a Chloroplastic Viroid: Implications for Their Genesis and for Pathogenesis. Plos One 4, e7539-e7539.

Diaz-Pendon, J.A., Li, F., Li, W.X., and Ding, S.W. (2007). Suppression of antiviral silencing by cucumber mosaic virus $2 \mathrm{~b}$ protein in Arabidopsis is associated with drastically reduced accumulation of three classes of viral small interfering RNAs. Plant Cell 19, 2053-2063.

Dolgosheina, E.V., Morin, R.D., Aksay, G., Sahinalp, S.C., Magrini, V., Mardis, E.R., Mattsson, J., and Unrau, P.J. (2008). Conifers have a unique small RNA silencing signature. RNA 14, 1508-1515.

Donaire, L., Barajas, D., Martinez-Garcia, B., Martinez-Priego, L., Pagan, I., and Llave, C. (2008). Structural and genetic requirements for the biogenesis of tobacco rattle virus-derived small interfering RNAs. Journal of Virology 82, 5167-5177.

Douglas, R.N., Wiley, D., Sarkar, A., Springer, N., Timmermans, M.C.P., and Scanlon, M.J. (2010). ragged seedling2 Encodes an ARGONAUTE7-Like Protein Required for Mediolateral Expansion, but Not Dorsiventrality, of Maize Leaves. Plant Cell 22, 1441-1451.

Dunoyer, P., Himber, C., and Voinnet, O. (2005). DICER-LIKE 4 is required for RNA interference and produces the 21 -nucleotide small interfering RNA component of the plant cell-to-cell silencing signal. Nature Genetics 37, 1356-1360.

Dunoyer, P., Schott, G., Himber, C., Meyer, D., Takeda, A., Carrington, J.C., and Voinnet, O. (2010). Small RNA Duplexes Function as Mobile Silencing Signals Between Plant Cells. Science 328, 912-916.

Eamens, A.L., Kim, K.W., Curtin, S.J., and Waterhouse, P.M. (2012a). DRB2 Is Required for MicroRNA Biogenesis in Arabidopsis thaliana. Plos One 7, e35933-e35933.

Eamens, A.L., Smith, N.A., Curtin, S.J., Wang, M.B., and Waterhouse, P.M. (2009). The Arabidopsis thaliana double-stranded RNA binding protein DRB1 directs guide strand selection from microRNA duplexes. RNA 15, 2219-2235.

Eamens, A.L., Wook Kim, K., and Waterhouse, P.M. (2012b). DRB2, DRB3 and DRB5 function in a non-canonical microRNA pathway in Arabidopsis thaliana. Plant Signaling and Behavior 7, 1224-1229.

Earley, K.W., and Poethig, R.S. (2011). Binding of the cyclophilin 40 ortholog SQUINT to Hsp90 protein is required for SQUINT function in Arabidopsis. Journal of Biological Chemistry 286, 38184-38189.

Eun, C., Lorkovic, Z.J., Naumann, U., Long, Q., Havecker, E.R., Simon, S.A., Meyers, B.C., Matzke, A.J.M., and Matzke, M. (2011). AGO6 Functions in RNA-Mediated Transcriptional Gene Silencing in Shoot and Root Meristems in Arabidopsis thaliana. Plos One 6, e25730-e25730.

Fang, Y., and Spector, D.L. (2007). Identification of nuclear dicing bodies containing proteins for microRNA biogenesis in living Arabidopsis plants. Current Biology 17, 818-823.

Fatyol, K., Ludman, M., and Burgyan, J. (2016). Functional dissection of a plant Argonaute. Nucleic Acids Research 44, 1384-1397.

Finnegan, E.J., Margis, R., and Waterhouse, P.M. (2003). Posttranscriptional gene silencing is not compromised in the Arabidopsis CARPEL FACTORY (DICER-LIKE1) mutant, a homolog of dicer-1 from Drosophila. Current Biology 13, 236-240. 
Franco-Zorrilla, J.M., Valli, A., Todesco, M., Mateos, I., Puga, M.I., Rubio-Somoza, I., Leyva, A., Weigel, D., Garcia, J.A., and Paz-Ares, J. (2007). Target mimicry provides a new mechanism for regulation of microRNA activity. Nature Genetics 39, 1033-1037.

Fusaro, A.F., Matthew, L., Smith, N.A., Curtin, S.J., Dedic-Hagan, J., Ellacott, G.A., Watson, J.M., Wang, M.B., Brosnan, C., Carroll, B.J., et al. (2006). RNA interference-inducing hairpin RNAs in plants act through the viral defence pathway. EMBO Rep 7, 1168-1175.

Galun, E. (2005). RNA silencing in plants. In Vitro Cellular \& Developmental Biology-Plant 41, $113-123$.

Garcia-Ruiz, H., Takeda, A., Chapman, E.J., Sullivan, C.M., Fahlgren, N., Brempelis, K.J., and Carrington, J.C. (2010). Arabidopsis RNA-Dependent RNA Polymerases and Dicer-Like Proteins in Antiviral Defense and Small Interfering RNA Biogenesis during Turnip Mosaic Virus Infection. Plant Cell 22, 481-496.

Garcia, D., Collier, S.A., Byrne, M.E., and Martienssen, R.A. (2006). Specification of leaf polarity in Arabidopsis via the trans-acting siRNA pathway. Current Biology 16, 933-938.

German, M.A., Pillay, M., Jeong, D.H., Hetawal, A., Luo, S., Janardhanan, P., Kannan, V., Rymarquis, L.A., Nobuta, K., German, R., et al. (2008). Global identification of microRNA-target RNA pairs by parallel analysis of RNA ends. Nature Biotechnology 26, 941-946.

Giner, A., Lakatos, L., Garcia-Chapa, M., Lopez-Moya, J.J., and Burgyan, J. (2010). Viral Protein Inhibits RISC Activity by Argonaute Binding through Conserved WG/GW Motifs. Plos Pathogens 6, 1-13.

Guo, X.Y., Zhang, Z.L., Gerstein, M.B., and Zheng, D.Y. (2009). Small RNAs Originated from Pseudogenes: cis- or trans-Acting? Plos Computational Biology 5, e1000449-e1000449.

Hall, I.M., Shankaranarayana, G.D., Noma, K., Ayoub, N., Cohen, A., and Grewal, S.I. (2002). Establishment and maintenance of a heterochromatin domain. Science 297, 2232-2237.

Havecker, E.R., Wallbridge, L.M., Hardcastle, T.J., Bush, M.S., Kelly, K.A., Dunn, R.M., Schwach, F., Doonan, J.H., and Baulcombe, D.C. (2010). The Arabidopsis RNA-Directed DNA Methylation Argonautes Functionally Diverge Based on Their Expression and Interaction with Target Loci. Plant Cell 22, 321-334.

He, X.J., Hsu, Y.F., Zhu, S.H., Wierzbicki, A.T., Pontes, O., Pikaard, C.S., Liu, H.L., Wang, C.S., Jin, H.L., and Zhu, J.K. (2009). An Effector of RNA-Directed DNA Methylation in Arabidopsis Is an ARGONAUTE 4-and RNA-Binding Protein. Cell 137, 498-508.

Henderson, I.R., Zhang, X., Lu, C., Johnson, L., Meyers, B.C., Green, P.J., and Jacobsen, S.E. (2006). Dissecting Arabidopsis thaliana DICER function in small RNA processing, gene silencing and DNA methylation patterning. Nature Genetics 38, 721-725.

Herr, A.J., Jensen, M.B., Dalmay, T., and Baulcombe, D.C. (2005). RNA polymerase IV directs silencing of endogenous DNA. Science 308, 118-120.

Himber, C., Dunoyer, P., Moissiard, G., Ritzenthaler, C., and Voinnet, O. (2003). Transitivity-dependent and -independent cell-to-cell movement of RNA silencing. The EMBO journal 22, 4523-4533.

Horwich, M.D., Li, C.J., Matranga, C., Vagin, V., Farley, G., Wang, P., and Zamore, P.D. (2007). The Drosophila RNA methyltransferase, DmHen1, modifies germline piRNAs and single-stranded siRNAs in RISC. Current Biology 17, 1265-1272.

Howell, M.D., Fahlgren, N., Chapman, E.J., Cumbie, J.S., Sullivan, C.M., Givan, S.A., Kasschau, K.D., and Carrington, J.C. (2007). Genome-wide analysis of the RNA-DEPENDENT RNA POLYMERASE6/DICER-LIKE4 pathway in Arabidopsis reveals dependency on miRNA- and tasiRNA-directed targeting. Plant Cell 19, 926-942. 
Huang, S.Q., Peng, J., Qiu, C.X., and Yang, Z.M. (2009). Heavy metal-regulated new microRNAs from rice. Journal of inorganic biochemistry 103, 282-287.

Hunter, C., Sun, H., and Poethig, R.S. (2003). The Arabidopsis heterochronic gene ZIPPY is an ARGONAUTE family member. Current Biology 13, 1734-1739.

Iki, T., Yoshikawa, M., Meshi, T., and Ishikawa, M. (2012). Cyclophilin 40 facilitates HSP90-mediated RISC assembly in plants. The EMBO journal 31, 267-278.

Jackson, J.P., Lindroth, A.M., Cao, X., and Jacobsen, S.E. (2002). Control of CpNpG DNA methylation by the KRYPTONITE histone H3 methyltransferase. Nature 416, 556-560.

Jakubiec, A., Yang, S.W., and Chua, N.H. (2012). Arabidopsis DRB4 protein in antiviral defense against Turnip yellow mosaic virus infection. Plant Journal 69, 14-25.

Jaskiewicz, L., and Filipowicz, W. (2008). Role of Dicer in posttranscriptional RNA silencing. Current topics in microbiology and immunology 320, 77-97.

Jaubert, M., Bhattacharjee, S., Mello, A.F.S., Perry, K.L., and Moffett, P. (2011). ARGONAUTE2 Mediates RNA-Silencing Antiviral Defenses against Potato virus X in Arabidopsis. Plant Physiology 156, 1556-1564.

Jeong, D.H., Thatcher, S.R., Brown, R.S.H., Zhai, J.X., Park, S., Rymarquis, L.A., Meyers, B.C., and Green, P.J. (2013). Comprehensive Investigation of MicroRNAs Enhanced by Analysis of Sequence Variants, Expression Patterns, ARGONAUTE Loading, and Target Cleavage. Plant Physiology 162, 1225-1245.

Ji, L.J., Liu, X.G., Yan, J., Wang, W.M., Yumul, R.E., Kim, Y.J., Dinh, T.T., Liu, J., Cui, X., Zheng, B.L., et al. (2011). ARGONAUTE10 and ARGONAUTE1 Regulate the Termination of Floral Stem Cells through Two MicroRNAs in Arabidopsis. Plos Genetics 7, e1001358-e1001358.

Jones, L., Keining, T., Eamens, A., and Vaistij, F.E. (2006). Virus-induced gene silencing of Argonaute genes in Nicotiana benthamiana demonstrates that extensive systemic silencing requires Argonaute1-like and Argonaute4-like genes. Plant Physiology 141, 598-606.

Jones, L., Ratcliff, F., and Baulcombe, D.C. (2001). RNA-directed transcriptional gene silencing in plants can be inherited independently of the RNA trigger and requires Met1 for maintenance. Current Biology 11, 747-757.

Jover-Gil, S., Candela, H., Robles, P., Aguilera, V., Barrero, J.M., Micol, J.L., and Ponce, M.R. (2012). The MicroRNA Pathway Genes AGO1, HEN1 and HYL1 Participate in Leaf Proximal-Distal, Venation and Stomatal Patterning in Arabidopsis. Plant and Cell Physiology 53, 1322-1333.

Kanno, T., Aufsatz, W., Jaligot, E., Mette, M.F., Matzke, M., and Matzke, A.J. (2005a). A SNF2-like protein facilitates dynamic control of DNA methylation. EMBO Rep 6, 649-655.

Kanno, T., Huettel, B., Mette, M.F., Aufsatz, W., Jaligot, E., Daxinger, L., Kreil, D.P., Matzke, M., and Matzke, A.J. (2005b). Atypical RNA polymerase subunits required for RNA-directed DNA methylation. Nature Genetics 37, 761-765.

Kanno, T., Mette, M.F., Kreil, D.P., Aufsatz, W., Matzke, M., and Matzke, A.J. (2004). Involvement of putative SNF2 chromatin remodeling protein DRD1 in RNA-directed DNA methylation. Current Biology 14, 801-805.

Kapoor, M., Arora, R., Lama, T., Nijhawan, A., Khurana, J.P., Tyagi, A.K., and Kapoor, S. (2008). Genome-wide identification, organization and phylogenetic analysis of Dicer-like, Argonaute and RNA-dependent RNA Polymerase gene families and their expression analysis during reproductive development and stress in rice. BMC Genomics 9, 451-451. 
Kasschau, K.D., Fahlgren, N., Chapman, E.J., Sullivan, C.M., Cumbie, J.S., Givan, S.A., and Carrington, J.C. (2007). Genome-wide profiling and analysis of Arabidopsis siRNAs. Plos Biology 5, e57.

Kidner, C.A., and Martienssen, R.A. (2004). Spatially restricted microRNA directs leaf polarity through ARGONAUTE1. Nature 428, 81-84.

Komiya, R., Ohyanagi, H., Niihama, M., Watanabe, T., Nakano, M., Kurata, N., and Nonomura, K. (2014). Rice germline-specific Argonaute MEL1 protein binds to phasiRNAs generated from more than 700 lincRNAs. Plant Journal 78, 385-397.

Kozomara, A., and Griffiths-Jones, S. (2014). miRBase: annotating high confidence microRNAs using deep sequencing data. Nucleic Acids Res 42, D68-73.

Kravchik, M., Damodharan, S., Stav, R., and Arazi, T. (2014a). Generation and characterization of a tomato DCL3-silencing mutant. Plant Science 221, 81-89.

Kravchik, M., Sunkar, R., Damodharan, S., Stav, R., Zohar, M., Isaacson, T., and Arazi, T. (2014b). Global and local perturbation of the tomato microRNA pathway by a trans-activated DICER-LIKE 1 mutant. Journal of Experimental Botany 65, 725-739.

Kumar, S.P., Pandya, H.A., and Jasrai, Y.T. (2014). A computational model for non-conserved mature miRNAs from the rice genome. Sar and Qsar in Environmental Research 25, 205-220.

Lee, W.C., Lu, S.H., Lu, M.H., Yang, C.J., Wu, S.H., and Chen, H.M. (2015). Asymmetric bulges and mismatches determine 20-nt microRNA formation in plants. RNA Biology 12, 1054-1066.

Leibman, D., Wolf, D., Saharan, V., Zelcer, A., Arazi, T., Yoel, S., Gaba, V., and Gal-On, A. (2011). A High Level of Transgenic Viral Small RNA Is Associated with Broad Potyvirus Resistance in Cucurbits. Molecular Plant-Microbe Interactions 24, 1220-1238.

Li, C.F., Pontes, O., El-Shami, M., Henderson, I.R., Bernatavichute, Y.V., Chan, S.W., Lagrange, T., Pikaard, C.S., and Jacobsen, S.E. (2006). An ARGONAUTE4-containing nuclear processing center colocalized with Cajal bodies in Arabidopsis thaliana. Cell 126, 93-106.

Li, H., Xu, L., Wang, H., Yuan, Z., Cao, X.F., Yang, Z.N., Zhang, D.B., Xu, Y.Q., and Huang, H. (2005a). The putative RNA-dependent RNA polymerase RDR6 acts synergistically with ASYMMETRIC LEAVES1 and 2 to repress BREVIPEDICELLUS and MicroRNA165/166 in Arabidopsis leaf development. Plant Cell 17, 2157-2171.

Li, J., Reichel, M., and Millar, A.A. (2014). Determinants beyond both complementarity and cleavage govern microR159 efficacy in Arabidopsis. Plos Genetics 10, e1004232.

Li, J., Yang, Z., Yu, B., Liu, J., and Chen, X. (2005b). Methylation protects miRNAs and siRNAs from a 3'-end uridylation activity in Arabidopsis. Current Biology 15, 1501-1507.

Li, S.B., Liu, L., Zhuang, X.H., Yu, Y., Liu, X.G., Cui, X., Ji, L.J., Pan, Z.Q., Cao, X.F., Mo, B.X., et al. (2013). MicroRNAs Inhibit the Translation of Target mRNAs on the Endoplasmic Reticulum in Arabidopsis. Cell 153, 562-574.

Liang, D., White, R.G., and Waterhouse, P.M. (2012). Gene silencing in Arabidopsis spreads from the root to the shoot, through a gating barrier, by template-dependent, nonvascular, cell-to-cell movement. Plant Physiology 159, 984-1000.

Lin, D., Xiang, Y., Xian, Z., and Li, Z. (2016). Ectopic expression of SlAGO7 alters leaf pattern and inflorescence architecture and increases fruit yield in tomato. Physiologia plantarum.

Lindroth, A.M., Cao, X., Jackson, J.P., Zilberman, D., McCallum, C.M., Henikoff, S., and Jacobsen, S.E. (2001). Requirement of CHROMOMETHYLASE3 for maintenance of CpXpG methylation. Science 292, 2077-2080. 
Liu, B., Chen, Z.Y., Song, X.W., Liu, C.Y., Cui, X., Zhao, X.F., Fang, J., Xu, W.Y., Zhang, H.Y., Wang, X.J., et al. (2007). Oryza sativa dicer-like4 reveals a key role for small interfering RNA silencing in plant development. Plant Cell 19, 2705-2718.

Liu, Q., Wang, F., and Axtell, M.J. (2014). Analysis of complementarity requirements for plant microRNA targeting using a Nicotiana benthamiana quantitative transient assay. Plant Cell 26, 741-753.

Liu, Q.L., Yao, X.Z., Pi, L.M., Wang, H., Cui, X.F., and Huang, H. (2009a). The ARGONAUTE10 gene modulates shoot apical meristem maintenance and establishment of leaf polarity by repressing miR165/166 in Arabidopsis. Plant Journal 58, 27-40.

Liu, Q.P., Feng, Y., and Zhu, Z.J. (2009b). Dicer-like (DCL) proteins in plants. Functional \& Integrative Genomics 9, 277-286.

Llave, C., Xie, Z., Kasschau, K.D., and Carrington, J.C. (2002). Cleavage of Scarecrow-like mRNA targets directed by a class of Arabidopsis miRNA. Science 297, 2053-2056.

Lobbes, D., Rallapalli, G., Schmidt, D.D., Martin, C., and Clarke, J. (2006). SERRATE: a new player on the plant microRNA scene. EMBO Rep 7, 1052-1058.

Lu, C., Tej, S.S., Luo, S., Haudenschild, C.D., Meyers, B.C., and Green, P.J. (2005). Elucidation of the small RNA component of the transcriptome. Science 309, 1567-1569.

Ma, X.F., Nicole, M.C., Meteignier, L.V., Hong, N., Wang, G.P., and Moffett, P. (2015). Different roles for RNA silencing and RNA processing components in virus recovery and virus-induced gene silencing in plants. Journal of Experimental Botany 66, 919-932.

Malagnac, F., Bartee, L., and Bender, J. (2002). An Arabidopsis SET domain protein required for maintenance but not establishment of DNA methylation. The EMBO journal 21, 6842-6852.

Mallory, A.C., and Bouche, N. (2008). MicroRNA-directed regulation: to cleave or not to cleave. Trends in Plant Science 13, 359-367.

Mallory, A.C., Elmayan, T., and Vaucheret, H. (2008). MicroRNA maturation and action - the expanding roles of ARGONAUTEs. Current Opinion in Plant Biology 11, 560-566.

Mallory, A.C., Reinhart, B.J., Jones-Rhoades, M.W., Tang, G., Zamore, P.D., Barton, M.K., and Bartel, D.P. (2004). MicroRNA control of PHABULOSA in leaf development: importance of pairing to the microRNA 5' region. The EMBO journal 23, 3356-3364.

Manavella, P.A., Hagmann, J., Ott, F., Laubinger, S., Franz, M., Macek, B., and Weigel, D. (2012). Fast-Forward Genetics Identifies Plant CPL Phosphatases as Regulators of miRNA Processing Factor HYL1. Cell 151, 859-870.

Margis, R., Fusaro, A.F., Smith, N.A., Curtin, S.J., Watson, J.M., Finnegan, E.J., and Waterhouse, P.M. (2006). The evolution and diversification of Dicers in plants. FEBS Letters 580, 2442-2450.

Margulis, L., and Schwartz, K.V. (1998). Five kingdoms : an illustrated guide to the phyla of life on earth, 3rd edn (New York: W.H. Freeman).

Marin-Gonzalez, E., and Suarez-Lopez, P. (2012). "And yet it moves": Cell-to-cell and long-distance signaling by plant microRNAs. Plant Science 196, 18-30.

Marrocco, K., Criqui, M.C., Zervudacki, J., Schott, G., Eisler, H., Parnet, A., Dunoyer, P., and Genschik, P. (2012). APC/C-Mediated Degradation of dsRNA-Binding Protein 4 (DRB4) Involved in RNA Silencing. Plos One 7, e35173-e35173.

Matzke, M.A., and Birchler, J.A. (2005). RNAi-mediated pathways in the nucleus. Nature Reviews Genetics 6, 24-35. 
Matzke, M.A., Kanno, T., and Matzke, A.J. (2015). RNA-Directed DNA Methylation: The Evolution of a Complex Epigenetic Pathway in Flowering Plants. Annual review of plant biology 66, 243-267.

McCue, A.D., Panda, K., Nuthikattu, S., Choudury, S.G., Thomas, E.N., and Slotkin, R.K. (2015). ARGONAUTE 6 bridges transposable element mRNA-derived siRNAs to the establishment of DNA methylation. The EMBO journal 34, 20-35.

McHale, N.A., and Koning, R.E. (2004). MicroRNA-directed cleavage of Nicoltiana sylvestris PHAVOLUTA mRNA regulates the vascular cambium and structure of apical Meristems. Plant Cell 16, 1730-1740.

Meng, Y., and Shao, C. (2012). Large-scale identification of mirtrons in Arabidopsis and rice. Plos One 7, e31163.

Mette, M.F., Aufsatz, W., van der Winden, J., Matzke, M.A., and Matzke, A.J. (2000). Transcriptional silencing and promoter methylation triggered by double-stranded RNA. The EMBO journal 19, 5194-5201.

Mi, S., Cai, T., Hu, Y., Chen, Y., Hodges, E., Ni, F., Wu, L., Li, S., Zhou, H., Long, C., et al. (2008). Sorting of small RNAs into Arabidopsis argonaute complexes is directed by the 5' terminal nucleotide. Cell 133, 116-127.

Migicovsky, Z., and Kovalchuk, I. (2014). Transgenerational changes in plant physiology and in transposon expression in response to UV-C stress in Arabidopsis thaliana. Plant Signaling and Behavior 9, e976490-e976490.

Migicovsky, Z., and Kovalchuk, I. (2015). Transgenerational inheritance of epigenetic response to cold in Arabidopsis thaliana. Biocatalysis and Agricultural Biotechnology 4, 1-10.

Migicovsky, Z., Yao, Y., and Kovalchuk, I. (2014). Transgenerational phenotypic and epigenetic changes in response to heat stress in Arabidopsis thaliana. Plant Signaling and Behavior 9.

Minoia, S., Carbonell, A., Di Serio, F., Gisel, A., Carrington, J.C., Navarro, B., and Flores, R. (2014). Specific Argonautes Selectively Bind Small RNAs Derived from Potato Spindle Tuber Viroid and Attenuate Viroid Accumulation In Vivo. Journal of Virology 88, 11933-11945.

Mirzaei, K., Bahramnejad, B., Shamsifard, M.H., and Zamani, W. (2014). In Silico Identification, Phylogenetic and Bioinformatic Analysis of Argonaute Genes in Plants. International Journal of Genomics 2014, 967461-967461.

Mlotshwa, S., Pruss, G.J., Peragine, A., Endres, M.W., Li, J.J., Chen, X.M., Poethig, R.S., Bowman, L.H., and Vance, V. (2008). DICER-LIKE2 Plays a Primary Role in Transitive Silencing of Transgenes in Arabidopsis. Plos One 3, e1755-e1755.

Moissiard, G., Parizotto, E.A., Himber, C., and Voinnet, O. (2007). Transitivity in Arabidopsis can be primed, requires the redundant action of the antiviral Dicer-like 4 and Dicer-like 2, and is compromised by viral-encoded suppressor proteins. RNA 13, 1268-1278.

Moissiard, G., and Voinnet, O. (2006). RNA silencing of host transcripts by cauliflower mosaic virus requires coordinated action of the four Arabidopsis Dicer-like proteins (Retracted article. See vol. 112, pg. E4818, 2015). Proc Natl Acad Sci U S A 103, 19593-19598.

Montgomery, T.A., Howell, M.D., Cuperus, J.T., Li, D.W., Hansen, J.E., Alexander, A.L., Chapman, E.J., Fahlgren, N., Allen, E., and Carrington, J.C. (2008). Specificity of ARGONAUTE7-miR390 interaction and dual functionality in TAS3 trans-acting siRNA formation. Cell 133, 128-141.

Morel, J.B., Godon, C., Mourrain, P., Beclin, C., Boutet, S., Feuerbach, F., Proux, F., and Vaucheret, H. (2002). Fertile hypomorphic ARGONAUTE (ago1) mutants impaired in post-transcriptional gene silencing and virus resistance. Plant Cell 14, 629-639. 
Mourrain, P., Beclin, C., Elmayan, T., Feuerbach, F., Godon, C., Morel, J.B., Jouette, D., Lacombe, A.M., Nikic, S., Picault, N., et al. (2000). Arabidopsis SGS2 and SGS3 genes are required for posttranscriptional gene silencing and natural virus resistance. Cell 101, 533-542.

Muangsan, N., Beclin, C., Vaucheret, H., and Robertson, D. (2004). Geminivirus VIGS of endogenous genes requires SGS2/SDE1 and SGS3 and defines a new branch in the genetic pathway for silencing in plants. Plant Journal 38, 1004-1014.

Mukherjee, K., Campos, H., and Kolaczkowski, B. (2013). Evolution of Animal and Plant Dicers: Early Parallel Duplications and Recurrent Adaptation of Antiviral RNA Binding in Plants. Molecular Biology and Evolution 30, 627-641.

Nagano, H., Fukudome, A., Hiraguri, A., Moriyama, H., and Fukuhara, T. (2014). Distinct substrate specificities of Arabidopsis DCL3 and DCL4. Nucleic Acids Research 42, 1845-1856.

Nagasaki, H., Itoh, J.-I., Hayashi, K., Hibara, K.-I., Satoh-Nagasawa, N., Nosaka, M., Mukouhata, M., Ashikari, M., Kitano, H., Matsuoka, M., et al. (2007). The small interfering RNA production pathway is required for shoot meristern initiation in rice. Proc Natl Acad Sci U S A 104, 14867-14871.

Nakazawa, Y., Hiraguri, A., Moriyama, H., and Fukuhara, T. (2007). The dsRNA-binding protein DRB4 interacts with the Dicer-like protein DCL4 in vivo and functions in the trans-acting siRNA pathway. Plant Molecular Biology 63, 777-785.

Odokonyero, D., Mendoza, M.R., Alvarado, V.Y., Zhang, J.T., Wang, X.F., and Scholthof, H.B. (2015). Transgenic down-regulation of ARGONAUTE2 expression in Nicotiana benthamiana interferes with several layers of antiviral defenses. Virology 486, 209-218.

Ogwok, E., Ilyas, M., Alicai, T., Rey, M.E.C., and Taylor, N.J. (2016). Comparative analysis of virus-derived small RNAs within cassava (Manihot esculenta Crantz) infected with cassava brown streak viruses. Virus Research 215, 1-11.

Oliver, C., Santos, J.L., and Pradillo, M. (2014). On the role of some ARGONAUTE proteins in meiosis and DNA repair in Arabidopsis thaliana. Frontiers in Plant Science 5, 177-177.

Olmedo-Monfil, V., Duran-Figueroa, N., Arteaga-Vazquez, M., Demesa-Arevalo, E., Autran, D., Grimanelli, D., Slotkin, R.K., Martienssen, R.A., and Vielle-Calzada, J.P. (2010). Control of female gamete formation by a small RNA pathway in Arabidopsis. Nature 464, 628-U200.

Onodera, Y., Haag, J.R., Ream, T., Nunes, P.C., Pontes, O., and Pikaard, C.S. (2005). Plant nuclear RNA polymerase IV mediates siRNA and DNA methylation-dependent heterochromatin formation. Cell 120, 613-622.

Palmer, J.D., Soltis, D.E., and Chase, M.W. (2004). The plant tree of life: an overview and some points of view. American journal of botany 91, 1437-1445.

Papp, I., Mette, M.F., Aufsatz, W., Daxinger, L., Schauer, S.E., Ray, A., van der Winden, J., Matzke, M., and Matzke, A.J.M. (2003). Evidence for nuclear processing of plant micro RNA and short interfering RNA precursors. Plant Physiology 132, 1382-1390.

Parent, J.S., Bouteiller, N., Elmayan, T., and Vaucheret, H. (2015). Respective contributions of Arabidopsis DCL2 and DCL4 to RNA silencing. Plant Journal 81, 223-232.

Park, M.Y., Wu, G., Gonzalez-Sulser, A., Vaucheret, H., and Poethig, R.S. (2005). Nuclear processing and export of microRNAs in Arabidopsis. Proc Natl Acad Sci U S A 102, 3691-3696.

Park, W., Li, J.J., Song, R.T., Messing, J., and Chen, X.M. (2002). CARPEL FACTORY, a Dicer homolog, and HEN1, a novel protein, act in microRNA metabolism in Arabidopsis thaliana. Current Biology 12, 1484-1495. 
Pelissier, T., Clavel, M., Chaparro, C., Pouch-Pelissier, M.N., Vaucheret, H., and Deragon, J.M. (2011). Double-stranded RNA binding proteins DRB2 and DRB4 have an antagonistic impact on polymerase IV-dependent siRNA levels in Arabidopsis. RNA 17, 1502-1510.

Pelissier, T., Thalmeir, S., Kempe, D., Sanger, H.L., and Wassenegger, M. (1999). Heavy de novo methylation at symmetrical and non-symmetrical sites is a hallmark of RNA-directed DNA methylation. Nucleic Acids Research 27, 1625-1634.

Pelissier, T., and Wassenegger, M. (2000). A DNA target of $30 \mathrm{bp}$ is sufficient for RNA-directed DNA methylation. RNA 6, 55-65.

Penterman, J., Zilberman, D., Huh, J.H., Ballinger, T., Henikoff, S., and Fischer, R.L. (2007). DNA demethylation in the Arabidopsis genome. Proc Natl Acad Sci U S A 104, 6752-6757.

Peragine, A., Yoshikawa, M., Wu, G., Albrecht, H.L., and Poethig, R.S. (2004). SGS3 and SGS2/ SDE1/RDR6 are required for juvenile development and the production of trans-acting siRNAs in Arabidopsis. Genes \& Development 18, 2368-2379.

Pontes, O., Li, C.F., Nunes, P.C., Haag, J., Ream, T., Vitins, A., Jacobsen, S.E., and Pikaard, C.S. (2006). The Arabidopsis chromatin-modifying nuclear siRNA pathway involves a nucleolar RNA processing center. Cell 126, 79-92.

Pontier, D., Picart, C., Roudier, F., Garcia, D., Lahmy, S., Azevedo, J., Alart, E., Laudie, M., Karlowski, W.M., Cooke, R., et al. (2012). NERD, a Plant-Specific GW Protein, Defines an Additional RNAi-Dependent Chromatin-Based Pathway in Arabidopsis. Molecular Cell 48, 121-132.

Pouch-Pelissier, M.-N., Pelissier, T., Elmayan, T., Vaucheret, H., Boko, D., Jantsch, M.F., and Deragon, J.-M. (2008). SINE RNA Induces Severe Developmental Defects in Arabidopsis thaliana and Interacts with HYL1 (DRB1), a Key Member of the DCL1 Complex. Plos Genetics 4, e1000096-e1000096.

Qu, F., Ye, X.H., Hou, G.C., Sato, S., Clemente, T.E., and Morris, T.J. (2005). RDR6 has a broad-spectrum but temperature-dependent antiviral defense role in Nicotiana benthamiana. Journal of Virology 79, 15209-15217.

Qu, F., Ye, X.H., and Morris, T.J. (2008). Arabidopsis DRB4, AG01, AG07, and RDR6 participate in a DCL4-initiated antiviral RNA silencing pathway negatively regulated by DCL1. Proc Natl Acad Sci U S A 105, 14732-14737.

Raghuram, B., Sheikh, A.H., Rustagi, Y., and Sinha, A.K. (2015). MicroRNA biogenesis factor DRB1 is a phosphorylation target of mitogen activated protein kinase MPK3 in both rice and Arabidopsis. FEBS Journal 282, 521-536.

Raja, P., Jackel, J.N., Li, S.Z., Heard, I.M., and Bisaro, D.M. (2014). Arabidopsis Double-Stranded RNA Binding Protein DRB3 Participates in Methylation-Mediated Defense against Geminiviruses. Journal of Virology 88, 2611-2622.

Rajagopalan, R., Vaucheret, H., Trejo, J., and Bartel, D.P. (2006). A diverse and evolutionarily fluid set of microRNAs in Arabidopsis thaliana. Genes \& Development 20, 3407-3425.

Reinhart, B.J., Weinstein, E.G., Rhoades, M.W., Bartel, B., and Bartel, D.P. (2002). MicroRNAs in plants. Genes \& Development 16, 1616-1626.

Reis, R.S., Eamens, A.L., Roberts, T.H., and Waterhouse, P.M. (2016). Chimeric DCL1-Partnering Proteins Provide Insights into the MicroRNA Pathway. Frontiers in Plant Science 6.

Reis, R.S., Eamens, A.L., and Waterhouse, P.M. (2015a). Missing Pieces in the Puzzle of Plant MicroRNAs. Trends in Plant Science 20, 721-728. 
Reis, R.S., Hart-Smith, G., Eamens, A.L., Wilkins, M.R., and Waterhouse, P.M. (2015b). Gene regulation by translational inhibition is determined by Dicer partnering proteins. Nature Plants 1 .

Reis, R.S., Hart-Smith, G., Eamens, A.L., Wilkins, M.R., and Waterhouse, P.M. (2015c). MicroRNA Regulatory Mechanisms Play Different Roles in Arabidopsis. Journal of Proteome Research 14, 4743-4751.

Ren, G.D., Xie, M., Zhang, S.X., Vinovskis, C., Chen, X.M., and Yu, B. (2014). Methylation protects microRNAs from an AGO1-associated activity that uridylates 5' RNA fragments generated by AGO1 cleavage. Proc Natl Acad Sci U S A 111, 6365-6370.

Rhoades, M.W., Reinhart, B.J., Lim, L.P., Burge, C.B., Bartel, B., and Bartel, D.P. (2002). Prediction of plant microRNA targets. Cell 110, 513-520.

Rodriguez, R.E., Mecchia, M.A., Debernardi, J.M., Schommer, C., Weigel, D., and Palatnik, J.F. (2010). Control of cell proliferation in Arabidopsis thaliana by microRNA miR396. Development 137, 103-112.

Rogers, K., and Chen, X.M. (2013). Biogenesis, Turnover, and Mode of Action of Plant MicroRNAs. Plant Cell 25, 2383-2399.

Roodbarkelari, F., Du, F., Truernit, E., and Laux, T. (2015). ZLL/AGO10 maintains shoot meristem stem cells during Arabidopsis embryogenesis by down-regulating ARF2-mediated auxin response. BMC Biology 13, 74-74.

Salgado, P.S., Koivunen, M.R.L., Makeyev, E.V., Bamford, D.H., Stuart, D.I., and Grimes, J.M. (2006). The structure of an RNAi polymerase links RNA silencing and transcription. Plos Biology 4, 2274-2281.

Schauer, S.E., Jacobsen, S.E., Meinke, D.W., and Ray, A. (2002). DICER-LIKE1: blind men and elephants in Arabidopsis development. Trends in Plant Science 7, 487-491.

Schiebel, W., Haas, B., Marinkovic, S., Klanner, A., and Sanger, H.L. (1993). RNA-directed RNA polymerase from tomato leaves. II. Catalytic in vitro properties. Journal of Biological Chemistry 268, 11858-11867.

Schiebel, W., Pelissier, T., Riedel, L., Thalmeir, S., Schiebel, R., Kempe, D., Lottspeich, F., Sanger, H.L., and Wassenegger, M. (1998). Isolation of an RNA-Directed RNA polymerase-specific cDNA clone from tomato. Plant Cell 10, 2087-2101.

Schwab, R., Palatnik, J.F., Riester, M., Schommer, C., Schmid, M., and Weigel, D. (2005). Specific effects of microRNAs on the plant transcriptome. Developmental Cell 8, 517-527.

Shao, C.G., Chen, M., and Meng, Y.J. (2013). A reversed framework for the identification of microRNA-target pairs in plants. Briefings in Bioinformatics 14, 293-301.

Shao, F.J., and Lu, S.F. (2013). Genome-wide identification, molecular cloning, expression profiling and posttranscriptional regulation analysis of the Argonaute gene family in Salvia miltiorrhiza, an emerging model medicinal plant. BMC Genomics 14, 512-512.

Shao, F.J., and Lu, S.F. (2014). Identification, Molecular Cloning and Expression Analysis of Five RNA-Dependent RNA Polymerase Genes in Salvia miltiorrhiza. Plos One 9, e95117-e95117.

Shi, Z.Y., Wang, J., Wan, X.S., Shen, G.Z., Wang, X.Q., and Zhang, J.L. (2007). Over-expression of rice OsAGO7 gene induces upward curling of the leaf blade that enhanced erect-leaf habit. Planta 226, 99-108.

Shivaprasad, P.V., Rajeswaran, R., Blevins, T., Schoelz, J., Meins, F., Hohn, T., and Pooggin, M.M. (2008). The CaMV transactivator/viroplasmin interferes with RDR6-dependent trans-acting and secondary siRNA pathways in Arabidopsis. Nucleic Acids Research 36, 5896-5909. 
Silva, T.F., Romanel, E.A.C., Andrade, R.R.S., Farinelli, L., Osteras, M., Deluen, C., Correa, R.L., Schrago, C.E.G., and Vaslin, M.F.S. (2011). Profile of small interfering RNAs from cotton plants infected with the polerovirus Cotton leafroll dwarf virus. BMC Molecular Biology 12, 40-40.

Singh, M., Goel, S., Meeley, R.B., Dantec, C., Parrinello, H., Michaud, C., Leblanc, O., and Grimanelli, D. (2011). Production of Viable Gametes without Meiosis in Maize Deficient for an ARGONAUTE Protein. Plant Cell 23, 443-458.

Smith, M.R., Willmann, M.R., Wu, G., Berardini, T.Z., Moller, B., Weijers, D., and Poethig, R.S. (2009). Cyclophilin 40 is required for microRNA activity in Arabidopsis. Proc Natl Acad Sci U S A 106, 5424-5429.

Song, X.W., Li, P.C., Zhai, J.X., Zhou, M., Ma, L.J., Liu, B., Jeong, D.H., Nakano, M., Cao, S.Y., Liu, C.Y., et al. (2012). Roles of DCL4 and DCL3b in rice phased small RNA biogenesis. Plant Journal 69, 462-474.

Souret, F.F., Kastenmayer, J.P., and Green, P.J. (2004). AtXRN4 degrades mRNA in Arabidopsis and its substrates include selected miRNA targets. Molecular Cell 15, 173-183.

Sunkar, R., and Zhu, J.K. (2004). Novel and stress-regulated microRNAs and other small RNAs from Arabidopsis. Plant Cell 16, 2001-2019.

Svoboda, P., and Cara, A.D. (2006). Hairpin RNA: a secondary structure of primary importance. Cell Mol Life Sci 63, 901-908.

Takeda, A., Iwasaki, S., Watanabe, T., Utsumi, M., and Watanabe, Y. (2008). The mechanism selecting the guide strand from small RNA duplexes is different among Argonaute proteins. Plant and Cell Physiology 49, 493-500.

Thomas, C.L., Jones, L., Baulcombe, D.C., and Maule, A.J. (2001). Size constraints for targeting post-transcriptional gene silencing and for RNA-directed methylation in Nicotiana benthamiana using a potato virus X vector. Plant Journal 25, 417-425.

Tkaczuk, K.L., Obarska, A., and Bujnicki, J.M. (2006). Molecular phylogenetics and comparative modeling of HEN1, a methyltransferase involved in plant microRNA biogenesis. BMC Evolutionary Biology 6, 6-6.

Tucker, M.R., Okada, T., Hu, Y., Scholefield, A., Taylor, J.M., and Koltunow, A.M. (2012). Somatic small RNA pathways promote the mitotic events of megagametogenesis during female reproductive development in Arabidopsis. Development 139, 1399-1404.

Tucker, M.R., Roodbarkelari, F., Truernit, E., Adamski, N.M., Hinze, A., Lohmuller, B., Wurschum, T., and Laux, T. (2013). Accession-specific modifiers act with ZWILLE/ARGONAUTE10 to maintain shoot meristem stem cells during embryogenesis in Arabidopsis. BMC Genomics 14, 809-809.

Tworak, A., Urbanowicz, A., Podkowinski, J., Kurzynska-Kokorniak, A., Koralewska, N., and Figlerowicz, M. (2016). Six Medicago truncatula Dicer-like protein genes are expressed in plant cells and upregulated in nodules. Plant Cell Reports 35, 1043-1052.

Urayama, S., Moriyama, H., Aoki, N., Nakazawa, Y., Okada, R., Kiyota, E., Miki, D., Shimamoto, K., and Fukuhara, T. (2010). Knock-down of OsDCL2 in Rice Negatively Affects Maintenance of the Endogenous dsRNA Virus, Oryza sativa Endornavirus. Plant and Cell Physiology 51, $58-67$.

Vaistij, F.E., Jones, L., and Baulcombe, D.C. (2002). Spreading of RNA targeting and DNA methylation in RNA silencing requires transcription of the target gene and a putative RNA-dependent RNA polymerase. Plant Cell 14, 857-867. 
Van Ex, F., Jacob, Y., and Martienssen, R.A. (2011). Multiple roles for small RNAs during plant reproduction. Current Opinion in Plant Biology 14, 588-593.

Vaucheret, H. (2005). MicroRNA-dependent trans-acting siRNA production. Science's STKE : signal transduction knowledge environment 2005, pe43-pe43.

Vaucheret, H. (2008). Plant ARGONAUTES. Trends in Plant Science 13, 350-358.

Vaucheret, H., Vazquez, F., Crete, P., and Bartel, D.P. (2004). The action of ARGONAUTE1 in the miRNA pathway and its regulation by the miRNA pathway are crucial for plant development. Genes \& Development 18, 1187-1197.

Vazquez, F. (2006). Arabidopsis endogenous small RNAs: highways and byways. Trends in Plant Science 11, 460-468.

Vazquez, F., Gasciolli, V., Crete, P., and Vaucheret, H. (2004). The nuclear dsRNA binding protein HYL1 is required for MicroRNA accumulation and plant development, but not posttranscriptional transgene silencing. Current Biology 14, 346-351.

Vongs, A., Kakutani, T., Martienssen, R.A., and Richards, E.J. (1993). Arabidopsis thaliana DNA methylation mutants. Science 260, 1926-1928.

Wang, X.B., Jovel, J., Udomporn, P., Wang, Y., Wu, Q.F., Li, W.X., Gasciolli, V., Vaucheret, H., and Ding, S.W. (2011). The 21-Nucleotide, but Not 22-Nucleotide, Viral Secondary Small Interfering RNAs Direct Potent Antiviral Defense by Two Cooperative Argonautes in Arabidopsis thaliana. Plant Cell 23, 1625-1638.

Wang, X.B., Wu, Q.F., Ito, T., Cillo, F., Li, W.X., Chen, X.M., Yu, J.L., and Ding, S.W. (2010). RNAi-mediated viral immunity requires amplification of virus-derived siRNAs in Arabidopsis thaliana. Proc Natl Acad Sci U S A 107, 484-489.

Wassenegger, M., Heimes, S., Riedel, L., and Sanger, H.L. (1994). RNA-directed de novo methylation of genomic sequences in plants. Cell 76, 567-576.

Wassenegger, M., and Krczal, G. (2006). Nomenclature and functions of RNA-directed RNA polymerases. Trends in Plant Science 11, 142-151.

Wei, L.Y., Gu, L.F., Song, X.W., Cui, X.K., Lu, Z.K., Zhou, M., Wang, L.L., Hu, F.Y., Zhai, J.X., Meyers, B.C., et al. (2014). Dicer-like 3 produces transposable element-associated 24-nt siRNAs that control agricultural traits in rice. Proc Natl Acad Sci U S A 111, 3877-3882.

Wei, W., Ba, Z., Gao, M., Wu, Y., Ma, Y., Amiard, S., White, C.I., Rendtlew Danielsen, J.M., Yang, Y.G., and Qi, Y. (2012). A role for small RNAs in DNA double-strand break repair. Cell 149, $101-112$.

Wu, J.G., Yang, Z.R., Wang, Y., Zheng, L.J., Ye, R.Q., Ji, Y.H., Zhao, S.S., Ji, S.Y., Liu, R.F., Xu, L., et al. (2015). Viral-Inducible Argonaute 18 Confers Broad-Spectrum Virus Resistance in Rice by Sequestering A Host MicroRNA. Elife 4.

Wu, L., Mao, L., and Qi, Y.J. (2012). Roles of DICER-LIKE and ARGONAUTE Proteins in TAS-Derived Small Interfering RNA-Triggered DNA Methylation. Plant Physiology 160, 990-999.

Wu, L., Zhang, Q.Q., Zhou, H.Y., Ni, F.R., Wu, X.Y., and Qi, Y.J. (2009). Rice MicroRNA Effector Complexes and Targets. Plant Cell 21, 3421-3435.

Wu, L., Zhou, H.Y., Zhang, Q.Q., Zhang, J.G., Ni, F.R., Liu, C., and Qi, Y.J. (2010). DNA Methylation Mediated by a MicroRNA Pathway. Molecular Cell 38, 465-475.

Xie, M., and Yu, B. (2015). siRNA-directed DNA Methylation in Plants. Current Genomics 16, 23-31. 
Xie, Z.X., Allen, E., Wilken, A., and Carrington, J.C. (2005). DICER-LIKE 4 functions in trans-acting small interfering RNA biogenesis and vegetative phase change in Arabidopsis thaliana. Proc Natl Acad Sci U S A 102, 12984-12989.

Xie, Z.X., Johansen, L.K., Gustafson, A.M., Kasschau, K.D., Lellis, A.D., Zilberman, D., Jacobsen, S.E., and Carrington, J.C. (2004). Genetic and functional diversification of small RNA pathways in plants. Plos Biology 2, 642-652.

Xie, Z.X., Kasschau, K.D., and Carrington, J.C. (2003). Negative feedback regulation of Dicer-Like1 in Arabidopsis by microRNA-guided mRNA degradation. Current Biology 13, 784-789.

Xie, Z.X., Khanna, K., and Ruan, S.L. (2010). Expression of microRNAs and its regulation in plants. Seminars in Cell \& Developmental Biology 21, 790-797.

Yang, L., Liu, Z.Q., Lu, F., Dong, A.W., and Huang, H. (2006). SERRATE is a novel nuclear regulator in primary microRNA processing in Arabidopsis. Plant Journal 47, 841-850.

Yang, L., Wu, G., and Poethig, R.S. (2012). Mutations in the GW-repeat protein SUO reveal a developmental function for microRNA-mediated translational repression in Arabidopsis. Proc Natl Acad Sci U S A 109, 315-320.

Yang, S.J., Carter, S.A., Cole, A.B., Cheng, N.H., and Nelson, R.S. (2004). A natural variant of a host RNA-dependent RNA polymerase is associated with increased susceptibility to viruses by Nicotiana benthamiana. Proc Natl Acad Sci U S A 101, 6297-6302.

You, C.X., Zhao, Q., Wang, X.F., Xie, X.B., Feng, X.M., Zhao, L.L., Shu, H.R., and Hao, Y.J. (2014). A dsRNA-binding protein MdDRB1 associated with miRNA biogenesis modifies adventitious rooting and tree architecture in apple. Plant Biotechnology Journal 12, 183-192.

Yu, B., Yang, Z., Li, J., Minakhina, S., Yang, M., Padgett, R.W., Steward, R., and Chen, X. (2005). Methylation as a crucial step in plant microRNA biogenesis. Science 307, 932-935.

Yu, D.Q., Fan, B.F., MacFarlane, S.A., and Chen, Z.X. (2003). Analysis of the involvement of an inducible Arabidopsis RNA-dependent RNA polymerase in antiviral defense. Molecular Plant-Microbe Interactions 16, 206-216.

Zhai, L., Sun, W., Zhang, K., Jia, H., Liu, L., Liu, Z., Teng, F., and Zhang, Z. (2014). Identification and characterization of Argonaute gene family and meiosis-enriched Argonaute during sporogenesis in maize. J Integr Plant Biol 56, 1042-1052.

Zhang, H., Xia, R., Meyers, B.C., and Walbot, V. (2015). Evolution, functions, and mysteries of plant ARGONAUTE proteins. Current Opinion in Plant Biology 27, 84-90.

Zhang, W.X., Gao, S., Zhou, X.F., Xia, J., Chellappan, P., Zhou, X.A., Zhang, X.M., and Jin, H.L. (2010). Multiple distinct small RNAs originate from the same microRNA precursors. Genome Biology 11, R81-R81.

Zhang, X., Henderson, I.R., Lu, C., Green, P.J., and Jacobsen, S.E. (2007). Role of RNA polymerase IV in plant small RNA metabolism. Proc Natl Acad Sci U S A 104, 4536-4541.

Zhang, X., Zhao, H., Gao, S., Wang, W.C., Katiyar-Agarwal, S., Huang, H.D., Raikhel, N., and Jin, H. (2011). Arabidopsis Argonaute 2 regulates innate immunity via miRNA393(*)-mediated silencing of a Golgi-localized SNARE gene, MEMB12. Molecular Cell 42, 356-366.

Zhang, X.C., Zhang, X.F., Singh, J., Li, D.W., and Qu, F. (2012). Temperature-Dependent Survival of Turnip Crinkle Virus-Infected Arabidopsis Plants Relies on an RNA Silencing-Based Defense That Requires DCL2, AG02, and HEN1. Journal of Virology 86, 6847-6854.

Zhao, X., Zhang, H., and Li, L. (2013). Identification and analysis of the proximal promoters of microRNA genes in Arabidopsis. Genomics 101, 187-194. 
Zheng, B., Wang, Z., Li, S., Yu, B., Liu, J.-Y., and Chen, X. (2009). Intergenic transcription by RNA Polymerase II coordinates Pol IV and Pol V in siRNA-directed transcriptional gene silencing in Arabidopsis. Genes \& Development 23, 2850-2860.

Zheng, X.W., Zhu, J.H., Kapoor, A., and Zhu, J.K. (2007). Role of Arabidopsis AGO6 in siRNA accumulation, DNA methylation and transcriptional gene silencing. The EMBO journal 26, 1691-1701.

Zhou, C., Han, L., Fu, C., Wen, J., Cheng, X., Nakashima, J., Ma, J., Tang, Y., Tan, Y., Tadege, M., et al. (2013). The Trans-Acting Short Interfering RNA3 Pathway and NO APICAL MERISTEM Antagonistically Regulate Leaf Margin Development and Lateral Organ Separation, as Revealed by Analysis of an argonaute7/lobed leaflet1 Mutant in Medicago truncatula. Plant Cell $25,4845-4862$.

Zhou, Y., Honda, M., Zhu, H., Zhang, Z., Guo, X., Li, T., Li, Z., Peng, X., Nakajima, K., Duan, L., et al. (2015). Spatiotemporal Sequestration of miR165/166 by Arabidopsis Argonaute10 Promotes Shoot Apical Meristem Maintenance. Cell Reports 10, 1819-1827.

Zhu, H., Zhou, Y., Castillo-Gonzalez, C., Lu, A., Ge, C., Zhao, Y.T., Duan, L., Li, Z., Axtell, M.J., Wang, X.J., et al. (2013). Bidirectional processing of pri-miRNAs with branched terminal loops by Arabidopsis Dicer-like1. Nature Structural \& Molecular Biology 20, 1106-1115.

Zhu, H.L., Hu, F.Q., Wang, R.H., Zhou, X., Sze, S.H., Liou, L.W., Barefoot, A., Dickman, M., and Zhang, X.R. (2011). Arabidopsis Argonaute10 Specifically Sequesters miR166/165 to Regulate Shoot Apical Meristem Development. Cell 145, 242-256.

Zilberman, D., Cao, X.F., Johansen, L.K., Xie, Z.X., Carrington, J.C., and Jacobsen, S.E. (2004). Role of arabidopsis ARGONAUTE4 in RNA-directed DNA methylation triggered by inverted repeats. Current Biology 14, 1214-1220. 



\title{
RNAi AND mIRNA PATHWAYS IN PLANTS II - MOBILITY OF SMALL RNAS
}

Keywords: dsRNA, miRNA, siRNA, Dicer, Argonaute, RdRP, systemic RNAi, phloem

\section{PETR SVOBODA}

Institute of Molecular Genetics, Academy of Sciences of the Czech Republic, Videnska 1083, 14220 Prague 4, Czech Republic

Correspondence to: Petr Svoboda, Institute of Molecular Genetics ASCR, Videnska 1083, 14220 Prague 4, Czech Republic, tel. \#+420 241063147, e-mail: svobodap@img.cas.cz.

\begin{abstract}
RNA silencing denotes sequence-specific repression mediated by small RNAs. Plants have arguably the most complex RNA silencing among eukaryots because of existence of many paralogs of key protein factors, which form in an intricate network of primary and secondary small RNAs, which mediate transcriptional and post-transcriptional effects, which target endogenous protein-coding gene expression, serve as a form of innate immunity targeting viruses, and protect genome integrity by repressing retrotransposons. The complexity of RNA silencing was introduced in an accompanying review. Here, I will focus on mobility of small RNAs in plants, which allows for silencing effects occurring at a different place than where the silencing was initiated. I will discuss different types of mobility of different classes of small RNAs across plant tissues and their biological implications.
\end{abstract}

\section{Introduction}

RNA silencing in plants is highly complex thanks to existence of many Dicer, Argonaute, and RNA-dependent RNA polymerase (RdRP) paralogs, which fucntion in a network of primary and secondary small RNAs, which mediate transcriptional and post-transcriptional effects (Fig. 1). The nomenclature of small RNAs in plants is complex and arbitrary; small RNAs include miRNAs and various types of siRNAs produced from dsRNA, which are distinguished by their origin (viral siRNA), biogenesis (phased siRNAs) or their effect (heterochromatinizing siRNAs). RNA silencing in plants can be divided in three to four main systems: miRNA, RNAi/ post-transcriptional gene silencing (PTGS), antiviral defense/virus-induced gene silencing (VIGS), and transcriptional gene silencing (TGS) (Bologna and Voinnet, 2014; Bonnet et al., 2006; Borges and Martienssen, 2015; Carbonell and Carrington, 2015; Chen, 2009; Galun, 2005; Mallory et al., 2008; Van Ex et al., 2011; Vazquez, 2006; Zhang 


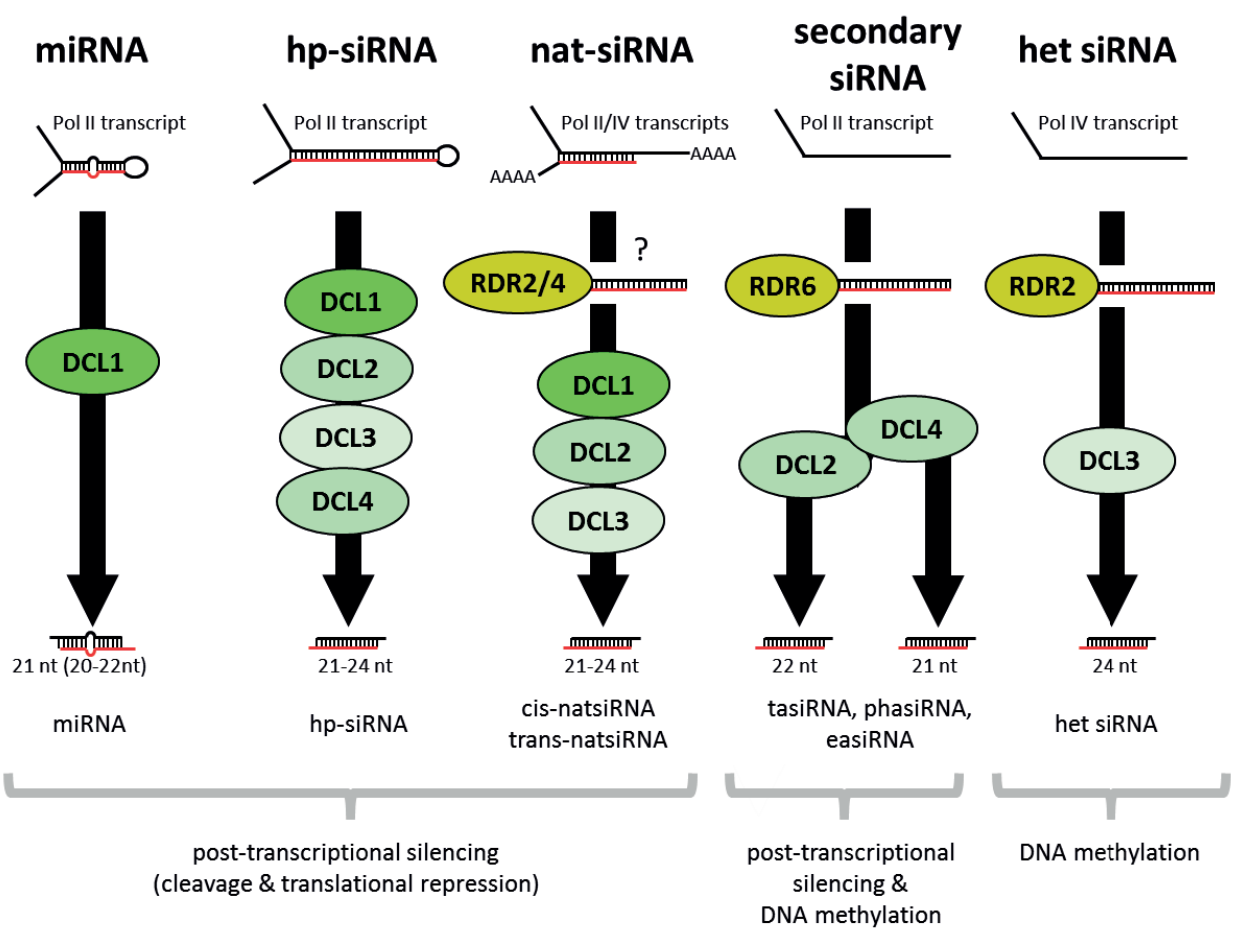

Figure 1 Complexity of small RNAs in plants. The scheme was adapted from (Borges and Martienssen, 2015)

et al., 2015). The complexity of small RNA biology was reviewed in the first plant RNA silencing review (Svoboda, 2019). Here, I will focus on mobility of small RNAs in plants.

\section{Plant anatomy and features relevant for movement of molecules}

Plants are unique in several aspects when compared to cells of other eukaryotic organisms. Plant cell features important for intercellular exchange of molecules include a polysaccharide cell wall and plasmodesmata. The latter are microscopic channels traversing cell walls of plant cells allowing for movement (symplastic movement or symplast) of molecules between adjacent cells (reviewed in Maule, 2008; Maule et al., 2011). An alternative to the symplastic movement, which involves cell cytoplasm, is apoplastic movement (apoplast) where molecules move through cell walls and intercellular space.

A flowering plant (Fig. 2) consists of three anatomical systems - root, shoot, and inflorescence. It is anchored by a root, absorbs water and minerals from the grounds and transports them through the xylem vasculature through the plant and into leaves, which are the main photosynthetic organs. Sugars produced in leaves are transported around through the phloem vasculature. 
Structurally, plant tissues are divided into meristems (containing actively dividing cells, thus being the primary place of plant growth) and permanent tissues classified according to their shapes and intercellular space.

Long distance movement of molecules occurs through the aforementioned vascular system, which transports water, mineral, signaling molecules, nutrients, and other molecules. In particular, phloem is the main avenue for transporting organic molecules (discussed in (Atkins et al., 2011; De Schepper et al., 2013; Turgeon and Wolf, 2009)). There is a large number of mobile macromolecules in plants. In an effort to provide a systematic catalogue of mobile macromolecules, a database PlaMoM was compiled, which provides convenient and interactive search tools allowing users to retrieve, to analyze and also to predict mobile RNAs/proteins (Guan et al., 2017). The current version compiles a total of 17,991 mobile macromolecules from 14 plant species/ecotypes and is available at: http://www.systembioinfo.org/plamom/.

\section{Distribution of RNA silencing pathways across plant tissues}

Plants expressed multiple AGOs, DCLs and RDRs, which support different types of RNA silencing pathways employing different types of small RNAs (Fig. 1). Plant small RNAs are typically methylated at the 3' end, which protects them from degradation (Li et al., 2005). Plant RNA silencing pathways can be divided into miRNA and siRNA pathways, which use distinct small RNA substrates. From the perspective of small RNA populations originating from different substrates, two distinct types of small RNAs can be recognized when considering their sequence prediction (Fig. 3): The first type, exemplified by miRNAs, comprises small RNAs, which occur in cells in many identical copies (i.e. with the same sequence). The second type, exemplified by siRNAs derived from dsRNA, is characterized by existence of populations of small RNAs with variable sequences, which originate from a longer sequence. Although their sequences could be determined, their individual annotation is pointless. One kilobase of dsRNA theoretically produces nearly thousand small RNAs differing at their 5' ends where each one of them could regulate a different set of genes (there is 4096 possible of a hexamer sequence). These small RNAs usually function as a defense system operating on the basis (nearly) perfect complementarity.

miRNA (Fig. 4) and related pathways function post-transcriptionally and include the canonical miRNA pathway with 21nt miRNAs (utilizing DCL1 and AGO1) and its alternatives employing other DCL and AGO proteins and longer hairpin substrates.

siRNA pathways use either exogenous substrates (antiviral defense and transgene silencing, Fig. 5) or various dsRNAs derived from genomic sequences. Various biogenesis pathways produce primary and secondary siRNAs of various types and lengths $(21,22$, or $24 \mathrm{nt}$ ), which mediate post-transcriptional or transcriptional silencing. siRNA lengths are determined by the processing Dicer paralogue. $21 \mathrm{nt}$ siRNAs can be produced by DCL4 or DCL1, 22nt siRNAs by DCL2, and 24 nt by DCL3.

There is a large volume of literature concerning NGS analysis of small RNA populations from different tissues in different plant species. A large volume of NGS data can be accessed through Plant MPSS (massively parallel signature sequencing) databases website 


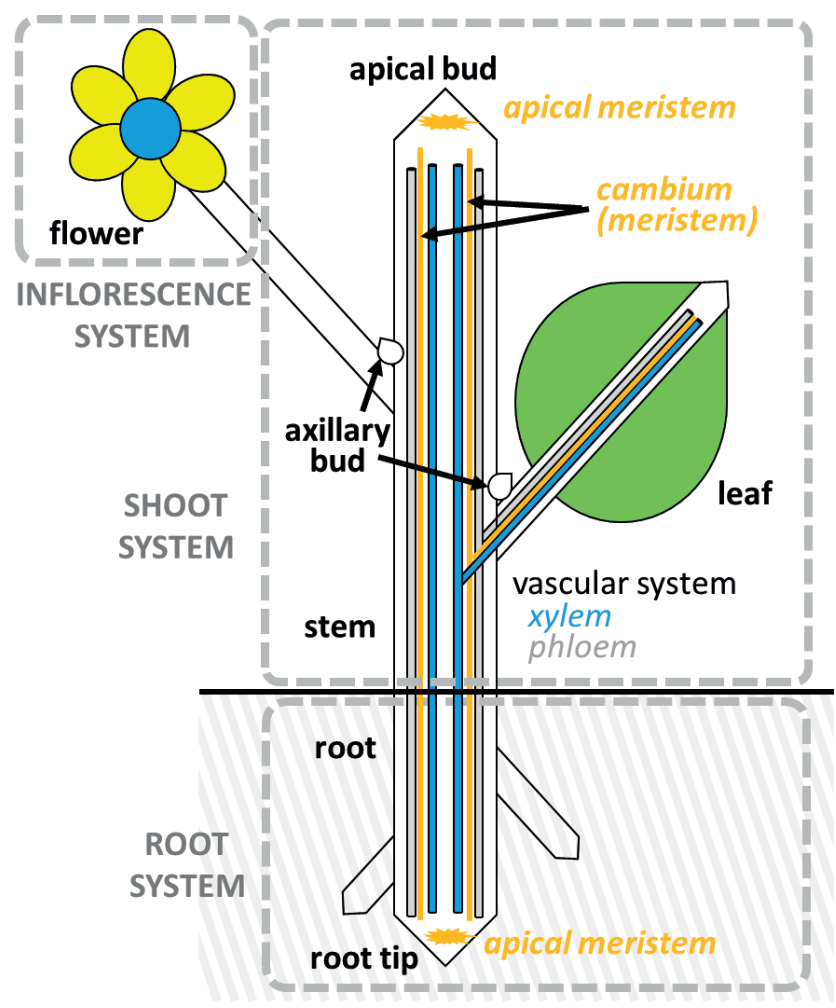

Figure 2 A schematic overview of plant body organization.

(https://mpss.danforthcenter.org/), which was introduced in 2005 (Nakano et al., 2006) and has been continuously updated and expanded. It currently contains small RNA data from 19 plant species. Most datasets in the database (six) come from analysis of small RNAs in rice. These rice datasets combine the expression atlas of rice mRNAs and small RNAs (Nobuta et al., 2007), with analysis of small RNAs during development where different pools of phasiRNAs were identified (Fei et al., 2016), a comprehensive analysis of small RNAs in different tissues under normal conditions and stress (Jeong et al., 2011) and unpublished NGS data from different rice tissues and AGO immunoprecipitates.

These and other data from rice (e.g. (Heisel et al., 2008)) reveal common and tissue-specific populations of small RNAs. The existence of tissue-specific small RNAs shows that systemic RNA silencing co-exists with RNA silencing mechanisms restricted to specific tissues. Several factors may underlie tissue specificity of particular small RNA mechanisms. These include (i) tissue-restricted expression of proteins involved in biogenesis and activity of particular small RNA class, (ii) tissue-restricted presence of substrates for biogenesis of small RNAs, and (iii) restriction of mobility/spreading of small RNAs, which could differ between different cell types. Next, I will briefly discuss selected specific examples, which provide an insight into the complexity of the issue: 


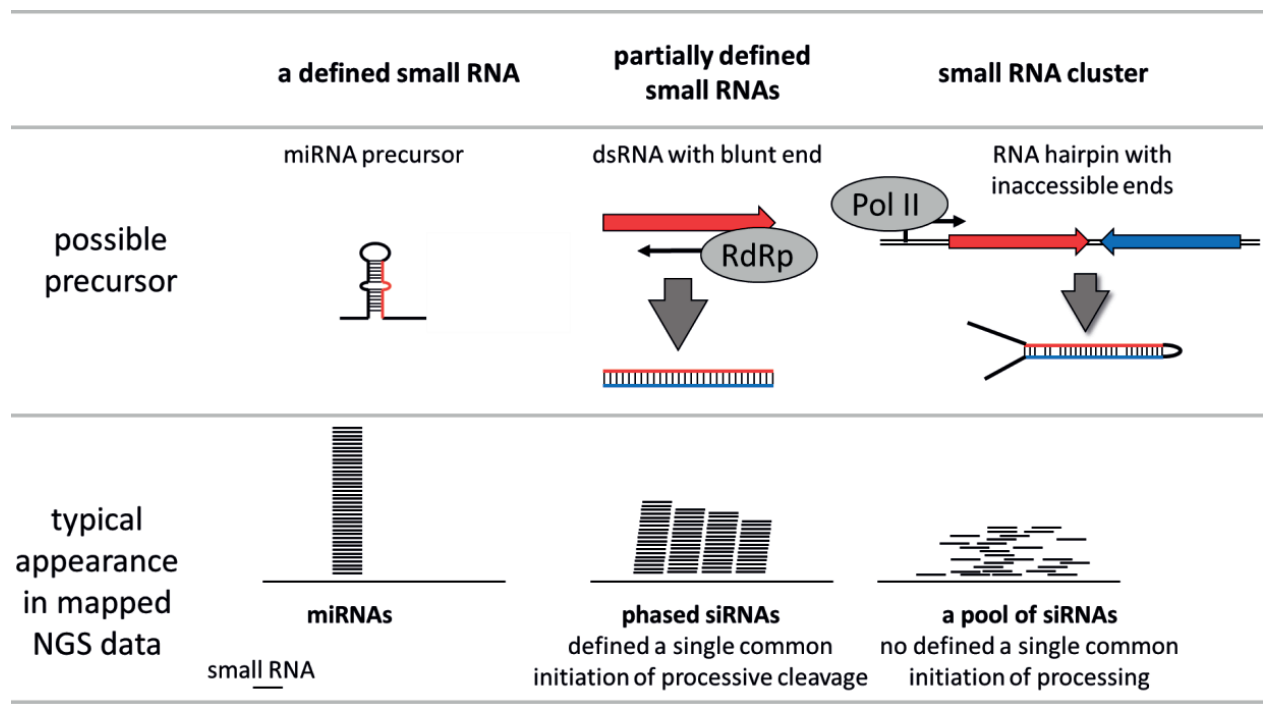

Figure 3 Distinct types of small RNAs differing in occurrence of RNAs with identical sequences

\section{Tissue-restricted expression of protein factors involved in small RNA biogenesis and activity}

While many genes encoding factors involved in small RNA biogenesis (e.g. Dicers, RDRs) and function (e.g. Argonautes) of small RNAs are ubiquitously expressed, some exhibit preferential or tissue restricted expression. For example, a systematic analysis of gene expression was done in Arabidopsis and rice (Kapoor et al., 2008), where microarray profiling identified several differentially expressed factors during development and in different tissues. Similar data could also be extracted from available NGS profiling of mRNAs at the aforementioned website (https://mpss.danforthcenter.org/). In general, these expression data have predictive value mainly for specific Argonaute paralogs, which host unique small RNA pools and have highly restricted expression patterns.

\section{Tissue-restricted presence of substrates for biogenesis of small RNAs}

This factor involves localization of exogenous small RNA substrates to different tissues (i.e. viral infections and transgene expression) or expression of endogenous small RNA substrates. This allows, for example to differentiate expression of subpopulations of small RNAs in time and space. A classic example is tissue-specific expression miRNAs, whose precursors are transcribed by polII polymerase and thus can exhibit tissue-restricted expression as mRNAs. As there is a large volume of the literature on miRNA expression in plants, which is beyond the scope of this report, I only select illustrative examples of analyses of tissue-specific expression of miRNAs in rice .(Mittal et al., 2013; Zhu et al., 2008), Nicotiana (Valoczi et al., 2006), and Arabidopsis (Grant-Downton et al., 2009; Valoczi et al., 
2006). Tissue-specific expression of small RNAs can be observed for tasiRNAs, which originate from specific loci (Marin et al., 2010; Zabala et al., 2012) or phasiRNA, where a specific miRNA initiates production of phasiRNAs (Fei et al., 2016).

\section{Restriction of mobility/spreading of small RNAs.}

Restriction of mobility assures that tissue/cell-specific expression will remain contained. There are several cell types, from which small RNAs do not seem to be spreading around. These include guard cells of stomata (Voinnet et al., 1998), endosperm (energy storage of the seed) (Hournard et al., 2007) or the seed coat (Tuteja et al., 2009).

\section{Movement of small RNAs between cells and tissues - miRNA mobility}

miRNAs can also be transported over long distances but this is not a universal rule for all miRNAs (Buhtz et al., 2008; de Felippes et al., 2011; Knauer et al., 2013; Lin et al., 2008). Analysis of the phloem sap of oilseed rape Brassica napus identified 32 annotated microRNAs (miRNAs) from 18 different families (Buhtz et al., 2008). In addition, the levels of three mature miRNAs known to respond to nutrient deprivation in non-vascular tissue, MIR395 (sulphate), MIR398 (copper) and MIR399 (phosphate), were increased in phloem sap during the growth of plants under the respective nutrient deprivation (Buhtz et al., 2008). Other known mobile miRNAs in Arabidopsis are MIR394, which is produced by the surface cell layer and contributes to shoot meristem formation (Knauer et al., 2013), and MIR165, which regulates differentiation (Miyashima et al., 2011).

MIR395 and MIR399 mobility through phloem was demonstrated in grafting experiments while MIR171 was not transported (Buhtz et al., 2010; Pant et al., 2008). Consistent with phloem movement, another analysis of miR399 movement using reciprocal grafting in Arabidopsis suggested that it moves from shoots to roots (Lin et al., 2008).

The basis of selectivity underlying miRNA mobility in plants remains unclear. It was shown $22 \mathrm{nt}$ artificial miRNAs derived from asymmetric duplexes mediate widespread silencing of their cognate gene more efficiently than $21 \mathrm{nt}$ siRNAs from symmetric duplexes (McHale et al., 2013).

\section{Movement of small RNAs between cells and tissues - systemic RNAi in plants}

RNAi can either act in a cell autonomous manner, i.e. affecting only cells directly exposed to dsRNA, or can propagate across cell boundaries. Early observations of co-suppression in petunia (Jorgensen, 1995; Napoli et al., 1990) and antiviral resistance in Nicotiana benthamiana (Ratcliff et al., 1997) suggested that RNA silencing in plants includes a mobile silencing signal. Several studies subsequently confirmed that transgene-induced silencing is mobile (Palauqui et al., 1997; Voinnet and Baulcombe, 1997; Voinnet et al., 1998, 2016). These experiments used grafting and agroinfiltration strategies to show that silencing 
spreads from the lower silenced leaves to the upper non-silenced leaves (Palauqui et al., 1997; Voinnet and Baulcombe, 1997). Based on the distance of silencing spread, short range and long range/systemic spread can be distinguished (Fig. 6).

\section{Short range silencing}

Short range silencing manifests as RNA silencing in a defined area. It was shown that a short-distance spreading of RNA silencing, once initiated from a small group of cells, can spread over 10-15 cells independently of the presence of cognate transcripts (Himber et al., 2003). Short range silencing was observed for both transgenes and endogenous genes (Dunoyer et al., 2007; Kalantidis et al., 2006; Ryabov et al., 2004; Schwach et al., 2005; Smith et al., 2007).

Regarding the nature of the short-range mobile signal, it is still a matter of debate. Initially, it was proposed that short range spreading involves DCL3-dependent 24nt siRNAs (Hamilton et al., 2002; Molnar et al., 2010). Subsequently, DCL4-dependent $21 \mathrm{nt}$ siRNA were also implicated in short-range spreading (Dunoyer et al., 2007; Dunoyer et al., 2005; Himber et al., 2003; Smith et al., 2007). It was shown that they are sufficient for the limited moving of the silencing and that the short range silencing is independent of an RdRP SDE1 and helicase SDE3 (Himber et al., 2003). Furthermore, it was suggested that the mobile signal is are siRNAs themselves ant not their longer precursors and AGO-bound single strand molecules but this evidence came in one of the recently retracted papers (discussed in Addendum at the end of the review).

Besides 21 siRNAs, several other types of plant small RNAs can spread cell-to-cell. These include miRNAs (discussed separately further below), tasiRNAs of the TAS3 locus (Chitwood et al., 2009; de Felippes et al., 2011; Marin et al., 2010; Schwab et al., 2009).

In terms of the mechanism of cell-to-cell silencing movement, early studies implied that post-transcriptional silencing spreads through plasmodesmata (Palauqui et al., 1997; Voinnet et al., 1998). However, the amount of evidence for cell-to-cell silencing movement through plasmodesmata is rather moderate. Support for the symplastic movement through plasmodesmata comes from an observation that symplastically isolated guard cells of stomata escape short-range spreading (Himber et al., 2003; Kalantidis et al., 2006; Voinnet et al., 1998). Furthermore, analysis of short-range spreading in Arabidopsis embryos, suggested that the spread is affected by and positively correlates with plasmodesma aperture (Kobayashi and Zambryski, 2007). While these results are in agreement with the assumption that short-range spreading of silencing occurs through diffusion and plasmodesmata, alternative routes, such as secretory vesicles should still not be excluded.

In addition, genetic analysis revealed several factors involved for cell-to-cell silencing movement (summarized in Table 1). Some of these factors were already mentioned above. Interestingly, among the factors whose mutations reduce cell-to-cell silencing movements were also RDR2 CLSY1, and NRPD1a, which are required for 24nt siRNA-mediated transcriptional silencing (Dunoyer et al., 2007; Smith et al., 2007). It was suggested that these factors might function in reception and/or downstream functional integration of mobile siRNAs in recipient cells (Brosnan and Voinnet, 2011). Another nuclear factor whose mutation affects cell-to-cell silencing movement is JMJ14, a H3K4 histone 


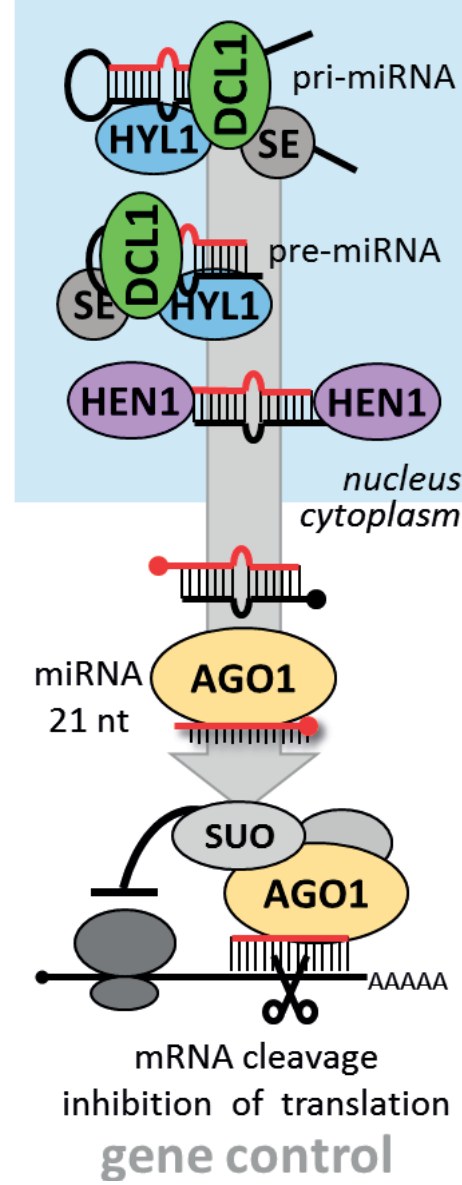

Figure 4 Plant miRNA pathway

demethylase implicated in non-CpG DNA methylation (Searle et al., 2010). However, short-range movement of tasiRNAs does not seem to require RDR2, CLSY1, NRPD1a, and JMJ14.

Taken together, while there is evidence that DCL4-dependent $21 \mathrm{nt}$ siRNAs are the mobile signal in cell-to-cell movement, cell-to-cell silencing mobility might also concern DCL3-dependent 24-nt siRNAs and other RNA molecules.

\section{Extended short-range silencing}

In addition to short-range spreading of RNA silencing, a long-range cell-to-cell movement process was also reported that occurs as a relay amplification, involving the combined activity of an RdRP SDE1 and helicase SDE3 (Himber et al., 2003). This is essentially an extended version of short-range silencing described above where the mobile signal is amplified along the way through production of secondary small RNAs (Fig. 6). This amplification process, is known as "transitivity" (Melnyk et al., 2011b). Thus, extensive and limited cell-to-cell movements of silencing are triggered by the same molecules, occur within the same tissues and likely recruit the same plasmodesmata channels (Himber et al., 2003).

\section{Long range/systemic silencing}

Plants exhibit systemic RNA silencing, which can move over long distance. This implies that a mobile silencing signal from the source tissue enters the vascular system, it is transported and then enters the recipient tissue or cells (Fig. 7). Early studies implied that post-transcriptional silencing spreads over long distance through phloem (Palauqui et al., 1997; Voinnet et al., 1998) (of note is that xylem sap, which transports water and ions, does not carry RNA (Buhtz et al., 2008)).

It was shown that transgene-specific post-transcriptional silencing is transmitted by grafting from silenced stocks to non-silenced scions (Palauqui et al., 1997). The transmission of co-suppression occurs when silenced stocks and non-silenced target scions are physically separated by up to $30 \mathrm{~cm}$ of stem lacking cognate RNAs (Palauqui et al., 1997). The systemic movement of the silencing signal takes days and typically moves from leaves (photosynthetic source) to roots and growing points (sucrose sinks) (Voinnet et al., 1998); this flow is characteristic of phloem (discussed in (De Schepper et al., 2013; Turgeon and Wolf, 2009)). Furthermore, phloem transport block by cadmium inhibits systemic silencing 


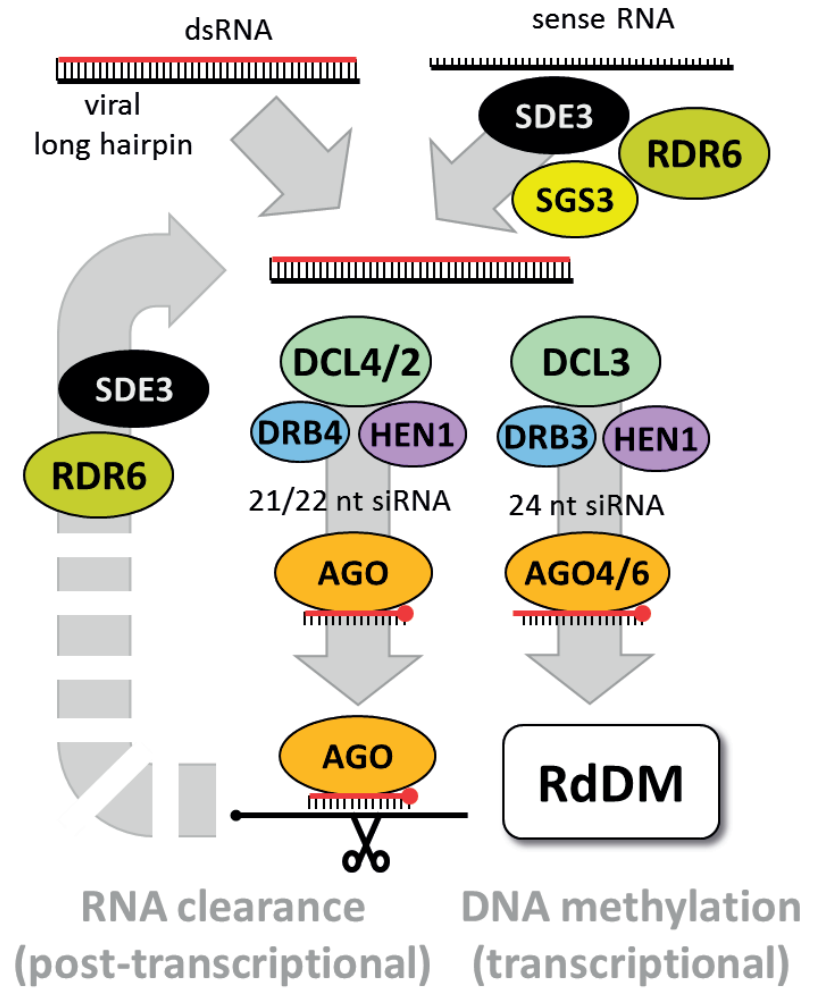

Figure 5 "RNAi module" of RNA silencing in Arabidopsis.

The key step in the RNAi module is conversion of different dsRNA into small RNAs by one of the DCL proteins, among which dominate DCL2/3/4. Some dsRNA is produced by RDR6 either as the initial trigger or as an amplification step where AGO-targeted RNAs are converted to dsRNA, which is processed into secondary siRNAs. If the targeting by AGO is precisely defined (e.g. by miRNA), the secondary siRNAs would be phased. DCL3-generated $24 \mathrm{nt}$ siRNAs can induce RdDM.

spread (Beclin et al., 1998; Ghoshroy et al., 1998). Later, it was also shown that phloem flow strongly influences the systemic spread of silencing in Nicotiana benthamiana and that the direction of systemic spread of silencing from inducer to sensor can be manipulated by altering sink/source relations in the plant (Tournier et al., 2006). Thus, systemic silencing is not an accidental stochastic process, but can be predicted according to the phloem flow direction (Tournier et al., 2006).

The systemic mobile signal could be various forms of RNA - small RNAs or their precursors. Identification of the systemic RNA silencing signal included analyses of the phloem sap and RNA behaviour upon various grafting experiments

Analysis of phloem sap found presence of siRNAs and miRNAs (Buhtz et al., 2008; Yoo et al., 2004). Subsequent grafting experiments suggested that all classes of small RNAs could be mobile while the systemic silencing signal was proposed to be the $24 \mathrm{nt}$ siRNA species (Himber et al., 2003; Melnyk et al., 2011a; Molnar et al., 2010). However, grafting 

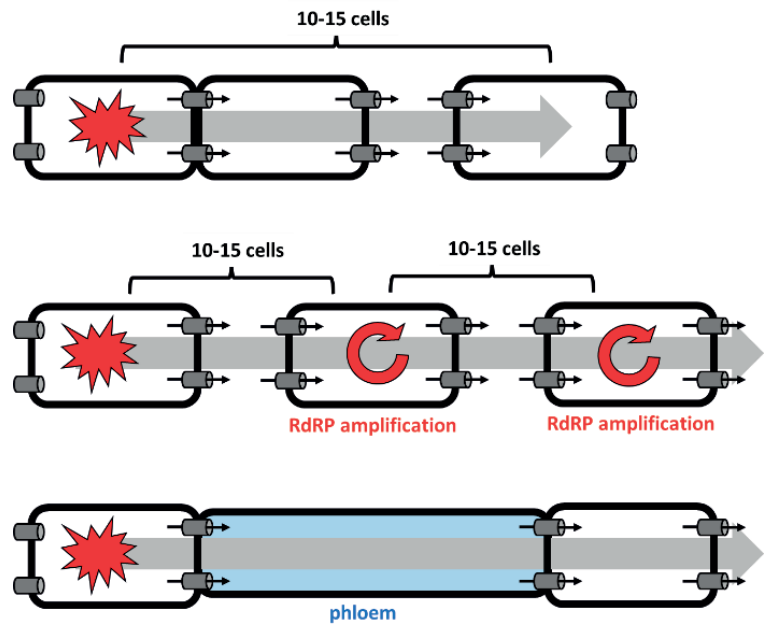

Figure 6 Movement of RNA silencing in plants.

In short-distance cell-to-cell movement, small RNAs move from the source cells where silencing was initiated (explosion pictogram) through plasmodesmata (symplastic route). Plasmodesmata are schematically depicted as tunnels in cell walls. Mobility is limited to $10-15$ cells. In long-range cell-cell movement, small RNA move again via plasmodesmata. Amplification (cycling arrow) of small RNAs by transitive small RNA production utilizing an RdRP activity extends the range of mobility far beyond the first 10-15 cells. In systemic movement, small RNAs enter the phloem and are transported to distant tissues

experiment with rootstocks lacking DCL2, 3, and 4 showed that such rootstocks still transmit the systemic silencing signal (Brosnan et al., 2007). This suggests that the silencing signal could be either long dsRNA precursor or siRNAs generated by DCL1, which normally generates miRNAs. Mobility of small RNAs was supported by NGS analysis of tissues lacking DCL2, 3, and 4, which could identify long dsRNA-derived 21, 22, and 24nt siRNAs (Molnar et al., 2010). Furthermore, the situation is complicated by secondary siRNAs, which emerge in the systemically silenced scion and degrade the target mRNA (Shimamura et al., 2007).

In any case, it is generally accepted that 24nt siRNA species are associated with systemic RNA silencing in plants but little is known concerning the exact form of systematically moving silencing RNAs. Since phloem transportation can accommodate relatively large molecules including mRNAs (reviewed in (Atkins et al., 2011; Hannapel et al., 2013)) mobility of small RNA precursors should not be excluded.

\section{Protein factors involved in systemic RNA silencing}

Consistent with involvement of 24nt siRNAs, four genes involved in a chromatin silencing pathway;NRPD1a, RDR2, and DCL3, are required for reception of long-distance mRNA silencing and an AGO4 mutant is also partially compromised in the reception of silencing (Brosnan et al., 2007). These data were corroborated by another study, which linked CLSY1, AGO4, RDR2, and NRPD1a to silencing spread (Smith et al., 2007). DCL3 appears to be the 
only DCL protein essential for systemic silencing (Melnyk et al., 2011a). In addition, RDR6 was implicated in systemic silencing where it seems to be involved in the perception of the silencing signal (Schwach et al., 2005) and its maintenance (Himber et al., 2003; Vaistij et al., 2002). RDR6 is amplifying decapped mRNAs cleaved by $24 \mathrm{nt}$ siRNAs. Subsequently. DCL4 and DCL2 act hierarchically to produce 21- and 22-nt siRNAs guiding mRNA cleavage mRNA degradation (Brosnan et al., 2007). Taken together, it appears that the systemic silencing employs both, post-transcriptional and transcriptional RNA silencing mechanisms. However, their integration in the systemic silencing process is still a matter of debate.

Interestingly, biochemical analysis of pumpkin (Cucurbita maxima) identified CmPSRP1 (PHLOEM SMALL RNA BINDING PROTEIN 1), which binds 25nt single-strand small RNAs in pumpkin and is a candidate protein for trafficking small single-strand RNA molecules (Yoo et al., 2004). Although there is additional supporting biochemical evidence for the role of CmPSRP1 in systemic silencing, there is no genetic support and its homologues across the plant kingdom are yet to be identified (Ham et al., 2014).

\section{Directionality of systemic RNA silencing}

Systemic silencing frequently flows in the common source-to-sink direction. This, however, does not preclude spreading in the opposite direction, which has also been observed. Below, I will discuss directionality of spreading between main plant parts.

\section{Shoot-to-root}

Depletion of shoot small RNAs corresponded to reduction of 24-nt small RNAs in wildtype roots, indicating that shoot-derived small RNAs contribute to the total root small RNA population as small RNAs in these grafted plants move predominantly from shoot to root following source-sink gradients (Molnar et al., 2010). This is was corroborated by a later study, which showed in Arabidopsis thaliana that 24-nt small RNAs are mobile from shoot to root (Melnyk et al., 2011b). This was further extended by showing that RNA-dependent DNA methylation in root tissues (occurring predominantly in non-CpG contexts) depends on mobile small RNAs from the shoot (Lewsey et al., 2016).

\section{Root-to-shoot:}

Upward (root-to-shoot) systemic silencing has also been reported from both, Nicotiana and Arabidopsis (Kasai et al., 2011; Kasai et al., 2013; Liang et al., 2012). For example, post-transcriptional silencing of an endogenous gene in the shoot was observed in Nicotiana when a wild-type scion was grafted on a rootstock synthesizing a siRNA signal (Kasai et al., 2011). The signal can spread from a rootstock can travel quite far as demonstrated in cherry, where siRNAs were detected $1.2 \mathrm{~m}$ from the graft union (Zhao and Song, 2014).

To what extent this type of spreading employs phloem is not clear. As mentioned above, while phloem flow the direction of systemic spread, it can be manipulated (Tournier et al., 2006). However, a study in Arabidopsis suggested that root-to-shoot signal movement might occur through plasmodesmata and not through the phloem (Liang et al., 2012). 


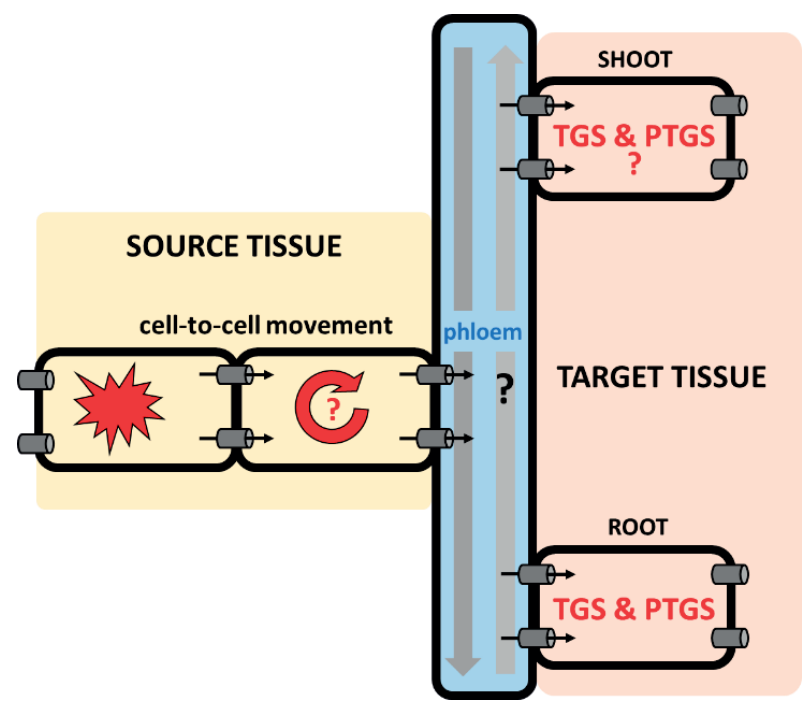

Figure 7 Systemic silencing mobility

Systemic silencing is initiated in the source tissue. The mobile silencing signal enters phloem and moves to the target tissue. Systemic silencing can occur in both directions, i.e. shoot-to-root and rootto-shoot. The shoot-to-root direction is following the common path of sugars to "sink" tissues. However, there is also evidence that systemic silencing can propagate in root-to-shoot direction.

Importantly, root-to-shoot spreading of RNA silencing is not a universal plant mechanism in vivo. It was shown that by RNA-mediated gene silencing signals are not graft transmissible from the rootstock to the scion in greenhouse-grown apple plants Malus sp. (Flachowsky et al., 2012). Further research is needed to reveal the molecular mechanisms and biological role(s) of the root-to-shoot (rootstock-scion) transmission of RNA silencing.

\section{Flower/seed concerning silencing spreading}

Some of the references addressed spreading of silencing in reproductive organs and seeds. It was shown that gamete formation requires AGO9, which controls female gamete formation in a non-cell autonomous manner (Olmedo-Monfil et al., 2010). In grafted Arabidopsis and Nicotiana the systemic silencing penetrated male sporogenic tissues suggesting that plants harbour an endogenous long-distance small RNA transport pathway facilitating siRNA signalling into meiotically active cells. (Zhang et al., 2014). Regarding seeds, an experiment targeting RNAi to the endosperm showed that RNAi was restricted to the endosperm tissue and that transitive RNAi did not occur (Hournard et al., 2007). Similarly, RNAi was observed to remain contained in the seed coat and did not spread to cotyledons and vegetative tissues (Tuteja et al., 2009). 
Table 1 Mutations that affect the cell-to-cell RNA silencing spreading phenotype in Arabidopsis adopted from (Melnyk et al., 2011b)

\begin{tabular}{|c|c|c|c|c|}
\hline \multicolumn{5}{|c|}{ Mutations that affect the cell-to-cell RNA silencing spreading phenotype in Arabidopsis } \\
\hline \multicolumn{5}{|c|}{ Mutations that reduce RNA silencing spread } \\
\hline mutation & type & gene & note & reference \\
\hline agol & inhib. & AGO1 & Argonaute, $21 \mathrm{nt}$ siRNA pathway & (Dunoyer et al., 2007) \\
\hline clsyl & inhib. & CLSY1 & SNF2-domain-containing protein & (Smith et al., 2007) \\
\hline$d c l 1$ & inhib. & DCL1 & $\begin{array}{l}\text { Dicer, miRNA \& } 21 \mathrm{nt} \text { siRNA } \\
\text { pathway }\end{array}$ & (Dunoyer et al., 2007) \\
\hline$d c l 4$ & inhib. & DCL4 & Dicer, $21 \mathrm{nt}$ siRNA pathway & (Dunoyer et al., 2005; Smith et al., 2007) \\
\hline$f_{c a}$ & inhib. & FCA & & (Baurle et al., 2007) \\
\hline$f p a$ & inhib. & FPA & & (Baurle et al., 2007) \\
\hline fy & inhib. & FY & & (Manzano et al., 2009) \\
\hline jmj14 & inhib. & JMJ14 & $\begin{array}{l}\text { histone H3K4 demethylase } \\
\text { linked to non-CpG methylation }\end{array}$ & (Searle et al., 2010) \\
\hline henl & inhib. & HEN1 & small RNA methylase & (Dunoyer et al., 2007) \\
\hline hprl & inhib. & HPR1 & RNA trafficking protein & (Jauvion et al., 2010) \\
\hline $\begin{array}{l}\text { nrpdl/ } \\
\text { smdl }\end{array}$ & inhib. & NPRD1 & plant RNA pol-IV component & $\begin{array}{l}\text { (Brosnan et al., 2007; Dunoyer et al., } \\
\text { 2007; Dunoyer et al., 2005; Smith et al., } \\
\text { 2007) }\end{array}$ \\
\hline$n r p d 2 a$ & inhib. & NRPDA2 & & (Smith et al., 2007) \\
\hline$r d r 2 / s m d 2$ & inhib. & RDR2 & plant RNA pol-IV component & $\begin{array}{l}\text { (Brosnan et al., 2007; Dunoyer et al., } \\
\text { 2007; Dunoyer et al., 2005; Smith et al., } \\
\text { 2007) }\end{array}$ \\
\hline $\operatorname{smd3}$ & inhib. & SMD3 & silencing movement deficient & (Dunoyer et al., 2005) \\
\hline texl & stim & TEX1 & mRNA export complex & (Yelina et al., 2010) \\
\hline \multirow[t]{2}{*}{$\operatorname{ago4}$} & stim & \multirow[t]{2}{*}{ AGO4 } & Argonaute, & (Searle et al., 2010; Smith et al., 2007) \\
\hline & inhib & & $\begin{array}{l}\text { partially compromised reception } \\
\text { of silencin }\end{array}$ & (Brosnan et al., 2007) \\
\hline \multirow[t]{2}{*}{$d c l 3^{b}$} & stim & \multirow[t]{2}{*}{ DCL3 } & & (Smith et al., 2007) \\
\hline & inhib & & & (Brosnan et al., 2007) \\
\hline
\end{tabular}

\section{Summary}

Taken together, it seems that there is no an exclusive mobile small RNA species. Rather, there may be more forms of small mobile RNAs. At the moment, there is no definite consensus on specific mechanism(s) regulating sorting of small RNAs into mobile and cell-autonomous small RNAs.

\section{Addendum}

I feel obliged to mention an incident which stirred the RNA silencing field and affected the literature on systemic RNA silencing in plants. In January 2015, a number of publications 
authored by Olivier Voinnet was brought up as potentially containing manipulated images at a server PubPeer.com. Among these were highly cited publications that laid foundations of RNA silencing research in plants. A subsequent ethical committee investigation concluded that publications indeed contained manipulated figures. the following Voinnet's publications had to be corrected or retracted:

\section{Retractions:}

1. Brigneti G.et al.,EMBO J (1998)

2. Voinnet, O.et al., Plant J. 33: 949-56 (2003)

3. Dunoyer P.et al., Plant Cell 16: 1235-50 (2004)

4. Moissiard G. and Voinnet O., Proc. Nat. Acad. Sci.USA 103 (51): 19593 (2006)

5. Dunoyer P.et al., EMBO J (2010)

6. Dunoyer P. et al., Science 328 (5980), 912 (2010)

7. Ciaudo C.et al., PLoS Genetics 9(11): e1003791 (2013)

8. Sansregret R.et al., PLoS Pathogens 1(9): e1005207 (2013)

\section{Corrections:}

1. Ruiz MT et al, Plant Cell10: 937-946 (1998)

2. Voinnet O, et al., Nat.l Acad. Sci.USA 96:14147-14152; (1999)

4. Dunoyer P.et al., Plant J. 29: 555-67 (2002)

3. Hamilton A.et al., EMBO J (2002)

5. Parizotto E.A.et al., Genes \& Development 18: 2237-42 (2004) - 2nd

Correction:

8. Deleris A. et al, Science 313(5783): 68-71 (2006)

6. Dunoyer P.et al., Nature Genetics 38: 258-63 (2006)

7. Navarro L. et al., Science 312(5772): 436-439 (2006)

9. Moissiard et al, RNA 13: 1268-1278 (2007)

10. Haas G. et al, EMBO J 27, 2102-2112 (2008)

11. Ciaudo C.et al., PLoS Genetics 5(8): e1000620 (2009)

12. Bennasser Y et al., Nat. Struct. Mol. Biol. 18, 323-327 (2011)

13. Jay F, et al. (2011) PLoS Pathog 7(5): e1002035

14. Brodersen P.et al., Proc. Nat.l Acad. Sci. USA 109: 1778-83 (2012)

15. Gibbings D. et al, Nature Cell Biology 14, 1314-1321 (2012)

16. Schott G. et al, EMBO J 31, 2553-2565 (2012)

17. Boccara M.,et al., PLoS Pathogens 10(1): e1003883 (2014)

Further details about the affair can be found, for example, here: http://retractionwatch.com/category/by-author/olivier-voinnet/ http://labtimes.org/editorial/e_594.lasso http://labtimes.org/editorial/e_600.lasso http://labtimes.org/editorial/e_623.lasso http://labtimes.org/editorial/e_624.lasso 


\section{Acknowledgement}

I would like to thank my colleagues Jan Paces, Miloslav Nic, and Tomas Novotny for help with collecting literature for the review. The review content was produced under a contract OC/EFSA/GMO/2015/01-CT 01 with European Food Safety Authority (EFSA); the opinions expressed are those of the contractor only and do not represent EFSA's official position. Publication of the review was funded by LO1220 and LM2015063 by the Ministry of Education, Youth and Sports.

\section{References}

Atkins, C.A., Smith, P.M., and Rodriguez-Medina, C. (2011). Macromolecules in phloem exudates--a review. Protoplasma 248, 165-172.

Baurle, I., Smith, L., Baulcombe, D.C., and Dean, C. (2007). Widespread role for the flowering-time regulators FCA and FPA in RNA-mediated chromatin silencing. Science 318, 109-112.

Beclin, C., Berthome, R., Palauqui, J.C., Tepfer, M., and Vaucheret, H. (1998). Infection of tobacco or Arabidopsis plants by CMV counteracts systemic post-transcriptional silencing of nonviral (trans) genes. Virology 252, 313-317.

Borges, F., and Martienssen, R.A. (2015). The expanding world of small RNAs in plants. Nature Reviews Molecular Cell Biology 16, 727-741.

Brosnan, C.A., Mitter, N., Christie, M., Smith, N.A., Waterhouse, P.M., and Carroll, B.J. (2007). Nuclear gene silencing directs reception of long-distance mRNA silencing in Arabidopsis. America 104, 14741-14746.

Brosnan, C.A., and Voinnet, O. (2011). Cell-to-cell and long-distance siRNA movement in plants: mechanisms and biological implications. Curr Opin Plant Biol 14, 580-587.

Buhtz, A., Pieritz, J., Springer, F., and Kehr, J. (2010). Phloem small RNAs, nutrient stress responses, and systemic mobility. BMC Plant Biol 10, 64 .

Buhtz, A., Springer, F., Chappell, L., Baulcombe, D.C., and Kehr, J. (2008). Identification and characterization of small RNAs from the phloem of Brassica napus. Plant J 53, 739-749.

Chitwood, D.H., Nogueira, F.T., Howell, M.D., Montgomery, T.A., Carrington, J.C., and Timmermans, M.C. (2009). Pattern formation via small RNA mobility. Genes Dev 23, 549-554.

de Felippes, F.F., Ott, F., and Weigel, D. (2011). Comparative analysis of non-autonomous effects of tasiRNAs and miRNAs in Arabidopsis thaliana. Nucleic Acids Research 39, 2880-2889.

De Schepper, V., De Swaef, T., Bauweraerts, I., and Steppe, K. (2013). Phloem transport: a review of mechanisms and controls. J Exp Bot 64, 4839-4850.

Dunoyer, P., Himber, C., Ruiz-Ferrer, V., Alioua, A., and Voinnet, O. (2007). Intra- and intercellular RNA interference in Arabidopsis thaliana requires components of the microRNA and heterochromatic silencing pathways. Nature Genetics 39, 848-856.

Dunoyer, P., Himber, C., and Voinnet, O. (2005). DICER-LIKE 4 is required for RNA interference and produces the 21-nucleotide small interfering RNA component of the plant cell-to-cell silencing signal. Nature Genetics 37, 1356-1360. 
Fei, Q., Yang, L., Liang, W., Zhang, D., and Meyers, B.C. (2016). Dynamic changes of small RNAs in rice spikelet development reveal specialized reproductive phasiRNA pathways. J Exp Bot 67, 6037-6049.

Flachowsky, H., Trankner, C., Szankowski, I., Waidmann, S., Hanke, M.V., Treutter, D., and Fischer, T.C. (2012). RNA-mediated gene silencing signals are not graft transmissible from the rootstock to the scion in greenhouse-grown apple plants Malus sp. Int J Mol Sci 13, 9992-10009.

Ghoshroy, S., Freedman, K., Lartey, R., and Citovsky, V. (1998). Inhibition of plant viral systemic infection by non-toxic concentrations of cadmium. Plant J 13, 591-602.

Grant-Downton, R., Le Trionnaire, G., Schmid, R., Rodriguez-Enriquez, J., Hafidh, S., Mehdi, S., Twell, D., and Dickinson, H. (2009). MicroRNA and tasiRNA diversity in mature pollen of Arabidopsis thaliana. Bmc Genomics 10, 643-643.

Guan, D., Yan, B., Thieme, C., Hua, J., Zhu, H., Boheler, K.R., Zhao, Z., Kragler, F., Xia, Y., and Zhang, S. (2017). PlaMoM: a comprehensive database compiles plant mobile macromolecules. Nucleic Acids Res 45, D1021-D1028.

Ham, B.K., Li, G., Jia, W., Leary, J.A., and Lucas, W.J. (2014). Systemic delivery of siRNA in pumpkin by a plant PHLOEM SMALL RNA-BINDING PROTEIN 1-ribonucleoprotein complex. Plant J 80, 683-694.

Hamilton, A., Voinnet, O., Chappell, L., and Baulcombe, D. (2002). Two classes of short interfering RNA in RNA silencing. Embo Journal 21, 4671-4679.

Hannapel, D.J., Sharma, P., and Lin, T. (2013). Phloem-mobile messenger RNAs and root development. Front Plant Sci 4, 257.

Heisel, S.E., Zhang, Y.J., Allen, E., Guo, L., Reynolds, T.L., Yang, X., Kovalic, D., and Roberts, J.K. (2008). Characterization of Unique Small RNA Populations from Rice Grain. Plos One 3, e2871-e2871.

Himber, C., Dunoyer, P., Moissiard, G., Ritzenthaler, C., and Voinnet, O. (2003). Transitivity-dependent and -independent cell-to-cell movement of RNA silencing. The EMBO journal 22, 4523-4533.

Hournard, N.M., Mainville, J.L., Bonin, C.P., Huang, S., Luethy, M.H., and Malvar, T.M. (2007). High-lysine corn generated by endosperm-specific suppression of lysine catabolism using RNAi. Plant Biotechnology Journal 5, 605-614.

Jauvion, V., Elmayan, T., and Vaucheret, H. (2010). The conserved RNA trafficking proteins HPR1 and TEX1 are involved in the production of endogenous and exogenous small interfering RNA in Arabidopsis. Plant Cell 22, 2697-2709.

Jeong, D.H., Park, S., Zhai, J., Gurazada, S.G., De Paoli, E., Meyers, B.C., and Green, P.J. (2011). Massive analysis of rice small RNAs: mechanistic implications of regulated microRNAs and variants for differential target RNA cleavage. Plant Cell 23, 4185-4207.

Jorgensen, R.A. (1995). Cosuppression, flower color patterns, and metastable gene expression States. Science 268, 686-691.

Kalantidis, K., Tsagris, M., and Tabler, M. (2006). Spontaneous short-range silencing of a GFP transgene in Nicotiana benthamiana is possibly mediated by small quantities of siRNA that do not trigger systemic silencing. Plant Journal 45, 1006-1016.

Kapoor, M., Arora, R., Lama, T., Nijhawan, A., Khurana, J.P., Tyagi, A.K., and Kapoor, S. (2008). Genome-wide identification, organization and phylogenetic analysis of Dicer-like, Argonaute and RNA-dependent RNA Polymerase gene families and their expression analysis during reproductive development and stress in rice. BMC Genomics 9, 451. 
Kasai, A., Bai, S.L., Li, T.Z., and Harada, T. (2011). Graft-Transmitted siRNA Signal from the Root Induces Visual Manifestation of Endogenous Post-Transcriptional Gene Silencing in the Scion. Plos One 6, e16895-e16895.

Kasai, A., Sano, T., and Harada, T. (2013). Scion on a Stock Producing siRNAs of Potato Spindle Tuber Viroid (PSTVd) Attenuates Accumulation of the Viroid. Plos One 8, e57736-e57736.

Knauer, S., Holt, A.L., Rubio-Somoza, I., Tucker, E.J., Hinze, A., Pisch, M., Javelle, M., Timmermans, M.C., Tucker, M.R., and Laux, T. (2013). A Protodermal miR394 Signal Defines a Region of Stem Cell Competence in the Arabidopsis Shoot Meristem. Developmental Cell 24, 125-132.

Kobayashi, K., and Zambryski, P. (2007). RNA silencing and its cell-to-cell spread during Arabidopsis embryogenesis. Plant Journal 50, 597-604.

Lewsey, M.G., Hardcastle, T.J., Melnyk, C.W., Molnar, A., Valli, A., Urich, M.A., Nery, J.R., Baulcombe, D.C., and Ecker, J.R. (2016). Mobile small RNAs regulate genome-wide DNA methylation. America 113, E801-E810.

Li, J., Yang, Z., Yu, B., Liu, J., and Chen, X. (2005). Methylation protects miRNAs and siRNAs from a 3'-end uridylation activity in Arabidopsis. Curr Biol 15, 1501-1507.

Liang, D.C., White, R.G., and Waterhouse, P.M. (2012). Gene Silencing in Arabidopsis Spreads from the Root to the Shoot, through a Gating Barrier, by Template-Dependent, Nonvascular, Cell-to-Cell Movement. Plant Physiology 159, 984-1000.

Lin, S.I., Chiang, S.F., Lin, W.Y., Chen, J.W., Tseng, C.Y., Wu, P.C., and Chiou, T.J. (2008). Regulatory network of microRNA399 and PHO2 by systemic signaling. Plant Physiology 147, 732-746.

Manzano, D., Marquardt, S., Jones, A.M., Baurle, I., Liu, F., and Dean, C. (2009). Altered interactions within FY/AtCPSF complexes required for Arabidopsis FCA-mediated chromatin silencing. Proc Natl Acad Sci U S A 106, 8772-8777.

Marin, E., Jouannet, V., Herz, A., Lokerse, A.S., Weijers, D., Vaucheret, H., Nussaume, L., Crespi, M.D., and Maizel, A. (2010). miR390, Arabidopsis TAS3 tasiRNAs, and their AUXIN RESPONSE FACTOR targets define an autoregulatory network quantitatively regulating lateral root growth. Plant Cell 22, 1104-1117.

Maule, A.J. (2008). Plasmodesmata: structure, function and biogenesis. Curr Opin Plant Biol 11, 680-686.

Maule, A.J., Benitez-Alfonso, Y., and Faulkner, C. (2011). Plasmodesmata - membrane tunnels with attitude. Curr Opin Plant Biol 14, 683-690.

McHale, M., Eamens, A.L., Finnegan, E.J., and Waterhouse, P.M. (2013). A 22-nt artificial microRNA mediates widespread RNA silencing in Arabidopsis. Plant Journal 76, 519-529.

Melnyk, C.W., Molnar, A., Bassett, A., and Baulcombe, D.C. (2011a). Mobile 24 nt Small RNAs Direct Transcriptional Gene Silencing in the Root Meristems of Arabidopsis thaliana. Current Biology 21, 1678-1683.

Melnyk, C.W., Molnar, A., and Baulcombe, D.C. (2011b). Intercellular and systemic movement of RNA silencing signals. The EMBO journal 30, 3553-3563.

Mittal, D., Mukherjee, S.K., Vasudevan, M., and Mishra, N.S. (2013). Identification of Tissue-Preferential Expression Patterns of Rice miRNAs. Journal of Cellular Biochemistry 114, 2071-2081. Miyashima, S., Koi, S., Hashimoto, T., and Nakajima, K. (2011). Non-cell-autonomous microRNA165 acts in a dose-dependent manner to regulate multiple differentiation status in the Arabidopsis root. Development 138, 2303-2313. 
Molnar, A., Melnyk, C.W., Bassett, A., Hardcastle, T.J., Dunn, R., and Baulcombe, D.C. (2010). Small Silencing RNAs in Plants Are Mobile and Direct Epigenetic Modification in Recipient Cells. Science 328, 872-875.

Nakano, M., Nobuta, K., Vemaraju, K., Tej, S.S., Skogen, J.W., and Meyers, B.C. (2006). Plant MPSS databases: signature-based transcriptional resources for analyses of mRNA and small RNA. Nucleic Acids Res 34, D731-735.

Napoli, C., Lemieux, C., and Jorgensen, R. (1990). Introduction of a Chimeric Chalcone Synthase Gene into Petunia Results in Reversible Co-Suppression of Homologous Genes in trans. Plant Cell 2, 279-289.

Nobuta, K., Venu, R.C., Lu, C., Belo, A., Vemaraju, K., Kulkarni, K., Wang, W.Z., Pillay, M., Green, P.J., Wang, G.L., et al. (2007). An expression atlas of rice mRNAs and small RNAs. Nature Biotechnology 25, 473-477.

Olmedo-Monfil, V., Duran-Figueroa, N., Arteaga-Vazquez, M., Demesa-Arevalo, E., Autran, D., Grimanelli, D., Slotkin, R.K., Martienssen, R.A., and Vielle-Calzada, J.P. (2010). Control of female gamete formation by a small RNA pathway in Arabidopsis. Nature 464, 628-U200.

Palauqui, J.C., Elmayan, T., Pollien, J.M., and Vaucheret, H. (1997). Systemic acquired silencing: transgene-specific post-transcriptional silencing is transmitted by grafting from silenced stocks to non-silenced scions. The EMBO journal 16, 4738-4745.

Pant, B.D., Buhtz, A., Kehr, J., and Scheible, W.R. (2008). MicroRNA399 is a long-distance signal for the regulation of plant phosphate homeostasis. Plant Journal 53, 731-738.

Ratcliff, F., Harrison, B.D., and Baulcombe, D.C. (1997). A similarity between viral defense and gene silencing in plants. Science 276, 1558-1560.

Ryabov, E.V., van Wezel, R., Walsh, J., and Hong, Y. (2004). Cell-to-cell, but not long-distance, spread of RNA silencing that is induced in individual epidermal cells. Journal of Virology 78 , 3149-3154.

Schwab, R., Maizel, A., Ruiz-Ferrer, V., Garcia, D., Bayer, M., Crespi, M., Voinnet, O., and Martienssen, R.A. (2009). Endogenous TasiRNAs Mediate Non-Cell Autonomous Effects on Gene Regulation in Arabidopsis thaliana. Plos One 4, e5980-e5980.

Schwach, F., Vaistij, F.E., Jones, L., and Baulcombe, D.C. (2005). An RNA-dependent RNA polymerase prevents meristem invasion by potato virus $\mathrm{X}$ and is required for the activity but not the production of a systemic silencing signal. Plant Physiology 138, 1842-1852.

Searle, I.R., Pontes, O., Melnyk, C.W., Smith, L.M., and Baulcombe, D.C. (2010). JMJ14, a JmjC domain protein, is required for RNA silencing and cell-to-cell movement of an RNA silencing signal in Arabidopsis. Genes \& Development 24, 986-991.

Shimamura, K., Oka, S., Shimotori, Y., Ohmori, T., and Kodama, H. (2007). Generation of secondary small interfering RNA in cell-autonomous and non-cell autonomous RNA silencing in tobacco. Plant Molecular Biology 63, 803-813.

Smith, L.M., Pontes, O., Searle, L., Yelina, N., Yousafzai, F.K., Herr, A.J., Pikaard, C.S., and Baulcombe, D.C. (2007). An SNF2 protein associated with nuclear RNA silencing and the spread of a silencing signal between cells in Arabidopsis. Plant Cell 19, 1507-1521.

Svoboda, P. (2019). RNAi and miRNA pathways in plants I - molecular mechanisms.

Tournier, B., Tabler, M., and Kalantidis, K. (2006). Phloem flow strongly influences the systemic spread of silencing in GFP Nicotiana benthamiana plants. Plant Journal 47, 383-394. 
Turgeon, R., and Wolf, S. (2009). Phloem transport: cellular pathways and molecular trafficking. Annu Rev Plant Biol 60, 207-221.

Tuteja, J.H., Zabala, G., Varala, K., Hudson, M., and Vodkin, L.O. (2009). Endogenous, Tissue-Specific Short Interfering RNAs Silence the Chalcone Synthase Gene Family in Glycine max Seed Coats. Plant Cell 21, 3063-3077.

Vaistij, F.E., Jones, L., and Baulcombe, D.C. (2002). Spreading of RNA targeting and DNA methylation in RNA silencing requires transcription of the target gene and a putative RNA-dependent RNA polymerase. Plant Cell 14, 857-867.

Valoczi, A., Varallyay, E., Kauppinen, S., Burgyan, J., and Havelda, Z. (2006). Spatio-temporal accumulation of microRNAs is highly coordinated in developing plant tissues. Plant Journal 47, $140-151$.

Voinnet, O., and Baulcombe, D.C. (1997). Systemic signalling in gene silencing. Nature 389, 553.

Voinnet, O., Vain, P., Angell, S., and Baulcombe, D.C. (1998). Systemic spread of sequence-specific transgene RNA degradation in plants is initiated by localized introduction of ectopic promoterless DNA. Cell 95, 177-187.

Voinnet, O., Vain, P., Angell, S., and Baulcombe, D.C. (2016). Systemic Spread of Sequence-Specific Transgene RNA Degradation in Plants Is Initiated by Localized Introduction of Ectopic Promoterless DNA. Cell 166, 779.

Yelina, N.E., Smith, L.M., Jones, A.M., Patel, K., Kelly, K.A., and Baulcombe, D.C. (2010). Putative Arabidopsis THO/TREX mRNA export complex is involved in transgene and endogenous siRNA biosynthesis. Proc Natl Acad Sci U S A 107, 13948-13953.

Yoo, B.C., Kragler, F., Varkonyi-Gasic, E., Haywood, V., Archer-Evans, S., Lee, Y.M., Lough, T.J., and Lucas, W.J. (2004). A systemic small RNA signaling system in plants. Plant Cell 16, 1979-2000.

Zabala, G., Campos, E., Varala, K.K., Bloomfield, S., Jones, S.I., Win, H., Tuteja, J.H., Calla, B., Clough, S.J., Hudson, M., et al. (2012). Divergent patterns of endogenous small RNA populations from seed and vegetative tissues of Glycine max. Bmc Plant Biology 12, 177-177.

Zhang, W.N., Kollwig, G., Stecyk, E., Apelt, F., Dirks, R., and Kragler, F. (2014). Graft-transmissible movement of inverted-repeat-induced siRNA signals into flowers. Plant Journal 80, 106-121.

Zhao, D., and Song, G.Q. (2014). Rootstock-to-scion transfer of transgene-derived small interfering RNAs and their effect on virus resistance in nontransgenic sweet cherry. Plant Biotechnol J 12, 1319-1328.

Zhu, Q.H., Spriggs, A., Matthew, L., Fan, L.J., Kennedy, G., Gubler, F., and Helliwell, C. (2008). A diverse set of microRNAs and microRNA-like small RNAs in developing rice grains. Genome Research 18, 1456-1465. 



\title{
SPECIFICITY OF TARGETING IN RNAi AND MIRNA PATHWAYS
}

Keywords: RNAi, siRNA, miRNA, Argonaute, off-targeting

\author{
PETR SVOBODA \\ Institute of Molecular Genetics, Academy of Sciences of the Czech Republic, \\ Videnska 1083, 14220 Prague 4, Czech Republic \\ Correspondence to: Petr Svoboda, Institute of Molecular Genetics ASCR, \\ Videnska 1083, 14220 Prague 4, Czech Republic, tel. \# +420 241063147, \\ e-mail: svobodap@img.cas.cz.
}

\begin{abstract}
This review systematically covers sequence-specific gene regulation by miRNAs and dsRNA-derived siRNAs in animals and plants from the perspective of target RNA recognition, potential for non-target (off-target) effects, and reliable determination of biological effects of small RNAs in animals and plants. I will review sequence complementarity between target RNA and small RNA (siRNA or miRNA), including tolerance to mismatches, parameters influencing sequence complementarity (and target recognition and repression) and discuss specificity of targeting by miRNAs and off-targeting by siRNAs. In addition, I will discuss reliable identification of target RNAs (and, eventually, biological effects). Accordingly, the text is divided into the following four sections: (I) Small RNA:target RNA base pairing, (II) Other key factors influencing target recognition and repression, (III) Off-targeting - causes and remedies, (III) Smal RNA target identification.
\end{abstract}

\section{Introduction}

Within the complex world of RNA silencing, two related yet distinct pathways exist in animals and plants: RNA interference (RNAi) and microRNA (miRNA) pathways (Fig. 1). Both pathways employ small RNAs loaded on Argonaute proteins as sequence-specific guides for post-transcriptional repression. The elementary difference between these two pathways is that miRNA pathways employ genome-encoded small RNAs with defined sequences (i.e. miRNAs can be annotated) while RNAi is initiated by processing long double-stranded RNA (dsRNA) into a mixture of short interfering RNAs (siRNAs). Thus, the miRNA pathway in a cell employs a population of miRNA molecules that can be clustered based on unique sequences, corresponding to specific positions in miRNA precursors. In other words, the major distinction between RNAi and miRNA pathways is the origin of small RNAs and their information content. In terms of their mode of action, siRNAs and miRNAs can be in some cases indistinguishable. 


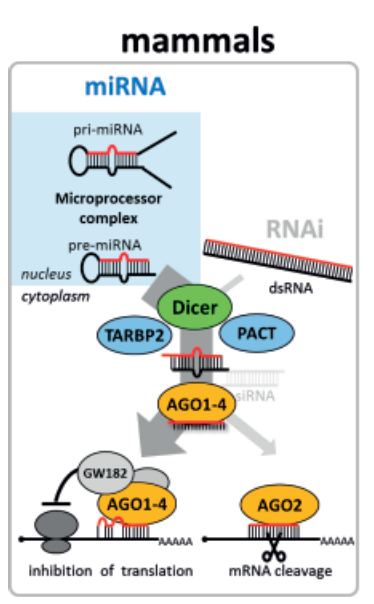

\section{Drosophila (Arthropoda)}

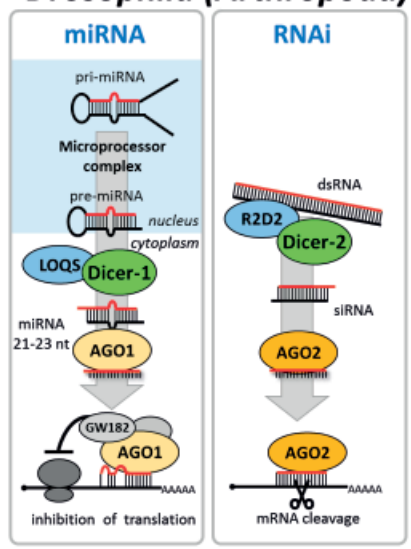

\section{Caenorhabditis (Nematoda)}
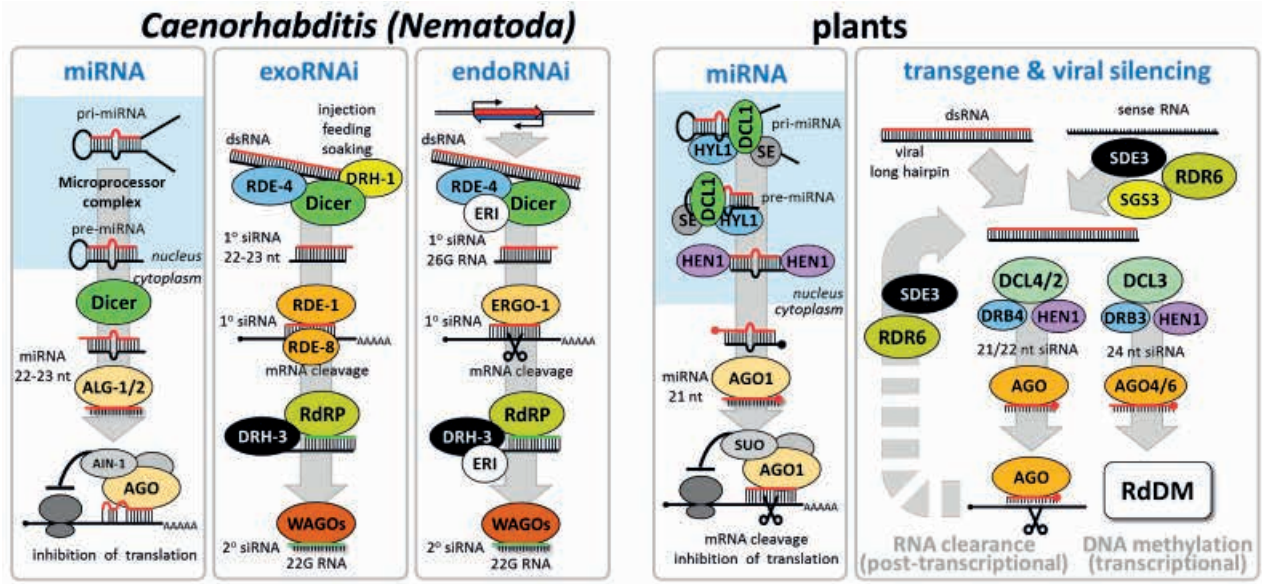

Figure 1 miRNA and RNAi pathways in animals and plants

The schemes depict key components of miRNA and RNAi pathways in the main eukaryotic model systems

In animals, the miRNA pathway, which is primarily a gene-regulating pathway, is highly conserved. The canonical miRNA biogenesis is a spatially separated into two-steps. The first step takes place in the nucleus where RNase III Drosha, a component of the Microprocessor complex, releases a precursor miRNA (pre-miRNA) from a primary miRNA transcript (pri-miRNA). Next, a pre-miRNA is transported to the cytoplasm where it is cleaved by a second RNase III Dicer. Dicer releases a miRNA duplex of which one strand will be loaded on an Argonaute protein. The miRNA pathway in plants operates similarly but employs only a single nuclear Dicer-like 1 (DCL1) RNase III to produce pre-miRNAs and miRNAs. The second important difference is the methylation of plant miRNAs at their 3' end mediated by HEN1 methyltransferase. 
The RNAi pathway is much more diverse across animals and plants. It is conceivable given the antiviral role of RNAi where the parasite:host interactions can accelerate evolution of RNAi pathways in different taxons. Despite the differences, RNAi and miRNA pathways share common features, which include biogenesis of small RNAs involving Dicer and effector complexes containing an Argonaute protein carrying a small RNA. Argonaute proteins are composed of four main domains: the central PAZ domain, the C-terminal PIWI (P-element induced wimpy testis), the N-terminal domain, and the MID domain between PAZ and PIWI domains (Fig. 2). The PIWI domain has an RNase H-like fold and carries a "slicer" activity (Ma et al., 2005; Parker et al., 2004; Song et al., 2004; Yuan et al., 2005). Argonaute proteins fall into three distinct groups (reviewed in Faehnle and Joshua-Tor, 2007): (1) AGO proteins, found in all kingdoms, (2) PIWI proteins found in animals, and (3) WAGO proteins found only in nematodes.

From the mechanistic perspective, post-transcriptional repression by small RNAs employs two distinct yet related (and often overlapping) modes of action:

Direct endonucleolytic RNA cleavage mediated by the so-called "slicer" activity of an Argonaute protein. This mode of action needs two conditions to be met: (i) the Argonaute protein has the slicer activity (not all family members have it) and (ii) there needs to be extensive base pairing between the Argonaute-bound small RNA and the cognate RNA. Extensive base pairing positions the cognate RNA such that it can be sliced in the position corresponding the middle of the guiding small RNA. This mode of action has been traditionally associated with RNAi and will be refered to as "RNAi-like" targeting. However, it should be pointed out that the two conditions for RNA-like targeting do not exclude miRNAs and, in fact, it is well established that miRNAs loaded on a slicing Argonaute would guide slicing of perfectly complementary cognate RNAs.

Indirect mRNA destabilization, which is found when an Argonaute protein lacks the slicing activity or the base pairing is incomplete and prevents positioning of the cognate RNA (typically lack of base pairing in the middle of the small RNA:target RNA duplex. In these cases, Argonaute-bound small RNAs provide sufficiently stable interaction for target recognition while the repression is mediated by Argonaute-interacting partners. While the precise mode of action is still debated and may vary between different cell types and model systems, it seems to be coupled with common mechanisms of mRNA destabilization, i.e. deadenylation and decapping.

At least four types of RNAi \& miRNA pathway combinations can be recognized in animals and plants (Fig. 1):

(I) overlapping miRNA and RNAi pathways with a single-set of Dicer and Argonaute proteins and without an RNA-dependent RNA polymerase (RdRP) - typical for vertebrates especially mammals. The molecular machinery in the cell primarily produces miRNAs but it can also support canonical RNAi, which can be observed under rare circumstances. The term RNAi in mammals is commonly used for RNAi-like cleavage mediated by a siRNA loaded on AGO2. However, siRNAs are being loaded on all four mammalian AGO protein (Meister et al., 2004), and once loaded, their behavior is indistinguishable from miRNAs. This functional overlap at the level of the effector complex is the major source of the so-called "off-targeting" phenomenon where siRNAs target also other mRNAs through miRNA-like mode of action. 
(II) separated miRNA and RNAi pathways with dedicated Dicer and Argonaute proteins (no RdRPs). This arrangement is observed in Arthropods (Drosophila).

(III) distinct miRNA pathway and a complex RNAi system employing RdRp(s) sharing a single Dicer. This arrangement is observed in nematodes where expansion of Argonaute proteins created a highly complex RNA silencing system

(IV) separated miRNA pathway and a complex RNAi system employing RdRp(s) with multiple Dicer and Argonaute proteins. This arrangement is observed in plants.

Mechanistical aspects of target recognition and its specificity will be discussed next.

\section{Small RNA:target RNA base pairing}

A small RNA loaded on an Argonaute protein functions as a guide selectively recognizing cognate RNAs through sequence complementarity. Sequence complementarity can be high (full or almost full) or partial. High sequence complementarity operates in RNAi-mediated innate immunity and genome defense where it is desirable to degrade all nucleic acids with highly similar sequences. High sequence complementarity is also observed for many plant miRNAs, which could be, at least in part, a consequence of their evolution (Allen et al., 2004; Llave et al., 2002). Animal miRNAs and some plant miRNAs have typically partial sequence complementarity, which seems to be non-randomly distributed along a small RNA (reviewed, for example in Bartel, 2009). Partial complementarity could be seen as a minimal requirement for functional target recognition formed by natural selection. However,

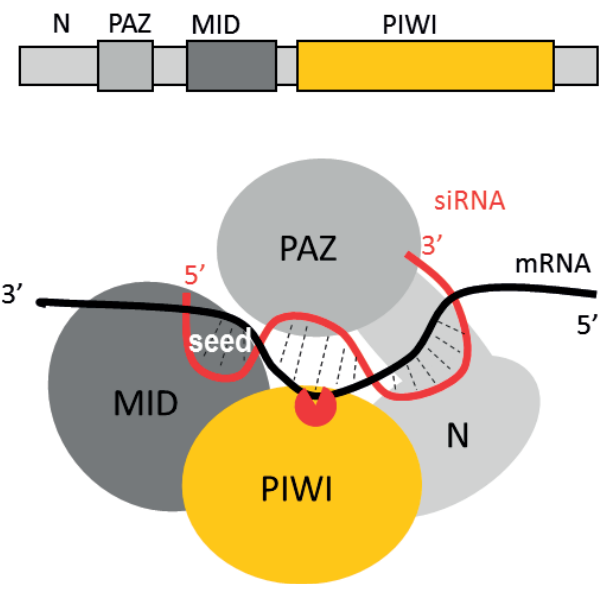

Figure 2 Argonaute protein structure

Schematic domain organization of an Argonaute protein. The scheme shows how a siRNA-loaded Argonaute cleaves a perfectly complementary RNA, which becomes accessible by the catalytical center in the PIWI domain upon base pairing with a small RNA. Nucleotides 2-8 of the small RNA initiate the interaction with the cognate RNA and form the so-called "seed", which has a highly predictive value for miRNA binding sites and siRNA off-targeting. The cognate mRNA is cleaved in the middle of the base paired sequence by the slicer activity depicted as a red pac-man. 
before addressing small RNA:target RNA complementarity, I will review the structure of Argonaute proteins and its implications for base pairing and target recognition. The reason is that structural analyses of Argonaute proteins provided important insights into the mechanism of how an Argonaute-loaded small RNA recognizes and binds its target.

\section{Structural insights into target recognition by Argonaute-bound small RNAs}

The one of the fundamental steps in deciphering rules governing target recognition and repression in RNA silencing is understanding the structure of a cognate RNA bound to a guide RNA loaded on an Argonaute protein. The pioneering structural analysis of fulllength Argonaute proteins has been carried out on crystalized archaeal proteins from Pyrococcus furiosus (Song et al., 2004), Aquifex aeolicus (Yuan et al., 2005), Archaeglobus fulgidus (Ma et al., 2005; Parker et al., 2005), and Thermus thermophiles (Wang et al., 2008; Wang et al., 2009) and, subsequently on human AGO1 and AGO2 proteins (Elkayam et al., 2012; Faehnle et al., 2013; Nakanishi et al., 2013; Schirle et al., 2016; Schirle and MacRae, 2012; Schirle et al., 2015; Schirle et al., 2014).

Structural analysis of archaeal proteins revealed that Argonaute proteins are composed of four main domains: the central PAZ domain, the C-terminal PIWI, the N-terminal domain, and the MID domain between PAZ and PIWI domains. A small RNA is anchored with its 3 ' end in the PAZ domain and the 5' end in a binding pocket between the MID domain and the PIWI domain (Fig. 2). Human AGO1 and AGO2 proteins also show this organization (Elkayam et al., 2012; Faehnle et al., 2013; Nakanishi et al., 2013; Schirle et al., 2016; Schirle and MacRae, 2012; Schirle et al., 2015; Schirle et al., 2014). While both proteins accommodate siRNAs and miRNAs, only AGO2 has the slicer activity (Liu et al., 2004; Meister et al., 2004). The crystal structure of human AGO2 revealed a bilobed molecule with a central cleft for binding guide and target RNAs (Elkayam et al., 2012; Schirle and MacRae, 2012; Schirle et al., 2015; Schirle et al., 2014). The crystal structures of human AGO1 bound to endogenous co-purified RNAs or loaded with miRNA (let-7) are very similar to the structures of AGO2 despite the fact that AGO1 lacks the slicer activity (Faehnle et al., 2013; Nakanishi et al., 2013).

The key observation coming from the structural analysis is that nucleotides 2 to 6 of a guide RNA are positioned in an A-form conformation for base pairing with target messenger RNAs (Elkayam et al., 2012; Faehnle et al., 2013; Nakanishi et al., 2013; Schirle et al., 2016; Schirle and MacRae, 2012; Schirle et al., 2015; Schirle et al., 2014) (Fig. 3). An RNA molecule can occur in many three dimensional conformations because there are multiple angles along which it can rotate its parts. Accordingly, initiation of base pairing requires proper conformation of two RNA molecules in order to initiate formation of hydrogen bonds between two complementary molecules. An Argonaute protein facilitates base pairing between a small RNA and a complementary RNA (= target recognition) by exposing nucleotides 2-6 arranged in a conformation needed for proper base pairing.

Between nucleotides 6 and 7, there is a kink that may function in miRNA target recognition or release of sliced RNA products. (Schirle and MacRae, 2012). Crystallization of loaded human AGO2 in the presence of target RNA sequences suggested a stepwise mechanism for interaction with cognate RNAs. First, AGO2 exposes guide nucleotides (nt) 2 to 
A

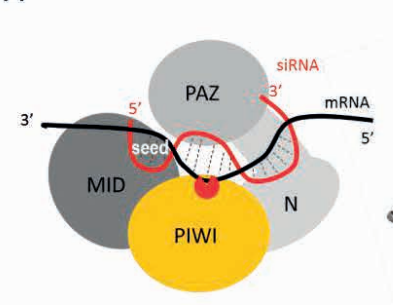

B

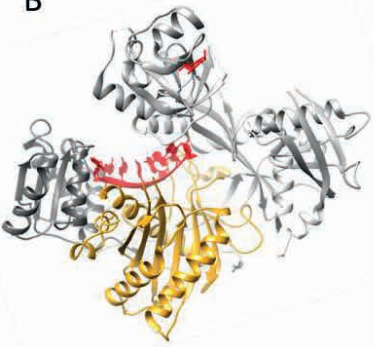

C

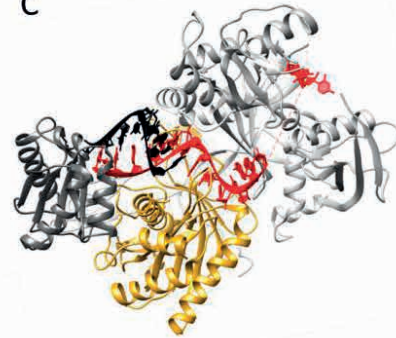

Figure 3 Crystal structures of Argonautes with bound RNAs

(A) A schematic depiction of AGO2 domain organization. (B) AGO2 with bound small RNA (in red), visible is the seed in A conformation (Schirle et al., 2014). (C) AGO2 loaded with a small RNA (in red) interacting with a target RNA (in black) (Schirle et al., 2014). Data for visualization were obtained from wwPDB and displayed in UCSF Chimera.

5 for initial target pairing, which then promotes conformational changes that expose nt 2 to 8 and 13 to 16 for further target recognition (Schirle et al., 2014). miRNA binding seem to lock the otherwise flexible AGO2 enzyme in a stable conformation (Elkayam et al., 2012). The structure of human Ago2 bound to miR-20a implies that the miRNA is anchored at both ends by the MID and PAZ domains with several kinks and turns along the binding groove (Elkayam et al., 2012). Spurious slicing of miRNA targets is avoided through an inhibitory coordination of one catalytic magnesium ion (Schirle et al., 2014). Evolutionary changes that rendered hAGO1 inactive included a mutation of a catalytic tetrad residue and mutations on a loop near the actives site (Faehnle et al., 2013; Nakanishi et al., 2013). Importantly, the PIWI domain contains tandem tryptophan-binding pockets, that function in recruitment of glycine-tryptophan-182 (GW182) or other tryptophan-rich cofactors (Schirle and MacRae, 2012). Computer simulation of the structural and functional dynamics of human $\mathrm{AGO} 2$ and the interaction mechanism with siRNAs confirmed that AGO2 adopts two conformations such as "open" and "close" and the PAZ is a highly flexible region. (Bhandare and Ramaswamy, 2016). Models of miRNA-loaded Argonautes imply that Argonautes adopt variable conformations at distinct target sites that generate distorted, imperfect miRNA-target duplexes where structural distortions are better tolerated in solvent-exposed seed and 3'-end regions than in the central duplex region (Gan and Gunsalus, 2015).

Structural analysis also clarified the effect of the first nucleotide in the cognate site, which does not base pair with the loaded small RNA because the first nucleotide of the small RNA (frequently U) is buried in the 5' end-binding pocket. Yet, it was observed that interaction with the cognate site is enhanced by adenosine in the position 1 of a miRNA binding site; the structural analysis revealed that the adenosine in the mRNA is recognized indirectly by $\mathrm{AGO} 2$ through a hydrogen-bonding network of water molecules that preferentially interacts with the N6 amine on the adenine base (Schirle et al., 2015). Importantly, N6 adenosine methylation blocks recognition of the adenosine, which might reflect a possible mechanism for regulating of miRNA binding through covalent modification of miRNA binding sites (Schirle et al., 2015). 


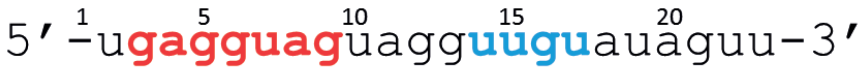 \\ seed $(2-8)$ \\ central $\prod_{\text {3' supplementary }}$}

Figure 4 Small RNA domains.

Small RNAs loaded onto AGO proteins can be divided into modules including the 5 ' the anchor, seed sequence, central part, 3' supplementary sequence, and tail (Wee et al., 2012).

These data provide structural foundations of many features of target recognition and can be used for computer simulations of miRNA-target interaction in the context of the loaded Argonaute structure. In fact, an algorithm MiREN, which builds and scores three-dimensional models of the ternary complex formed by AGO, a miRNA and $22 \mathrm{nt}$ of a target mRNA, can be used to assess the likelihood that an RNA molecule is the target of a given miRNA (Leoni and Tramontano, 2016).

Importantly, they also explain features associated with different regions of miRNA and siRNA sequences that were identified in kinetic and bioinformatics studies. Taken together, crystal structures of AGO2 explain the nucleotide-pairing patterns that emerged during previous studies of miRNA sequences, namely analyses of conservations of miRNA binding sites and biochemical analyses of target recognition, which are discussed later.

\section{small RNA:target RNA base pairing}

Sequence complementarity between a small RNA and its target RNA can be full (or almost full) or partial. Full complementarity is typically associated with siRNAs while partial with miRNAs although imperfect base pairing of siRNAs and perfect base pairing of miRNAs also occur. To provide a framework for this section, I first review the full-complementarity, then the partial complementarity involving base pairing of 5' small RNA nucleotides (the seed, Fig. 4) and then seedless (non-canonical, non-seed) interactions and their implications on target recognition, prediction and effective repression. Importantly, target mRNAs are as efficiently repressed by microRNA-binding sites in the 5 'UTR as in the 3 'UTR as shown in experiments in cultured human cells (Lytle et al., 2007).

\section{siRNA complementarity and sequence features}

RNAi efficiency correlates well with the binding energy of a siRNA to its mRNA target (Muckstein et al., 2006). While full complementarity yields a perfect duplex in which all nucleotides participate seemingly equally, some positive correlations were identified between positions of specific nucleotides and siRNA suppressing efficiency. These features may reflect positive effects on Argonaute loading (strand selection) as well as on target recognition. Analysis of the efficiency of $\sim 600$ siRNAs suggested higher siRNA efficiency with $\mathrm{A} / \mathrm{U}$ at positions 10 and 19 , a G/C at position 1, and more than three A/Us between positions 13 and 19, in the sense strand of the siRNA sequence (Jagla et al., 2005). Furthermore, specific residues at every third position of an siRNA influence its efficient RNAi 
activity, which might reflect interaction with TARBP2 during formation of the RNA-induced silencing complex (RISC) (Katoh and Suzuki, 2007).

Target recognition by siRNAs is highly specific. However, discrimination of RNAi between two sequences differing by a single nucleotide varies according to the position of the mismatch. A systematic analysis of single-nucleotide mutations in target sites of a functionally validated siRNA showed that the position of the mismatched base pair and the identity of the nucleotides forming the mismatch matter for effective silencing (Du et al., 2005). A:C mismatches were, in addition to the G:U wobble base pairs, surprisingly well tolerated and target sites containing such mismatches were silenced almost as efficiently as with full complementarity (Du et al., 2005). G:U wobble base pairing in the central part of the antisense strand caused a pronounced decrease in activity, while mutations at the 5, and 3'ends were well-tolerated (Holen et al., 2005). Interestingly, analysis of siRNA selectivity suggested that siRNAs with G:U wobble base pairs or a mismatches located in the "seed" are discriminating less between perfect and mismatched target than those in which the mismatch was located 3' to the seed (nucleotides 9-14); this region is critical for target cleavage but not siRNA binding (Schwarz et al., 2006).

\section{miRNAs with extensive base pairing}

Target recognition by miRNAs in plants is commonly thought to involve extensive base pairing and RNAi-like cleavage of the target (reviewed in Axtell, 2013; Wang et al., 2015). This notion stems from the perfect complementarity between miR171 and its SCARECROW-LIKE (SCL) mRNA target, which was the first identified miRNA:mRNA interaction in plants (Llave et al., 2002). However, the perfect complementarity is rather an exception as most of the identified miRNA targets in plant cells have some imperfect base pairing (summarized in (Jones-Rhoades and Bartel, 2004; Jones-Rhoades et al., 2006)). Extensive base pairing and microRNA-directed RNAi-like cleavage exists also in animals but it is rare; one of the exceptional cases is HOXB8 mRNA cleavage by miR-196 (Yekta et al., 2004)

Mismatches to the miRNA 5' regions strongly reduce repression but are found in several natural miRNA-binding sites while miRNA binding with a few mismatches to the miRNA 3 ' regions are common in plants and are often equally (or even more) effective as perfectly matched sites (Liu et al., 2014b). Central mismatches interfere with repression (Liu et al., 2014b). However, miR398 in Arabidopsis binds 5'UTR of the blue copper-binding protein mRNA with a bulge of six nucleotides opposite to the 5' region of the miRNA (Brousse et al., 2014). These and other studies led to consensus base pairing rules for a functional plant miRNA-target interaction: little tolerance for mismatches at positions $2-13$, with especially little tolerance of mismatches at positions 9-11, and more tolerance of mismatches at positions 1, and 14-21 (Wang et al., 2015). This is in contrast with animal miRNAs where pairing at positions 2-7 can be sufficient for a functional interaction (Bartel, 2009).

High sequence complementarity in mammals may be coupled with Argonaute "unloading". It was found that highly complementary target RNAs significantly accelerate release of the guide RNA from Ago2. Unloading can be enhanced by mismatches between the 
target and miRNA's 5' end and attenuated by mismatches to miRNA's 3' end (De et al., 2013).

\section{Imperfect base pairing of miRNAs}

Animal miRNAs typically base pair imperfectly with their targets. Target sites can be grouped into two broad categories. 5' dominant sites have sufficient complementarity to the miRNA 5' end to function with little or no support from pairing to the miRNA 3' end. Indeed, sites with $3^{\prime}$ pairing below the random noise level are functional given a strong 5 ' end. In contrast, 3' compensatory sites have insufficient 5' pairing and require strong 3 ' pairing for function (Brennecke et al., 2005). Accordingly, I will separately discuss the canonical base pairing involving miRNA's 5' end (the seed) and the non-canonical (seedless) interactions. I will start with the canonical interaction involving base pairing of the seed because it is the most studied and integrates knowledge from structural studies as well as sequence analyses.

\section{Seed-involving interactions}

The seed sequence concept emerged already during pioneering work on miRNA annotation where it became apparent that miRNAs form families sharing 5' sequences (Lagos-Quintana et al., 2001; Lau et al., 2001; Lee and Ambros, 2001). Then it became clear that the seed sequence is a strong predictor for miRNA targets (Lewis et al., 2005; Sood et al., 2006) as well as for siRNA off-targeting (Jackson et al., 2006b). An analysis of more than 18,000 high-confidence miRNA-mRNA interactions suggested that binding of most miRNAs includes the 5' seed region, while around $60 \%$ of seed interactions contained bulged or mismatched nucleotides (Helwak et al., 2013). The molecular mechanism of miRNA and target recognition (reviewed in Bartel, 2009) provides an explanation for the significance of the seed sequence and, while there are also small RNA:target mRNA interactions that do not involve the seed sequence, the concept of the seed is sufficient to explain that any AGO-loaded small RNA in any cell type has the potential to interact with hundreds and thousands of different mRNAs. In fact the estimates for human mRNAs targeted by miRNAs are between 30 and $>60 \%$ (Friedman et al., 2009; Lewis et al., 2005).

The seed region is generally defined as a 7 nt region mapping to positions $2-8$ and it strongly confers specificities of animal miRNAs to their mRNA targets. There is a high functional cost of even single nucleotide changes within seed regions, which is consistent with their high sequence conservation among miRNA families both within and between species and suggests processes that may underlie the evolution of miRNA regulatory control (Hill et al., 2014). The target specificity determined by the seed has evolutionary and biological implications because single nucleotide polymorphisms in canonical miRNA binding sites would affect miRNA-mediated regulations, a notion supported also by experimental data (Afonso-Grunz and Muller, 2015; Vosa et al., 2015).

The canonical 7nt seed can be divided into several types (Ellwanger et al., 2011). More specifically, the core seeds have been described as a 6-mer (bases 2-7), 7-mer ("7-mer-A1" being bases $1-7$, and " 7 -mer-m 8 " being bases $2-8$ ), and 8 -mer (bases 1-8); sometimes the 
7-mer-A1 and 8-mer seeds are required to have an adenine, 'A', as the first nucleotide types (Bartel, 2009; Ellwanger et al., 2011).

Longer seeds, i.e. seeds of 7 or 8 nucleotides in length are more evolutionarily conserved than shorter ones (Ellwanger et al., 2011). Longer seeds confer higher specificity and repression. It was reported that the extent of the seed match has a strong impact on resulting target repression: single 8 mer seed match mediates down-regulation comparable to two 7 mer seed matches (Nielsen et al., 2007). However, others did not observe a linear relationship between seed length and miRNA expression dysregulation, which does not support the hypothesis the seed region length alone influences mRNA repression. (Mullany et al., 2016)

In any case, the majority of functional target sites seems formed by less specific seeds of only $6 \mathrm{nt}$ indicating a crucial biological role of this type (Ellwanger et al., 2011). In fact, pairing at positions $2-7$ is sufficient for a functional interaction of animal miRNAs with their targets (Bartel, 2009). In contrast, seed pairing does not appear to be critical for land plant miRNAs (Liu et al., 2014b).

The minimal requirement for miRNA:mRNA interactions in animals explains the large numbers of targets of animal miRNAs and the fact that, the majority of functional sites is poorly detected by common prediction methods (Ellwanger et al., 2011). While the initial studies suggested that average miRNAs have approximately 100 target sites (Brennecke et al., 2005), subsequent bioinformatics and experimental identification of miRNA targets suggest even higher number of target sites.

There are several targeting determinants that enhance seed match-associated mRNA repression, including the presence of adenosine opposite miRNA base 1 (this functionality is explained by Argonaute protein structure (Schirle et al., 2015)) and of adenosine or uridine opposite miRNA base 9 , independent of complementarity to the siRNA/miRNA (Lewis et al., 2005; Nielsen et al., 2007). Furthermore, seed-based canonical target recognition was dependent on the GC content of the miRNA seed - low GC content in the seed was coupled with non-canonical target recognition. (Wang, 2014). Additional reported determinants beyond seed pairing include: AU-rich nucleotide composition near the site, proximity to sites for co-expressed miRNAs (which leads to cooperative action), proximity to residues pairing to miRNA nucleotides 13-16, positioning within the $3^{\prime} \mathrm{UTR}$ at least $15 \mathrm{nt}$ from the stop codon, and positioning away from the center of long UTRs (Grimson et al., 2007).

\section{Non-canonical - non-seed interactions}

There is large variety of miRNA-target duplex structures, which include seedless interactions (reviewed in Cipolla, 2014; Seok et al., 2016a). The existence of seedless interactions explains reports that perfect seed pairing is not a generally reliable predictor for miRNA-target interactions (Didiano and Hobert, 2006). Despite attempts to classify non-canonical interactions (Xu et al., 2014b) and tertiary structure-based modelling of miRNA interactions (Gan and Gunsalus, 2015), bioinformatic prediction of non-canonical interactions is far from ideal. A solution is integration of bioinformatic target prediction with biochemically identified miRNA binding sites. Such analyses suggested that most miRNA targets were of a non-canonical type, i.e. not involving perfect complementarity in the seed region 
(Khorshid et al., 2013; Wang, 2014). Importantly, analysis of AGO-associated mRNAs that lack seed complementarity with miRNAs suggested that AGO might have its own binding preference within target mRNAs, independent of guide miRNAs (Li et al., 2014). A structurally accessible and evolutionarily conserved region ( $\sim 10$ nucleotides in length) was identified that alone can accurately predict AGO-mRNA associations, independent of the presence of miRNA binding sites (Li et al., 2014). In any case, the impact of non-canonical targeting regarding target downregulation is not fully resolved (Khorshid et al., 2013; Martin et al., 2014; Wang, 2014).

\section{Other important factors influencing target recognition and repression}

It is important to recognize that sequence complementarity between a small RNA and its putative target is not sufficient to make any prediction about silencing of the target because there are other important factors at play. The two most important are discussed in the next two sections are (I) the binding site accessibility and (II) stoichiometry between a small RNA ant its target (or binding kinetics). Other factors, which might contribute to silencing in a context-dependent manner are, for example, alternative polyadenylation and arrangement of miRNA binding sites in 3'UTRs might cause different effects in different cells (Hon and Zhang, 2007; Majoros and Ohler, 2007; Nam et al., 2014a). In particular, there was a strong preference reported for targets to be located in close vicinity of the stop codon and the polyadenylation sites. (Majoros and Ohler, 2007).

\section{Binding site accessibility}

Mere sequence complementarity is not a sufficient predictor whether base pairing will occur in vivo. RNA molecules always form secondary structures and, in the cellular context, a number of proteins interacts with RNA molecules. Accordingly, secondary structures or RNA binding proteins may prevent base pairing of two complementary sequences. The issue of sequence accessibility was recognized during early RNAi experiments with stochastic knockdown efficiency. When searching for factors influencing knock-down efficiency, attention turned to the local RNA structure at siRNA target sites and it was demonstrated that local RNA target structure is an important factor for siRNA efficacy (Schubert et al., 2005). Accordingly, siRNA design tools started to accommodate not only properties of siRNAs but also properties of the target site because it strongly increased efficiency of designed siRNAs (Heale et al., 2005, 2006; Shao et al., 2007; Tafer et al., 2008).

Systematic investigation of siRNA:target RNA interactions and the effect of local secondary structures provided also insights into the molecular mechanism of target recognition. It was shown in vitro and in vivo, that the accessibility of the target site correlates directly with the efficiency of cleavage, demonstrating that RISC is not unfolding structured RNA (Ameres et al., 2007). During target recognition, RISC transiently contacts single-stranded RNA nonspecifically and promotes siRNA-target RNA annealing (Ameres et al., 2007).The seed of Argonaute-associated siRNA creates a thermodynamic threshold that determines the stable association of RISC and the target RNA (Ameres et al., 2007). 
The same principles apparently apply for miRNA-mediated repression (Long et al., 2007; Xu et al., 2014b). Mutations diminishing target accessibility substantially reduce microRNA-mediated translational repression, with effects comparable to those of mutations that disrupt sequence complementarity (Kertesz et al., 2007).

\section{small RNA:target RNA stoichiometry and binding kinetics}

The second critical factor for target repression is stoichiometry between a small RNA ant its target. This is especially important for the miRNA-like type of target repression because a miRNA must remain associated with its target RNA in order to induce its translational repression and degradation. Thus, suppression of a specific mRNA by a miRNA requires enough miRNA molecules that would assure enough interactions with binding sites in that particular RNA while these binding sites essentially compete with all binding sites for that miRNA in the transcriptome.

\section{Biochemical analyses of stoichiometry and kinetics}

Kinetic data should be taken as a biochemical range for any hypotheses concerning target recognition and biological effects of small RNAs in the context of loaded RISC. Among these is a detailed kinetic study of Drosophila and mouse AGO2 RISCs (Wee et al., 2012).

It was shown that siRNA-programmed RISC is a classical Michaelis-Menten enzyme in the presence of ATP (Haley and Zamore, 2004). In the absence of ATP, the rate of multiple rounds of catalysis is limited by release of the cleaved products (Haley and Zamore, 2004). Kinetic analysis suggests that different regions of the siRNA play distinct roles in the cycle of target recognition, cleavage, and product release (Haley and Zamore, 2004). Later, it was shown that Argonaute divides its RNA guide into domains with distinct functions and RNA-binding properties. (Wee et al., 2012) According to this analysis, small RNAs loaded onto AGO proteins are actually composed of five distinct modules the anchor, seed, central, 3' supplementary, and tail (Fig. 4) (Wee et al., 2012). Bases near the siRNA 5' end disproportionately contribute to target RNA-binding energy, whereas base pairs formed by the central and 3' regions of the siRNA provide a helical geometry required for catalysis (Haley and Zamore, 2004). Mouse AGO2, which mainly mediates miRNA-directed repression in vivo, dissociates rapidly and with similar rates for fully paired and seed-matched targets (Wee et al., 2012). An important conclusion from this study is that low-abundant miRNAs are unlikely to contribute much biologically meaningful regulation because they are present at a concentration less than their KD for seed-matching targets, which are in a picomolar range (Wee et al., 2012). Another study characterized siRNA binding, target RNA recognition, sequence-specific cleavage and product release by recombinant human Ago 2 (hAgo2). This yielded a minimal mechanistic model describing fundamental steps during RNAi, which is consistent with a "two-state" model of RISC action (Deerberg et al., 2013). Finally, it was found that $\mathrm{Mg} 2+$ concentration, influences AGO2 structural flexibility and is important for its catalytic/functional activity, with low $[\mathrm{Mg} 2+]$ favoring greater Ago2 flexibility (e.g., greater entropy) and less miRNA/mRNA duplex stability, thus favoring slicing(Nam et al., 2014b). 
Importantly, it seems that miRNA:mRNA stoichiometry cannot be simply determined by quantifying RNAs. Quantification of Argonaute-associated endogenous miRNAs or exogenous siRNAs in cultured cells suggested that only a small proportion (even $<10 \%$ ) of such small RNAs is loaded on Argonautes (Janas et al., 2012; Stalder et al., 2013). Furthermore, a substantial percentage of the miRNA pool associated with mRNAs without Argonautes (Janas et al., 2012; Stalder et al., 2013). It was also found that endogenous human miRNAs vary widely, by $>100$-fold, in their level of RISC association and show that the level of Ago binding is a better indicator of inhibitory potential than is the total level of miRNA expression (Flores et al., 2014). Together, these data indicate that the level of RISC association of a given endogenous miRNA is regulated by the available RNA targetome and predicts miRNA function. (Flores et al., 2014).

\section{Small RNA:target RNA binding single-molecule analysis}

Recent advances in single-molecule analysis brought also single- molecule data about RISC:target interaction, which is consistent with other biochemical data and the two state model for Argonaute action (Li and Zhang, 2012; Zander et al., 2014).

Loaded AGO2 utilizes short RNAs as specificity determinants with thermodynamic and kinetic properties more typical of RNA-binding proteins. A small RNA loaded on Argonaute does not follows rules by which free oligonucleotides find, bind, and dissociate from complementary nucleic acid sequences (Salomon et al., 2015). This is conceivable given the fixed "A" conformation of the seed of a small RNA loaded on an Argonaute protein.

Single-molecule fluorescence experiments using a minimal RISC (a small RNA and AGO2) showed that target binding starts at the seed region of the guide RNA (Chandradoss et al., 2015; Jo et al., 2015a; Jo et al., 2015b). AGO2 initially scans for target sites with complementarity to nucleotides $2-4$ of the miRNA. This initial transient interaction propagates into a stable association when target complementarity extends to nucleotides $2-8$. This stepwise recognition process is coupled to lateral diffusion of AGO2 along the target RNA, which promotes the target search by enhancing the retention of AGO2 on the RNA (Chandradoss et al., 2015). Stable RISC binding is thus efficiently established with the seed match only, providing a potential explanation for the seed-match rule of miRNA target selection (Chandradoss et al., 2015; Jo et al., 2015a; Jo et al., 2015b). Mouse AGO2 binds tighter to miRNA targets than its RNAi cleavage product, even though the cleaved product contains more base pairs (Salomon et al., 2015). Annealing between miRNA and its target with poor seed match proceeds in a stepwise way, which is in accordance with the increase in the number of conformational states of miRNA-target duplex accommodated by the miRISC, suggesting the structural plasticity of human miRISC to conciliate the mismatches in seed region (Li and Zhang, 2012)

Target cleavage required extensive sequence complementarity and accelerated coreRISC dissociation for recycling (Jo et al., 2015a) and sensitively depended on the sequence (Jo et al., 2015b). While RISC generally releases the 5' cleavage fragment from the guide 3 ' supplementary region first and then the 3 ' fragment from the seed region. This order can be reversed by extreme stabilization of the 3' supplementary region or mismatches in the seed region. Therefore, the release order of the two cleavage fragments is influenced by the 
stability in each region, in contrast to the unidirectional base pairing propagation from the seed to the 3' supplementary region upon target recognition.(Yao et al., 2015).

\section{Off-targeting - causes and remedies}

Off-targeting effects surfaced as a major issue in RNAi experiments when the effects of RNAi treatment were systematically analyzed (Fedorov et al., 2006; Jackson et al., 2003; Lin et al., 2005; Scacheri et al., 2004; Snove and Holen, 2004). One of the most revealing data came from mammalian cells transfected with different siRNAs targeting the same gene, which were systematically analyzed using microarrays (Jackson et al., 2003). Using 16 different siRNAs against IGF1R and 8 different siRNAs against MAPK14, strong siRNA-specific expression changes were found in transfected cells with only a few genes regulated in common by siRNAs targeting the same gene. Off-targeting effects were also found also in other animal models (Ma et al., 2006) and plants (Xu et al., 2006). In fact, off-targeting causes a significant bias in high-throughput RNAi screens (Ma et al., 2006)

Off-targeting is concentration dependent, it could be attributed to both siRNA strands, and a portion of off-targeting appears to be caused by partial complementarity between a siRNA and its target, reminiscent of the 5' seed regions of miRNAs (Aleman et al., 2007; Birmingham et al., 2006; Jackson et al., 2003; Jackson et al., 2006b; Qiu et al., 2007). In some cases of off-targeting, no correlation between predicted and actual off-target effects was reported (Hanning et al., 2013). However, this probably reflects problems of accurate miRNA target prediction rather than the absence of miRNA-like off-targeting. In any case, a recent systematic analysis of off-targeting effects confirmed that strength of base pairing in the siRNA seed region is the primary factor determining the efficiency of off-target silencing (Kamola et al., 2015)

The main cause of off-targeting is miRNA-like behavior of siRNAs. It was experimentally demonstrated in mammalian cells that siRNAs can function as miRNAs (Doench et al., 2003) and that siRNAs imperfectly matching endogenous mRNAs repress translation (Martin and Caplen, 2006; Saxena et al., 2003) suggesting that miRNAs and siRNAs use similar if not identical, mechanisms for target repression (Zeng et al., 2003). The current view of mammalian RNAi is that experimental RNAi induced with a siRNA or shRNA hijacks the molecular machinery dedicated to the miRNA pathway (reviewed in Svoboda, 2014). Consequently, some degree of off-targeting likely occurs in every RNAi experiment.

Importantly, experimental RNAi can also cause artifacts through saturation of the miRNA pathway, which essentially suppresses normal miRNA function (Khan et al., 2009). Exportin 5 seems to be a bottleneck for an effective RNA silencing (Lu and Cullen, 2004; Yi et al., 2005). Indeed, lethal non-specific effects observed with type I shRNAs delivered to the mouse liver by a viral vector were linked to the saturation of Exportin 5 (Grimm et al., 2006). Inhibition of Exportin 5 could also provide an explanation to early lethality defects observed during generation transgenic mice carrying class I shRNA expression cassette (Cao et al., 2005). 


\section{Suppression and by-passing off-targeting}

Off-targeting has been a recurring problem with RNAi experiments, especially in RNAi screens searching for novel regulators. Off-targeting was frequently causing false-positive results in such screens although this issue has been partially remedied (reviewed in Mohr et al., 2014; Petri and Meister, 2013). Below, I list options for dealing with off-targeting, which emerged from the literature review.

\section{Appropriate experimental design}

This is actually a simple solution, which emerged from initial experiments detecting off-targeting (reviewed in(Svoboda, 2007), which suggested that off-targeting operates through miRNA-like behavior of siRNAs and is concentration-dependent. Thus, a proper practice is to use the minimal effective siRNA concentration. Importantly, this step strongly reduces off-targeting but it does not eliminate it as the targeting siRNA is still present and functions as a miRNA (Jackson et al., 2003; Jackson et al., 2006b).

\section{Pools of SiRNA}

An extension of a strategy to lower siRNA concentration to the point that off-targeting effects in the model system become very low or even undetectable. If a pool of 10 siRNAs is used at the same total siRNA concentration, a single siRNA is having ten times lower concentration and causes lower off-targeting effects. One can produce an siRNA pool by an enzymatic digest of long dsRNA with Dicer or simply purchase a number of siRNAs targeting a single mRNA. In fact, some companies offer pre-made siRNA pools. A unique type of siRNA pools are siPools, which are produced by in vitro transcription of tandemly arrayed siRNA sequences (Hannus et al., 2014)

\section{Bioinformatics filtering}

Since the siRNA seed region is strongly associated with off-target silencing (Jackson et al., 2006b; Kamola et al., 2015), it could be used to filter RNAi screening data to reduce of off-target rates (Yilmazel et al., 2014; Zhong et al., 2014). In fact, revised analysis of RNAi screens could identify functionally relevant genes suppressed by off-targeting (Adams et al., 2015; Lin et al., 2007; Singh et al., 2015).

\section{Better small RNA design}

Understanding of the molecular mechanism of RNAi is also reflected in constantly improving siRNA design which aims at providing siRNAs specifically silencing a gene of interest with little or no off-target effects and no cell toxicity (reviewed in Ahmed et al., 2015; Tafer, 2014). Improved siRNA design can reduce off-targeting in several ways. First, siRNAs designed for efficient strand selection would have reduced off-targeting caused by AGO-loaded passenger strand. It was also found that increased siRNA duplex stability 
correlates with reduced off-target and elevated on-target effects (Petri et al., 2011). This can be, for example, influenced by the seed binding energy and seed composition, which would determine the pool of potential binding sites in the transcriptome and the difference between on-target and off-target RNAs (Das et al., 2013a; Das et al., 2013b). Adaptations of siRNA/shRNA design to reduce off-target effects include weak base pairing in both seed and 3 ' regions (Gu et al., 2014) and evaluation of potential cross-hybridization candidates (Anderson et al., 2008; Yamada and Morishita, 2005). Reduced off-targeting features were subsequently integrated into siRNA design tools such as siDirect (Naito and Ui-Tei, 2013; Naito et al., 2009).

\section{Mismatch introduction}

Mismatch introduction into siRNA at the positions 2 of the base pairing also weakens off-targeting (Dua et al., 2011; Li et al., 2015)

\section{Chemical modifications of small RNAs}

The discovery that off-targeting involves miRNA-like behavior of siRNAs prompted research on chemical modifications that would reduce miRNA-like behavior while not interfering with desired RNAi effects (Chiu and Rana, 2003). A thorough review of the chemical modifications is beyond the scope of this report but can be found elsewhere (Engels, 2013; Nolte et al., 2013; Peacock et al., 2011; Snove and Rossi, 2006). There are two common strategies, to reduce off-targeting - (I) Chemical modifications on the passenger strand preventing its loading, hence eliminating off-targeting caused by the passenger strand (Chen et al., 2008; Snead et al., 2013) and (II) Chemical modifications in the seed region, which interfere with miRNA-like target recognition but do not prevent specific RNAi targeting. Different chemistry was used for chemical modification of siRNAs with reduced off-targeting effects including unlocked nucleic acid (UNA) modification (Snead et al., 2013), locked nucleic acid (LNA) modification (Fluiter et al., 2009), 2'-O-methyl ribosyl (Chen et al., 2008; Jackson et al., 2006a), or abasic nucleotides (Seok et al., 2016b).

Position of the modification on the guiding strand is important for reduced off-targeting. While it is usually involving seed, the modified nucleotide may vary. For example, 2'-O-methyl ribosyl substitution at position 2 in the guide strand reduces most off-target effects caused by complementarity to the seed region of the siRNA guide strand (Jackson et al., 2006a). At the same time, an abasic nucleotide at the position 6 in the guide strand also eliminates miRNA-like off-target repression but preserves near-perfect on-target activity (80-100\%) (Seok et al., 2016b).

\section{Discerning specific RNAi phenotypes from off-targeting effects}

While strategies for suppressing off-targeting effects clearly reduce experimental artifacts, off-targeting should be seen as a type of noise in RNAi experiments that cannot be completely eliminated. Assuming that some off-targeting occurs in every RNAi experiment, one can focus on a more important issue: how to identify biologically relevant effects 
of off-targeting (phenotype) and separate them from the specific RNAi effect caused by knock-down of the desired gene. The idea is simple - while one can try to minimize off-targeting effects, the risk cannot be completely eliminated. Thus, it is equally important to use an appropriate experimental design, which allows to distinguish between off-targeting and specific RNAi effects. The two possible strategies were proposed a decade ago and were named "the two R's": rescue and redundancy (Echeverri et al., 2006).

The principle of the rescue strategy is expressing an RNAi-resistant version of the targeted gene. If a phenotype is caused by the gene knock-down, it should be rescued. It can be either mutated such that the base pairing with a short RNA is eliminated. One can, for example target 3'UTR and use a different one in the rescue construct or mutate/degenerate appropriate codon positions if targeting CDS. This strategy is powerful because it accepts all effects in an RNAi experiment and tests the contribution of the specific gene knock-down. For recent information on design of the rescue system see, for example, (Kumar, 2015)

The second strategy is based on phenotype redundancy. Two or more RNAi triggers with different sequences (i.e. specific siRNAs or shRNAs) producing the same phenotype decrease the probability that a phenotype would be caused by off-targeting. However, some common phenotypes (e.g. slower growth, apoptosis, and developmental arrest) may be a frequent off-targeting phenotype induced by different RNAi triggers, so the redundancy strategy would be less powerful than the rescue strategy described above. However, for some purposes (e.g. high-throughput RNAi screening), it might be easier to implement the redundancy strategy as a control for off-targeting than the rescue strategy.

Importantly, "non-targeting" controls (e.g. siRNAs with a random sequence or targeting non-expressed genes such as EGFP or luciferase) cannot be used controls for off-targeting for reasons mentioned above. It is a frequent misconception ignoring the fact that off-targeting is individual to each RNAi trigger because it is sequence-specific. "Non-targeting" siRNAs or shRNAs RNAs may serve as controls for the sequence-independent effects, such as interferon response and saturation of RNA silencing with an excess of exogenous short RNAs. If a small RNA is needed as a control for off-targeting, one may only use a pool of scrambled small RNAs, which would have highly diluted off-targeting effects.

\section{Target identification - in silico \& experimental approaches}

Target identification is a common issue in the small RNA field (reviewed for example in Pasquinelli, 2012; Tarang and Weston, 2014). Target identification can utilize bioinformatic analysis, experimental analysis or their combination. Briefly, bioinformatic analysis primarily implements the canonical seed-match model, evolutionary conservation, and binding energy, which are often complemented by neural networks trained on sets of experimental data in order to optimize filtering parameters. Some bioinformatics tools also consider non-canonical binding sites. Importantly, a mere presence of a miRNA binding site is insufficient for predicting target regulation as additional factors influence the regulation, including the above-mentioned accessibility of a binding site and stoichiometry between a miRNA and its targets. In the end, experimental verification of microRNA targets is essential, prediction alone is insufficient (Law et al., 2013). 


\section{Target prediction in silico}

Identification of many miRNAs in model organisms prompted development of bioinformatics tools for prediction of targeted mRNAs (Enright et al., 2003; Grun et al., 2005; John et al., 2004; Kiriakidou et al., 2004; Krek et al., 2005; Lewis et al., 2003; Stark et al., 2003). A number of bioinformatics tools emerged for miRNA analysis and target prediction (a comprehensive overview of all miRNA analysis tools is provided at https://tools4mirs .org/, for recent reviews on bioinformatic target prediction see, for example, (Elton and Yalowich, 2015; Lagana, 2015; Li and Zhang, 2015; Ristevski, 2015). A searchable database of systematically annotated miRNA tools can be found here: https://tools4mirs.org /software/target_prediction/.

Importantly, accurate bioinformatic prediction of miRNA-mediated repression is still problematic. This was shown, for example, during experiments with systematically generated artificial miRNAs targeting a desired gene (Arroyo et al., 2014). It turned out that seed-based artificial miRNA design was highly inefficient, as the majority of miRNAs with even perfect seed matches did not repress either target. Moreover, commonly used target prediction programs had problems to discriminate effective artificial miRNAs from ineffective ones, indicating that current algorithms do not fully accommodate important miRNA features allowing for designing artificial miRNAs (Arroyo et al., 2014). Another unresolved issue is reliable prediction of non-canonical (non-seed) miRNA binding sites as most algorithms are based on detection of seed-based miRNA binding sites.

\section{Common target prediction tools for animal miRNAs}

Among the prediction tools, several can be highlighted. These include Targetscan, miRanda, DIANA-microT, PicTAR, whose predictions were integrated into the miRBase, the central annotation database for miRNAs (Kozomara and Griffiths-Jones, 2014) and were also repeatedly evaluated in benchmark studies (Alexiou et al., 2009; Ding et al., 2012; Majoros et al., 2013; Peterson et al., 2014; Xu et al., 2014a). I add to these also MIRZA as it is one of the most recent algorithms, which in many aspects outperforms the other ones (Gumienny and Zavolan, 2015).

\section{Targetscan (http://www.targetscan.org/vert_71/)}

Targetscan is one of the most popular miRNA target prediction tools and its predictions are integrated in the miRBase (Kozomara and Griffiths-Jones, 2014). It is being developed in David Bartel's laboratory as a tool for miRNA target prediction for over a decade (Lewis et al., 2005; Lewis et al., 2003). It predicts biological targets of miRNAs by searching for the presence of conserved $8 \mathrm{mer}, 7 \mathrm{mer}$, and $6 \mathrm{mer}$ sites that match the seed region of each miRNA but there is also an optional search for poorly conserved sites. Its development included also scoring for binding sites with mismatches in the seed region that are compensated by 3' end pairing (Friedman et al., 2009) an improved quantitative model of canonical targeting (Agarwal et al., 2015) and addition other features. The current version considers a site type and fourteen other features and, according to authors, 
it outperforms other tools and matches high-throughput in vivo crosslinking approaches (Agarwal et al., 2015).

\section{DIANA-MicroT (http://diana.imis.athena-innovation.gr/DianaTools/index.php)}

DIANA-MicroT target prediction tools are another popular source for miRNA target prediction whose predictions are integrated with miRBase (Kozomara and Griffiths-Jones, 2014). DIANA-MicroT tools are being developed in Artemis Hatzigeorgiou's laboratory for over a decade (Alexiou et al., 2010; Kiriakidou et al., 2004; Maragkakis et al., 2009; Maragkakis et al., 2011; Megraw et al., 2007; Paraskevopoulou et al., 2013a; Paraskevopoulou et al., 2013b; Paraskevopoulou et al., 2016; Reczko et al., 2011; Sethupathy et al., 2006; Vergoulis et al., 2012; Vlachos et al., 2012). MicroT is specifically trained on a positive and a negative set of miRNA binding sites located in 3'-UTR and CDS regions. DIANA Tools offer target prediction algorithms (microT $\mathrm{v} 4$ and microT-CDS), databases of experimentally verified miRNA targets on coding and non-coding RNAs (TarBase v7.0 and LncBase), and tools for assessment of biological impacts of miRNAs (mirPath). In addition, the Web Server (v5.0) supports workflows enabling to perform complex functional miRNA analyses.

\section{Pictar (http://www.pictar.org/)}

Pictar is an algorithm for the identification of microRNA targets from Nikolaus Rajewsky's laboratory (Grun et al., 2005; Krek et al., 2005). Its predictions are also integrated with miRBase (Kozomara and Griffiths-Jones, 2014). Pictar offers for searching of targets of annotated miRNAs or mRNAs. Pictar predicts targets based on complementarity in a $7 \mathrm{nt}$ seed region, takes into account conservation and uses hidden Markov model approach to produce the final score. In contrast to Targetscan and DIANA-MicroT, Pictar has not been intensely developed. While it represents one of the older and simpler target prediction algorithms it is quite accurate prediction tool (Alexiou et al., 2009).

\section{miRanda at microRNA.org - Targets and Expression (http://www.microrna.org/)}

miRanda belongs among the pioneering target prediction algorithms (Enright et al., 2003; John et al., 2004). Its latest version miRanda-miRSVR (Betel et al., 2010; Betel et al., 2008) is integrated into target predictions at http://www.microrna.org where one can search predictions for annotated miRNAs in the main experimental model organisms. These predictions are also integrated with miRBase (Kozomara and Griffiths-Jones, 2014). miRanda analyses miRNA sequence complementarity with 3'UTRs and evaluates binding energy, conservation and binding site position in the 3'UTR. miRanda-miRSVR also identifies non-canonical and non-conserved sites (Betel et al., 2010; Betel et al., 2008).

\section{MIRZA \& MIRZA-G (http://www.sib.swiss/zavolan-mihaela/services)}

These tools are being developed in Mihaela Zavolan's lab and can be used for the prediction of miRNA targets and siRNA off-targets on a genome-wide scale. MIRZA is biophysical 
model of microRNA-target interaction that enables accurate identification of microRNA targets, particularly from Argonaute-CLIP data (Khorshid et al., 2013). MIRZA-G employs both the MIRZA biophysical model as well as other features to predict microRNA target sites genome-wide (Gumienny and Zavolan, 2015). MIRZA-G performed better on a benchmark test than Targetscan Context+ and DIANA-microT-v3 (Gumienny and Zavolan, 2015) making it a good choice for predicting canonical and non-canonical miRNA target sites as well as siRNA off-target sites.

\section{Target prediction for plant miRNAs}

Target prediction for plant miRNAs differs from prediction of miRNA targets in animals because of frequent highly complementary targets of plant miRNAs. A pioneering study of miRNA-mediated repression in plants revealed near-perfect complementarity between Arabidopsis miRNAs and their targets suggests suggesting that many plant miRNAs act similarly to siRNAs and direct mRNA cleavage (Rhoades et al., 2002). Consequently, miRNA target prediction in plants (for a recent review, see, for example, (Mishra et al., 2015)) is routinely performed as a relatively simple search for highly complementary mRNA sequences without a specialized target prediction algorithm. For example, Singh et al. (Singh et al., 2016) used for miRNA target prediction in ginger (Zingiber officinale) the following three simple criteria, which could be written into a simple search script:

1) not more than four mismatches allowed between predicted mRNAs and target gene.

2) no mismatches allowed for 10th and 11th positions of complementary site (a cleavage site).

3) maximum $4 \mathrm{GU}$ pair was allowed in the complimentary alignment.

Some authors even use for searching sequence similarity between a plant miRNA and mRNAs the Basic Local Alignment Search Tool algorithm (Huang et al., 2014a). A specific plant-miRNA-target analysis server is psRNATarget: a plant small RNA target analysis server (Dai and Zhao, 2011), which can be used not only for miRNAs but also for other plant small RNA analysis (Guzman et al., 2013; Huang et al., 2014b; Kumar et al., 2014). Other authors use general target prediction algorithms such as Miranda or RNAhybrid either alone (Shweta and Khan, 2014) or in more complex arrangements (Kurubanjerdjit et al., 2013).

A systematic evaluation of tools to predict targets of miRNAs and siRNAs in plants was provided by Srivastava et al. who compared 11 computational tools in identifying genomewide targets in Arabidopsis and other plants. Among them, Targetfinder was the most efficient in predicting 'true-positive' targets in Arabidopsis miRNA-mRNA interactions but performed much worse when analyzing data from non-Arabidopsis species. (Srivastava et al., 2014). Furthermore, combination of Targetfinder and psRNATarget provides high true positive coverage, whereas the intersection of psRNATarget and Tapirhybrid outputs deliver highly 'precise' predictions. All evaluated tools yielded a large number of 'false negative' predictions in non-Arabidopsis datasets (Srivastava et al., 2014).

Targets of plant miRNAs, that induce sequence-specific RNAi-like cleavage, can be further identified by employing degradome sequencing, a method determining RNA termini. 
Thus, in mRNAs cleaved by a miRNA after its $10^{\text {th }}$ nucleotide, one would observe alignment of RNA termini matching the predicted miRNA binding site. This strategy complementing bioinformatics description is further described in the following section.

\section{Common experimental approaches for identification of targets of small RNAs}

Bioinformatic target prediction is probabilistic. In other words, bioinformatics predictions identifies a set of putative small RNA targets, which fit certain set of criteria and are assigned a certain probability of being targeted by a specific small RNA. At the same time, each prediction yields positive and false negative results. A common problem in bioinformatic prediction is reliable prediction of non-canonical targets, whose recognition does not involve a complete seed match and, to a lesser extent, prediction of targets recognized through non-conserved binding sites. It is common that researchers aiming at target identification start with bioinformatics prediction and become entangled in the net of prediction tools and generate partially overlapping lists of predicted targets. This strategy is inherently biased towards canonical conserved miRNA binding sites and the highest scoring targets will have more than one such a site. However, this strategy is problematic for identification of the full set of targets.

Accordingly, more reliable identification of small RNA targets usually combines bioinformatics and experimental approaches. (Chen et al., 2015; Tarang and Weston, 2014; Thomson et al., 2011).

\section{High throughput expression analysis}

High throughput analysis (expression arrays, RNA sequencing or high-throughput proteomics) can complement target prediction in different ways. One can manipulate the miRNA pathway by various means (reviewed, for example in Svoboda, 2015), such as miRNA overexpression, knock-out or inhibition by complementary oligonucleotides (so-called antagomirs) and then identify correlations between target prediction and their actual behavior (e.g. Krutzfeldt et al., 2005; Lim et al., 2005; Sood et al., 2006). However, these strategies yield only correlative results, i.e. do not directly detect smallRNA:targetRNA interaction.

\section{Small RNA capture strategies}

These strategies can be used to identify either mRNAs bound by a small RNA or small RNAs bound to a selected mRNA. Identification of targets of a small RNA employs delivery of a tagged small RNA (e.g. biotinylated miRNA) followed by an affinity capture to co-purify targets (Baigude et al., 2012; Orom and Lund, 2007; Tan and Lieberman, 2016). These strategies are prone to artifacts because delivery of biotinylated small RNAs can create nonphysiological conditions and affinity purification could be influenced by the binding site context.

Identification of small RNAs bound to a selected mRNA(e.g. miR-CATCH or miRIP methods) employs capture of a selected RNA with associated small RNA using a complementary 
oligonucleotide, which can be used for affinity capture (e.g. a biotinylated complementary oligonucleotide or a complementary oligonucleotide covalently bound to a magnetic bead) (Su et al., 2015; Vencken et al., 2015). This strategy is suitable for detailed analysis of miRNA-mediated regulation of a specific mRNA but not for a transcriptome-wide target assessment. One variant employs a tested mRNA fused with a common sequence (EGFP) allowing for using the same set of biotinylated DNA anti-sense oligonucleotides for analyzing different mRNAs allowing for increasing the throughput (Wei et al., 2014).

\section{miRNA extension}

This strategy employs miRNA extension with a reverse transcriptase on endogenous target mRNAs. Purified hybrid 3'-cDNA-miRNA-5' molecules are used in a second round of reverse transcription and sequenced (Vatolin et al., 2006). However, this method is prone to artifacts stemming from the variability of miRNA:target mRNA base pairing, which would result in highly variable efficiency of reverse transcription priming.

\section{Immunoprecipitation of small RNA:target RNA complexes}

There is a large number of immunoprecipitation strategies aimed at purifying small RNA:target RNA complexes, usually by immunoprecipitating them through an Argonaute protein. Initial experiments immunoprecipitated native Argonaute complexes without including a cross-linking step; immunopurified RNAs were analyzed on microarrays (Easow et al., 2007; Hendrickson et al., 2008; Karginov et al., 2007). An adaptation of Argonaute immunoprecipitation for detection of specific miRNA targets is a RIP competition assay wherein anti-miR is titrated into cytosolic extracts prior to Argonaute immunoprecipitation. Direct target transcripts displaced by anti-miR are then identified based on their depletion from IP fraction (Androsavich and Chau, 2014)

The immunoprecipitation strategy was further developed into a number of methods for isolation of small RNAs bound to their targets, which include high throughput sequencing of crosslinking immunoprecipitation (HITS-CLIP) and crosslinking ligation and sequencing of hybrids (CLASH) methods (reviewed more detail in Broughton and Pasquinelli, 2016; Jaskiewicz et al., 2012).

There are several modifications of the basic HITS-CLIP. An improvement of the basic HITS-CLIP approach (Chi et al., 2009; Zisoulis et al., 2010) came with crosslinking based on photoactivatable nucleosides such as 4-thiouridine, a CLIP modification known as photoactivatable-ribonucleoside-enhanced crosslinking and immunoprecipitation (PAR-CLIP) (Hafner et al., 2010; Hafner et al., 2012). PAR-CLIP offers more efficient crosslinking, hence up to three orders of magnitude better RNA recovery than HITS-CLIP (Hafner et al., 2010). Furthermore, PAR-CLIP also allows for precise localization of miRNA binding site as cross-linked 4-thiouridine marks the cross-linked site with frequent thymidine to cytidine change, which is revealed by deep sequencing (Hafner et al., 2010).

Another modified strategy is covalent ligation of endogenous Argonaute-bound RNAs crosslinking immunoprecipitation (CLEAR-CLIP) which enriches miRNAs ligated to their endogenous mRNA targets (Moore et al., 2015). CLEAR-CLIP approach is in principle 
the same as the above-mentioned CLASH (Helwak et al., 2013; Helwak and Tollervey, 2014). Adding ligation of miRNAs to their mRNA targets yields chimeric reads allowing for robust detection miRNA:target RNA interactions occurring in vivo.

Data mining of CLIP data provides not only a comprehensive list of miRNA:target mRNA interactions but also provides insights into the principles governing these interactions, which in turn facilitate further improvement of target prediction algorithms. For example, an in vivo C. elegans data set and reanalysis of published mammalian AGO-CLIP data yielded approximately 17,000 miRNA:target site interactions. This strategy identified canonical, noncanonical, and nonconserved miRNA:targets with about $80 \%$ of miRNA interactions having perfect or partial seed complementarity (Grosswendt et al., 2014). Another comprehensive analysis of 34 Argonaute HITS-CLIP datasets from human and mouse cells revealed that many heteroduplexes are "non-canonical" i.e. their seed region comprises G:U and bulge combinations (Clark et al., 2014).

CLIP strategies are nowadays popular for high-throughput analysis of physiological miRNA targets (Chi et al., 2012; Chi et al., 2009; Clark et al., 2014; Grosswendt et al., 2014; Haecker and Renne, 2014; Hafner et al., 2010; Imig et al., 2015; Leung et al., 2011; Liu et al., 2014a; Marin et al., 2012; Zisoulis et al., 2010) and it is accompanied with a number of algorithms and databases facilitating identification of miRNA targets in high-throughput CLIP data (Balaga et al., 2012; Bandyopadhyay et al., 2015; Chou et al., 2013; Erhard et al., 2013; Guo et al., 2015; Hsieh and Wang, 2011; Hsu et al., 2015; Liu et al., 2013; Paraskevopoulou et al., 2013a; Rennie et al., 2014; Wang et al., 2013; Wang et al., 2014; Xie et al., 2014; Yang et al., 2011).

\section{Degradome analysis}

It was mentioned above in the section discussing target prediction for plant miRNAs that when small RNA-target RNA interaction results in RNAi-like cleavage, identification of targets can be experimentally augmented by degradome sequencing. This sequencing allows for identification of RNA termini, including those created by RNAi-like cleavage. Thus, if there are mRNAs cleaved by a miRNA after its $10^{\text {th }}$ nucleotide, one would observe alignment of RNA termini matching the predicted miRNA binding site. Degradome analysis usually defines a category of transcripts predicted to be endonucleolytically cleaved and then are cleavage positions compared to predicted miRNA binding sites (Ding et al., 2016; Fan et al., 2016; Li and Sunkar, 2013; Shao et al., 2013; Wang et al., 2016; Xing et al., 2014). Degradome analysis and target prediction has been integrated in to a web resource comPARE for plant miRNA target analysis (Kakrana et al., 2014). Degradome analysis can be also used in animals to identify rare miRNA targets suppressed by slicing (Park et al., 2013).

\section{Conclusions}

Given the nature of the interaction between small RNAs and their target RNAs, target prediction will always yield putative targets with partial complementary. For seed-mediated interactions, there can easily be hundreds of targets predicted for any small RNA acting 
as a miRNA. This is due to the combination of the following facts: a) a specific hexamer sequence occurs in a random sequence with a theoretical frequency of 1/4096 and b) exons of protein-coding genes constitute 70-80 megabases of well-annotated mammalian genomes (and exome size of eukaryotic genomes might not be dramatically smaller than that). Therefore, a hexamer would occur in a mammalian exonic sequence on average $\sim 20$ $000 \mathrm{x}$ and if $1 \%$ of these hexamers would fit other target site prediction criteria, that would leave on average 200 potential binding sites.

The bottom line is that applying a minimal base pairing criterion for miRNA-like interaction will identify a number of potential targets in any eukaryotic organism. At the same time, sequence based target prediction is insufficient to assess whether there will be target repression induced by a specific small RNA when introduced into an animal or mammal because there is a number of other critical parameters, which must be considered. Two of them stand out above anything else: 1) the amount of the specific small RNA loaded on Argonaute proteins, and 2) target site accessibility. Thus, target assessment of small RNAs needs to address these two parameters. While target accessibility can be considered a relatively common feature for all organisms since the same rules would apply for RNA folding and interference caused by RNA binding proteins (translation machinery etc.), loading of a small RNA onto Argonaute proteins depends on factors which may dramatically differ between different organisms. For example, organisms that exhibit environmental RNAi would be much more prone to the uptake of small RNAs. Factors such as length, chemical modifications, or terminal nucleotides of a small RNA, could underlie differences in sorting and loading to the various Argonaute proteins. Thus, possible fates of a specific small RNA in a specific organism are difficult to predict and should be tested experimentally. The most informative parameter is the amount (number of molecules) of a small RNA in question, which would be loaded on an Argonaute protein (e.g. AGO1 and AGO2 in animals), because it could be compared with known kinetic data to assess the strength of potential repressive effects it could achieve in vivo.

\section{Acknowledgement}

I would like to thank my colleagues Jan Paces, Miloslav Nic, and Tomas Novotny for help with collecting literature for the review and Jana Kubikova for help with figure preparation. The review content was produced under a contract OC/EFSA/GMO/2015/01-CT 01 with European Food Safety Authority (EFSA); the opinions expressed are those of the contractor only and do not represent EFSA's official position. Publication of the review was funded by LO1220 and LM2015063 by the Ministry of Education, Youth and Sports.

\section{References}

Adams, R., Nicke, B., Pohlenz, H.D., and Sohler, F. (2015). Deciphering Seed Sequence Based Off-Target Effects in a Large-Scale RNAi Reporter Screen for E-Cadherin Expression. Plos One 10, e0137640-e0137640. 
Afonso-Grunz, F., and Muller, S. (2015). Principles of miRNA-mRNA interactions: beyond sequence complementarity. Cellular and Molecular Life Sciences 72, 3127-3141.

Agarwal, V., Bell, G.W., Nam, J.W., and Bartel, D.P. (2015). Predicting effective microRNA target sites in mammalian mRNAs. Elife 4.

Ahmed, F., Dai, X.B., and Zhao, P.X. (2015). Bioinformatics Tools for Achieving Better Gene Silencing in Plants. In Plant Gene Silencing: Methods and Protocols, pp. 43-60.

Aleman, L.M., Doench, J., and Sharp, P.A. (2007). Comparison of siRNA-induced off-target RNA and protein effects. Rna-a Publication of the Rna Society 13, 385-395.

Alexiou, P., Maragkakis, M., Papadopoulos, G.L., Reczko, M., and Hatzigeorgiou, A.G. (2009). Lost in translation: an assessment and perspective for computational microRNA target identification. Bioinformatics 25, 3049-3055.

Alexiou, P., Vergoulis, T., Gleditzsch, M., Prekas, G., Dalamagas, T., Megraw, M., Grosse, I., Sellis, T., and Hatzigeorgiou, A.G. (2010). miRGen 2.0: a database of microRNA genomic information and regulation. Nucleic Acids Research 38, D137-D141.

Allen, E., Xie, Z.X., Gustafson, A.M., Sung, G.H., Spatafora, J.W., and Carrington, J.C. (2004). Evolution of microRNA genes by inverted duplication of target gene sequences in Arabidopsis thaliana. Nature Genetics 36, 1282-1290.

Ameres, S.L., Martinez, J., and Schroeder, R. (2007). Molecular basis for target RNA recognition and cleavage by human RISC. Cell 130, 101-112.

Anderson, E.M., Birmingham, A., Baskerville, S., Reynolds, A., Maksimova, E., Leake, D., Fedorov, Y., Karpilow, J., and Khvorova, A. (2008). Experimental validation of the importance of seed complement frequency to siRNA specificity. Rna-a Publication of the Rna Society 14, 853-861.

Androsavich, J.R., and Chau, B.N. (2014). Non-inhibited miRNAs shape the cellular response to anti-miR. Nucleic Acids Research 42, 6945-6955.

Arroyo, J.D., Gallichotte, E.N., and Tewari, M. (2014). Systematic design and functional analysis of artificial microRNAs. Nucleic Acids Research 42, 6064-6077.

Axtell, M.J. (2013). Classification and comparison of small RNAs from plants. Annu Rev Plant Biol 64, 137-159.

Baigude, H., Ahsanullah, Li, Z., Zhou, Y., and Rana, T.M. (2012). miR-TRAP: a benchtop chemical biology strategy to identify microRNA targets. Angew Chem Int Ed Engl 51, 5880-5883.

Balaga, O., Friedman, Y., and Linial, M. (2012). Toward a combinatorial nature of microRNA regulation in human cells. Nucleic Acids Research 40, 9404-9416.

Bandyopadhyay, S., Ghosh, D., Mitra, R., and Zhao, Z.M. (2015). MBSTAR: multiple instance learning for predicting specific functional binding sites in microRNA targets. Scientific Reports $5,8004-8004$.

Bartel, D.P. (2009). MicroRNAs: Target Recognition and Regulatory Functions. Cell 136, 215-233.

Betel, D., Koppal, A., Agius, P., Sander, C., and Leslie, C. (2010). Comprehensive modeling of microRNA targets predicts functional non-conserved and non-canonical sites. Genome Biology 11, R90-R90.

Betel, D., Wilson, M., Gabow, A., Marks, D.S., and Sander, C. (2008). The microRNA.org resource: targets and expression. Nucleic Acids Research 36, D149-D153.

Bhandare, V., and Ramaswamy, A. (2016). Structural dynamics of human argonaute2 and its interaction with siRNAs designed to target mutant tdp43. Advances in Bioinformatics 2016, 8792814-8792814. 
Birmingham, A., Anderson, E.M., Reynolds, A., Ilsley-Tyree, D., Leake, D., Fedorov, Y., Baskerville, S., Maksimova, E., Robinson, K., Karpilow, J., et al. (2006). 3' UTR seed matches, but not overall identity, are associated with RNAi off-targets. Nature methods 3, 199-204.

Brennecke, J., Stark, A., Russell, R.B., and Cohen, S.M. (2005). Principles of MicroRNA-target recognition. Plos Biology 3, 404-418.

Broughton, J.P., and Pasquinelli, A.E. (2016). A tale of two sequences: microRNA-target chimeric reads. Genetics, selection, evolution : GSE 48, 31-31.

Brousse, C., Liu, Q.K., Beauclair, L., Deremetz, A., Axtell, M.J., and Bouche, N. (2014). A non-canonical plant microRNA target site. Nucleic Acids Research 42, 5270-5279.

Cao, W., Hunter, R., Strnatka, D., McQueen, C.A., and Erickson, R.P. (2005). DNA constructs designed to produce short hairpin, interfering RNAs in transgenic mice sometimes show early lethality and an interferon response. J Appl Genet 46, 217-225.

Chandradoss, S.D., Schirle, N.T., Szczepaniak, M., MacRae, I.J., and Joo, C. (2015). A Dynamic Search Process Underlies MicroRNA Targeting. Cell 162, 96-107.

Chen, L., Hou, J., and Liu, L. (2015). High-Throughput Techniques for Identifying microRNA Target Genes. Current Bioinformatics 10, 370-376.

Chen, P.Y., Weinmann, L., Gaidatzis, D., Pei, Y., Zavolan, M., Tuschl, T., and Meister, G. (2008). Strand-specific 5 '-O-methylation of siRNA duplexes controls guide strand selection and targeting specificity. Rna-a Publication of the Rna Society 14, 263-274.

Chi, S.W., Hannon, G.J., and Darnell, R.B. (2012). An alternative mode of microRNA target recognition. Nature Structural \& Molecular Biology 19, 321-U380.

Chi, S.W., Zang, J.B., Mele, A., and Darnell, R.B. (2009). Argonaute HITS-CLIP decodes microRNA-mRNA interaction maps. Nature 460, 479-486.

Chiu, Y.L., and Rana, T.M. (2003). siRNA function in RNAi: A chemical modification analysis. Rna-a Publication of the Rna Society 9, 1034-1048.

Chou, C.H., Lin, F.M., Chou, M.T., Hsu, S.D., Chang, T.H., Weng, S.L., Shrestha, S., Hsiao, C.C., Hung, J.H., and Huang, H.D. (2013). A computational approach for identifying microRNA-target interactions using high-throughput CLIP and PAR-CLIP sequencing. Bmc Genomics 14, S2-S2.

Cipolla, G.A. (2014). A non-canonical landscape of the microRNA system. Frontiers in genetics 5, 337-337.

Clark, P.M., Loher, P., Quann, K., Brody, J., Londin, E.R., and Rigoutsos, I. (2014). Argonaute CLIPSeq reveals miRNA targetome diversity across tissue types. Scientific Reports 4, 5947-5947.

Dai, X.B., and Zhao, P.X. (2011). psRNATarget: a plant small RNA target analysis server. Nucleic Acids Research 39, W155-W159.

Das, S., Ghosal, S., Chakrabarti, J., and Kozak, K. (2013a). SeedSeq: Off-Target Transcriptome Database. Biomed Research International 2013, 905429-905429.

Das, S., Ghosal, S., Kozak, K., and Chakrabarti, J. (2013b). An siRNA designing tool with a unique functional off-target filtering approach. Journal of Biomolecular Structure \& Dynamics 31, 1343-1357.

De, N., Young, L., Lau, P.W., Meisner, N.C., Morrissey, D.V., and MacRae, I.J. (2013). Highly Complementary Target RNAs Promote Release of Guide RNAs from Human Argonaute2. Molecular Cell 50, 344-355.

Deerberg, A., Willkomm, S., and Restle, T. (2013). Minimal mechanistic model of siRNA-dependent target RNA slicing by recombinant human Argonaute 2 protein. America 110, 17850-17855. 
Didiano, D., and Hobert, O. (2006). Perfect seed pairing is not a generally reliable predictor for miRNA-target interactions. Nature Structural \& Molecular Biology 13, 849-851.

Ding, J., Zhou, S., and Guan, J. (2012). Finding microRNA targets in plants: current status and perspectives. Genomics, proteomics \& bioinformatics 10, 264-275.

Ding, X.L., Li, J.J., Zhang, H., He, T.T., Han, S.H., Li, Y.W., Yang, S.P., and Gai, J.Y. (2016). Identification of miRNAs and their targets by high-throughput sequencing and degradome analysis in cytoplasmic male-sterile line NJCMS1A and its maintainer NJCMS1B of soybean. Bmc Genomics $17,24-24$.

Doench, J.G., Petersen, C.P., and Sharp, P.A. (2003). siRNAs can function as miRNAs. Genes \& Development $17,438-442$.

Du, Q., Thonberg, H., Wang, J., Wahlestedt, C., and Liang, Z.C. (2005). A systematic analysis of the silencing effects of an active siRNA at all single-nucleotide mismatched target sites. Nucleic Acids Research 33, 1671-1677.

Dua, P., Yoo, J.W., Kim, S., and Lee, D.K. (2011). Modified siRNA Structure With a Single Nucleotide Bulge Overcomes Conventional siRNA-mediated Off-target Silencing. Molecular Therapy 19, 1676-1687.

Easow, G., Teleman, A.A., and Cohen, S.M. (2007). Isolation of microRNA targets by miRNP immunopurification. Rna-a Publication of the Rna Society 13, 1198-1204.

Echeverri, C.J., Beachy, P.A., Baum, B., Boutros, M., Buchholz, F., Chanda, S.K., Downward, J., Ellenberg, J., Fraser, A.G., Hacohen, N., et al. (2006). Minimizing the risk of reporting false positives in large-scale RNAi screens. Nat Methods 3, 777-779.

Elkayam, E., Kuhn, C.D., Tocilj, A., Haase, A.D., Greene, E.M., Hannon, G.J., and Joshua-Tor, L. (2012). The structure of human argonaute-2 in complex with miR-20a. Cell 150, 100-110.

Ellwanger, D.C., Buttner, F.A., Mewes, H.W., and Stumpflen, V. (2011). The sufficient minimal set of miRNA seed types. Bioinformatics 27, 1346-1350.

Elton, T.S., and Yalowich, J.C. (2015). Experimental procedures to identify and validate specific mRNA targets of miRNAs. EXCLI journal 14, 758-790.

Engels, J.W. (2013). Gene silencing by chemically modified siRNAs. New Biotechnology 30, 302-307.

Enright, A.J., John, B., Gaul, U., Tuschl, T., Sander, C., and Marks, D.S. (2003). MicroRNA targets in Drosophila. Genome biology 5, R1-R1.

Erhard, F., Dolken, L., Jaskiewicz, L., and Zimmer, R. (2013). PARma: identification of microRNA target sites in AGO-PAR-CLIP data. Genome Biology 14, R79-R79.

Faehnle, C.R., Elkayam, E., Haase, A.D., Hannon, G.J., and Joshua-Tor, L. (2013). The making of a slicer: activation of human Argonaute-1. Cell Rep 3, 1901-1909.

Faehnle, C.R., and Joshua-Tor, L. (2007). Argonautes confront new small RNAs. Curr Opin Chem Biol 11, 569-577.

Fan, G.Q., Li, X.Y., Deng, M.J., Zhao, Z.L., and Yang, L. (2016). Comparative Analysis and Identification of miRNAs and Their Target Genes Responsive to Salt Stress in Diploid and Tetraploid Paulownia fortunei Seedlings. Plos One 11, e0149617-e0149617.

Fedorov, Y., Anderson, E.M., Birmingham, A., Reynolds, A., Karpilow, J., Robinson, K., Leake, D., Marshall, W.S., and Khvorova, A. (2006). Off-target effects by siRNA can induce toxic phenotype. Rna-a Publication of the Rna Society 12, 1188-1196. 
Flores, O., Kennedy, E.M., Skalsky, R.L., and Cullen, B.R. (2014). Differential RISC association of endogenous human microRNAs predicts their inhibitory potential. Nucleic Acids Research 42, 4629-4639.

Fluiter, K., Mook, O.R.F., and Baas, F. (2009). The Therapeutic Potential of LNA-Modified siRNAs: Reduction of Off-Target Effects by Chemical Modification of the siRNA Sequence. In Methods in Molecular Biology, pp. 189-203.

Friedman, R.C., Farh, K.K.H., Burge, C.B., and Bartel, D.P. (2009). Most mammalian mRNAs are conserved targets of microRNAs. Genome Research 19, 92-105.

Gan, H.H., and Gunsalus, K.C. (2015). Assembly and analysis of eukaryotic Argonaute-RNA complexes in microRNA-target recognition. Nucleic Acids Research 43, 9613-9625.

Grimm, D., Streetz, K.L., Jopling, C.L., Storm, T.A., Pandey, K., Davis, C.R., Marion, P., Salazar, F., and Kay, M.A. (2006). Fatality in mice due to oversaturation of cellular microRNA/short hairpin RNA pathways. Nature 441, 537-541.

Grimson, A., Farh, K.K.H., Johnston, W.K., Garrett-Engele, P., Lim, L.P., and Bartel, D.P. (2007). MicroRNA targeting specificity in mammals: Determinants beyond seed pairing. Molecular Cell 27, 91-105.

Grosswendt, S., Filipchyk, A., Manzano, M., Klironomos, F., Schilling, M., Herzog, M., Gottwein, E., and Rajewsky, N. (2014). Unambiguous Identification of miRNA: Target Site Interactions by Different Types of Ligation Reactions. Molecular Cell 54, 1042-1054.

Grun, D., Wang, Y.L., Langenberger, D., Gunsalus, K.C., and Rajewsky, N. (2005). MicroRNA target predictions across seven Drosophila species and comparison to mammalian targets. Plos Computational Biology 1, 51-66.

Gu, S., Zhang, Y., Jin, L., Huang, Y., Zhang, F.J., Bassik, M.C., Kampmann, M., and Kay, M.A. (2014). Weak base pairing in both seed and 3 ' regions reduces RNAi off-targets and enhances si/ shRNA designs. Nucleic Acids Research 42, 12169-12176.

Gumienny, R., and Zavolan, M. (2015). Accurate transcriptome-wide prediction of microRNA targets and small interfering RNA off-targets with MIRZA-G. Nucleic Acids Research 43, 1380-1391.

Guo, Z.W., Xie, C., Yang, J.R., Li, J.H., Yang, J.H., and Zheng, L.M. (2015). MtiBase: a database for decoding microRNA target sites located within CDS and 5 ' UTR regions from CLIP-Seq and expression profile datasets. Database-the Journal of Biological Databases and Curation 2015.

Guzman, F., Almerao, M.P., Korbes, A.P., Christoff, A.P., Zanella, C.M., Bered, F., and Margis, R. (2013). Identification of potential miRNAs and their targets in Vriesea carinata (Poales, Bromeliaceae). Plant Science 210, 214-223.

Haecker, I., and Renne, R. (2014). HITS-CLIP and PAR-CLIP advance viral miRNA targetome analysis. Critical Reviews in Eukaryotic Gene Expression 24, 101-116.

Hafner, M., Landthaler, M., Burger, L., Khorshid, M., Hausser, J., Berninger, P., Rothballer, A., Ascano, M., Jr., Jungkamp, A.C., Munschauer, M., et al. (2010). Transcriptome-wide identification of RNA-binding protein and microRNA target sites by PAR-CLIP. Cell 141, 129-141.

Hafner, M., Lianoglou, S., Tuschl, T., and Betel, D. (2012). Genome-wide identification of miRNA targets by PAR-CLIP. Methods 58, 94-105.

Haley, B., and Zamore, P.D. (2004). Kinetic analysis of the RNAi enzyme complex. Nature Structural \& Molecular Biology 11, 599-606.

Hanning, J.E., Saini, H.K., Murray, M.J., van Dongen, S., Davis, M.P.A., Barker, E.M., Ward, D.M., Scarpini, C.G., Enright, A.J., Pett, M.R., et al. (2013). Lack of correlation between predicted and 
actual off-target effects of short-interfering RNAs targeting the human papillomavirus type 16 E7 oncogene. British Journal of Cancer 108, 450-460.

Hannus, M., Beitzinger, M., Engelmann, J.C., Weickert, M.T., Spang, R., Hannus, S., and Meister, G. (2014). siPools: highly complex but accurately defined siRNA pools eliminate off-target effects. Nucleic Acids Research 42, 8049-8061.

Heale, B.S.E., Soifer, H.S., Bowers, C., and Rossi, J.J. (2005). siRNA target site secondary structure predictions using local stable substructures. Nucleic Acids Research 33, 1-10.

Heale, B.S.E., Soifer, H.S., Bowers, C., and Rossi, J.J. (2006). siRNA target site secondary structure predictions using local stable substructures (vol 33, pg 30, 2005). Nucleic Acids Research 34, 4653-4653.

Helwak, A., Kudla, G., Dudnakova, T., and Tollervey, D. (2013). Mapping the Human miRNA Interactome by CLASH Reveals Frequent Noncanonical Binding. Cell 153, 654-665.

Helwak, A., and Tollervey, D. (2014). Mapping the miRNA interactome by cross-linking ligation and sequencing of hybrids (CLASH). Nature Protocols 9, 711-728.

Hendrickson, D.G., Hogan, D.J., Herschlag, D., Ferrell, J.E., and Brown, P.O. (2008). Systematic Identification of mRNAs Recruited to Argonaute 2 by Specific microRNAs and Corresponding Changes in Transcript Abundance. Plos One 3, e2126-e2126.

Hill, C.G., Jabbari, N., Matyunina, L.V., and McDonald, J.F. (2014). Functional and Evolutionary Significance of Human MicroRNA Seed Region Mutations. Plos One 9, e115241-e115241.

Holen, T., Moe, S.E., Sorbo, J.G., Meza, T.J., Ottersen, O.P., and Klungland, A. (2005). Tolerated wobble mutations in siRNAs decrease specificity, but can enhance activity in vivo. Nucleic Acids Research 33, 4704-4710.

Hon, L.S., and Zhang, Z.M. (2007). The roles of binding site arrangement and combinatorial targeting in microRNA repression of gene expression. Genome Biology 8, R166-R166.

Hsieh, W.J., and Wang, H.Y. (2011). Human microRNA target identification by RRSM. Journal of Theoretical Biology 286, 79-84.

Hsu, S.D., Huang, H.Y., Chou, C.H., Sun, Y.M., Hsu, M.T., and Tsou, A.P. (2015). Integrated analyses to reconstruct microRNA-mediated regulatory networks in mouse liver using high-throughput profiling. Bmc Genomics 16, S12-S12.

Huang, Y., Zou, Q., Sun, X.H., and Zhao, L.P. (2014a). Computational Identification of MicroRNAs and Their Targets in Perennial Ryegrass (Lolium perenne). Applied Biochemistry and Biotechnology 173, 1011-1022.

Huang, Y., Zou, Q., and Wang, Z.B. (2014b). Computational identification of miRNA genes and their targets in mulberry. Russian Journal of Plant Physiology 61, 537-542.

Imig, J., Brunschweiger, A., Brummer, A., Guennewig, B., Mittal, N., Kishore, S., Tsikrika, P., Gerber, A.P., Zavolan, M., and Hall, J. (2015). miR-CLIP capture of a miRNA targetome uncovers a lincRNA H19-miR-106a interaction. Nature Chemical Biology 11, 107-U143.

Jackson, A.L., Bartz, S.R., Schelter, J., Kobayashi, S.V., Burchard, J., Mao, M., Li, B., Cavet, G., and Linsley, P.S. (2003). Expression profiling reveals off-target gene regulation by RNAi. Nature Biotechnology 21, 635-637.

Jackson, A.L., Burchard, J., Leake, D., Reynolds, A., Schelter, J., Guo, J., Johnson, J.M., Lim, L., Karpilow, J., Nichols, K., et al. (2006a). Position-specific chemical modification of siRNAs reduces "off-target" transcript silencing. Rna-a Publication of the Rna Society 12, 1197-1205. 
Jackson, A.L., Burchard, J., Schelter, J., Chau, B.N., Cleary, M., Lim, L., and Linsley, P.S. (2006b). Widespread siRNA “off-target” transcript silencing mediated by seed region sequence complementarity. Rna-a Publication of the Rna Society 12, 1179-1187.

Jagla, B., Aulner, N., Kelly, P.D., Song, D., Volchuk, A., Zatorski, A., Shum, D., Mayer, T., De Angelis, D.A., Ouerfelli, O., et al. (2005). Sequence characteristics of functional siRNAs. Rna-a Publication of the Rna Society 11, 864-872.

Janas, M.M., Wang, B., Harris, A.S., Aguiar, M., Shaffer, J.M., Subrahmanyam, Y.V., Behlke, M.A., Wucherpfennig, K.W., Gygi, S.P., Gagnon, E., et al. (2012). Alternative RISC assembly: binding and repression of microRNA-mRNA duplexes by human Ago proteins. RNA 18, 2041-2055.

Jaskiewicz, L., Bilen, B., Hausser, J., and Zavolan, M. (2012). Argonaute CLIP - A method to identify in vivo targets of miRINAs. Methods 58, 106-112.

Jo, M.H., Shin, S., Jung, S.R., Kim, E., Song, J.J., and Hohng, S. (2015a). Human Argonaute 2 Has Diverse Reaction Pathways on Target RNAs. Molecular Cell 59, 117-124.

Jo, M.H., Song, J.-J., and Hohng, S. (2015b). Single-molecule fluorescence measurements reveal the reaction mechanisms of the core-RISC, composed of human Argonaute 2 and a guide RNA. BMB Reports 48, 643-644.

John, B., Enright, A.J., Aravin, A., Tuschl, T., Sander, C., and Marks, D.S. (2004). Human MicroRNA targets. Plos Biology 2, 1862-1879.

Jones-Rhoades, M.W., and Bartel, D.P. (2004). Computational identification of plant microRNAs and their targets, including a stress-induced miRNA. Mol Cell 14, 787-799.

Jones-Rhoades, M.W., Bartel, D.P., and Bartel, B. (2006). MicroRNAS and their regulatory roles in plants. Annu Rev Plant Biol 57, 19-53.

Kakrana, A., Hammond, R., Patel, P., Nakano, M., and Meyers, B.C. (2014). sPARTA: a parallelized pipeline for integrated analysis of plant miRNA and cleaved mRNA data sets, including new miRNA target-identification software. Nucleic Acids Research 42, e139-e139.

Kamola, P.J., Nakano, Y., Takahashi, T., Wilson, P.A., and Uitei, K. (2015). The siRNA Non-seed Region and Its Target Sequences Are Auxiliary Determinants of Off-Target Effects. Plos Computational Biology 11, e1004656-e1004656.

Karginov, F.V., Conaco, C., Xuan, Z., Schmidt, B.H., Parker, J.S., Mandel, G., and Hannon, G.J. (2007). A biochemical approach to identifying microRNA targets. America 104, 19291-19296.

Katoh, T., and Suzuki, T. (2007). Specific residues at every third position of siRNA shape its efficient RNAi activity. Nucleic Acids Research 35, e27-e27.

Kertesz, M., Iovino, N., Unnerstall, U., Gaul, U., and Segal, E. (2007). The role of site accessibility in microRNA target recognition. Nature Genetics 39, 1278-1284.

Khan, A.A., Betel, D., Miller, M.L., Sander, C., Leslie, C.S., and Marks, D.S. (2009). Transfection of small RNAs globally perturbs gene regulation by endogenous microRNAs. Nature Biotechnology 27, 549-U592.

Khorshid, M., Hausser, J., Zavolan, M., and Van Nimwegen, E. (2013). A biophysical miRNA-mRNA interaction model infers canonical and noncanonical targets. Nature Methods 10, 253-255.

Kiriakidou, M., Nelson, P.T., Kouranov, A., Fitziev, P., Bouyioukos, C., Mourelatos, Z., and Hatzigeorgiou, A. (2004). A combined computational-experimental approach predicts human microRNA targets. Genes \& Development 18, 1165-1178.

Kozomara, A., and Griffiths-Jones, S. (2014). miRBase: annotating high confidence microRNAs using deep sequencing data. Nucleic Acids Res 42, D68-73. 
Krek, A., Grun, D., Poy, M.N., Wolf, R., Rosenberg, L., Epstein, E.J., MacMenamin, P., da Piedade, I., Gunsalus, K.C., Stoffel, M., et al. (2005). Combinatorial microRNA target predictions. Nature Genetics 37, 495-500.

Krutzfeldt, J., Rajewsky, N., Braich, R., Rajeev, K.G., Tuschl, T., Manoharan, M., and Stoffel, M. (2005). Silencing of microRNAs in vivo with 'antagomirs'. Nature 438, 685-689.

Kumar, D. (2015). Synthetic Gene Complementation to Determine Off-Target Silencing. In Plant Gene Silencing: Methods and Protocols, pp. 281-293.

Kumar, D., Singh, D., Kanodia, P., Prabhu, K.V., Kumar, M., and Mukhopadhyay, K. (2014). Discovery of novel leaf rust responsive microRNAs in wheat and prediction of their target genes. Journal of Nucleic Acids 2014, 570176-570176.

Kurubanjerdjit, N., Huang, C.H., Lee, Y.L., Tsai, J.J.P., and Ng, K.L. (2013). Prediction of microRNA-regulated protein interaction pathways in Arabidopsis using machine learning algorithms. Computers in Biology and Medicine 43, 1645-1652.

Lagana, A. (2015). Computational Prediction of microRNA Targets. In Microrna: Basic Science: From Molecular Biology to Clinical Practice, pp. 231-252.

Lagos-Quintana, M., Rauhut, R., Lendeckel, W., and Tuschl, T. (2001). Identification of novel genes coding for small expressed RNAs. Science 294, 853-858.

Lau, N.C., Lim, L.P., Weinstein, E.G., and Bartel, D.P. (2001). An abundant class of tiny RNAs with probable regulatory roles in Caenorhabditis elegans. Science 294, 858-862.

Law, P.T., Qin, H., Chan, T.F., and Wong, N. (2013). Experimental verification of microRNA targets is essential, prediction alone is insufficient. Carcinogenesis 34, 723-723.

Lee, R.C., and Ambros, V. (2001). An extensive class of small RNAs in Caenorhabditis elegans. Science 294, 862-864.

Leoni, G., and Tramontano, A. (2016). A structural view of microRNA-target recognition. Nucleic acids research.

Leung, A.K.L., Young, A.G., Bhutkar, A., Zheng, G.X., Bosson, A.D., Nielsen, C.B., and Sharp, P.A. (2011). Genome-wide identification of Ago2 binding sites from mouse embryonic stem cells with and without mature microRNAs. Nature Structural \& Molecular Biology 18, 237-U309.

Lewis, B.P., Burge, C.B., and Bartel, D.P. (2005). Conserved seed pairing, often flanked by adenosines, indicates that thousands of human genes are microRNA targets. Cell 120, 15-20.

Lewis, B.P., Shih, I.H., Jones-Rhoades, M.W., Bartel, D.P., and Burge, C.B. (2003). Prediction of mammalian microRNA targets. Cell 115, 787-798.

Li, C., Liu, Z., Yang, F., Liu, W., Wang, D., Dong, E., Wang, Y., Wu, C.I., and Lu, X. (2015). siRNAs with decreased off-target effect facilitate the identification of essential genes in cancer cells, pp. 21603-21613.

Li, J.J., Kim, T.H., Nutiu, R., Ray, D., Hughes, T.R., and Zhang, Z.L. (2014). Identifying mRNA sequence elements for target recognition by human Argonaute proteins. Genome Research 24, 775-785.

Li, Y., and Zhang, C.Y. (2012). Analysis of MicroRNA-Induced Silencing Complex-Involved MicroRNA-Target Recognition by Single-Molecule Fluorescence Resonance Energy Transfer. Analytical Chemistry 84, 5097-5102.

Li, Y., and Zhang, Z.L. (2015). Computational Biology in microRNA. Wiley Interdisciplinary Reviews-Rna 6, 435-452. 
Li, Y.F., and Sunkar, R. (2013). Global identification of small RNA targets in plants by sequencing sliced ends of messenger RNAs, pp. 119-129.

Lim, L.P., Lau, N.C., Garrett-Engele, P., Grimson, A., Schelter, J.M., Castle, J., Bartel, D.P., Linsley, P.S., and Johnson, J.M. (2005). Microarray analysis shows that some microRNAs downregulate large numbers of target mRNAs. Nature 433, 769-773.

Lin, X., Morgan-Lappe, S., Huang, X., Li, L., Zakula, D.M., Vernetti, L.A., Fesik, S.W., and Shen, Y. (2007). 'Seed' analysis of off-target siRNAs reveals an essential role of Mcl-1 in resistance to the small-molecule Bcl-2/Bcl-X-L inhibitor ABT-737. Oncogene 26, 3972-3979.

Lin, X.Y., Ruan, X., Anderson, M.G., McDowell, J.A., Kroeger, P.E., Fesik, S.W., and Shen, Y. (2005). siRNA-mediated off-target gene silencing triggered by a $7 \mathrm{nt}$ complementation. Nucleic Acids Research 33, 4527-4535.

Liu, C.C., Mallick, B., Long, D., Rennie, W.A., Wolenc, A., Carmack, C.S., and Ding, Y. (2013). CLIP-based prediction of mammalian microRNA binding sites. Nucleic Acids Research 41, e138-e138.

Liu, C.C., Rennie, W.A., Mallick, B., Kanoria, S., Long, D., Wolenc, A., Carmack, C.S., and Ding, Y. (2014a). MicroRNA binding sites in C. elegans 3 ' UTRs. Rna Biology 11, 693-701.

Liu, J., Carmell, M.A., Rivas, F.V., Marsden, C.G., Thomson, J.M., Song, J.J., Hammond, S.M., Joshua-Tor, L., and Hannon, G.J. (2004). Argonaute2 is the catalytic engine of mammalian RNAi. Science 305, 1437-1441.

Liu, Q.K., Wang, F., and Axtell, M.J. (2014b). Analysis of Complementarity Requirements for Plant MicroRNA Targeting Using a Nicotiana benthamiana Quantitative Transient Assay. Plant Cell 26, 741-753.

Llave, C., Xie, Z., Kasschau, K.D., and Carrington, J.C. (2002). Cleavage of Scarecrow-like mRNA targets directed by a class of Arabidopsis miRNA. Science 297, 2053-2056.

Long, D., Lee, R., Williams, P., Chan, C.Y., Ambros, V., and Ding, Y. (2007). Potent effect of target structure on microRNA function. Nature Structural \& Molecular Biology 14, 287-294.

Lu, S., and Cullen, B.R. (2004). Adenovirus VA1 noncoding RNA can inhibit small interfering RNA and MicroRNA biogenesis. J Virol 78, 12868-12876.

Lytle, J.R., Yario, T.A., and Steitz, J.A. (2007). Target mRNAs are repressed as efficiently by microRNA-binding sites in the 5 ' UTR as in the 3 ' UTR. America 104, 9667-9672.

Ma, J.B., Yuan, Y.R., Meister, G., Pei, Y., Tuschl, T., and Patel, D.J. (2005). Structural basis for 5 '-end-specific recognition of guide RNA by the A-fulgidus Piwi protein. Nature 434, 666-670.

Ma, Y., Creanga, A., Lum, L., and Beachy, P.A. (2006). Prevalence of off-target effects in Drosophila RNA interference screens. Nature 443, 359-363.

Majoros, W.H., Lekprasert, P., Mukherjee, N., Skalsky, R.L., Corcoran, D.L., Cullen, B.R., and Ohler, U. (2013). MicroRNA target site identification by integrating sequence and binding information. Nat Methods 10, 630-633.

Majoros, W.H., and Ohler, U. (2007). Spatial preferences of microRNA targets in 3 ' untranslated regions. Bmc Genomics 8, 152-152.

Maragkakis, M., Reczko, M., Simossis, V.A., Alexiou, P., Papadopoulos, G.L., Dalamagas, T., Giannopoulos, G., Goumas, G., Koukis, E., Kourtis, K., et al. (2009). DIANA-microT web server: elucidating microRNA functions through target prediction. Nucleic Acids Research 37, W273-W276. 
Maragkakis, M., Vergoulis, T., Alexiou, P., Reczko, M., Plomaritou, K., Gousis, M., Kourtis, K., Koziris, N., Dalamagas, T., and Hatzigeorgiou, A.G. (2011). DIANA-microT Web server upgrade supports Fly and Worm miRNA target prediction and bibliographic miRNA to disease association. Nucleic Acids Research 39, W145-W148.

Marin, R.M., Voellmy, F., von Erlach, T., and Vanicek, J. (2012). Analysis of the accessibility of CLIP bound sites reveals that nucleation of the miRNA:mRNA pairing occurs preferentially at the 3 '-end of the seed match. Rna-a Publication of the Rna Society 18, 1760-1770.

Martin, H.C., Wani, S., Steptoe, A.L., Krishnan, K., Nones, K., Nourbakhsh, E., Vlassov, A., Grimmond, S.M., and Cloonan, N. (2014). Imperfect centered miRNA binding sites are common and can mediate repression of target mRNAs. Genome Biology 15, R51-R51.

Martin, S.E., and Caplen, N.J. (2006). Mismatched siRNAs downregulate mRNAs as a function of target site location. Febs Letters 580, 3694-3698.

Megraw, M., Sethupathy, P., Corda, B., and Hatzigeorgiou, A.G. (2007). miRGen: a database for the study of animal microRNA genomic organization and function. Nucleic Acids Research 35, D149-D155.

Meister, G., Landthaler, M., Patkaniowska, A., Dorsett, Y., Teng, G., and Tuschl, T. (2004). Human Argonaute2 mediates RNA cleavage targeted by miRNAs and siRNAs. Mol Cell 15, 185-197.

Mishra, A.K., Duraisamy, G.S., and Matousek, J. (2015). Discovering MicroRNAs and Their Targets in Plants. Critical Reviews in Plant Sciences 34, 553-571.

Mohr, S.E., Smith, J.A., Shamu, C.E., Neumuller, R.A., and Perrimon, N. (2014). RNAi screening comes of age: improved techniques and complementary approaches. Nature Reviews Molecular Cell Biology 15, 591-600.

Moore, M.J., Scheel, T.K.H., Luna, J.M., Park, C.Y., Fak, J.J., Nishiuchi, E., Rice, C.M., and Darnell, R.B. (2015). miRNA-target chimeras reveal miRNA 3 '-end pairing as a major determinant of Argonaute target specificity. Nature Communications 6, 8864-8864.

Muckstein, U., Tafer, H., Hackermuller, J., Bernhart, S.H., Stadler, P.F., and Hofacker, I.L. (2006). Thermodynamics of RNA-RNA binding. Bioinformatics 22, 1177-1182.

Mullany, L.E., Herrick, J.S., Wolff, R.K., and Slattery, M.L. (2016). MicroRNA Seed Region Length Impact on Target Messenger RNA Expression and Survival in Colorectal Cancer. PloS one 11, e0154177-e0154177.

Naito, Y., and Ui-Tei, K. (2013). Designing functional siRNA with reduced off-target effects. Methods in Molecular Biology 942, 57-68.

Naito, Y., Yoshimura, J., Morishita, S., and Ui-Tei, K. (2009). siDirect 2.0: updated software for designing functional siRNA with reduced seed-dependent off-target effect. Bmc Bioinformatics 10, 392-392.

Nakanishi, K., Ascano, M., Gogakos, T., Ishibe-Murakami, S., Serganov, A.A., Briskin, D., Morozov, P., Tuschl, T., and Patel, D.J. (2013). Eukaryote-Specific Insertion Elements Control Human ARGONAUTE Slicer Activity. Cell Reports 3, 1893-1900.

Nam, J.W., Rissland, O.S., Koppstein, D., Abreu-Goodger, C., Jan, C.H., Agarwal, V., Yildirim, M.A., Rodriguez, A., and Bartel, D.P. (2014a). Global Analyses of the Effect of Different Cellular Contexts on MicroRNA Targeting. Molecular Cell 53, 1031-1043.

Nam, S., Ryu, H., Son, W.J., Kim, Y.H., Kim, K.T., Balch, C., Nephew, K.P., and Lee, J. (2014b). $\mathrm{Mg} 2+$ Effect on Argonaute and RNA Duplex by Molecular Dynamics and Bioinformatics Implications. Plos One 9, e109745-e109745. 
Nielsen, C.B., Shomron, N., Sandberg, R., Hornstein, E., Kitzman, J., and Burge, C.B. (2007). Determinants of targeting by endogenous and exogenous microRNAs and siRNAs. Rna-a Publication of the Rna Society 13, 1894-1910.

Nolte, A., Ott, K., Rohayem, J., Walker, T., Schlensak, C., and Wendel, H.P. (2013). Modification of small interfering RNAs to prevent off-target effects by the sense strand. New Biotechnology 30 , 159-165.

Orom, U.A., and Lund, A.H. (2007). Isolation of microRNA targets using biotinylated synthetic microRNAs. Methods 43, 162-165.

Paraskevopoulou, M.D., Georgakilas, G., Kostoulas, N., Reczko, M., Maragkakis, M., Dalamagas, T.M., and Hatzigeorgiou, A.G. (2013a). DIANA-LncBase: experimentally verified and computationally predicted microRNA targets on long non-coding RNAs. Nucleic Acids Research 41, D239-D245.

Paraskevopoulou, M.D., Georgakilas, G., Kostoulas, N., Vlachos, I.S., Vergoulis, T., Reczko, M., Filippidis, C., Dalamagas, T., and Hatzigeorgiou, A.G. (2013b). DIANA-microT web server v5.0: service integration into miRNA functional analysis workflows. Nucleic Acids Research 41, W169-W173.

Paraskevopoulou, M.D., Vlachos, I.S., Karagkouni, D., Georgakilas, G., Kanellos, I., Vergoulis, T., Zagganas, K., Tsanakas, P., Floros, E., Dalamagas, T., et al. (2016). DIANA-LncBase v2: indexing microRNA targets on non-coding transcripts. Nucleic Acids Research 44, D231-D238.

Park, J.H., Ahn, S., Kim, S., Lee, J., Nam, J.W., and Shin, C. (2013). Degradome sequencing reveals an endogenous microRNA target in C. elegans. Febs Letters 587, 964-969.

Parker, J.S., Roe, S.M., and Barford, D. (2004). Crystal structure of a PIWI protein suggests mechanisms for siRNA recognition and slicer activity. Embo Journal 23, 4727-4737.

Parker, J.S., Roe, S.M., and Barford, D. (2005). Structural insights into mRNA recognition from a PIWI domain-siRNA guide complex. Nature 434, 663-666.

Pasquinelli, A.E. (2012). MicroRNAs and their targets: recognition, regulation and an emerging reciprocal relationship. Nat Rev Genet 13, 271-282.

Peacock, H., Kannan, A., Beal, P.A., and Burrows, C.J. (2011). Chemical Modification of siRNA Bases To Probe and Enhance RNA Interference. Journal of Organic Chemistry 76, 7295-7300.

Peterson, S.M., Thompson, J.A., Ufkin, M.L., Sathyanarayana, P., Liaw, L., and Congdon, C.B. (2014). Common features of microRNA target prediction tools. Frontiers in genetics 5, 23-23.

Petri, S., Dueck, A., Lehmann, G., Putz, N., Rudel, S., Kremmer, E., and Meister, G. (2011). Increased siRNA duplex stability correlates with reduced off-target and elevated on-target effects. Rna 17 , 737-749.

Petri, S., and Meister, G. (2013). siRNA design principles and off-target effects. Methods in molecular biology (Clifton, NJ) 986, 59-71.

Qiu, S.B., Lane, T.R., and Yang, C.D. (2007). Efficient search algorithms for RNAi target detection. Journal of Supercomputing 42, 303-319.

Reczko, M., Maragkakis, M., Alexiou, P., Papadopoulos, G.L., and Hatzigeorgiou, A.G. (2011). Accurate microRNA Target Prediction Using Detailed Binding Site Accessibility and Machine Learning on Proteomics Data. Frontiers in genetics 2, 103-103.

Rennie, W., Liu, C.C., Carmack, C.S., Wolenc, A., Kanoria, S., Lu, J., Long, D., and Ding, Y. (2014). STarMir: a web server for prediction of microRNA binding sites. Nucleic Acids Research 42, W114-W118. 
Rhoades, M.W., Reinhart, B.J., Lim, L.P., Burge, C.B., Bartel, B., and Bartel, D.P. (2002). Prediction of plant microRNA targets. Cell 110, 513-520.

Ristevski, B. (2015). Overview of Computational Approaches for Inference of MicroRNA-Mediated and Gene Regulatory Networks. In Advances in Computers, Vol 97, pp. 111-145.

Salomon, W.E., Jolly, S.M., Moore, M.J., Zamore, P.D., and Serebrov, V. (2015). Single-Molecule Imaging Reveals that Argonaute Reshapes the Binding Properties of Its Nucleic Acid Guides. Cell 162, 84-95.

Saxena, S., Jonsson, Z.O., and Dutta, A. (2003). Small RNAs with imperfect match to endogenous mRNA repress translation - Implications for off-target activity of small inhibitory RNA in mammalian cells. Journal of Biological Chemistry 278, 44312-44319.

Scacheri, P.C., Rozenblatt-Rosen, O., Caplen, N.J., Wolfsberg, T.G., Umayam, L., Lee, J.C., Hughes, C.M., Shanmugam, K.S., Bhattacharjee, A., Meyerson, M., et al. (2004). Short interfering RNAs can induce unexpected and divergent changes in the levels of untargeted proteins in mammalian cells. America 101, 1892-1897.

Schirle, N.T., Kinberger, G.A., Murray, H.F., Lima, W.F., Prakash, T.P., and MacRae, I.J. (2016). Structural Analysis of Human Argonaute-2 Bound to a Modified siRNA Guide. J Am Chem Soc 138, 8694-8697.

Schirle, N.T., and MacRae, I.J. (2012). The Crystal Structure of Human Argonaute2. Science 336, 1037-1040.

Schirle, N.T., Sheu-Gruttadauria, J., Chandradoss, S.D., Joo, C., and MacRae, I.J. (2015). Water-mediated recognition of t1-adenosine anchors Argonaute2 to microRNA targets. Elife 4.

Schirle, N.T., Sheu-Gruttadauria, J., and MacRae, I.J. (2014). Structural basis for microRNA targeting. Science 346, 608-613.

Schubert, S., Grunweller, A., Erdmann, V.A., and Kurreck, J. (2005). Local RNA target structure influences siRNA efficacy: systematic analysis of intentionally designed binding regions. Journal of Molecular Biology 348, 883-893.

Schwarz, D.S., Ding, H.L., Kennington, L., Moore, J.T., Schelter, J., Burchard, J., Linsley, P.S., Aronin, N., Xu, Z.S., and Zamore, P.D. (2006). Designing siRNA that distinguish between genes that differ by a single nucleotide. Plos Genetics 2, 1307-1318.

Seok, H., Ham, J., Jang, E.S., and Chi, S.W. (2016a). MicroRNA Target Recognition: Insights from Transcriptome-Wide Non-Canonical Interactions. Molecules and cells.

Seok, H., Jang, E.-S., and Chi, S.W. (2016b). Rationally designed siRNAs without miRNA-like off-target repression. Bmb Reports 49, 135-136.

Sethupathy, P., Corda, B., and Hatzigeorgiou, A.G. (2006). TarBase: A comprehensive database of experimentally supported animal microRNA targets. Rna-a Publication of the Rna Society 12, 192-197.

Shao, C.G., Chen, M., and Meng, Y.J. (2013). A reversed framework for the identification of microRNA-target pairs in plants. Briefings in Bioinformatics 14, 293-301.

Shao, Y., Chan, C.Y., Maliyekkel, A., Lawrence, C.E., Roninson, I.B., and Ding, Y. (2007). Effect of target secondary structure on RNAi efficiency. Rna-a Publication of the Rna Society 13, $1631-1640$.

Shweta, and Khan, J.A. (2014). In silico prediction of cotton (Gossypium hirsutum) encoded microRNAs targets in the genome of Cotton leaf curl Allahabad virus. Bioinformation 10, 251-255. 
Singh, N., Srivastava, S., and Sharma, A. (2016). Identification and analysis of miRNAs and their targets in ginger using bioinformatics approach. Gene 575, 570-576.

Singh, S., Wu, X.Y., Ljosa, V., Bray, M.A., Piccioni, F., Root, D.E., Doench, J.G., Boehm, J.S., and Carpenter, A.E. (2015). Morphological Profiles of RNAi-Induced Gene Knockdown Are Highly Reproducible but Dominated by Seed Effects. Plos One 10, e0131370-e0131370.

Snead, N.M., Escamilla-Powers, J.R., Rossi, J.J., and McCaffrey, A.P. (2013). 5 ‘ Unlocked Nucleic Acid Modification Improves siRNA Targeting. Molecular Therapy-Nucleic Acids 2, e103-e103.

Snove, O., and Holen, T. (2004). Many commonly used siRNAs risk off-target activity. Biochemical and Biophysical Research Communications 319, 256-263.

Snove, O., and Rossi, J.J. (2006). Chemical modifications rescue off-target effects of RNAi. Acs Chemical Biology 1, 274-276.

Song, J.J., Smith, S.K., Hannon, G.J., and Joshua-Tor, L. (2004). Crystal structure of Argonaute and its implications for RISC slicer activity. Science 305, 1434-1437.

Sood, P., Krek, A., Zavolan, M., Macino, G., and Rajewsky, N. (2006). Cell-type-specific signatures of microRNAs on target mRNA expression. America 103, 2746-2751.

Srivastava, P.K., Moturu, T.R., Pandey, P., Baldwin, I.T., and Pandey, S.P. (2014). A comparison of performance of plant miRNA target prediction tools and the characterization of features for genome-wide target prediction. Bmc Genomics 15, 348-348.

Stalder, L., Heusermann, W., Sokol, L., Trojer, D., Wirz, J., Hean, J., Fritzsche, A., Aeschimann, F., Pfanzagl, V., Basselet, P., et al. (2013). The rough endoplasmatic reticulum is a central nucleation site of siRNA-mediated RNA silencing. EMBO J 32, 1115-1127.

Stark, A., Brennecke, J., Russell, R.B., and Cohen, S.M. (2003). Identification of Drosophila MicroRNA targets. Plos Biology 1, 397-409.

Su, X.P., Wang, H.M., Ge, W., Yang, M.J., Hou, J., Chen, T.Y., Li, N., and Cao, X.T. (2015). An In Vivo Method to Identify microRNA Targets Not Predicted by Computation Algorithms: p21 Targeting by miR-92a in Cancer. Cancer Research 75, 2875-2885.

Svoboda, P. (2007). Off-targeting and other non-specific effects of RNAi experiments in mammalian cells. Curr Opin Mol Ther 9, 248-257.

Svoboda, P. (2014). Renaissance of mammalian endogenous RNAi. FEBS Lett 588, 2550-2556.

Svoboda, P. (2015). A toolbox for miRNA analysis. FEBS Lett 589, 1694-1701.

Tafer, H. (2014). Bioinformatics of sirna design. Methods in Molecular Biology 1097, 477-490.

Tafer, H., Ameres, S.L., Obernosterer, G., Gebeshuber, C.A., Schroeder, R., Martinez, J., and Hofacker, I.L. (2008). The impact of target site accessibility on the design of effective siRNAs. Nature Biotechnology 26, 578-583.

Tan, S.M., and Lieberman, J. (2016). Capture and Identification of miRNA Targets by Biotin Pulldown and RNA-seq. In Post-Transcriptional Gene Regulation, 2nd Edition, pp. 211-228.

Tarang, S., and Weston, M.D. (2014). Macros in microRNA target identification A comparative analysis of in silico, in vitro, and in vivo approaches to microRNA target identification. Rna Biology 11, 324-333.

Thomson, D.W., Bracken, C.P., and Goodall, G.J. (2011). Experimental strategies for microRNA target identification. Nucleic Acids Research 39, 6845-6853.

Vatolin, S., Navaratne, K., and Weil, R.J. (2006). A novel method to detect functional microRNA targets. Journal of Molecular Biology 358, 983-996. 
Vencken, S., Hassan, T., McElvaney, N.G., Smith, S.G.J., and Greene, C.M. (2015). miR-CATCH: MicroRNA Capture Affinity Technology. In Rna Interference: Challenges and Therapeutic Opportunities, pp. 365-373.

Vergoulis, T., Vlachos, I.S., Alexiou, P., Georgakilas, G., Maragkakis, M., Reczko, M., Gerangelos, S., Koziris, N., Dalamagas, T., and Hatzigeorgiou, A.G. (2012). TarBase 6.0: capturing the exponential growth of miRNA targets with experimental support. Nucleic Acids Research 40, D222-D229.

Vlachos, I.S., Kostoulas, N., Vergoulis, T., Georgakilas, G., Reczko, M., Maragkakis, M., Paraskevopoulou, M.D., Prionidis, K., Dalamagas, T., and Hatzigeorgiou, A.G. (2012). DIANA miRPath v.2.0: investigating the combinatorial effect of microRNAs in pathways. Nucleic Acids Research 40, W498-W504.

Vosa, U., Esko, T., Kasela, S., and Annilo, T. (2015). Altered Gene Expression Associated with microRNA Binding Site Polymorphisms. Plos One 10, e0141351-e0141351.

Wang, F., Polydore, S., and Axtell, M.J. (2015). More than meets the eye? Factors that affect target selection by plant miRNAs and heterochromatic siRNAs. Current Opinion in Plant Biology 27, 118-124.

Wang, P., Ning, S.W., Wang, Q.H., Li, R.H., Ye, J.R., Zhao, Z.X.L., Li, Y., Huang, T., and Li, X. (2013). mirTarPri: Improved Prioritization of MicroRNA Targets through Incorporation of Functional Genomics Data. Plos One 8, e53685-e53685.

Wang, T., Chen, B.B., Kim, M.S., Xie, Y., and Xiao, G.H. (2014). A Model-Based Approach to Identify Binding Sites in CLIP-Seq Data. Plos One 9, e93248-e93248.

Wang, X.W. (2014). Composition of seed sequence is a major determinant of microRNA targeting patterns. Bioinformatics 30, 1377-1383.

Wang, Y., Li, L., Tang, S., Liu, J., Zhang, H., Zhi, H., Jia, G., and Diao, X. (2016). Combined small RNA and degradome sequencing to identify miRNAs and their targets in response to drought in foxtail millet. BMC Genetics 17, 57-57.

Wang, Y.L., Juranek, S., Li, H.T., Sheng, G., Tuschl, T., and Patel, D.J. (2008). Structure of an argonaute silencing complex with a seed-containing guide DNA and target RNA duplex. Nature 456, 921-U972.

Wang, Y.L., Juranek, S., Li, H.T., Sheng, G., Wardle, G.S., Tuschl, T., and Patel, D.J. (2009). Nucleation, propagation and cleavage of target RNAs in Ago silencing complexes. Nature 461, 754-U753.

Wee, L.M., Flores-Jasso, C.F., Salomon, W.E., and Zamore, P.D. (2012). Argonaute Divides Its RNA Guide into Domains with Distinct Functions and RNA-Binding Properties. Cell 151, 1055-1067.

Wei, K., Yan, F., Xiao, H., Yang, X.X., Xie, G.E., Xiao, Y., Wang, T.T., Xun, Y., Huang, Z.Q., Han, M., et al. (2014). Affinity Purification of Binding miRNAs for Messenger RNA Fused with a Common Tag. International Journal of Molecular Sciences 15, 14753-14765.

Xie, P., Liu, Y., Li, Y.D., Zhang, M.Q., and Wang, X.W. (2014). MIROR: a method for cell-type specific microRNA occupancy rate prediction. Molecular Biosystems 10, 1377-1384.

Xing, L.B., Zhang, D., Li, Y.M., Zhao, C.P., Zhang, S.W., Shen, Y.W., An, N., and Han, M.Y. (2014). Genome-wide identification of vegetative phase transition-associated microRNAs and target predictions using degradome sequencing in Malus hupehensis. Bmc Genomics 15, 1125-1125.

Xu, P., Zhang, Y.J., Kang, L., Roossinck, M.J., and Mysore, K.S. (2006). Computational estimation and experimental verification of off-target silencing during posttranscriptional gene silencing in plants. Plant Physiology 142, 429-440. 
Xu, W.L., San Lucas, A., Wang, Z.X., and Liu, Y. (2014a). Identifying microRNA targets in different gene regions. Bmc Bioinformatics 15, S4-S4.

Xu, W.L., Wang, Z.X., and Liu, Y. (2014b). The Characterization of microRNA-Mediated Gene Regulation as Impacted by Both Target Site Location and Seed Match Type. Plos One 9, e108260-e108260.

Yamada, T., and Morishita, S. (2005). Accelerated off-target search algorithm for siRNA. Bioinformatics 21, 1316-1324.

Yang, J.H., Li, J.H., Shao, P., Zhou, H., Chen, Y.Q., and Qu, L.H. (2011). starBase: a database for exploring microRNA-mRNA interaction maps from Argonaute CLIP-Seq and Degradome-Seq data. Nucleic Acids Research 39, D202-D209.

Yao, C.Y., Sasaki, H.M., Ueda, T., Tomari, Y., and Tadakuma, H. (2015). Single-Molecule Analysis of the Target Cleavage Reaction by the Drosophila RNAi Enzyme Complex. Molecular Cell 59, 125-132.

Yekta, S., Shih, I.H., and Bartel, D.P. (2004). MicroRNA-directed cleavage of HOXB8 mRNA. Science 304, 594-596.

Yi, R., Doehle, B.P., Qin, Y., Macara, I.G., and Cullen, B.R. (2005). Overexpression of exportin 5 enhances RNA interference mediated by short hairpin RNAs and microRNAs. Rna 11, 220-226.

Yilmazel, B., Hu, Y.H., Sigoillot, F., Smith, J.A., Shamu, C.E., Perrimon, N., and Mohr, S.E. (2014). Online GESS: prediction of miRNA-like off-target effects in large-scale RNAi screen data by seed region analysis. Bmc Bioinformatics 15, 192-192.

Yuan, Y.R., Pei, Y., Ma, J.B., Kuryavyi, V., Zhadina, M., Meister, G., Chen, H.Y., Dauter, Z., Tuschl, T., and Patel, D.J. (2005). Crystal structure of A. aeolicus argonaute, a site-specific DNA-guided endoribonuclease, provides insights into RISC-mediated mRNA cleavage. Mol Cell 19, 405-419.

Zander, A., Holzmeister, P., Klose, D., Tinnefeld, P., and Grohmann, D. (2014). Single-molecule FRET supports the two-state model of argonaute action. RNA Biology 11, 45-56.

Zeng, Y., Yi, R., and Cullen, B.R. (2003). MicroRNAs and small interfering RNAs can inhibit mRNA expression by similar mechanisms. America 100, 9779-9784.

Zhong, R., Kim, J., Kim, H.S., Kim, M., Lum, L., Levine, B., Xiao, G.H., White, M.A., and Xie, Y. (2014). Computational detection and suppression of sequence-specific off-target phenotypes from whole genome RNAi screens. Nucleic Acids Research 42, 8214-8222.

Zisoulis, D.G., Lovci, M.T., Wilbert, M.L., Hutt, K.R., Liang, T.Y., Pasquinelli, A.E., and Yeo, G.W. (2010). Comprehensive discovery of endogenous Argonaute binding sites in Caenorhabditis elegans. Nature Structural \& Molecular Biology 17, 173-U176. 


\title{
EXTRACELLULAR SMALL RNAS AND THEIR TRANSFER BETWEEN SPECIES AND KINGDOMS
}

Keywords: dsRNA, miRNA, siRNA, xenomiR, systemic RNAi, environmental RNAi

\section{PETR SVOBODA}

Institute of Molecular Genetics, Academy of Sciences of the Czech Republic, Videnska 1083, 14220 Prague 4, Czech Republic

Correspondence to: Petr Svoboda, Institute of Molecular Genetics ASCR, Videnska 1083, 14220 Prague 4, Czech Republic, tel. \# +420 241063147, e-mail: svobodap@img.cas.cz.

\begin{abstract}
RNA silencing is a common term for mechanisms where small RNAs guide repression of other RNAs (mRNAs, retrotransposon RNAs, viral RNAs) in a sequence-specific manner. RNA silencing exists among eukaryotic organisms where the core mechanisms evolved into many variants with different biological functions. The two most common RNA silencing pathways are microRNA (miRNA) and RNA interference (RNAi). While the miRNA pathway serves for negative regulation of endogenous genes, RNAi pathway usually functions as a form of protection against parasitic sequences although it also targets endogenous genes in some cases. Notably, RNA silencing does not often operate in a cell-autonomous manner. In a number of cases, small RNAs were detected in extracellular environment and were observed to pass through cells and even to be transferred between organisms. This review provides an overview of "mobile" small RNAs, with a particular focus on miRNAs and short interfering RNAs (siRNA) of the RNAi pathways, which exert their effects in different species than they originate from.
\end{abstract}

\section{Introduction}

RNA silencing utilizes small RNAs as sequence-specific guides for repression of cognate RNAs. The two most common small RNA pathways are microRNA (miRNA) and RNA interference (RNAi) pathways (Fig. 1). miRNA and RNAi are typically presented in a cell autonomous manner, i.e. repression remains restricted to cells directly exposed to dsRNA. However, small RNA-mediated repression can propagate across cell boundaries. There are two distinct modes of non-cell autonomous RNAi (Fig. 2, (Whangbo and Hunter, 2008)): (1) systemic RNAi includes processes where a silencing signal spreads from a cell across cellular boundaries into other cells and (2) environmental RNAi, which involves processes where dsRNA is taken up by a cell from the environment. Both modes can be combined in different ways. For example, gene silencing by soaking C. elegans in dsRNA solution is a combination 


\section{MiRNA}

gene repression

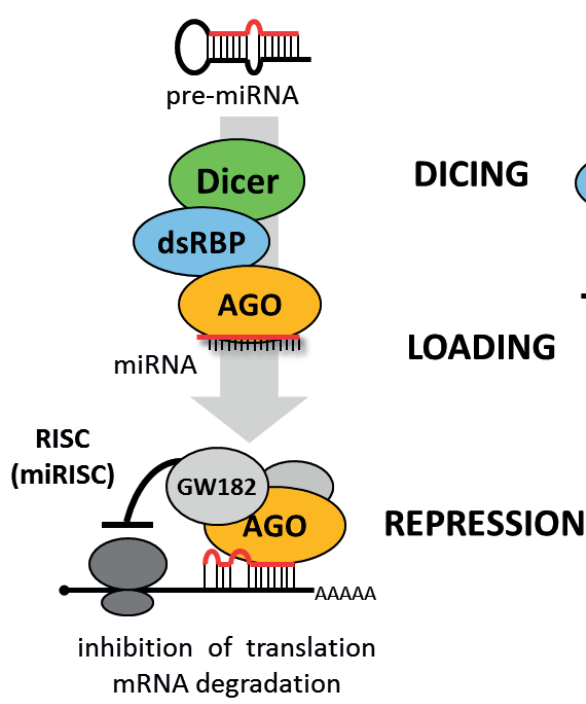

RNAi

viruses, convergent transcription, inverted repeat transcription, $\mathrm{RdRp}$ activity, artificial

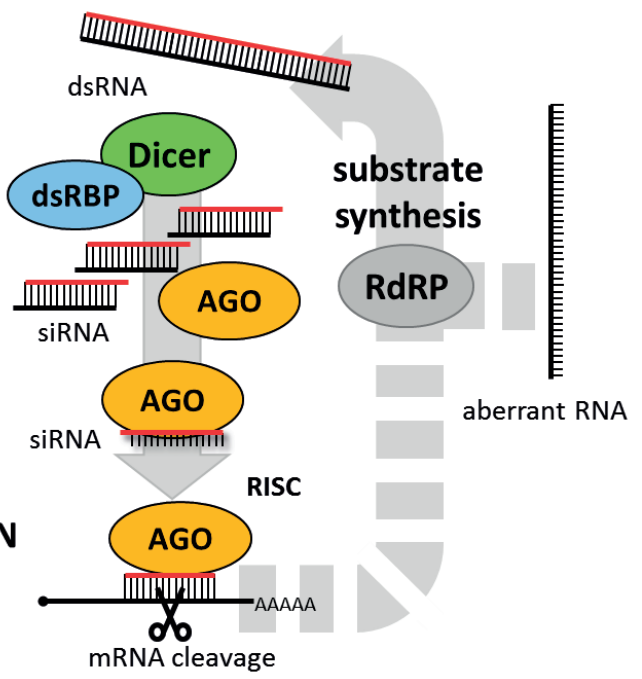

Figure 1 Schematic overview of miRNA and RNAi pathways. miRNA pathway is shown from the moment of miRNA precursor (pre-miRNA) processing by Dicer. miRNAs are genome-encoded and pre-miRNAs are produced from long primary transcripts with local small hairpin structures, which are processed to give rise to pre-miRNA. While both pathways show distinct modes of repression, miRNAs cully complementary to cognate mRNAs, which are common in plants but rare in mammals, can also induce RNAi-like cleavage. At the same time, siRNAs with partial complementarity can induce miRNA-like translational repression in mammalian cells. RdRP component of RNAi is present in some taxons (plants, nematodes) but some species execute RNAi without it (vertebrates, insects).

of environmental and systemic RNAi (Tabara et al., 1998). Non-cell autonomous RNAi has been discovered also in parasitic nematodes (Geldhof et al., 2007), hydra (Chera et al., 2006), planaria (Newmark et al., 2003; Orii et al., 2003), insects (Tomoyasu et al., 2008; Xu and Han, 2008), or plants (Himber et al., 2003). An extreme case would be trans-species (or even trans-kingdom) effect where dsRNA or small RNAs (miRNA or siRNA) produced in one organism (e.g. plant) would induce silencing in another organism (e.g. insect pest).

\section{Horizontal transfer of small RNAs and its possible consequences}

\section{Plant-pathogen transfer \& artificial plant resistance to pathogens}

RNA silencing can spread out of a plant as demonstrated by RNAi-inducing transgenes, which can extend their effects into nematodes (Huang et al., 2006), insects (Baum et al., 


\section{cell autonomous \\ RNA silencing}
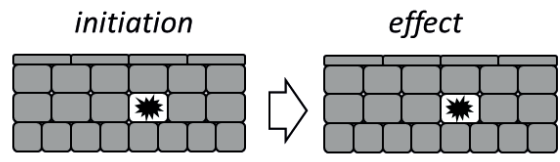

example

mammals
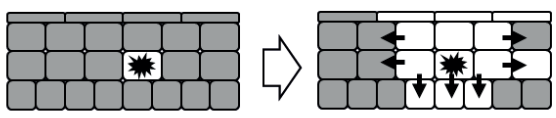

\section{C. elegans}

some Arthropods (Tribolium)

plants

RNA silencing

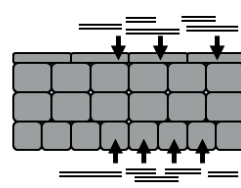

plant tissue

environmental

RNA silencing

trans-species

RNA silencing
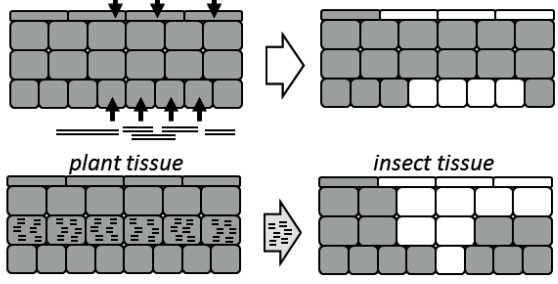

\section{C. elegans}

insects

\section{"RNAi-pesticide"}

Figure 2 Cell autonomous and cell non-autonomous RNAi

RNAi can either act in a cell autonomous manner, i.e. affecting only cells where RNA silencing was initiated. Cell non-autonomous RNA silencing types include systemic, where the RNAi effects propagates across cellular boundaries (e.g. mediated by transport of small RNAs) or environmental where the silencing RNA (e.g. dsRNA substrate) can be absorbed from the environment. Systemic and environmental RNAi can coexist and contribute to trans-species silencing where, for example, small RNAs produced in one species induce silencing in another one.

2007; Mao et al., 2007), parasitic fungi (Nowara et al., 2010) or another plant through a parasitic plant intermediate (Melnyk et al., 2011). Thus, trans-kingdom RNAi combining systemic \& environmental RNAi has biotechnological implications as it allows for producing a genetically engineered plant, which can be resistant to a specific virus or even induce an RNAi effect in a selected recipient (Mansoor et al., 2006). This can be explored, for example, for producing plants expressing dsRNA and selectively targeting RNAi-sensitive pests with an outcome of choice, e.g. repelling the pest, immobilizing it, sterilizing it (Bhatia et al., 2012), or killing it (Baum et al., 2007; Kola et al., 2016). Given the genome sequence diversity and relatively high sequence-specificity of RNAi, RNAi offers an adjustable selectivity for pest control. At the same time, this new technology might raise safety concerns. If the small RNAs can spread, could an RNAi-inducing transgene in a plant affect also non-targeted organisms?

The issue is essentially equivalent to off-targeting effects of RNAi brought to a species level. RNAi-off-targeting is a manifestation of the fact that partial base pairing can also induce RNA silencing. Consequently, some level of off-targeting in RNA silencing is inevitable. However, off-target effects can be suppressed to the point that they are not phenotypically manifested. The key factors reducing RNAi off-targeting effects in trans-kingdom RNAi are (i) the composition of the small RNA population (Fig. 3) and (ii) amount 
of small RNAs loaded on effector complexes, which would exhibit miRNA-like reduced sequence-specificity (i.e. seed match). It can be inferred directly from the molecular mechanism of RNAi and miRNA pathways that a homogeneous small RNA population where all small RNAs have the same sequence (e.g. represented by a single specific siRNA delivery) has a higher off-target risk than a mixture of small RNAs with different sequences (e.g. produced from a long dsRNA).

In transmission of RNA silencing from one species to another, the amount of small RNAs loaded on effector complexes in the recipient species will be influenced by (i) the nature of the transferred silencing molecule (small RNA, long dsRNA), (ii) efficiency of the transfer, and (iii) efficiency of effector complex formation in the recipient species. Thus, species, which can absorb long dsRNA and have systemic RNAi like C. elegans (Tabara et al., 1998) are more prone to exhibit specific silencing effects as well as off-target effects. At the same time, significant off-target effects require specific conditions in terms of stoichiometry between small RNAs and their targets, which are unlikely to be met upon RNAi induction with long dsRNA.

\section{Extracellular miRNAs in mammals and their transfer}

Release of miRNAs from mammalian cells is a well-known phenomenon. Here, I will highlight features of circulating miRNAs, which are important in the context of this review.

Extracellular circulating mammalian miRNAs were reported in 2008 when they were found in serum of lymphoma patients; they were immediately recognized as potential non-invasive biomarkers for cancer diagnostics and treatment (Lawrie et al., 2008). In fact, the bulk of the circulating RNA literature concerns biomarker potential of circulating miRNAs, which is of high clinical relevance but outside the scope of this report. Extracellular miRNAs were identified in a broad range of biological fluids (Baglio et al., 2015; Dismuke et al., 2015; Huang et al., 2013; Izumi et al., 2015; Kropp et al., 2014; Pegtel et al., 2011; Wu et al., 2015). Apart from plasma, circulating miRNAs in milk are also intensely studied (Alsaweed et al., 2016a, b, c; Benmoussa et al., 2016; Izumi et al., 2012; Izumi et al., 2015; Kuruppath et al., 2013; Sun et al., 2013; Xi et al., 2016).

miRNAs were identified in the cargo of exosomes, membranous vesicles 40 to $100 \mathrm{~nm}$ in diameter, which are constitutively released by almost all cell types and are found essentially in every biological fluid (reviewed, for example, in Rak, 2013; Yoon et al., 2014). Exosomal vesicles can carry miRNAs as well as siRNAs (reviewed in Lasser, 2012) - in the latter case, exosomes were adapted as a delivery tool for siRNAs (reviewed in Kumar et al., 2015). However, most individual exosomes in standard preparations do not seem to contain biologically significant numbers of miRNAs (Chevillet et al., 2014) and 95-99\% of circulating miRNA were reported to be associated with AGO proteins and not in extracellular vesicles (Arroyo et al., 2011; Turchinovich et al., 2011).

The molecular mechanism of miRNA release, whether as a cargo in a vesicle or not, is unclear and so is the function of circulating miRNAs, if there is any. Two distinct hypotheses were formulated concerning existence of circulating miRNAs (for a detailed review, see (Turchinovich et al., 2016)): 


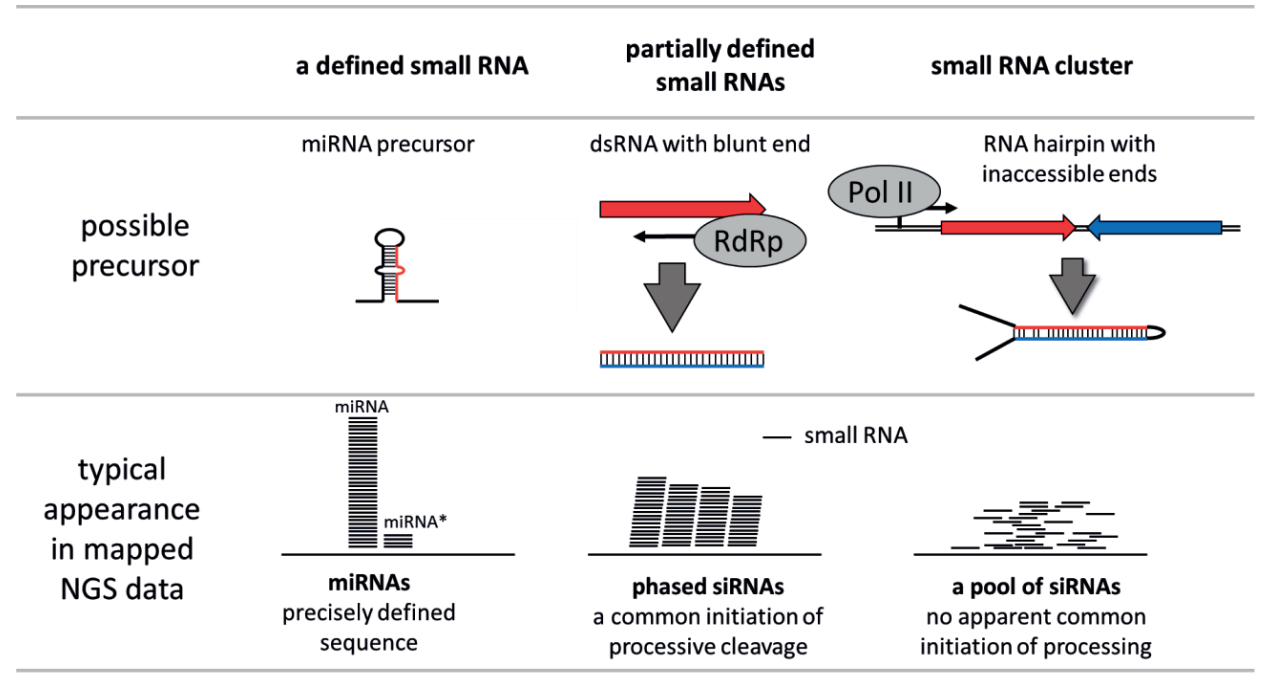

Figure 3 Different structures of small RNA populations from different precursors.

Two distinct types of small RNA populations exist: Highly homogeneous small RNAs, exemplified by miRNAs, which are present in cells in many (e.g. 103-104) identical copies, i.e. having the same sequence, which is "annotateable". Their biological effect on gene expression is partially predictable even if there is only a partial sequence complementarity (typically 6-7 nucleotides at the 5' end). The second type, exemplified by siRNAs derived from various dsRNA, is characterized by existence of populations of small RNAs with many different sequences, which originate from a longer dsRNA. Although such a population can be sequenced to identify individual sequences, their individual annotation is pointless and target prediction is restricted to high complementarity of the substrate dsRNA sequence.

Cell-to-cell communication hypothesis: According to this hypothesis, miRNAs can mediate cell-to-cell signalling. While the hypothesis is supported by circumstantial and correlative evidence (reviewed in Turchinovich et al., 2016), a mechanism for sorting, mobility, and targeting by mobile miRNAs and their function in vivo are unclear. This hypothesis has to face existing kinetic data (Wee et al., 2012) either by providing reliable evidence that sufficient quantities of miRNAs reach recipient cells or by identifying some non-canonical signaling function.

Cellular by-product hypothesis: This hypothesis states that circulating miRNAs are a non-specific noise resulting from cell physiology and turnover of biological material in the organism. As such, circulating miRNAs do not have a specific cell-to-cell communication function and their role in regulating gene expression in other cells is negligible or even non-existent. While this hypothesis might be consistent with the kinetic data (Wee et al., 2012), one cannot exclude a possibility that the "noise" of circulating miRNAs could yield specific biological roles during evolution. In fact, emergence of such an adaptation could be expected.

The scenario where the bulk of circulating miRNAs would represent a consequence of cell physiology while a limited number of circulating miRNAs would represent functionally relevant adaptations might reflect the real situation. In any case, extensive and 
rigorous research is needed to address the problem. This means in particular designing experiments aimed at proving either hypothesis wrong by deciphering underlying molecular mechanisms.

Finally, extracellular mammalian miRNAs can be transmitted from one animal to another or even between species. For example, placental miRNAs were found circulating in maternal plasma (Chim et al., 2008). It has also been reported that food-borne plant miRNAs are able to regulate gene expression in the liver (Zhang et al., 2012a) or miRNAs from consumed milk might contribute to circulating miRNAs in plasma (Baier et al., 2014) or that exosomes secreted by nematode parasites transfer small RNAs to mammalian cells (Buck et al., 2014). The issue of xenogenic miRNAs is addressed in detail next.

\section{Exogenic (nutritional, xenogenic) miRNAs in mammals}

In 2012, the article by Zhang et al. proposed that miRNAs from ingested plants could traverse into the bloodstream and suppress genes in the liver (Zhang et al., 2012a). The report sparked an ongoing debate because of potential implications these data could have.

It should be pointed out that, while the article reported unexpected and surprising results, it was not breaking any conceptual dogma. The idea that information could be transmitted from food in a form of a large organic molecule that would traverse into the human organism has been an integral part of the prion hypothesis, which brought a concept of foodborne infectious particles made only of proteins (prions, reviewed, for example in Peggion et al., 2016)). The prion hypothesis, for which Stanley Prusiner received a Nobel Prize in 1997 , is nowadays a biology textbook knowledge. Furthermore, cross-kingdom regulation by small RNAs was discovered in RNA silencing field already in its early years - long dsRNA expressed in bacteria could induce repression of worm genes with complementary sequences when worms were fed with such bacteria (Timmons and Fire, 1998). Furthermore, it was already well known in 2012 that feeding on a plant carrying an RNAi-inducing transgene can induce RNAi in nematodes, insects, or fungi (Baum et al., 2007; Huang et al., 2006; Mao et al., 2007; Nowara et al., 2010). Thus, the article by Zhang et al. was not bringing a major shift in existing paradigms but was rather extending knowledge by reporting an example of a miRNA activity transferred from plants to mammals through feeding.

\section{The critical issues}

The main controversy of the field of mammalian nutritional miRNAs is that its claims are inconsistent with the molecular mechanism of the mammalian miRNA pathway. Namely, there are three areas, which need to be further investigated and receive solid experimental support:

(i) Mechanism of the transport. It is unknown how plant miRNAs would be transported from the intestinal lumen into liver cells across cell membranes. Mammals have no systemic RNAi. miRNA pathway mobility is also limited. It is known that miRNAs can be released from cells and be detected in blood plasma (discussed above). While circulating miRNAs offer interesting biomarkers, their functional significance is still 
unclear and the mechanism of their sorting and release is also not understood. What is missing is the molecular mechanism transporting miRNAs from the intestinal lumen into the blood and into target cells.

(ii) Effector complex structure. It is unknown how plant miRNAs could regulate mammalian genes in mammalian cells. miRNA-mediated repression requires miRNAs to be loaded on AGO proteins. Thus, a plant miRNA operating in mammalian cells must either remain with its plant AGO protein, which would have to be compatible with mammalian components required for miRNA function, or be unloaded and reloaded onto a mammalian Argonaute protein. Importantly, plant miRNAs are methylated at their 3' end while mammalian miRNAs are not. Data by Zhang et al. suggest that plant miRNAs detected in mammalian serum are indeed methylated (Figure 1 in (Zhang et al., 2012a)). However, mammalian 3' small RNA methylation concerns only piRNAs, a distinct class of small RNAs produced from sense and antisense RNAs. piRNAs are longer (24-30 nt) than siRNAs or miRNAs and are loaded onto a distinct subfamily of Argonaute proteins named PIWI (reviewed in Peters and Meister, 2007). PIWI proteins are expressed in the germline but not in mammalian somatic cells. In contrast, miRNA and siRNA-binding Argonaute proteins (AGO subfamily) do not bind small RNAs methylated at the 3' end. (Tian et al., 2011). Yet, it has been reported that MIR168 is bound by murine AGO2.

(iii) Targeting stoichiometry. The canonical miRNA-mediated post-transcriptional regulation requires appropriate stoichiometry between miRNAs and their targets. However, the evidence that plant miRNAs would reach such physiologically active levels in liver cells is questionable. Plant miRNAs were detected in the serum at femtomolar concentrations $(\sim 3-16 \mathrm{fM})$, which was one order of magnitude less than the serum level of endogenous miRNAs (Zhang et al., 2012a). Total circulating miRNA concentration in human plasma ranges were estimated to be $68-250 \mathrm{fM}$ (Williams et al., 2013). At this concentration, human circulating miRNAs are considered unlikely to regulate gene expression by canonical miRNA activity as miRNAs are thought to require intracellular levels greater than 1000 copies per cell to exert measurable activity (Brown et al., 2007; Hafner et al., 2011). In fact, miR-122, a highly abundant miRNA regulating gene expression in the liver, has been estimated to be expressed at 120,000 molecules/ hepatocyte (one of the highest miRNA amounts/cell) while less expressed miR-33 and miR-16 were estimated to be expressed at 1,200 and 11,000 copies/hepatocyte, respectively (Bissels et al., 2009; Denzler et al., 2014). These numbers contrasts with estimates for xenogenic miRNAs. As 10 femtomoles of miRNAs are approximately $6 \times 10^{9}$ molecules, an extracellular concentration of $10 \mathrm{fM}$ corresponds to 6 molecules per nanoliter of serum. A somatic cell volume is up to several picoliters. Thus, unless there is a yet unknown active transport of circulating miRNAs into hepatocytes, xenogenic miRNAs entering the circulatory system have no capacity to regulate gene expression by canonical miRNA activity in the liver. However, Zhang et al. argue that MIR168a abundance in the liver tissue is equivalent to 853 copies per cell. The ongoing debate on stoichiometry, which is discussed later, has been inconclusive. Furthermore, a critical review of the results is compromised by the fact that some of the critical NGS datasets were not publicly released; hence, their analysis cannot be independently validated. 


\section{Exogenic miRNA transfer literature}

As of December 2016 when the EFSA report was being written (Paces et al., 2017), there were 227 citations of the original article, which I were reviewed. Among the 227, 51 original research articles were identified, which were related to xenogenic and circulating miRNAs. From these, I focus here on those directly relevant to the concept of plant-mammal miRNA transmission. Literature concerning the existence and function of xenogenic miRNAs includes:

- The original study (Zhang et al., 2012a). Using NGS, they identified plant miRNAs in mammalian sera. Unfortunately, the information provided was only partial. The entire data normalization was described as: For normalization, the sequencing frequency of each plant miRNA was normalized to the total amount of mammalian miRNAs. (Zhang et al., 2012a) while the availability of original sequencing data in a public repository was not indicated, thus not allowing for an independent assessment of sequence analysis and quantification. Different quantification strategy could be a potential source of discordance with other published data, which emerged in the literature (Chen et al., 2013).

- The patent: WO 2012135820A2 Edible transgenic plants as oral delivery vehicles for RNA-based therapeutics filled by Eric Lam, Rutgers University. The claim is based on an experiment where feeding rabbits or mice with transgenic tomato producing siRNA against $\mathrm{HCV}$ yielded detectable $\mathrm{HCV}$ siRNAs and tomato RNAs in mammalian blood and tissues. This work was not published in a peer review journal, the patent documentation contains a small RNA northern blot with a faint signal, RT-PCR detection of tomato RNAs in mammalian samples and analysis of RNAi-mediated repression in cultured HepG2 cells.

- A survey of human plasma for microRNA biomarkers in NGS datasets from human plasma revealed circulating RNAs appearing to originate from exogenous species including bacteria, fungi, and other species (Wang et al., 2012). Interestingly, authors could detect RNA fragments from common food where American samples exhibited higher abundance of corn over rice sequences while a control Chinese sample (SRR332232) showed a reversed trend (Wang et al., 2012). Authors also report detection of minuscule amounts of plant miRNAs in human samples 6-16 reads of MIR168 per sample were observed at total sequencing depths of 18-28 million reads; public availability of raw data was not indicated. Notably, authors also indicate low fraction of mapable sequences - they observed less than $1.5 \%$ of processed reads mapping to human miRNAs (Wang et al., 2012). Thus, calculating miRNA RPM values (reads per million) from the mapped reads or miRNA reads could yield two orders of magnitude differences.

- The same group, which published the original xenomiR study, recently published another article proposing that a plant miRNA MIR2911 from honeysuckle (Lonicera japonica), a traditional Chinese medicine herb, can traverse into mouse lungs and inhibit Influenza A (Zhou et al., 2015). In this case, MIR2911 concentration was estimated to reach $1 \mathrm{pM}$ in plasma after feeding mice honeysuckle decoction, which is the range of endogenous circulating miRNAs. MIR2911 was detected in the mouse by NGS (acc. no. GSE55268), qPCR, and in situ hybridization. The estimated amount of MIR2911 in lung cell was 
300-400, suggesting that MIR2911 operates within the physiological range of the canonical miRNA pathway (Zhou et al., 2015).

- Vicki Vance's group reported that oral administration of 2'-O-methylated mammalian miRNAs, which have tumor suppressor properties (miR-34a, miR-143, and miR145) results in reduced intestinal tumor burden in mice (Mlotshwa et al., 2015). This work is also linked to two issues patents and one pending patent application, all entitled "Compositions and Methods for the Modulation of Gene Expression in Plants": US patent application no. 13/282,680, Canadian patent no. 2,276,233 and Australian patent no. AU 2003/254052 B2.

- A third report of xenogenic miRNA regulation suggests that plant miRNA MIR159 is present in human plasma and its level inversely correlates with breast cancer incidence. MIR159 was identified in the serum by NGS (GSE55268). A synthetic mimic of MIR159 was capable of inhibiting cell proliferation in breast cancer cells but not non-cancerous mammary epithelial cells (Chin et al., 2016). Authors claim, "These results demonstrate for the first time that a plant miRNA can inhibit cancer growth in mammals."

- Work by Zhang et al. used miRNA target prediction in Arabidopsis and humans to assemble a hypothetical cross-species regulatory network, which they further analyzed. They proposed that the cross-species regulatory network contains three core module functionally linked to ion transport, metabolic process and stress response (Zhang et al., 2016). However, this study provides a strategy to explore possible cross-species miRNA regulations rather than providing evidence that such regulations exist.

Against these publications stands a number of reports, which question the idea of functional xenogenic miRNAs. For example:

- Analysis of 83 NGS datasets (identified in the report) revealed presence of plant-derived MIRNAs among which dominated MIR 168 from monocot plants. However, the highest observed ratio of plant miRNAs/animal miRNAs is $0.456 \%$, which is 10 times lower than a figure of $\sim 5 \%$ reported by Zhang et al. (Zhang et al., 2012a). These data suggests that the observed plant miRNAs in animal small RNA datasets can originate in the process of sequencing, and that accumulation of plant miRNAs via dietary exposure is not universal in animals (Zhang et al., 2012b).

- Snow et al. analyzed miRNA content in diets of humans, mice, and honeybees and examined transfer of several different miRNAs into the recipient organism: conserved and highly-expressed plant miRNAs (MIR156a, MIR159a and MIR169a) and conserved, abundant, and ubiquitous animal miRNA miR-21. Healthy human subjects routinely eating fruits replete in MIR156a, MIR159a and MIR169a had undetectable levels of plant-derived miRNAs in their plasma (as measured by qPCR). Similarly, miR-21 levels were negligible in plasma or tissues of miR-21/- mice after oral diets containing miR-21. The same was observed for MIR156a, MIR159a and MIR169a in mice and honeybee tissues. This study concludes that: " horizontal delivery of microRNAs via typical dietary ingestion is neither a robust nor a frequent mechanism to maintain steady-state microRNA levels in a variety of model animal organisms, thus defining the biological limits of these molecules in vivo." (Snow et al., 2013)

- Importantly, an attempt to replicate the claims in the original xenogenic miRNA paper failed (Dickinson et al., 2013). This article prompted a comment by authors of the original 


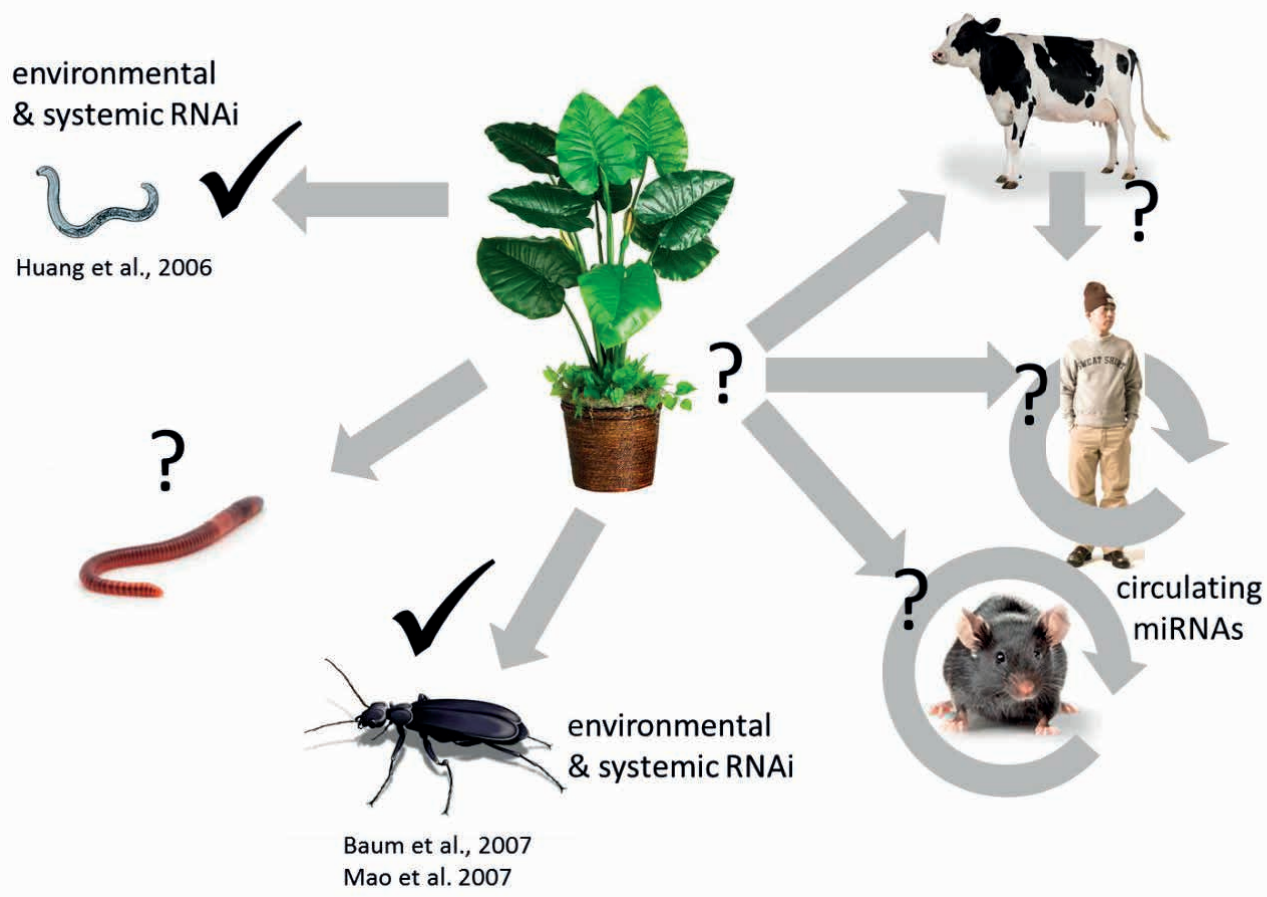

Figure 4 Mobile RNA silencing

The scheme depicts confirmed trans-species/trans-kingdom silencing where silencing of parasitic nematodes or insect pests can be induced by RNA produced in plants. Transmission of miRNAs and induction of silencing into other species remains controversial although existence of circulating miRNAs in humans and mice is well documented.

xenogenic paper pointing out several discrepancies in data and that experimental design used by Dickinson et al. for NGS analysis was suboptimal and could produce artifacts (Chen et al., 2013). Unfortunately, one cannot reanalyze both datasets because only the one from Dickinson et al. was made available (SRP028401).

- A feeding study on Macaca nemestrina failed to provide support for xenogenic miRNAs in blood plasma in response to dietary intake of a plant miRNA-rich food source. Putative xenogenic miRNAs were detected real-time quantitative PCR and droplet digital PCR (Witwer et al., 2013). Detected levels of miRNAs were minimal and/or amplification was non-specific. Detection was largely unreliable for plant miRNAs 156, 160, $166,167,168$ and 172 despite the relative abundance of all but miR172 in a soy- and fruit-substance that was administered to the macaque subjects (Witwer et al., 2013).

- Bioinformatic analysis of NGS data for diet-derived miRNAs in deep-sequencing libraries also did not provide a support for the "xenomiR hypothesis". Authors provide evidence that cross-contamination during library preparation can be a source of exogenous RNAs and that such a cross-contamination could be identified in an NGS study of Amphioxus performed by the group, which published the first xenogenic miRNA article 
in 2012 (Tosar et al., 2014). The original data from the Zhang et al. 2012 article could not be examined for cross-contamination. Tossar et al. conclude that cross-contamination between samples from the same organism can go completely unnoticed, possibly affecting conclusions derived from NGS transcriptomics (Tosar et al., 2014).

Taken together, the field remains split since the original report (Zhang et al., 2012a). The essential questions concerning the existence of the proposed mechanism emerged already in 2012. Further research is necessary to clarify the basis of the aforementioned contradictory observations. At the moment, there is no consensus regarding specific aspects of the xenomiR hypothesis, i.e.:

- the mechanism of transport of a plant miRNAs from food into the blood.

- amounts of plant miRNAs reaching the mammalian circulation system and tissues

- the nature of the ribonucleoprotein complexes, in which they exist in the plasma

- the mechanism of entrance (accumulation?) of xenomiRs into cells

- the nature of the ribonucleoprotein complexes, in which xenomiRs exist in the cell Remarkably, the simplicity of the xenomiR hypothesis where an oral uptake of miRNAs in food results in post-transcriptional repression in tissues contrasts with more than a decade of development of small RNA-based therapeutics, which still struggles with efficiency of uptake, distribution, and targeting. However, the current stage of (incomplete) knowledge precludes strong conclusions regarding the future of the xenomiR hypothesis.

\section{Conclusions}

Mobile RNA silencing exists in plants and some animals (Fig. 4). RNA silencing, and RNAi in particular, operates with unique (though not absolute) selectivity and is being explored in many different ways in biomedicine and biotechnology. In some cases, RNA silencing can spread across cellular boundaries, which can extend into systemic silencing. Systemic silencing is restricted to specific taxons and RNA silencing pathways. While the framework for understanding the molecular basis of systemic silencing in different model systems was established, the knowledge is still fragmented and far from complete. Even less clear is the horizontal transmission of RNA silencing, especially into species, where primarily cell-autonomous silencing is expected.

\section{Acknowledgement}

I would like to thank my colleagues Jan Paces, Miloslav Nic, Tomas Novotny, and Eliska Sovobodova for help with collecting literature for the review. The review content was produced under a contract OC/EFSA/GMO/2015/01-CT 01 with European Food Safety Authority (EFSA); the opinions expressed are those of the contractor only and do not represent EFSA's official position. Publication of the review was funded by LO1220 and LM2015063 by the Ministry of Education, Youth and Sports. 


\section{References}

Alsaweed, M., Lai, C.T., Hartmann, P.E., Geddes, D.T., and Kakulas, F. (2016a). Human Milk Cells and Lipids Conserve Numerous Known and Novel miRNAs, Some of Which Are Differentially Expressed during Lactation. Plos One 11.

Alsaweed, M., Lai, C.T., Hartmann, P.E., Geddes, D.T., and Kakulas, F. (2016b). Human Milk Cells Contain Numerous miRNAs that May Change with Milk Removal and Regulate Multiple Physiological Processes. International Journal of Molecular Sciences 17.

Alsaweed, M., Lai, C.T., Hartmann, P.E., Geddes, D.T., and Kakulas, F. (2016c). Human milk miRNAs primarily originate from the mammary gland resulting in unique miRNA profiles of fractionated milk. Scientific Reports 6 .

Arroyo, J.D., Chevillet, J.R., Kroh, E.M., Ruf, I.K., Pritchard, C.C., Gibson, D.F., Mitchell, P.S., Bennett, C.F., Pogosova-Agadjanyan, E.L., Stirewalt, D.L., et al. (2011). Argonaute2 complexes carry a population of circulating microRNAs independent of vesicles in human plasma. Proc Natl Acad Sci U S A 108, 5003-5008.

Baglio, S.R., Rooijers, K., Koppers-Lalic, D., Verweij, F.J., Pérez Lanzón, M., Zini, N., Naaijkens, B., Perut, F., Niessen, H.W.M., Baldini, N., et al. (2015). Human bone marrow- and adipose-mesenchymal stem cells secrete exosomes enriched in distinctive miRNA and tRNA species. Stem Cell Research and Therapy 6.

Baier, S.R., Nguyen, C., Xie, F., Wood, J.R., and Zempleni, J. (2014). MicroRNAs Are Absorbed in Biologically Meaningful Amounts from Nutritionally Relevant Doses of Cow Milk and Affect Gene Expression in Peripheral Blood Mononuclear Cells, HEK-293 Kidney Cell Cultures, and Mouse Livers. Journal of Nutrition 144, 1495-1500.

Baum, J.A., Bogaert, T., Clinton, W., Heck, G.R., Feldmann, P., Ilagan, O., Johnson, S., Plaetinck, G., Munyikwa, T., Pleau, M., et al. (2007). Control of coleopteran insect pests through RNA interference. Nat Biotechnol 25, 1322-1326.

Benmoussa, A., Lee, C.H.C., Laffont, B., Savard, P., Laugier, J., Boilard, E., Gilbert, C., Fliss, I., and Provost, P. (2016). Commercial Dairy Cow Milk microRNAs Resist Digestion under Simulated Gastrointestinal Tract Conditions. Journal of Nutrition 146, 2206-2215.

Bhatia, V., Bhattacharya, R., Uniyal, P.L., Singh, R., and Niranjan, R.S. (2012). Host Generated siRNAs Attenuate Expression of Serine Protease Gene in Myzus persicae. Plos One 7, e46343-e46343.

Bissels, U., Wild, S., Tomiuk, S., Holste, A., Hafner, M., Tuschl, T., and Bosio, A. (2009). Absolute quantification of microRNAs by using a universal reference. RNA 15, 2375-2384.

Brown, B.D., Gentner, B., Cantore, A., Colleoni, S., Amendola, M., Zingale, A., Baccarini, A., Lazzari, G., Galli, C., and Naldini, L. (2007). Endogenous microRNA can be broadly exploited to regulate transgene expression according to tissue, lineage and differentiation state. Nat Biotechnol 25, 1457-1467.

Buck, A.H., Coakley, G., Simbari, F., McSorley, H.J., Quintana, J.F., Le Bihan, T., Kumar, S., Abreu-Goodger, C., Lear, M., Harcus, Y., et al. (2014). Exosomes secreted by nematode parasites transfer small RNAs to mammalian cells and modulate innate immunity. Nature Communications 5 .

Chen, X., Zen, K., and Zhang, C.-Y. (2013). Lack of detectable oral bioavailability of plant microRNAs after feeding in mice Reply. Nature Biotechnology 31, 967-969. 
Chera, S., de Rosa, R., Miljkovic-Licina, M., Dobretz, K., Ghila, L., Kaloulis, K., and Galliot, B. (2006). Silencing of the hydra serine protease inhibitor Kazall gene mimics the human SPINK1 pancreatic phenotype. J Cell Sci 119, 846-857.

Chevillet, J.R., Kang, Q., Ruf, I.K., Briggs, H.A., Vojtech, L.N., Hughes, S.M., Cheng, H.H., Arroyo, J.D., Meredith, E.K., Gallichotte, E.N., et al. (2014). Quantitative and stoichiometric analysis of the microRNA content of exosomes. Proc Natl Acad Sci U S A 111, 14888-14893.

Chim, S.S., Shing, T.K., Hung, E.C., Leung, T.Y., Lau, T.K., Chiu, R.W., and Lo, Y.M. (2008). Detection and characterization of placental microRNAs in maternal plasma. Clinical chemistry 54 , 482-490.

Chin, A.R., Fong, M.Y., Somlo, G., Wu, J., Swiderski, P., Wu, X., and Wang, S.E. (2016). Cross-kingdom inhibition of breast cancer growth by plant miR159. Cell Research 26, 217-228.

Denzler, R., Agarwal, V., Stefano, J., Bartel, D.P., and Stoffel, M. (2014). Assessing the ceRNA hypothesis with quantitative measurements of miRNA and target abundance. Mol Cell 54, 766-776.

Dickinson, B., Zhang, Y., Petrick, J.S., Heck, G., Ivashuta, S., and Marshall, W.S. (2013). Lack of detectable oral bioavailability of plant microRNAs after feeding in mice. Nature Biotechnology 31, 965-967.

Dismuke, W.M., Challa, P., Navarro, I., Stamer, W.D., and Liu, Y.T. (2015). Human aqueous humor exosomes. Experimental Eye Research 132, 73-77.

Geldhof, P., Visser, A., Clark, D., Saunders, G., Britton, C., Gilleard, J., Berriman, M., and Knox, D. (2007). RNA interference in parasitic helminths: current situation, potential pitfalls and future prospects. Parasitology 134, 609-619.

Hafner, M., Renwick, N., Brown, M., Mihailovic, A., Holoch, D., Lin, C., Pena, J.T., Nusbaum, J.D., Morozov, P., Ludwig, J., et al. (2011). RNA-ligase-dependent biases in miRNA representation in deep-sequenced small RNA cDNA libraries. RNA 17, 1697-1712.

Himber, C., Dunoyer, P., Moissiard, G., Ritzenthaler, C., and Voinnet, O. (2003). Transitivity-dependent and -independent cell-to-cell movement of RNA silencing. The EMBO journal 22, 4523-4533.

Huang, G., Allen, R., Davis, E.L., Baum, T.J., and Hussey, R.S. (2006). Engineering broad root-knot resistance in transgenic plants by RNAi silencing of a conserved and essential root-knot nematode parasitism gene. Proc Natl Acad Sci U S A 103, 14302-14306.

Huang, X.Y., Yuan, T.Z., Tschannen, M., Sun, Z.F., Jacob, H., Du, M.J., Liang, M.H., Dittmar, R.L., Liu, Y., Liang, M.Y., et al. (2013). Characterization of human plasma-derived exosomal RNAs by deep sequencing. BMC Genomics 14, 319-319.

Izumi, H., Kosaka, N., Shimizu, T., Sekine, K., Ochiya, T., and Takase, M. (2012). Bovine milk contains microRNA and messenger RNA that are stable under degradative conditions. Journal of Dairy Science 95, 4831-4841.

Izumi, H., Tsuda, M., Sato, Y., Kosaka, N., Ochiya, T., Iwamoto, H., Namba, K., and Takeda, Y. (2015). Bovine milk exosomes contain microRNA and mRNA and are taken up by human macrophages. Journal of Dairy Science 98, 2920-2933.

Kola, V.S.R., Renuka, P., Padmakumari, A.P., Mangrauthia, S.K., Balachandran, S.M., Babu, V.R., and Madhav, M.S. (2016). Silencing of CYP6 and APN Genes Affects the Growth and Development of Rice Yellow Stem Borer, Scirpophaga incertulas. Frontiers in Physiology 7, 20-20.

Kropp, J., Salih, S.M., and Khatib, H. (2014). Expression of microRNAs in bovine and human pre-implantation embryo culture media. Frontiers in Genetics 5, 91-91. 
Kumar, L., Verma, S., Vaidya, B., and Gupta, V. (2015). Exosomes: Natural Carriers for siRNA Delivery. Current Pharmaceutical Design 21, 4556-4565.

Kuruppath, S., Kumar, A., Modepalli, V.N., Ngo Khanh, P., Gras, S.L., and Lefevre, C. (2013). Buffalo Milk Transcriptomics. Buffalo Bulletin 32, 796-804.

Lasser, C. (2012). Exosomal RNA as biomarkers and the therapeutic potential of exosome vectors. Expert Opinion on Biological Therapy 12, S189-S197.

Lawrie, C.H., Gal, S., Dunlop, H.M., Pushkaran, B., Liggins, A.P., Pulford, K., Banham, A.H., Pezzella, F., Boultwood, J., Wainscoat, J.S., et al. (2008). Detection of elevated levels of tumour-associated microRNAs in serum of patients with diffuse large B-cell lymphoma. British journal of haematology 141, 672-675.

Mansoor, S., Amin, I., Hussain, M., Zafar, Y., and Briddon, R.W. (2006). Engineering novel traits in plants through RNA interference. Trends Plant Sci 11, 559-565.

Mao, Y.B., Cai, W.J., Wang, J.W., Hong, G.J., Tao, X.Y., Wang, L.J., Huang, Y.P., and Chen, X.Y. (2007). Silencing a cotton bollworm P450 monooxygenase gene by plant-mediated RNAi impairs larval tolerance of gossypol. Nat Biotechnol 25, 1307-1313.

Melnyk, C.W., Molnar, A., and Baulcombe, D.C. (2011). Intercellular and systemic movement of RNA silencing signals. The EMBO journal 30, 3553-3563.

Mlotshwa, S., Pruss, G.J., MacArthur, J.L., Endres, M.W., Davis, C., Hofseth, L.J., Pena, M.M., and Vance, V. (2015). A novel chemopreventive strategy based on therapeutic microRNAs produced in plants. Cell Research 25, 521-524.

Newmark, P.A., Reddien, P.W., Cebria, F., and Sanchez Alvarado, A. (2003). Ingestion of bacterially expressed double-stranded RNA inhibits gene expression in planarians. Proc Natl Acad Sci U S A 100 Suppl 1, 11861-11865.

Nowara, D., Gay, A., Lacomme, C., Shaw, J., Ridout, C., Douchkov, D., Hensel, G., Kumlehn, J., and Schweizer, P. (2010). HIGS: host-induced gene silencing in the obligate biotrophic fungal pathogen Blumeria graminis. Plant Cell 22, 3130-3141.

Orii, H., Mochii, M., and Watanabe, K. (2003). A simple "soaking method" for RNA interference in the planarian Dugesia japonica. Dev Genes Evol 213, 138-141.

Paces, J., Nic, M., Novotny, T., and Svoboda, P. (2017). Literature review of baseline information to support the risk assessment of RNAi-based GM plants. EFSA Supporting Publications 14, 315 .

Peggion, C., Bertoli, A., and Sorgato, M.C. (2016). Almost a century of prion protein(s): From pathology to physiology, and back to pathology. Biochem Biophys Res Commun.

Pegtel, D.M., van de Garde, M.D.B., and Middeldorp, J.M. (2011). Viral miRNAs exploiting the endosomal-exosomal pathway for intercellular cross-talk and immune evasion. Biochimica Et Biophysica Acta-Gene Regulatory Mechanisms 1809, 715-721.

Peters, L., and Meister, G. (2007). Argonaute proteins: Mediators of RNA silencing. Molecular Cell 26, 611-623.

Rak, J. (2013). Extracellular vesicles - biomarkers and effectors of the cellular interactome in cancer. Frontiers in Pharmacology 4, 21-21.

Snow, J.W., Hale, A.E., Isaacs, S.K., Baggish, A.L., and Chan, S.Y. (2013). Ineffective delivery of diet-derived microRNAs to recipient animal organisms. Rna Biology 10, 1107-1116.

Sun, Q., Chen, X., Yu, J., Zen, K., Zhang, C.-Y., and Li, L. (2013). Immune modulatory function of abundant immune-related microRNAs in microvesicles from bovine colostrum. Protein \& Cell 4, $197-210$. 
Tabara, H., Grishok, A., and Mello, C.C. (1998). RNAi in C. elegans: soaking in the genome sequence. Science 282, 430-431.

Tian, Y., Simanshu, D.K., Ma, J.B., and Patel, D.J. (2011). Structural basis for piRNA 2'-O-methylated 3'-end recognition by Piwi PAZ (Piwi/Argonaute/Zwille) domains. Proc Natl Acad Sci U S A 108, 903-910.

Timmons, L., and Fire, A. (1998). Specific interference by ingested dsRNA. Nature 395, 854.

Tomoyasu, Y., Miller, S.C., Tomita, S., Schoppmeier, M., Grossmann, D., and Bucher, G. (2008). Exploring systemic RNA interference in insects: a genome-wide survey for RNAi genes in Tribolium. Genome Biol 9, R10.

Tosar, J.P., Rovira, C., Naya, H., and Cayota, A. (2014). Mining of public sequencing databases supports a non-dietary origin for putative foreign miRNAs: underestimated effects of contamination in NGS. Rna-a Publication of the Rna Society 20, 754-757.

Turchinovich, A., Tonevitsky, A.G., and Burwinkel, B. (2016). Extracellular miRNA: A Collision of Two Paradigms. Trends Biochem Sci 41, 883-892.

Turchinovich, A., Weiz, L., Langheinz, A., and Burwinkel, B. (2011). Characterization of extracellular circulating microRNA. Nucleic Acids Research 39, 7223-7233.

Wang, K., Li, H., Yuan, Y., Etheridge, A., Zhou, Y., Huang, D., Wilmes, P., and Galas, D. (2012). The Complex Exogenous RNA Spectra in Human Plasma: An Interface with Human Gut Biota? Plos One 7.

Wee, L.M., Flores-Jasso, C.F., Salomon, W.E., and Zamore, P.D. (2012). Argonaute Divides Its RNA Guide into Domains with Distinct Functions and RNA-Binding Properties. Cell 151, 1055-1067. Whangbo, J.S., and Hunter, C.P. (2008). Environmental RNA interference. Trends Genet 24, 297-305. Williams, Z., Ben-Dov, I.Z., Elias, R., Mihailovic, A., Brown, M., Rosenwaks, Z., and Tuschl, T. (2013). Comprehensive profiling of circulating microRNA via small RNA sequencing of cDNA libraries reveals biomarker potential and limitations. Proc Natl Acad Sci U S A 110, 4255-4260.

Witwer, K.W., McAlexander, M.A., Queen, S.E., and Adams, R.J. (2013). Real-time quantitative PCR and droplet digital PCR for plant miRNAs in mammalian blood provide little evidence for general uptake of dietary miRNAs Limited evidence for general uptake of dietary plant xenomiRs. Rna Biology 10, 1080-1086.

Wu, G.P., Yang, G.H., Zhang, R.X., Xu, G.Y., Zhang, L., Wen, W., Lu, J.B., Liu, J.Y., and Yu, Y. (2015). Altered microRNA Expression Profiles of Extracellular Vesicles in Nasal Mucus From Patients With Allergic Rhinitis. Allergy Asthma \& Immunology Research 7, 449-457.

Xi, Y., Jiang, X., Li, R., Chen, M., Song, W., and Li, X. (2016). The levels of human milk microRNAs and their association with maternal weight characteristics. European Journal of Clinical Nutrition 70, 445-449.

Xu, W., and Han, Z. (2008). Cloning and phylogenetic analysis of sid-1-like genes from aphids. J Insect Sci 8, 1-6.

Yoon, Y.J., Kim, O.Y., and Gho, Y.S. (2014). Extracellular vesicles as emerging intercellular communicasomes. BMB Reports 47, 531-539.

Zhang, H., Li, Y., Liu, Y., Liu, H., Wang, H., Jin, W., Zhang, Y., Zhang, C., and Xu, D. (2016). Role of plant MicroRNA in cross-species regulatory networks of humans. Bmc Systems Biology 10.

Zhang, L., Hou, D.X., Chen, X., Li, D.H., Zhu, L.Y., Zhang, Y.J., Li, J., Bian, Z., Liang, X.Y., Cai, X., et al. (2012a). Exogenous plant MIR168a specifically targets mammalian LDLRAP1: evidence of cross-kingdom regulation by microRNA. Cell Research 22, 107-126. 
Zhang, Y., Wiggins, B.E., Lawrence, C., Petrick, J., Ivashuta, S., and Heck, G. (2012b). Analysis of plant-derived miRNAs in animal small RNA datasets. Bmc Genomics 13.

Zhou, Z., Li, X., Liu, J., Dong, L., Chen, Q., Liu, J., Kong, H., Zhang, Q., Qi, X., Hou, D., et al. (2015). Honeysuckle-encoded atypical microRNA2911 directly targets influenza A viruses. Cell Research 25, 39-49. 